\title{
SCREENING STUDY FOR WASTE BIOMASS TO ETHANOL PRODUCTION FACILITY USING THE AMOCO PROCESS IN NEW YORK STATE
}

Appendices to the Final Report

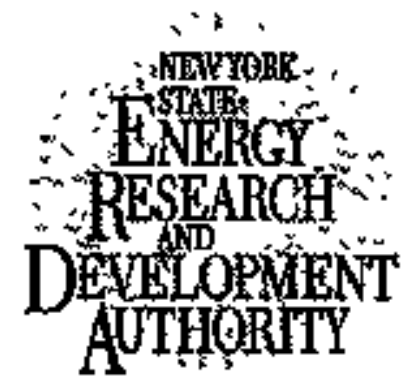


The New York State Energy Research and Development Authority (NYSERDA) is a public benefit corporation created in 1975 by the New Youk \$rate Legislature. A 13-member board governs NYSERDA.

NYSERDA's primary mission is to carry out a brosd program of energy research, development and demonstration projects designed to derelop and apply efficient tectanologies to help ensure that New York bas secure and econoruical future supplies of energy, while protecting environmental values and promoting conomic growth.

NYSERDA derives its basic research revenues from an assessment levied on the intrastate sales of New York State's investor-owned electric and gas utillites. Additional research dollars come from limited corporate funds and a voltemiary annual contribution by the New York Power Authority.

In its research program, NYSERDA stresses consultation and collaboration with other organizations, including utilities, universities, industries, private engineering and scientific research fums, local goventments, and State and Federal agencies. These efforts stretch NYSERDA's limticed research funds and ensure the inyolvement of those who can use the results of the research.

NYSERDA also has responsibility for:

- Managing the 3,300-acre Westem New York Nuclear Service Center at West Valley 35 miles south of Buffalo, the site of a former comtmercial nuclear tuel reprocessing plant and a low-level radioactive waste disposal area. These responsibilities includer

- Participaling in the West Valley Demonstration Profect, a joint Federal/State effort to solidify the high-level radioaclive wastes left over from the reprocessing operation and to clean up the facilities used.

- Maintaining the portion of the site not being used in the Demonstralion Project, including the shu-down low-level radioaclive waste disposal area.

- Issuing tex-exempt bonds to finence facilities for electric and gas utilities and energy projects for private companies.

- Consurcing and operating facilities for disposal of low-level radioaclive wastes produced in New York State, once the disposal method and sile decisions have been made by the Satte Low-Level Radioaclive Waște Siling Commission and approvals have been issured by State regulatory autborites.

- Managing a 365-acre portion of a Superfund clean-up site in Malka, 20 miles north of Albany. Part of the site was once owned by the Federal goveminent. Portions of it have been used by the Federal govenment and its contractors since the 1940 s for activities thal have included rocket engine and fuel testing, weapons testing, and space research.

For more information, contact the Technical Commumicalions Unit, NYSERDA, 2 Empire State Plaza, Sutle 1901, Albany, New York 12223-1253, (518) 465-6251.

State of New York George E. Palaki, Governor
Energy Research and Devetopment Authority F. Whilam Valentino, President 


\section{A NYSERDA Report in Brief}

Report:

Screening Study to Site a Biomass-to-Ethanol Production Facilizy Report 95-13

Project Manoger: Judy Jarnefeld

Contractor:

Stome Webster Engineering Corporation

Background: In 1994, the New York City Department of Samitation (NYCDOS) intended to solicit proposels for

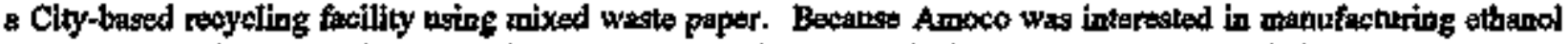
from biomass, it proposed to do a siting sereen in NYC, after which the study was expanded to include upstate. locations as weli.

Objectives: Tho objectivo was to identify and evaluate two sites in New York City and three sites in other New York State urban centers that would be appropriate for construction and long-term operation of a financially attractive and environmentally sound waste biomess-to-ethan of production facility using Amoco's biomass conversion technology (the "Amoco Process"),

R\&D Results: Siting information was gathered and used to identify two sites in New York City: Arthur Kill and Procter \& Gamble, and 23 sites in four upatate urban centers (Buffalo, Rochester, Symacuse, and Albany). Many site and business issues applied generically to all areas of the State. For example, process requirements affecting site selection and coonomic evaluation included minimum feedstock supply and quality; product and waste yislds: emission charateristics; acreage and preferred shape of site; generic equipment costs; and utility, chemical, transportation, and storage requirements. Community issues included zoning, permitting, and onvironmental impacts.

Minimum feedstock supply for an ecopomical plaut was determined to be 300 tons/day (tpd). Wood, paper shitge, and yard waste were considered as supplements. Feedstocks in each of the four upstete regions were ayailable to support a 500-tpd plant, but no larger; New York City could support a plant twice that size. Financial evaluations inciuded cost of site work, utility hoakups, and tipping foes. Capital costs wert similer for all feedstocks studjed. For both upstate and New York City, two approaches were studied: on-site oxidation of the solid by-product to generate energy, or transportation off site. In New Yotk City, totel sapital cost, includieg the process island, balance of plant, site lmprovements, and land costa, were \$139 tmillion for on-site and \$102 million for off-sile oxidution. For a 1000-tpd plant in NYC, economies of scate provide costs of $\$ 223$ million and $\$ 169$ million, respectively. Upstate, the costs are $\$ 113$ to $\$ 117$ million with on-site oxidation, and $\$ 84$ to $\$ 88$ million without oxidation. (Labor cests varied among regions.) For Syracuse, the most economical upstate region, a biomess lipping tee of $\$ 35.59 /$ ton was required to reach an ethanol sale price of $\$ 1.20 /$ gallon. In New York City, \$38/ton is veeded to reach $\$ 1.20 /$ gallon at the 500 -tpd size, while only $\$ 6 /$ ton is needed for the 1000 -tpd plant.

The NYCDOS solicitation has not yet been issued, as markets for scrap paper have changed since 1994. This change in plans makes construction of an ethanol ficility by Amseo in New York City unlikely, as paper supplies would be difficult to secure without NYCDOS aid. Upstate resnlts, without economic development inceptives, also did not appear compelling.

Copies A vallable: A limited number of copies of the full report are available from New York State Energy Research and Development Authority, 2 Empire Stake Plaza, Suite 1901, Albany, New Yort 12223-1253; (\$18) 4656251, sxt. 241 . 


\title{
SCREENING STUDY FOR WASTE BIOMASS TO ETHANOL PRODUCTION FACILITY USING THE AMOCO PROCESS IN NEW YORK STATE
}

\author{
Appendices to the \\ Final Report \\ Prepared for \\ THE NEW YORK STATE \\ ENERGY RESEARCH AND DEVELOPMENT AUTHORITY \\ Project Manager \\ Judy Jarnefeld \\ and
}

NATYONAY RENEWABLE ENERGY LABOHATORY AMOCO NYNEX CHAMPION INTERNATIONAL STONE \& WEBSTER DEVELOPMENT CORPORATION

Prepared by

STONE \& WGBSTER ENGINEERING CORPORATION

One Penn Plaza

250 West 34th Street

New York, NY 10119-2998

Project Manager

Henry Gastwirth

3163-ERTER-ER-94

NYSERDA

Report 95-13-Apps . 
NOTICE

This repon thats joepartal by Stooe \& Webster Engineering Corporation (the "Contractur") in the course of perfonming work contracted for and sponsored by the New York State Energy Resparch and I Jevelopiment Authority and the National Renewable Energy Lathoralury (hereafter the "Sponson"). The upinions expressed it this report do not necessarily reflect those of the Sponsors or the State of New York, and

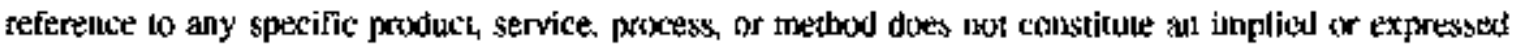
recommendation or endorsement of il. Further, the Spoussors and the Stale of New York matke no warrunties of representations, expressed or Emplied, as to the flaness for particular purpose or merchatulability of any product, anparalus. or service, or the usefuluess, completethess, or accuracy of any processes, inethods, or other infornation costained, described, disclosed, or referred tu in this report. The Simonsers, the Stale of New York, and the Conbraclor make no representation that the use of any product, anpiudus, proxess.

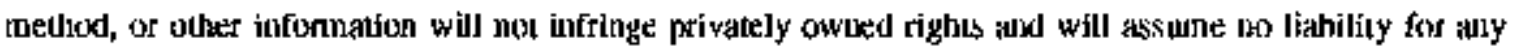

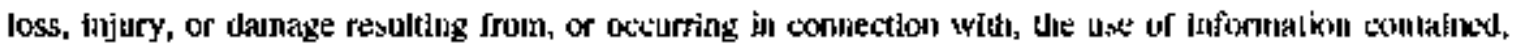
described, disclosed, or refierted to in this report. 


\section{DISCLAIMER}

Portions of this document may be illegible in electronic image products. Images are produced from the best available original document. 


\section{ABSTRACT}

This report evaluates the economic feasibility of locating biomass-to-ethanol waste conversion facilities in New York State. Part I of the sudy evaluates 74 potential sites in New York City and identifies two preferred sites on Staten Island - the Proctor \& Gamble and the Arthur Kill sites - for further consideration. Part II evaluates upstate New York and determines that four regions surrounding the urban centers of Albany, Buffalo, Rochester, and Syracuse provide suitable areas from which to select specific sites for further consideration. A separate appendix provides supplemental material supporting the evaluations.

A conceptual design and economic viability evaluation were developed for a minimum-size facility capable of processing $\mathbf{5 0 0}$ tons per day (tpd) of biomass consisting of wood or paper, or a combination ot the two for upstate regions. The facility would use Amoco's biomass conversion technology and produce 49,000 gallons per day of ethanol and approximately 300 tpd of lignin solid by-product. For New York City, a 1000-tpd processing facility was also evaluated to examine effects of economies of scale.

The reports evaluate the feasibility of building a biomass conversion facility in terms of city and state economic, envirosmental, and community factors. Given the data obtained to date, including changing costs for feedstock and ethanol, the project is marginally attractive. A facility should be as large as possible and located in a New York State Economic Development Zone to take advantage of economic incentives. The facility should have on-site oxidation capabilities, which will make it more financially viable given the high cost of energy.

\section{KEY WORDS}

Amoco biomass to thanol conversion process

Recycling recovered paper

Minimum facility requirements

Site selection 


\section{ACKNOWLEDGEMENTS}

We gratefully acknowledge the substantive contribution of research, technical input, and funding or cost staring made to this study by the following organizations and personnel:

Stone \& Webster Engineering Corporation

Stone \& Webster Development Corporation

The New York State Energy Research and

Development Authority (The Energy Authority)

Nationai Renewable Energy Authority (NREL)

Amoco

Champion International Corporation

NYNEX
Henry Gastwith

Dr. Ernest Zabolotny

Hope Hartley Stidstone

Judy Jarnefeld

Dianne Koepping

Joseph Masin

Richard Piela

John Balaguer

John Halenas 
SCREENING STUDY FOR WASTE BIOMASS TO ETHANOL PRODUCTION FACILITY USING THE AMOCO PROCESS IN NEW YORK STATE

PART IV.

APPENDICES 
June 20, 1994

Henry Gastwirth

Stone \& Webster Engineering Corporation

250 West 34th Street

New York, New York 10119-2998

\section{PROCESS DESIGN CONCERNS AND WOOD FEED MATERIAL BALANCE}

I have competed estimates of the remaining design questions addressed in Task 1.1. The maximum moisture content of the paper that will not downgrade the ethanol product rate is about $60 \%$. There is not preferred shape to the site parcel, but a rectangular plot would probably be easier on which to arrange process piping. There are no particular soil drainage characteristics needed, but the underlying soil should be able to support the weights of process equipment. No process equipment has an unusually high loading requirement.

There are no special utility needs. Some of the tankage in the process is used to grow yeast aerobically, so a power outage for any substantial period of time would force the yeast into anaerobic, non-growth metabolism. This could reduce the ethanol vield. Another concern from a proilonged power outage would be settling of the contents of the fermenter vessels. The agitation supplied in our design is capable of maintaining the vessels in suspension, but may not be sufficient to resuspend a tank that has settled out. Appropriate standby equipment with steam drives may be appropriate in these services if power reliability is suspect at a site. There are no special transportation, water hardness, or storage requirements for ouf process. Some materials stored in the process, like the feed paper and the concentrated ethanol, are flammable, and some chemicals used have other materials handling safety requirements, but appropriate containment technologies have been incorporated into our design.

Also attached are the material balance and utility estimates for a $\mathbf{5 0 0}$ ton per day (dry basis) olant fed with wood chips. Please let me know if there are any other concerns for the timely completion of this siting study.

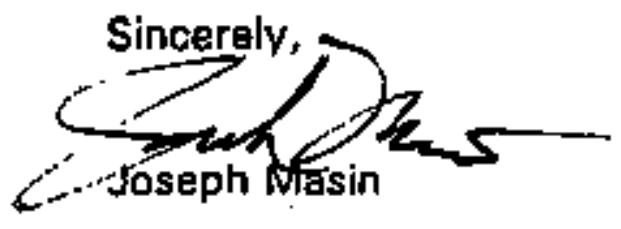

cc: R. H. Walker 


\section{ATTACHMENT 2-2}

June 13, 1994

H. Gastwith

Stone \& Webster Engineering Corporation

250 West 34th Street

New York, New York 101 19-2998

\section{STREAM COMPOSITIONS}

I have estimated stream compositlons for your use in assessing emissions and the heating value of the solids by-product. The following table gives ftow rates in pounds ger hour for the 500 Ton per day plant destgn transmitted previously.

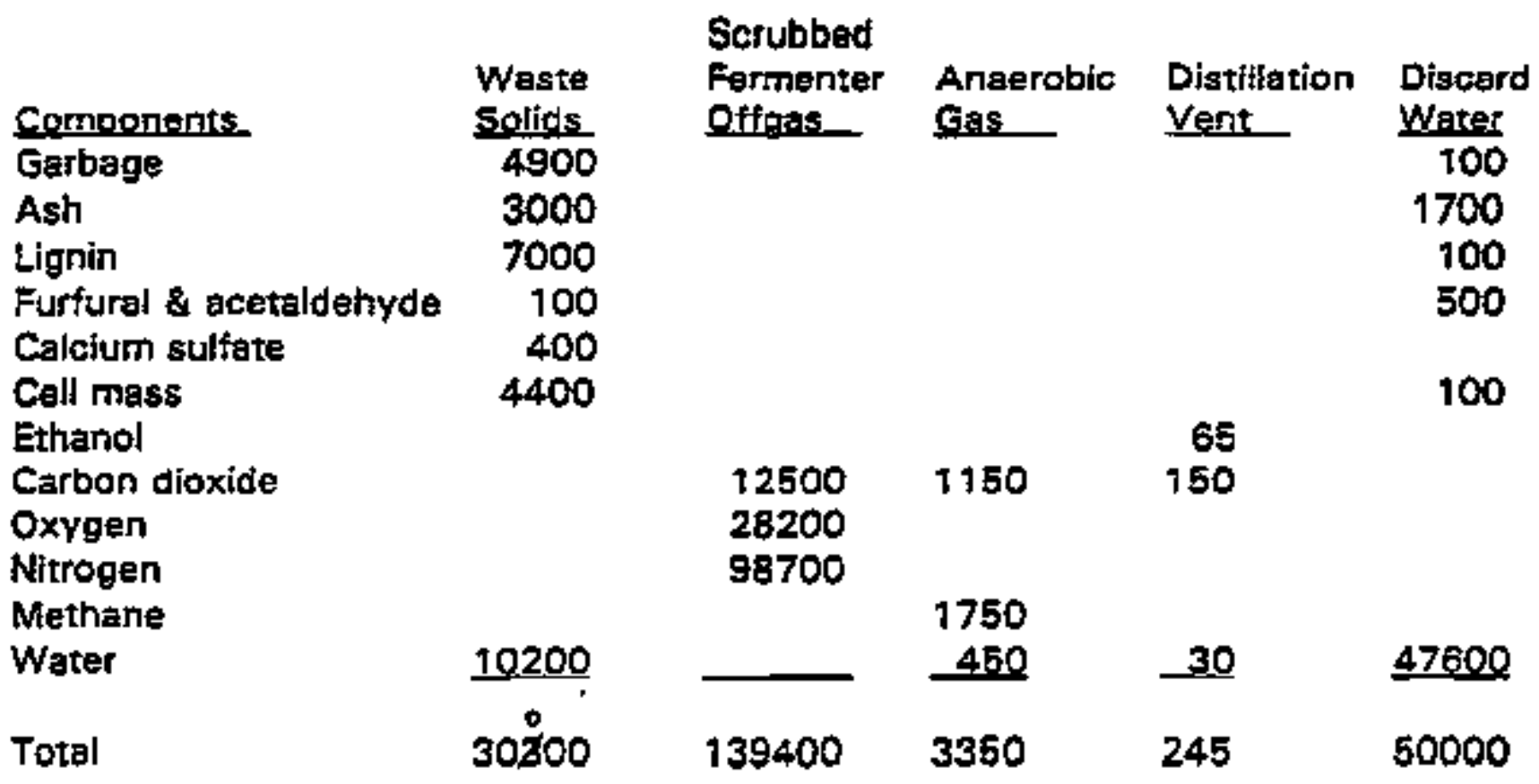

The estimated heating value of the waste solids is 4100 Btu per pound (wet basis) or 6100 Btu per pound (dry basis). Another byproduct is a fusel oil liquid stream that is oniy about 10 pounds per hour. The only other emisslons from the ethanol plant are fugitive emissions from unloading, shredding, and loading operations that can be minimized by proper equipment design and operation.

We have estimated thet a camplete grass roots plent will require about 86 workers: 10 in affice/management, 5 in lab, 6 th engineering, 55 hourly workers, and 10 in shipping/receiving/secutty. I am working on the other items that you requested.

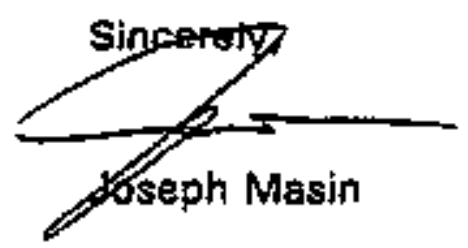

cc: A. H. Walker 
Tyehrolon onergitur

A prosece plant tor converting sellulosic material to ethanol requires beveral steps. We propose the following combination of procese stepe to give the most effective, reliable, and oconomical perforance:

(1) Peot storage, handing and preparation. This step in atrongly dependent on the feedstock chooen. The foed must bo eleaned and delivered to the procese in a form ouitable for further procersing. Rey concerns are to minimize water uge and to remove contaminante thet would bo detrimontal to downstream otepe. Dry ohredding or chipping are propoged for this project.

(2) Fratrantment. Thia step hydrolyged hemicellolose, exposes celluloap to further proceasing, and sterilizeg the foed. Protreatment will aloo phyeicaily diexupt tho otructurs of the feetatock, crateing high surface area for attaek by hydrolyais agenta. Amoco has developed unlgue pretreatment technology that optimizes procese conditlons and maximizeo the ultimate othanol yield.

(3) Hydzolysis. In this operation, eelluloge is convezted to glucore. Acid and sellulace anzyme are teed to acconpliah hydrolyoie. Hydrolysin may be conbined with pretreztment in some procesees, but is combined with fersientation (SSP) in thio proposal.

(4) Fermentation of glucode to ethanel using microorganigms. Normally, about half of the glucose mass in the feed if converted to carbon dioxiele, half to othanol, and emall amounte to mieroorganiem cell maga. This technology is similar to that used to convert corn starch Into ethanol.

Permentation of Elve carbon augars like xylose to ethanol will be considezed whire the feedstoek composition indicated that thin may b* eponomical. Itthough suth dual-fozmantation processes have not bein operated at lexpo ecale.

(5) Ethanol recovery. Digtillation id groposed, uaing technology commonly anployed by the corn-to-ethanol induatry, followed by the uae of molecular sieves to break the watnr-ethanol azeotrope.

(6) Solid-11quid geparation. Depending on the composition of the inlelal fadiatoek, unreacted onlida can be burned for procers heat and power, disposed of as a solid fuel or wate, or dried and oold for animal feed. Te propose ofther burning the solids or eeling them er fuel for othere, depending on site epacifice.

(7) Water eleanup and diepoeal or reuse. Host water is recycled, but any diecharged will mist clean water Act atandards.

(8) Production of fexmentation mleroorganiems and/or cellulase enzyme. These operatlons will not be ineluded at a high eost urban bitey oither mieroorganimm or enzyme can be purchaed.

Anoco has demonetrated all of these stepg both in the laboratory and at lta pilot plant with a veriety of papar and igricultural waste ford otocku. The etepe chosen for this project are ouperior to alternative technologies.

\section{Bariste of Altarnative Rachnologies}

A wide variety of procegaed have been propoesd for biomass-tonethenol conversion over the past 20 yearay none hapt proven economically attractive. Ethanol produced for motor tuel today is made from corr otareh. The process atepa for uging thig feedatoek are verY aimilar to those contanplated for 
celluloge coapertion, but etarch id much eabier to hydrolyze than aelluloge. The enzymo needed to hydrolyze otarch in low in cost and available $E \mathrm{rom}$ enveral cammerefal bources. Hermicelluloge id not hydralyzed, so fermentation of tivencarbon sugars is not a factor with corn processing. The major drawback to the use of corn le that it if a relatively expingive foidatock, in terms of both purchage cogt and the energy inpit reguired to grow 1t. Lowcost celluloos from paper or agricultural wastes has the potential to 1 splach $^{2}$ corn as the fendetodk of choies for ethanol production.

The appeal of being able to manufacture transportation tuels tron newable or waste biemage has regulted in ubetantlal research on procegeeg to make ethanol from raw waterials other than stareh and naturally ocenrying augarg. Although many of the proposed processed can be pade to tunction in the liboratory, mone to dite have met the oconomic, envtronmantal, and oparability stendares neceseary if a process th to bo commerelally $\forall$ iable. We believe thet tha propoead combingtion of otepe In thie project mey mast thoes deaired criteria on a elte epecific basig.

The hurdlee to comnercial viability are generally found in the pretreatment,

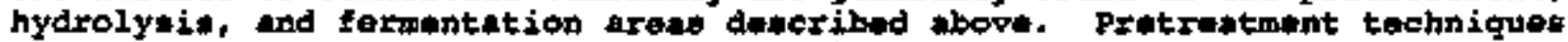
proposed and attempted in the past have included the uoe, fn varioue combinat lone, of aciet, batsos or othor chomicale, heat, and onzyner. Past

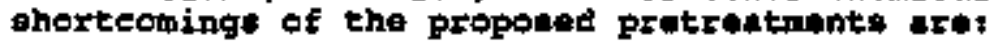

- The ues of acida, bases, or chepteali in other than catalytic cuantities creates either an environmental problen or subatantial recovery expente.

The ure of hent a a pretrentent 1 well known, but publighed resulta are inadequate fxom an economif vietpoint, not reprodufible, or coverad by patonte to which 1ietnes are not available. Fucthermore, the uae of heat, elther alone or in combination with actds, barea, or chemicals mugt bo caxefully controlled to avoid the formetion of undeairable, or evan toxic, byproducte which can heve a eevert inpact on proceag economice and compercisl viability.

- Enzymatic pretreatment hav not evolved jurficiently to provide an economic bagid for pretreatment since the raaction rated axe very blow thus adding to process expenie, and the byproducto are viewod by many ar greenhough gabee and thug not environmentaliy benign.

Hydrolyaie, Lf accompliehed woing strong atids, bagen, or other chemicals, auffere from the same drawbacks anumerated in the preceding diacusaion of protretitmnt techniquel. Enzymess can provide in envfrommentally bentgn approafh to hytrolyeis, but historically their ues has added too much to proceseing costs. Amoco has acches to low cott enzyal technology through its alliance with Iogen corporation.

The simultaneows hydrolysis and fermentation (SSP) of augara in a bingle unit operation has been advocated by hREt, and has advantagea over sequential hydrolysis and fecmentation operations in enzymatic hydrolysis since it helps avoid foedback inhibition caued by the buildup of intermediate sugar dimera, and keepe the avallability of fermentable sugare at low levela allowing favored organiem; to out-compute tramp organism. Since the practice in effect intaing the favored organish on tho "edge of gtarvation", precífion of process contcol id critical whan using this technigua, and nogative ssF axperiences are sometimes reported. Amoco has exporience with high precision profese control through ite operation of patroloum refineries and chtmical menufacture plants.

Fermentation proceneeg are complicated by the variety of wugart releawed when biomand ie hydrolyzed. The $m i x$ of five carbon and eix carbon sugars, as wall ad the specien within mach of these two clabeeg, varieg frow feedstock to peedetock. Anbeo, by vitue of tte analytical laboratory and ito crabA with tTEL, in able to identify all fermentablo ougara in a fapd stock. stnce the economice of a biorabe-to-ethanol procese are etrongly artected by the percent of total eugare converted to valuable producte and byprotucte, many approachas to the convertion of mixed eugar gtreamg to ethanol have been investigatad. Ethanol producing organisme survive bevt and are mot productive wher 
opntuming the sugarg to which theic metabolif processes are nulted. For ecme organieme the proferred gugar alx reduces to a aingle typa of bugar; for ane it ig a mixture of a few types.

Three approuches to the converston of broad epectrum eugar mixee heve been tried: mixed cultures of organiams, genettcaliy modified organibus with the motabolis pathway constructed to bindle vertoty of augarg, and the sonveraion of non-ferinentable eugare to ferwentable compounde.

- Mixed cultures preaent procega control problems, atince an environmont, uarally wbopthmim for elther organiom, nust be erested and abintajned that allows the degizable orgenibm typen to survire, but does not ancourage the growth of undeairable tramp organiams.

Ganattcaliy modifled arganiama (bactarla, but not necessarily yeanten) gonocally have reduced vitality, and thup provide an opportunity for undestrable organimms to take over the fexmentation, conaume the arallable vugar, and destroy project profitability. Wueb of the work on geneticaliy modifled bacteria has been tocuged on enteric organiems Iike B. colf, and Klebsiella, and Frytnia opeeied. sinoe these organiems do not bolong to the General2y Rogurded ad Safe (ORAs) gategory ogtabllahed by the Food and Drug Adininistration and the Erviromental protection

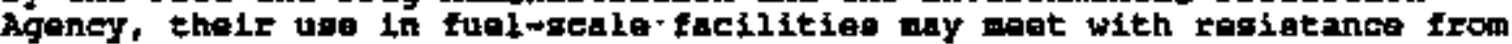
looal populations. Genetically aodifled bacterta 12ke NREL'a modified $z$. mobilitus or yeaseg like those belng doveloped by puxdue thikt qualify for GRAS atatise aro lese likely to have local acedptance problemi.

- The convergion of non-fecmentable sugarg to facmentable compound: (*.9. xyloae to xyluloge) has been deacribad in the literature and accomplitehed 1n the laboratory, but to achiere these typen of traneformat fond aither foquiros an additional formantation operation or. if achloved obalytioally, to generally 1 imited by uncavorable equilibriun constrainte.

A recent independent study of the most dineivend procoseas for blomar conversion to ethanol (Paclftc Intexnational Contoc for High Technology

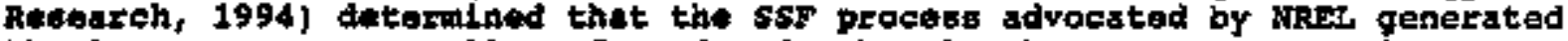
the lowest cogt por gallon of produced athanol; the Ampen procege is a dorivative of tho HRinh propase, noditlod to incorporate Amoco's unitgue skils and capabliztieg. It ghould be autable for the proposed New York oitka. 


\section{ATTACHMENT 2-3a}

Amoco Corporation

Ampoco Rasearch Canter

P.O. E0x 3011

Naparville. Illinois ENobs-7411

Alfernative Feedscok Development Department

fax: $708-420-5169$

August 17, 1994

Henry Gastwirth

Stone \& Webster Engineering Corporation

250 West 34th Street

New York, New York 101 is-2998

\section{PROCESS CAPITAL COST BREAK DOWN}

I have attempted to break down the capital cost estimate that I gave you into the categories listed in the Task 2 Statement of Work. The capital cost estimates that we have for our process were not broken down according to those categories, and the process equipment has also changed somewhat since we had a complete cost estimate made. Thus, this break down should be considered approximate. If we get any capital cost information as a result of the work that Stone \& Webster is doing on the Weyerhaeuser contract, we will confirm and/or update this break down then.

\begin{tabular}{|c|c|c|c|}
\hline Directs & MMs & Indirects & MMs \\
\hline Site Work & 0.0 & Construction Indirects & 3.7 \\
\hline Concrete & 3.7 & Start-Up & 0.0 \\
\hline Structural & 2.5 & Construction Mgt: & 1.4 \\
\hline Equipment & 12.4 & Engineering & 5.2 \\
\hline Piping & 3.1 & , Contingency & 6.2 \\
\hline Electricad & 2.5 & Env. Permitting & 2.3 \\
\hline Buildings & 0.0 & Insurance & 0.4 \\
\hline Instruments & 1.8 & Taxes & 0.6 \\
\hline Insulation & 1.2 & Plant Closure & 0.0 \\
\hline Land & 0.0 & Subtotal & 19.8 \\
\hline Subtotal & $\overline{27.2}$ & Total & 47.0 \\
\hline
\end{tabular}

Please call the if this break down does not appear to match your experience, and we can come up with a reasonable set to meet the requirements of Task 2.

Sincerely,

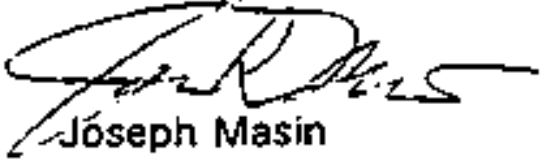

cc: R. H. Walker 
ATTACHUENT 2-4

GASES OF ESTIMATES

BUDGET ESTMMATE FOR BIOMASS TO ETHANOL. FACIUTY

WTH ON-STE OXIDATION

PART 1 - MY CITY PROJECT EVALUATION

1. Process tsand Cost adjusted to New York Cily Cost.

Escalatios - 1994 $\quad 47,000,000$ (19\% $=1,363,000$

Total Cos atter Escalation - \$47,000,000 + 1,363,000 =\$48,363,000

Assume Labor \& onathird of Total Cast - \$48,363,00013 = \$16,121,000

Labor factor between New York Cily \& Housion $=41,15116,45=2.23$

Tolat Labor Cosi Adjustment =\$16,121,000 (10 2.23 =\$35,949,600

Therefore Total adjusmont $=\$ 35,950,000-\$ 16,12 t, 000=\$ 19,829,000$

Total Adiusted N.Y,C. Cost $=\$ 46,360,000+\$ 19,829,000=\$ 68,192,000$

2.

BALANCE OF PLANT

a. Admin./Controllab. Bldg. - assume size; 100'x100'- 10000 st.e \$150is -

Concrete Foundation - 10,000 sl $\odot \$ 12$,outst -

Total

$\$ 1,500,000$

$\$ 120,000$

$\$ 1,620,000$

b. Haint JShop aldg. - assume size: $100 / \times 100^{\prime}=10000$ st $@ \$ 120.00 / s f-$

$\$ 1,200,000$

$\$ 150,000$

Tools and Equlpment -

Concrele Foundation - 10.000 어 @ 512 oonst -

$\$ 120,000$

Total

$\$ 1,470,000$

c. Plant Air Supply:

Two (2) Compressors =

$\$ 130,000$

Als Dryers a

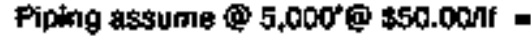

Total

$\$ 20,000$

S250.00010

$\$ 400,000$

d. Aarobtc Watertreatment:

Assume 苗 150,000 gal./day @ \$10.00/gal. =

$\$ 1,500,000$

e. Chilled/Cooling Water Supply.

Includes: Coolng Tower, Fdn,, Plping (250" c

$\$ 700,000$

f. Boller Feedwater (Demin,) allow:

$\$ 400,000$

g. Sold OxidalionfPower \& Distribulion;

$\$ 30,000,000$

h. Coxtreying Sysilo Oxidizer allow:

$\$ 1,500,000$ 
BASES DF ESTMMATES

GUDGET ESTIMATE FOH BIOAASS TO ETHANOL FACILITY

WITH ON-SITE OXIDATION

PAFT 1 - NY CITY PROJECT EVALUATION

\section{i. STIE MAPAOVEMENT}

1. Clearing \& Grubbing :

$\$ 50,000$

2. Sewer and Drainage :

Assume; 5300 w/g Dide \$ \$150.00/7

Manholes/C.B. - 10 ea. $@ \$ 12,000$

Total

$\$ 795,000$

$\$ 120,000$

$\$ 975,000$

3. Accoss Ptoactways \& Parking:

$\$ 800,000$

4. Piling assume whole sile:

Piles @ 22551/pile = 436, e00/2025 2,000 ga.

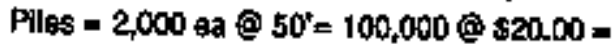

$\$ 2,000,000$

5. Dewalering allowance for excavation:

5500,000

6. Make-up \& Potabla Waler:

Plping \$ 5,300'@ \$100,00Nf

Motering \& Hook-ups

- Total

$\$ 590,000$

$\$ 70.000$

$\$ 600,000$

7. Sower Hook-ups for Bathroon,atc.antow.

$\$ 100,000$

8. Fire Water ; 5900 if @ \$250AI =

Hydrant 8 alsc. items =

Tolal

$\$ 1,325,000$

$\$ 75,000$

$\$ 1,400,000$

9. Sprinkler System whole site plus Standpipe 200,000 st $195.00 / s^{\prime}=$

$\$ 1,000,000$

10. Fencing \& Mics.Sthe work allow:

5270,000

11. Site Lighting:

436800 st $651.20=$

$\$ 510,001$

f. MARK-UPS:

Starl-up Cast $=1.5 \%$

Engineering $=10 \%$

Contingency $=20 \%$ 
ATTACHMENT 2-5

BASES OF ESTMMATES

BUOGET ESTTMATE FOP EIOMASS TO ETHANOL FACUTY WITHOUT OXIOATION PAFT 1 - NY CTTY PADFECT EVALUATION

1. Process Istand Cost adtused to New York City Cost.

Escalation - 1994 \$47,000,000 @ 2,94 - 1,363,000

Total Cos1 after Escalation - \$47,000,000 + 1,363,000 = \$48,365,000

Assume Labor @ onethird of Tolal Cast $-\$ 46,369,000 \%=\$ 16,121,000$

Labor Factor balween Now York Cily \& Houston = 41. 15/t8.45 = 2.23

Todal Labor Cos Adjustment = \$16,121,000 @ 2.23 = \$\$5,949,800

Therefiore Total adjusimant = \$35,950,000 - \$15,121,400 - \$1\$,829,0100

Total Aofusted N.Y.C. Cost $=548,363,000+\$ 19,2929,000-\$ 69,192,000$

2.

GALANCE OF PLANT

a. Adm|n./Controllab. Bldg. - asstme size: $100^{\prime} \times 100^{\prime}=10000$ st.9 \$150ts =

$\$ 1, \$ 00,000$

Concrete Foundation - 10,000 st @ \$12001st -

$\$ 120,000$

Total

$\$ 1,620,000$

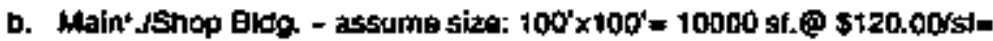
Tools and Equipment =

Conztete Foundation - 10,000 st 1 (12001st .

$\$ 1,200,000$

$\$ 150,000$

$\$ 120.000$

Total

$\$ 1,470,000$

c. Plant Air Supply.

Two (2) Compressors = Air Dryers :

Piping assume $5,000^{\circ}$ @ $\$ 50.00 \pi f=$

$\$ 130,000$

$\$ 20,000$

$\$ 250,000$

Total

$\$ 400,000$

d. Aerobic Watertreatment:

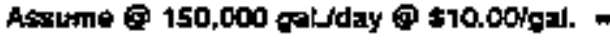

$\$ 1,500,000$

a. ChilledrCooting Waier Suppty:

Inctudes : Cooling Tower, Fon, Pioing 由.

$\$ 700,000$

t. Boiler Feedwater (Dernin,) allow;

$\$ 400,000$

9. Power \& Distribution:

$\$ 5,000,000$

n. Boiler Packaga :

$\$ 1,500,000$

I. Steam Turbing:

$\$ 250,000$ 


\section{BASES $O F$ ESTIMATES}

BUDGET ESTMMATE FOA BIOMASS TO ETHANOL FACALITY WITHOUT OXIDATION PAPT 1 - NY CITY PROJECT EVALUATION

\section{SITE RMPRONEMENT}

1. Cleasing \& Grubbing:

$\$ 50,000$

\section{Sewer and Drainage:}

Assume: 5300 'tig pipe Q $\$ 150.004 \mathrm{nt}$

ManholiserC.B. - 1000 . \$12,000

Total

$\$ 795,000$

$\$ 130.000$

$\$ 915,000$

3. Access Roatways \& Parking:

$\left(4300^{\prime} \times 20^{\circ}+20,000\right)=106,000$ sf $@ \$ 7.50=$

$\$ 800,000$

4. P*ing assumre whole she:

Piles @ 225st/pila - 436, B00/225- 2,000 ea.

Ptyes = 2,000 ga @ 50'- 100,000@ $\$ 20.00$ -

$\$ 2,000,000$

5. Dewalering allewance tor excavation:

5500,000

6. Makb-up \& Poteble Water:

Flphng @ 5,300'@ \$100,00Nt

$\$ \$ \$ 0,000$

batering \& Hook-ups

Tolal

$\$ 70,000$

$\$ 600,000$

7. Sower Hook-ups for Bathroom,elc.alkow:

$\$ 100,000$

8. Fire Water ; 5300 if @ \$2501i =

$\$ 1,325,000$

Hyourant \& Misc, ilems =

$\$ 75,000$

Tolat

$\$ 1,400,000$

9. Sprinkler System whole site plus Slanopipe 200,000 si 955.00 st -

$\$ 1.000 .000$

10. Fencing \& Nics.She work allow:

$\$ 270,000$

11. She Lighting :

436900 st $\$ t .20=$

$\$ 510,001$

k, MAFK-UPS:

Stant-up COSA $=1.5 \%$

Enginepering $=10 \%$

Conkingency $=\mathbf{2 0 \%}$ 


\title{
ATTACHMENT 2-6
}

\section{DEFINITIONS OF SITING CRITERIA}

\author{
June 21, 1994
}

\section{Threshoid Criteria}

To qualify for evaluation, a site must meet all the threshold criteria.

1. Size: The site must be at least ten usable acres.

2. Shape: The shape and slope of the site must allow for the layout of a conventlonal single-story paper-to-ethanol faciinty. (For example, a very narrow site would not qualify, oven if over ten acres.)

3. Natural Besources: If the site has natural resource areas, no port of the plant will be built on regulated wetlands or adjacent areas, or areas with endangered or threatened species, or sensitive habitat as listed by the New York State Department of Exvironmental Conservation. These areas do not count as usable acres when measuring the size of a site.

4. Availability: The site must be vacant, under-used, offered by its owner, or otherwise available. in some cases, sites that are available but already stated for other public uses will be deemed ineligible. For example, the Sotid Waste Management Plan - adopted by the City Council and approved by the New York State Department of Environmental Conservation - calls for development of a waste-to-energy facility at the Brooktyn Navy Yarcl. The site of this facility would be ireligible for consideration. A site will also be ruled out if its acquisition would require state or federal legistative action or in an advanced stage of development by private, city, state, or federat agenciess.

5. Zoning: The site must be located in a manufacturing zone. 


\section{Evaluation Criteria}

1. Industilal waterfont designation: The 1992 New York City Comprehensive Waterfront Plan designates six reaches of the city's waterfiont for future industrial uses and designates other reaches for non-industrial uses. It is deemed less desirable to locate a paper-to-ethanol facility at a site designated for a non-industrial use by the Waterfront Plan.

1 = Waterfiont Ptan designates the area of the site for non-industrial uses.

2 - Waterfont Plan designates the area for future industrial use (or does not encompass the area).

2. Site Zoning: Recognizes the advantages of industrial $(M)$ zoning.

1 - Site zoned $M-1$ for light manufacturing-

2 = Site zoned $M-2$ for medium manufacturing.

3 = Site zoned $M-3$ for heavy manufacturing.

3. Adjacent zoning: Recognizes the advantages of locating near other industrial uses and avoiding residential and pedestrian-oriented commercial zones like retail establishmesnts.

$1=$ Residential zons within 400 feet from site.

2 = Residential zone 401 to 800 feet or retail zone within 400 feet.

3 = No residential within 800 feet; retail zone 401 to 800 feet.

4 = All property within 800 feet zoned industriat (M-1, M-2, or M-3).

4. Natural Resources: As stated in the threshold criteria above, no part of the plant will be bust on regulated wetlands or adjacent areas, or on areas with endangered of threatened species or sensitive habitat listed by the New York State Department of Environmental Conservation. In addition, it is preferable to locate the plant away from these environmentally sensitive areas.

1 = Faciity would be less than 150 feet from such an area and within the jurisdiction of NYSDEC.

2 = Facility would be more than 150 feet from such an area or otherwise inland of the NYSDEC lurisdictional area. 
5. Residential densfty: The number of people living within a haif mile of the site, exctuding the population beyond any physical barrier. A physical barrier is a barrier such as a bocty of water, a major highway, or change in gracte that would block foot traffic that could be affected by the facility and that would terid to form a boundary beyond which impacts such as possible unsightliness, traffic or noise would probably not affect residents.

$1=$ More than 20000 residents.

$2=15001$ to 200000 residents.

$3=10001$ to 15000 residents.

$4=5000$ to 10000 residents

$\mathbf{5}=$ Fewer than $\mathbf{5 0 0 0}$ residents

6. Sensitive receptors nearby: The number of parks, hospitals, and schools, and sirnitar uses within a half mile of the site and within physical barriers to potential waste-to-energy impacts.

1 - More than 8 sensitive receptors within a half mile.

$2=5$ to $\mathbf{B}$ sensitive receptors within a halt mile.

$3=1$ to 4 sensitive receptors within a haff mile.

4 = No sensitive receptors within a half mile.

7. Rail access alfords another way of moving recyclables to customers and an opportunity to reduce the possible environmental impacts of trucking.

$1=$ No rail lines or spurs on or acjacent to the site.

2 = Inactive rail line or spur on or adjacent to the site.

$3=$ Active rail line or spur on or adjacent to thie site.

4 = Float bridge, pier, or bulkhead and active rail tine or spur on or adjacent to the site.

8. Barge access also gives an alternative means of taking recyclables to market and an opportunity to reduce the possible impacts of trucks.

1 F Not a waterfont site or a watertiont site with neither bulkhead nor pier.

2 = Waterfront site with either bulkhead or pier. 
9. Proximity to a through truck route: It is more desirabie for incoming or outgoing trucks to be on or near a through truck route than to have to travel through non-designated areas.

$1=$ Over 2000 feat to a through truck route access ramp.

2 = Site is 1001 to 2000 feet away from a through truck route access ramp.

$3=$ Site is 0 to 1000 feet away from a through truck route access ramp.

10. Site Expansion: Extra area beyond the minimum ten acres would allow for extra parking, storage and additional equipment to be added to the process in the future. Extra area would also allow more flexibility in operations. Sites are scored on the number of usable acres as follows.

1 E 10 acres or less.

$2=10.1$ to 20 acres.

$3=20.1$ to 30 acres.

$4=$ More than 30 acres.

11. Development time: Development time is a factor in costs as much as the price of land acquisition. 'The longer it takes to acquire a site or prepare it for construction, the more costly and less desirable the site. Impediments include the following:

- Presence of occupants (who may need relocation).

- Waterfront-related permits for development such as dredging and iulling-

- Unusually time-consuming land use approvals (special permits, rezoning. demapping).

- Acquisition from the federal government.

- Substantial or complex environmental cteanup.

- Multi-story đemolition.

Impediments apparent at the time of evaluation will be scored as follows:

1 = Fout or more of these impediments apply to the site.

2 = Any three apply.

3 = Any two apply.

4 = Any one applies. 
12. Capital cost of land acquisition: Costs vary depending upon whether the site is privately or publicly owned. Private sites are sold at market rate values, while public ones potentially can be well below market rate.

1 = Market rate or above

2 - Below market rate

13. Eliobility for economic incentives proprams: New businesses locating on designated sites in the city can become eligible for reductions in the costs of their utiflites.

1 = Site not elligible for economic incentives.

2 = Slte eligible for economic incentives.

siling \brooklyn \crit8.b93 


\section{ATTACHMENT 2-7}

\section{TECHNICAL PAPER}


wat

IMPLEMENTATION

OF A HYBRID

RANKING MODEL:

A CASE STUDY IN

SELECTING A WASTE

MANAGEMENT

PLANT WITH

MULTIPLE SITES

by

F. Keyler

R. Wrueke

P. Grulavary

Presented of

ECO WORL' 92 cONFERENC:

Woshington, DC

June 14-17, 1992

TP $92-49$

\section{Stone \&Webster}


The purpose of the study was to select a waste managenent plan that inchuded multiple sites, tectinclogies, and capacities.

In order to reduce a large inventory of sites, potential sites were screenad and ranked relying on envitonmental and engineering criteria. Final site selection was pertormed by framing over one hundred citywide sludge manegement plans consisting of site/technology/capacity components. These plans were fanked using a forced decision analysis approach, the Paired Comparison Technique (PCT).

The hybrid variation of the PCT ranking model, where siting and technology/capacity factors were integrated, provided a tool for selecting the best plan. The ranking process demonstrated that plans with fewer sites and technologies having larger capacities ranked higher.

\section{Introduction}

Since 1939, New York City has been disposing its sludgem the byproduct of the wastewater treatment process-in the ocean. In 1988, Congress passed the Ocean Dumping Ban Act (ODBA), which required all municipalities in the United States to cease ocean dumping and develop land-based solutions to shudge disposal.

Consequently, the city entered into a Consent Decree Enforcement Agreement with the U.S. Environmental Protection Agency (EPA) and the New York State Department of Environmental Conservation (DEC) providing a timetable for transference of sludge managernent from the ocean to the land. The consent decree timetable was linked to a strategic three-tiered plan, called the New York City Sludge Management Plan (SMP), which consists of an Immediate Range Plen, an Intermediate Range Plan, and a Long Range Plan.

The immediate Range Plan pertains to the construction of dewatering facilities at eight of the city s fourteen wastewater treatment plants. All of the dewatering plants are required to be in operation by June 1992. Dewatering is considered a prerequisite to land-based sludge management because the water content of the sludge is reduced to enable marageable handing and transportation of the resulting "studge cake".

Both the Long Range and lntermediate Rarge Plans relate to the development of land. based sludge management facilities for processing and/or disposal of the dewatered sludge. The developrnent and implementation of a long term reliable city-operated plan is anticipated to take several years. Therefore, in the Intermediate Plan, private contractors will manage the sludge for an interim period, until the Long Range Plan could be fully phased in and implemented in 1998.

This paper focuses on the Long Range Plan, with emphasis on the selection process for a preferreo program consisting of a deined mixture of sludge management sites, technologies, and capacities. 


\section{Overview of the Plan Selection Process}

Intilally, the Long Aange Plan evaluation process did not indicate specific commituments to sludge marragement technologies, the size or number of facilities, or their location. Planning guidelines evolved from several detailed independent studies covering technologies, siting, transportation, sludge quality and quartuty, regulations, and sludge markets. These studies resulted in the formulation of two important planning philosophies. First, that the preferred plan would beneficially reuse (recycle) sludgs; and second, that the management and marketing of the city's sludge would occur within the chy.

Having defined the guxidelines for selecting a plan, the building blocks of the city's sludige management plan, the sites, the technologies, and the capacities had to be integrated into alternatives. Based on market precictions of potentiad users within a 50-mile radius of the city, three beneficial technologies were selected; composting, thermal drying, and chenical stabilization. A detailed evaluation of different technology types within these three beneficial categories was carried out as part of a separate task study. Another study, which looked at the amount of projected studge production in the city in the years 2000 and 2020 , indicated that the city will produce approximately 450 and 500 dry tors per day (dtd) on an average annual basis, respectively. It was agreed that alternative plans would be framed around the 2020 projections with a progran capacity equal to approxirnately $200 \%$ of 2020 average production, i.e., 1,000 dtd.

In a third study, a pool of candidate sites was established after an exhaustive three. phased site selection process. The first phase began with a broad citywice mapping of vacant industrial land. ideally, the sludge processing plants would be located at the source of the sludge, the eight dewatering plants. However, the vacant land remaining at all but three of the dewatering plants was inadequate. Therefore, the majortity of processing capacity would have to be sited away from dewatering.

A three-acre minitrum siting requirement was established, based on the area requirement for processing the dewatered sludge from the dewatering plant with the lowest capacity, using thermal drying which consumes the least amourt of land. All industrially zoned vacant properties were screened from city agency property lists. An inventory of 73 sites resulted from this ettort.

After advertising for properties in the New York Times and meeting with city agencies, the site inventory was consolidated to 56 sites. The 56 sites were subsequently reduced to 36 sttes using three criteria: land use, transportation, and wetlands. In addition, a fow sites nominated by city agencies and elected officials were included.

In the second phase of the site selection process, in conjunctlon with a windshield survey, a more detailed list of criteria was used to screen the 36 sites, reducing the inventory to 26 sites. 
In the third phase of the site selection process, a detailed environmertal assessment was performed on all 26 sites and conceptual facility layouts prepared. A list of the issues addressed in the envitonmental assessment is shown in Attachment 1. These are categorized into stx major areas of concern:

- Transpoitation

- Reliability, Flexibility, and Operability

- Health and Environment

- Local Regulatory Concerns

- Public Acceptability

- Constructibitity

Also listed are issues used in the screening of sites in the first two phases of the site selection process. The list of issues demonstrates thet as the itventory of sites was reduced, the number of criteris used in screening increased. The amount of information used to define the criteria atso increased.

Following the detalied environmental assessment, 19 sites were selected for a more detailed anałysis that integrated site constraints with sludge management tectinologies.

\section{Framing of Plans}

The process of integrating sites with technologies and capacities to form long range plan options was described as "framing alternatives". The objective of this process was to develop a plan that would adiquately handle the clty's projected sludge capacity in the year 2020, as well as provide product fiexibility and some measure of reoundancy in case of plant fallures. Therefore, a plan would consist of some combination of sites, techrologies, and capacities in order to provide sufficient siudge processing capability.

Both centralized and decentralized plans were framed with site/technology/capacity combinations, called components (e.g., a 27 acre site with a composter at a capacity of $95 \mathrm{dry}$ tons per day). Centralization recuced the number of components in a framed plan aitemative, ignoring the five borough boundaries, and utilizing larger sites and capacities with muttiple technologies. In contrast, decentralized plans were borough-based, because components were framed around borough production. Thesa plans refiected a distribution of facilities among the boroughs in terms of equity and fair share. For both the centralized and decentralized plans, single and multiple technologies were framed at individual sites. 


\section{Long Range Plan Resource Issues Used to Evaluate Sites/Technologies/Capacities}

\section{PHASE 1 PHASE 2 PHASE 3}

\section{TRAMSPORTATION}

- Rali Access.

- Barge Access

$x$

- Truck Access.

$x$

- Materials Movement

$\mathbf{x}$

$\begin{array}{ll}x & x \\ x & x \\ x & x\end{array}$

2. RELIABILITY, FLEXIBILITY AND OPERAEILTYY CONCERNS

- Existing Infrastructure.

- Abidry to Expand

- Storage Capacity

- Ability to Divert

3. HEALTH AND ENVIRONMENTAL CONCERINS

- Distance to Wettands.............................. X

- Vegetation Cover

- Vegetation Maturity

- Slope to Sensitive Receptors...................

- Endangered Species ................................

- Significant Habitats.

4. LOCAL REGULATORY CONCERNS

- Zoning.

- Ownership

- Fair Share

- Competing Usas.

5. PUBLIC ACCEPTABIUTY CONCERNS

- Adjacent Property Use.

- Adventitious Recreatio

- Buffer Features.

- Distanceto Parks.

- Distance to Hospitals.

- Residential Magnitude

- Demographics.

- Distanceto Schools

\section{$x$}

$$
\text { COistance Scho }
$$

6. CONSTRUCTIBILITY

- Presance of Drums.

- Stained Soils.

- Soil Organic Content

- Slope to Surface Water.

$\begin{array}{lll}\mathbf{X} & \mathbf{x} & \mathbf{x} \\ \mathbf{x} & \mathbf{x} & \begin{array}{l}\mathbf{x} \\ \mathbf{x}\end{array} \\ \mathbf{x} & \mathbf{x} & \mathbf{x}\end{array}$

$\begin{array}{ccc}\mathbf{x} & \mathbf{x} & \mathbf{x} \\ \mathbf{x} & \mathbf{x} & \mathbf{x} \\ \mathbf{x} & \mathbf{x} \\ \mathbf{x} & \mathbf{x} & \mathbf{x} \\ \mathbf{x} & \mathbf{x} & \mathbf{x} \\ & \mathbf{x} & \mathbf{x} \\ \mathbf{x} & \mathbf{x} & \mathbf{x}\end{array}$

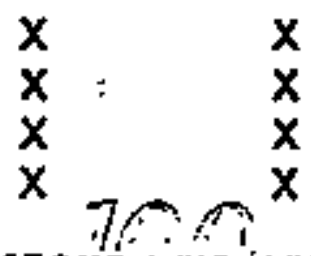


Each of the 19 sites used in the framing of aternatives was analyzed into a finite number of technology layouts and capacities (components). By evaluating many combinations and permutations, almost 130 plans were tramed. Approxirnately 100 of the framed attematives were centralized, and they included most component combinations. There were fewer decentralized plans framed because borough-wide plans were framed first, ranked, and only the highest ranking ones combined into citywide plans; otherwise framing alt the borough-wide plans would have resulted in hundreds of combinations.

\section{The Palred Compactson Technique (PCT) Ranking Model}

Once a range of alternative plans was defined, a system for objectively ranking the plans was devised so that the most desirable plans could undergo more detailed evaluation. The Patred Comparison Technique (PC) was the methodology selected to rank the plans.

The PCT wes developed by Dean and Nishry' in the mid 19605 as a model for prioritizing engineering projects from a range of alternatives. Subsequently, the approach has been used in environmental studies, and is particularty useftal for site selection'. The PCT (or some version of ti) has also been used by the US Army Corps of Engineers and the US Department of interior in evaluating alternatives for solid weste systems ${ }^{3}$, proposed highway alternattves", and water resource assessments and ptanning. Webster has applied the methodology to prioritize the upgrading, rehabilitation and expansion of Long Istand Rail Road parking facilities".

The PCT breaks down the decisiori-inaking process so that decisions or preferences are assigned to a number of selected attributes or criteria affecting an alternative. Once alternatives are established, a list of important decision-making criteria must be delined, and relative weights of importance assigned to them. The PCT becomes usehu in that orly two criteria are compared against each other at any one time, and a simple positive or negattive response is assigned. The term "forced decision analysis" has been coined to describe this procedure, because a dectsion maker is literally forced to make a decision between two alternattves. An example of the PCT and how scores are assigned to generic factors, and then how these scores are sumrned and the tactors weighted is illustrated in Attachment 2.

The first step in using the PCT make paired comparisons between each of the criteria of factors defined to assess the project alternatives. In Attachment 2, each factor was compared to every other factor, one factor at a time, hence the term paired comparison technique. Each time a comparison is made, a score of 0 (less desirable), I (more desirable), or 0.5 (no distinction) is assignad. A neutral factor is included for comparison. This factor serves as a dummy variable, so that no factor's sum of paired comparison scores is 0 . Even if a factor scored a 0 against all other factors in a category, that factor would score a 1 against the neutral factor. 


\section{Attachmont 2}

The Palred Comparison Technique

\section{Assigning Weights to Project Factors}

\begin{tabular}{|c|c|c|c|c|}
\hline \multirow[b]{2}{*}{ Factor $\left(F^{1}\right)$} & A1 & A2 & A3 & A4 \\
\hline & & & & nos \\
\hline A2 & & & & Hes \\
\hline A3 & & & & che \\
\hline A4 & & 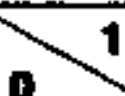 & & 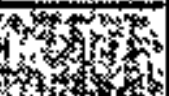 \\
\hline Neutral Fector & 01 & 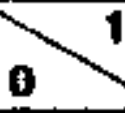 & & 01 \\
\hline $\operatorname{Sum}\left(s^{4}\right)$ & 2.0 & 3.5 & 3.5 & 1.0 \\
\hline Relattve Welght (Br) & 0.2 & 0.35 & 0.35 & 0.1 \\
\hline Percent & $20 \%$ & $35 \%$ & $35 \%$ & $10 \%$ \\
\hline
\end{tabular}

\begin{tabular}{|c|}
\hline TOTALS \\
\hline $10.0\left(\mathrm{~T}^{2}\right)$ \\
\hline $1.0^{3}$ \\
\hline $100 \%$ \\
\hline
\end{tabular}
1). $\Sigma F=5$
2). $T=\frac{\Sigma F(\Sigma F-1)}{2}=\frac{5 \times 4}{2}=10$
3). Musa sum to unily
4). Sum of scores dlvided from row and cohumn scores 
In the second step, all the scores for each factor are summed along the columns and rows. The individual summed scores for each factor are also totalled, and the relative weight calculated by dividing the factor scores by the totel of the individual factor scores. Thls total has been designated $T$ in Attachrnent 2. The sum of all the relative weights for each factor being considered should equal unity, and can be expressed as 100 percent. The total of all summed factor scores, result in the following formuta: $\mathbf{L F}$ (2F.1)/2, where $\Sigma F$ is equal to the number of factors considiared, including the neutral factor. Factors that are weighted higher have a greater influence on the final decision. The establishment of the relative weights for the factors is usually undertaken by indlviduals familiar with the project and the development of information about the same factors used to compare the project alternatives.

In the third step of the PCT the relative weights for a range of factors can be used to compare alternatives by applying the factors to data related to those factors. For example, when comparing atternatives an ecological factor addressing proximity to wetlands may be established, and be defined on a three point scale (e.g., within 100, 200, or 300 foet of a wetland). By obtaining the information about proxirnity to wetlands for all the alternatives being considered, values can be assigned to the wettand factor for each alternative. A weighted score can be derived by multiplying the wetland factor value for each alternative with a reletive weight established as described in the second step above. The weighted scores for all the factors would then be summed, and the alternative with the highest score would effectively be the most desirable. Alternatives with high data values for factors assigned high weights will tend to score well.

Gy methodically working through the three step PCT process, the technique provides for a method of diminishing subjectivity in selecting and ranking atternatives. However, in making the paired comparisons of factors, care should be taken in selecting a group of "experts" to make the comparisons, so as not to bias the weights. Consideration should be given to including not only those that are close to the project and highly farniliar with the issues, but also to have individuls that are familiar with the issues but not the project.

\section{Applying the PCT to the Alternative Plans}

In applying the PCT to ranking the alternative framed plans, 18 significant factors were selected. These tactors were called key determinant criteria and their selection was based on two qualities; first, the criteria had to be significant to the development of sludge management fecilities, and second, the data associated with the criteria had to be discriminatory. The 18 key determinant criteria were divided into six primary categories, some of which were similar to the categories of issues used during the site screening process (see Attachment 1).

The criteria inctuded both site and technology-related issues. Each of the key determinant criteria was defined, and a numerical neasure incorporated into the definition to quantify the determinant. For example, the key determinant "Barge Access" was based 
on a 4-point scoring system which takes into consideration not only whother a site is on the watertont, but also the condlition of existing piers.

The PCT was used in a two-stage process to weight the 19 key determinant criteria. Initfally, the slx primary categories were weighted, and then the category woights were distributed anong the criteria in the second step of the process. The six categories were weighted first because it would have been difficult to compare criteria concerning unreiated and diverse issues (e.g., "Ability to Expand vs. Land Use Compatiojility). Eeven members from the project tearn, both from the city and their consultants, used a scoring sheet (shown in Attachment 3) to assign weights to the categories and determinants. The project team members then met and a consensus was developed for the final weights.

The consensus of weights for the categories and determinants is presented in Attachment 4. The prinary category, Public Acceptance ranked highest (30\%), followed by Transportation (22\%), Local Aegulations (20\%), Ecology (15\%), Reliability, Flexibility, and Operability (10\%), and Project Economics (3\%).

\section{Ranking Components and Plans}

The ranking of the plans was carried out with the aid of a PC-based computer database software, dBase N (by Ashtor Tate). Because each of the plans consisted of several components (some decentralized plans contalined over 10 components), and each component had a value for each of the 18 determinants, thousands of data values had to be aralyzed and weighted according to the preestablished weights.

A total of 100 difierent components were distributed among the 130 plans, in various combinations. Using the computer, it was possible to develop a unique record for each of the 100 components that included a value for each of the 18 determinant criteria. The component records were copied and uniguely identified with a plan number. In this way a database of records consisting of component determinant velues, identified by plan number was created. A program was written in the sofwere to replicate the PCT, whereby each of the 100 components was ranked against eactl other using the database of determinant values and predetermined weights.

The ptan scores were derived by adding the indlviduad component scores for all 130 plans. Not all plans had the same mumber of components. Therefore, a weighted score was developed for each plan which was calculated on a capacity basis. Each component In a plan was multiplied by the proportion of processed sludige associated with each component to produce weighted component scores. These weighted component scores were then added to arrive at an average weighted score for the plans.

Attachment 5 shows how the plan scores were calculated from the individual component scores in a plan. The plan shown is the highest ranklng plan. 
Attachment 3

Ranking and Welghting of Prlmary Categorles and Key Determinants

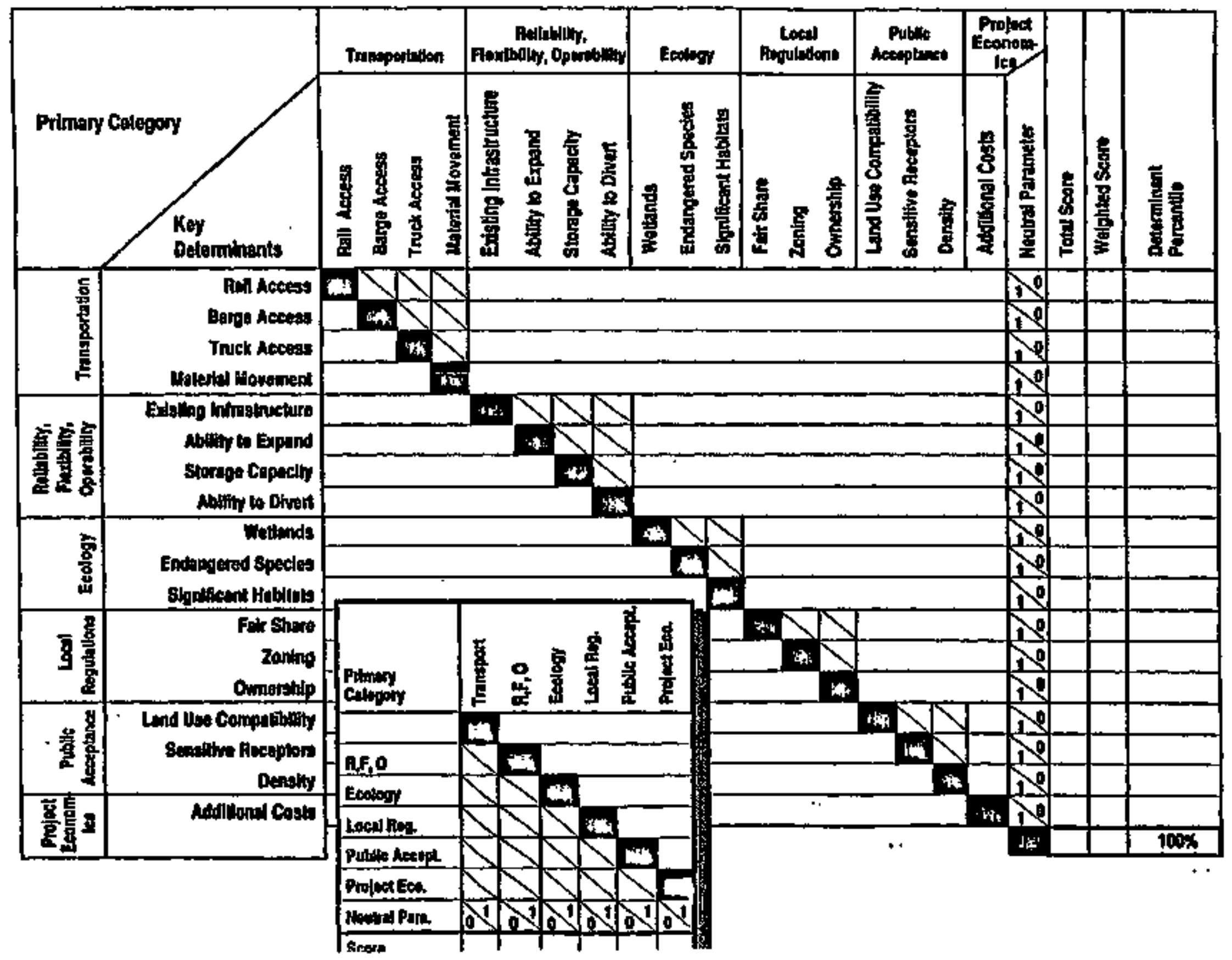




\section{Attachment 4}

\section{Key Determinant Weighting Results}

\begin{tabular}{|c|c|c|c|}
\hline PAamany Gatefory & & KEY DETEAMINANT & $\begin{array}{l}\text { RELATIVE } \\
\text { WEIGHTILAS }\end{array}$ \\
\hline Transportalion & (29) & $\begin{array}{l}\text { Rail Access } \\
\text { Barge Access } \\
\text { Proximity to Truck Roules } \\
\text { Meterlal Movement }\end{array}$ & $\begin{array}{l}\mathbf{3 . 3} \\
\mathbf{5 . 5} \\
\mathbf{7 . 7} \\
\mathbf{5 . 5}\end{array}$ \\
\hline $\begin{array}{l}\text { Relliability, Flexibillity, } \\
\text { and Operatabilliy }\end{array}$ & (10) & $\begin{array}{l}\text { Exlsting Intrastruclure } \\
\text { Abllity to Expand } \\
\text { Storage Capaclty } \\
\text { Abtlty to Divert }\end{array}$ & $\begin{array}{l}1.0 \\
3.0 \\
4.0 \\
2.0\end{array}$ \\
\hline Ecology & (15) & $\begin{array}{l}\text { Wetlands } \\
\text { Sensithe rrabltat } \\
\text { Endtangered Specles }\end{array}$ & $\begin{array}{l}5.0 \\
5.0 \\
5.0\end{array}$ \\
\hline Project Economics & (3) & Add'tlonal Costs & 3.0 \\
\hline Local Regulatlons & (20) & $\begin{array}{l}\text { Fair Share } \\
\text { Zonling } \\
\text { Ownership }\end{array}$ & $\begin{array}{r}10.0 \\
6.7 \\
3.3\end{array}$ \\
\hline Public Acceptance & (30) & $\begin{array}{l}\text { L.and Use Compatibillity } \\
\text { Sensitive Receplors } \\
\text { Densily }\end{array}$ & $\begin{array}{l}10.0 \\
10.0 \\
10.0\end{array}$ \\
\hline Total & $100 \%$ & & $100 \%$ \\
\hline
\end{tabular}




\section{Attachment 5 \\ Ranking of Plans by Weighted Scores}

\begin{tabular}{|c|c|c|c|c|}
\hline \multicolumn{5}{|c|}{ COMPONENTS } \\
\hline SITE UD. & TECHNOLOGY & $\begin{array}{l}\text { CAPACITY(C) } \\
\text { (Dy tons/day) }\end{array}$ & $\begin{array}{l}\text { RAW(R) } \\
\text { SCORE } \\
\end{array}$ & $\begin{array}{l}\text { WEVGHTED(W) } \\
\text { SCORE }\end{array}$ \\
\hline SI 36 & $\begin{array}{l}\text { Composting/Chemical } \\
\text { Stabilization }\end{array}$ & 400 & 1.116 & $4.933^{\star}$ \\
\hline$B \times 18$ & Thermal Drying & 150 & 1.156 & 1.915 \\
\hline Q7 & $\begin{array}{l}\text { Composting/Chemical } \\
\text { Stabilization }\end{array}$ & 120 & 1.165 & 1.544 \\
\hline M2 & Chemical Stabilization & 115 & 1.201 & 1.527 \\
\hline BK 23 & $\begin{array}{l}\text { Composting/Chemical } \\
\text { Stabilization }\end{array}$ & 120 & 1.043 & 1.383 \\
\hline & Total & $905(T)$ & & $11.302^{* \star}$ \\
\hline
\end{tabular}


The plan scores resulting from the ranking process indicated that the site-related determinants dominated the results. The Public Acceptence category witich accounted for 30 percart of a component's raw score wes particularly infiuential in the rankling. Components with sites having compatible land uses, being distant from sensitive receptors, and having a relatively low surrounding population density, ranked highty. Centralized plans ranked highest, but this was because their components comtzined stes which scored well in the Public Acceptence Category.

Pather than pick the highest ranking plan as the preferred plan, seven preferred plan alternatives were chosen tor additionat analysis regarding economic and transportetion feasibility. Selacted plans inchuded the highest ranking plans, both centrallized and decentralized, and the highest ranking plan with all waterfront sites (since barging was considered important to offset truck tratific). From these altematives several hybrid plans evolved, and once addítionad siting conflicts had been resolved, a final preferred plan was selected, and publichy announced.

\section{Conclusion}

Using a rationale and scientific method for screening, ranking, and selecting waste management sites has become an accepted practice for public agencies, whose decision. making methods are otten sub]ect to intense public scrutiny. in the case of New York City's sludge management plan, site selection and preferrex program identification constituted the initial steps taken in gaining public support of the plan. The plan was selected by framing a large number of atternative plans that consisted of site/technology/capacity "components". Criteria refiecting both site and technology/capaciky issues were chosen to rank the components and plans. The PCT becarne an effective tool in weighting the criteria in the process of ranking the plans.

The final prefecred plan that resulted from the framing and ranking of plans originally consisted of nine sites. As a result of the city's public approval process, the plan now has been recuced to five sites, one in each borough of the city. While the plan has changed, the fundamental guidelines and rules that were used in ranklng and selecting the sites have not. 


\section{BIBLOCFAPHY}

1. Dean, B.V. and M.J. Nishry. "Scoring and Profitability Models for Evaluating and Selecting Engineering Projects". J. Operations Res. Soc. Am. 13:550-559. t855.

2. Ash Management Study for Orange and Rockland Counties. Orange and Rockland Utilities. 1980.

3. Wenger, R.B. and C.R. Fhyner. Evaluation of Alternattves tor Solid Waste Systems". Joumal of Ervironmental Systems, V. 2, No. 2:89-108. 1972.

4. Odum, E.P., of at. Optimum Pathway Maurtx Analysis Approach to the Environmental Decision Maklng Process: Test Case: Aetative Impact of Proposed Highway Altematives". Institute of Ecology, University of Georgia, Athens, Georgla. $197 t$.

5. Soiomon, R.C., ot al. Water Resources Assessment Methctology (WRAM): Impact Assessment and Afternatives Evaluation". Report Y-77-1, U.S. Army Engineer Waterways Experiment Station, Vicksburg, Mississippi. 1977.

6. Dee, N., et al. "Emironmental Evaluation Systern for Water Resources Pianning". Finad Report, Battenle-Columbus Laboratories, Columbus, Ohio. 1973.

7. Parking Survey/Assessment inventory Final Report. Metropolitan Transit Authority (Long Island Rail Road). 1892. 


\section{GUIDELINES FOR PERFORMING \\ PAIRED COMPARISON TECHNIQUE}

WEIGHTING EVALUATION CRITERIA

Attached are the essential elements of the paired comparison technique (PCT) that are used in weighting evaluation criteria. The definitions of these criteria (Attachenent 1.5-1) must be carefully examined to assure an understanding of their interpretation and method of measurement. It should be noted that evaluation criteris are only applied to a site inventory that has been scteened initially with threshold criteria.

Attachment 1.5-4, the PCT Ranking and Weighting Matrix, is used to develop the weighted scores for each criteria. Personnel with multidisciplinary environmental and engineering backgrounds and a familiarity with the NYC Solid Waste Management Plan have participated in this weighting process.

The following steps are a guide for using the PCT matrix.

- The question to ask when scoring each comparison is, Which criteria is more important to the selection of an alternative site for a paper-to-ethanol bioconversion facility in the City of New York?"

- Comparisons are made, one at a time, between criteria by reiating those across the top with those down the side of the matrix.

- Each comparison has a point total of 1 to be awarded as a score of "0, 0.5, or 1". For example, when comparing Industrial Waterfront Designation to Site Zone, there are three choices. Either Industrial Waterfront Designation will be more important, and thus receive a score of "1", while Site Zone will receive a "O"; Industrial Waterfront Designation and Sife Zone will be equally important, and thus both receive a score of " $0 S^{\text {"; }}$ or Site Zone will be more important, and thus receive a score of " 1 ", while Industrial Waterfront Designation will receive a score of " 0 ".

- The score for Industrial Waterfront Designation belongs in the left half of the square where Industrial Waterfront Designation and Site Zone intersect along the side and across the top of the matrix, respectiveiy. The score for Site Zone belongs in the right half of that square. In Attachment 1.5-4, Site Zoning is considered more important than Industrial Waterfront Desfgnation and bas been given a score of " 1 " in the right half of the square. 
- This process is continued until all possible comparisons have been made.

- A neutral parameter is included so that no evaluation criteria will score a "O". Each criteria will score a " 1 " against the neutral parameter.

The method for determining the weight of each criteria is described below:

- The scores for each criteria are totaled and piaced in the column labeled Total Score." Both rows and columns are added together including the neutral parameter. As described above, points in the left half of the square correspond to the criteria listed along the side of the matrix, and points in the right balf correspond to criteria listed across the top. There are 91 comparisons to process with the 14 criteria including the neutral parameter. Because a score of one is the maximum allowed for each comparison, the total score for all criteria combined is 91 .

" In the column marked "Weighted Score", the total score for each criteria is divided by 91 . This weighted score is then multiplied by 100 and placed in the corresponding square of the column labeled "Criteria Percentile". 


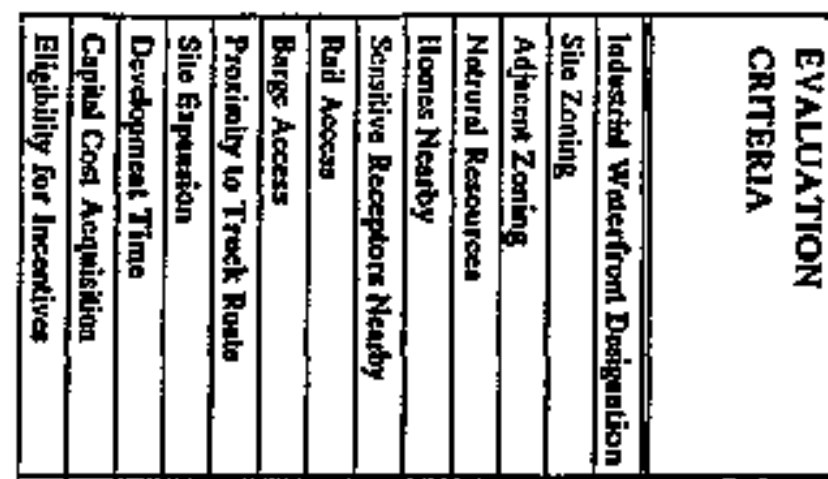

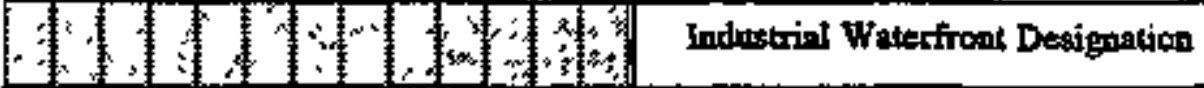

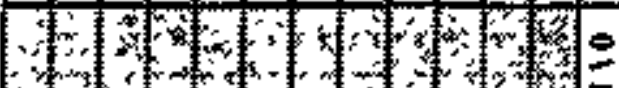

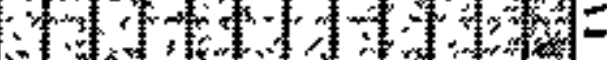

tom

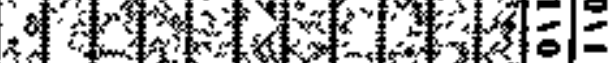

Site Zoning

Adjacent Zoning

(3)

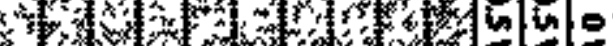

:

Natrural Rasutrces

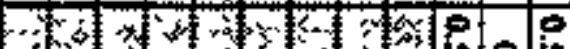

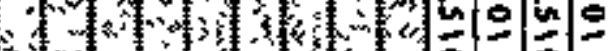

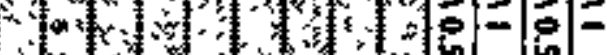

Homes Nearty

sin o

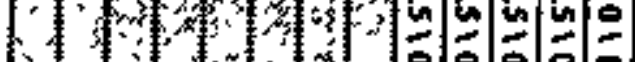

Sensitive Receptors Nearby

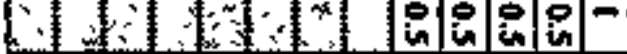

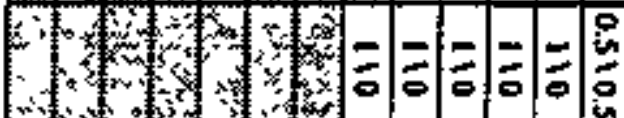

ind

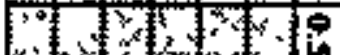

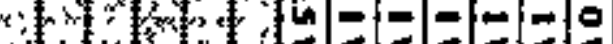

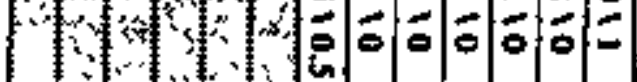

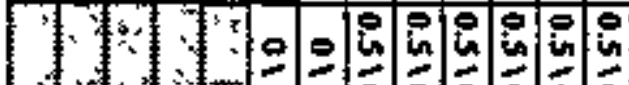

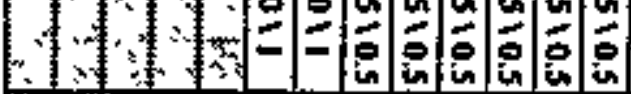

Rail Access

ove

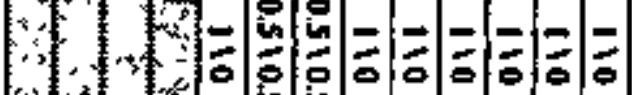

Barge Access

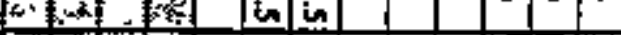

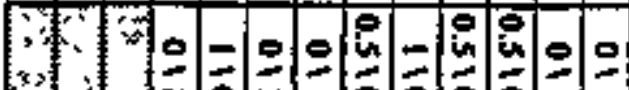

事挰 $=0$

Proximity to Truck Route

$\therefore-1=-0=$

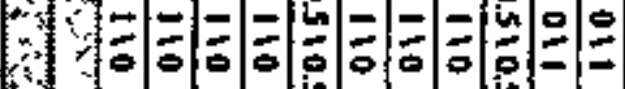

Capital Cost Acquisition

Axpo

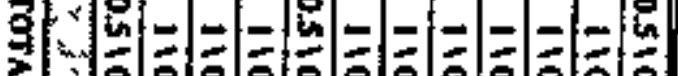

范

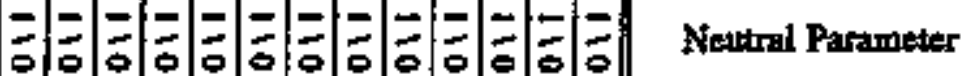

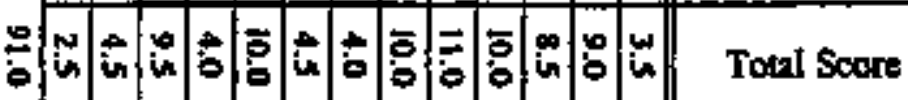

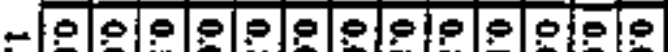

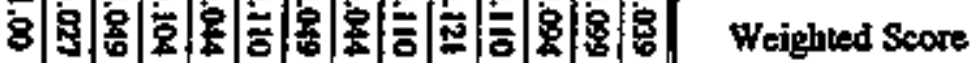

要 


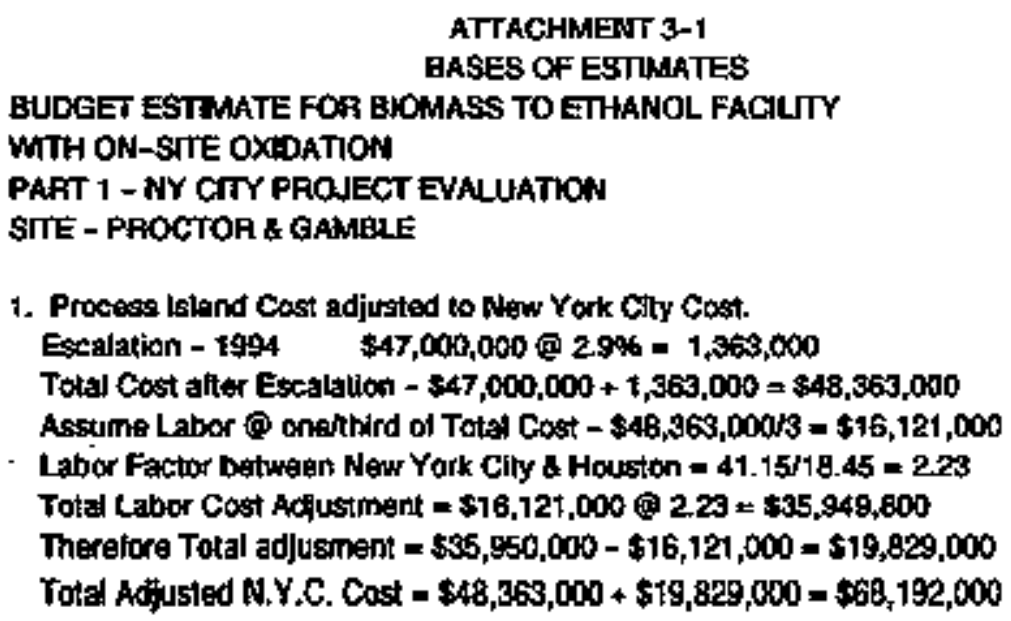

ATTACHMENT 3-1

BASES OF ESTINARTES

BUDGET ESTTMATE FOR BIOMASS TO ETHANOL FACILTY WTH ON-STE OXDATION

PART 1 - NY CITY PRONECT EVALUATION

\$TIE - PROCTOR \& GAMBLE

1. Process lstend Cast adjusted to Now York Cty Cost.

Escalation - 1994 \$47,006,000 (19 2,9\% - 1,3\%3,000

Total Cost after Escalalion - \$47,000,000+1,360,000 =\$48,363,010

Assume Labor @ one/third of Totes Cost - \$48,363,000r3 = \$16,121,000

Labior Factor Detwegn New Yark Cily \& Houston $=41.15 / 18.45=2.23$

Total Labor Cost Acqustinent $=\$ 16,12 t, 000$ @ 2.23 $=\$ 35,949,600$

Therelore Total adjusment $=\$ \$ 5,550,000-\$ 16,121,000=\$ 19,829,000$

Total Ad;usted N.Y.C. Cost $-\$ 48,363,000+\$ 19,829,000=\$ \$ 8,192,000$

2

BALANCE OF PLANT

g. Admin, /Control/Lab. Eldg. - assume slze: $100^{\prime} \times 100^{\prime}=10000$ st. $8150 / \mathrm{st}^{\prime}=$

$\$ 1,500,000$

Concrete Foundatios - 10,000 st @ \$12,00/st =

$\$ 120,000$

Tọla

$\$ 1,620,400$

b. Maint. Shop Eldg. - essumb size: 100'x100'= 10000 sl.@ $\$ 120.00$ ste Tools and Equipment -

$\$ 1,200,000$

$\$ \$ 50,000$

Concrets Foundation - to,0h0 st @ \$12.00/st =

$\$ 120,000$

Totat

$\$ 1,470,000$

c. Plant Air Supply.

Two (Z) Compressors =

$\$ 130,000$

Air Dryers =

Piping asstume fil 5,400@ \$50,0001 -

Total

$\$ 20,000$

$\$ 250,0.00$

$\$ 400,000$

d. Aerobic Watertreaimen:

Assume @ 150,000 gal.iday \$ \$10.00/gal. =

$\$ 1,500,000$

B. Chilled/Cooling Water Supply:

includes: Cooling Tower, Fdil., Pping $250 *$ =

$\$ 700,000$

c Boiler Feedwater (Demin.) allow:

$\$ 400,000$

g. Sold Oxidation/fower 8 Distribution;

$530,000,000$

h. Bailer Packagax

$\$ 1,000,000$

i. Steam Turbine:

$\$ 250,000$ 
BASES OF ESTTMATES

BUDGET ESTIMATE FOR BIONASS TO ETHANOL FACILTYY

WITH ON-STE OXDATION

PAFT 1 - NY CITY PAOJECT EVALUATION

STE - PAOCTOA \& GAMBLE

f. STIE IMPROVEMENT

1. Crearing \& Gnubbing :

$\$ 50,000$

2 Sewer and Dralnage:

Assuma: 5300'ułg pipe @ \$150.00:H

$\$ 795,000$

Manholes/C.A. - 10 a. @ \$12,00॰

$\$ 120,000$

$\$ 915,000$

3. Acress Poartways \& Parking:

T어례

$\$ 800,000$

4. Pillng assume whole site:

Piles @ 225stiplle $=436,200 / 225=2,000$ ea.

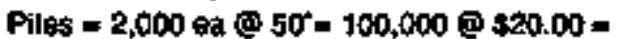

$\$ 2,000,000$

5. Dewatering allowance lor excenvalion:

$\$ 500,000$

6. Make-up \& Potable Water:

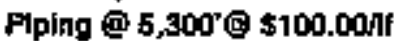

$\$ 530,000$

Matering \& Hook-ups

Tolat

$\$ 70,000$

$\$ 600,000$

7. Sewer Hook-ups tor Bathroom,etc.allow:

$\$ 100,000$

8. Fire Water : 5300 if $\$ \$ 250$ ll =

$\$ 1,325,000$

Hydranl \& Misc. Berns *

$\$ 75,060$

Total

$\$ 1,400,000$

9. Sprinkler Syglem whole site plus Standpipe 200,000 si @ \$5.00ist =

$\$ 1,000,000$

10. Fencing:

$\$ 70,000$

11. Gas Pipeling (ollow):

$\$ 150,000$

12. Sthe Utghing:

436800 \$f $\$ \$ 1.20=$

$\$ 510,002$

k. MAFK-UPS:

Stan-up COSt $=1.5 \%$

Expineering - 10\%

Contingency $=20 \%$ 


\section{ATTACHMENT 3-2}

BASES OF ESTMATES

BUDGET ESTIMATE FOR BIOMASS TO ETHANOL FACIUTY WITHOUT OXIOATION

PAFT 1 - NY CTTY PRONECT EVALUATION

SIE : PAOCTOR \& GAMELE

1. Process Lsland Cos adjusted to New York Chy Cost.

Excadailon - $1994 \quad 347,000,000 @ 2,9 \%=\$, 363,090$

Total Cost attex Escalation $-\$ 47,000,000+1,363,000=\$ 48,363,000$

Assume Labor @ onelthiro of Total Cost $-\$ 48,363,000 / 3=\$ 16,121,000$

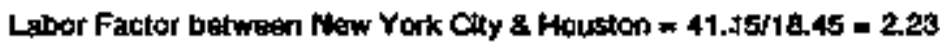

Total Labor Cost Adjustment $-\$ 16,121,000 @ 2.23=\$ 55,949,200$

Therefose Total adjusment $-\$ 35,950,000-\$ 16,121,000-\$ 19,829,000$

Total Adjusted N.Y,C. Cost $=\$ 4 B, 313,000+\$ 19,629,0000=\$ 68,192,000$

2.

BALANCE OF PLANT

a. Adrin./Controllab_Bldg, - assume size: $100^{\prime} \times 100^{\prime}=10000$ sf.@ $\$ 150 / s f=$ Concrede foundation $-10,000$ st $@ \$ 1200 \%$ st $=$

Total

$\$ 1, \$ 00,000$

$\$ 120,000$

$\$ 1,620,000$

b. Maint /Shop Bldg. - assume size: $100 \times 100=10000$ st. $\$ 120$,00/sf=

Tooks and Equipinent a

$\$ 1,200,000$

$\$ 150,000$

Contrete Foundation - 10,000 st 6s $\$ 12,004 \mathrm{sr}=$

$\$ 120,000$

Total

$\$ 1,470,000$

c. Pland Air Supply.

Two (2) Compressors Ar Dryers =

Piping assume o 5,000's \$50.00if =

Tolal

$\$ 150,000$

$\$ 20,000$

$\$ 250,090$

$\$ 400,000$

d. Aerobic Walertreatment:

Assume @ $150,000 \mathrm{gz} . \mathrm{kday}$ @ $510.00 / \mathrm{gal} .=$

$\$ 1,500,000$

e. Chilledeooling Water Supply.

Includes: Cooling Tower, Fin., Plping (9250' = \$700,000

t. Boilex Fengowater (Denin.) attow:

$\$ 400,000$

g. Power \& Distribution:

$\$ 5,000,000$

h. Bxilar Package:

$\$ 1,000,000$

i. Steam Turbine:

5250,000 


\section{BASES OF ESTINAKTES}

BUDGITT ESTHATE FOR BLOMABS TO ETHANOL FACM.TY WTHOUT OXIDATION PART 1 - NY CTYY PAOJECT EVALUATION

STE: PROCTOA \& GAMELE

\section{SITE MAFROVEMËNT}

1. Clearing \& Grubbing \& Demolilon:

$\$ 550,000$

2. Sewer and Dralnage:

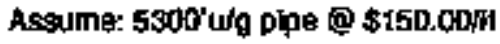
Manholesc.B. - 10 ea. $\$ \$ 12,000$

Total

$\$ 795,000$

$\$ 1200 \mathrm{HO}$

$\$ 915,000$

3. Access Rouarways \& Parklng:

$\left(4900^{\circ} \times 20^{\prime}+20,000\right)-106,000$ st $\$ \$ 7.50=$

$\$ 800,000$

4. Filing assuinf wholé slie:

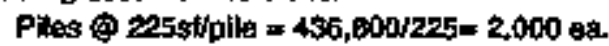

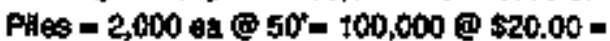

$\$ 2,0,010,000$

5. Dowalecing allowance for excevation:

$\$ 500,000$

5. Make-up \& Potable Water:

Piping@ 5,300'\$ \$100.00if

$\$ 550,000$

Melexing \& Hook-ups

Total

$\$ 70,000$

$\$ 600,000$

7. Sower Hook-Lps for Bethroom, 的callow:

$\$ 100,000$

8. Fre Waler : 5300 if @ \$250ift -

$\$ 1,325,000$

Hydrant \& Misc. Hems =

$\$ 75,000$

Total

$\$ 1,400,000$

9. Sprinkler Sysiem whode site plus Standpipe 200,0010 sf @ $\$ 5,00 / 3 t=$

$\$ 1,000,000$

10. Fancinos:

$\$ 70,000$

11, Gas Ppellne (allow):

$\$ 150,000$

12. Shie Lighting:

436800 sf $1 \$ 1.20=$

$\$ 510,002$

k. MAFK-UPS:

start-up cast $=1.5 \%$

Enginearing = 10\%

Corkipgency = 20\% 


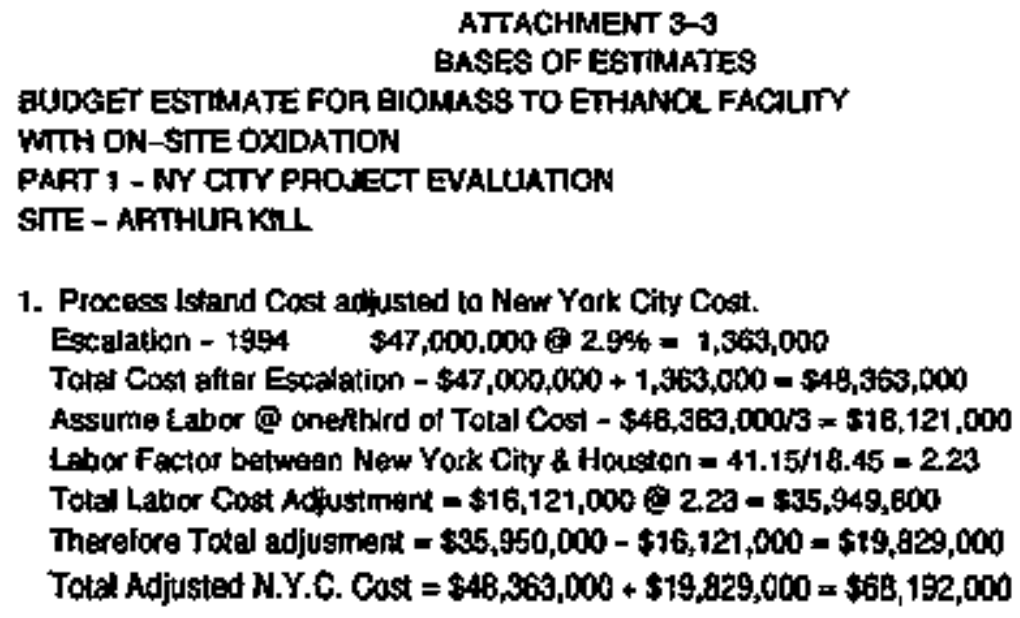

1. Process Istand Cost antusted to New Yark City Cost.

Escralation - $1994 \quad \$ 47,000,000$ (2,9\% $=1,369,000$

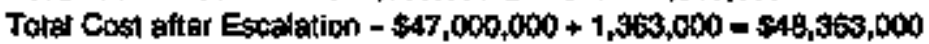

Assume Labor @ onetitird of Total Cos $-\$ 46,363,000 / 3=\$ 18,121,000$

Labor Factor between New Yosk City \& Housten = 41.15/18.45 = 2.23

Total Labior Cost Ad justmint $=\$ 16,121,000$ 2.23 $-\$ 35,949,600$

Therelore Tatel adjusment $=\$ 25,950,000-\$ 16,121,000=\$ 19,929,000$

Total Adjusted N.Y.C. Cost $=\$ 48,369,000+\$ 19,829,000=\$ 68,192,000$

2

BALANCE OF PLANT

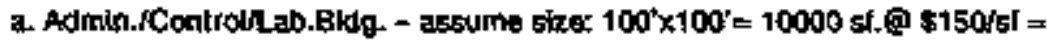
Concrete Foundation - 10,000 sf \& \$12,00rst =

b. Maint./Shoo Eldg. - assume size: $100^{\prime} \times 1001=10000$ st. $\$ 120.00 / s t=$

s1,200,000 Topls and Equipment =

$\$ 150,000$

Concrete Foundaton $-10,000$ sf (1) \$12,00/st =

$\$ 120,000$

Total

$\$ 1,470,000$

c. Plant Ait Supply.

Two (2) Compressors =

$\$ 130,000$

Alf Dryers =

Plping assume i 5,000 @ \$50.00Mt .

Total

$\$ 20,000$

$\$ 250,000$

$\$ 400,000$

d. Aeroble Watertreatment:

Assume o 150,000 gal_tday o $\$ 10.00 / 0 a t=$

$\$ 1,500,000$

e. Chilledrcoofing Water Supply:

Includes : Cooling Tower, Forn, Piping 일ㅇ' =

$\$ 700,000$

t. Boiler fetextwater (Demin.) strow:

$\$ 400,000$

g. Solid Oxidation'Power \& Disiribution:

$\$ 30,000,000$

n. Eojer Package:

$\$ 7,000,000$

i. Steam Tubine:

$\$ 250,000$ 
BUDGET ESTMMATE FOP BIDAASS TO ETHANOL FACHTY

WIIH ON-STTE OXIDATION

PART 1 - NY CITY PRONEGT EVALUATION

SITE - APTHUR KILL.

\section{J. SNTE IMPAOVEAENT}

1. Clearing \& Grubbing:

$\$ 50,000$

2 Sower and Drainage: *

Assime: 5300'w'g pipe i $\$ 150.004 f$

$\$ 795,000$

Manhales/C.B. - 10 ea. 원 $\$ 12,000$

Total

$\$ 120,000$

$\$ 915,000$

3. Access Foadways a Parking :

$\left\{4300^{\prime} \times 20^{2}+20,000\right)=106,000$ st in $\$ 7,50=$

$\$ 800,000$

4. Piling assume whole she:

Piles (2) 24sitpile $=436,800 / 225=2,060$ ea.

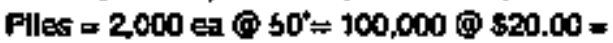

$\$ 2,000,000$

5. Dewalering allowance 'or excavation:

$\$ 500,000$

6. Make-up \& Potable Waler:

Piping $\odot$ 5,300'@ \$100.00月f

$55 \times 0,000$

Melering \& Hook-ups

Tolal

$\$ 70,000$

$\$ 600,000$

7. Sower Hook-ups for Bathroom,etc. allow:

$\$ 100,000$

g. Fire Water : 5800 it (1) 5250月 =

$\$ 1,325,000$

Hydrant a Mlsc. hems =

$\$ 75,000$

Toal

$\$ 1,400,000$

9. Sprinkler Syslem whote sitte phus Standpipe 200,000 st 0 s.001si =

$\$ 1,000,000$

10. Fencing:

$\$ 70,000$

11. Gos Ptpaline (allow):

$\$ 150,000$

12 sita Lighting:

496800 st $9 \$ 1.20=$

$\$ 510,002$

k. MAFK-UPS:

Start-4p Cost $=1.5 \%$

Enginearing = 10\%

Comingenty = 20\% 
ATTACHMENT 3-4

BASES OF ESTHATES

BUDGET ESTMAATE FOR BIOMASS TO EIHANDL FACIUTY WITHOUT OXDATION PART 1 - NY CITY PROIECT EVALUATION

SIE: AFTHUR KIEL

1. Process bsand Cost adjusted lo New York City Cost.

Excalation - $1994 \quad \$ 47,000,000 \div 2,9 \%=1,364,000$

Total Cost after Excalation $-\$ 47,000,000+1,363,000=\$ 48,363,000$

Assume Labor is onelthird of Folal Cost - \$48,363,000/3 =\$16,121,000

Labor Factor between Naw Yoik Clky \& Houston = 41.15/18.45 - 2.23

Total Labor Cost Ad]ustment $=\$ 16,121,000$ it 2,23 $=\$ \$ 5,949,800$

Therefore Total acfustment $=\$ \$ 3,950,000-\$ 16,121,000=\$ 19,829,000$

Total Adjusted N.Y.C. Cost $=\$ 48,363,000+\$ 19,829,000=\$ 68,192,000$

2.

BALANCE OF PLANT

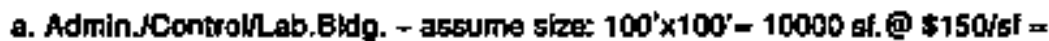
Concrota Foundation $-10,000$ st 6 \$12.00tst =

$\$ 1,500,000$

$\$ \$ 20,000$

Total

$\$ 1,620,000$

b. Maint JShop Bdg. - assume size: 107'x100'= 10000 s.@ \$120.00/sfe

Tooks and Equipment =

$\$ 1,200,000$

Concrete Foundation $+10,000$ sf if $\$ 12,00$ st $=$

$\$ 150,000$

$\$ 720,000$

Total

$\$ 1,470,000$

c. Plars Air Supply:

Two (2) Compressors =

$\$ 130,000$

Alr Dryers =

Piping assume is 5,000 @ \$50.00nt -

Total

520,000

$\$ 250,000$

$\$ 400,000$

d. Aerobic Watertreatment:

Assura @ 150,000 galuday @ \$10.00\%gal.

$\$ 1,500,000$

c. Chilled/Cooling Waler Sugply:

Includes: Cooling Tower, Fon., Piping P250' =

$\$ 700,000$

f. Boiler Feodwater (Dernin,) allowr.

$\$ 400,000$

p. Powar a Distribulion:

$\$ 5,000,000$

h. Boiler Package:

$\$ 1,000,000$

i. Steam Turbine:

$\$ 250,000$ 


\section{BASES OF ESTIMATES}

GUDGET ESTIMATE FOR BOMASS TO ETHANOL FACITY WTHHOUT OXDATION

PAFT 1 - NY CTTY PROJECT EVALISATION

GTE: ARTHURKLL

\section{J. \$ITE MPROVENENT}

1. Clearing \& Clubbing:

$\$ 50,000$

2. Sewer and Drainage:

Assumes 5300'uloplpe \$ \$150.00nf

$\$ 795,000$

Manholesc.8. - to ea f $\$ 12,000$

Tokal

$\$ 120,000$

\$915,000

3. Access Roadways \& Paiting:

$\left(4300^{\circ} \times 2 \% \cdot 20,000\right)=106,000$ st $\$ \$ 7.50=$

$\$ 900,000$

4. Piwng assume whole ste:

Piles o 245stiptte = 436,800r225- 2,000 6a.

Piles $=2,000$ ea $@ 50^{\prime}=100,000 \$ \$ 20,00=$

$\$ 2,000,000$

5. Dewatering alkowance bor exceravation:

$\$ 500,000$

6. Make-up \& Poteble Water:

Piplng (5,300' \$100.00,t

Hetering \& Hook-ups

Toral

$\$ 5 \$ 0,000$

$\$ 70,000$

$\$ 600,000$

7. Sewex Hook-ups tor Bahroom,etc.allow.

$\$ 100,000$

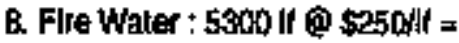

$\$ 1,325,000$

Hydram a Misc. hems =

Total

$\$ 75.000$

$\$ 1,400,000$

9. Sprinkler System whole slte plus Standplpe

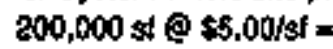

$\$ 1,000,000$

10. Fencling :

$\$ 70,600$

11. Cas Pipeline (allow)

$\$ 150,000$

12. Ste Lighting :

435800 혀 옹 51.20

$\$ \$ 10,002$

k. MARK-UPS:

Siart-up Cost $=1.5 \%$

Engmearing = 10\%

Comingency $=20$ 的 


\section{ATTACHMENT 4-1}

TABLES 46 TO 4-17

FIGURES 4-1 TO 4-16

PRO FORMA ANALYSIS 


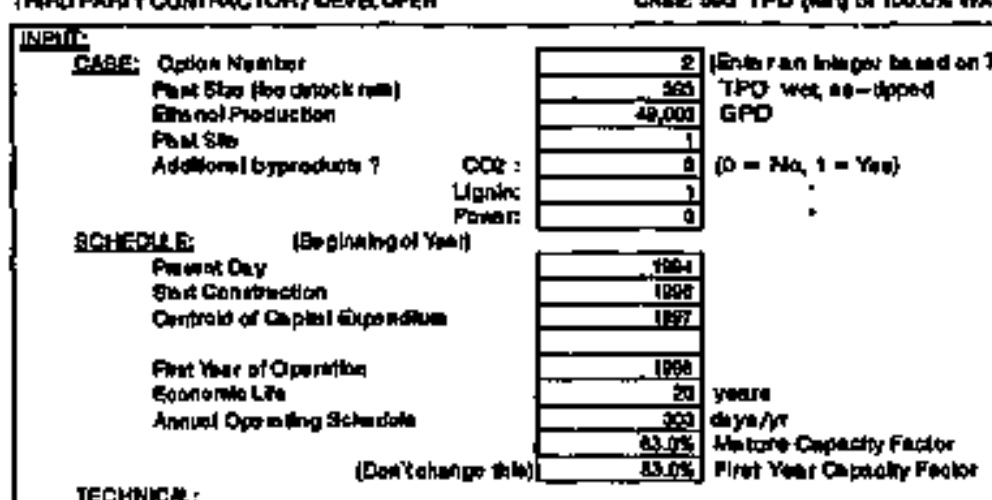

IEc단ㄷ.:

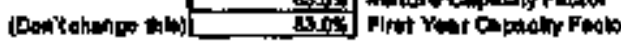

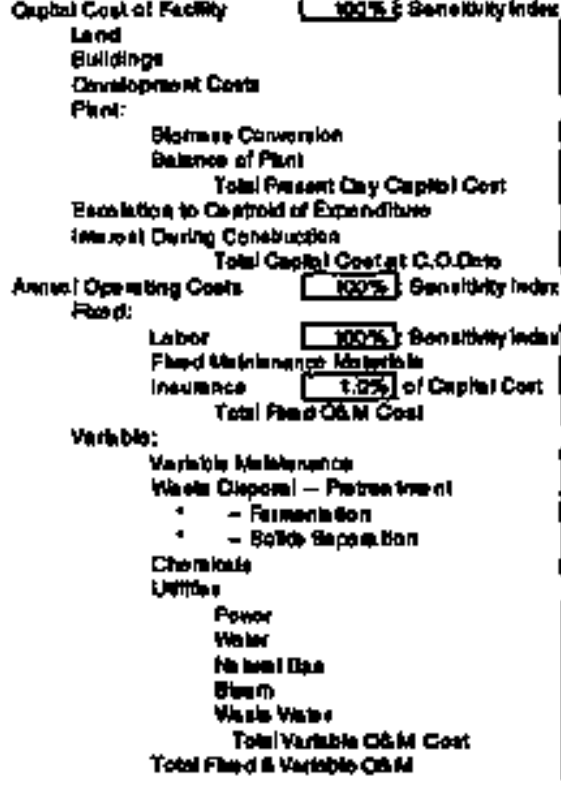

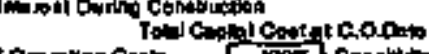

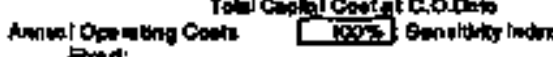

Fond:

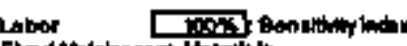

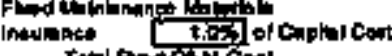

Vathole:

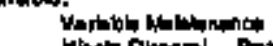
When Compon - Prim inent - - Farmemelon

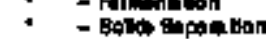
Cheniouth Intiltary Ponom tow

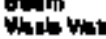
Toll Wurtuble dein con

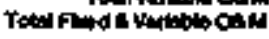

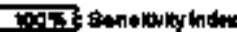

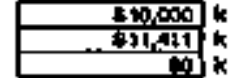

\section{- Eg-19: $k$}

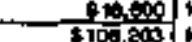

势
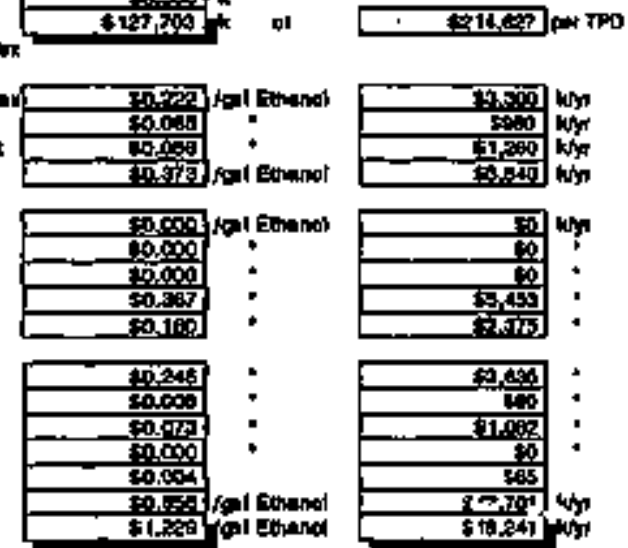

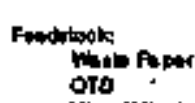

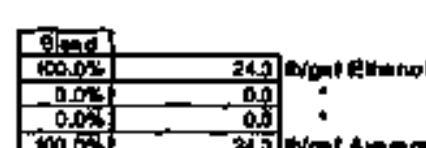

TopinoFm

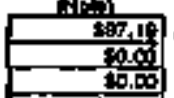

E-
Extrons:

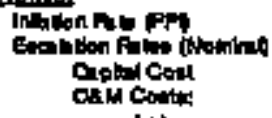

Labor

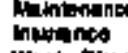

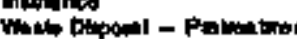
- Formanition

Crombers

Untor.

Prower

Nobrelent

Stan

Foodititi:

Gen

ninow:

山pois

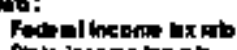

Exherthes

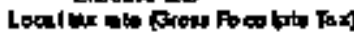

mopontrix fro

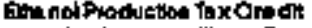

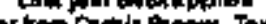

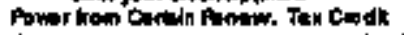

Aoply ?

Bild

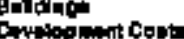

Const:

Blamill Gomwerton

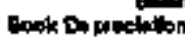

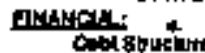

aning of Pant

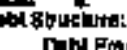

pub Fraction

Fineneing Tein

morinton ofteen

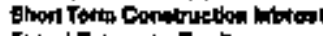

Whot Foburm on Eaphy
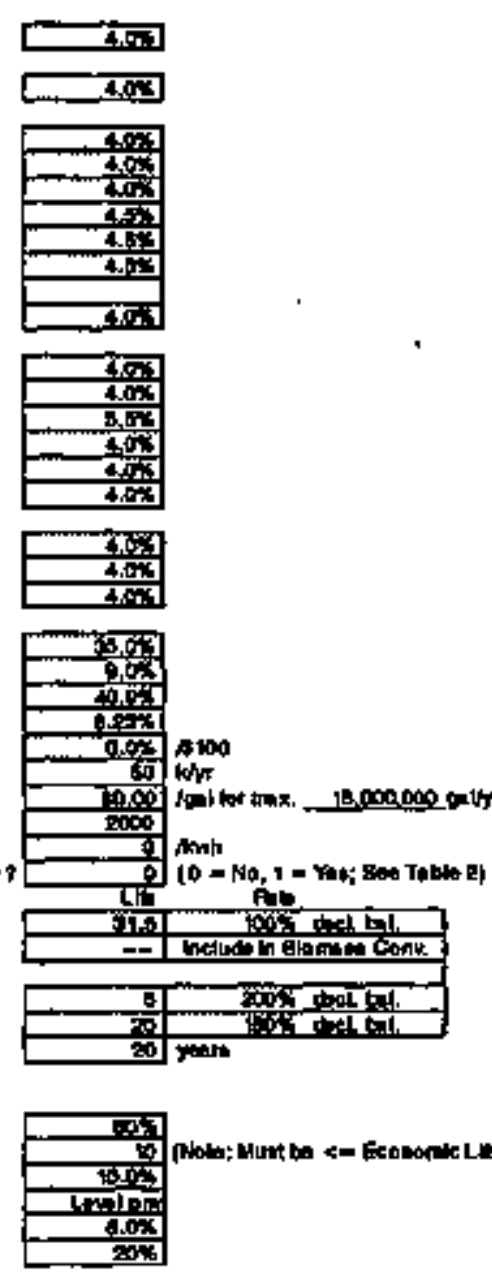

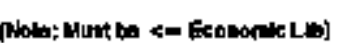

Ovtout
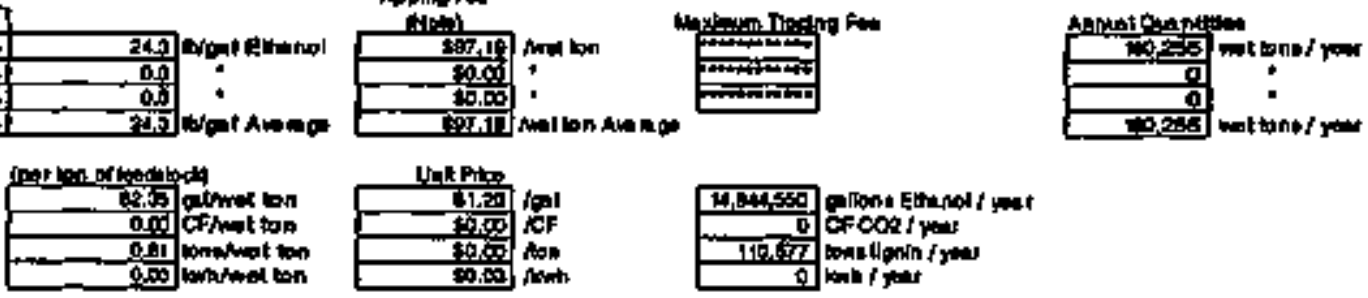

tom

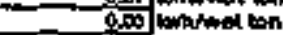

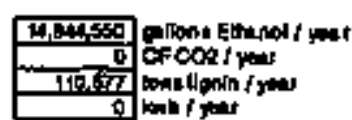

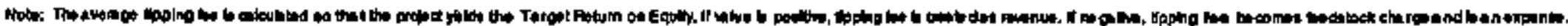




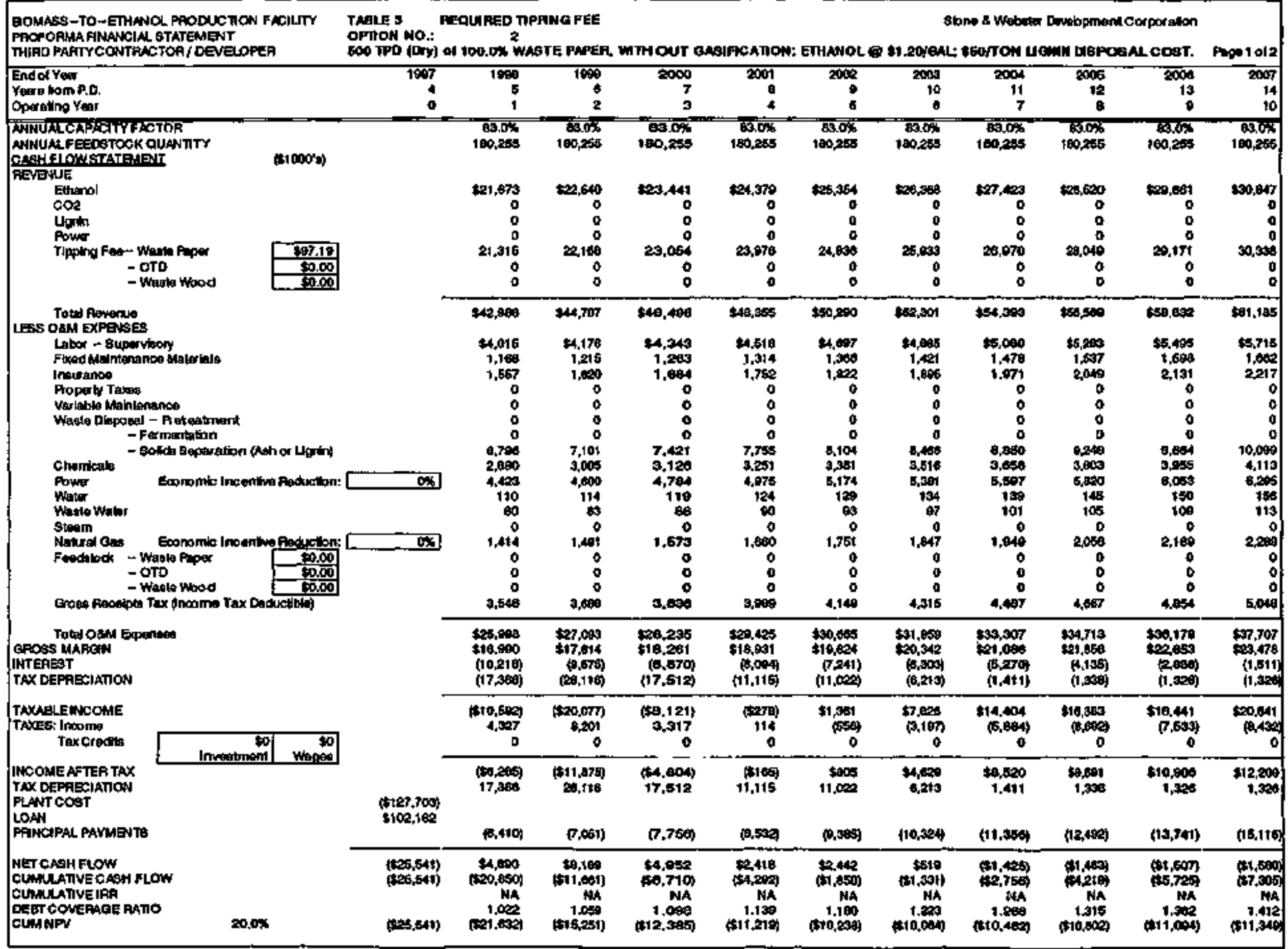




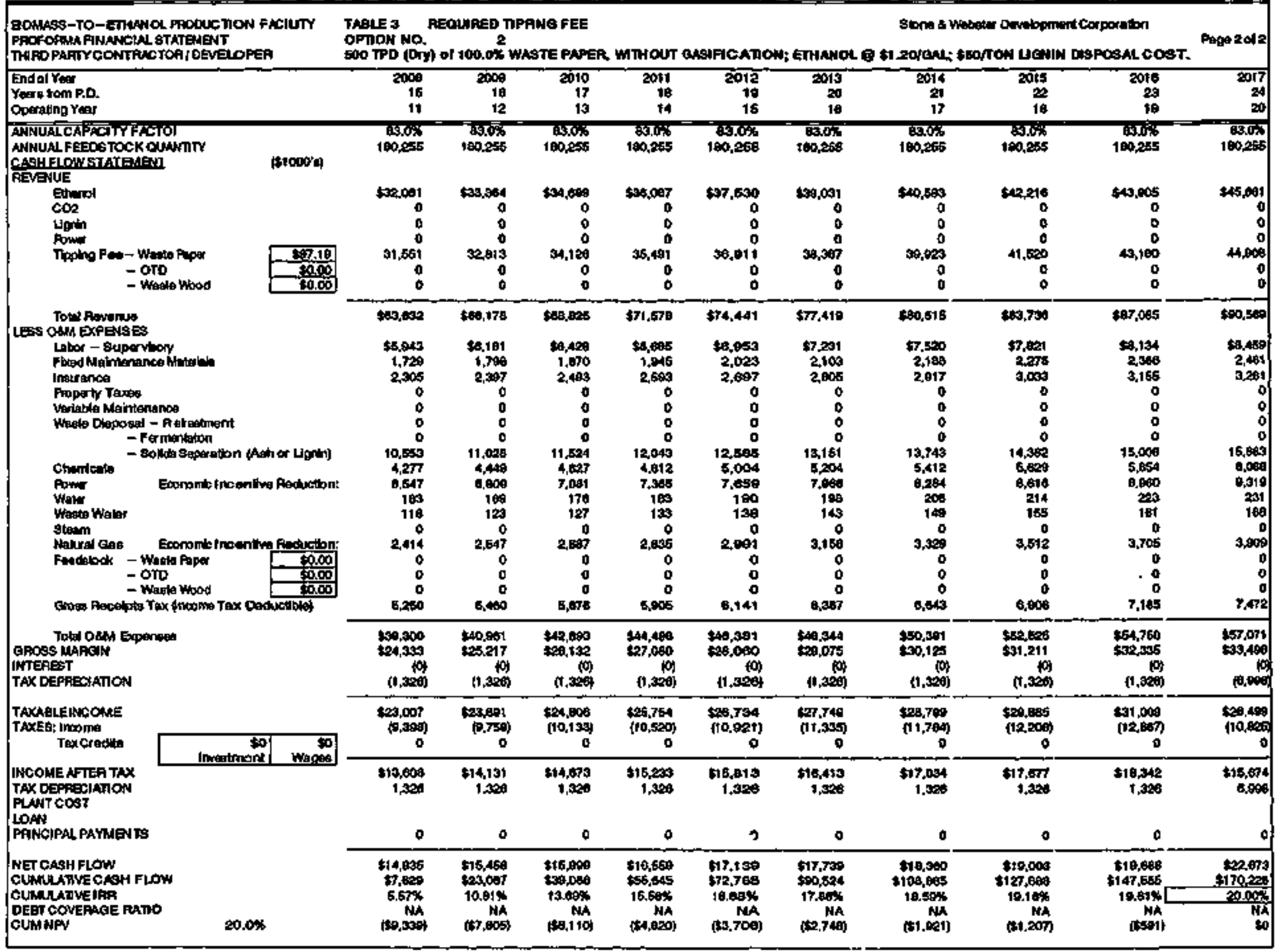




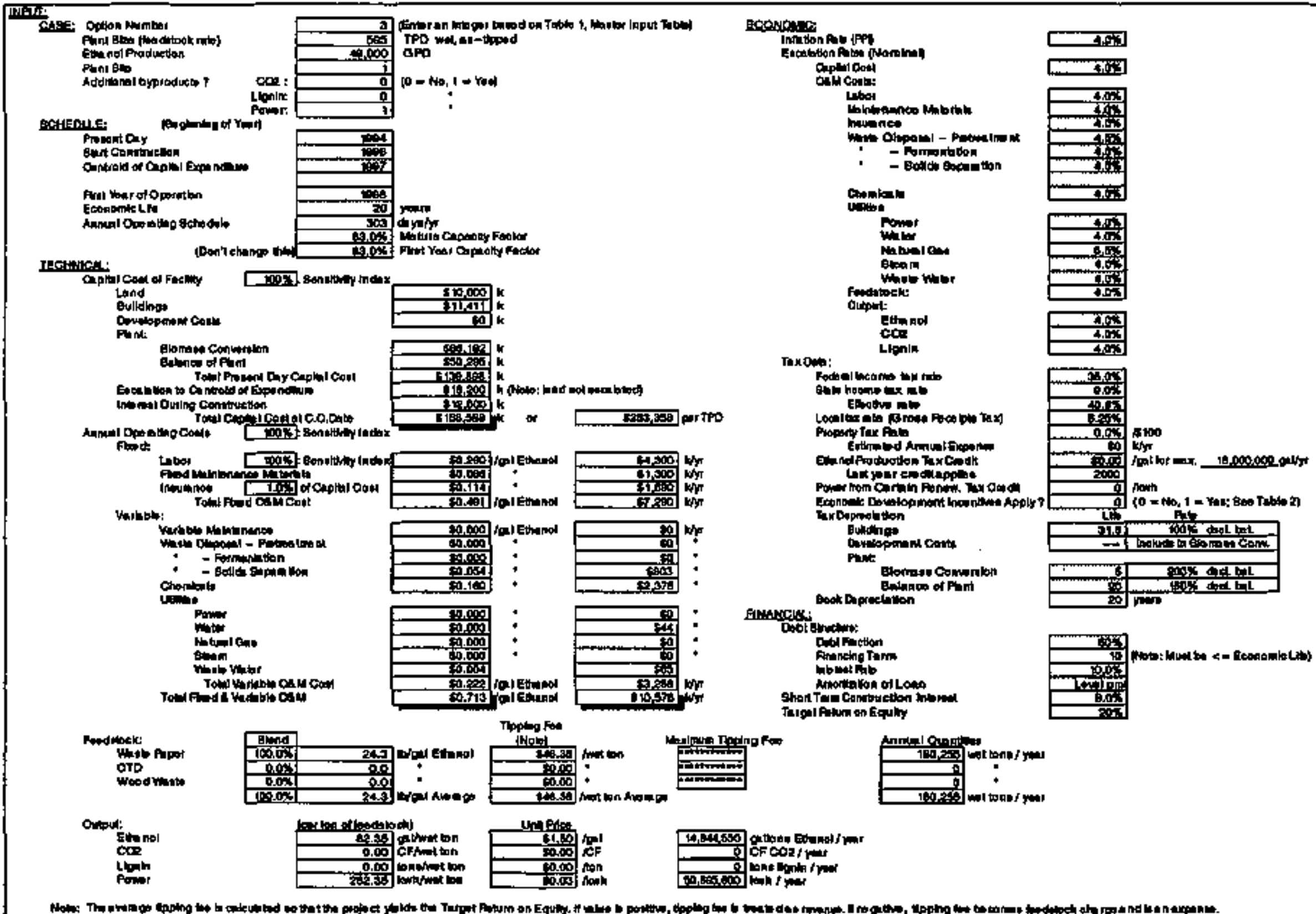




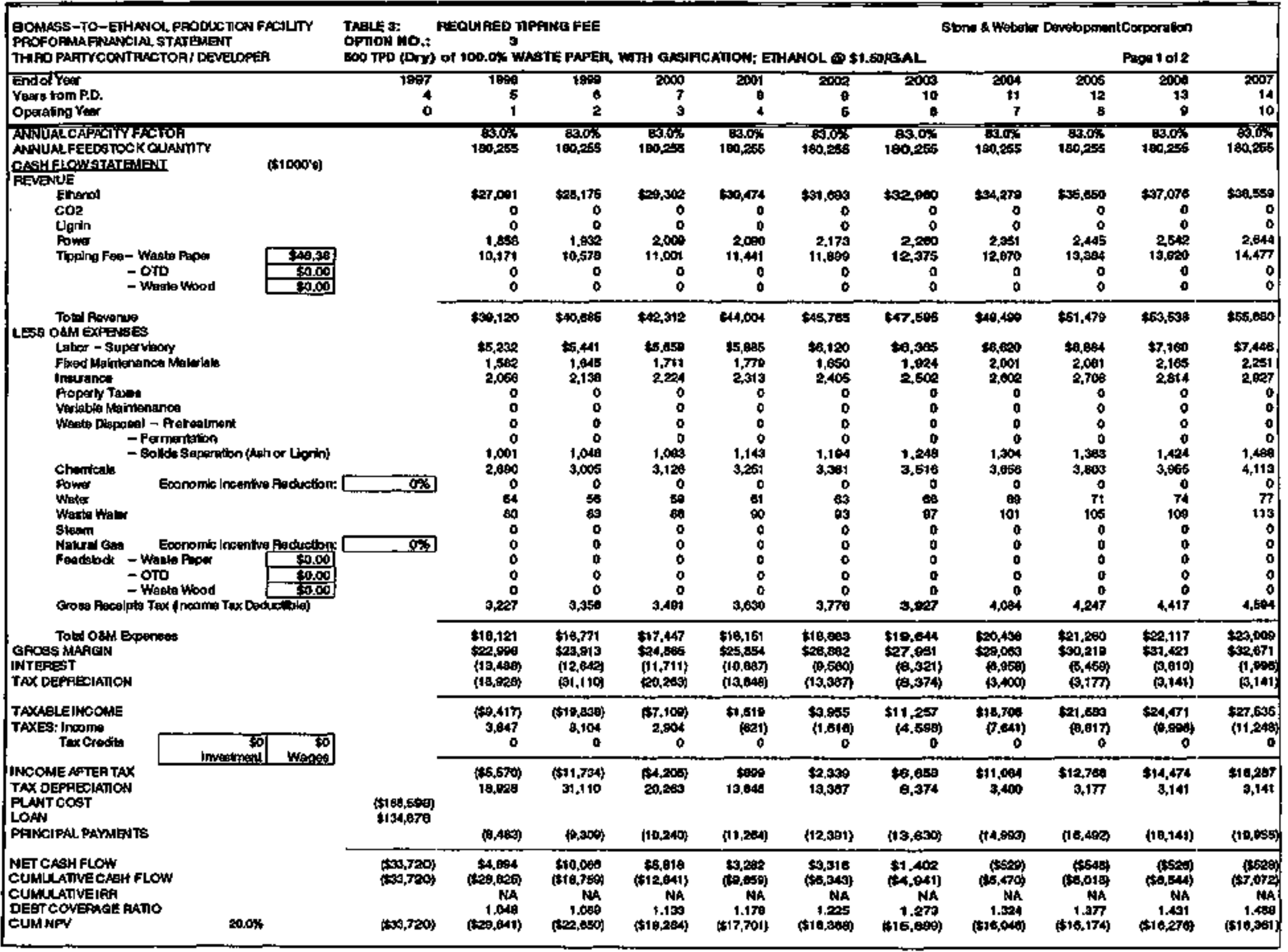




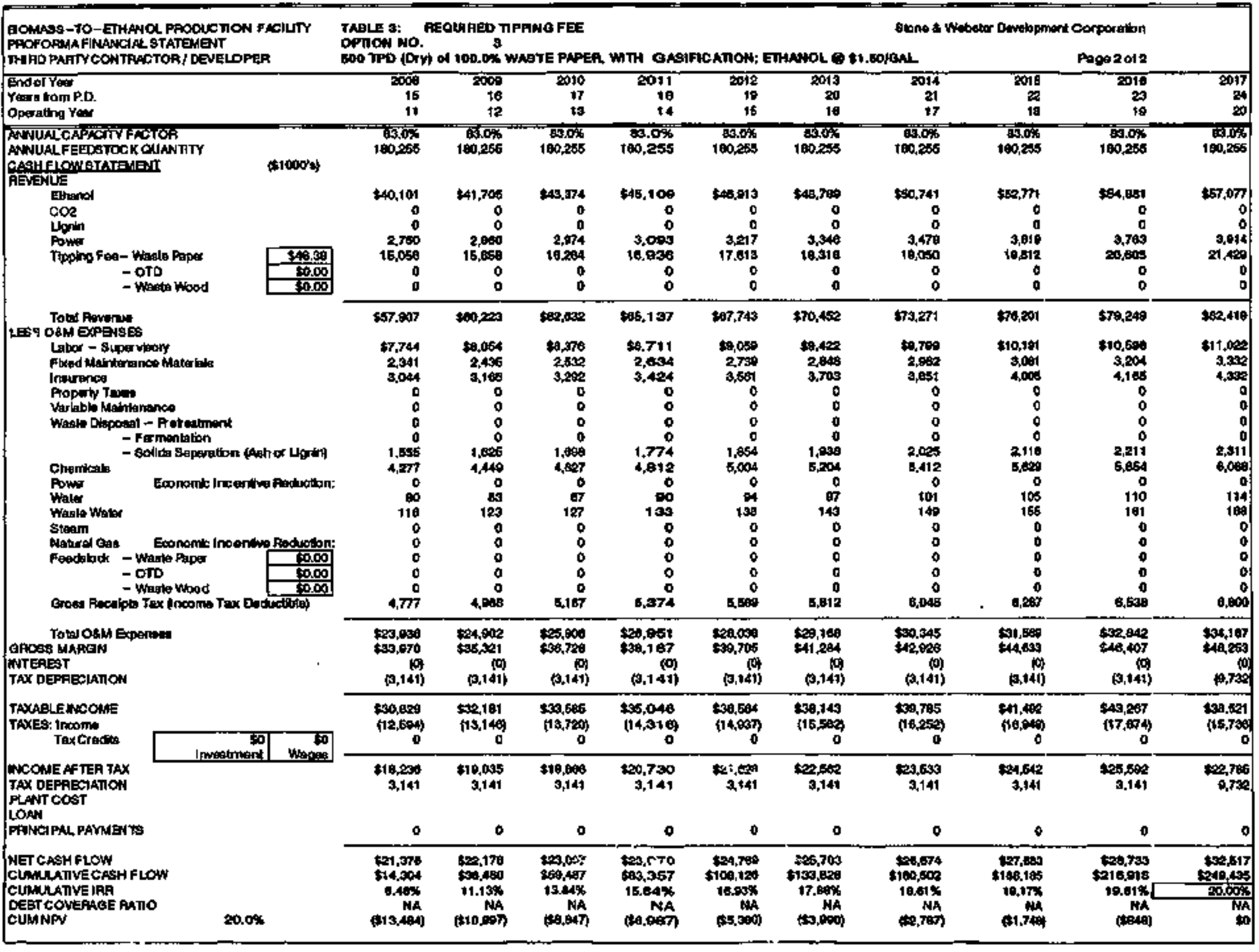




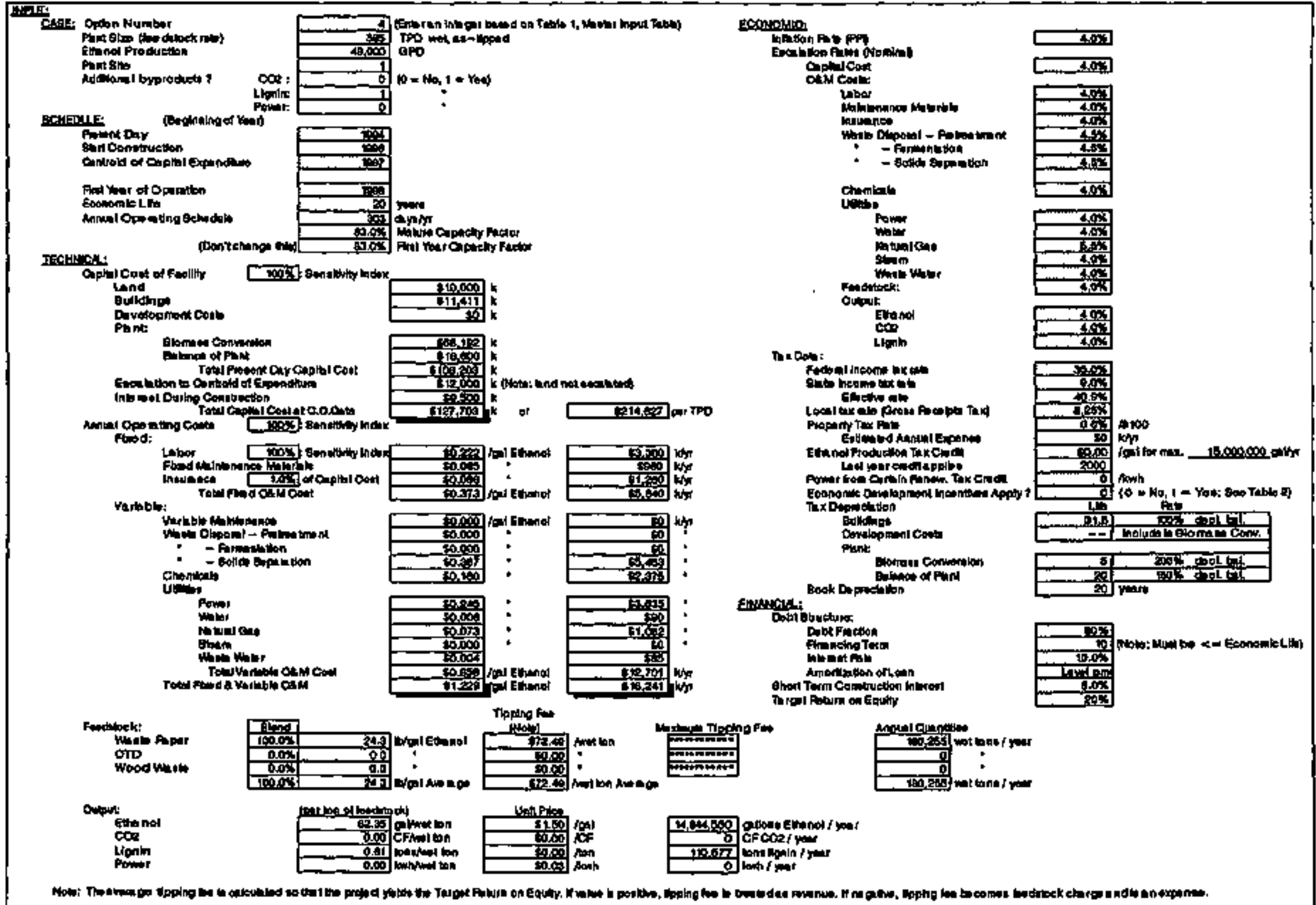




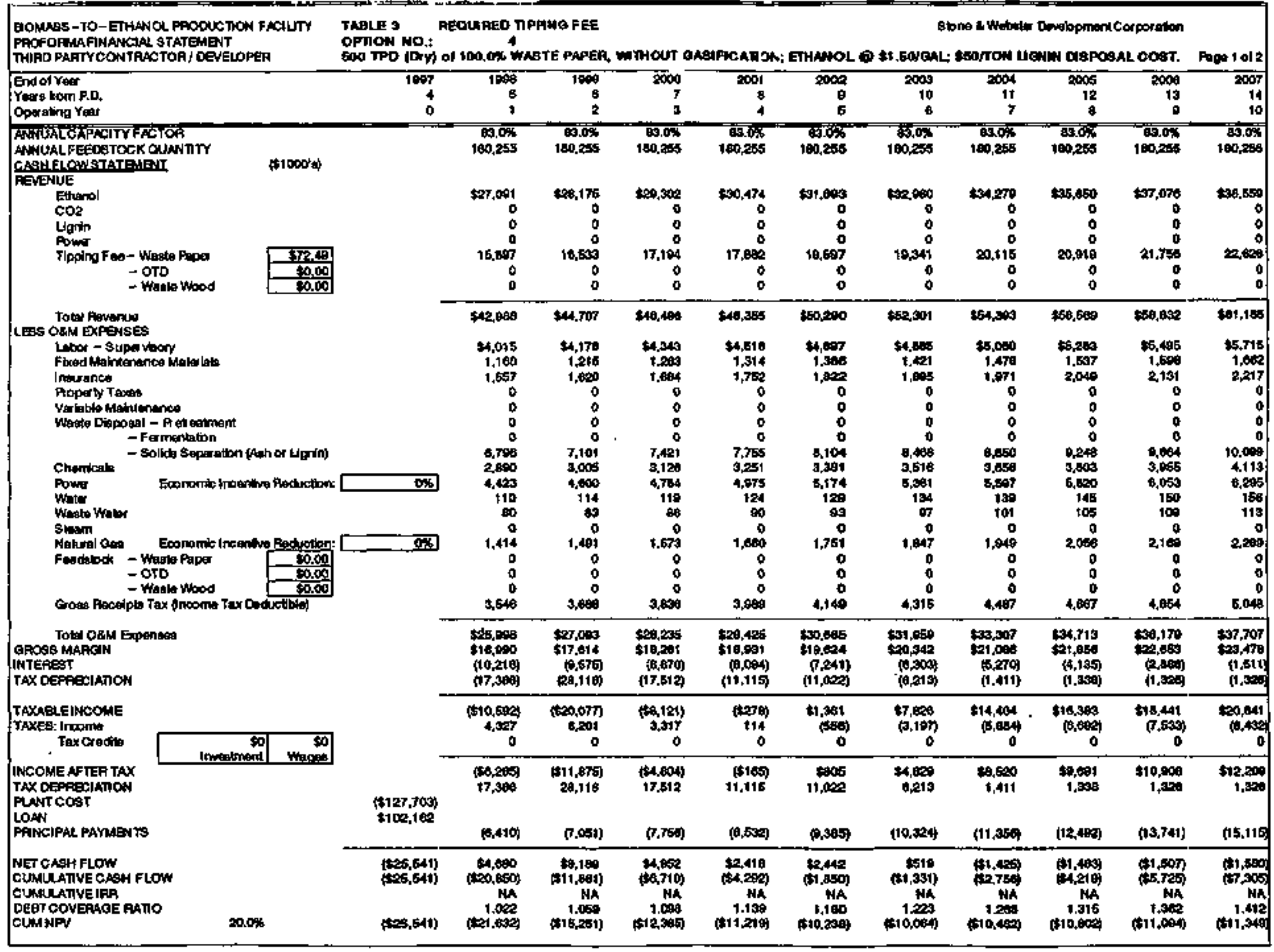




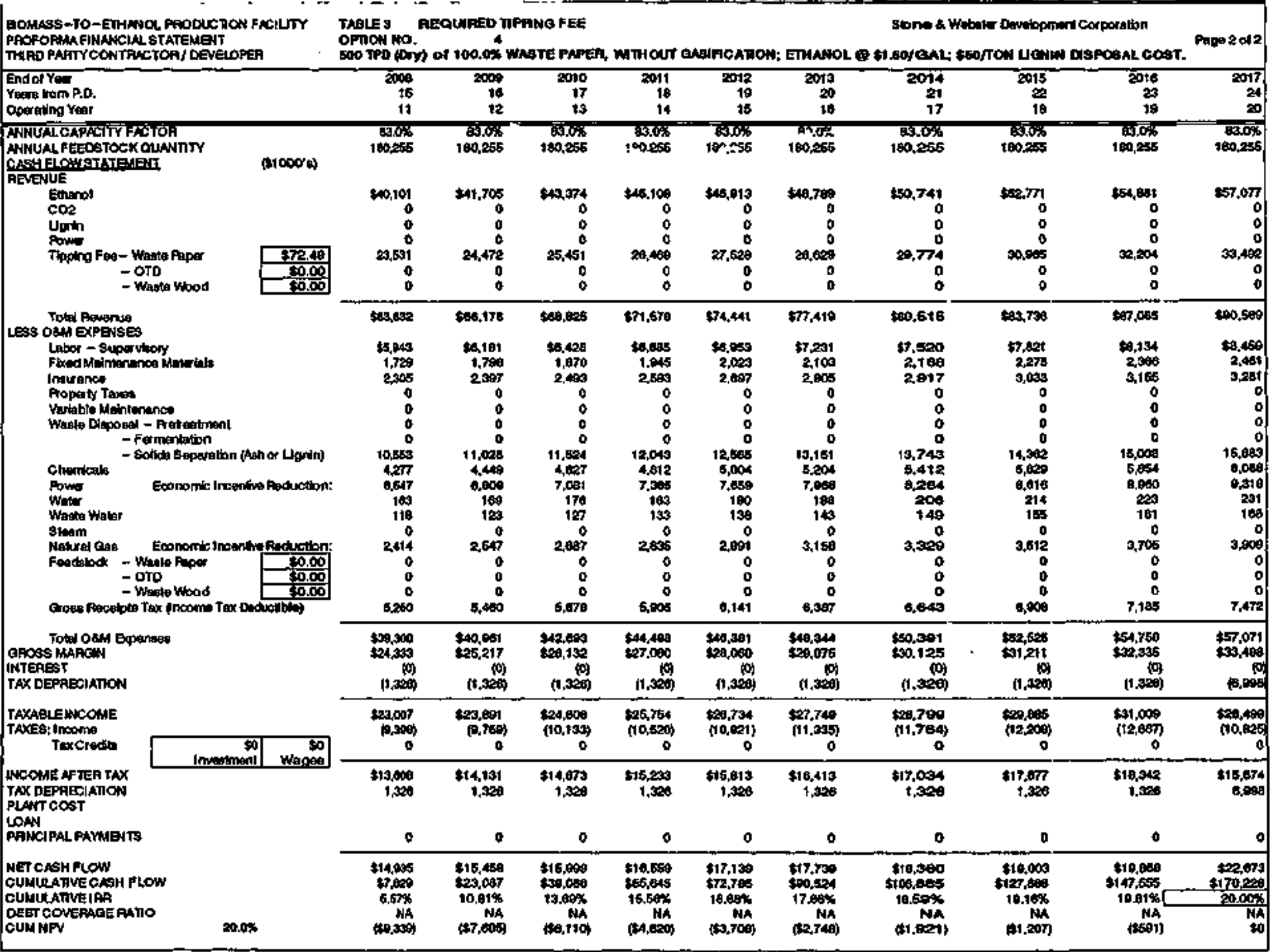




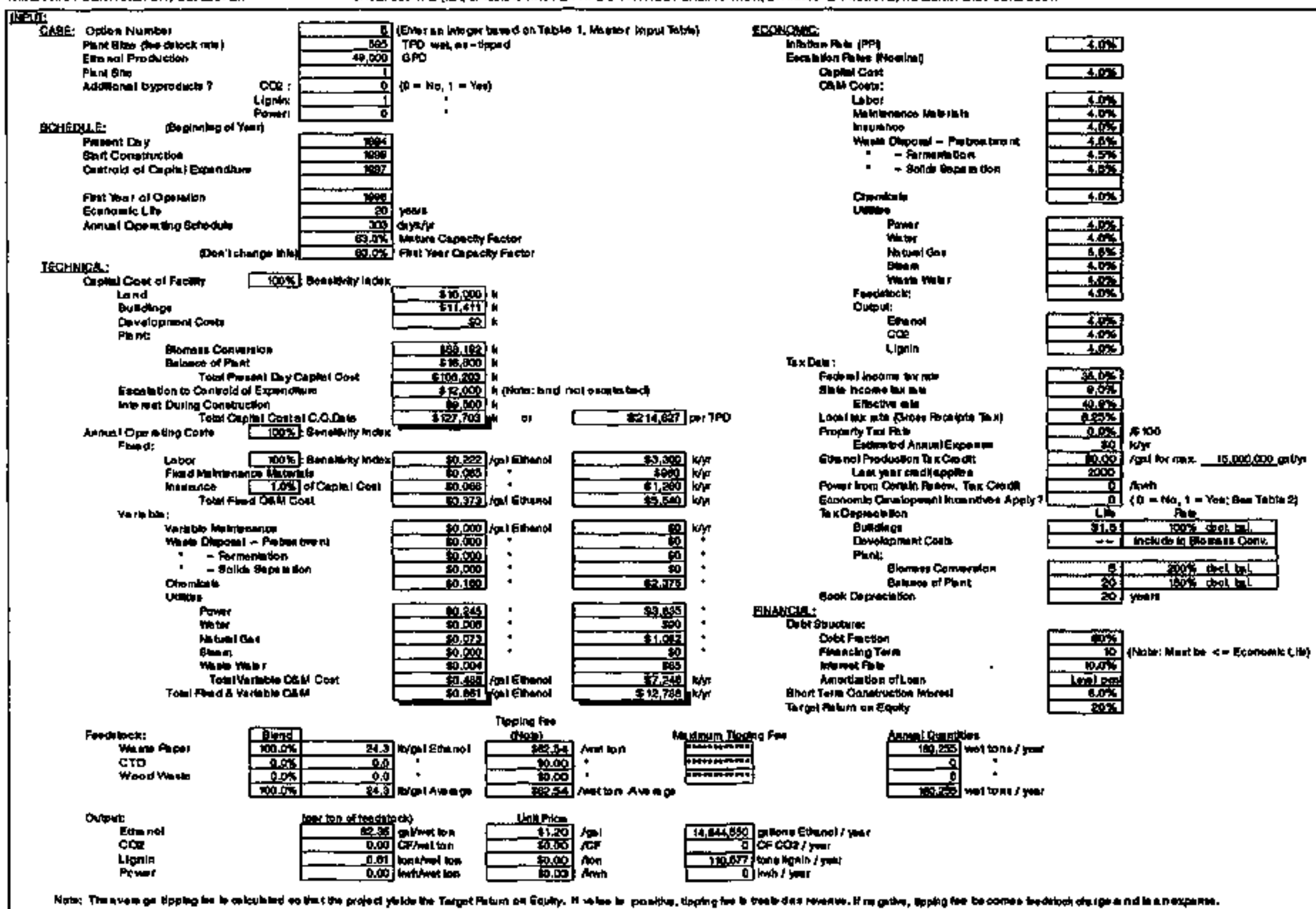




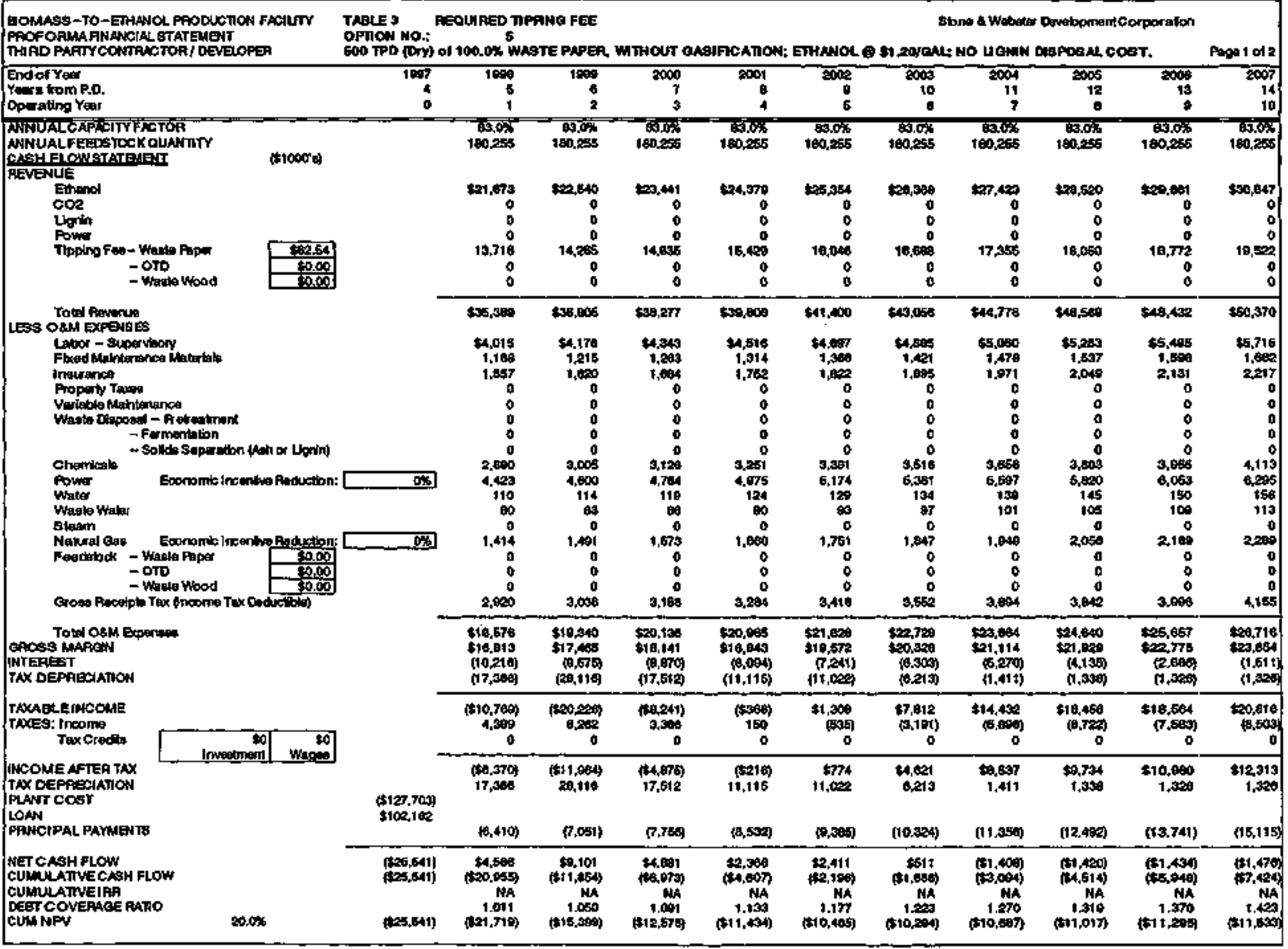




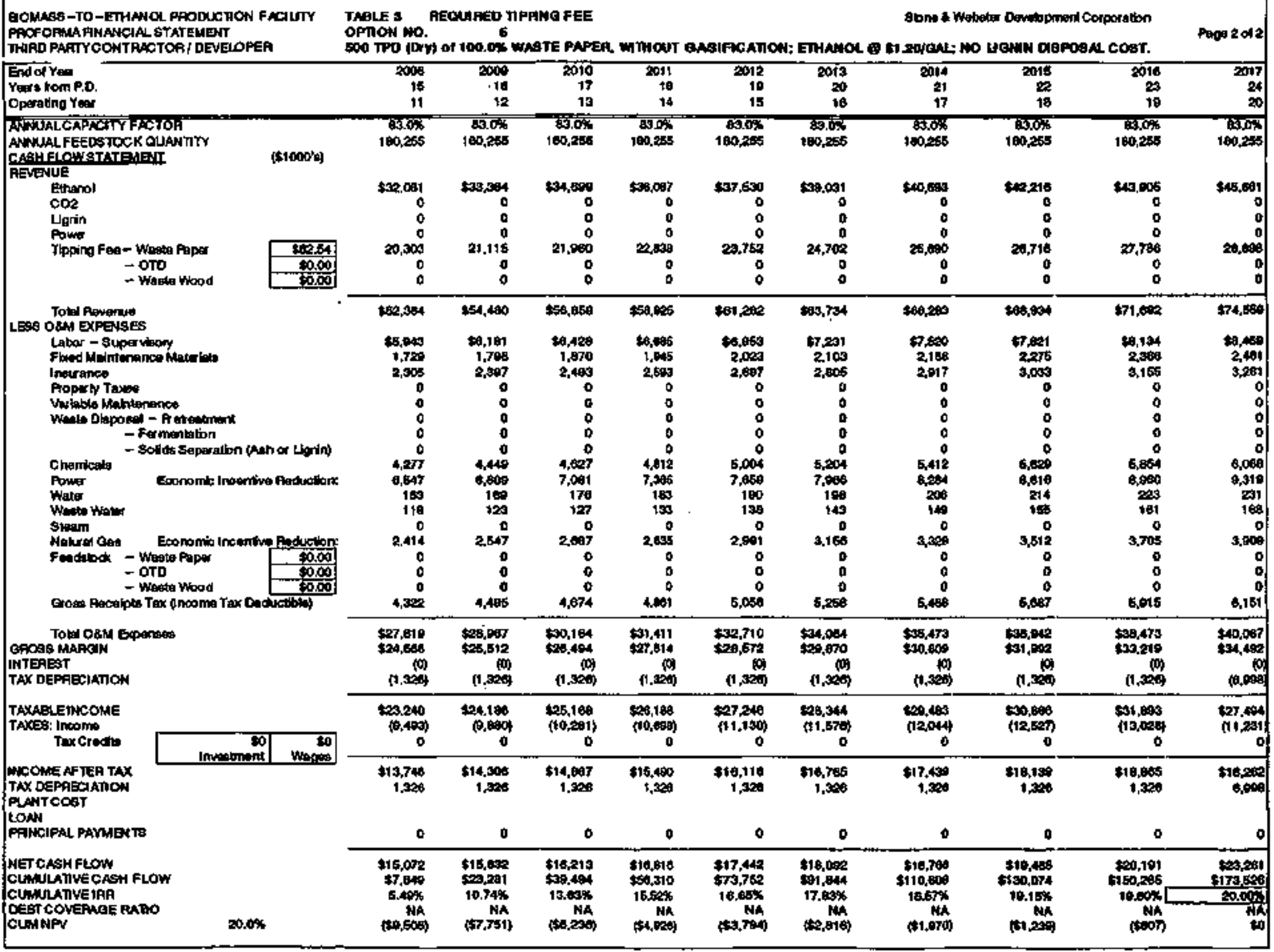


Fith Ware of Ophodbe

\section{Cones Opdon Number

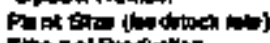 \\ Ethe nol Prodiction \\ Prent Eit: \\ Adationil bypotuch ? \\ thengin}

\section{Putinini Cay}

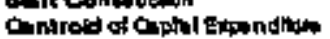

Feonontelis

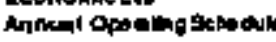
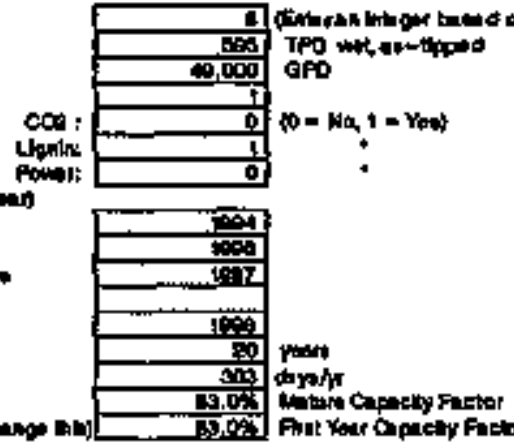

IEgendice

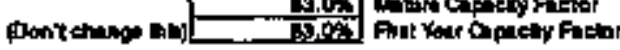

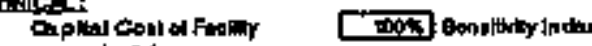

Oowationem corto

Punt:

Blomace Converion

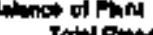

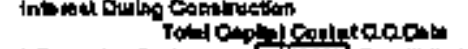

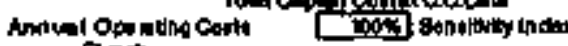
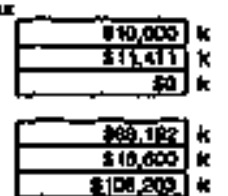

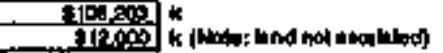

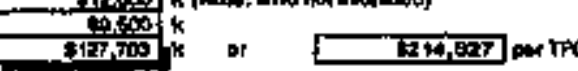

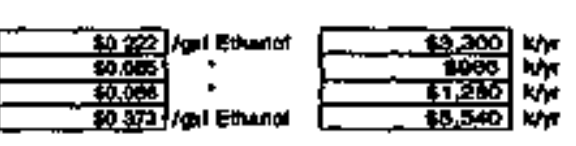
Varkbus Lever

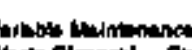

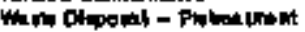
Climmots

Utionst.

pomar

Thionelone

mith unt

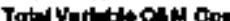

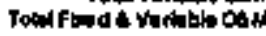
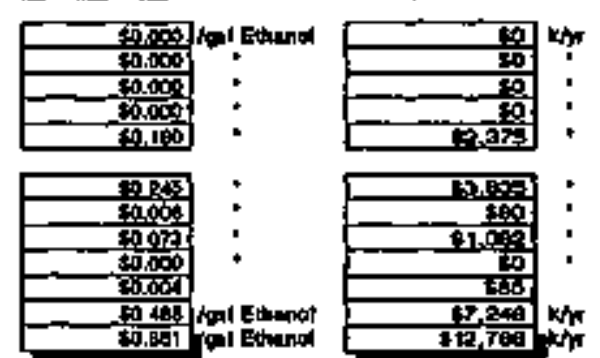

Fondorti:

$$
\text { Ord inter }
$$

Wrogut
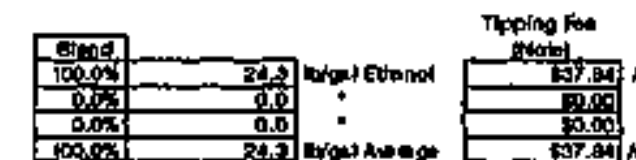

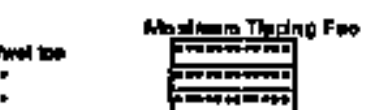

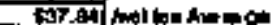

$$
\text { Lend }
$$

Interion

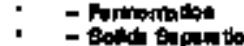

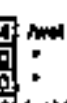

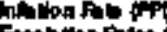

Dapulcoll

Lits:

Ineven

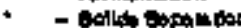

Chemblat:

Utillow

Power

Nhenalins

thenters

Fonotione

cesting

DrDat:

congan

Fadpel lators ak mo

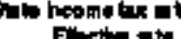

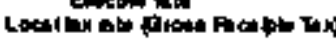

Propotity Tan Fe

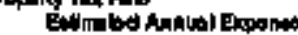

Ethenod Protuedion Tex C.edh

Lin wor ondikper

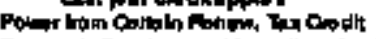

it ingintion Musty ?

xOnpectibs

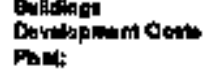

Bloment Carmotition

Ginswicis:

Book on pucistion

bit Anownes:

Dobt Finction

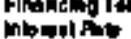

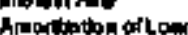

shen Trerm construetion Intwen:

herod Promem on fighy lobo:
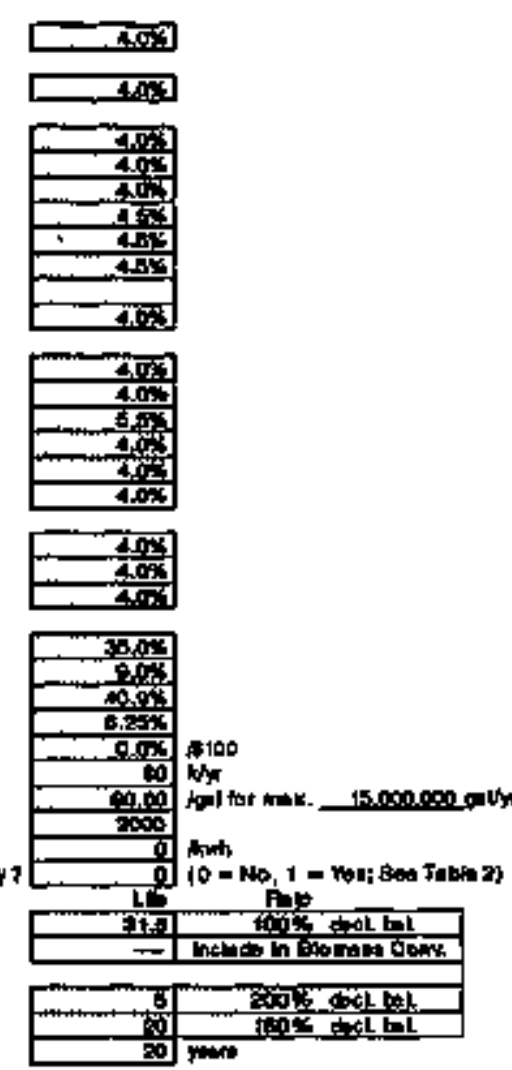

Onbut

Eganod
Cop
tionin
Pomern

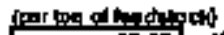

Un: this:

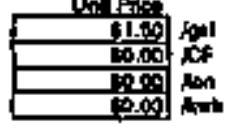

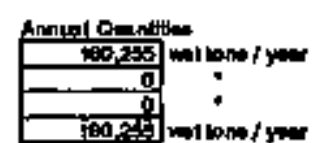

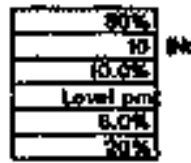

Whot Huel be se Feonomic (th)

然 


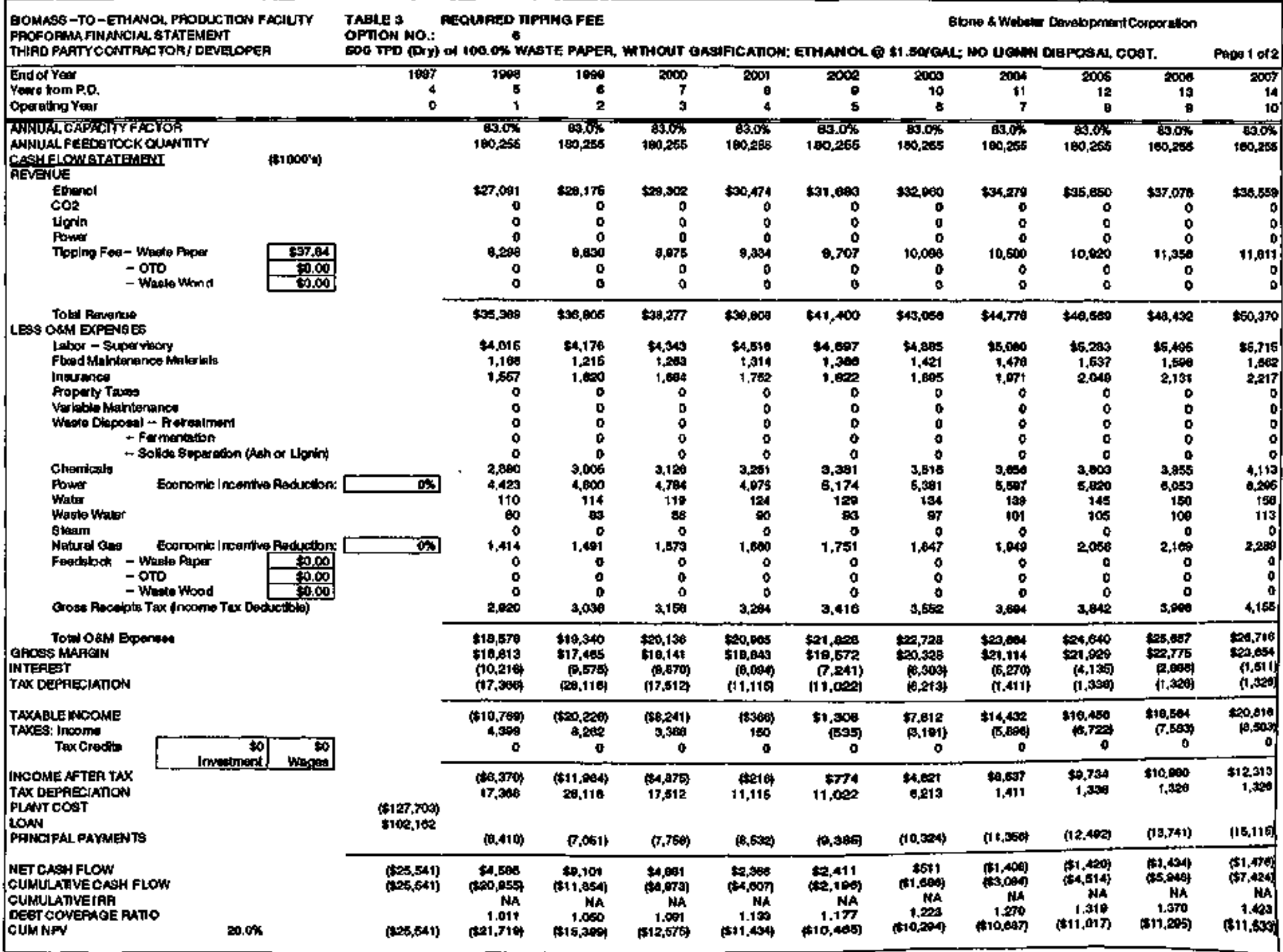




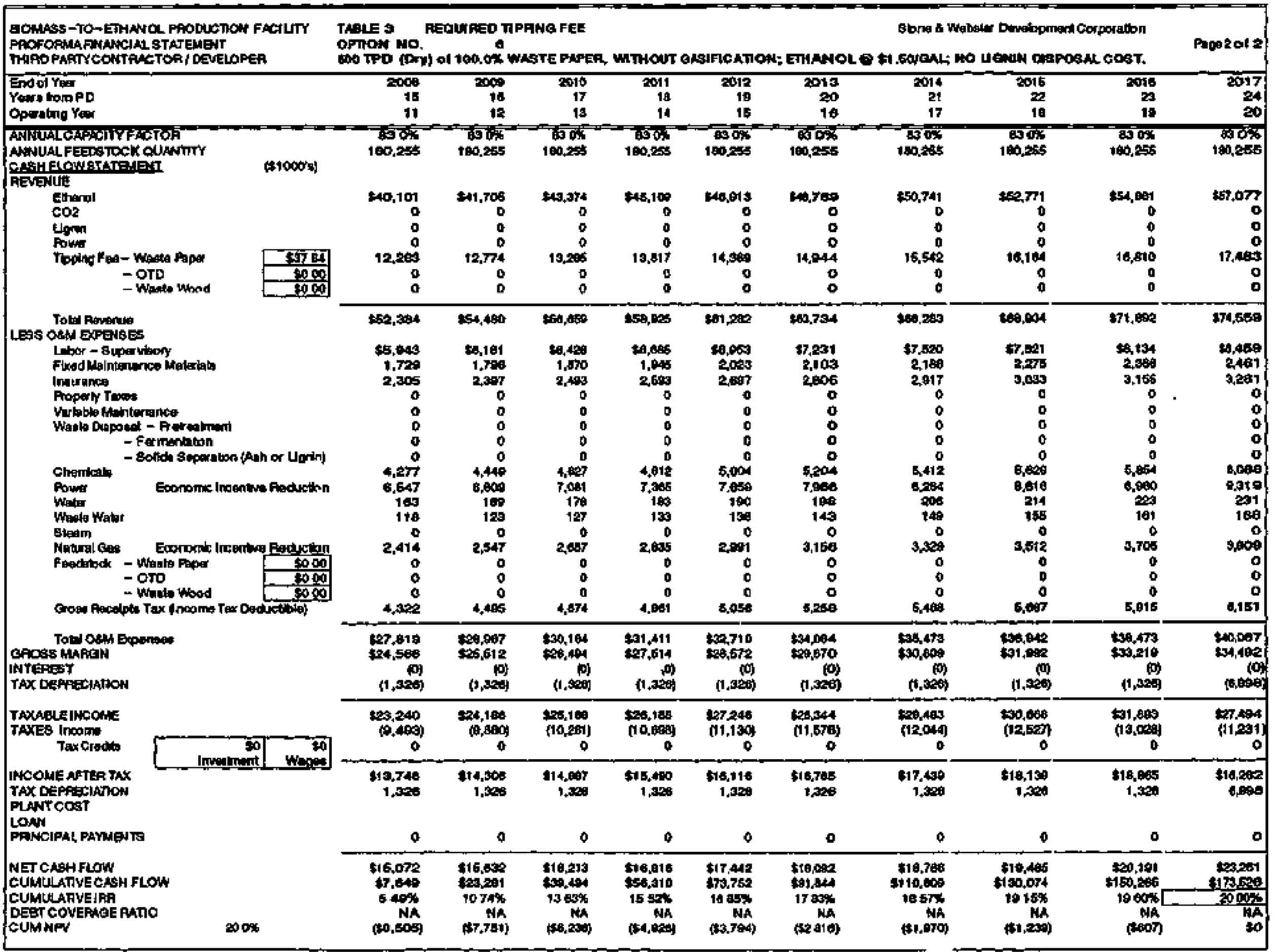




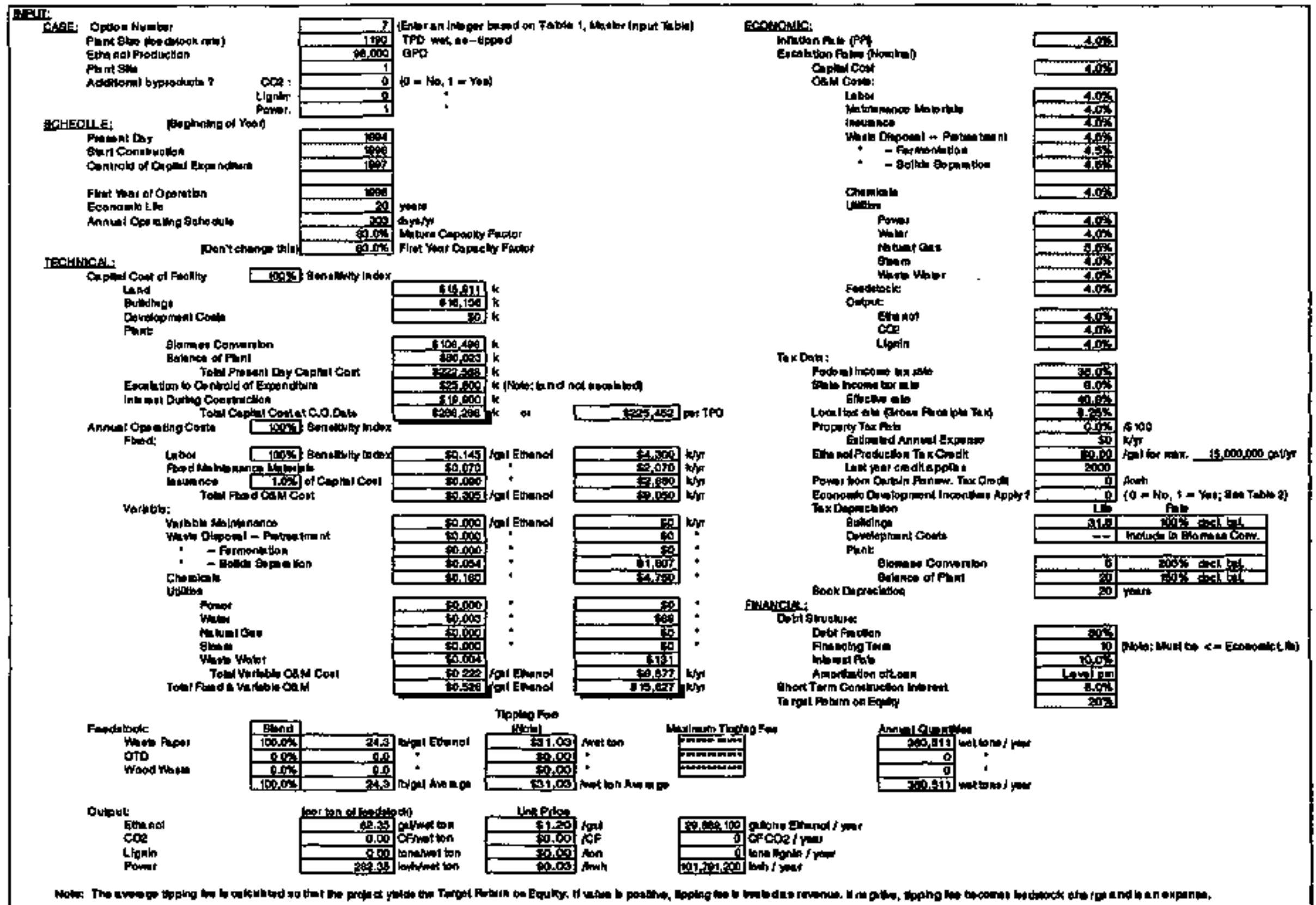




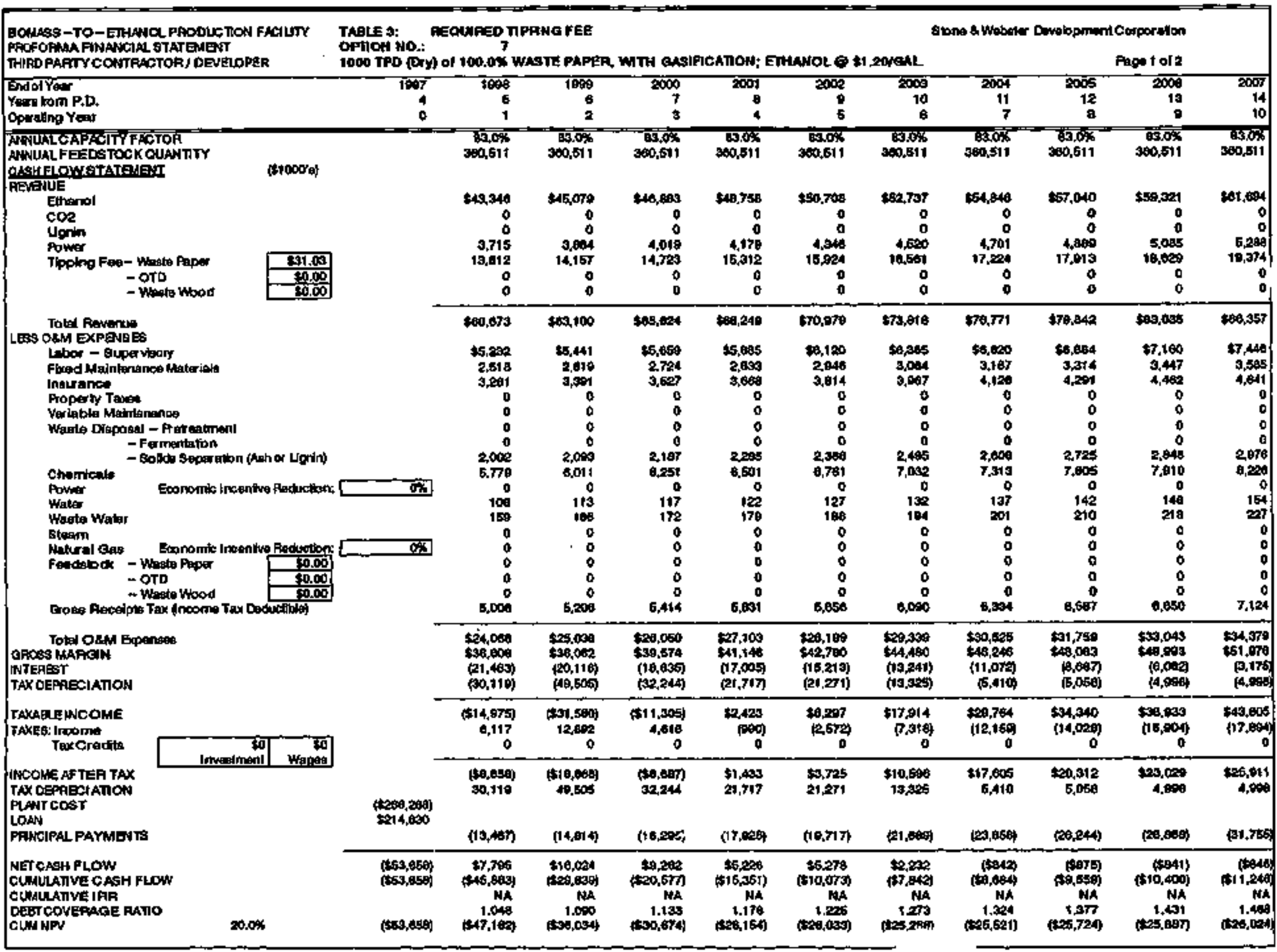




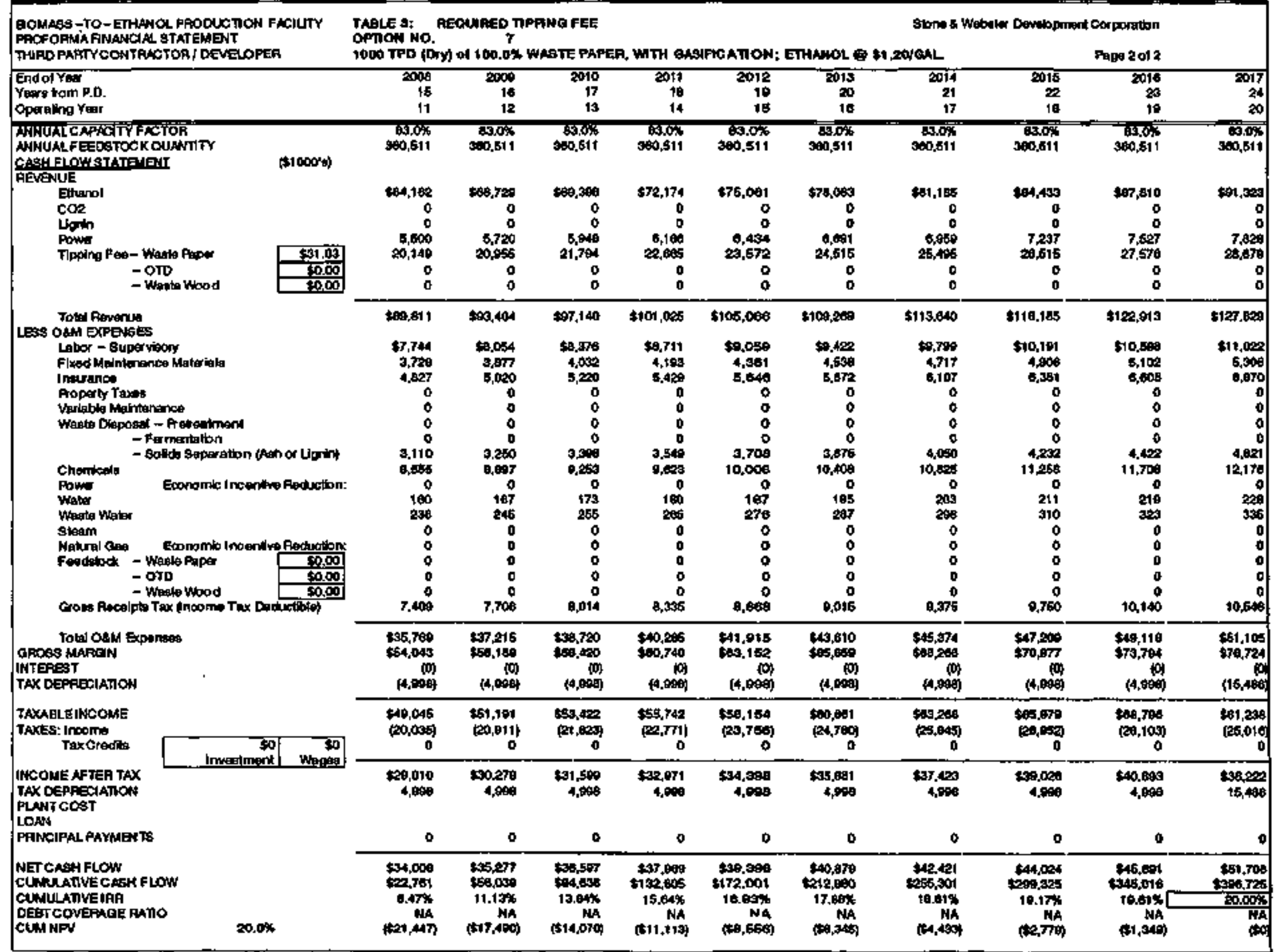




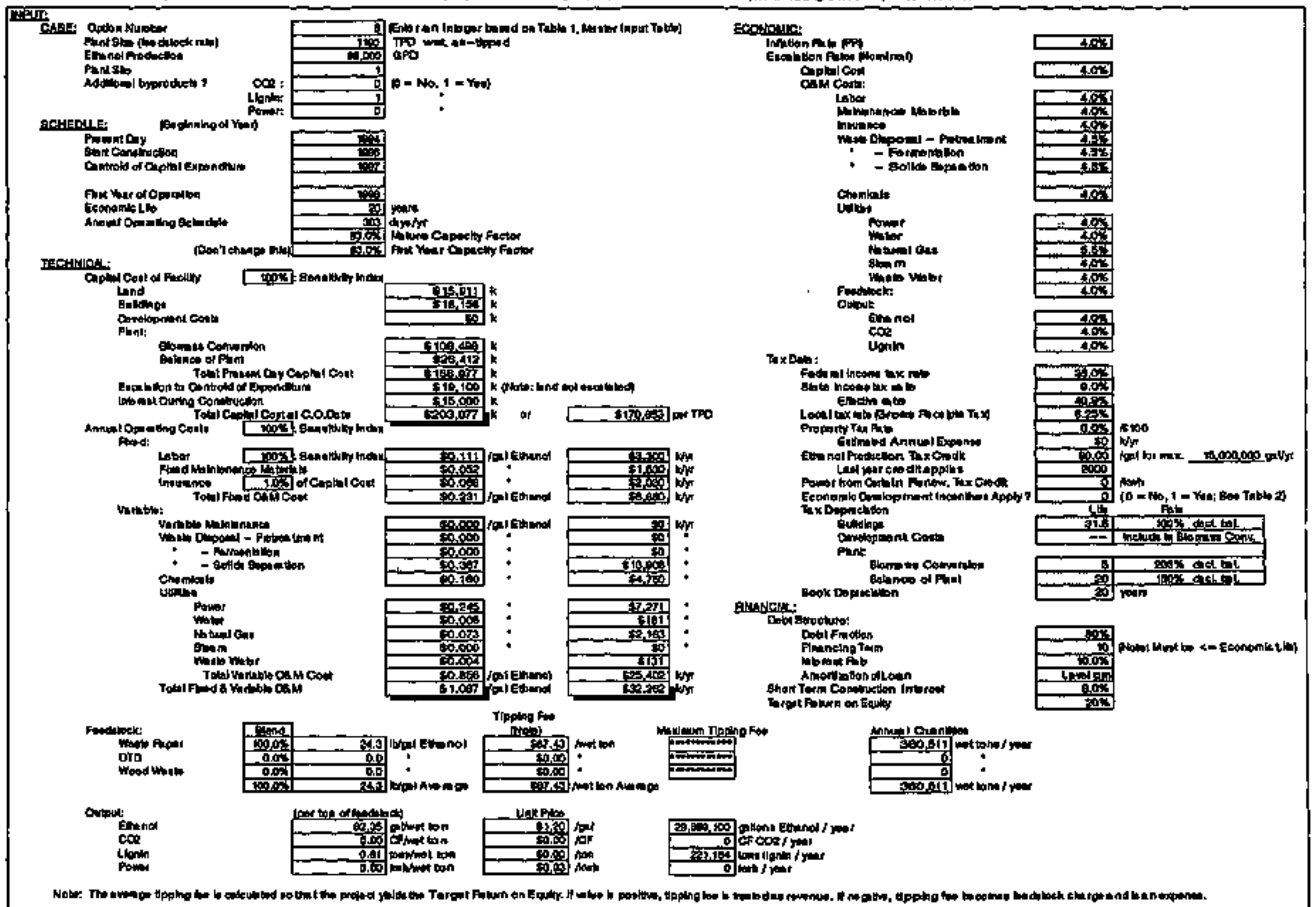




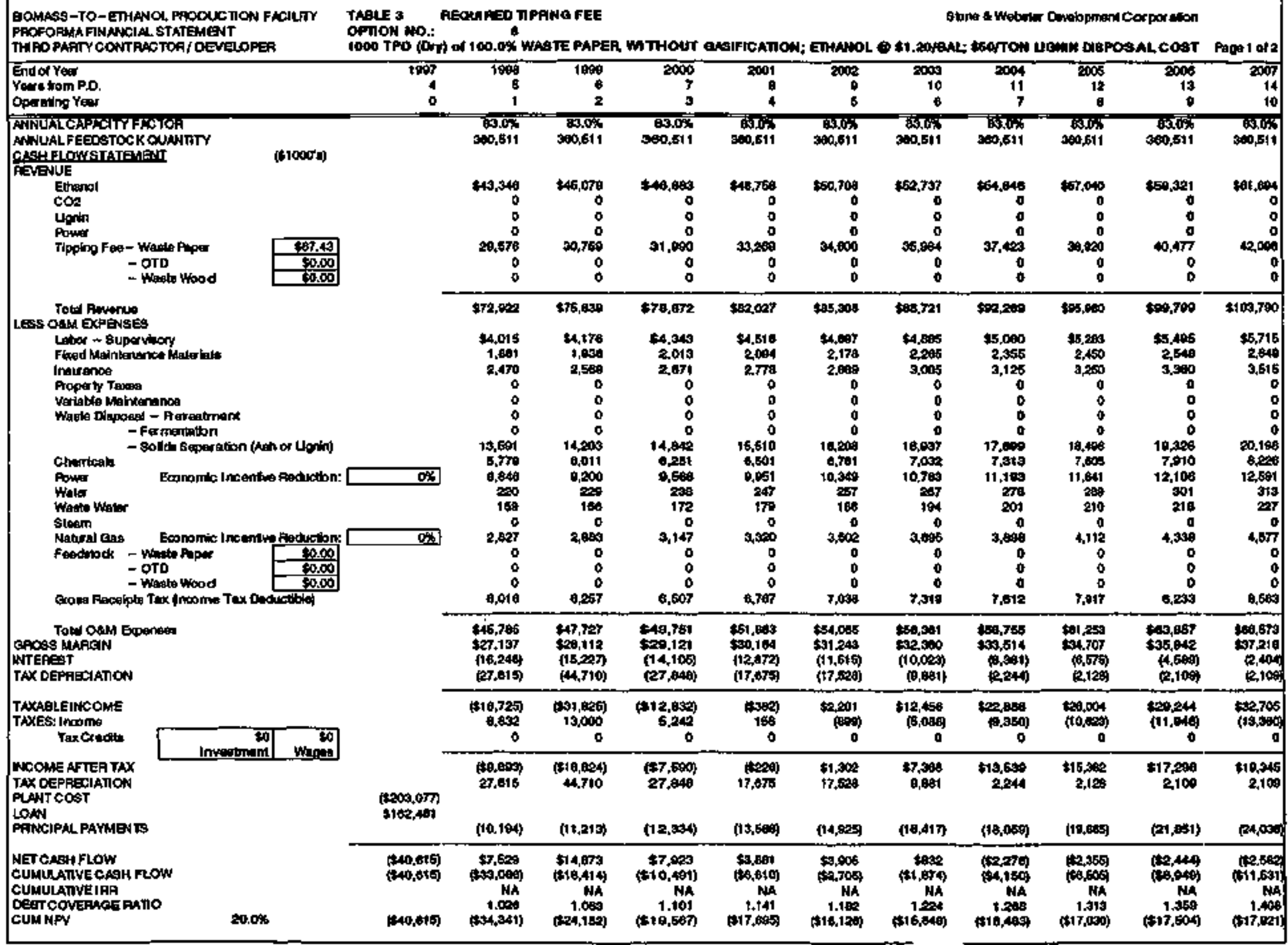




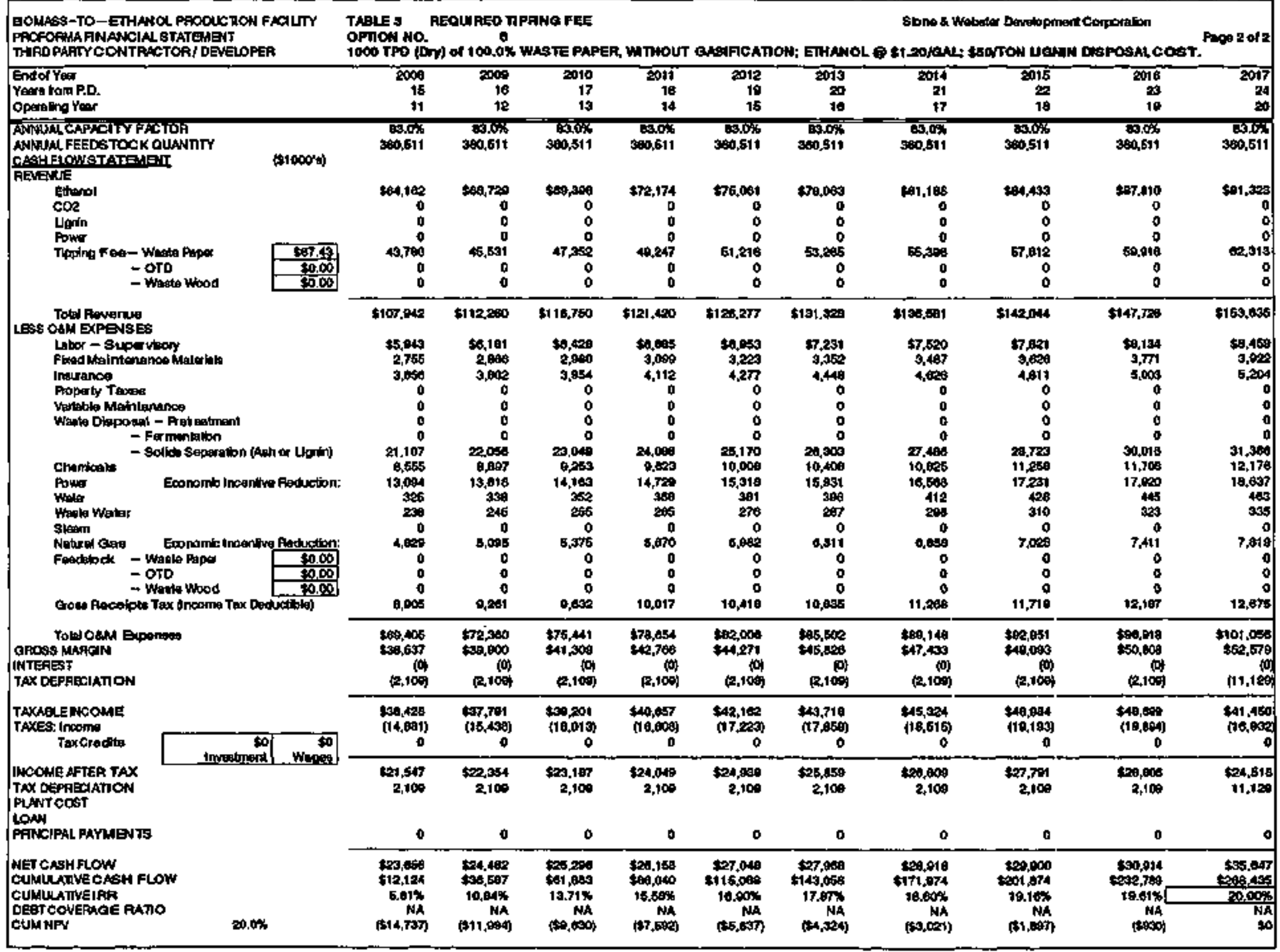




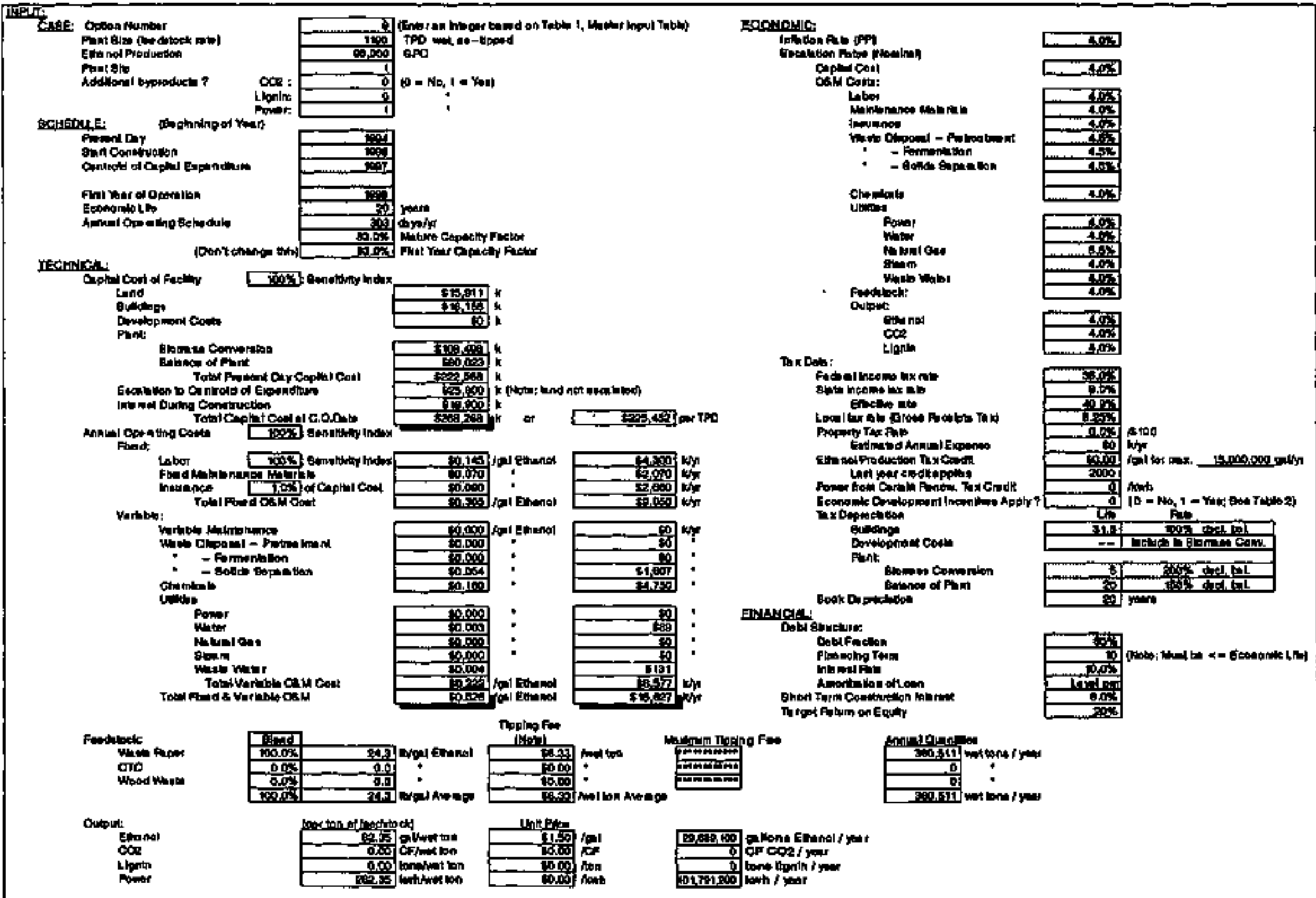

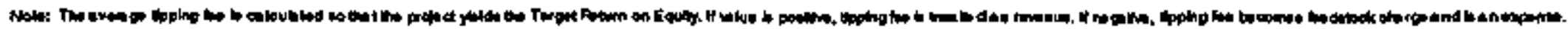




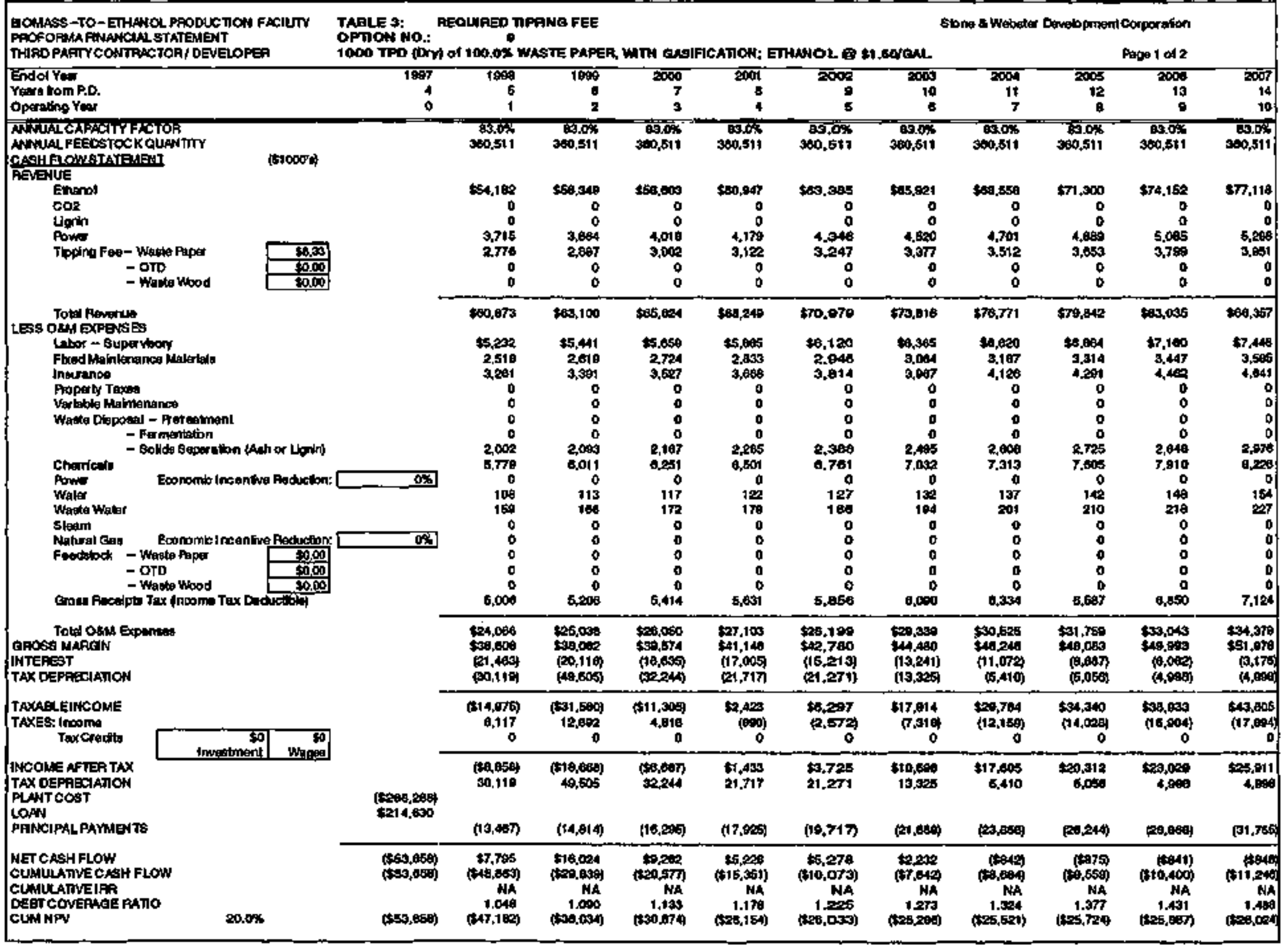




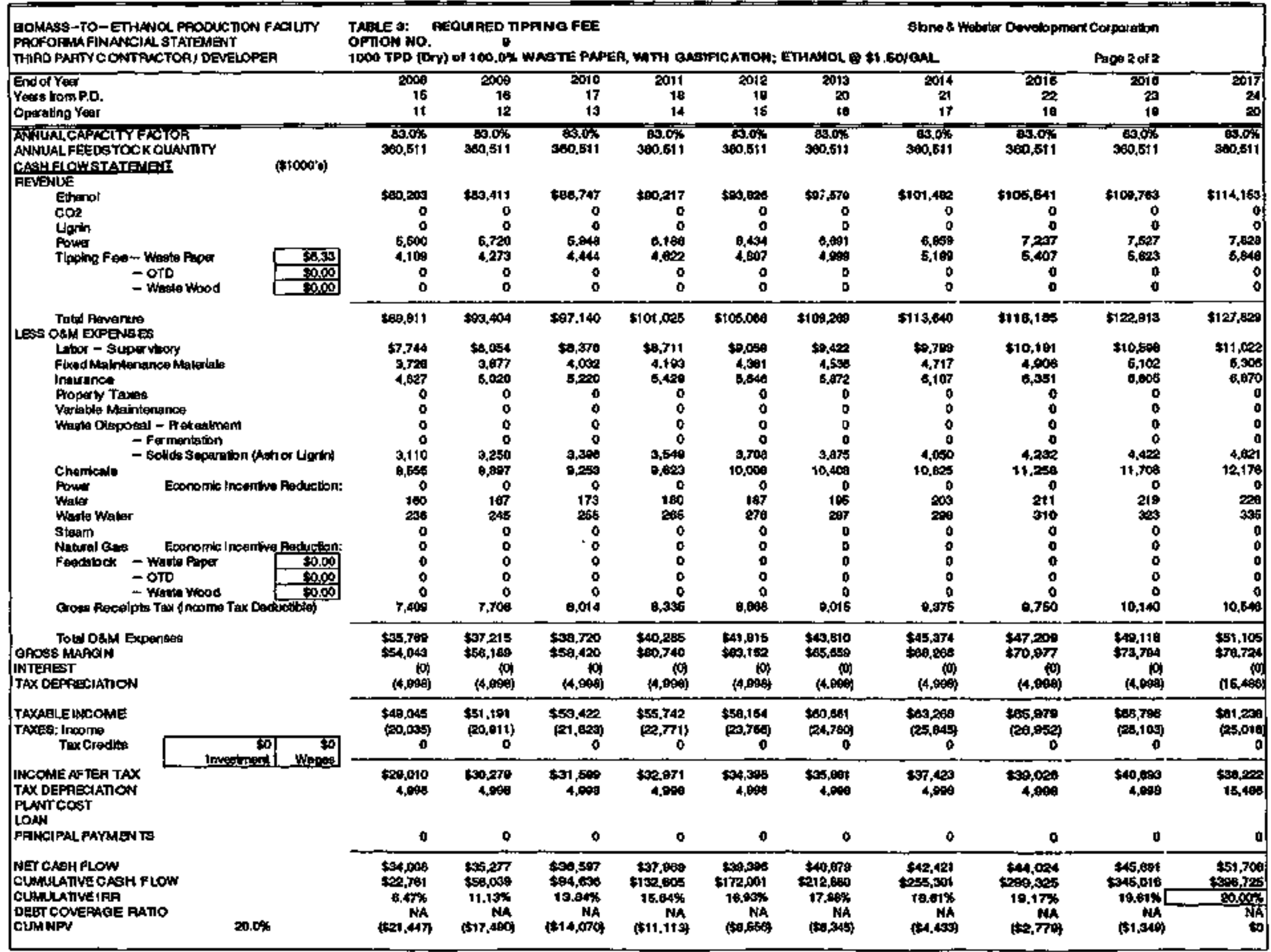




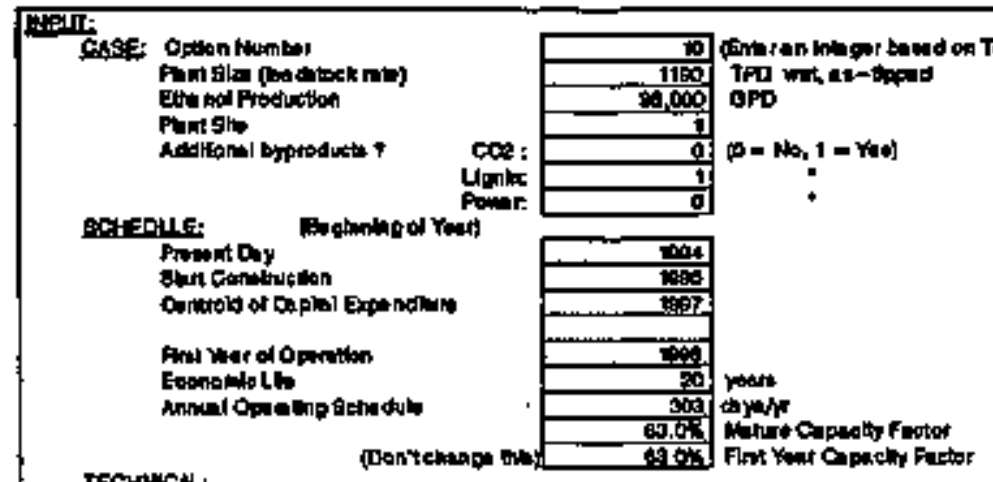

(Don tekeape why

IFCHAy

\section{Oaphalcont of Follty \\ bund

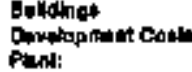

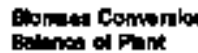 \\ Tod Prostant on cajial con

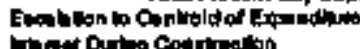

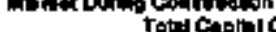

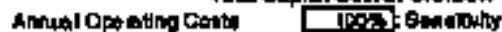 \\ Fheod:

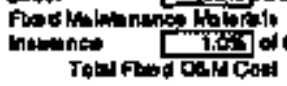

Verben:

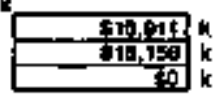

\section{-}

tion

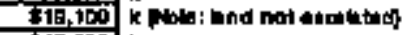

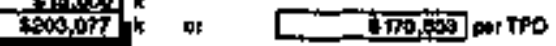
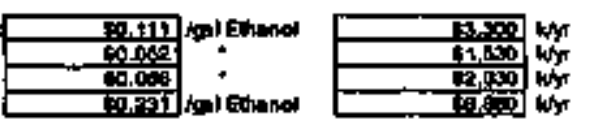

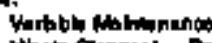

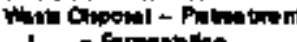

- - Fonmapion

Chenilutil

Pover

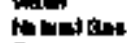

grom

Tortroterto

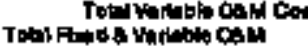

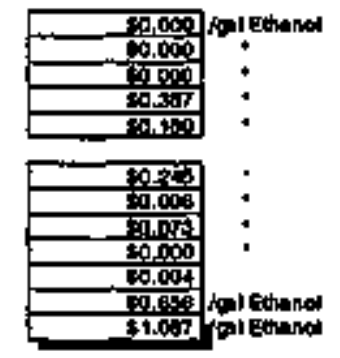

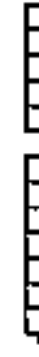

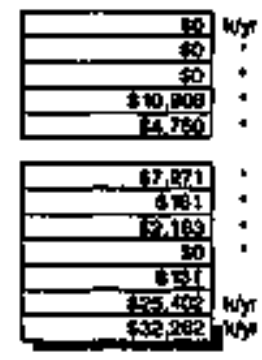

Thoping Fin
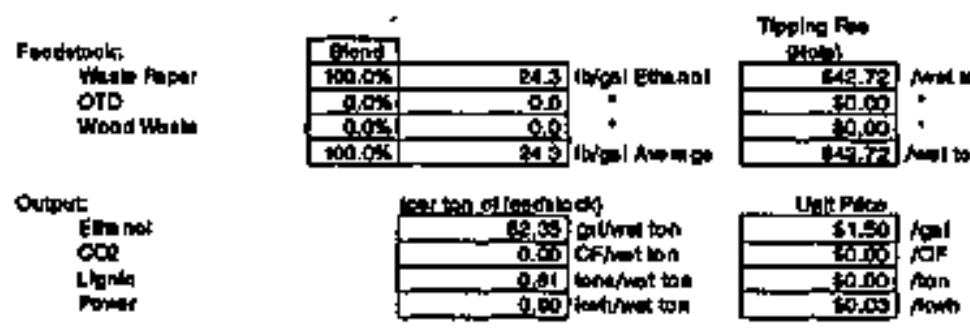

Con

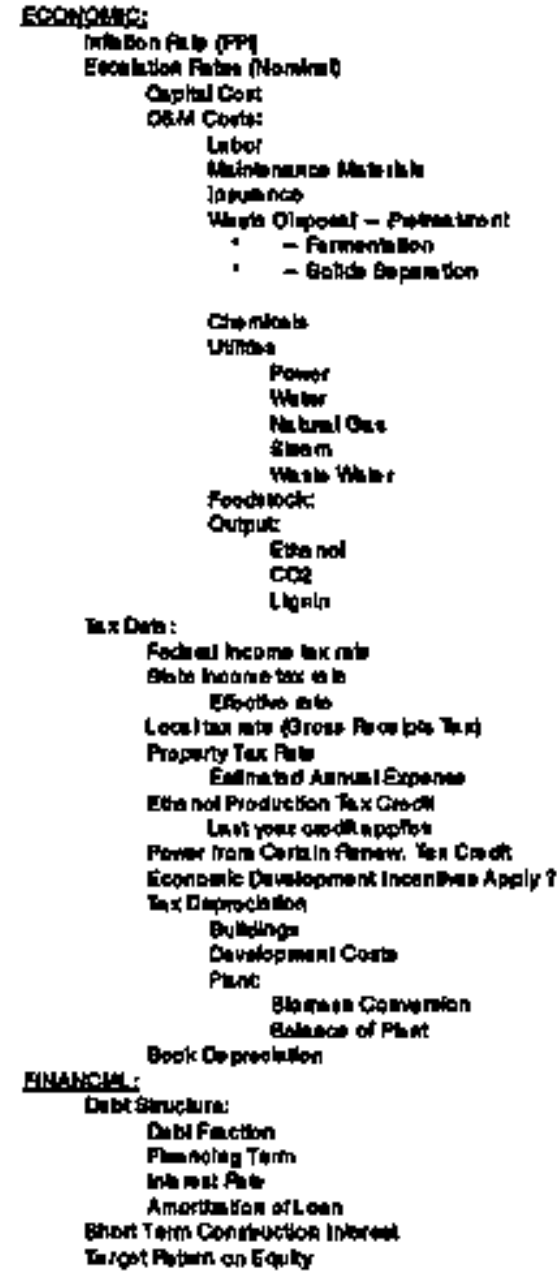

Ecomigutic

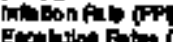

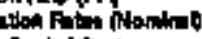

apen Conts:

Lntor

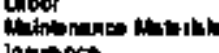

Jantancos

Whol olposer - Pumetront

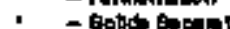

Cramionto

rom

Powor

Ptorlons

when wher

Foodanek

Cranol

Iotorin

Mxam:

Itorin

Rodal hedre wic nt

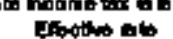

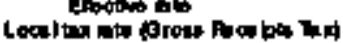

Propaty Tax pate

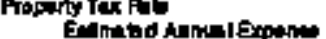

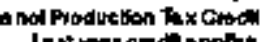

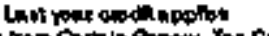

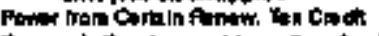

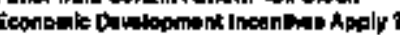

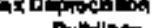

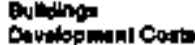

cavalog

ghanun comminan

Book Co procition

\section{Elsubers.}

1.

Dubfection

Plenolitg Thrm

Amertielsen of Loe

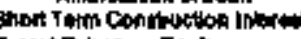

trest thitum on Equky
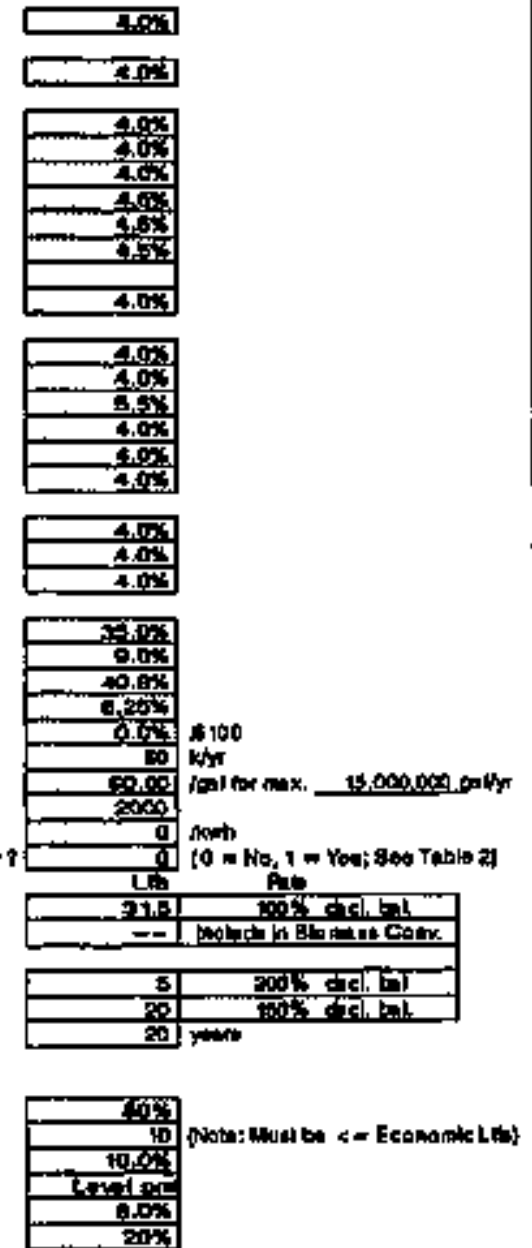


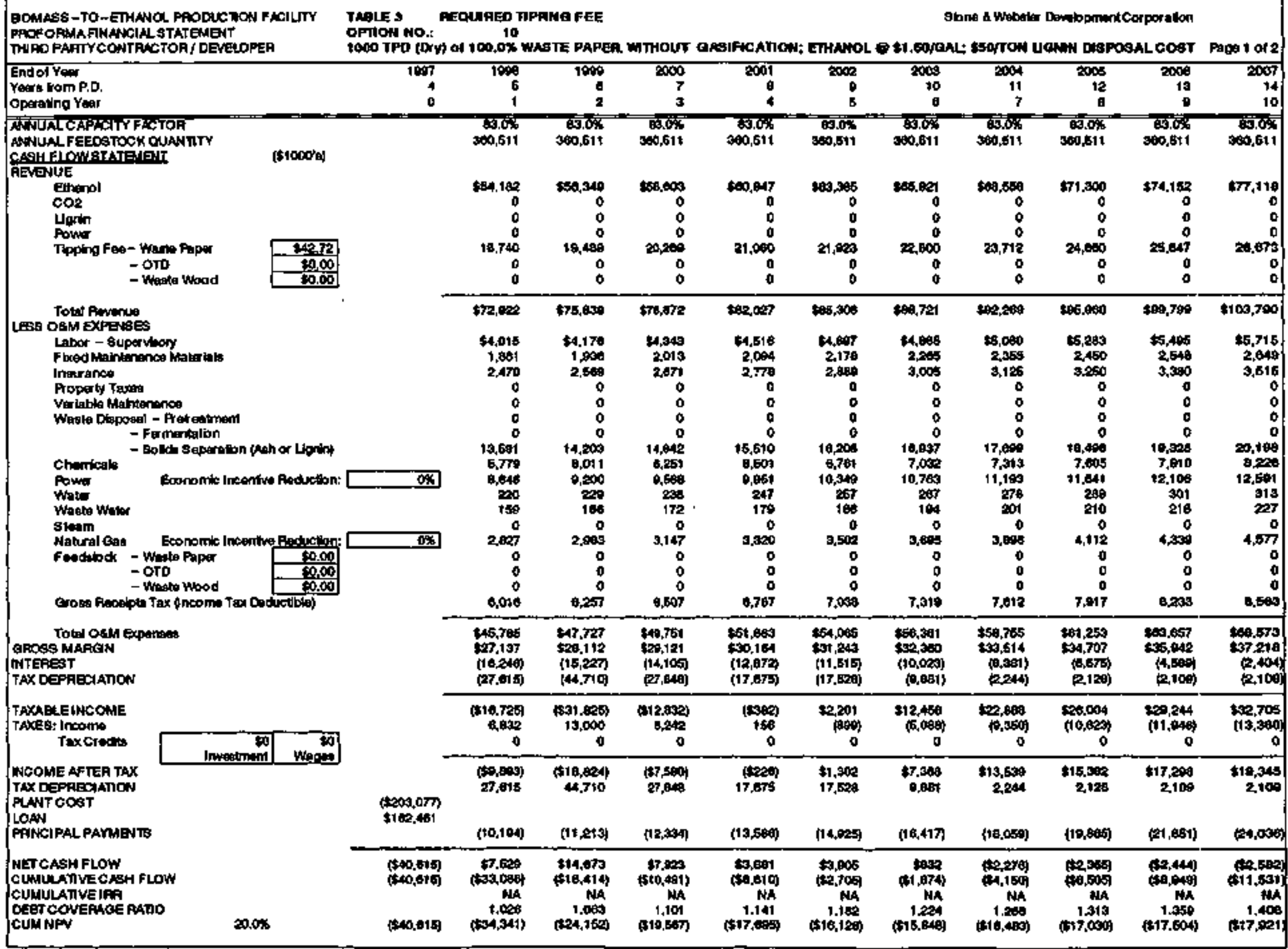




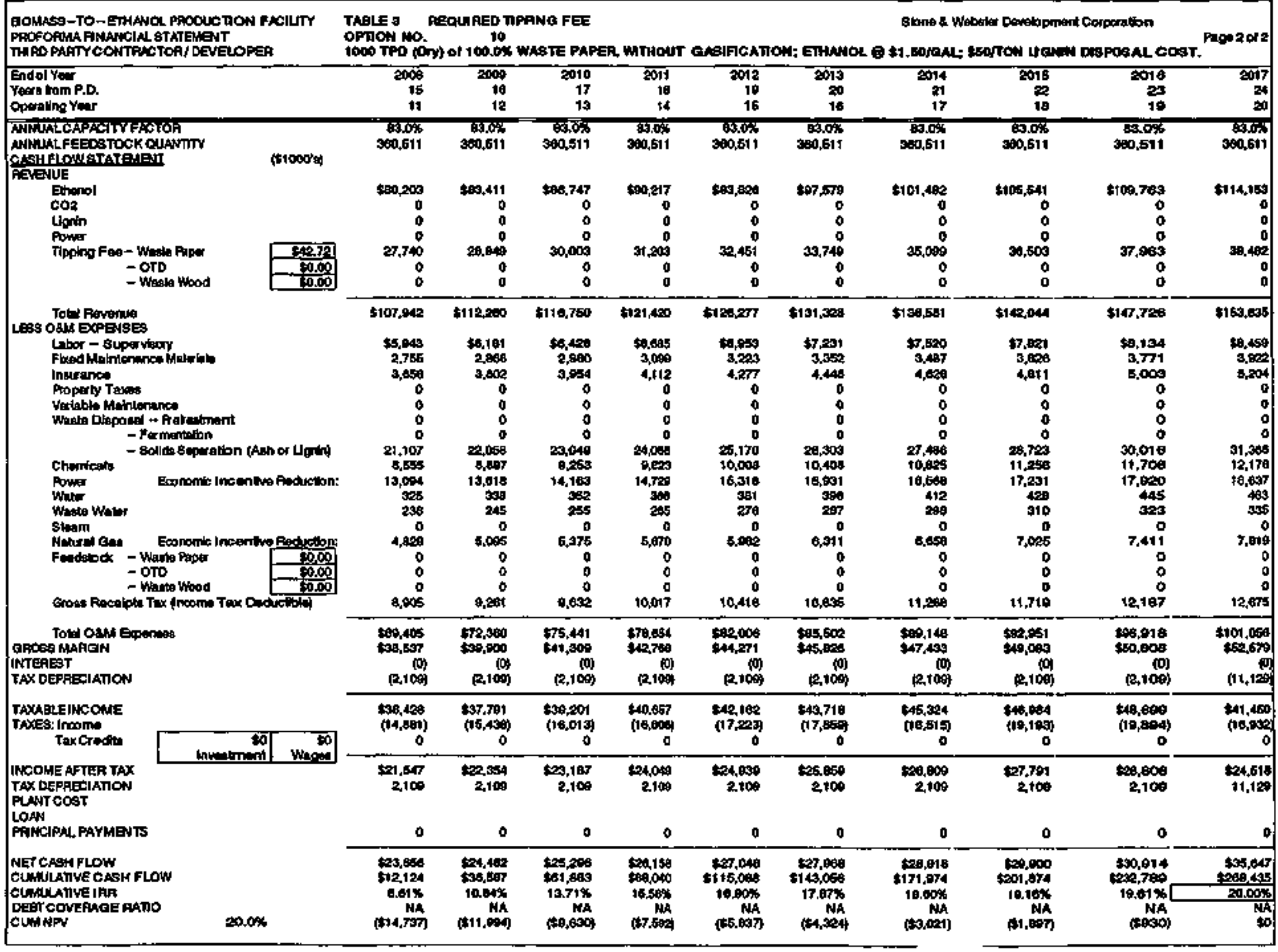




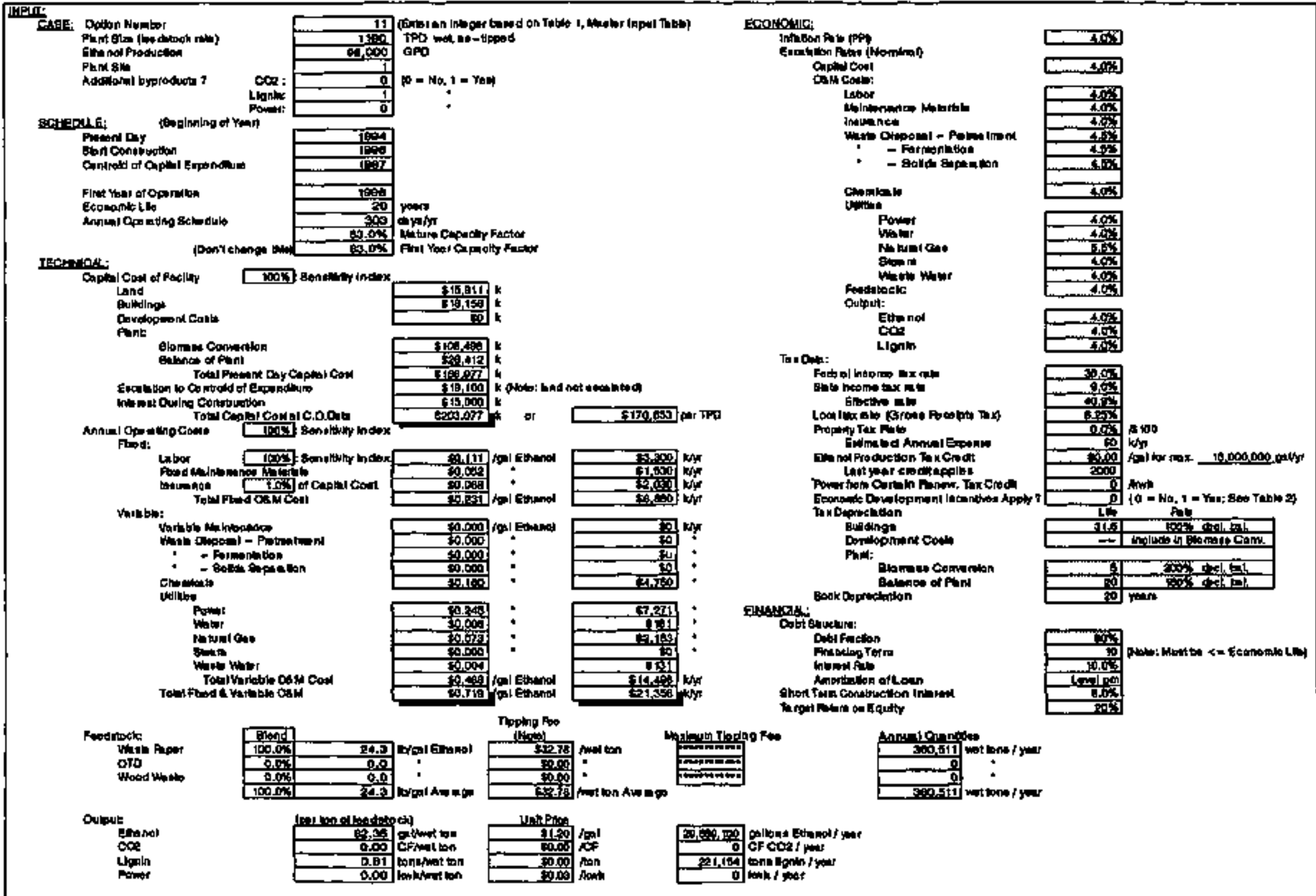

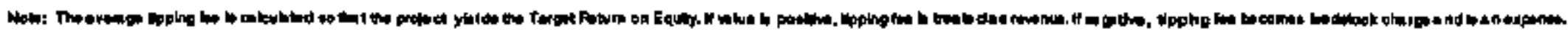




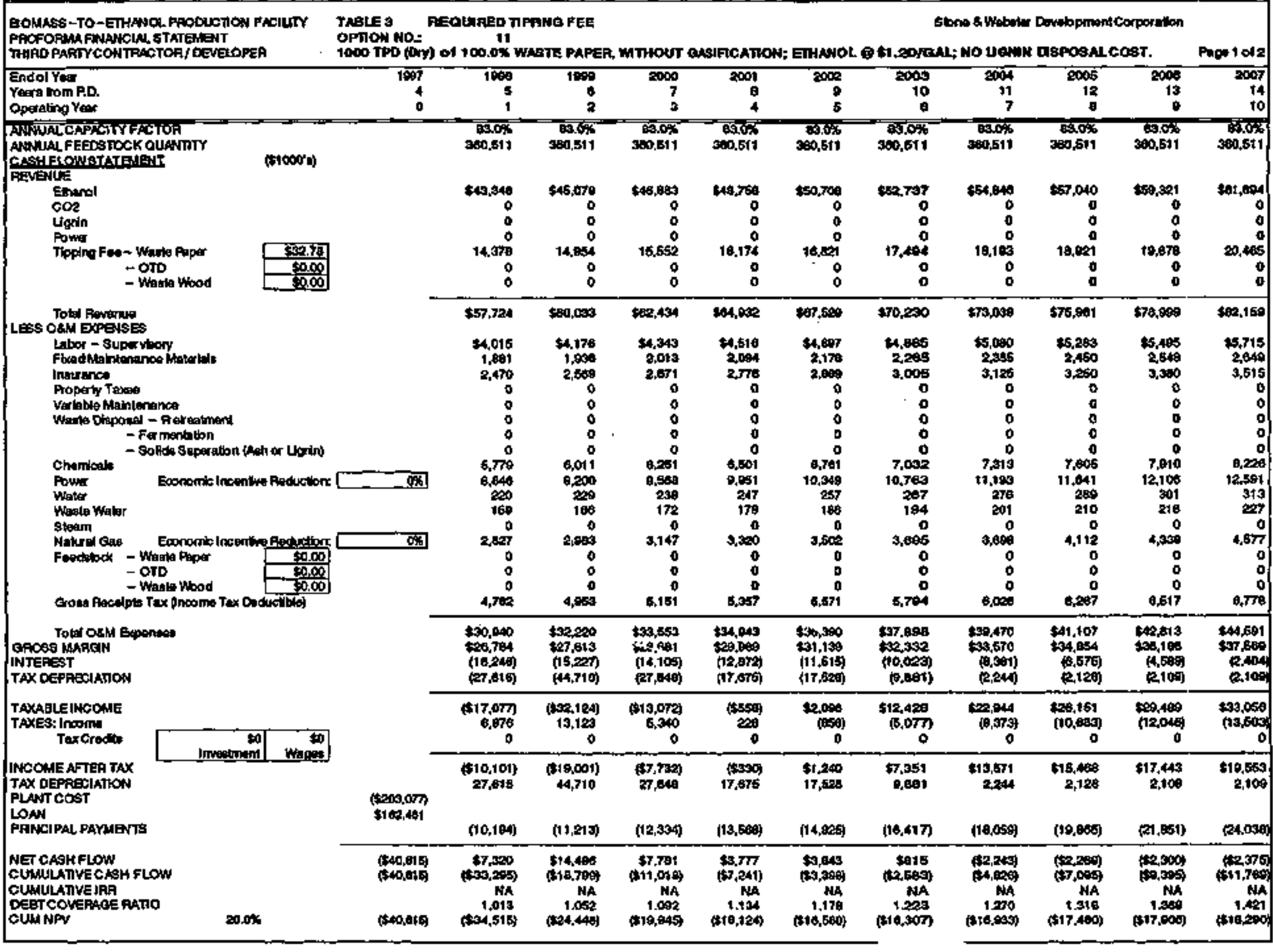




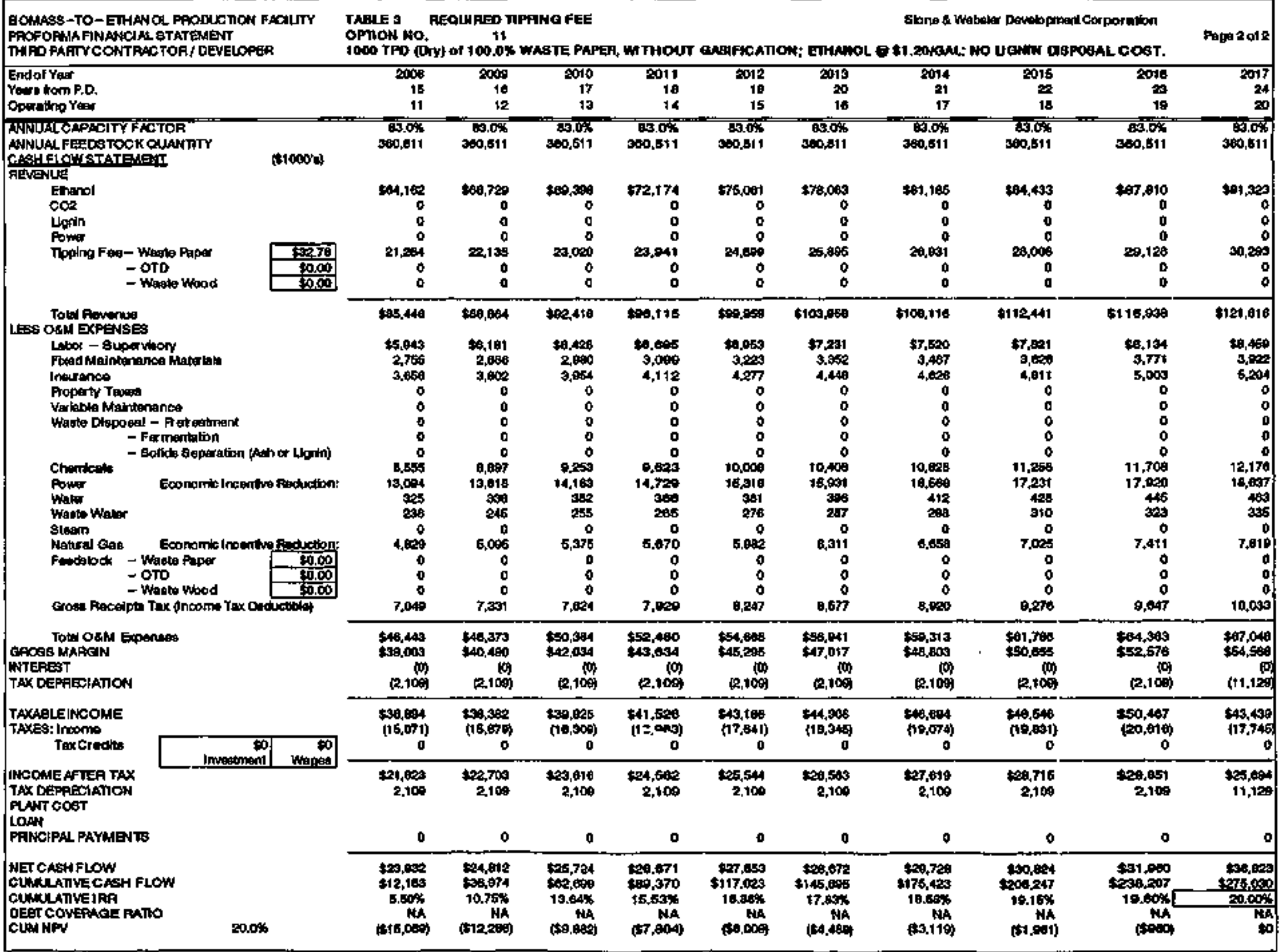




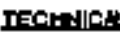

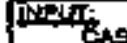

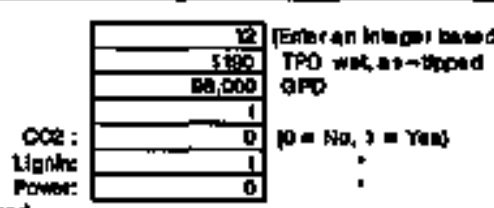

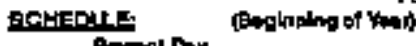

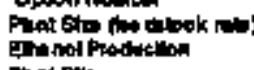 \\ fort then ot Opantion EconomkLLin

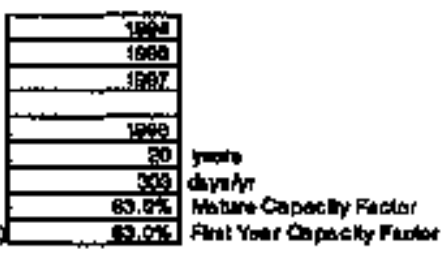 \\ pontenange wan}
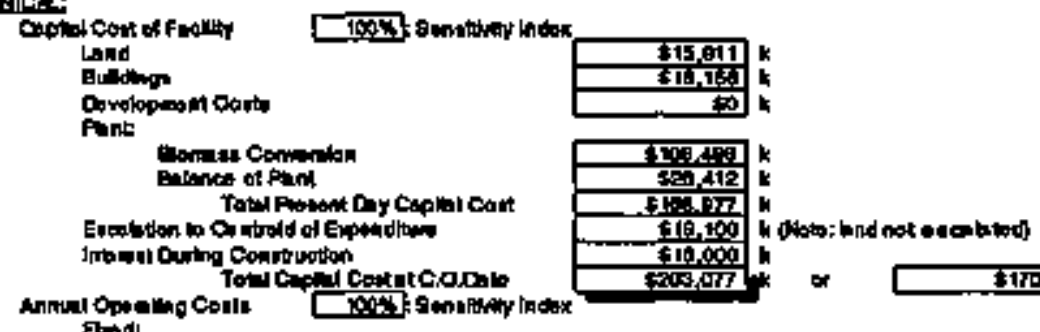

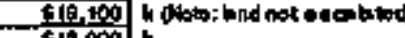

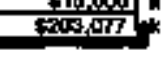

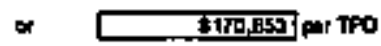

EConinowic

Oan Fition

Optajcont

ctuctosts:

Labor

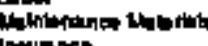

Invernet

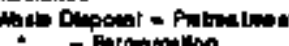

- - Bollo Boparatosa

Chambing

chater

Pond

Numalas

Fontiode

$\cos$

Throuk

Uonim

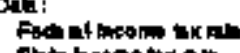

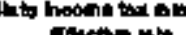

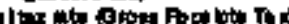

Propenty Tin Pat

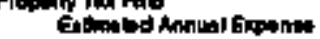

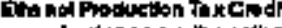

Lam your capiappat

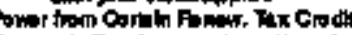

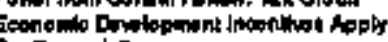

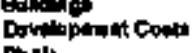

Phel ationes Combition Bock Doperition
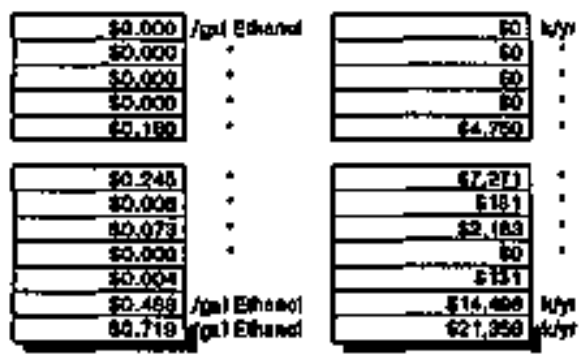

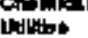

Painel

Mnciotion

Bite

Tow vatholo crat cont

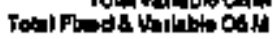

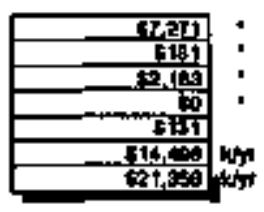

Exuscati:

o1 8yecture:

Dobifiection

Finantiag Tom

Anometop ol Loen

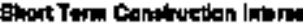

Terot Potson on Equitiv

Thping fin
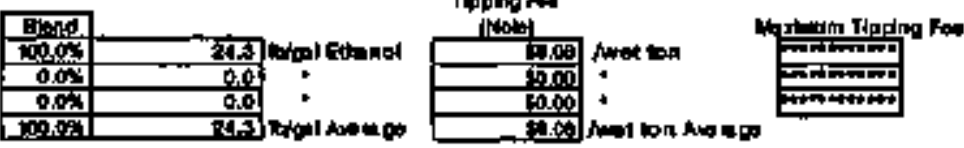

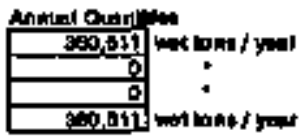

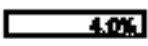

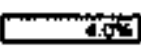

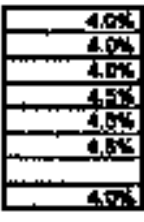

자.

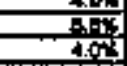

$1+\frac{40}{40}$
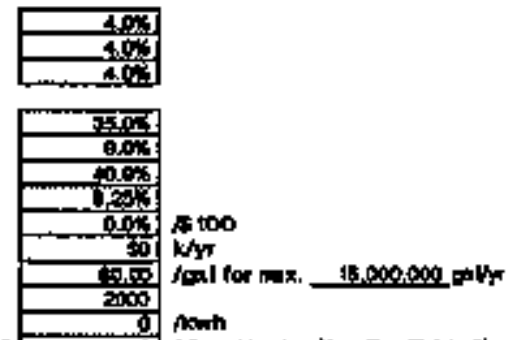
2nom

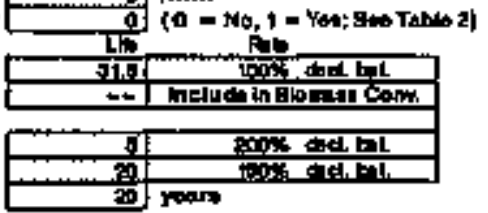

Fendutoek:
vinto Ampl
OTD

OTD

150.4.

저에

G.

Dulpox:

$\lim _{\operatorname{lin}}$

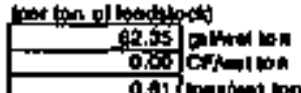

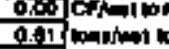

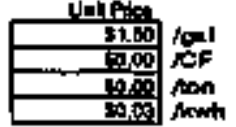

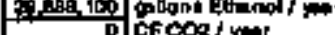 Dritat

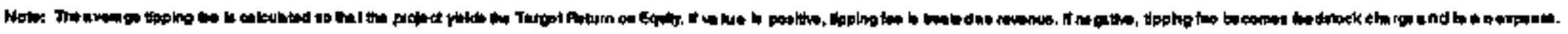




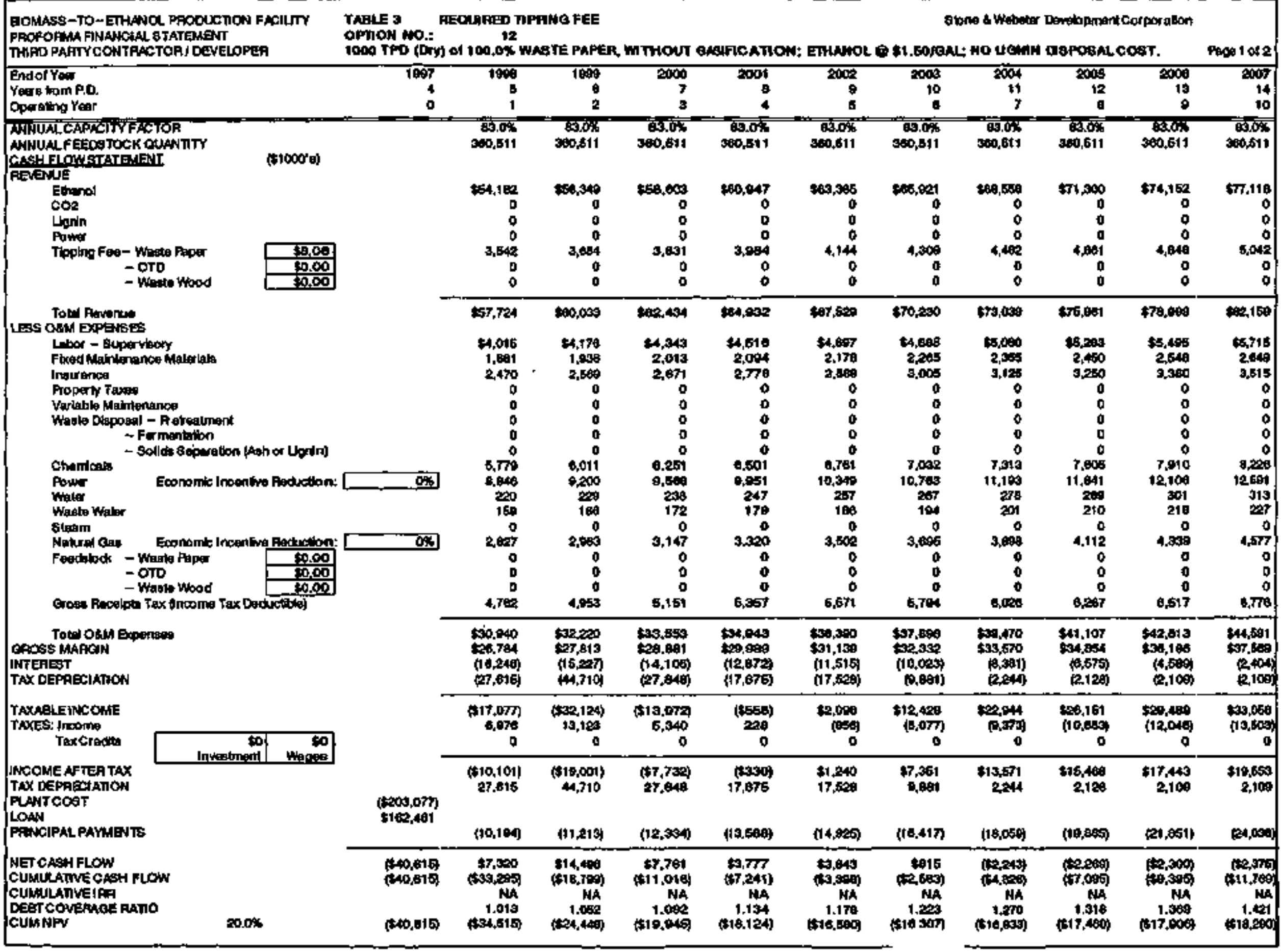




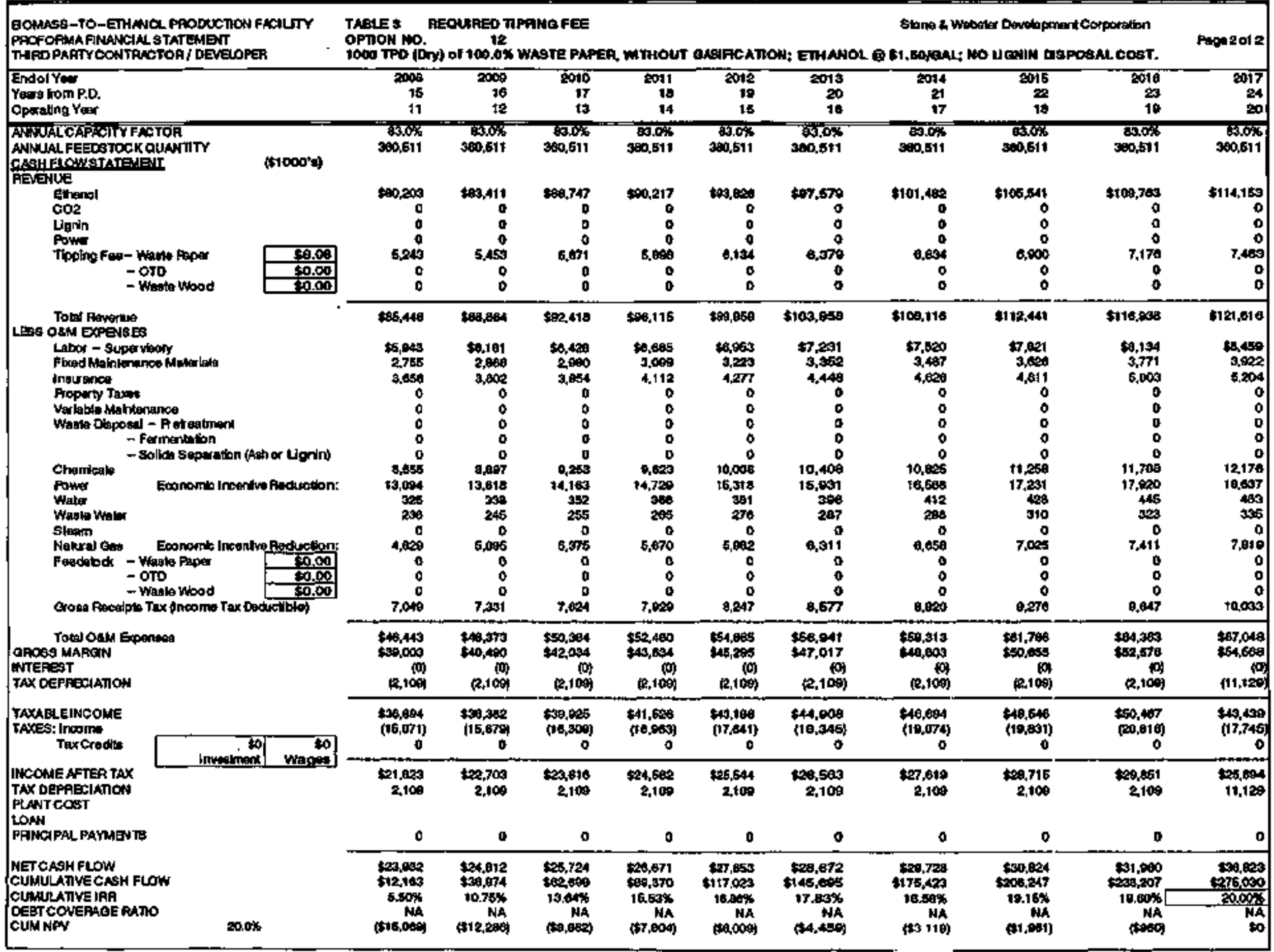




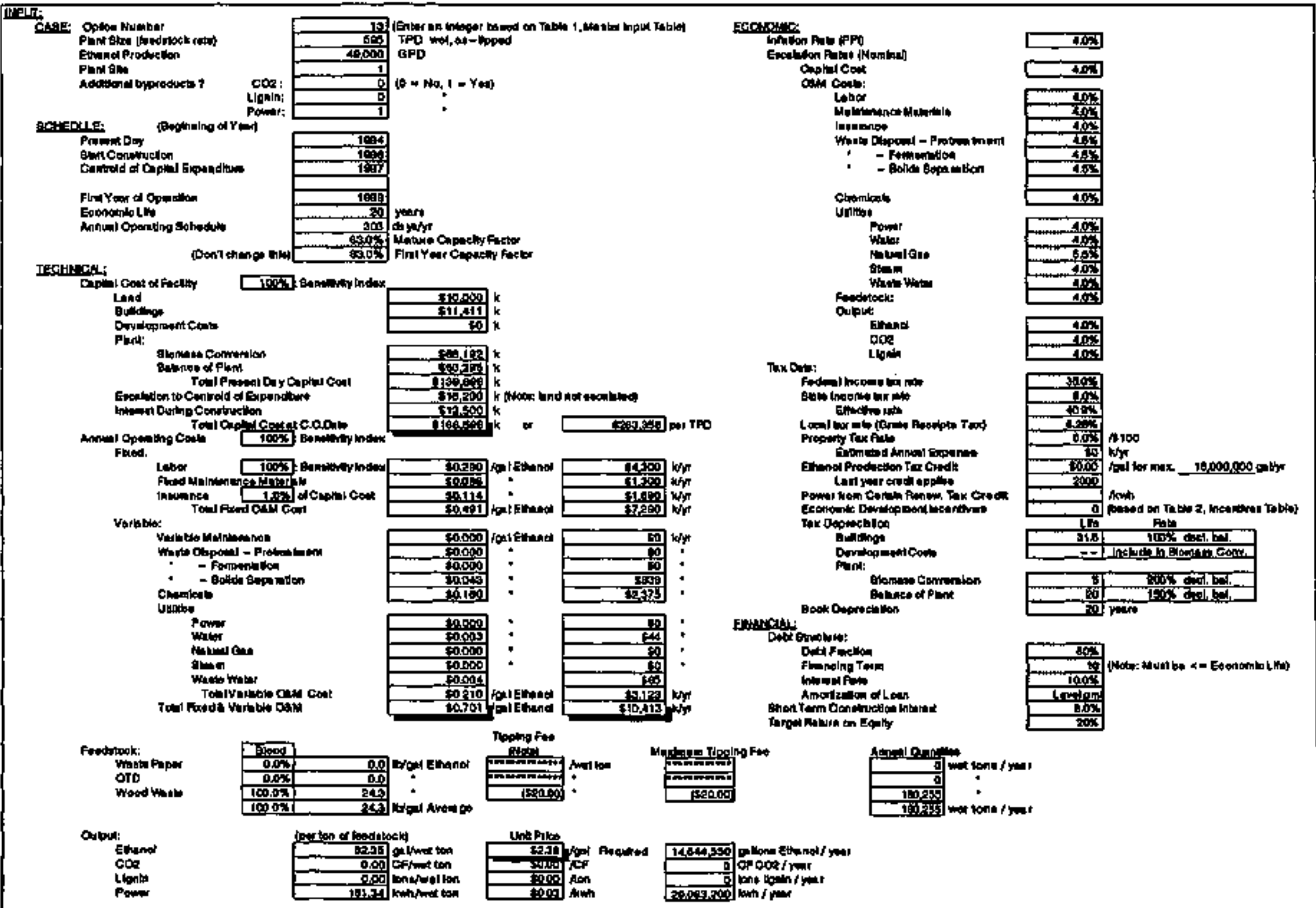




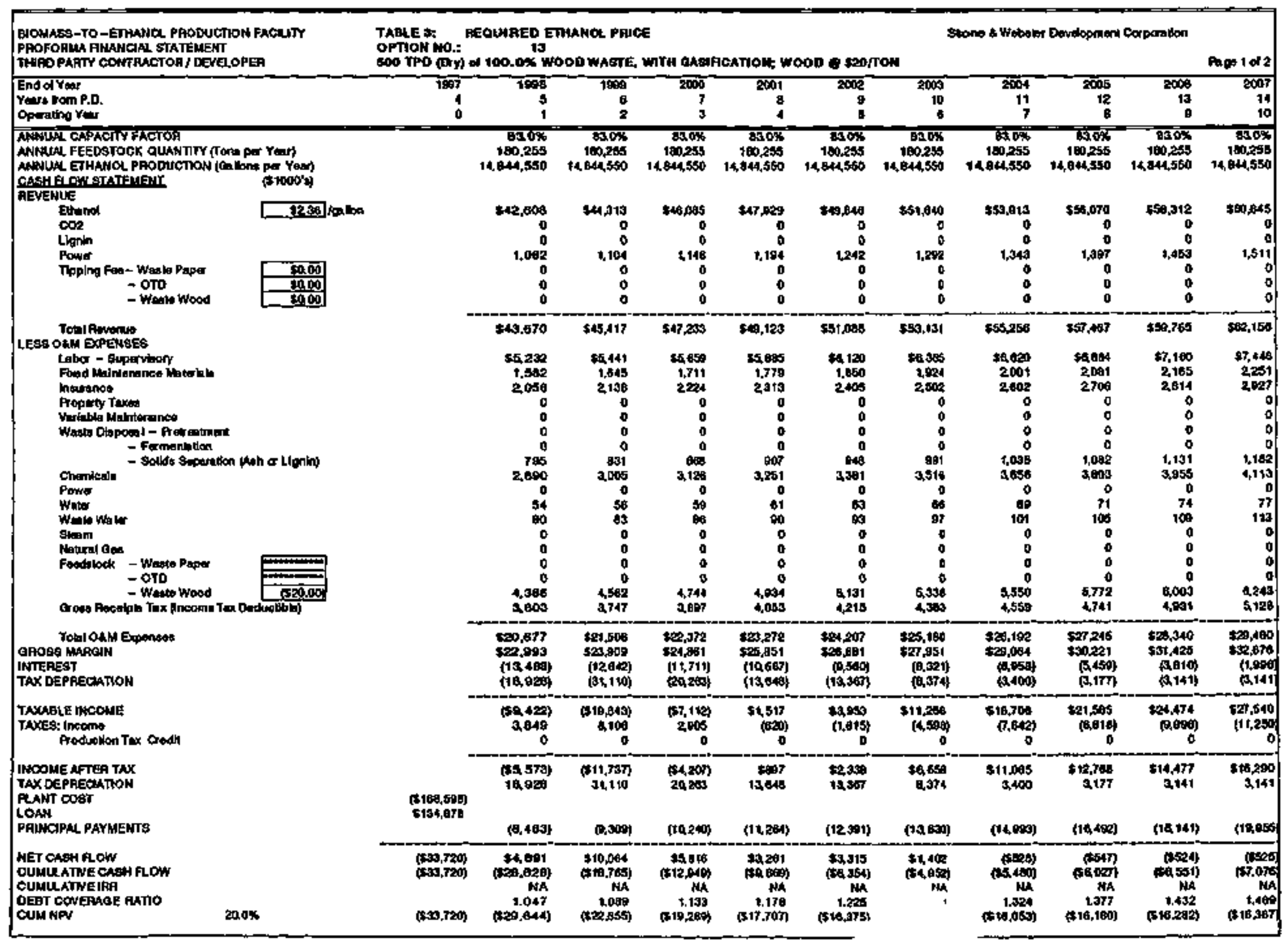




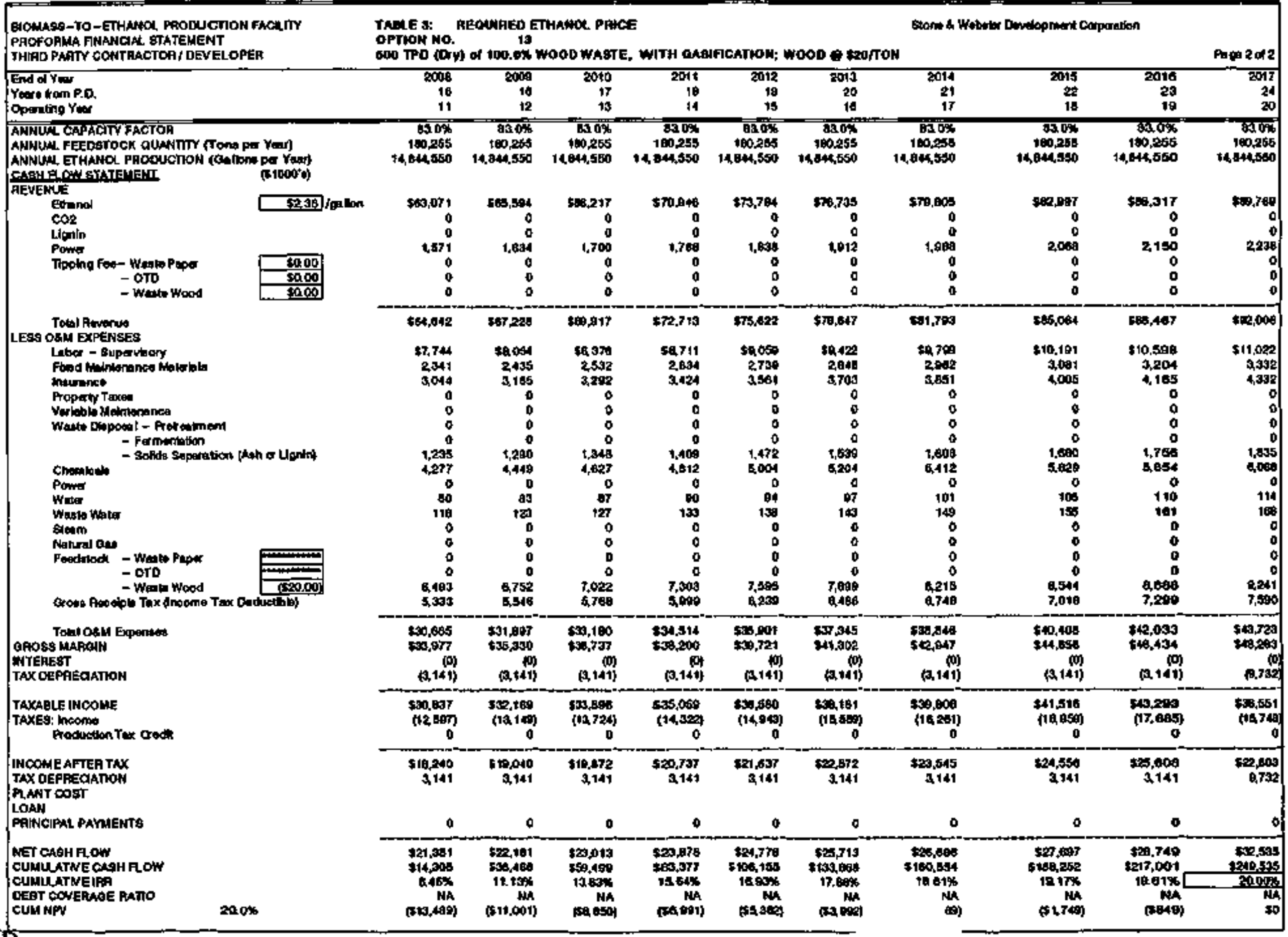




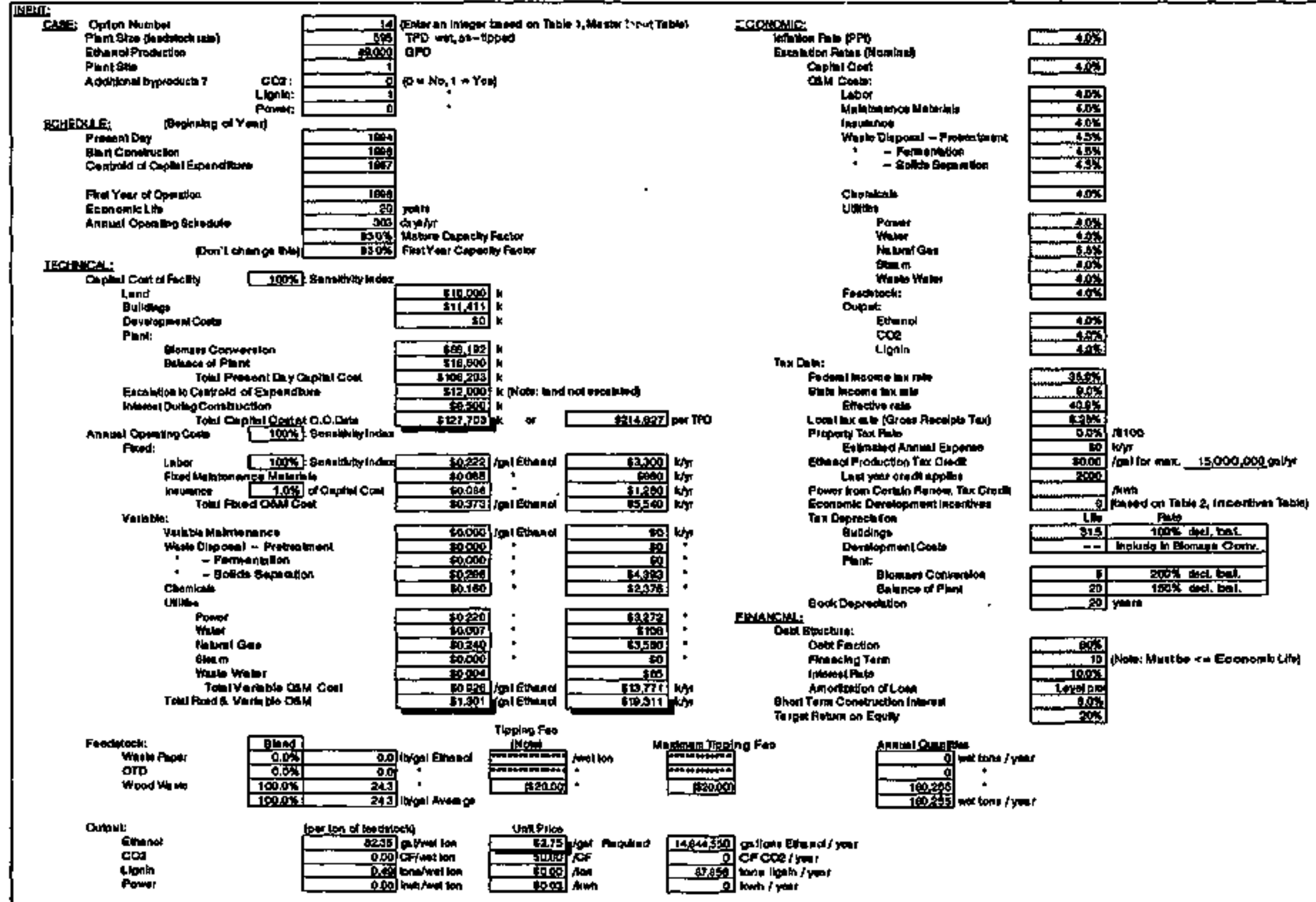




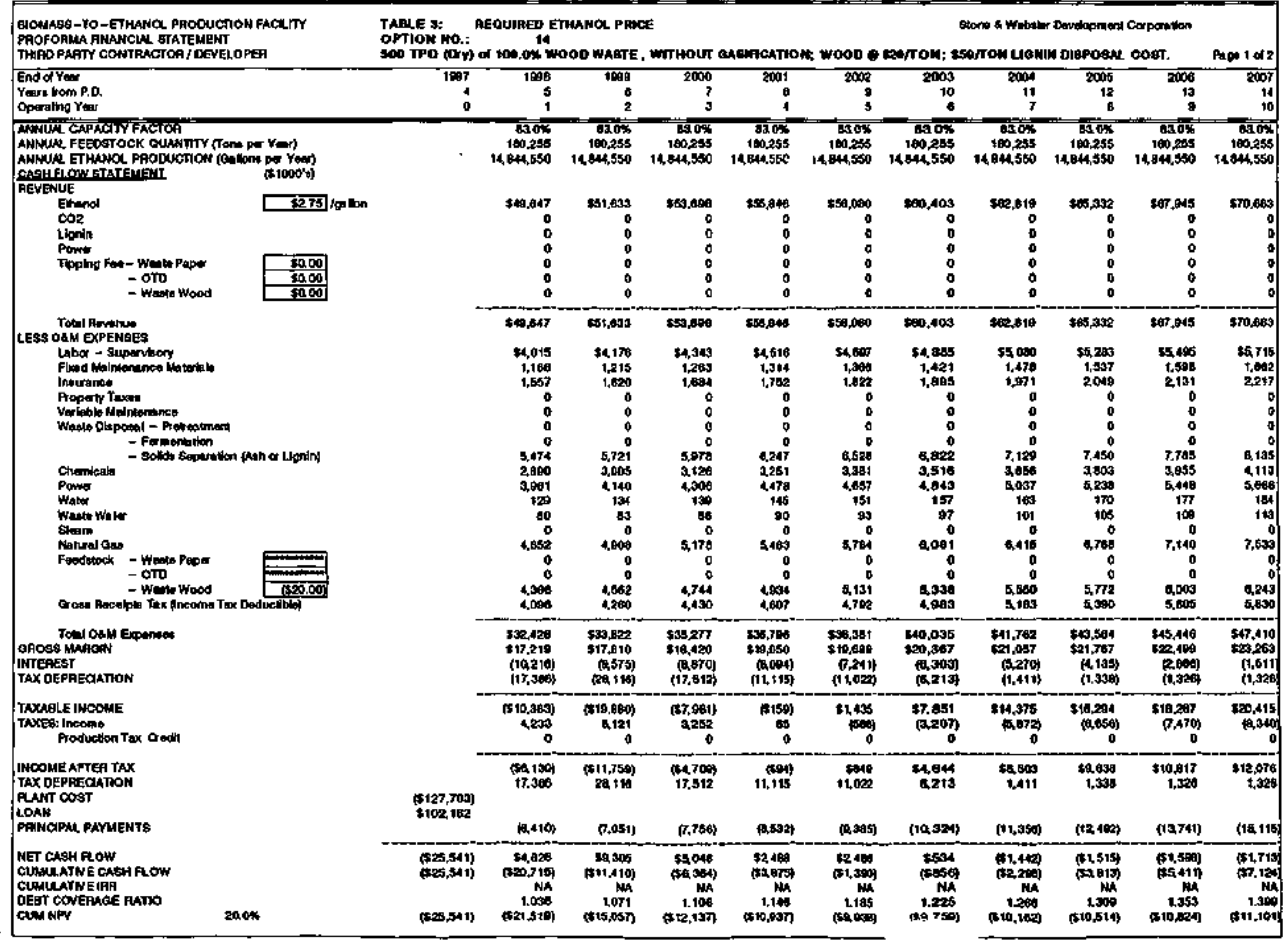




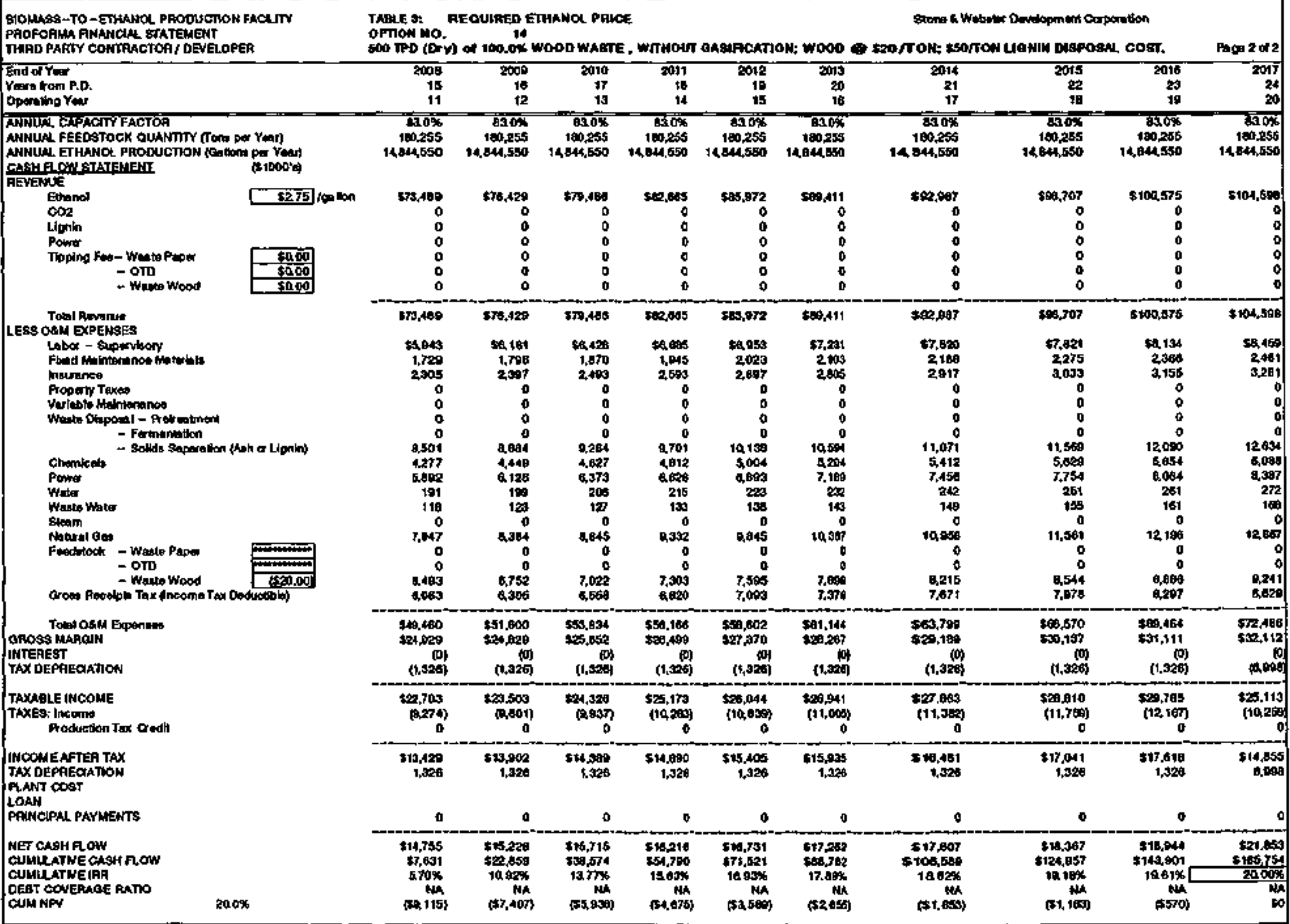




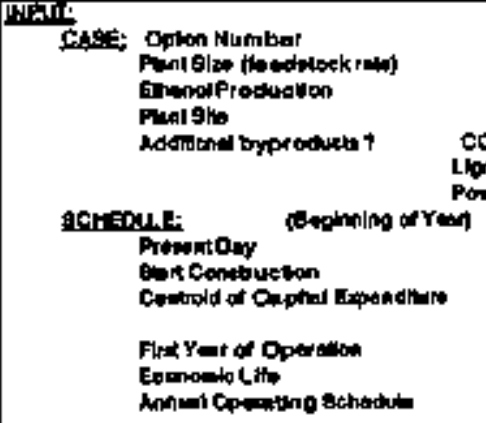

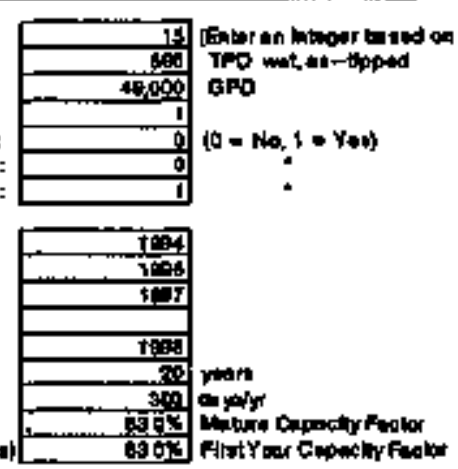

IEchнан.

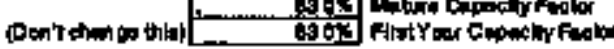
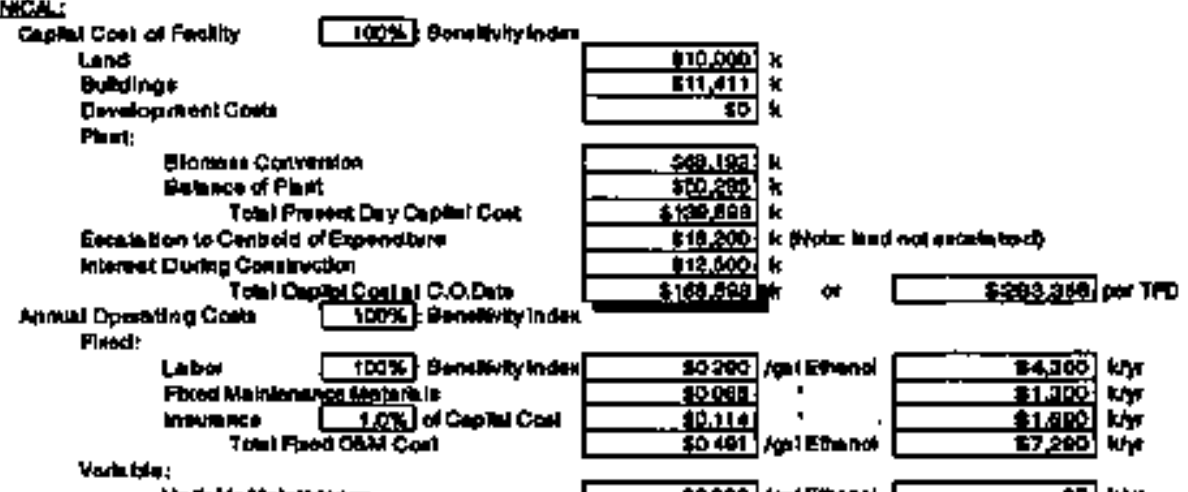

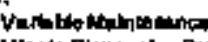

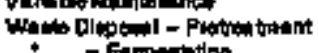
- Fompintition - Bothon Bumarod chnen

Power

Pown

mang

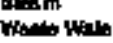

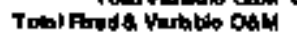
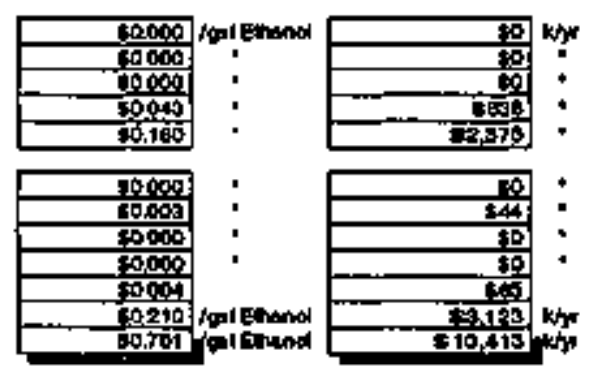

Elintrictal

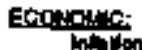

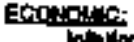

Intion An PPP Callow coll Caplalcous colls doost:

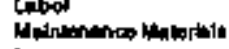

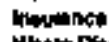

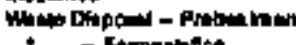

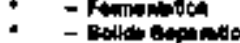

Chinelest

Un:mit

Punt

Notudat

stomen.

Fredation

Etane

cion

Thx Dow:

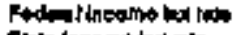

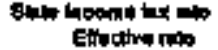

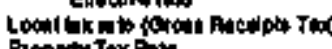

coputy Tax Pand

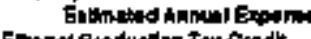

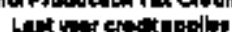

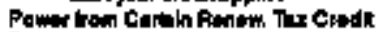

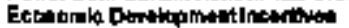

Tax Deproutition

gullding

Dovelopand cons

alomerescontwrion Book Daprocition

Dont gtovit:

Finachootor

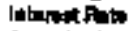

umortedion of Lom

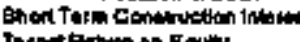

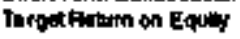
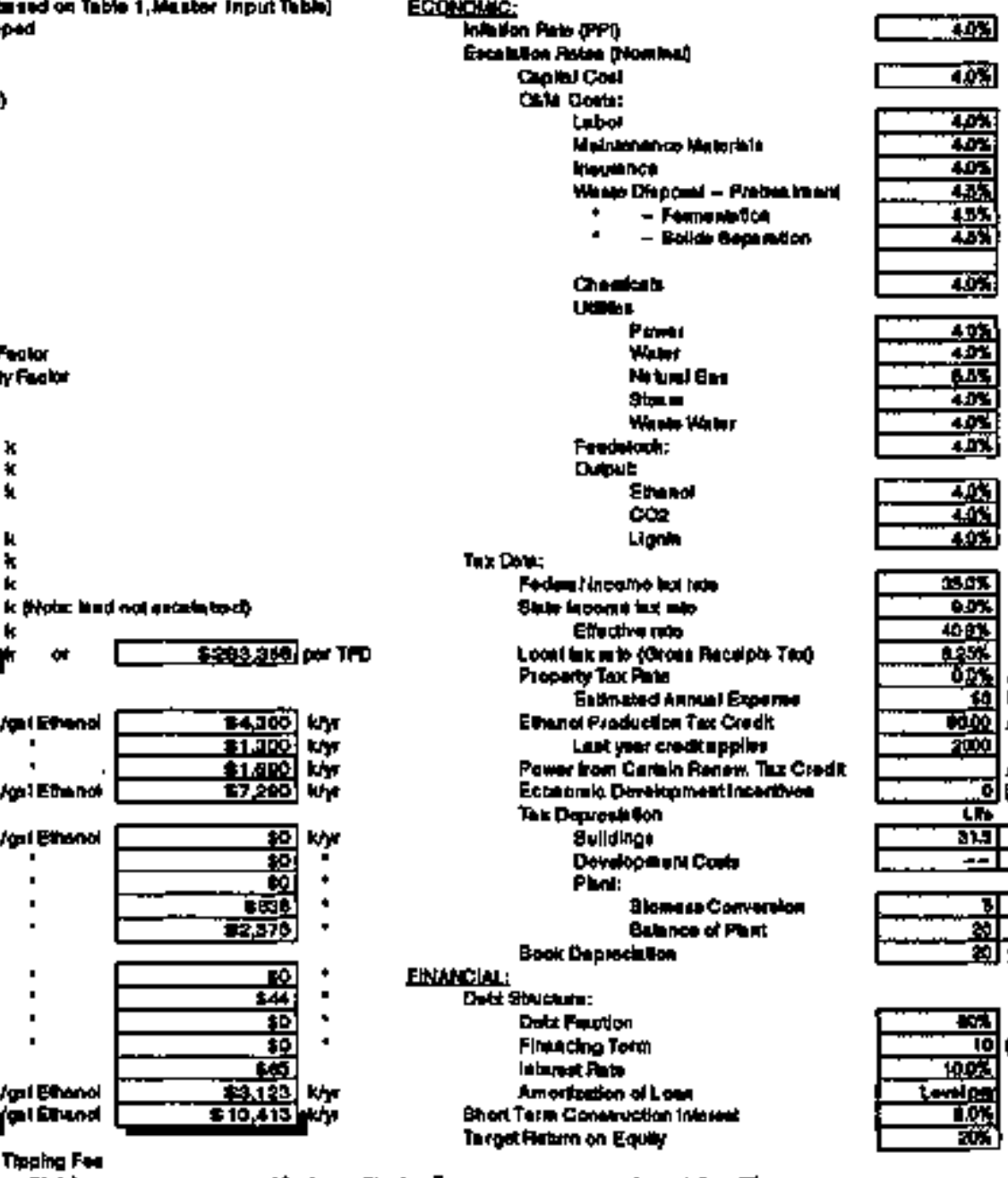

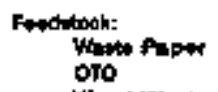

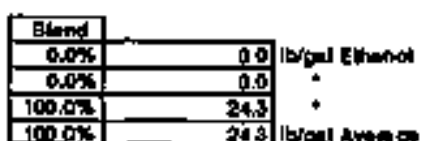

Tiopling Fu

Fintion
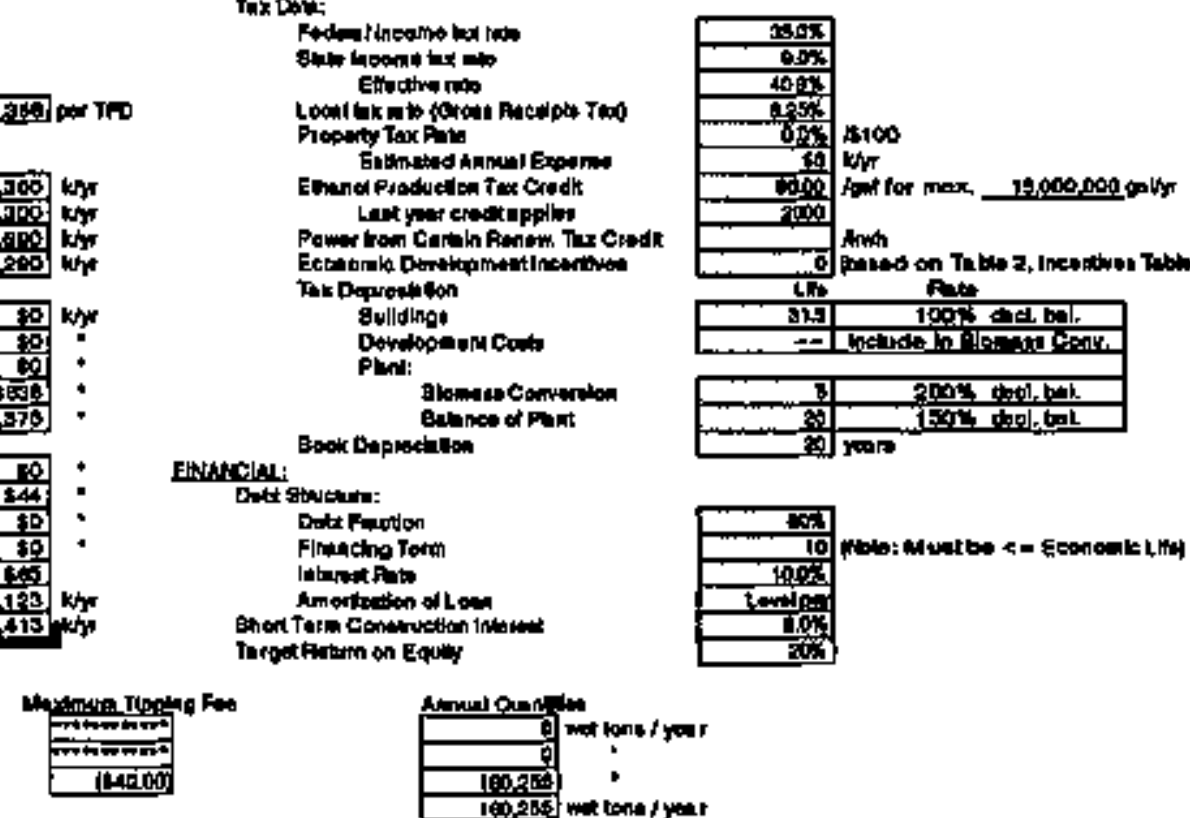

Hood nitor

[3000]

(Lerion)

Ouput

Exprat

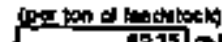

Whin Pitsots

toran

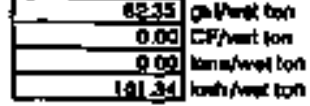

15.9

tong the

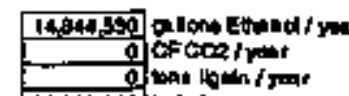

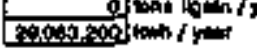

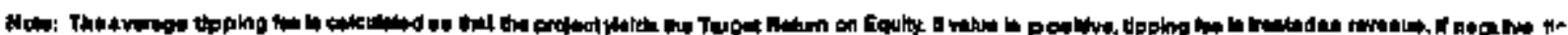




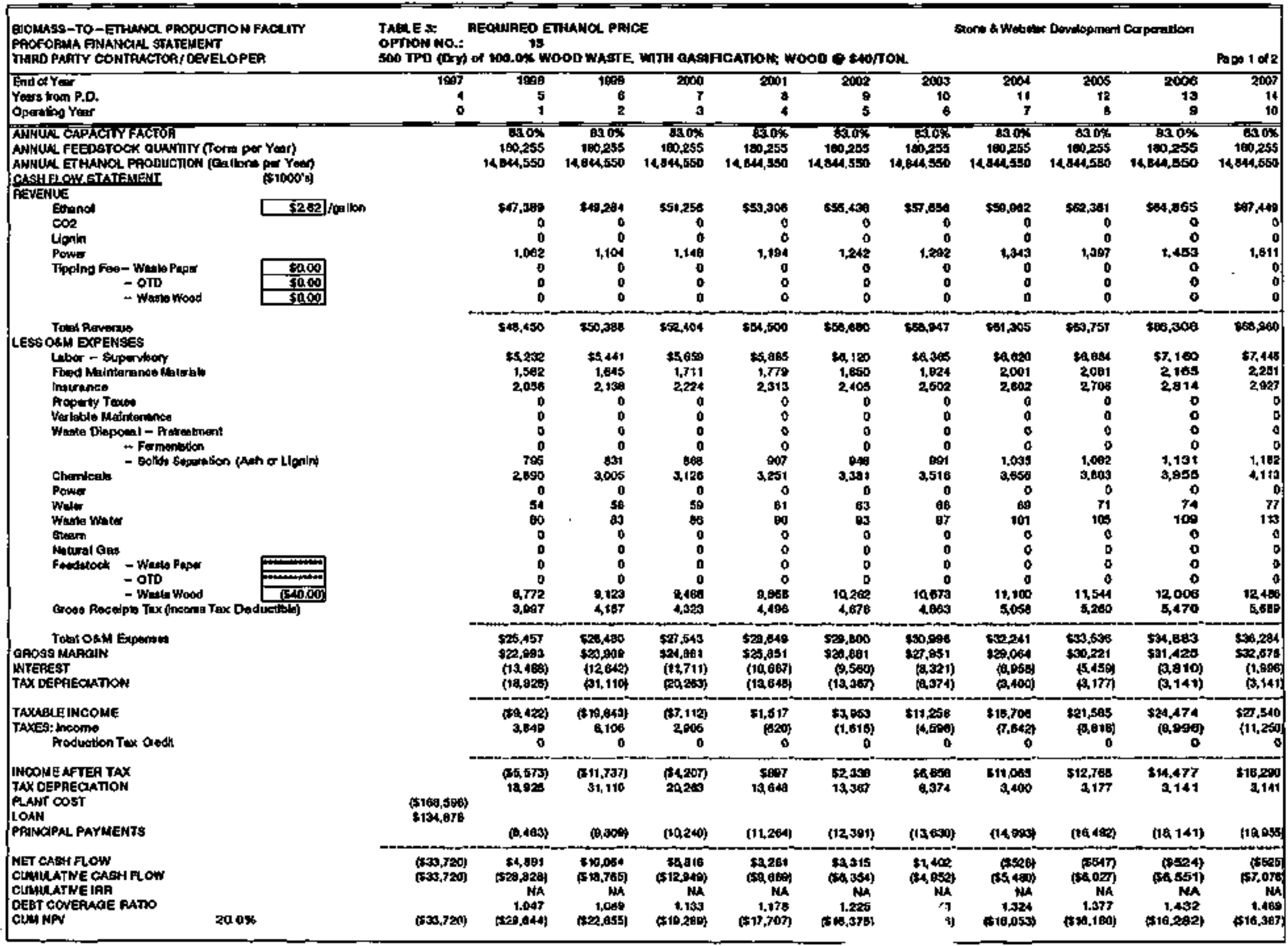




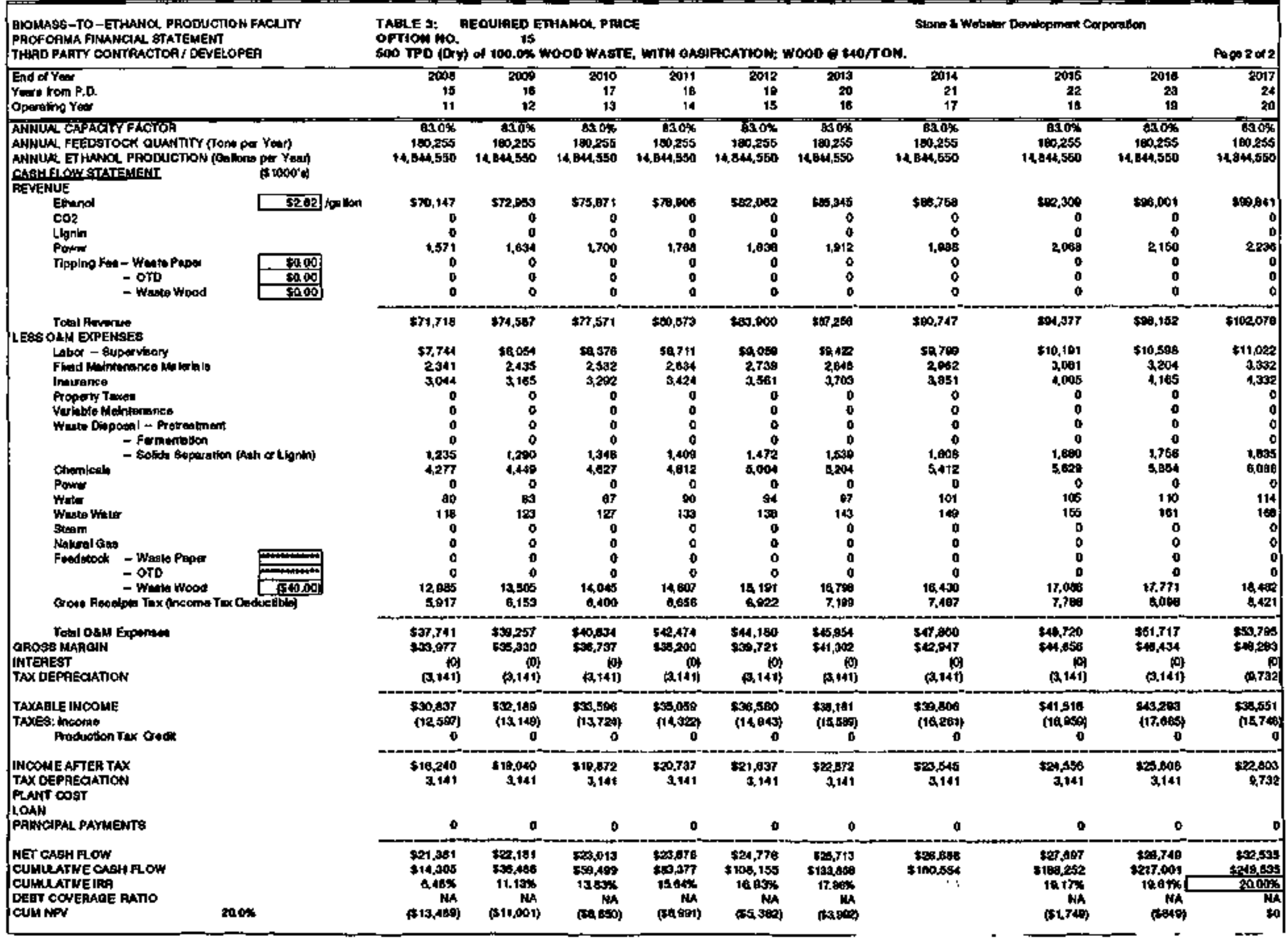




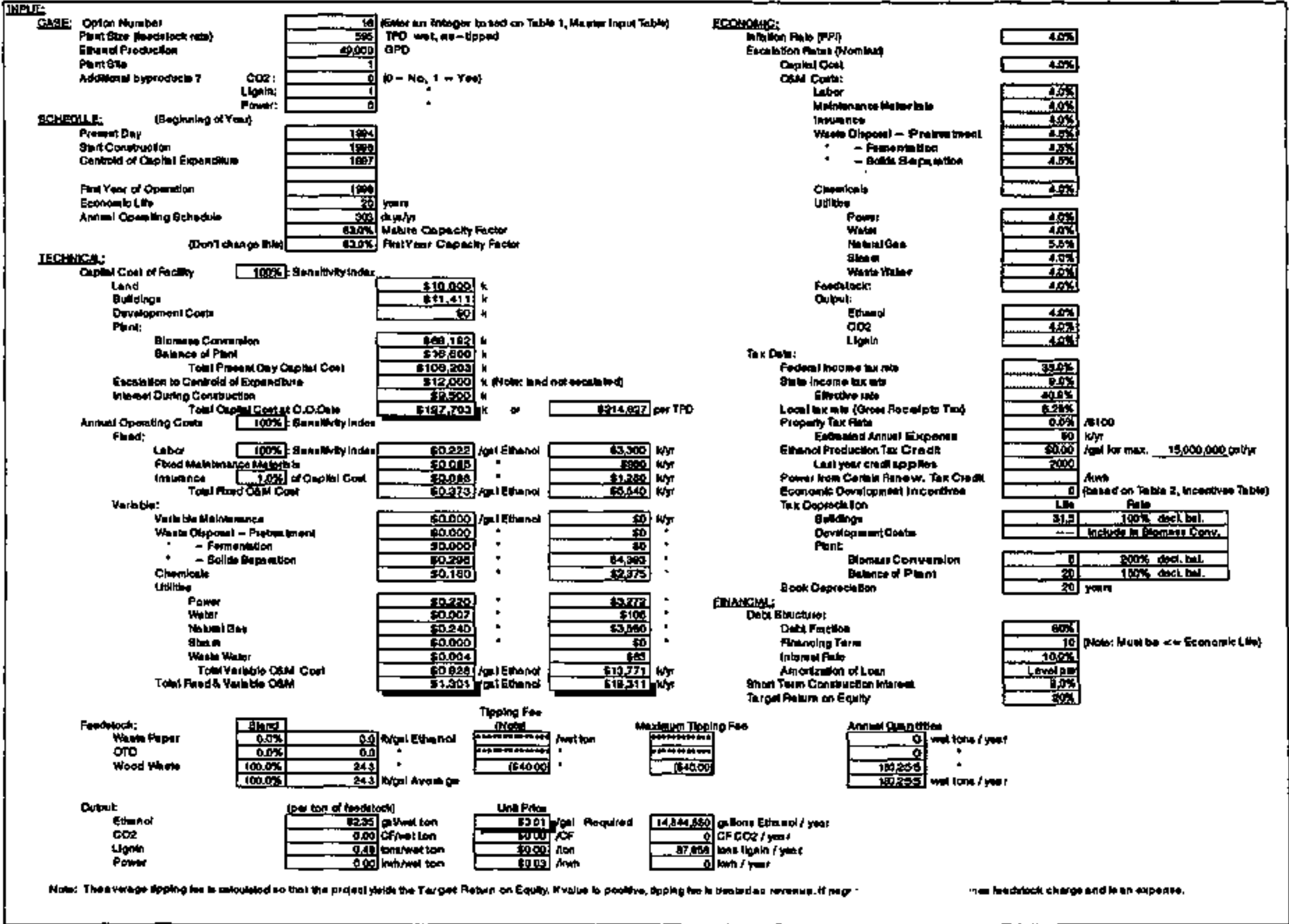




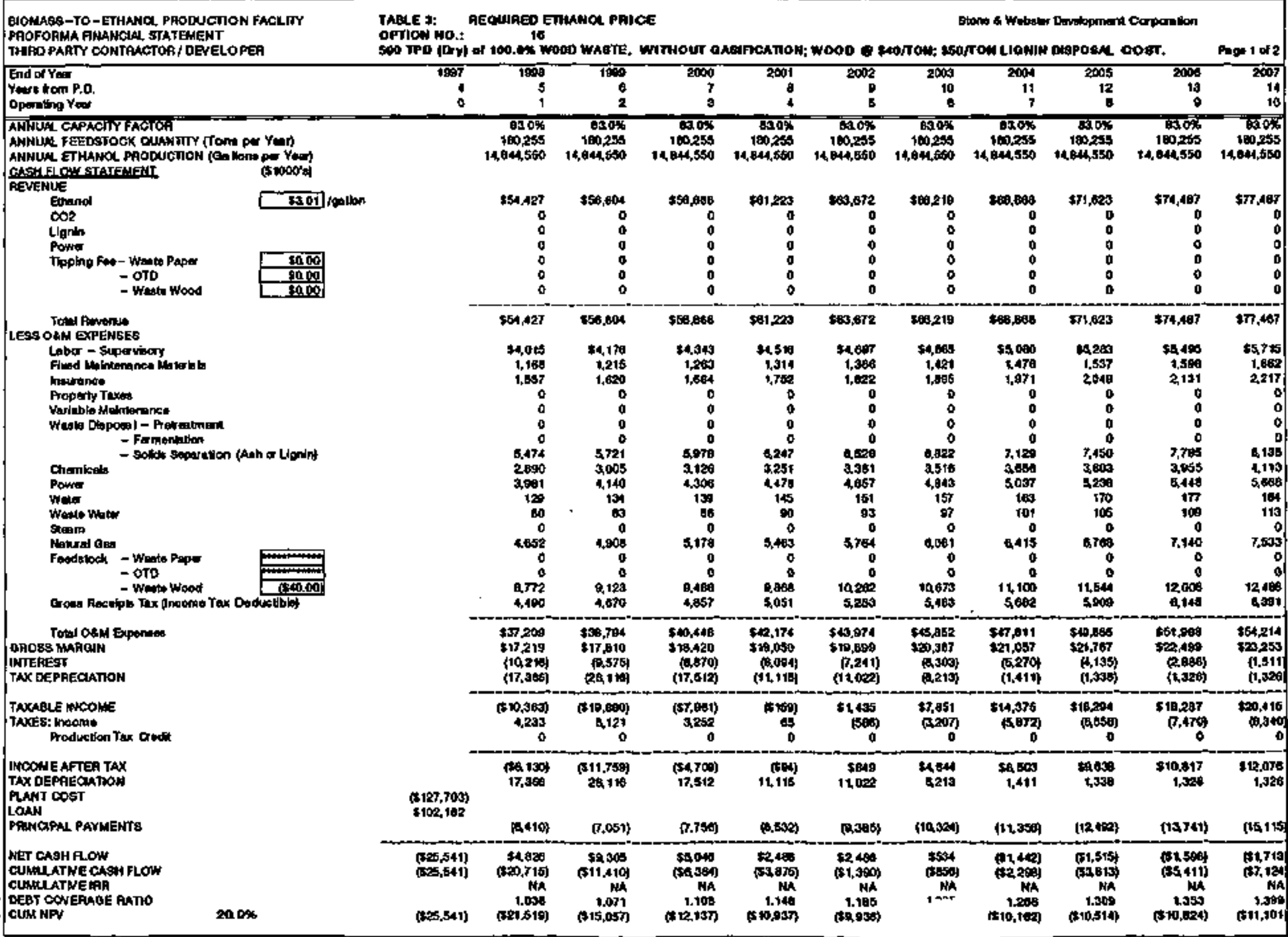




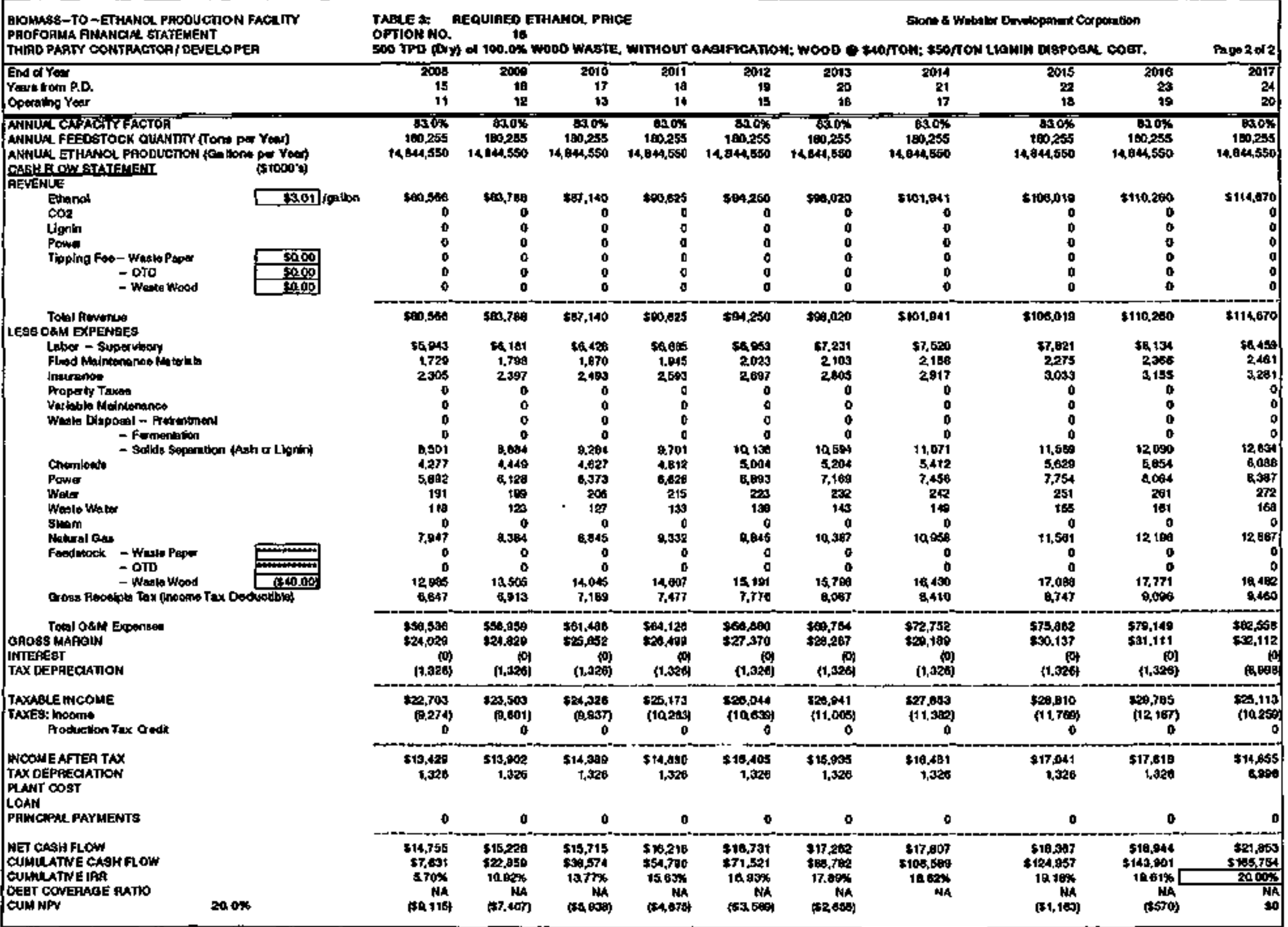




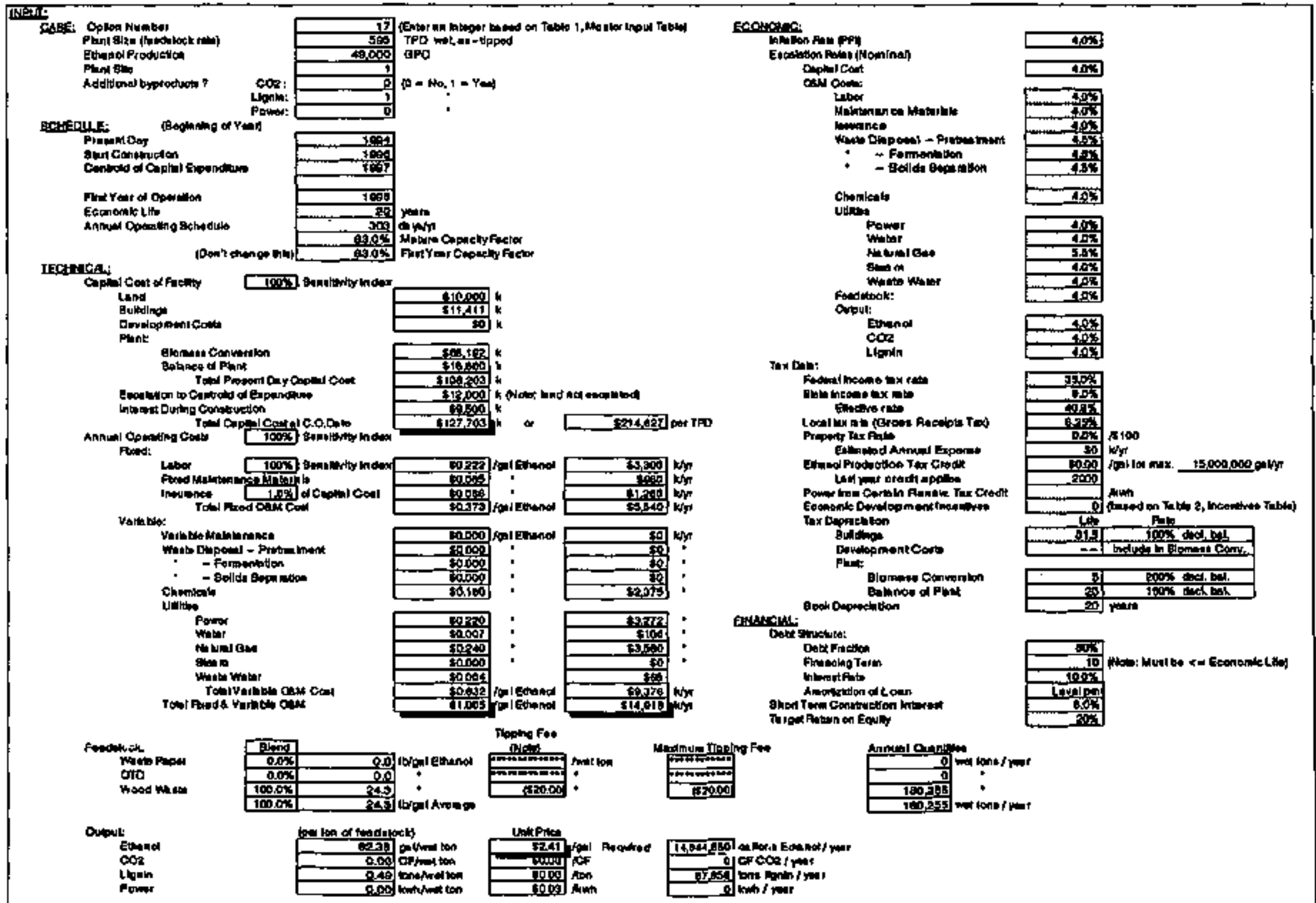

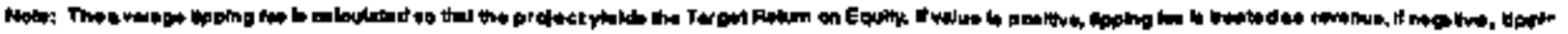




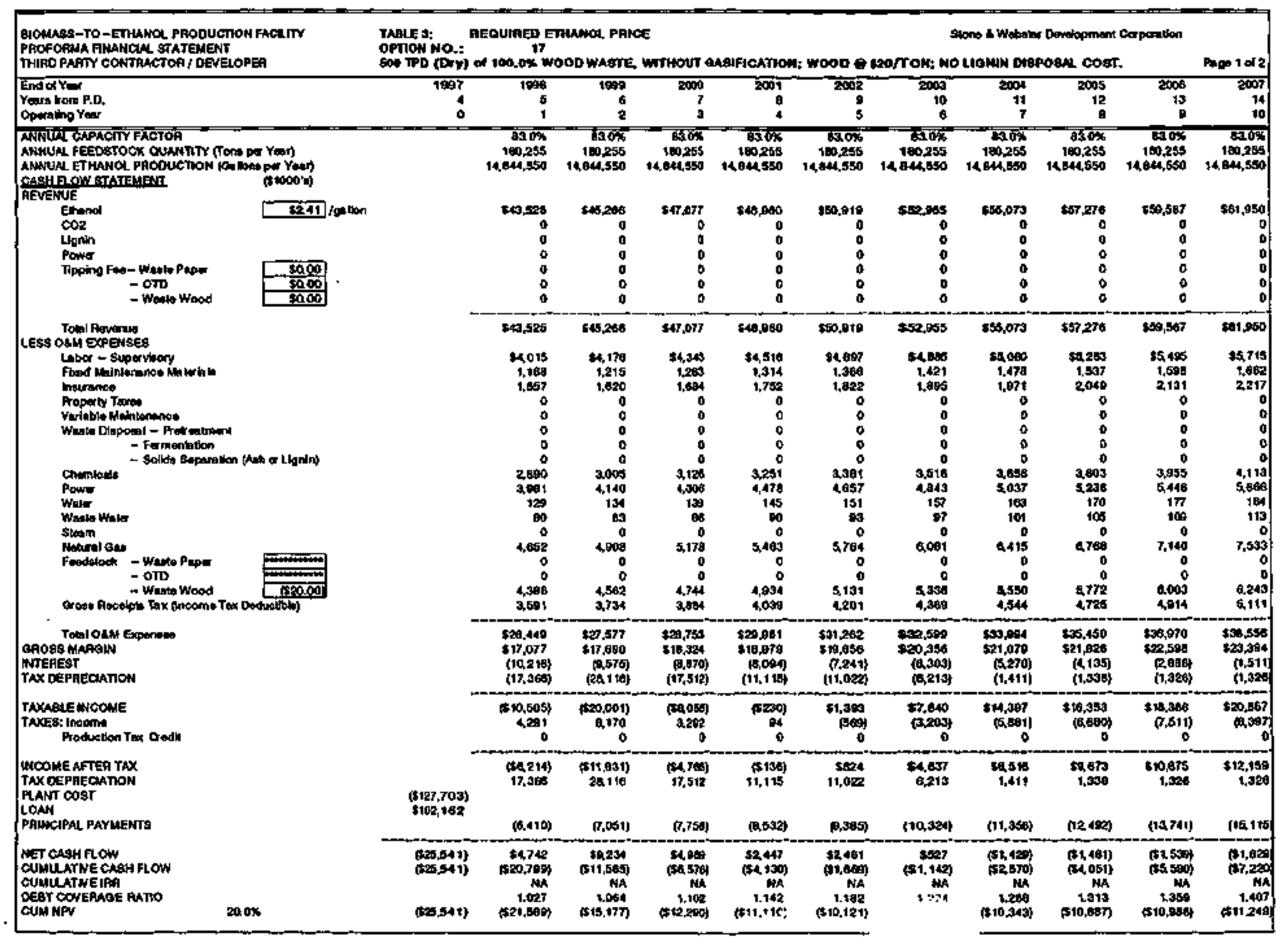




\begin{tabular}{|c|c|c|c|c|c|c|c|c|c|c|}
\hline 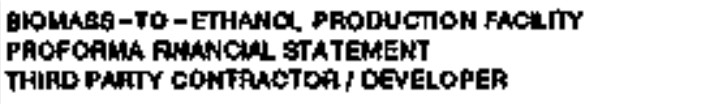 & \multicolumn{4}{|l|}{$\begin{array}{l}\text { TABLE 3: } \\
\text { OPTION No. } \\
\text { tho TPD tory }\end{array}$} & \multicolumn{5}{|c|}{ 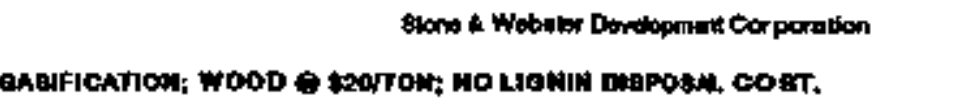 } & \multirow{2}{*}{$\begin{array}{r}\text { Papp } 2012 \\
2077 \\
24 \\
20\end{array}$} \\
\hline 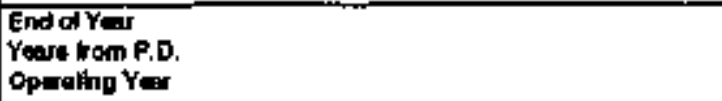 & $\begin{array}{r}2006 \\
15 \\
11\end{array}$ & $\begin{array}{r}20000 \\
18 \\
12\end{array}$ & $\begin{array}{r}2010 \\
17 \\
13\end{array}$ & $\begin{array}{r}2011 \\
16 \\
14\end{array}$ & $\begin{array}{r}2012 \\
10 \\
15\end{array}$ & $\begin{array}{r}2013 \\
29 \\
10\end{array}$ & $\begin{array}{r}2014 \\
21 \\
17\end{array}$ & $\begin{array}{r}2015 \\
22 \\
18\end{array}$ & $\begin{array}{r}2010 \\
29 \\
19\end{array}$ & \\
\hline 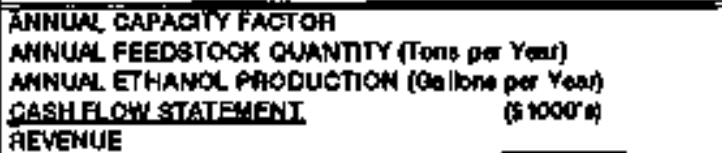 & $\begin{array}{r}180,255 \\
14640,556\end{array}$ & $\begin{array}{r}010 x \\
160,256 \\
14,64650\end{array}$ & $\begin{array}{r}430 \times \\
190,255 \\
14,64,550\end{array}$ & $\begin{array}{r}50.0 x \\
180,255 \\
14,04,550\end{array}$ & $\begin{array}{r}8,0 \% \\
160,255 \\
14,044,550\end{array}$ & $\begin{array}{r}0,6 \% \\
100,259 \\
14,640,590\end{array}$ & $\begin{array}{r}100 \% \\
1002254 \\
1404,550\end{array}$ & $\begin{array}{r}1,0 \times 5 \\
100,258 \\
1404,650\end{array}$ & $\begin{array}{r}506 \% \\
180,255 \\
1494050\end{array}$ & $\begin{array}{r}130 \times 6 \\
160,258 \\
14,94,550\end{array}$ \\
\hline 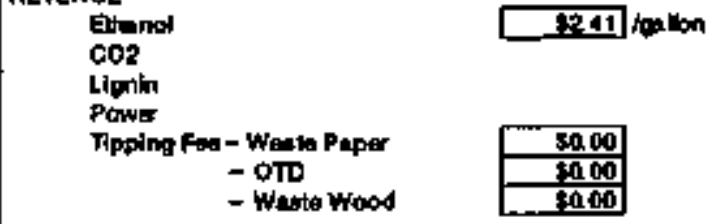 & $\begin{array}{r}\text { cos,42a } \\
0 \\
0 \\
0 \\
0 \\
0 \\
0\end{array}$ & $\begin{array}{r}060 \\
0 \\
0 \\
0 \\
0 \\
0 \\
0\end{array}$ & $\begin{array}{r}50,000 \\
0 \\
0 \\
0 \\
0 \\
0\end{array}$ & $\begin{array}{r}772,479 \\
0 \\
0 \\
0 \\
0 \\
0 \\
0\end{array}$ & $\begin{array}{r}\$ 75,972 \\
0 \\
0 \\
0 \\
0 \\
0 \\
0\end{array}$ & $\begin{array}{r}57897 \\
0 \\
0 \\
0 \\
0 \\
0 \\
0\end{array}$ & $\begin{array}{r}0 \\
0 \\
0 \\
0 \\
0 \\
0 \\
0\end{array}$ & $\begin{array}{r}\text { SAH.7to } \\
0 \\
0 \\
0 \\
0 \\
0 \\
0\end{array}$ & $\begin{array}{r}\$ 18,174 \\
0 \\
0 \\
0 \\
0 \\
0 \\
0\end{array}$ & \begin{tabular}{r|}
291.701 \\
0 \\
0 \\
0 \\
0 \\
0 \\
0
\end{tabular} \\
\hline 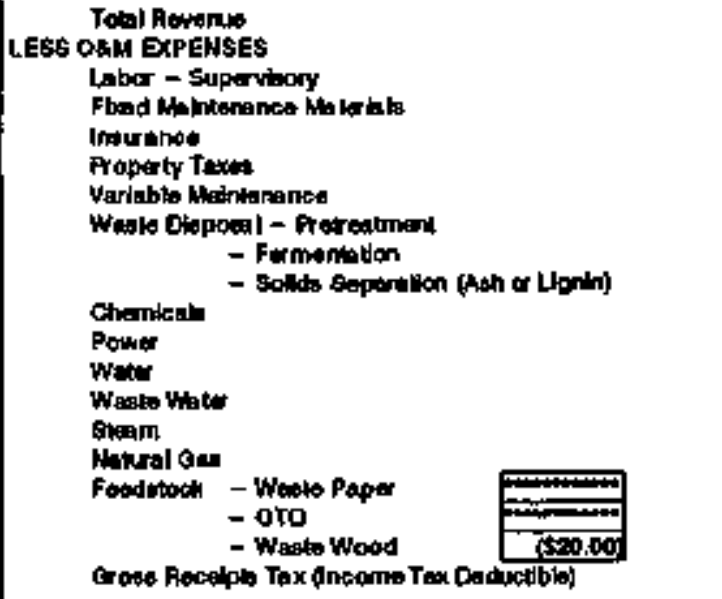 & 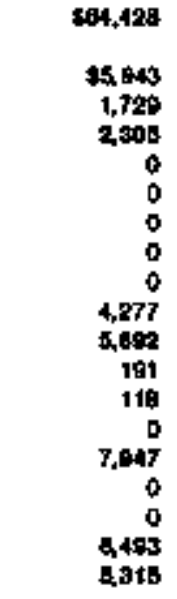 & 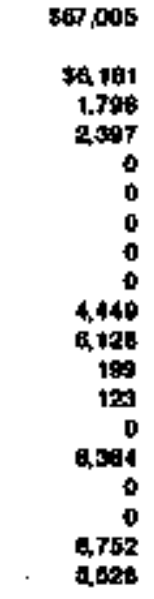 & 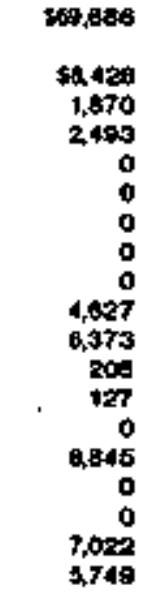 & $\begin{array}{r}\$ 72,473 \\
99,405 \\
1,945 \\
2,503 \\
0 \\
0 \\
0 \\
0 \\
0 \\
4,612 \\
0,620 \\
215 \\
190 \\
0 \\
9,332 \\
0 \\
0 \\
7,303 \\
4,970\end{array}$ & $\begin{array}{r}57 ., 372 \\
+9.959 \\
2,023 \\
2,007 \\
0 \\
0 \\
0 \\
0 \\
0 \\
5,004 \\
8,000 \\
220 \\
130 \\
0 \\
0,845 \\
0 \\
0 \\
7,605 \\
6,210\end{array}$ & $\begin{array}{r}50,397 \\
\$ 7.201 \\
2,108 \\
2,008 \\
0 \\
0 \\
0 \\
0 \\
0 \\
5,204 \\
7,160 \\
240 \\
144 \\
0 \\
19307 \\
0 \\
0 \\
7,009 \\
0,407\end{array}$ & $\begin{array}{r}501,522 \\
\$ 7,500 \\
2,189 \\
2,917 \\
0 \\
0 \\
0 \\
0 \\
0 \\
5412 \\
7,456 \\
242 \\
140 \\
0 \\
10,50 \\
0 \\
0 \\
2,215 \\
0,720\end{array}$ & $\begin{array}{r}50,783 \\
\$ 7,421 \\
2,275 \\
2,033 \\
0 \\
0 \\
0 \\
0 \\
0 \\
5,029 \\
7,754 \\
261 \\
153 \\
0 \\
11,561 \\
0 \\
0 \\
6,514 \\
6,05\end{array}$ & $\begin{array}{r}568,774 \\
56194 \\
2360 \\
3,155 \\
0 \\
0 \\
0 \\
0 \\
0 \\
5,054 \\
2004 \\
261 \\
161 \\
0 \\
12190 \\
0 \\
0 \\
7,096 \\
7,274\end{array}$ & 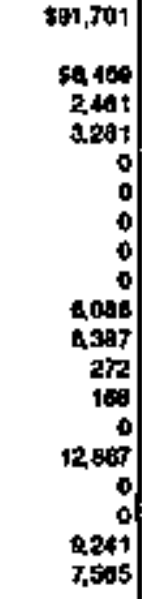 \\
\hline 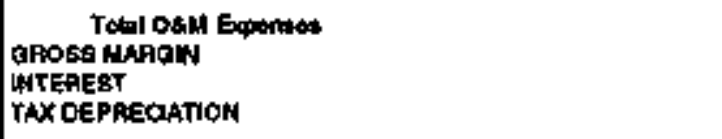 & $\begin{array}{r}\$ 40,211 \\
\$ 21,217 \\
(1,396)\end{array}$ & $\begin{array}{r}t+1,006 \\
5206,067 \\
(1,32)\end{array}$ & $\begin{array}{r}54,742 \\
524,944 \\
(0) \\
(1,326)\end{array}$ & 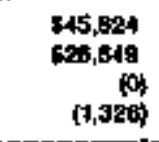 & $\begin{array}{r}\$ 7,500 \\
527,7: 3 \\
(1,320)\end{array}$ & $\begin{array}{r}\$ 49,840 \\
50,744 \\
(0) \\
(1,320)\end{array}$ & $\begin{array}{r}\$ \$ 1,702 \\
\$ 0,740 \\
(1,320)\end{array}$ & $\begin{array}{r}+54,016 \\
00,706 \\
(1,39)\end{array}$ & 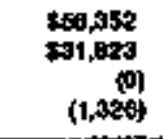 & 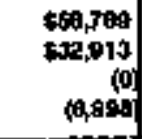 \\
\hline 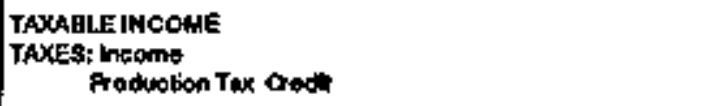 & $\begin{array}{r}52,691 \\
\{(9 ; 351\} \\
0\end{array}$ & $\begin{array}{c}00,741 \\
0,698) \\
0\end{array}$ & $\begin{array}{c}\text { ten, a17 } \\
(10,058) \\
0\end{array}$ & $\begin{array}{r}205,520 \\
114,424 \\
0\end{array}$ & $\begin{array}{r}\$ 20,457 \\
(10,007) \\
0\end{array}$ & $\begin{array}{r}27,420 \\
(11,201) \\
0\end{array}$ & $\begin{array}{c}\$ 20,414 \\
(11,60 \mathrm{r}) \\
0\end{array}$ & $\frac{12,0200}{0}$ & $\begin{array}{c}530,497 \\
(12,4 \$ 9) \\
0\end{array}$ & $\begin{array}{l}505914 \\
14500 \\
0\end{array}$ \\
\hline 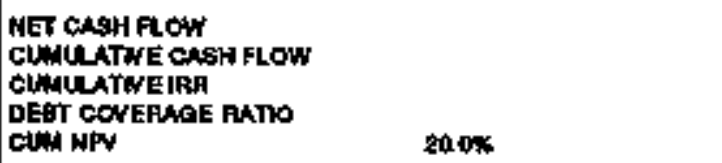 & 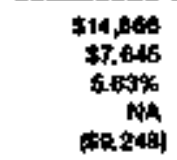 & $\begin{array}{r}515,399 \\
\$ 23,015 \\
10,96 \% \\
1 \% 4 \\
(\$ 7,524)\end{array}$ & $\begin{array}{r}\$ 15.917 \\
\$ 9.902 \\
1272 \% 6 \\
N A \\
1040)\end{array}$ & 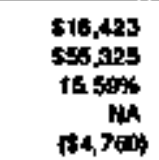 & 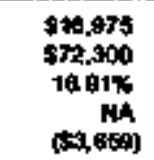 & $\begin{array}{r}\$ 17.516 \\
29.045 \\
17.07 \% \\
\text { NA } \\
92710)\end{array}$ & 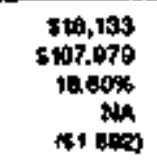 & $\begin{array}{r}5 \times, 740 \\
\$ 120,719 \\
1917 \% \\
174 \\
\$ 1,189\end{array}$ & 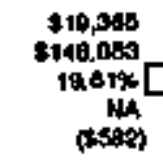 & 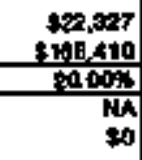 \\
\hline
\end{tabular}




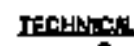

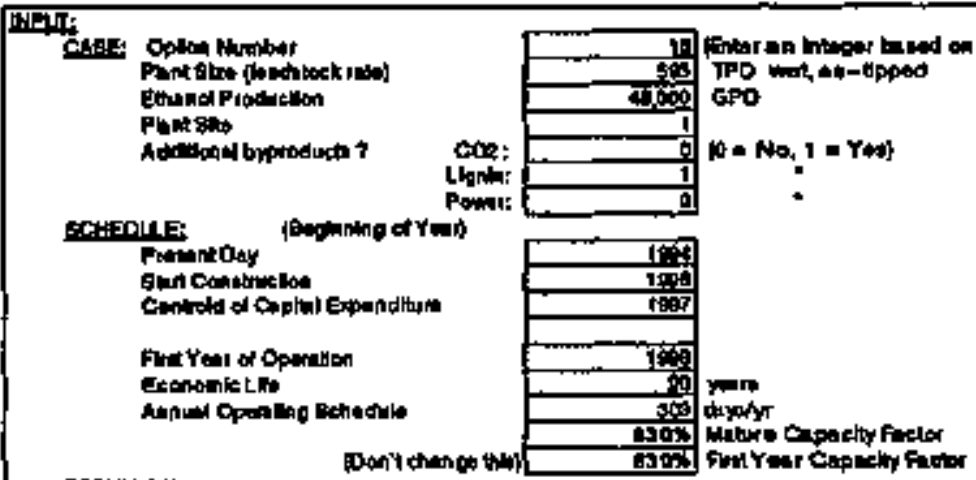

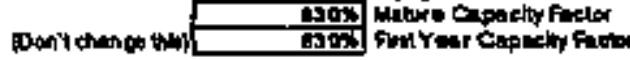

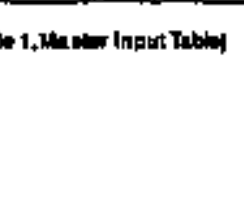

Bund

Butinge.

Drvolion

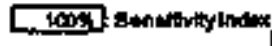

ancons

abmen conmenton

Thate PrM

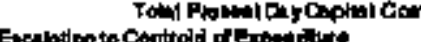

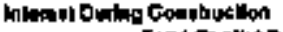

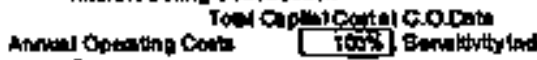

Fust:

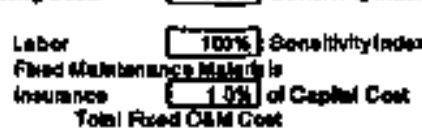

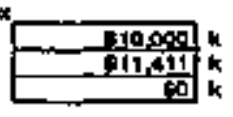

Verteth:

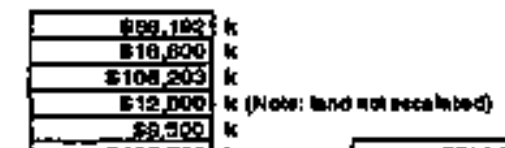

or retsogn por
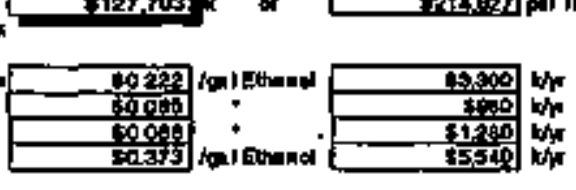

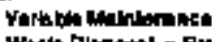

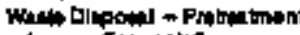
: Fonnimition Chonimater

Unim:

Pow

Nelumeres

anom

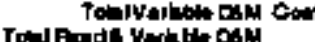
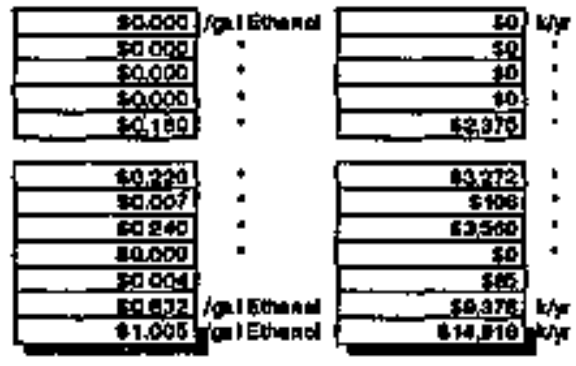

Thosine fos

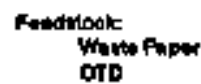

Wiv

nood wher

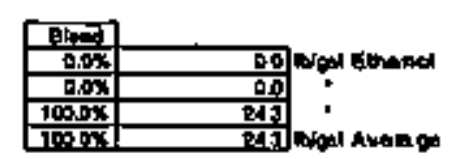

Anton

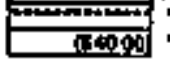

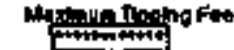 \\ 19000}

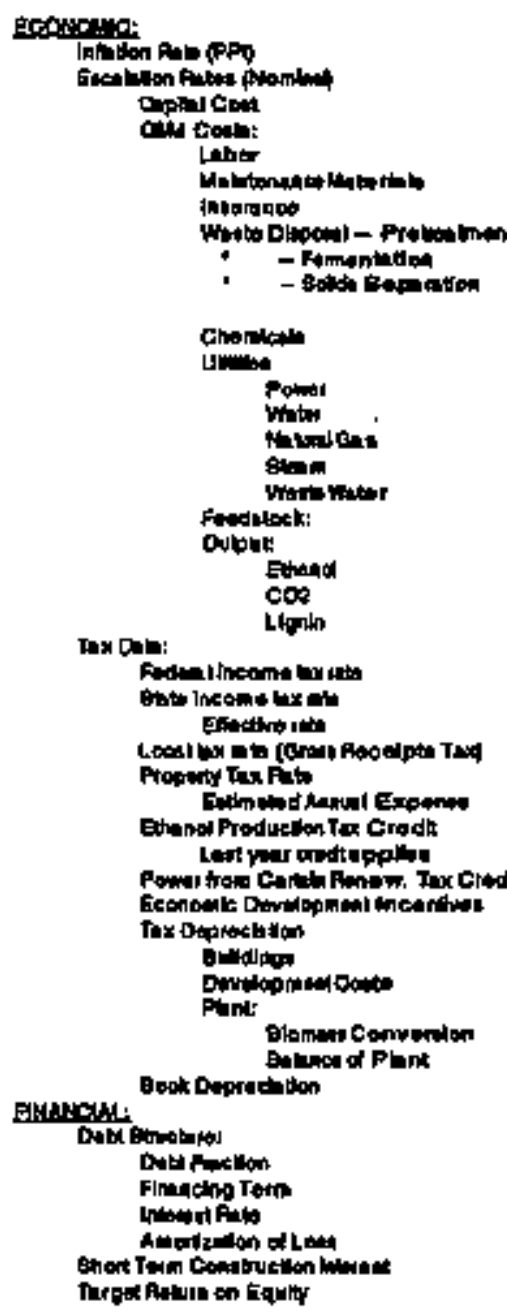

Eminewh.

terowno:

Irtidion Aus pPo

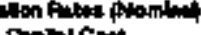

apd tout

Labt

inigrandos

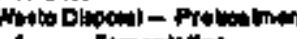

: $\quad$ - Fomentietlon

Chonketh

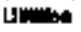

Pomits

thetriant

Stan

vatsmater

Forditox:

Ethand

Inx Dan:

LLFon

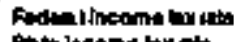

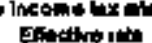

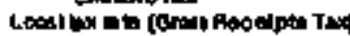

Propersy Tax Fat

Intmind hanul Exponon

anol Producton Ta. Cradk

Lent vir undtappling

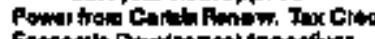

Thy Doprockltos

ond

Dmillop

Olomari Comporidon

Buluesed of Plent

Book Deprachidon

axis

Dut

Ineming Toin

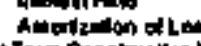

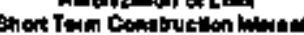

Thegex Autuls en Equity
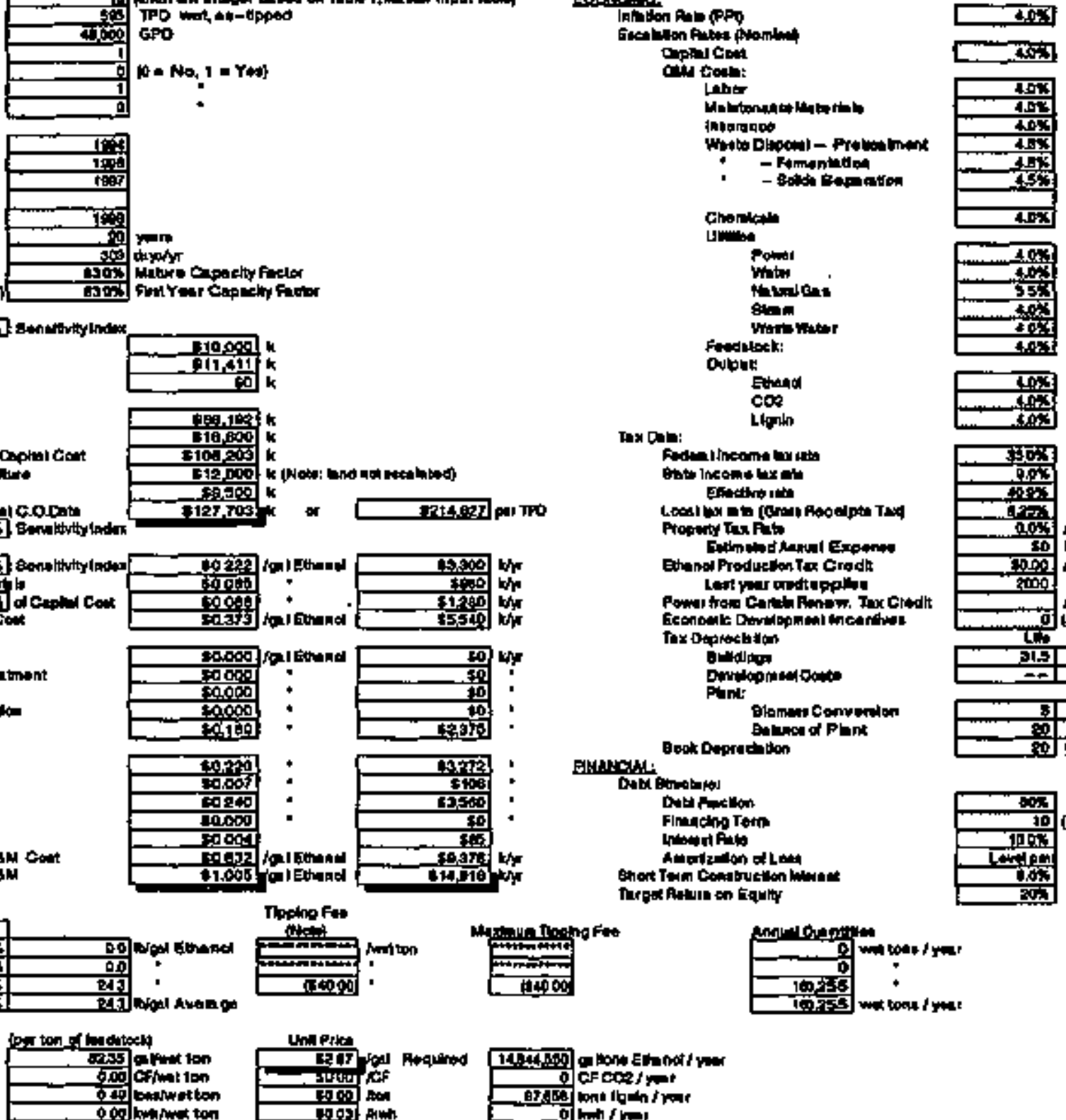

14:40. E. 


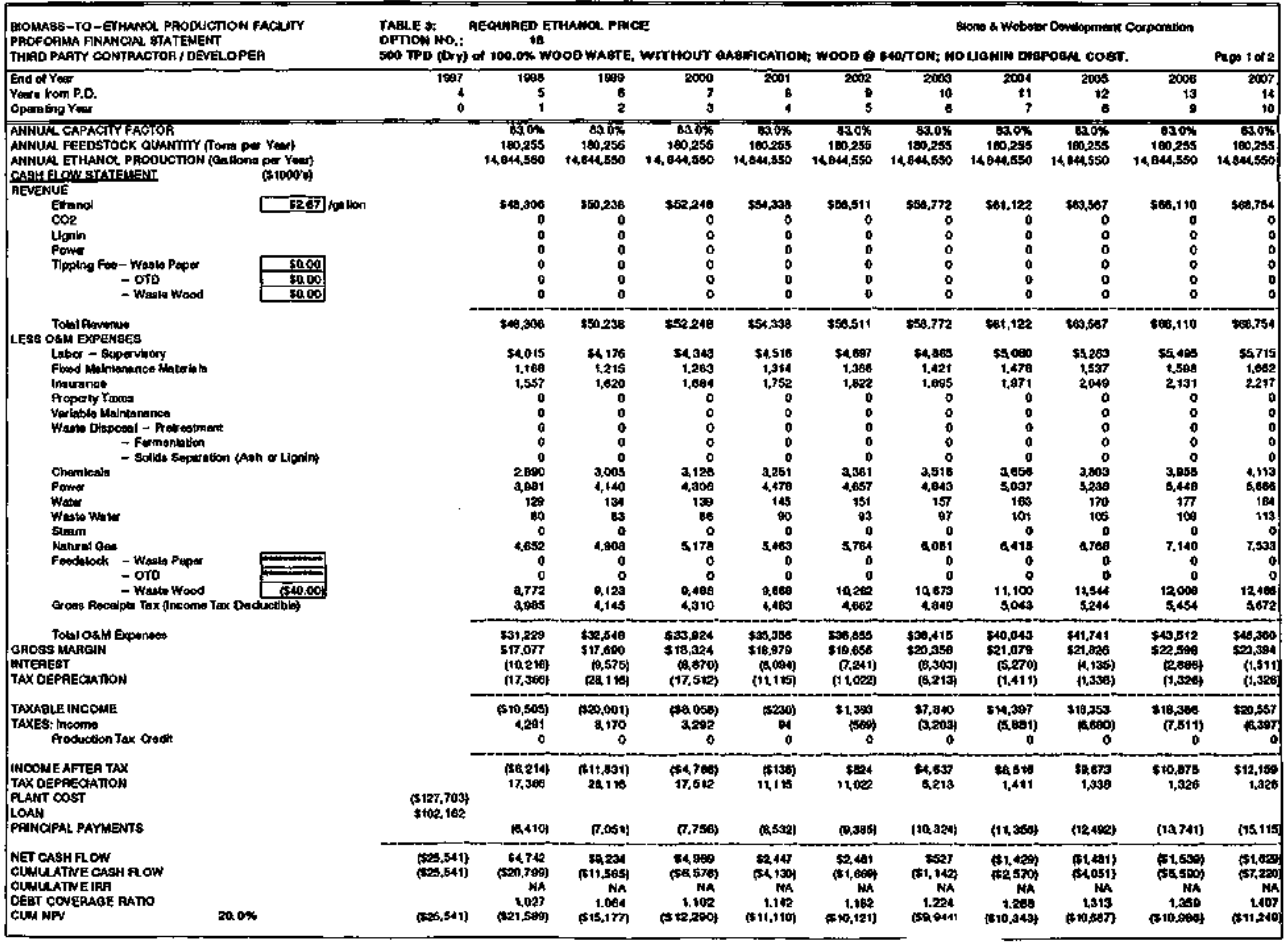




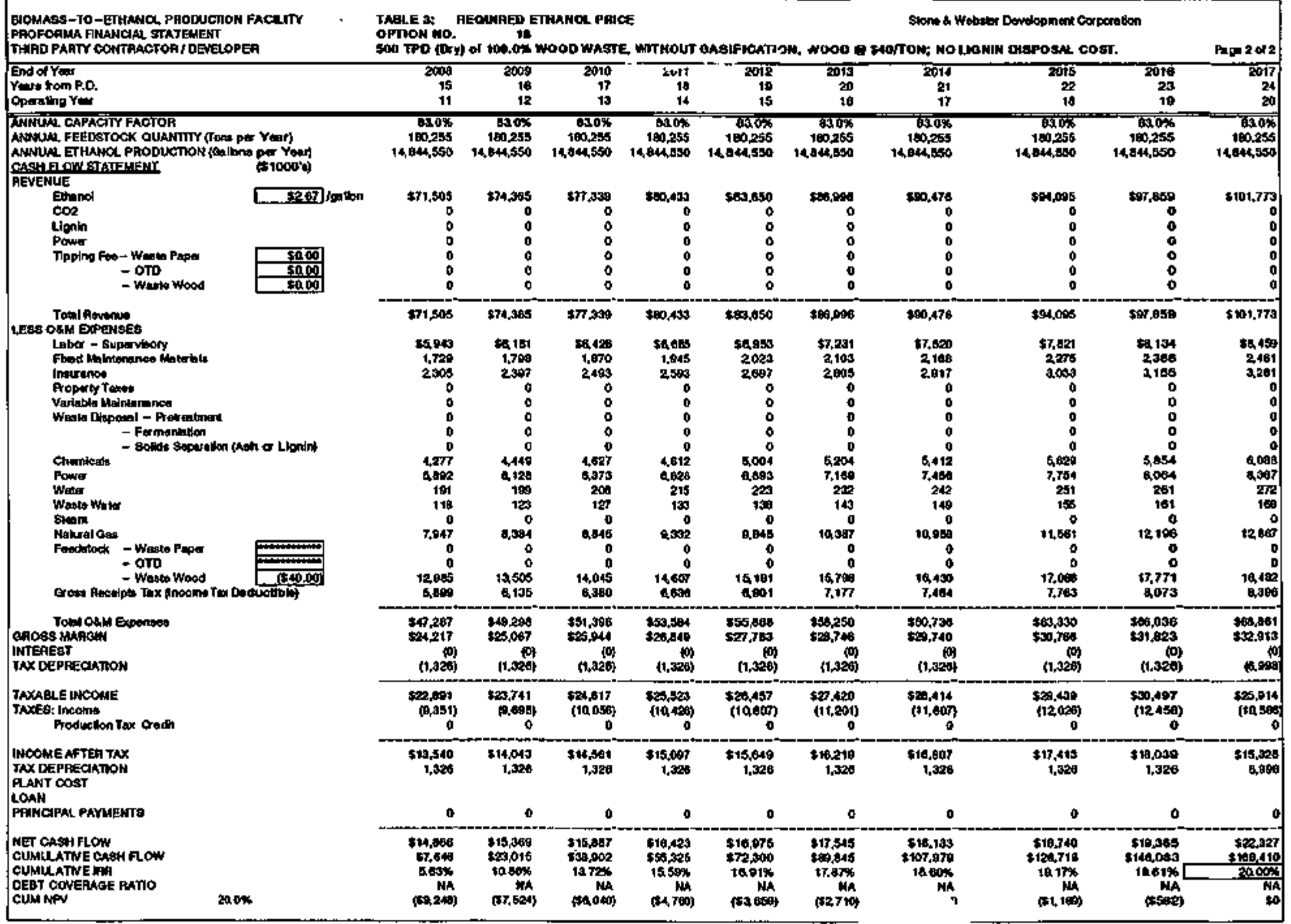




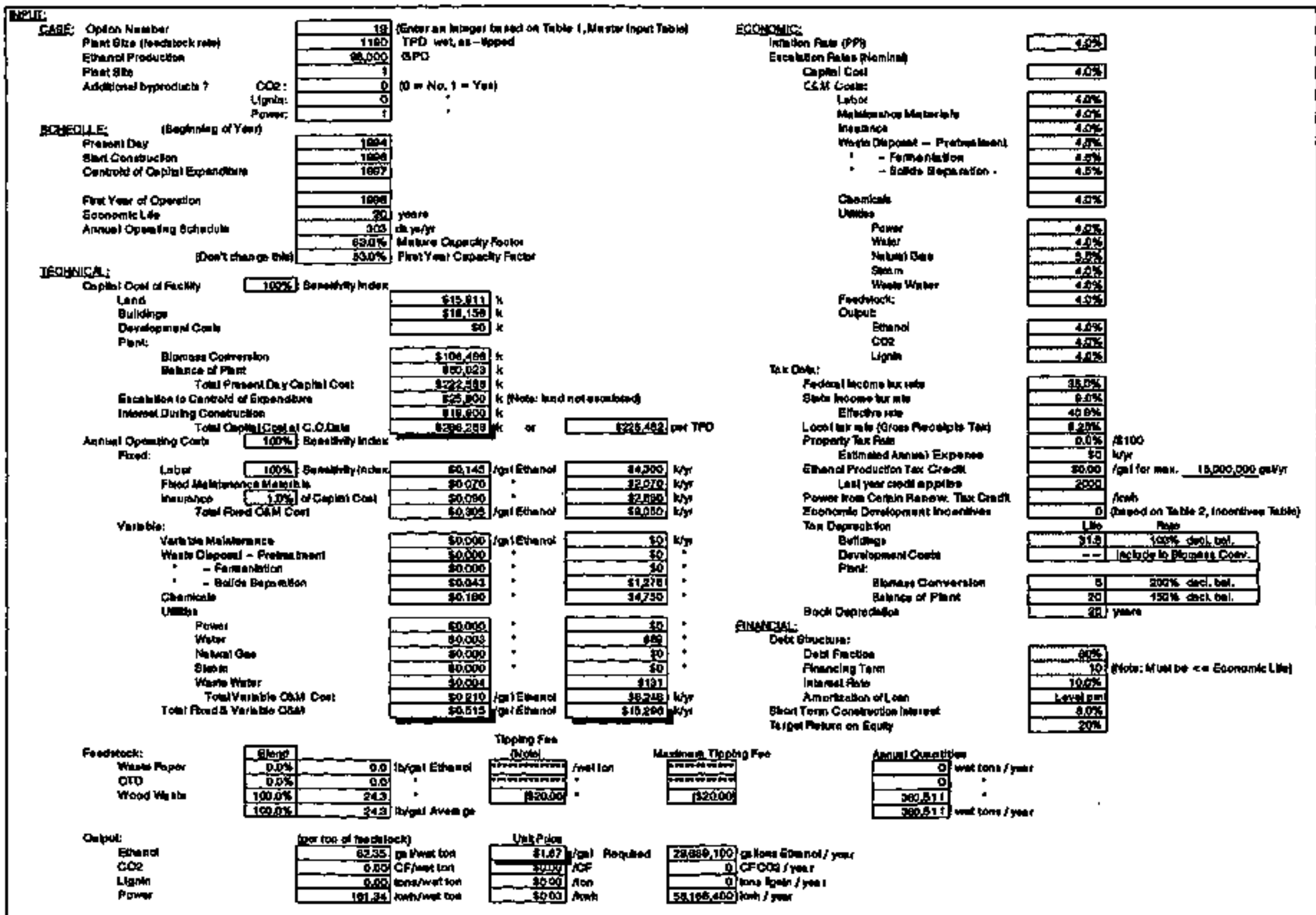

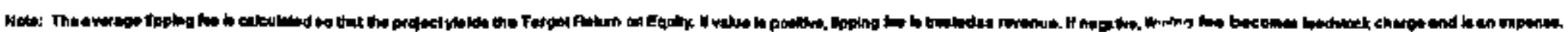




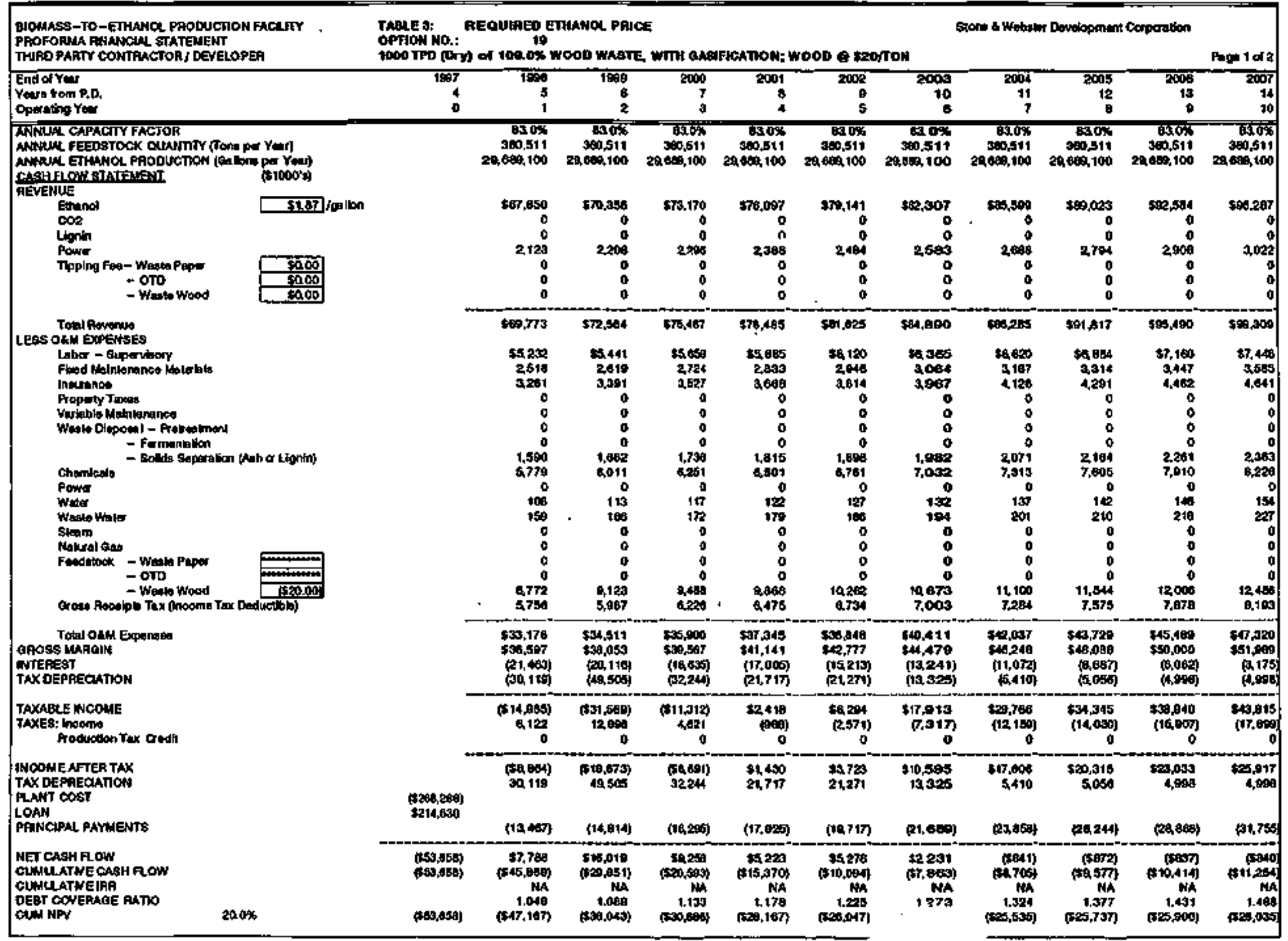




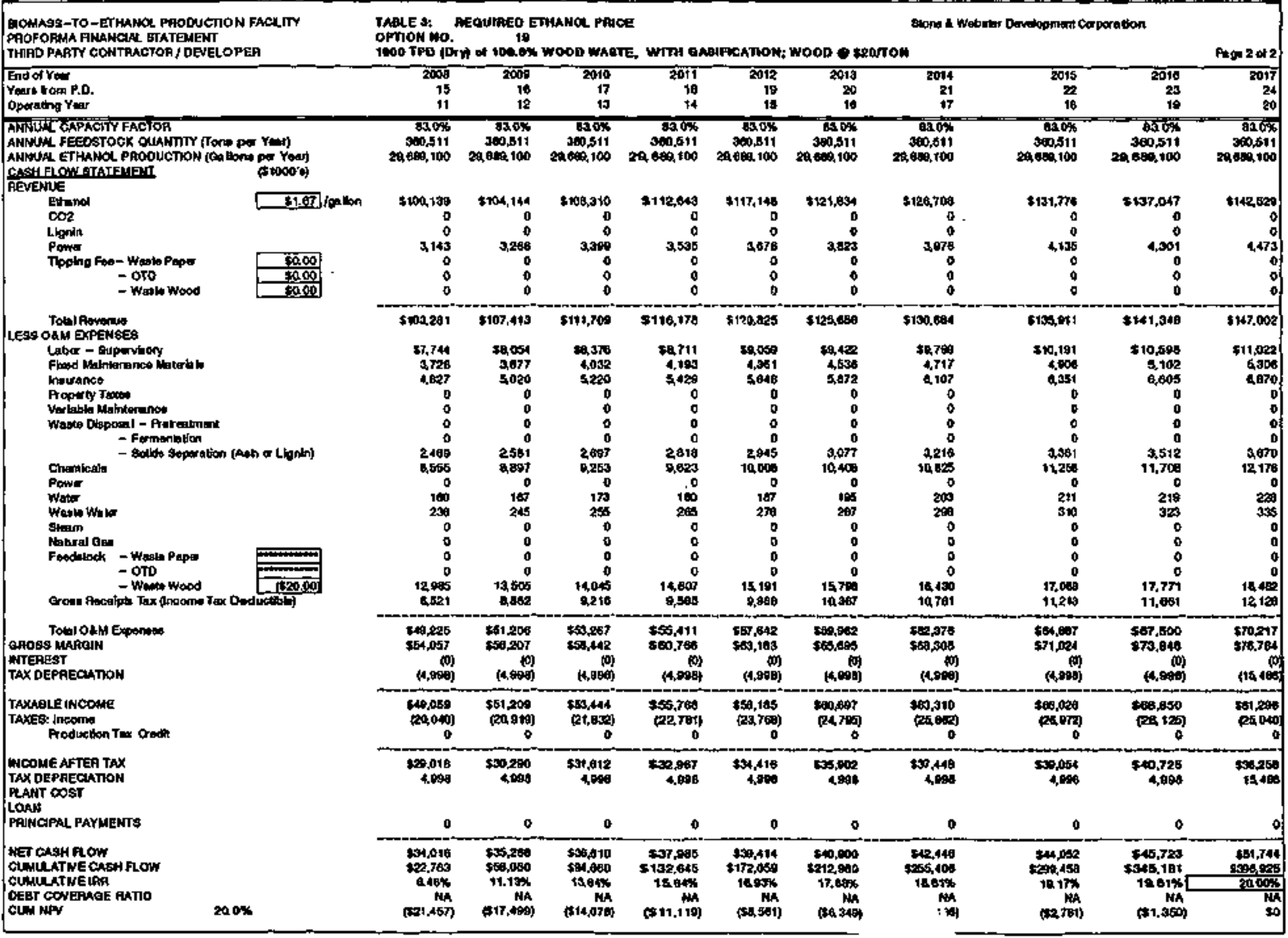




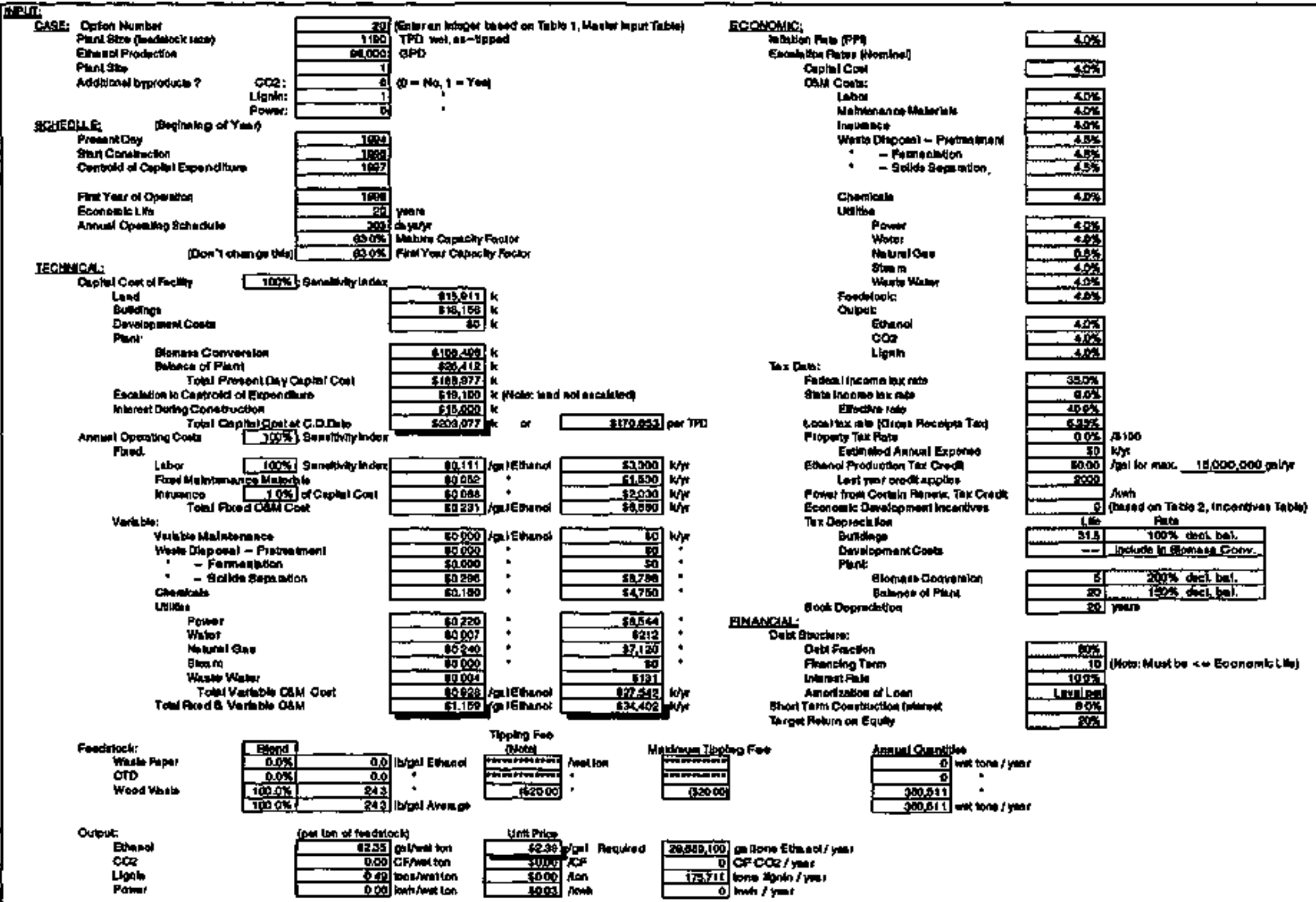

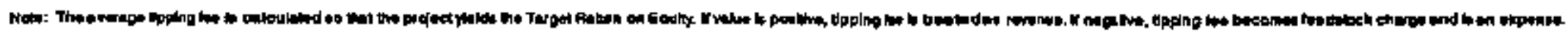




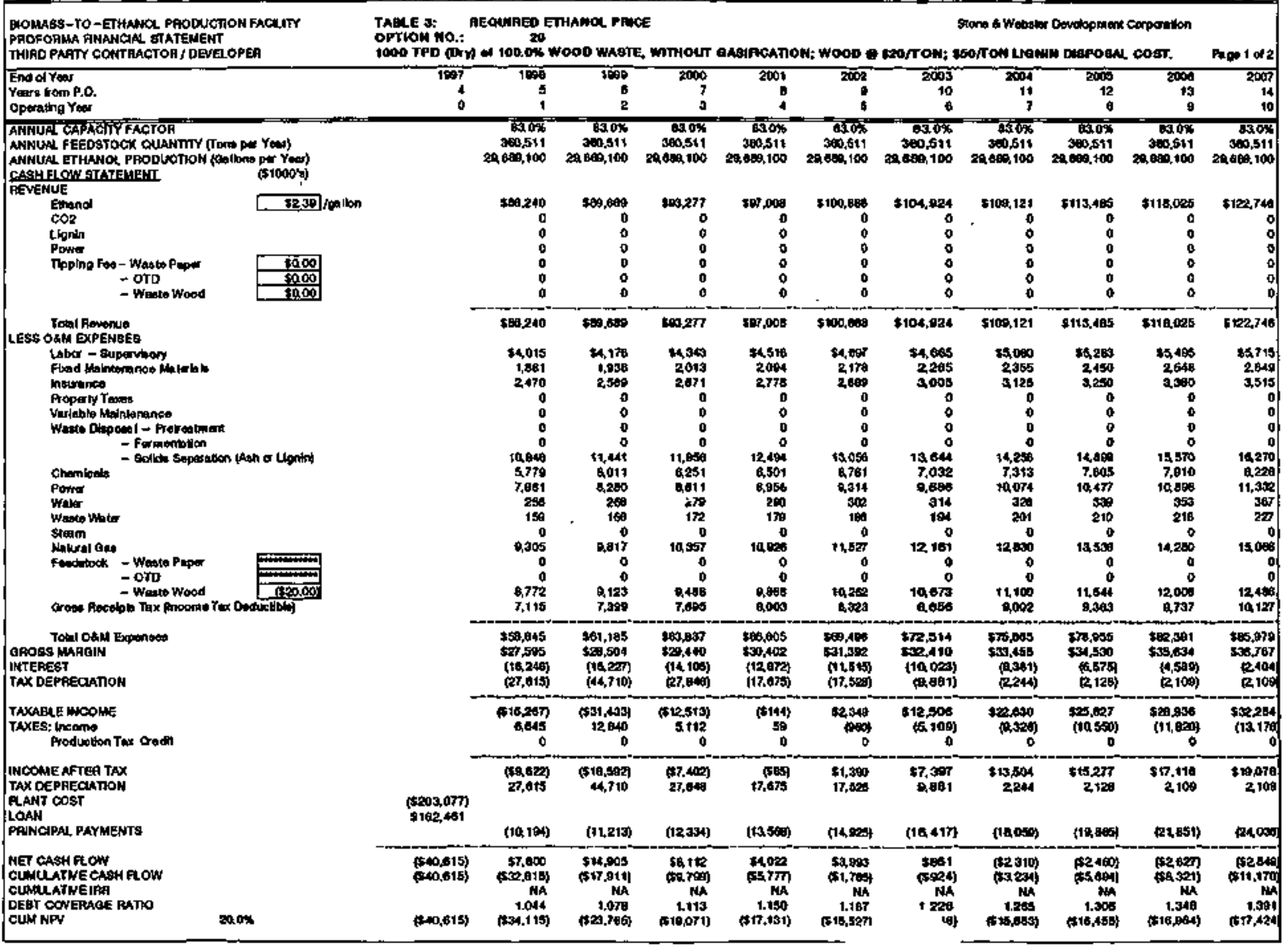




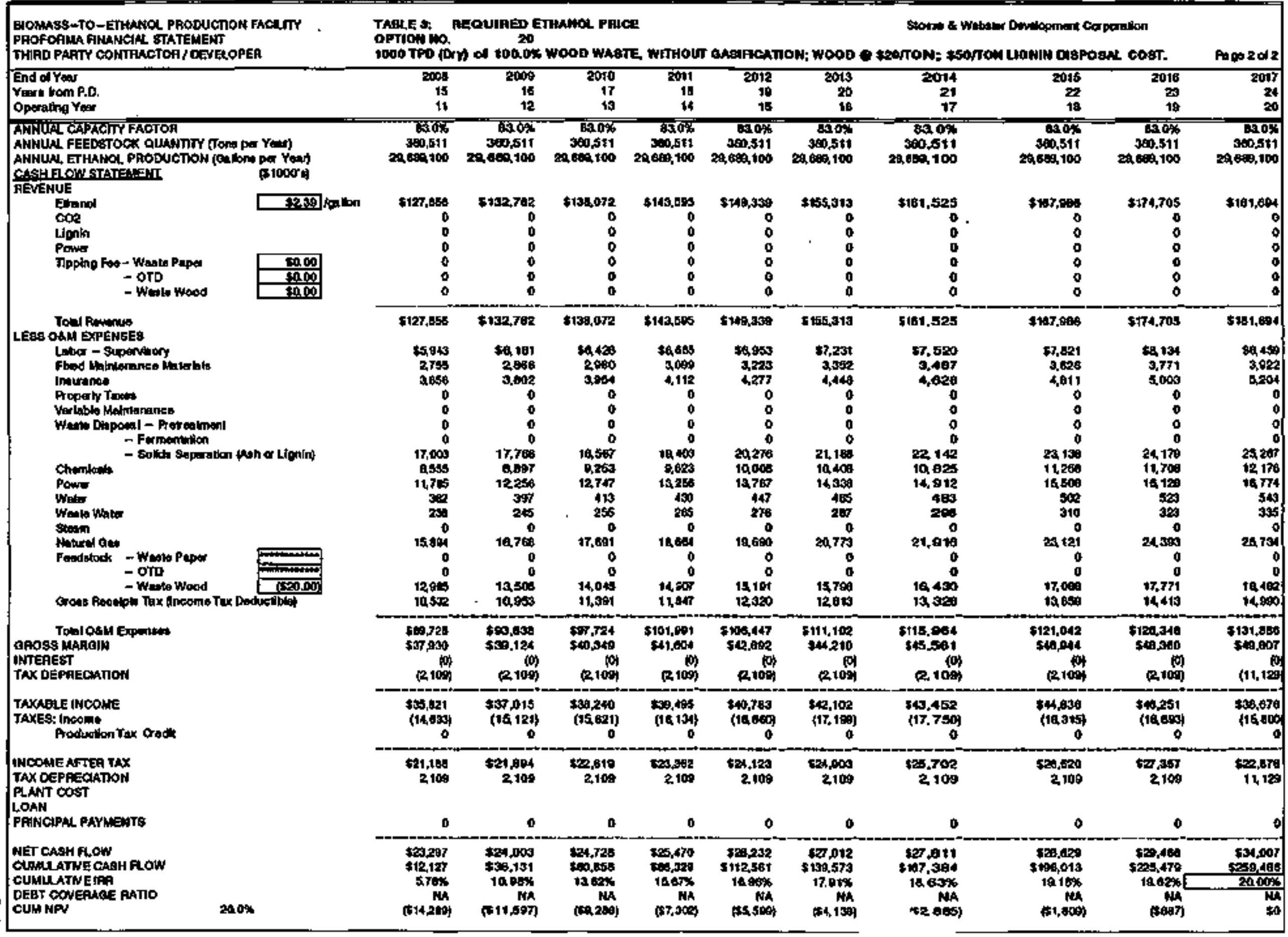




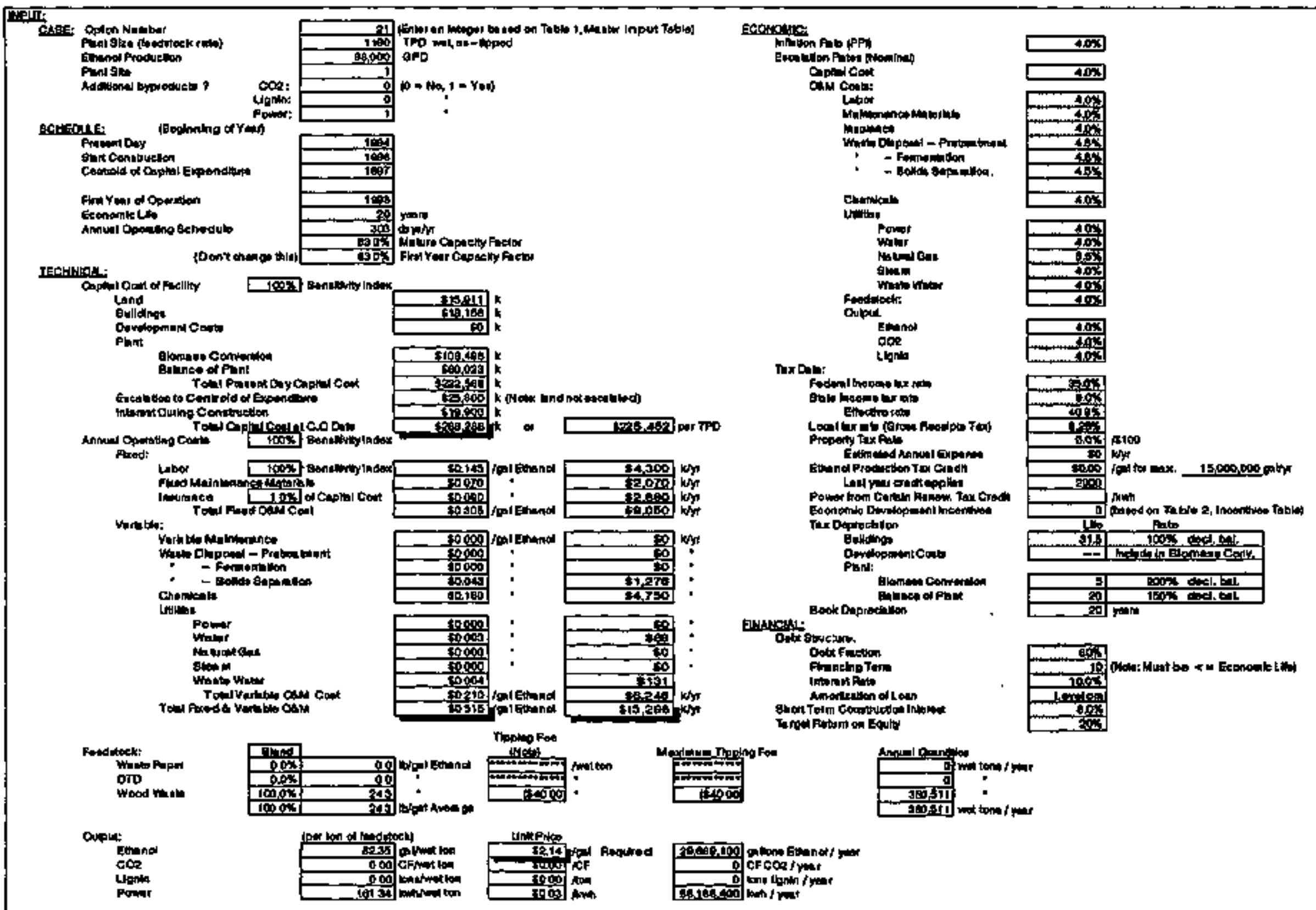

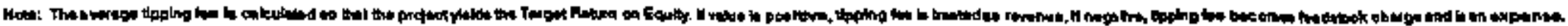




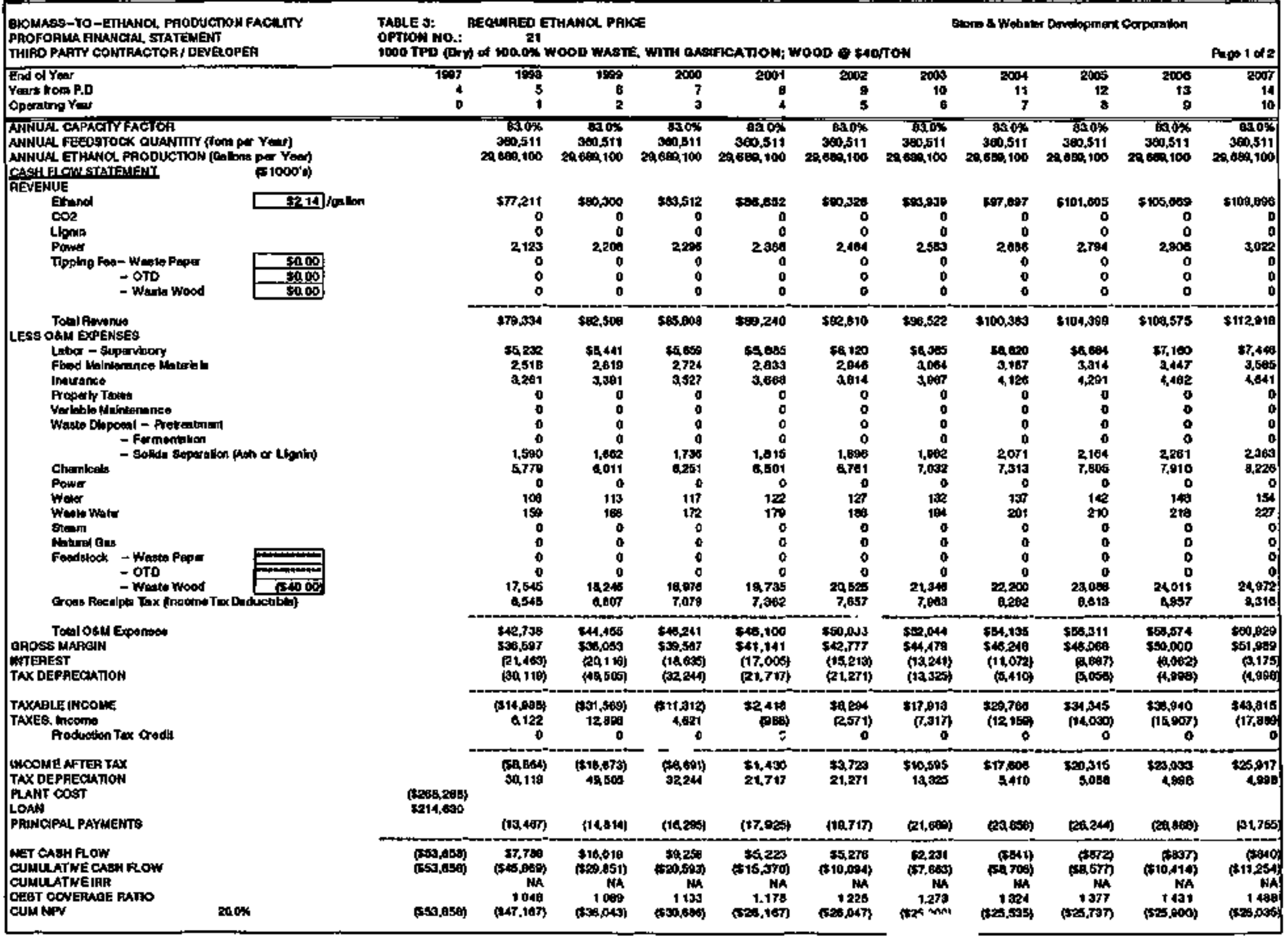




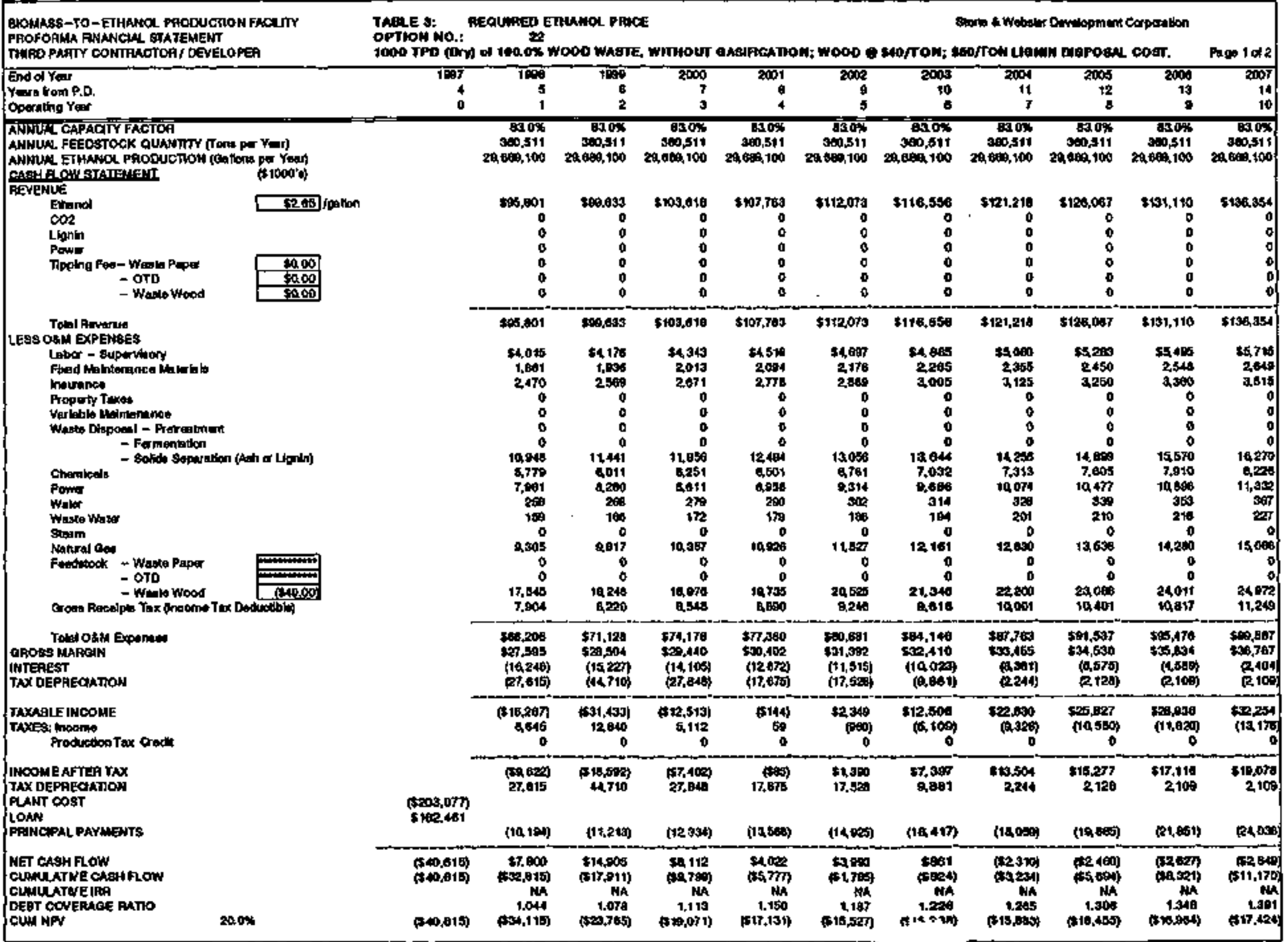




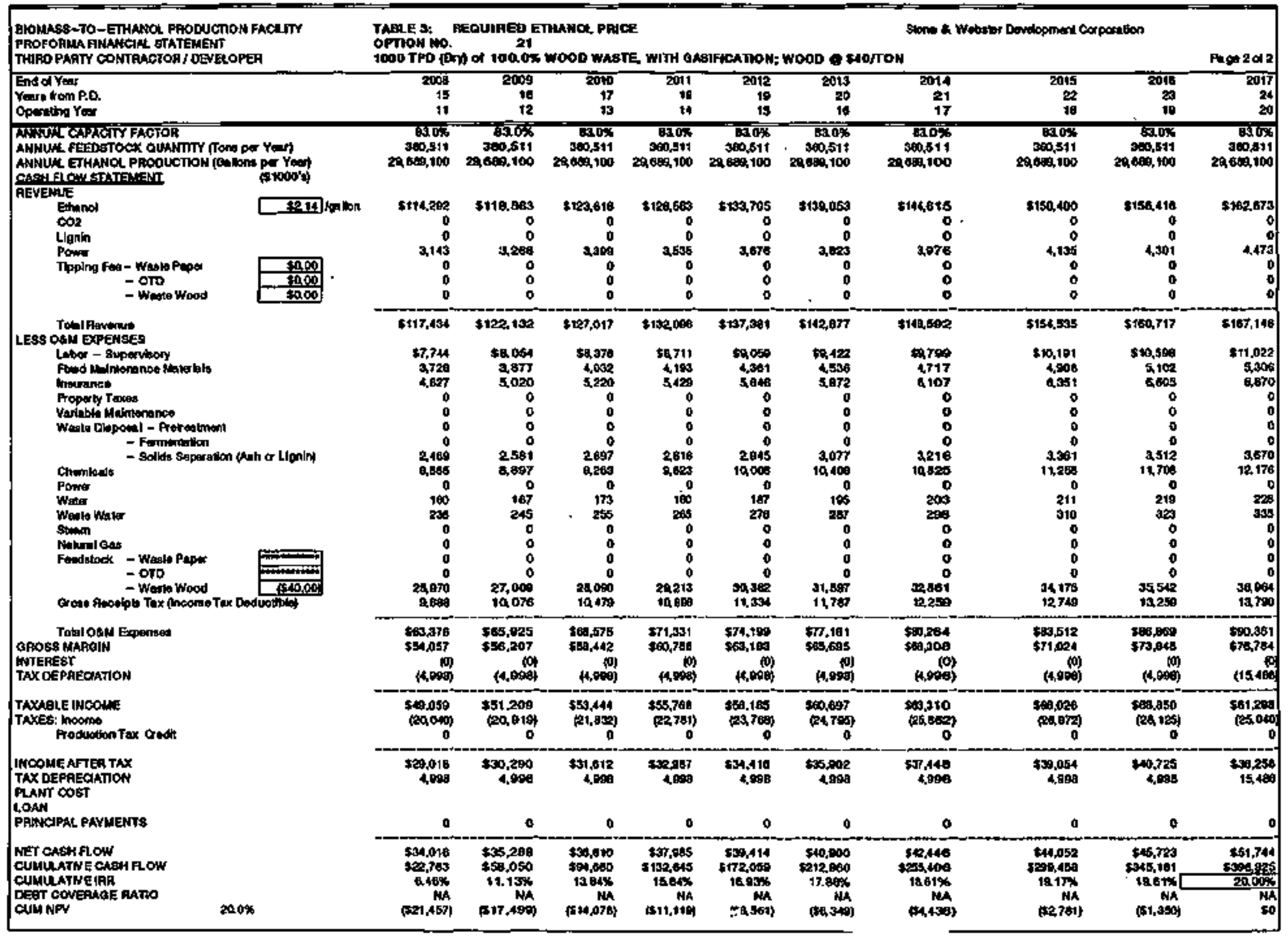




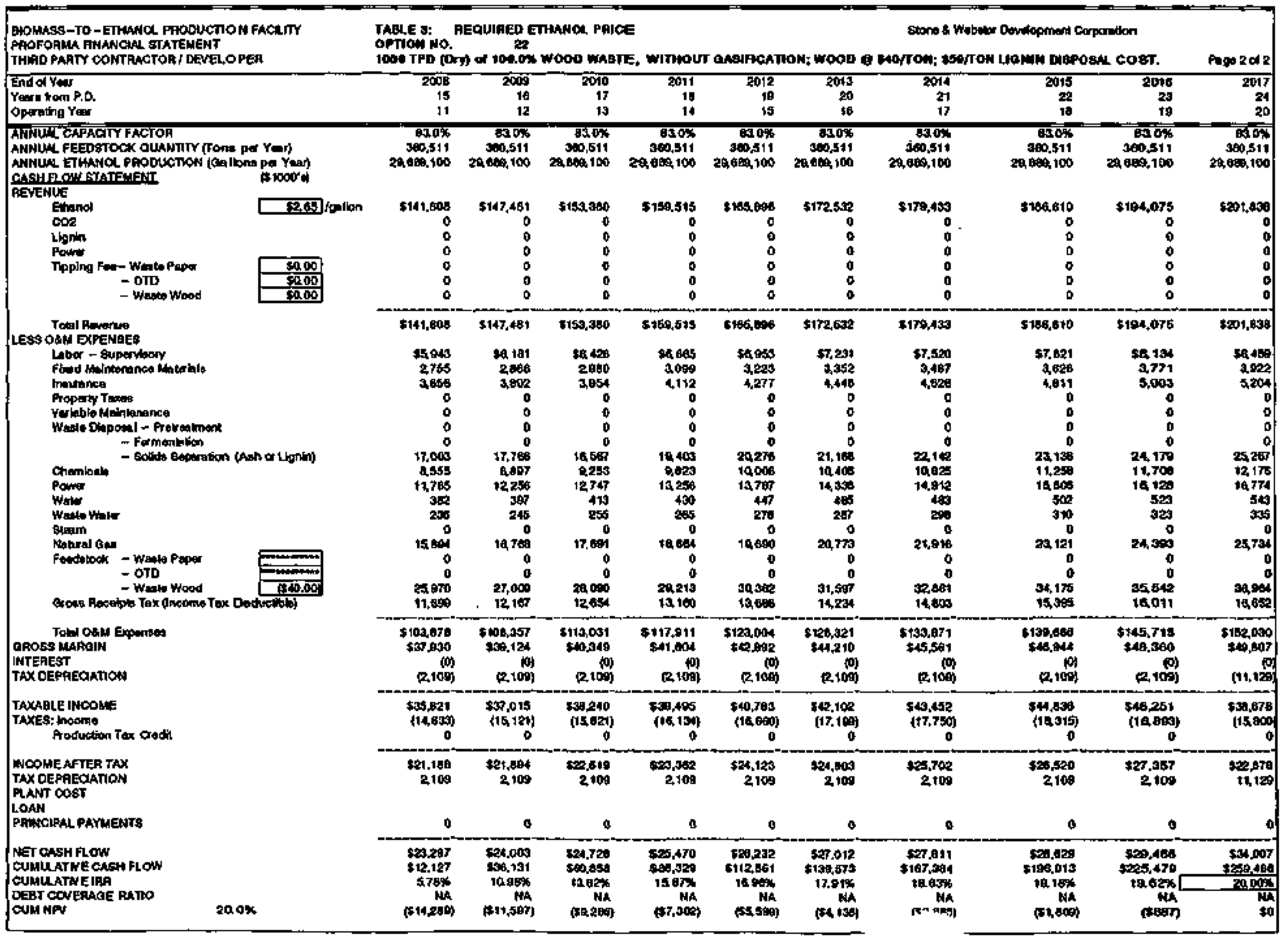




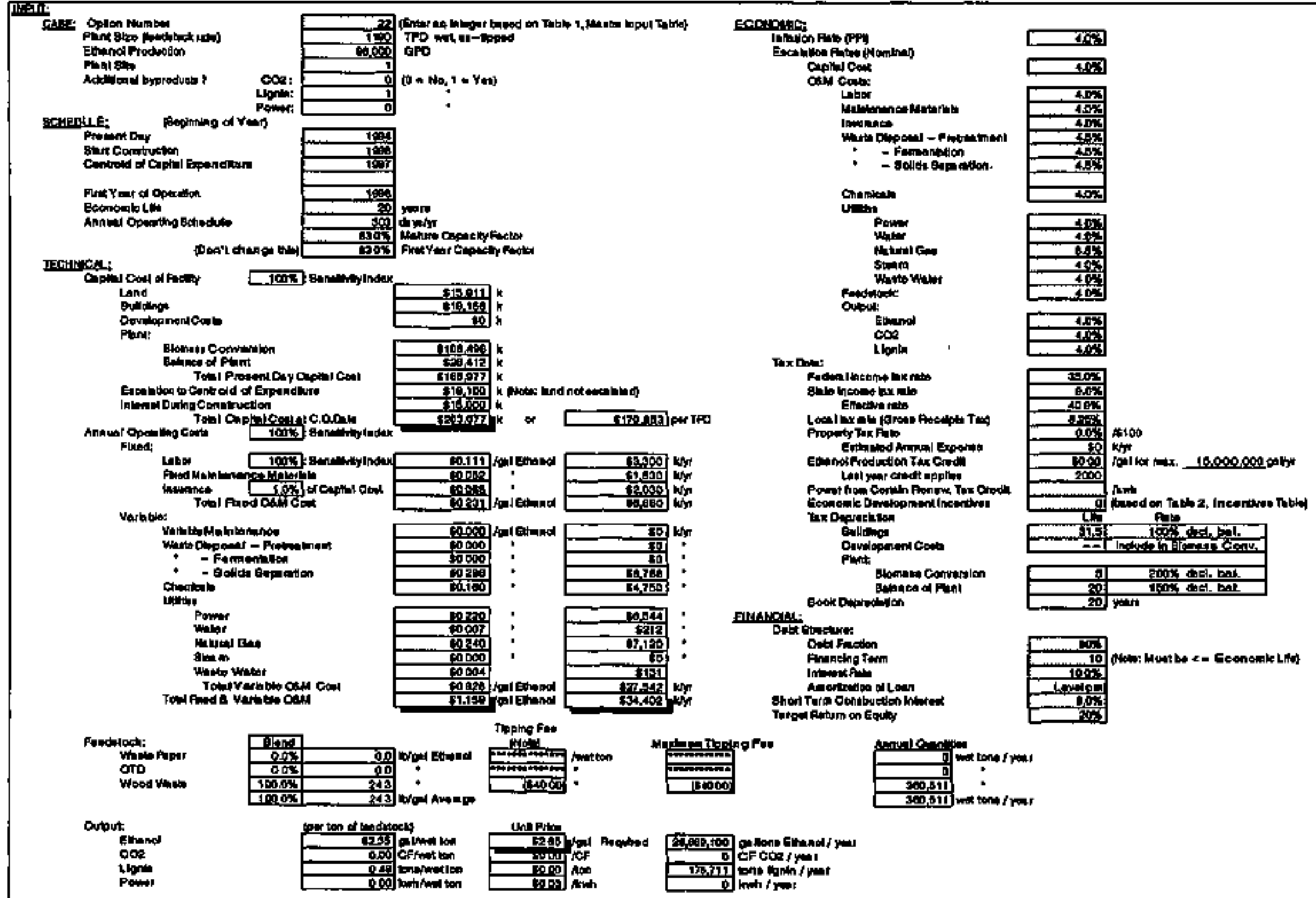

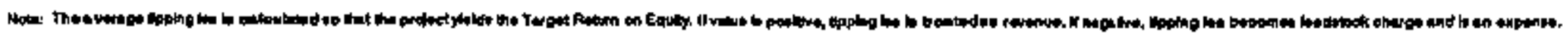




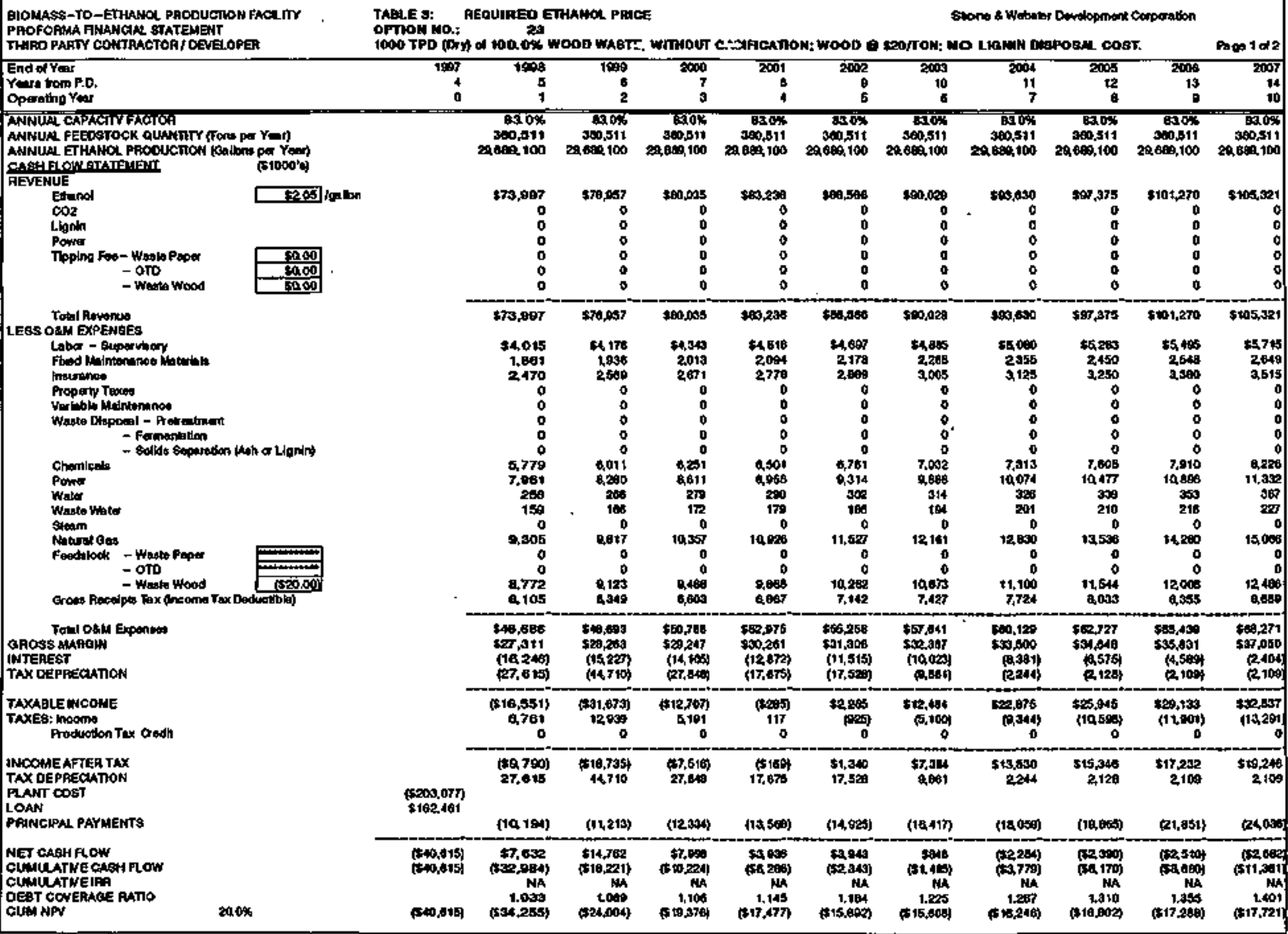




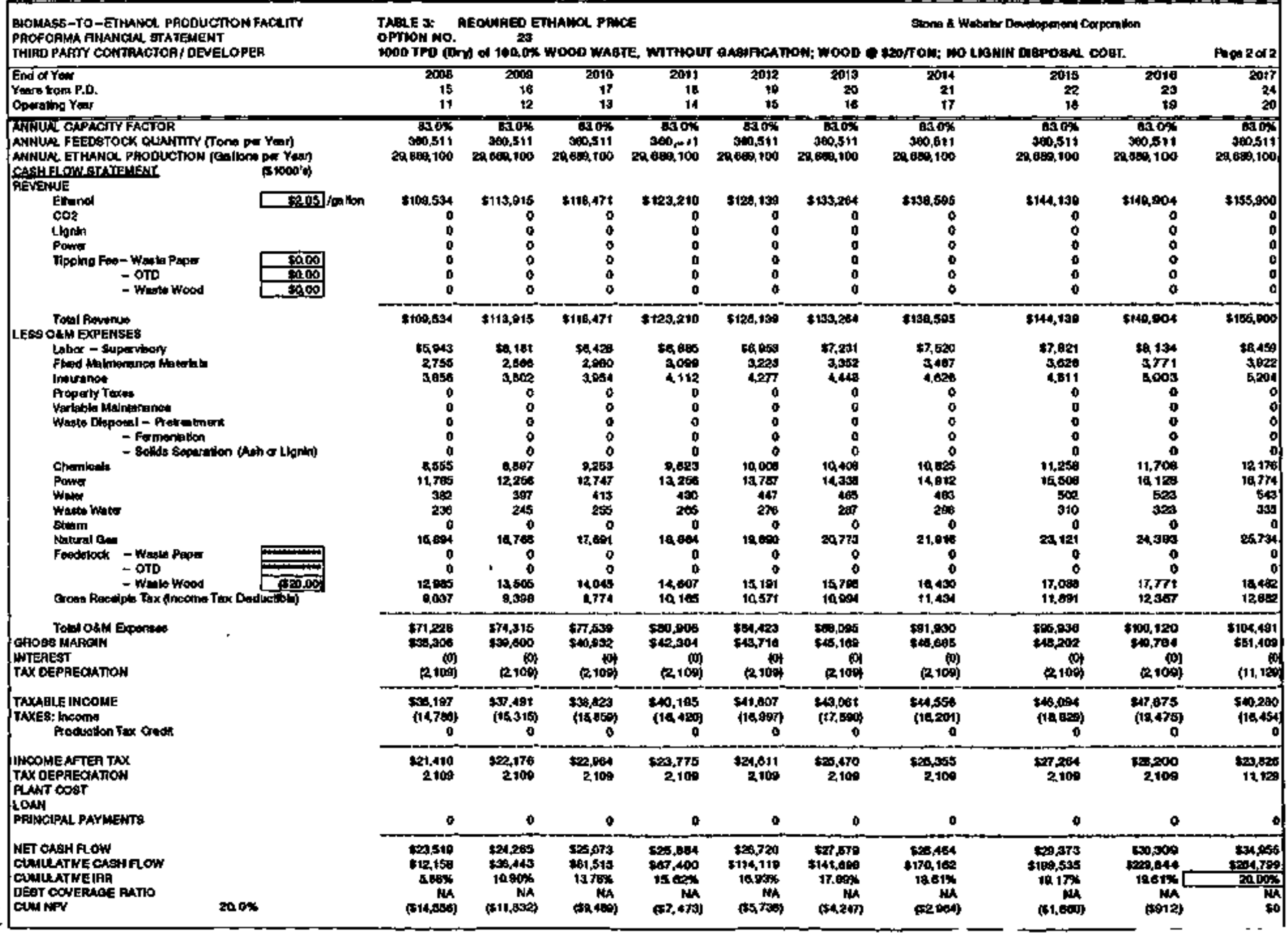




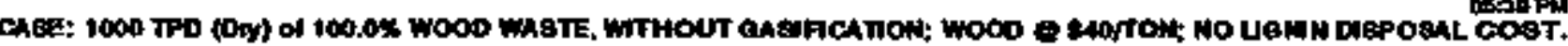

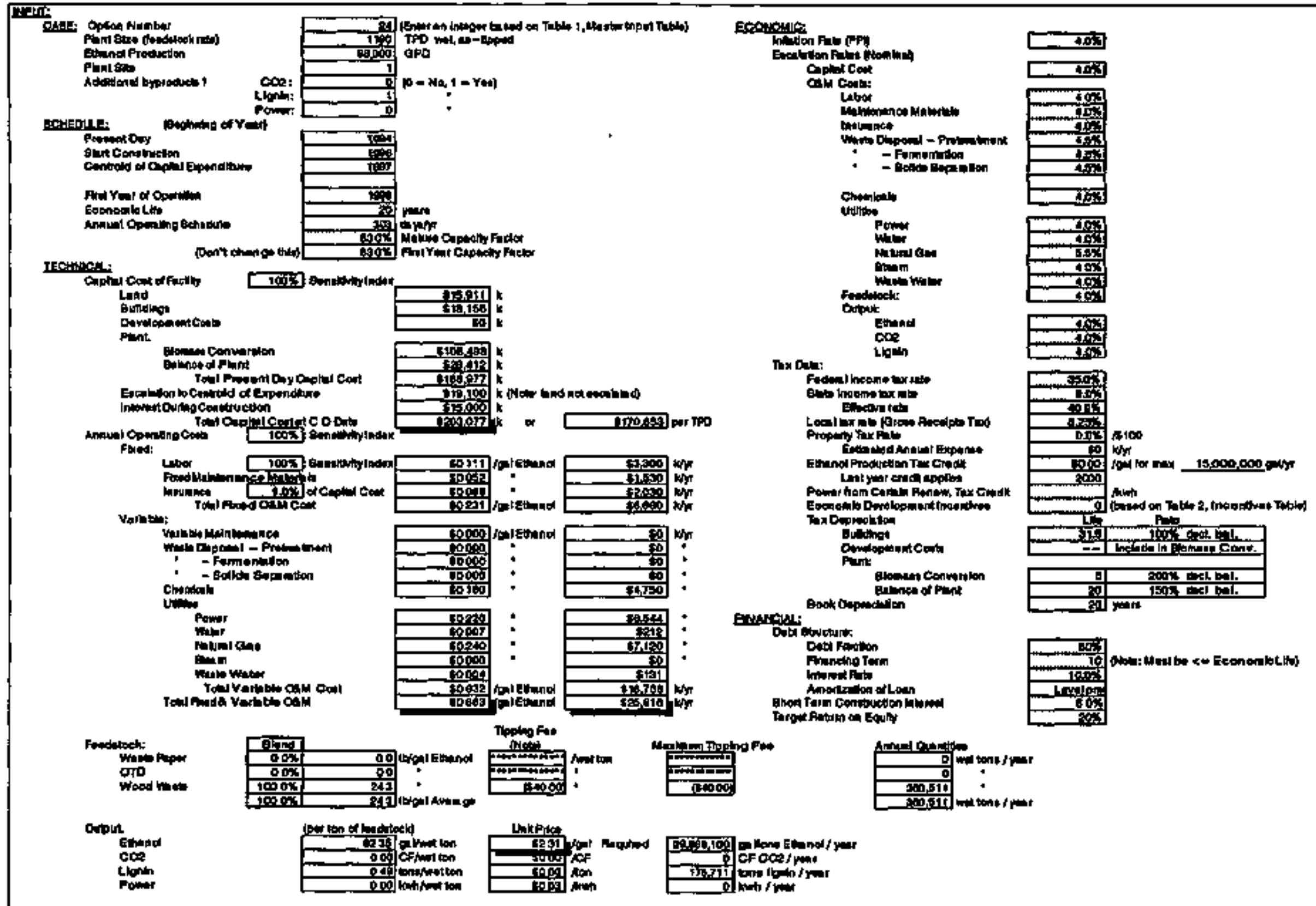

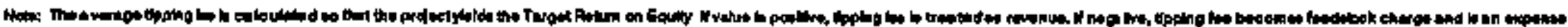




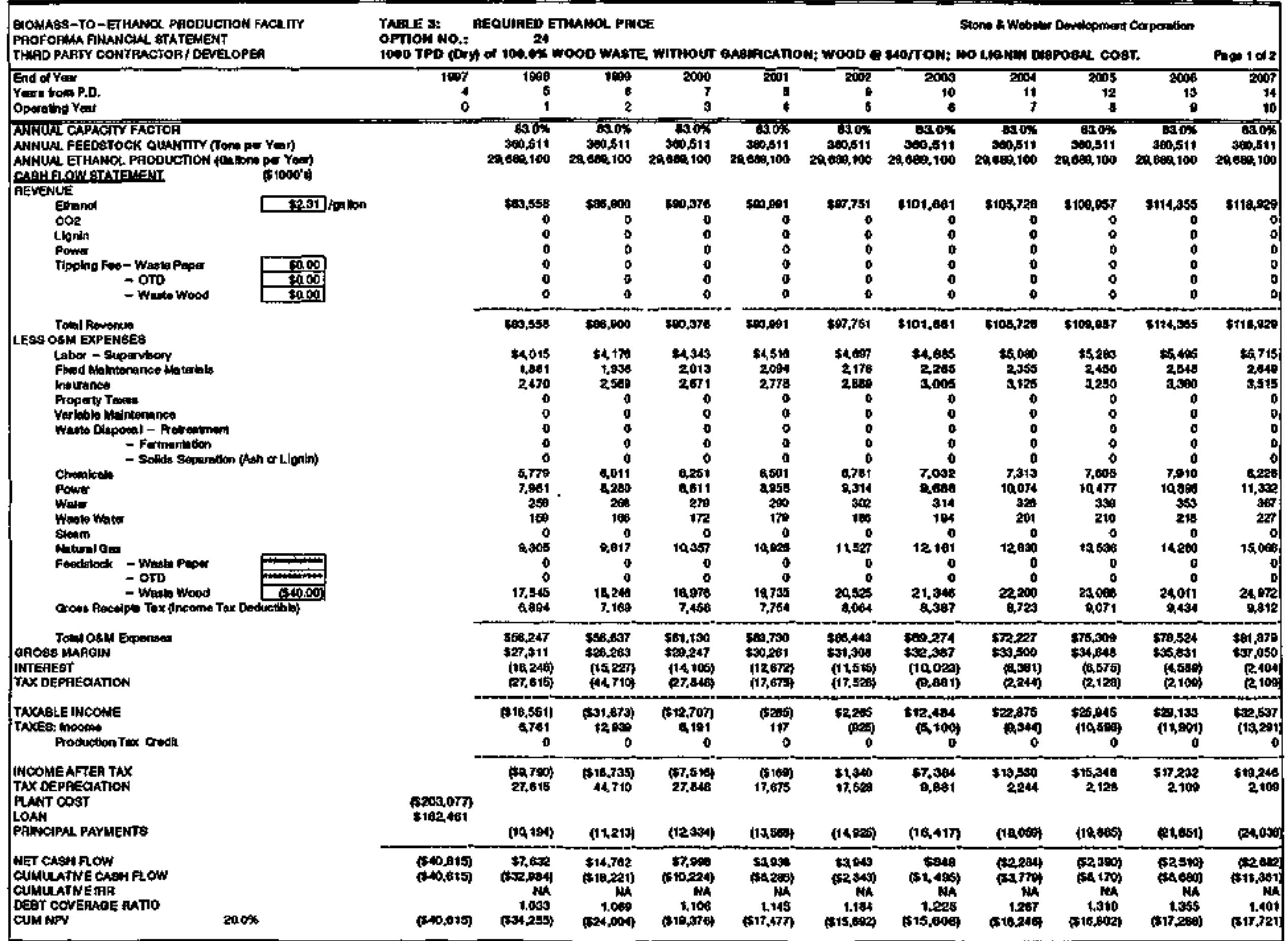




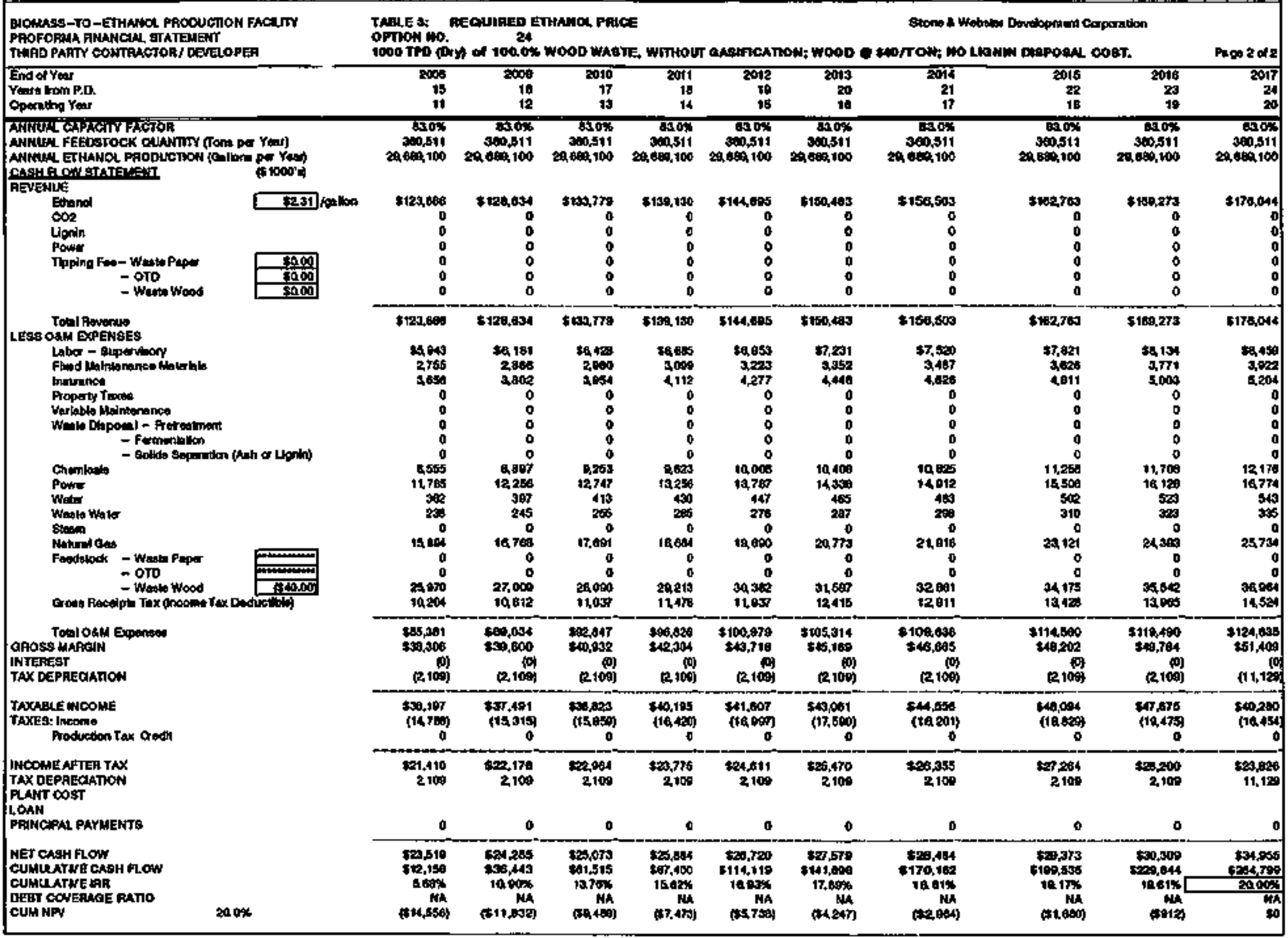




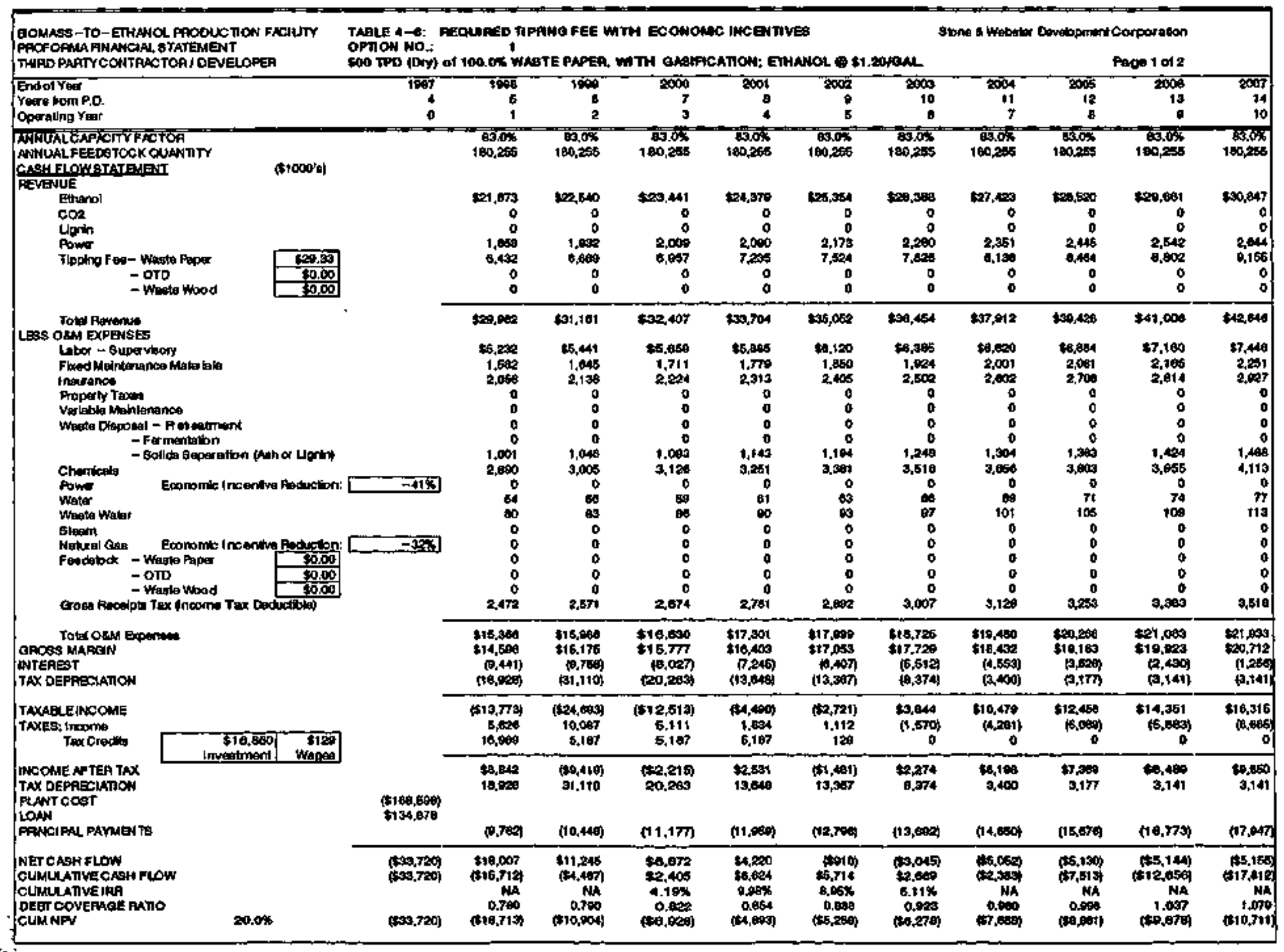




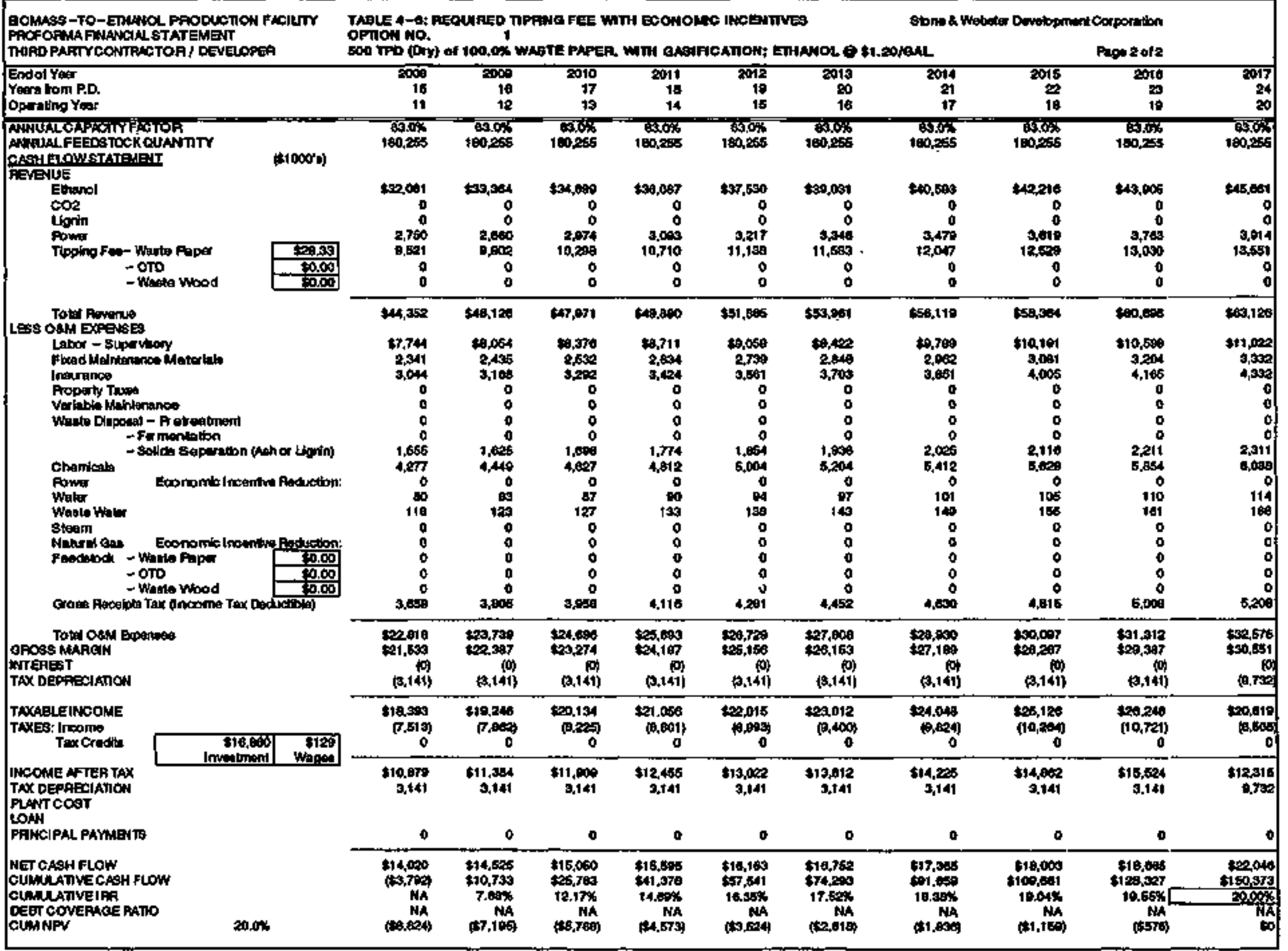




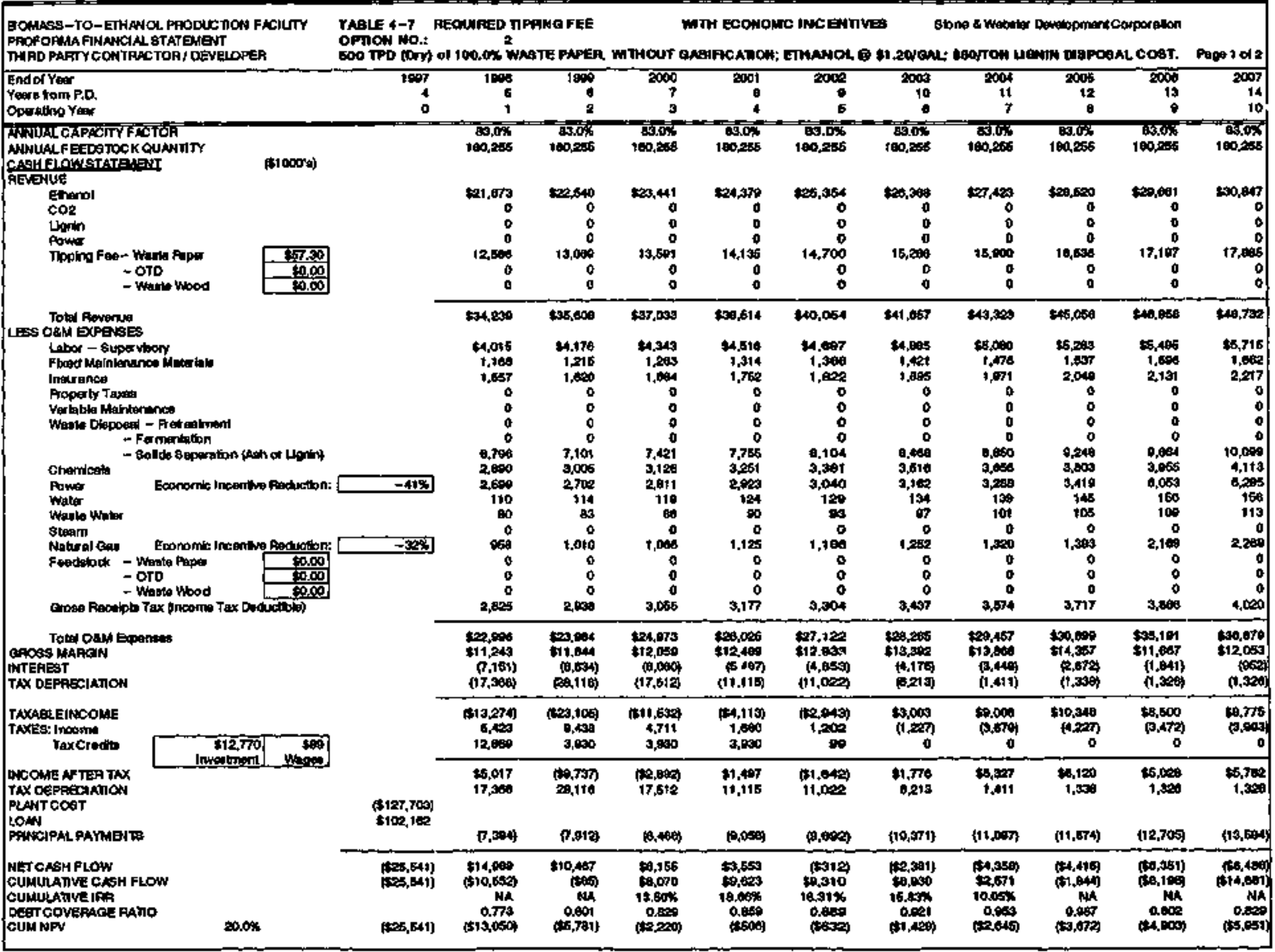




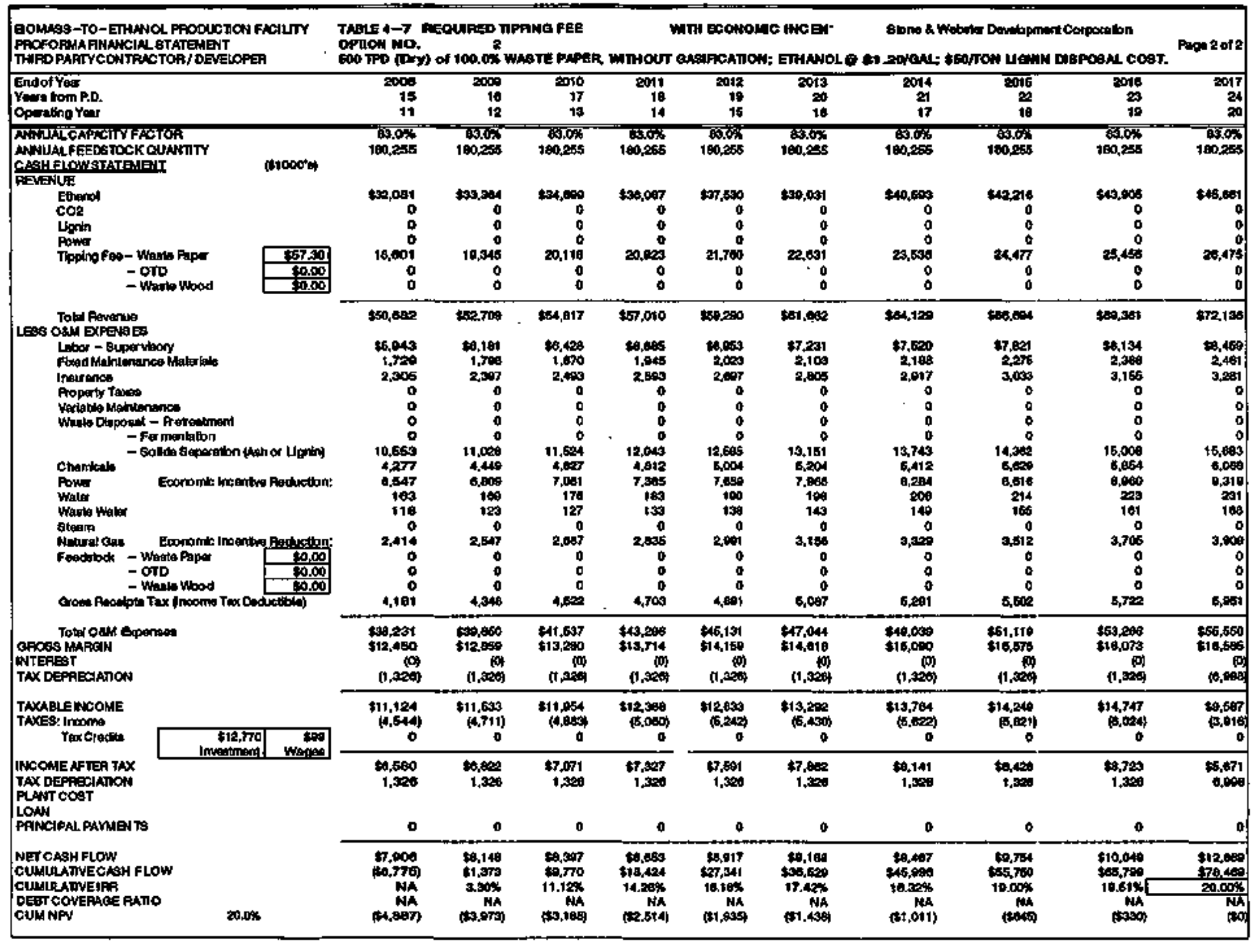




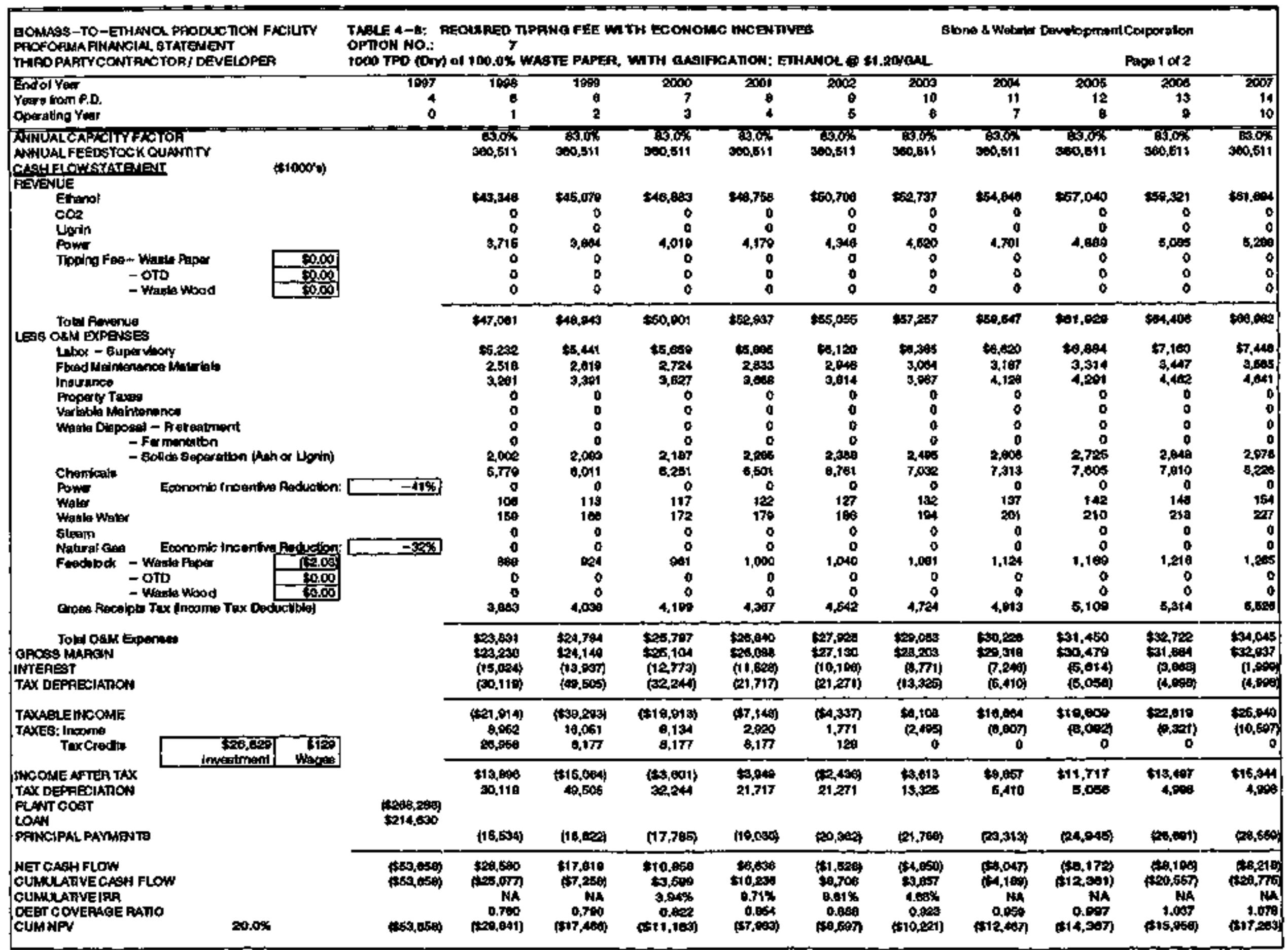




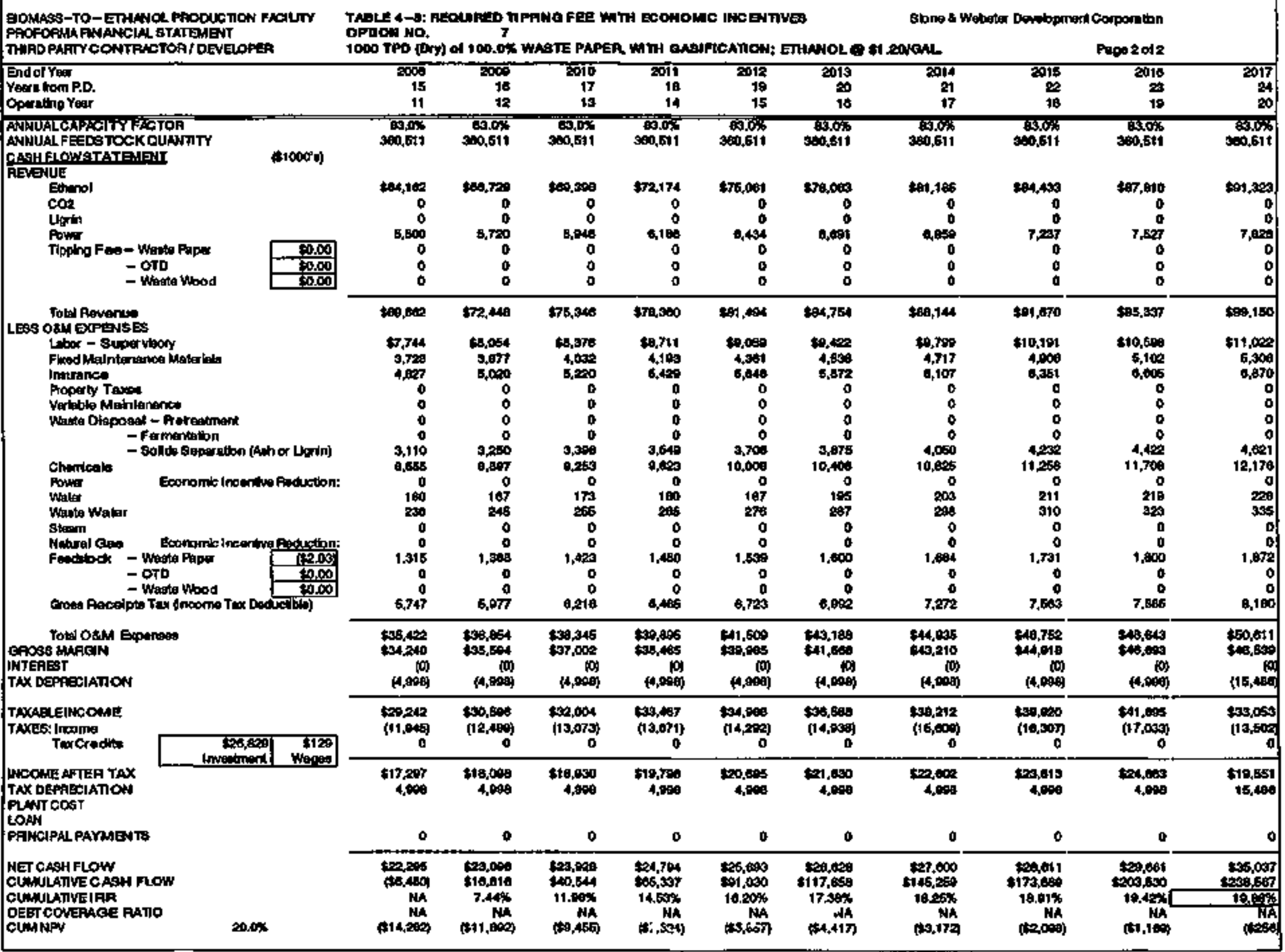




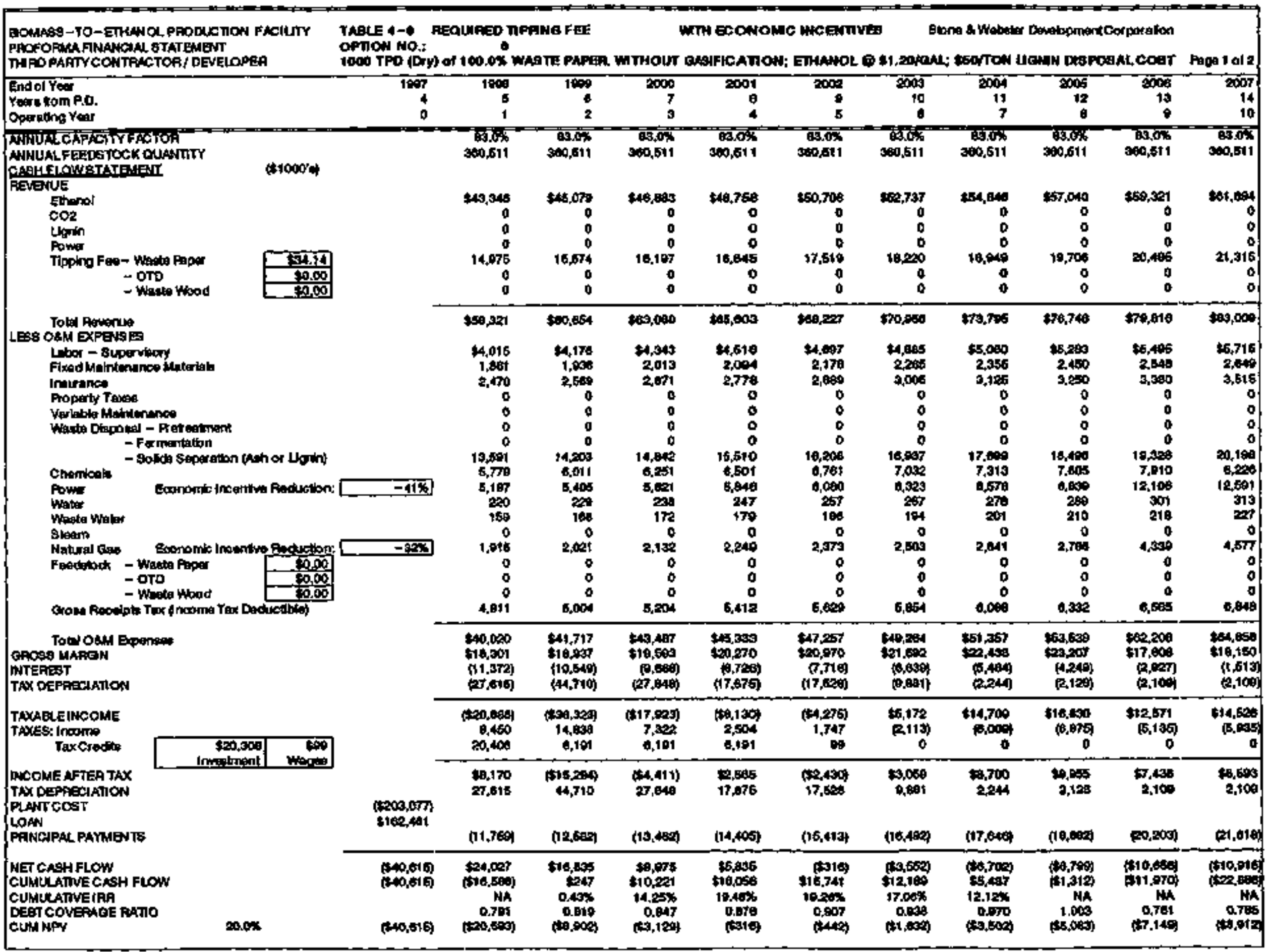




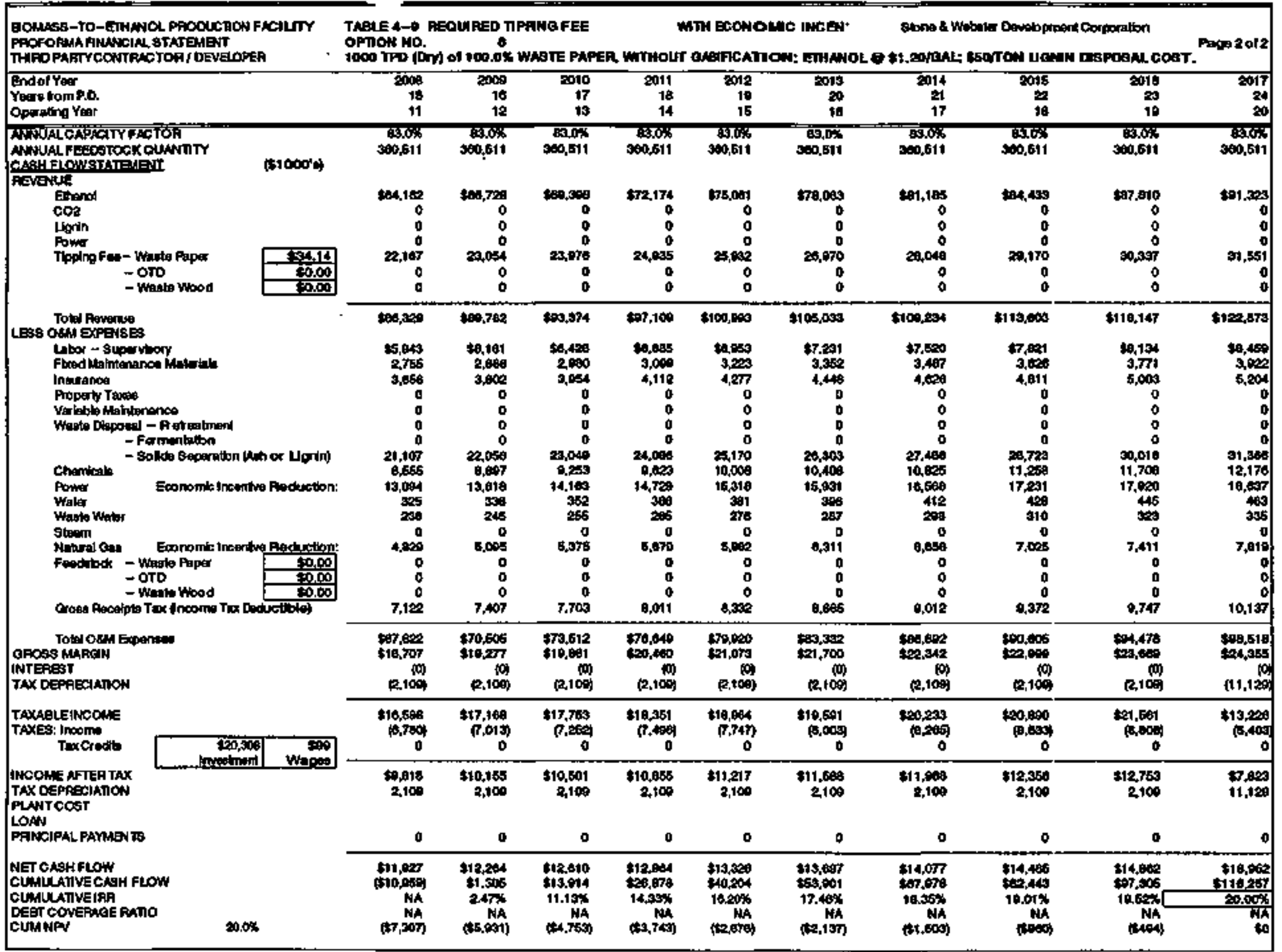




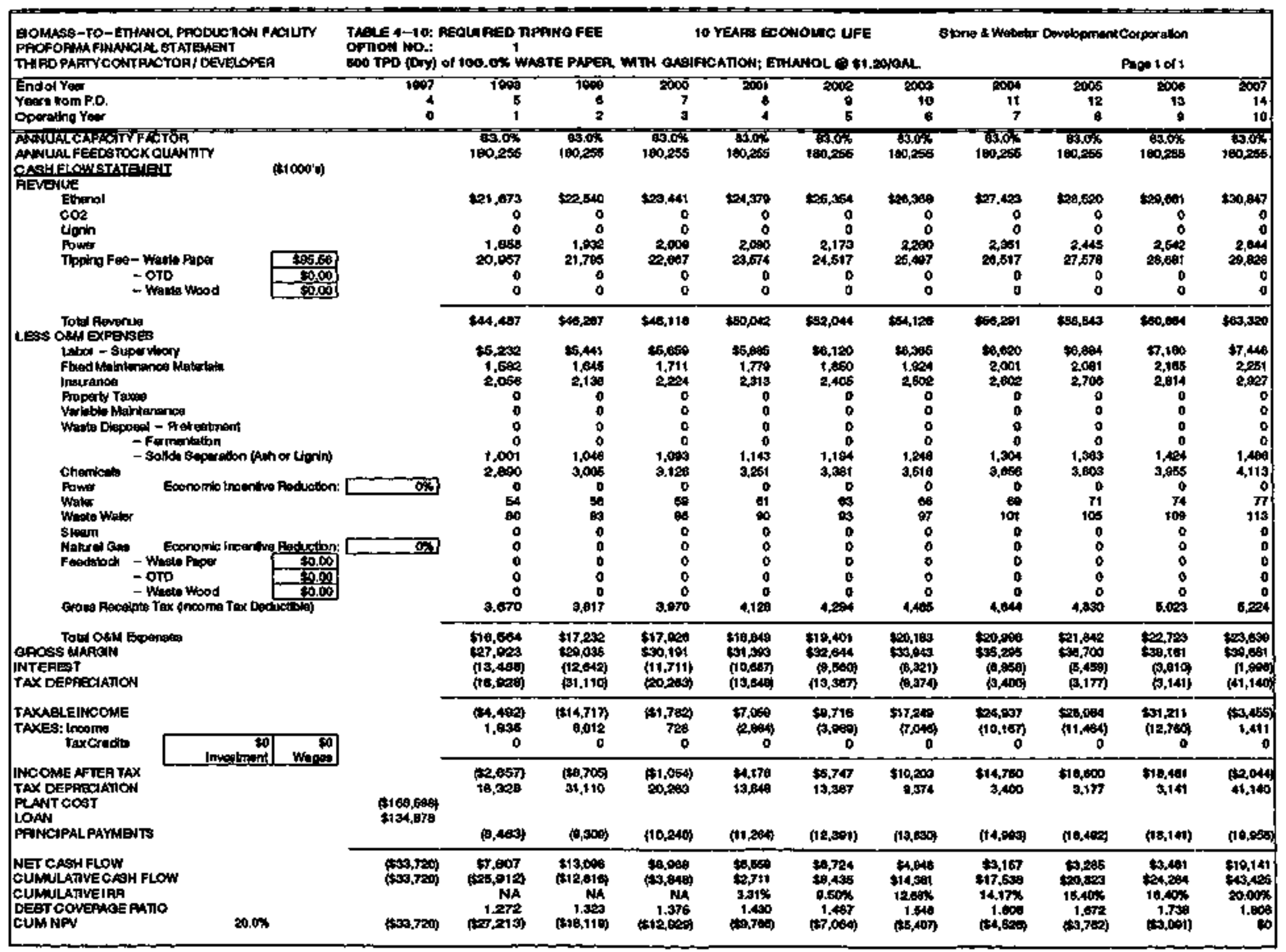




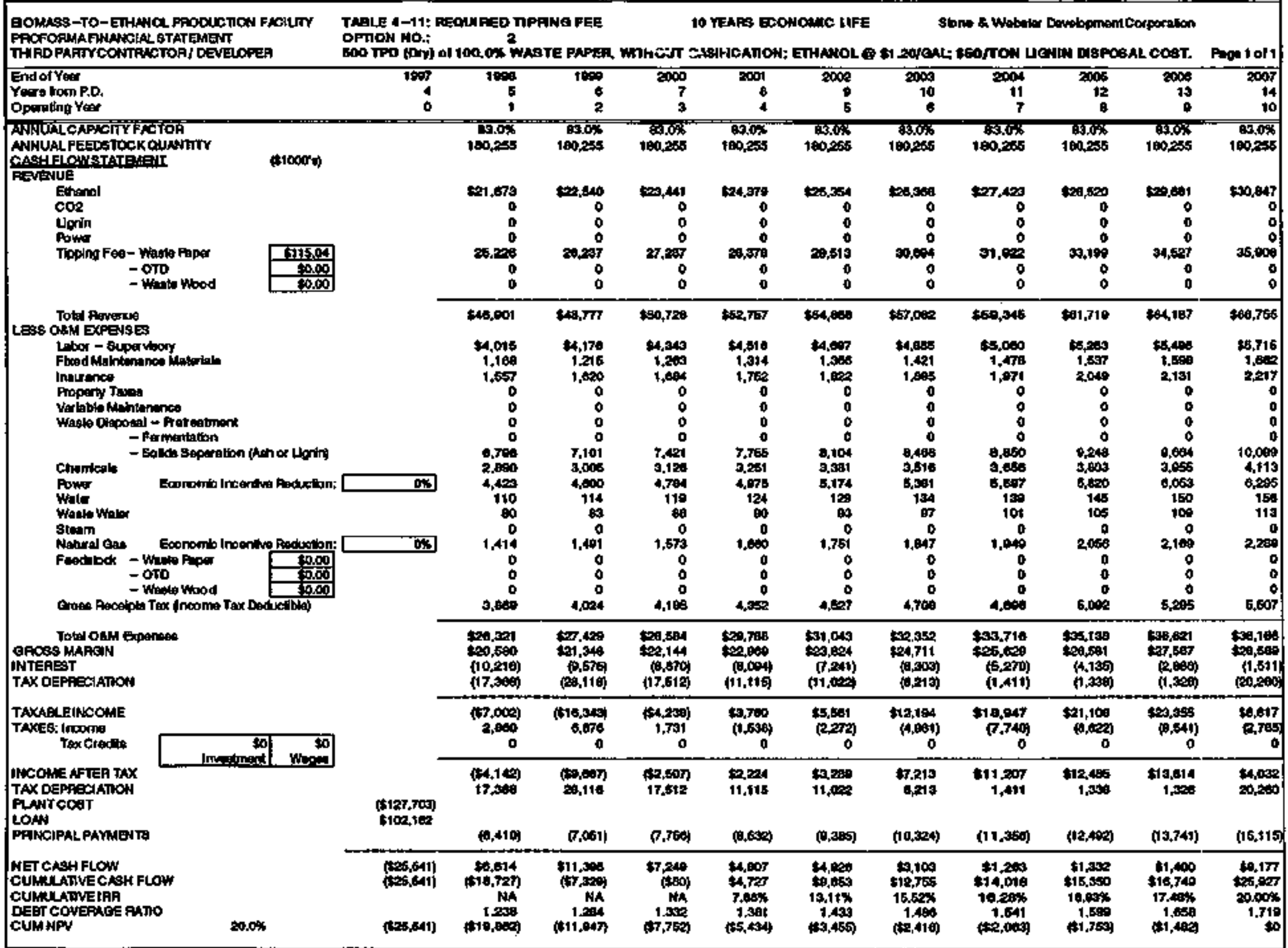




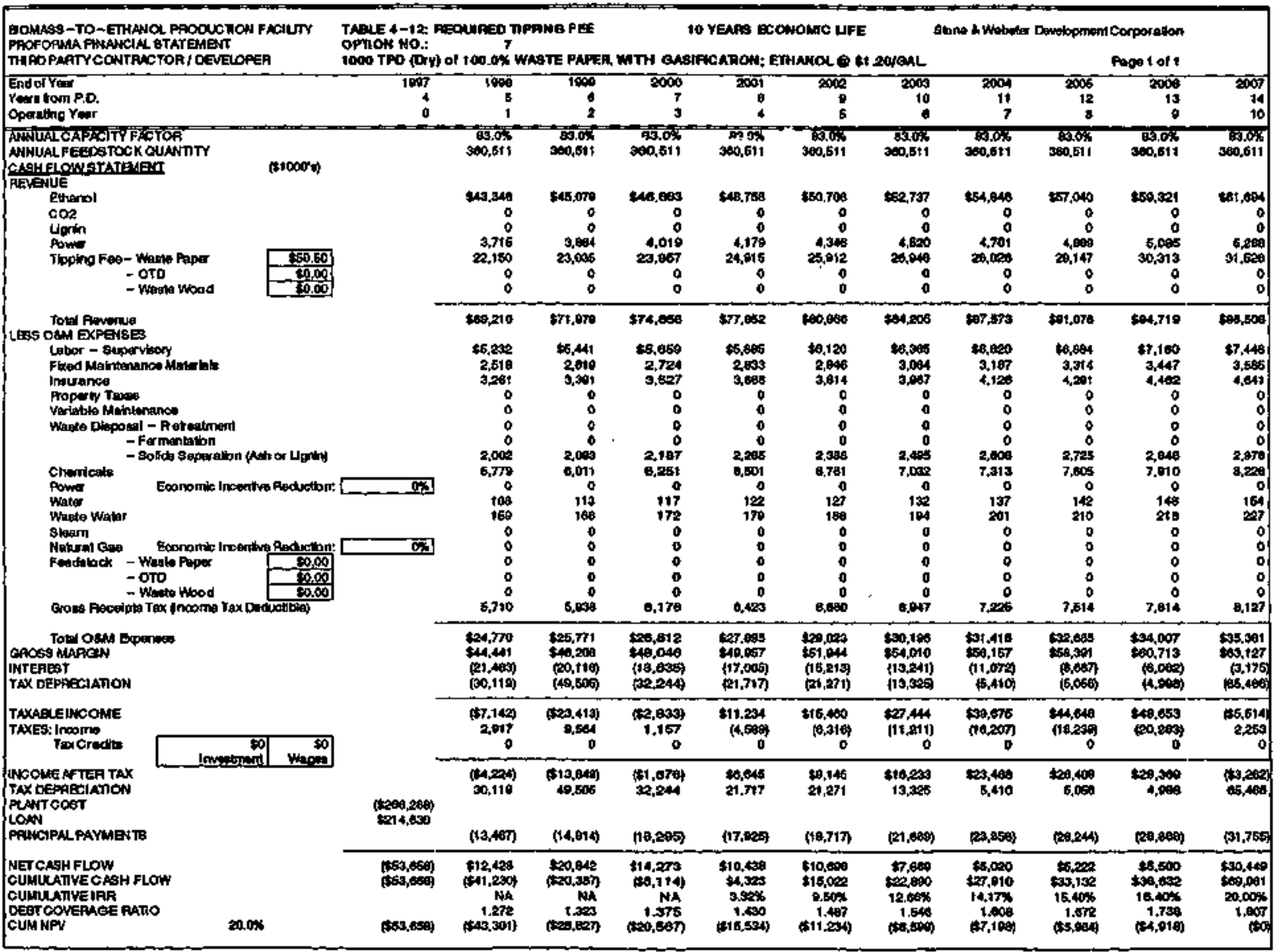




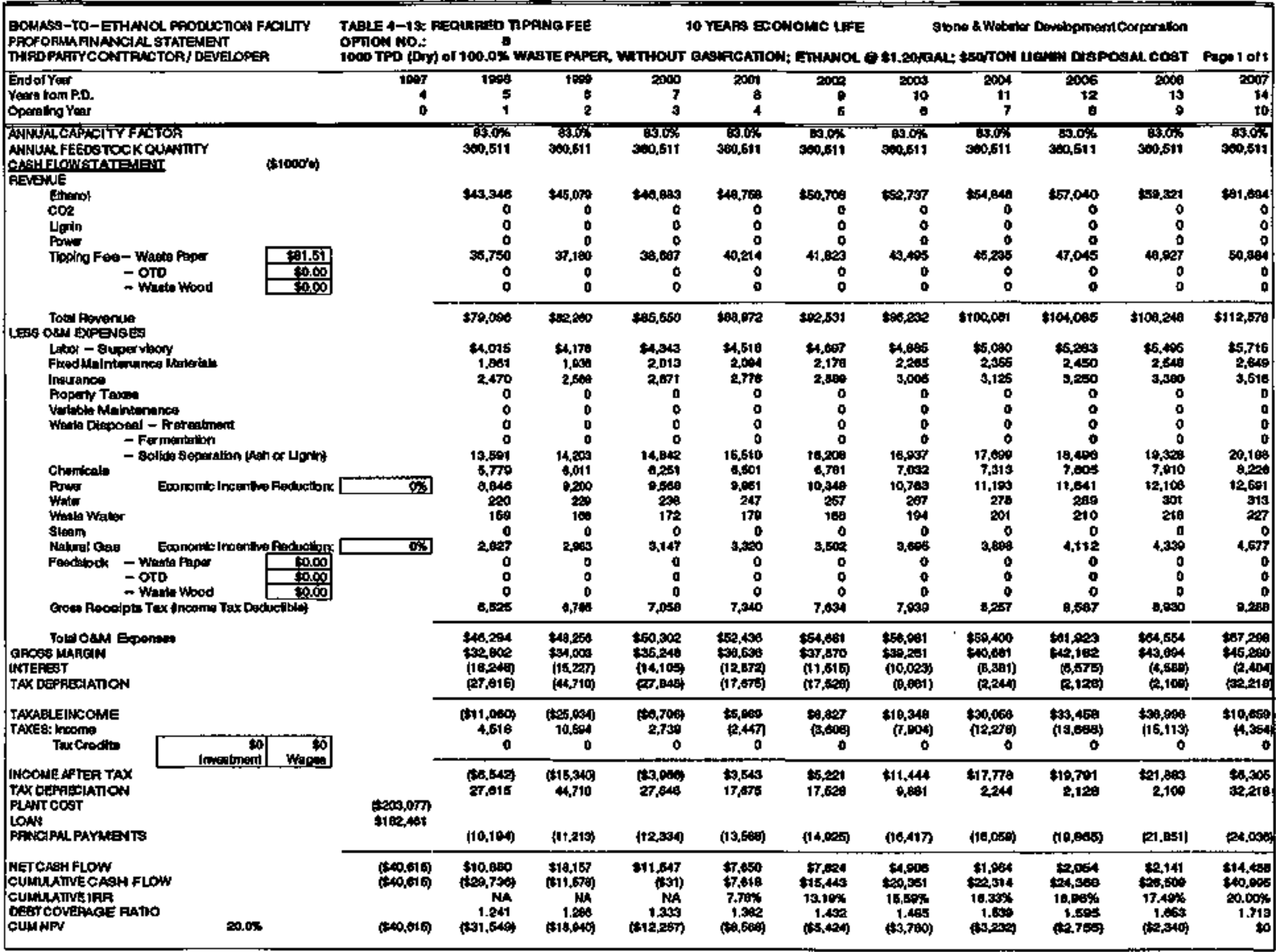




\begin{tabular}{|c|c|c|c|c|c|c|c|c|c|c|}
\hline \multirow{2}{*}{ 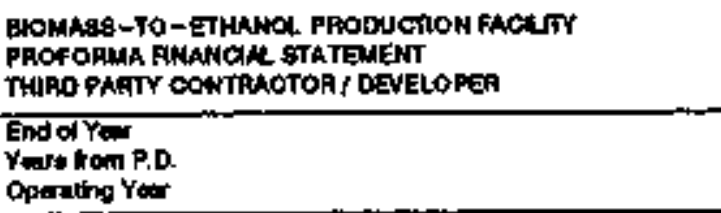 } & \multicolumn{9}{|c|}{ 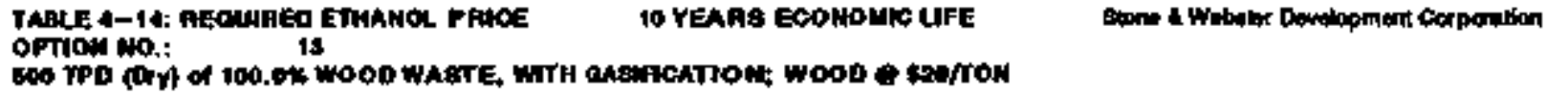 } & \multirow{2}{*}{$\begin{array}{r}\text { Pape } 1 \text { of } 11 \\
2007 \\
14 \\
10\end{array}$} \\
\hline & $\begin{array}{r}190 \\
5 \\
\end{array}$ & $\begin{array}{r}109 \\
2 \\
2\end{array}$ & $\frac{2000}{3}$ & $\begin{array}{r}2001 \\
8 \\
4\end{array}$ & $\begin{array}{r}2006 \\
6\end{array}$ & 10 & $\begin{array}{r}2004 \\
11 \\
7\end{array}$ & 12 & $\begin{array}{r}2066 \\
\$ 3 \\
0\end{array}$ & \\
\hline 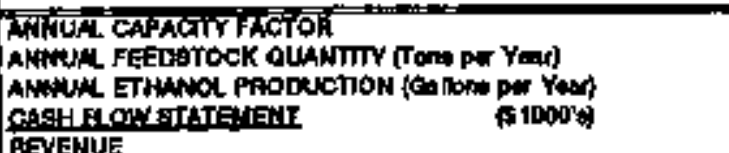 & $\begin{array}{r}160,258 \\
14,84,560\end{array}$ & $\begin{array}{r}180 \%, 255 \\
14,641,500\end{array}$ & 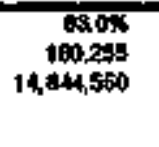 & $\begin{array}{r}50 \% \\
100,255 \\
14,844,560\end{array}$ & $\begin{array}{r}030 \% \\
100,255 \\
14104.560\end{array}$ & $\begin{array}{r}8006 \\
14005050\end{array}$ & $\begin{array}{r}1500,50 \\
1404,550\end{array}$ & $\begin{array}{r}1400,255 \\
1404,050\end{array}$ & 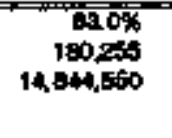 & $\begin{array}{r}180,25 \$ \\
1264,550\end{array}$ \\
\hline 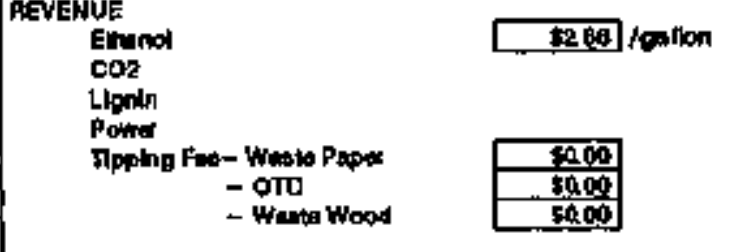 & $\begin{array}{r}247,975 \\
0 \\
0 \\
1,000 \\
0 \\
0 \\
0\end{array}$ & $\begin{array}{r}\$ 49,00 \% \\
0 \\
0 \\
1,+04 \\
0 \\
0 \\
0\end{array}$ & $\begin{array}{r}53,85 \\
0 \\
0 \\
1,169 \\
0 \\
0 \\
0\end{array}$ & $\begin{array}{r}+53.909 \\
0 \\
0 \\
1,194 \\
0 \\
0 \\
0\end{array}$ & $\begin{array}{r}560,120 \\
0 \\
0 \\
1,242 \\
0 \\
0 \\
0\end{array}$ & $\begin{array}{r}5 \times 0,373 \\
0 \\
0 \\
1,292 \\
0 \\
0 \\
0\end{array}$ & $\begin{array}{r}500,708 \\
0 \\
0 \\
1.349 \\
0 \\
0 \\
0\end{array}$ & $\begin{array}{r}663,136 \\
0 \\
0 \\
1,397 \\
0 \\
0 \\
0\end{array}$ & $\begin{array}{r}+06,061 \\
0 \\
0 \\
+, 453 \\
0 \\
0 \\
0\end{array}$ & \begin{tabular}{r|}
0 \\
0 \\
4511 \\
0 \\
0 \\
0
\end{tabular} \\
\hline 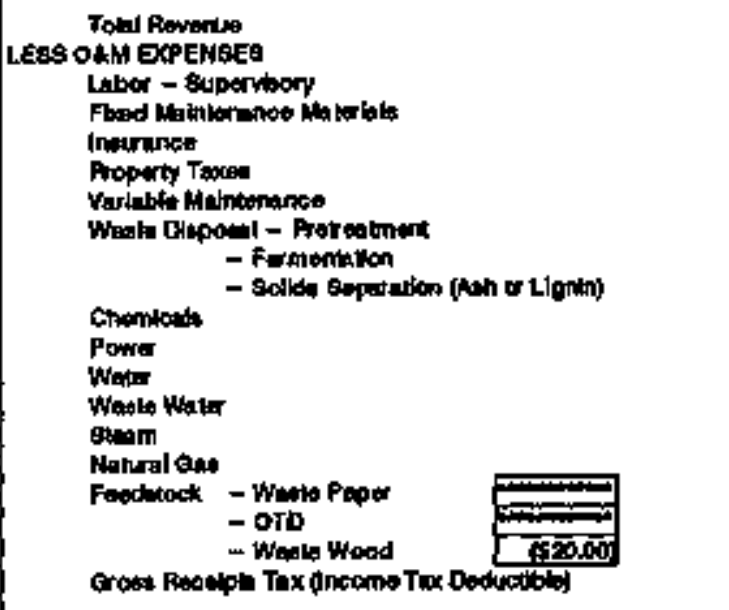 & $\begin{array}{r}54,040 \\
54,546 \\
1,590 \\
2,056 \\
0 \\
0 \\
0 \\
0 \\
708 \\
2090 \\
0 \\
54 \\
80 \\
0 \\
0 \\
0 \\
0 \\
4,306 \\
4,046\end{array}$ & $\begin{array}{r}5,, 001 \\
\$ 8,441 \\
1,645 \\
2,139 \\
0 \\
0 \\
0 \\
0 \\
005 \\
2005 \\
0 \\
59 \\
63 \\
0 \\
0 \\
0 \\
0 \\
4,562 \\
4,200\end{array}$ & 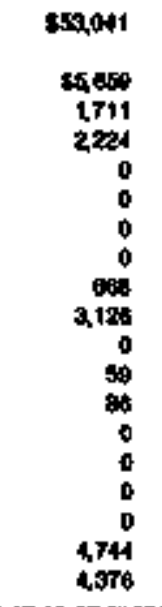 & $\begin{array}{r}556,163 \\
\$ 5,055 \\
1,770 \\
2,313 \\
0 \\
0 \\
0 \\
0 \\
0107 \\
3,251 \\
0 \\
01 \\
80 \\
0 \\
0 \\
0 \\
0 \\
4,934 \\
4,551\end{array}$ & $\begin{array}{r}58,370 \\
+, 120 \\
1.050 \\
2,405 \\
0 \\
0 \\
0 \\
0 \\
0 \\
045 \\
3,391 \\
0 \\
63 \\
93 \\
0 \\
0 \\
0 \\
0 \\
5,131 \\
4,730\end{array}$ & 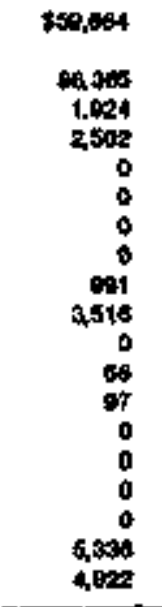 & 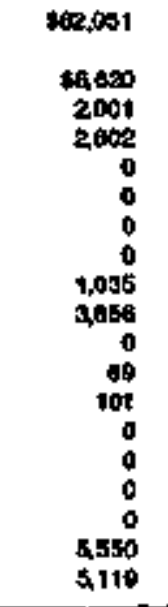 & 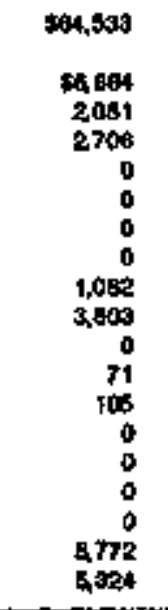 & $\begin{array}{r}\text { sar.114 } \\
57,100 \\
2,165 \\
2.614 \\
0 \\
0 \\
0 \\
0 \\
1.131 \\
3,055 \\
0 \\
74 \\
100 \\
0 \\
0 \\
0 \\
0 \\
4003 \\
408\end{array}$ & 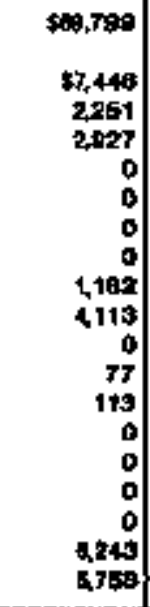 \\
\hline 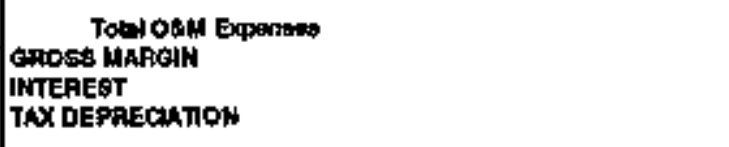 & $\begin{array}{l}121,+20 \\
127,020 \\
1+3,468) \\
(14,020)\end{array}$ & $\begin{array}{l}\$ 21,060 \\
20.003 \\
(32604) \\
(31,1+0)\end{array}$ & $\begin{array}{l}\$ 2,068 \\
\$ 00,100 \\
(11,711) \\
20,260)\end{array}$ & $\begin{array}{l}\$ 4,770 \\
591,303 \\
(1069) \\
(13646)\end{array}$ & 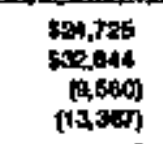 & 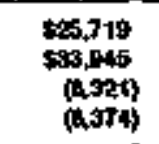 & $\begin{array}{l}50,7 \div 3 \\
(0,2906) \\
(1,400)\end{array}$ & $\begin{array}{l}\$ 2,828 \\
\$ 6,705 \\
4,450) \\
(0,177)\end{array}$ & 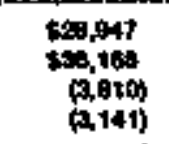 & 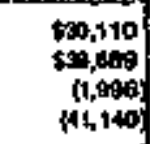 \\
\hline 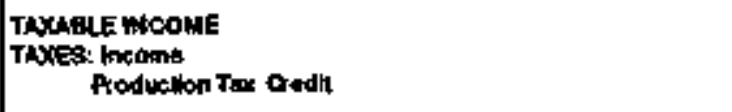 & $\begin{array}{r}(0,4907) \\
40166 \\
0\end{array}$ & $\begin{array}{r}+(4,710) \\
4010 \\
0\end{array}$ & $\begin{array}{r}\$ 1,78) \\
720\end{array}$ & $\begin{array}{c}\$ 7,059 \\
(2,064) \\
0\end{array}$ & $\begin{array}{c}\text { sartit } \\
0 \\
0\end{array}$ & $\begin{array}{r}0,04,25 t \\
0,045 \\
0\end{array}$ & 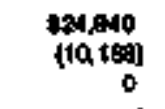 & $\begin{array}{c}0.060 \\
011,469) \\
0\end{array}$ & $\begin{array}{c}501,217 \\
(12,7=2) \\
0\end{array}$ & $\begin{array}{r}0.447 \\
0\end{array}$ \\
\hline $\begin{array}{l}\text { WCONE AFTEATAX } \\
\text { TAX OEPPECAATON } \\
\text { PLANT COST } \\
\text { LOAN } \\
\text { PANWCAPAL PAMUENTS }\end{array}$ & 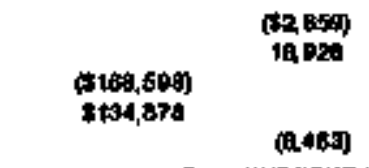 & $\begin{array}{l}(59706) \\
\$ 1,110\end{array}$ & $\begin{array}{l}\text { S1,056) } \\
24264\end{array}$ & $\begin{array}{l}14,175 \\
12,646\end{array}$ & $\begin{array}{l}18,748 \\
19.367\end{array}$ & $\begin{array}{r}50,504 \\
0,374\end{array}$ & $\begin{array}{r}\$ 14,752 \\
3 \times 00\end{array}$ & $\begin{array}{r}5 \times 0,609 \\
2,177\end{array}$ & $\begin{array}{r}510,40.4 \\
5,141\end{array}$ & $(19,950)$ \\
\hline 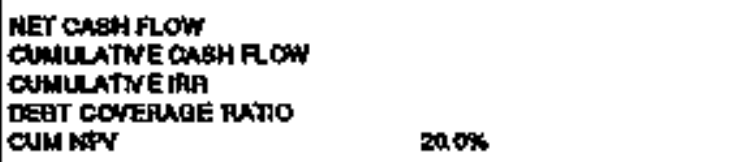 & 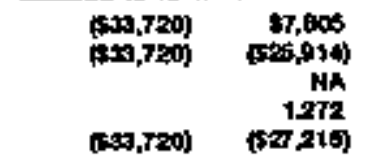 & 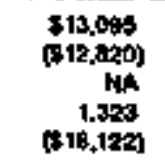 & 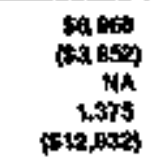 & $\begin{array}{c}3,559 \\
1270 \% \\
230 \% \\
1,430 \\
(52760)\end{array}$ & 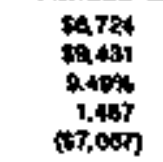 & 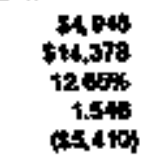 & $\begin{array}{r}19,150 \\
317,537 \\
14,174 \\
1.009 \\
51509\end{array}$ & 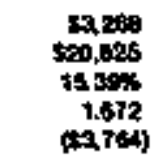 & $\begin{array}{l}2540 \\
524280 \\
1040 \% \\
1.739 \\
020004\end{array}$ & $\begin{array}{r}10,140 \\
20,136 \\
2000 \\
100 \\
10\end{array}$ \\
\hline
\end{tabular}




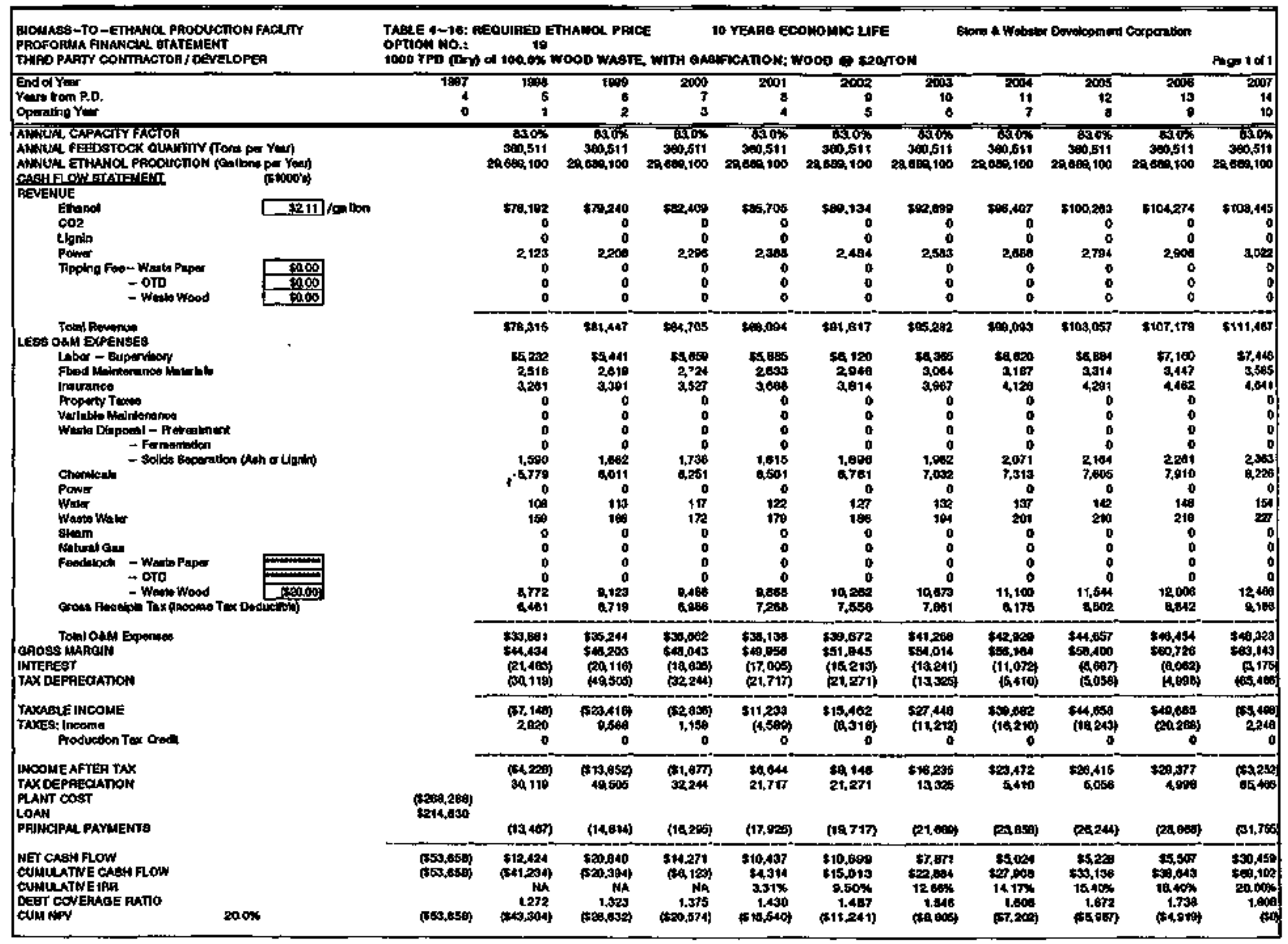




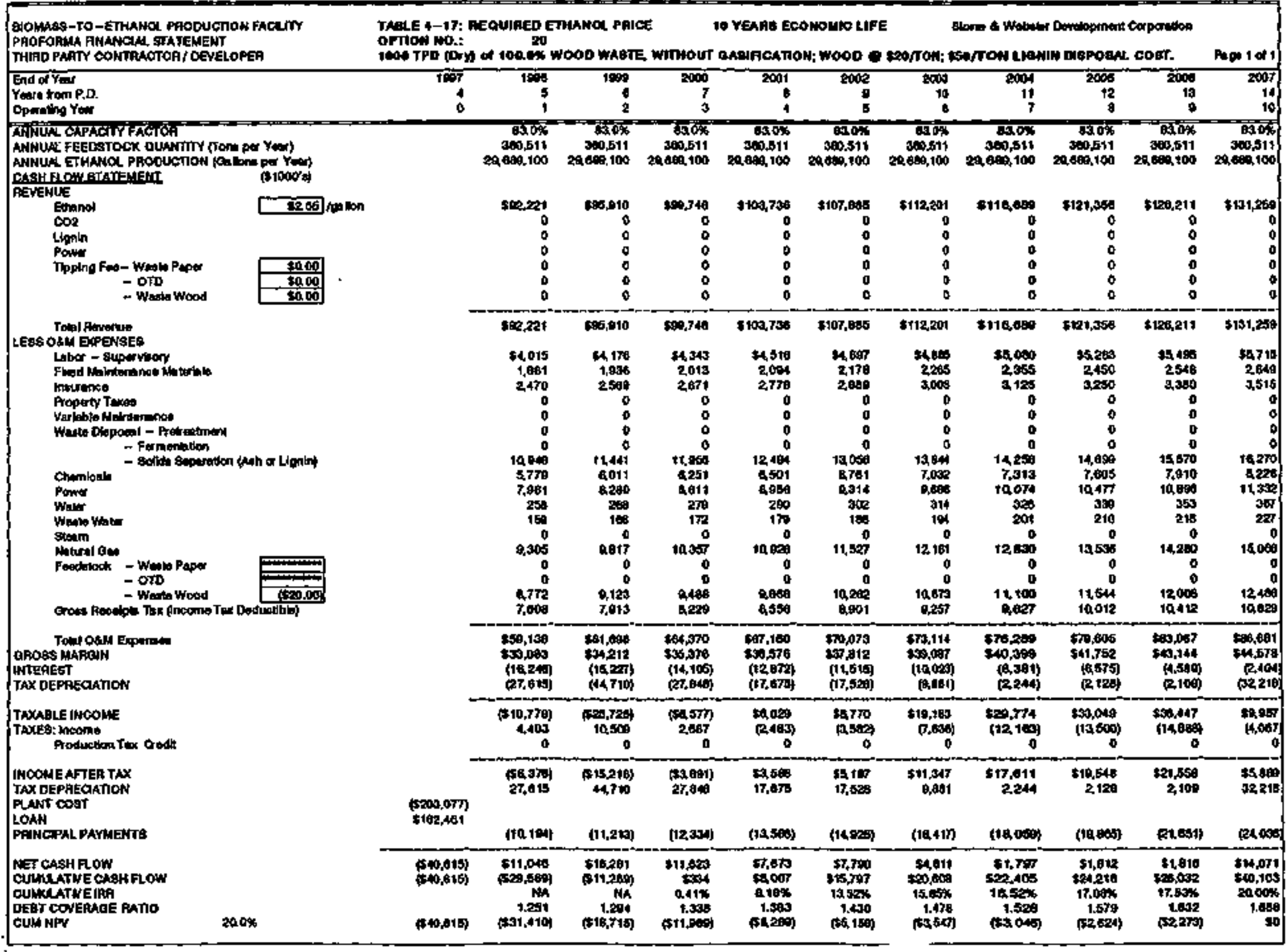




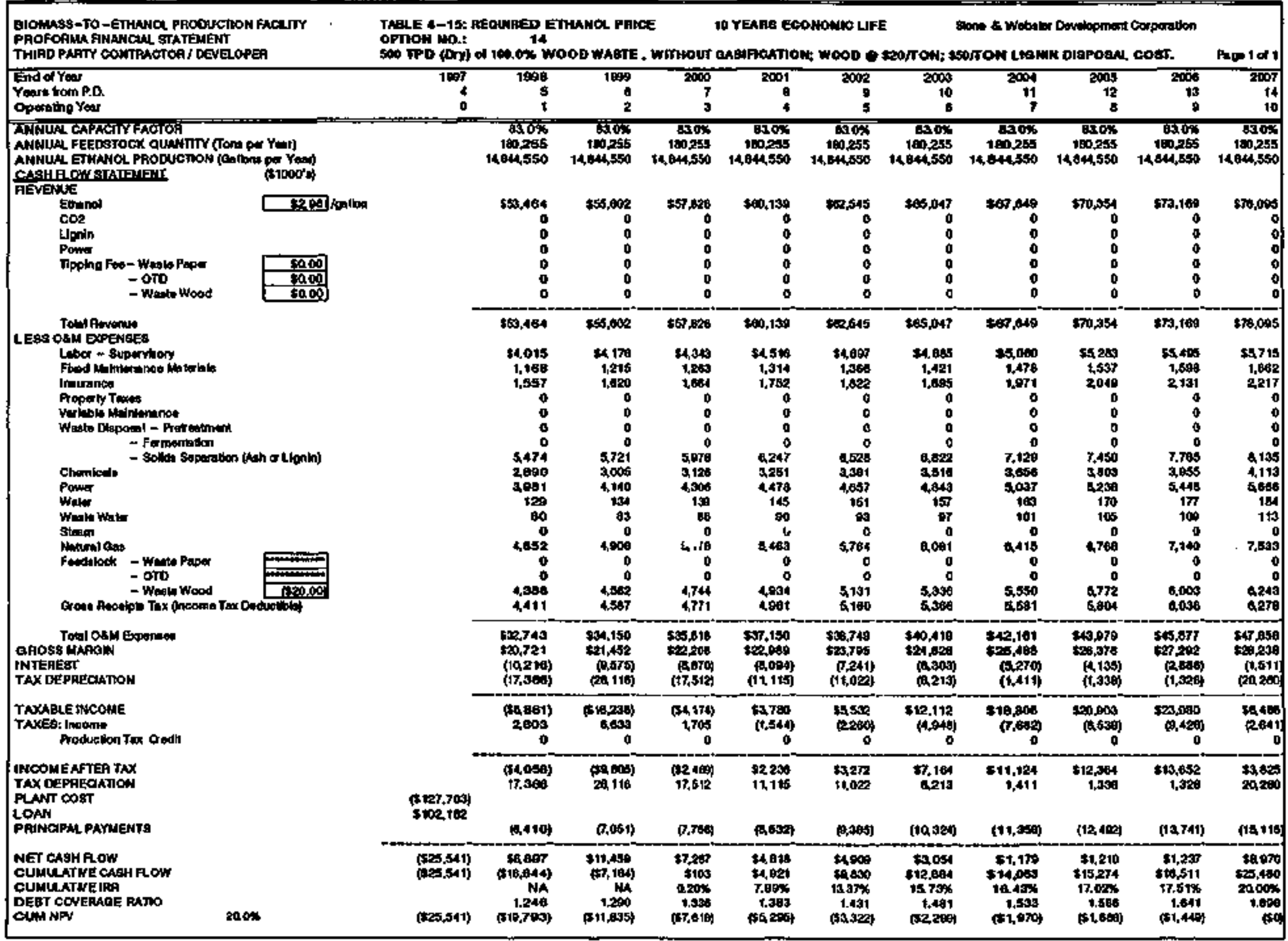




\section{REQUIRED TIPPING FEE vS. CAPITAL COST AS A PERCENTAGE OF ESTIMATED COST}

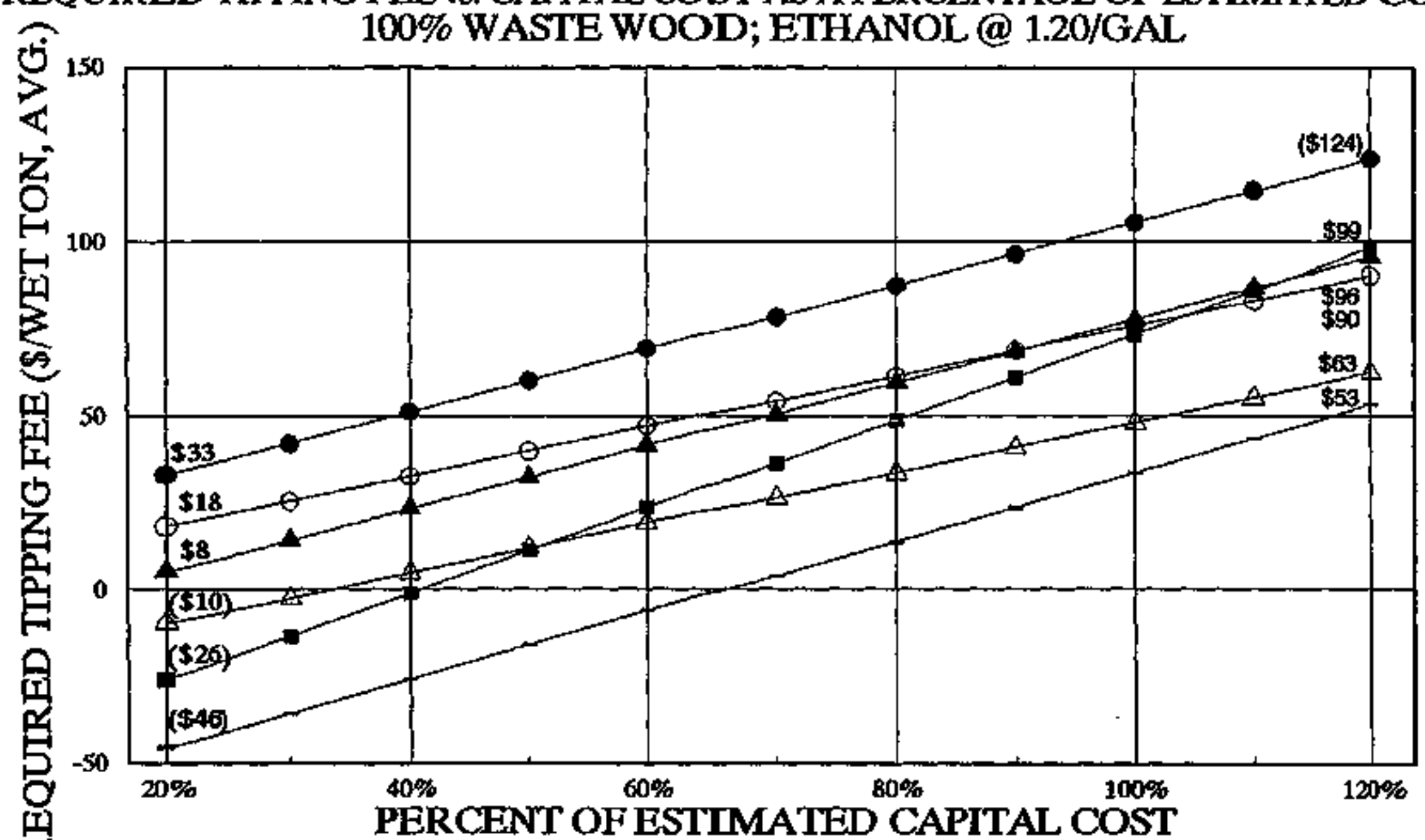

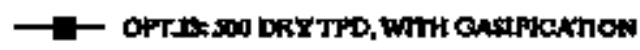

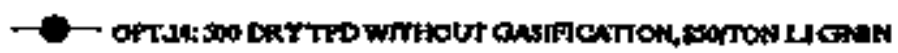

- OPCEN 1000 DNY TRD, WTH GASAFOXTHOW

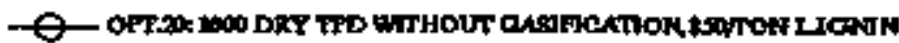

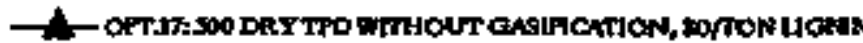

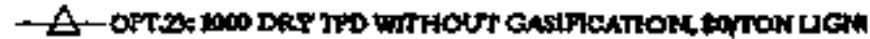

STONG \& WEBSTER DEVELOMMENT CORPORATYCN

FIGURE 4-1 


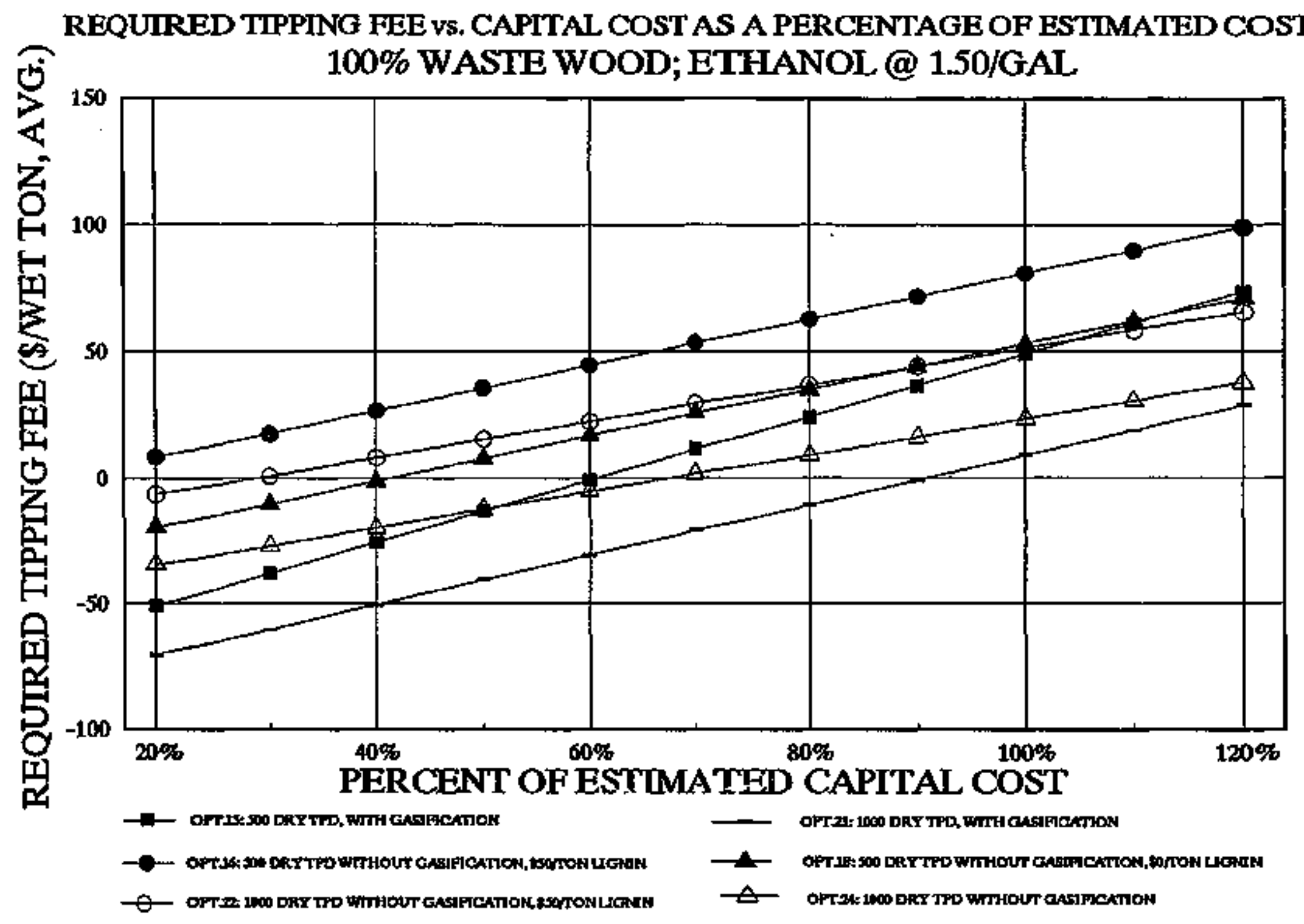

STONH \& WESTIER DEYHLOPMENT CORPORATION

FIGURE 4-2 


\section{REQUIRED TIPPING FEE vs. O\&M COST}

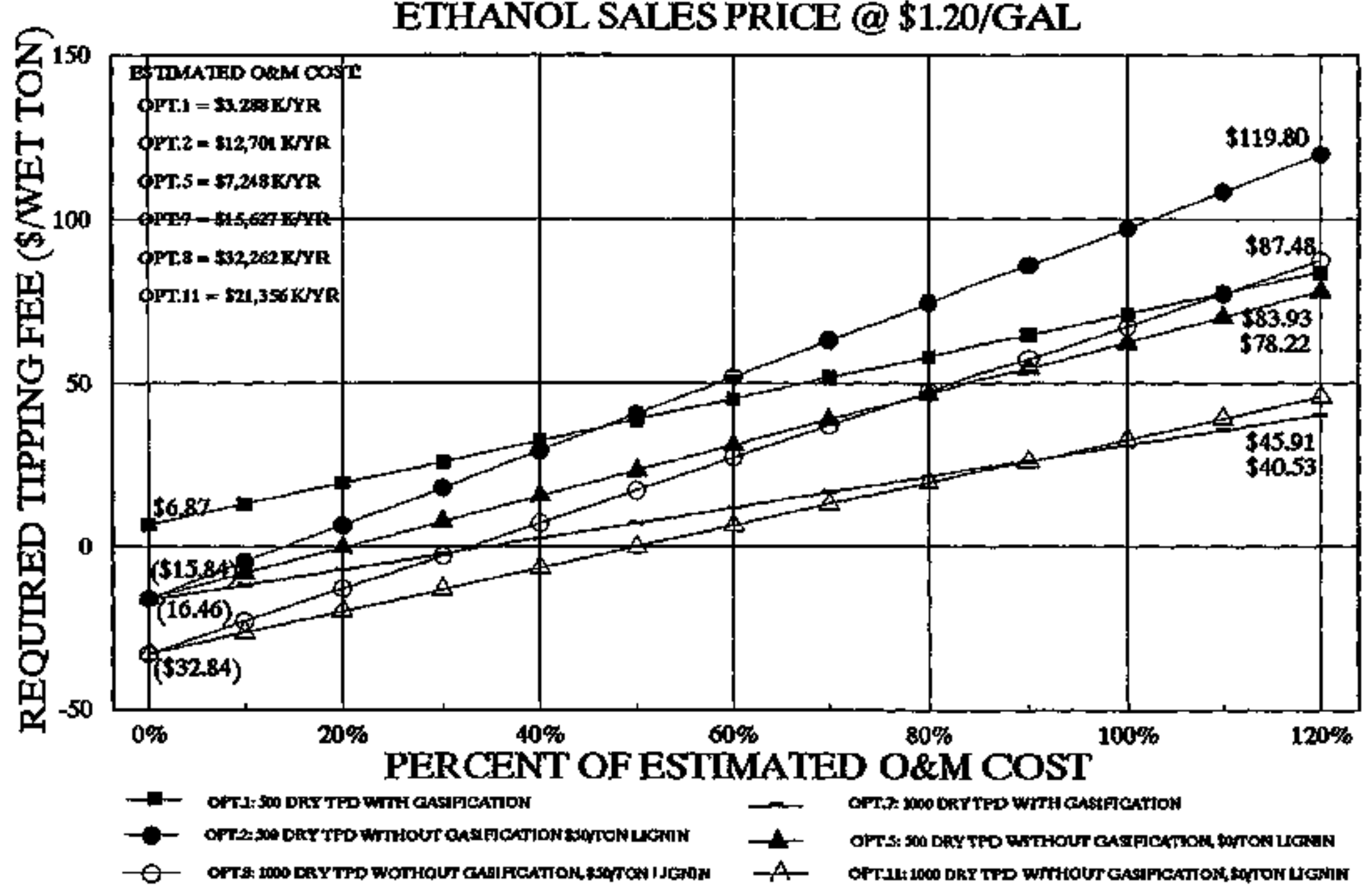




\section{REQUIRED TIPPING FEE vs. O\&M COST}

ETHANOL SALES PRICE @ \$1.50/GAL

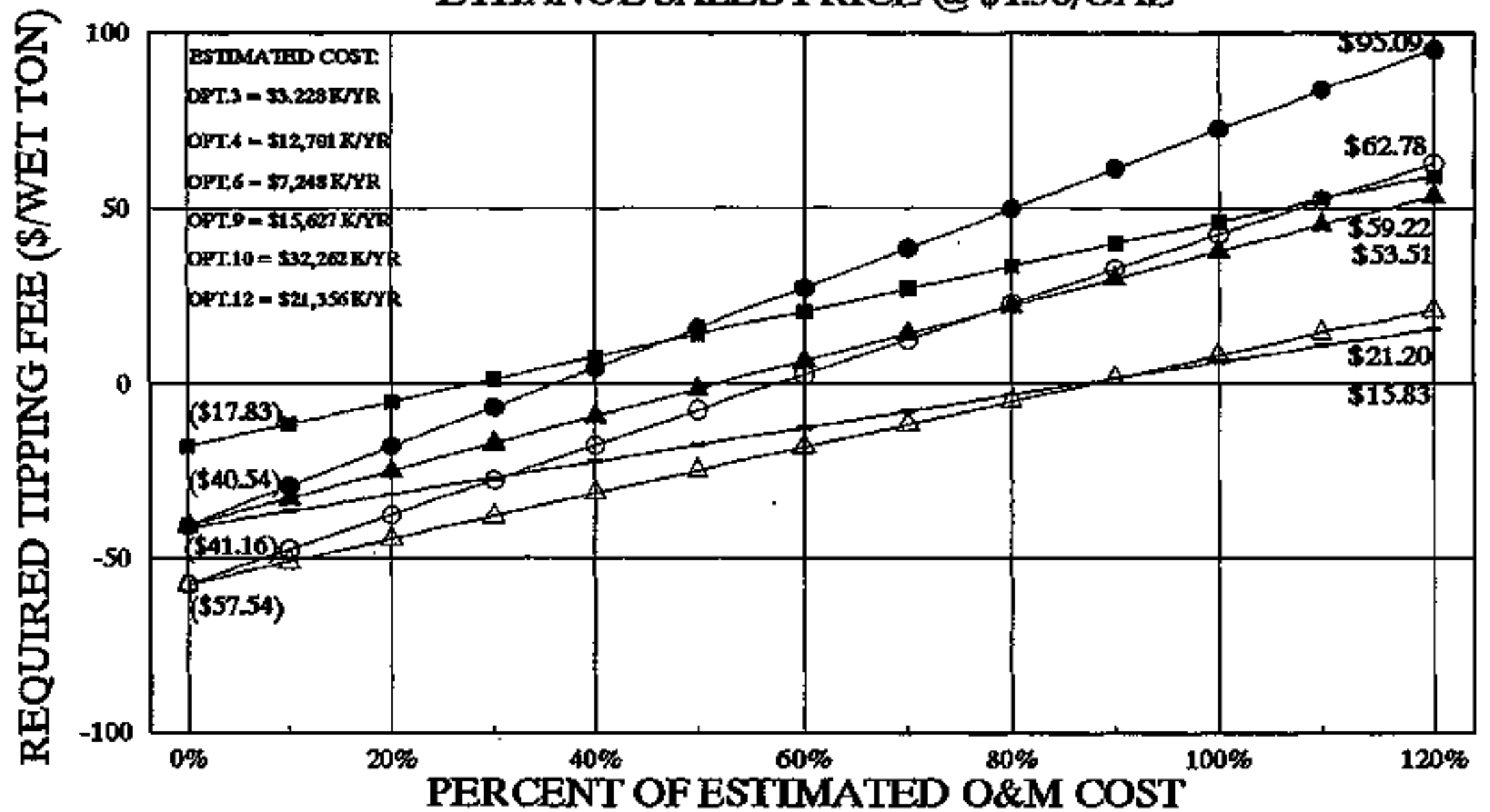

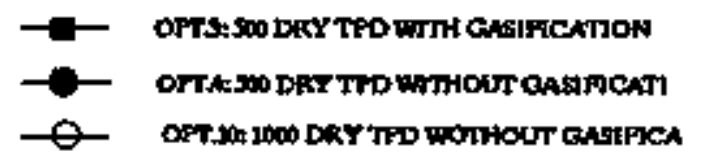

CAFTEAL COST AT10\% OPESTIMATED VALUI

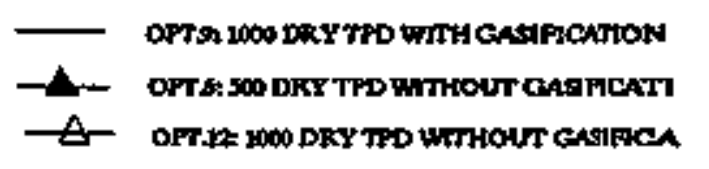

STONE \& WEASTER DEVEILRMIENTCORPORATION

FIGURE 4-4 


\section{REQUIRED TIPPING FEE vs. LABOR COST}

\section{0\% WASTE WOOD; ETHANOL @ \$1.20/GAL}

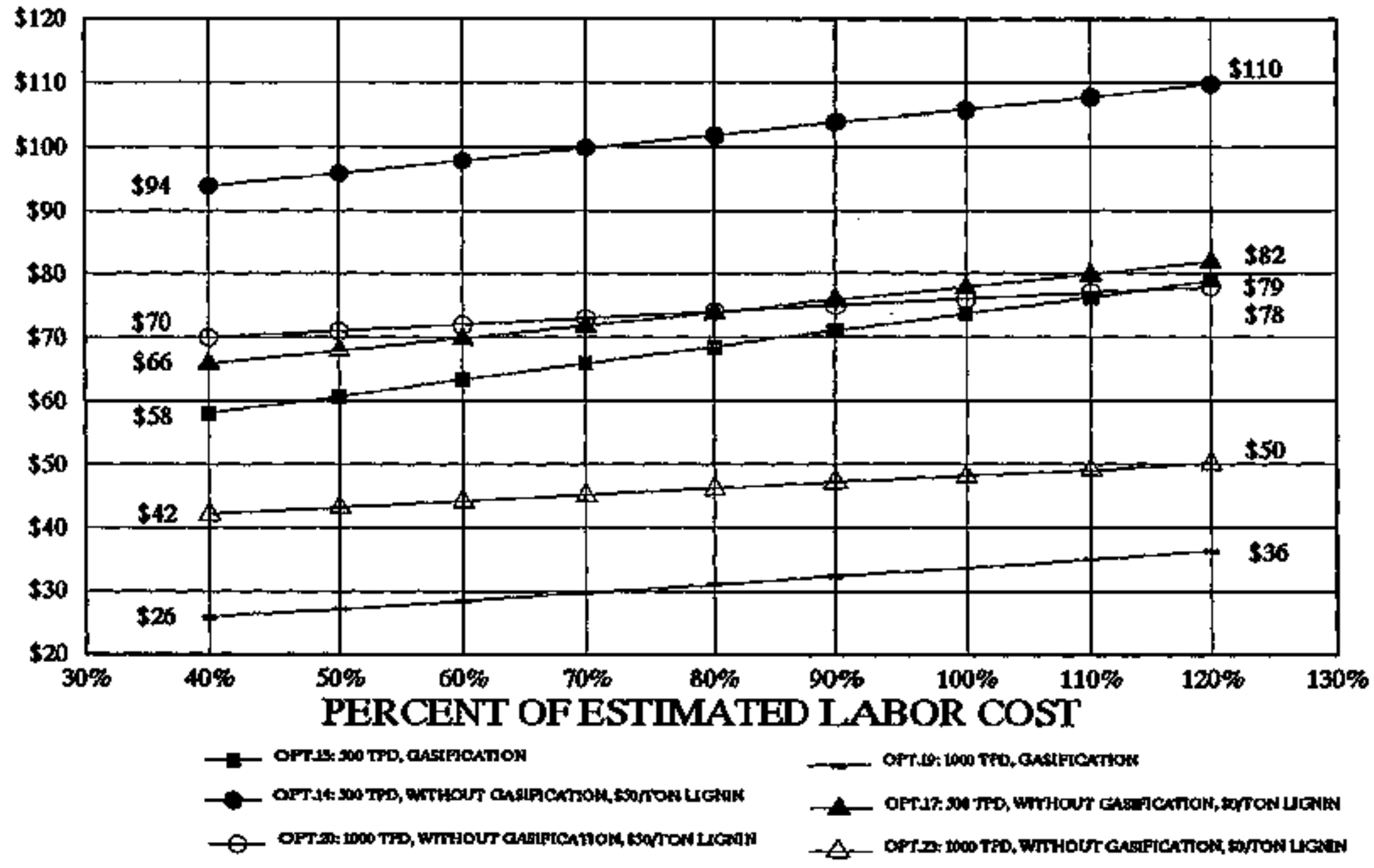


REQUIRED TIPPING FEE vs. LABOR COST

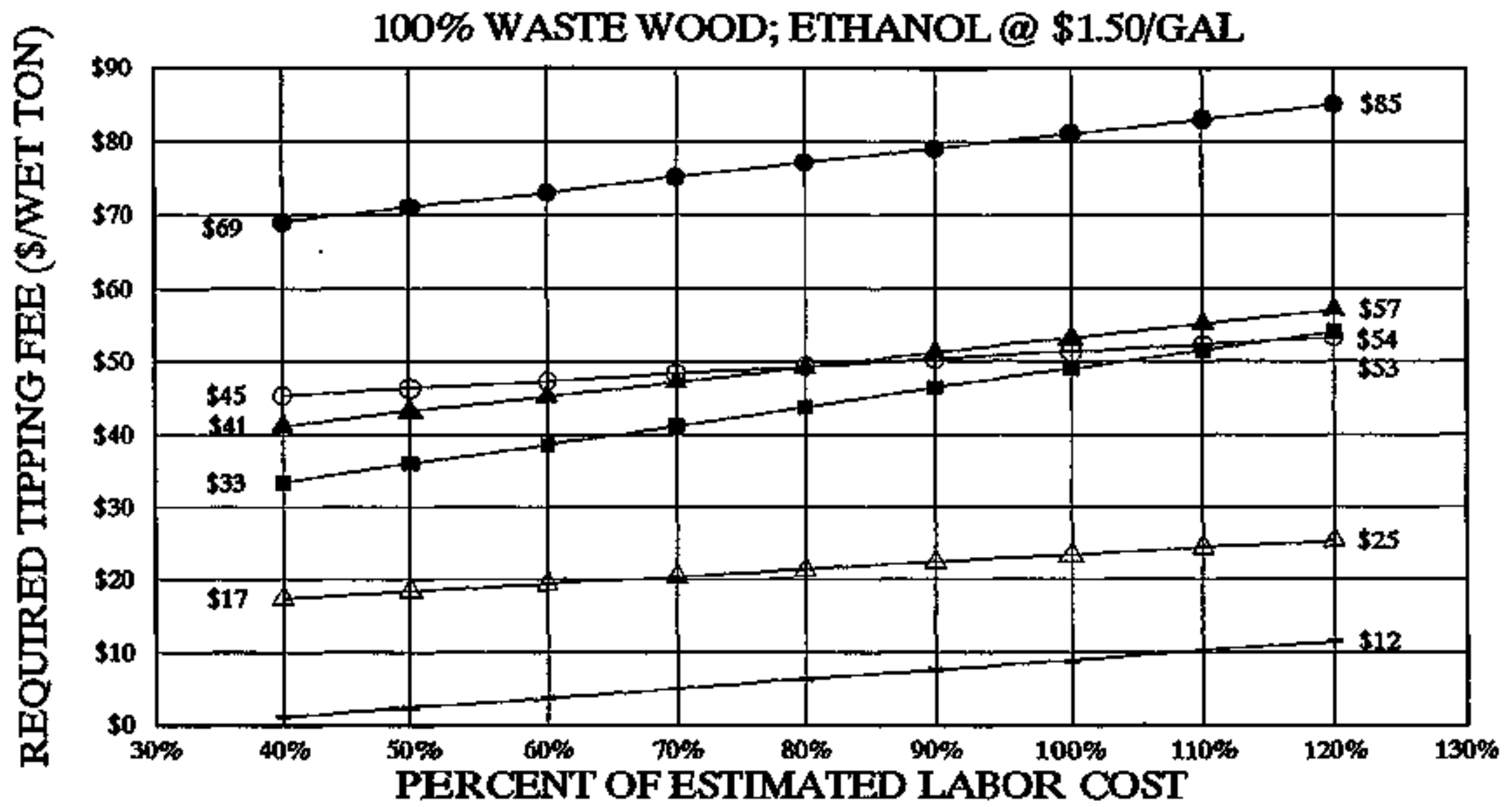

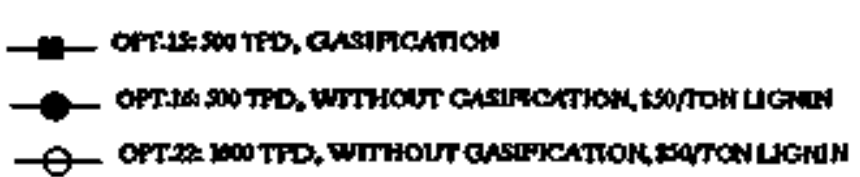

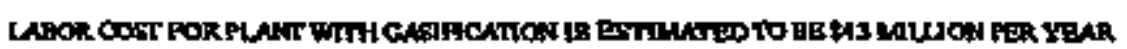

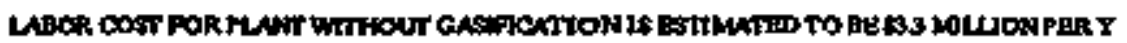

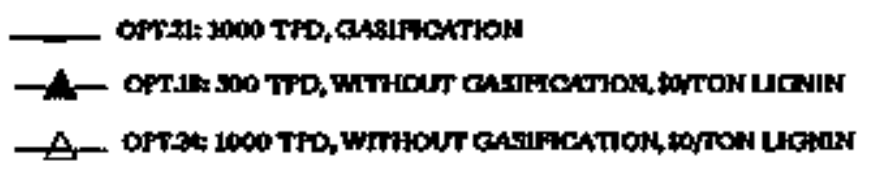

STCWE \& WEASTER DAVRLOPMENT CORPORAMON

FIGURE 4-6 


\section{REQUIRED TIPPING FEE vs. POWER PRICE}

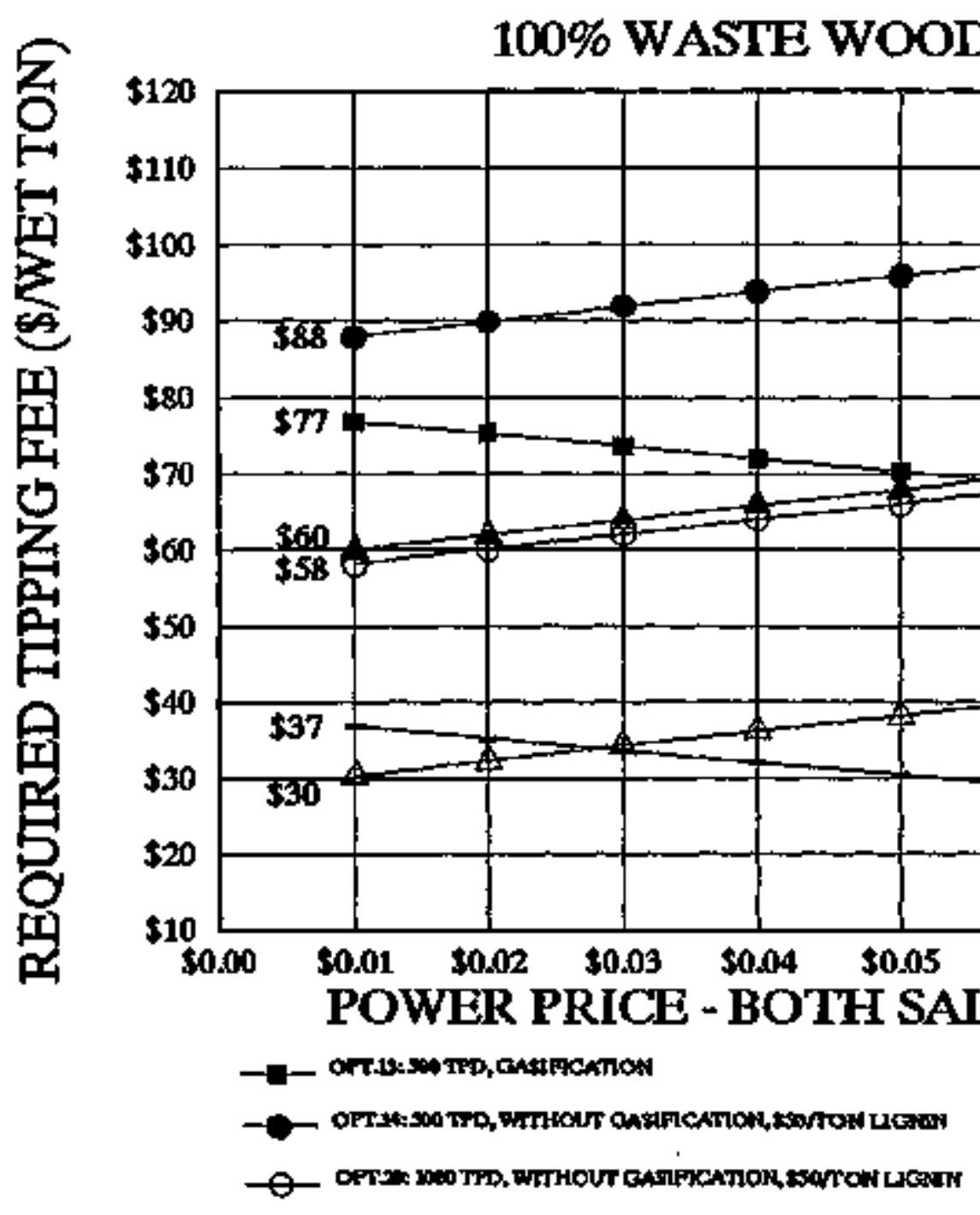


REQUIRED TIPPING FEE VS. POWER PRICE

突 100\% WASTE WOOD; ETHANOL @ \$1.50/GAL

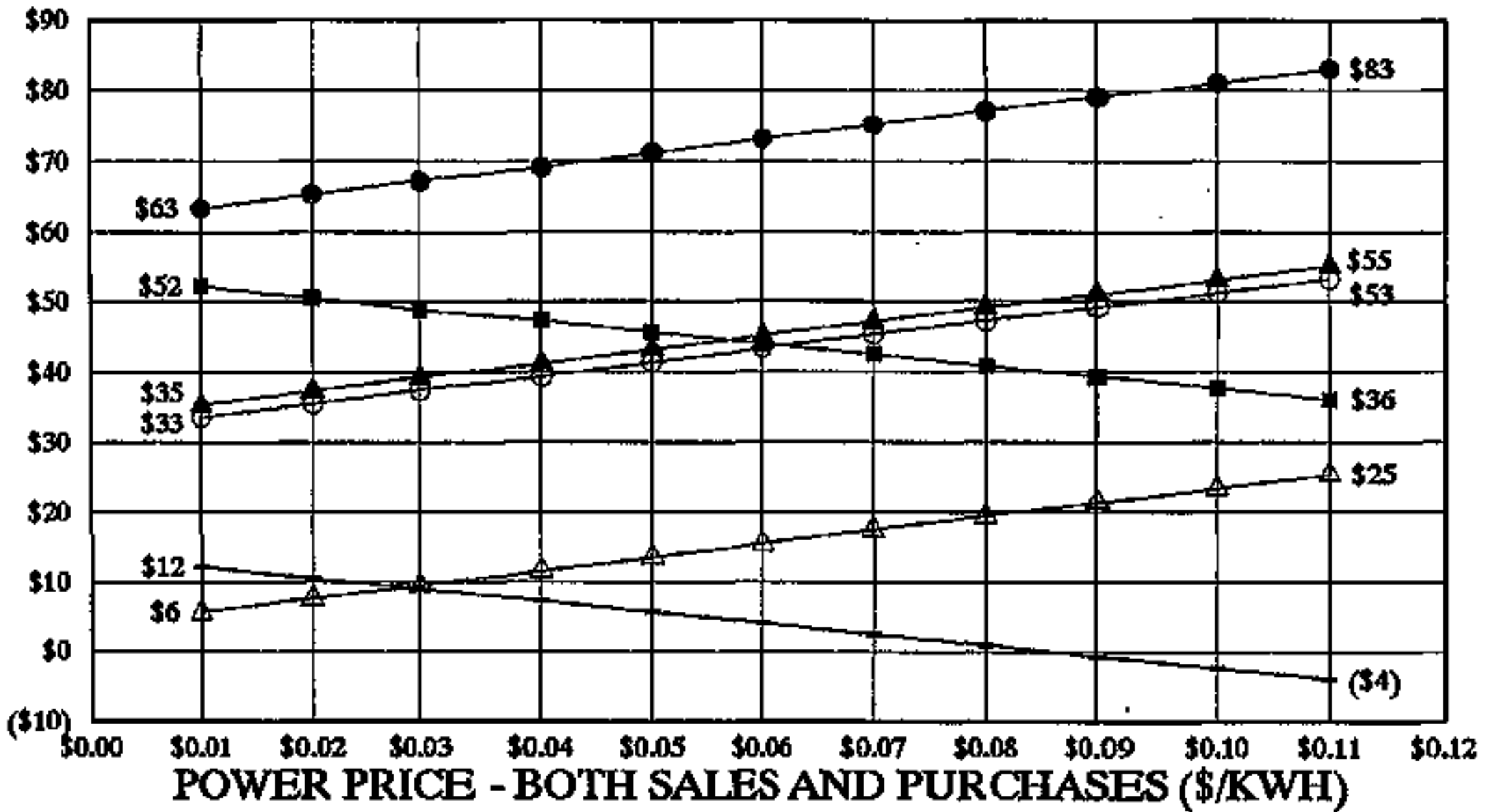

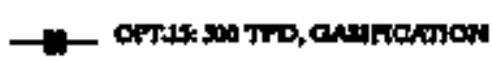

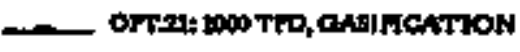

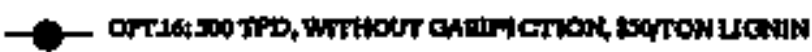

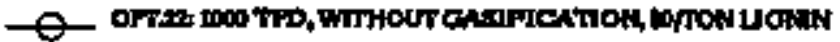

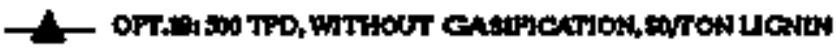

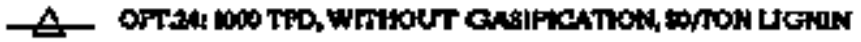

STONB \& WEDTER DEVEI OFAIRTT CORPORATION

FIGURE 4-8 


\section{REQUIRED TIPPING FEE vs. LIGNIN DISPOSAL COST}

100\% WASTE WOOD; ETHANOL @ \$1.20/GAL

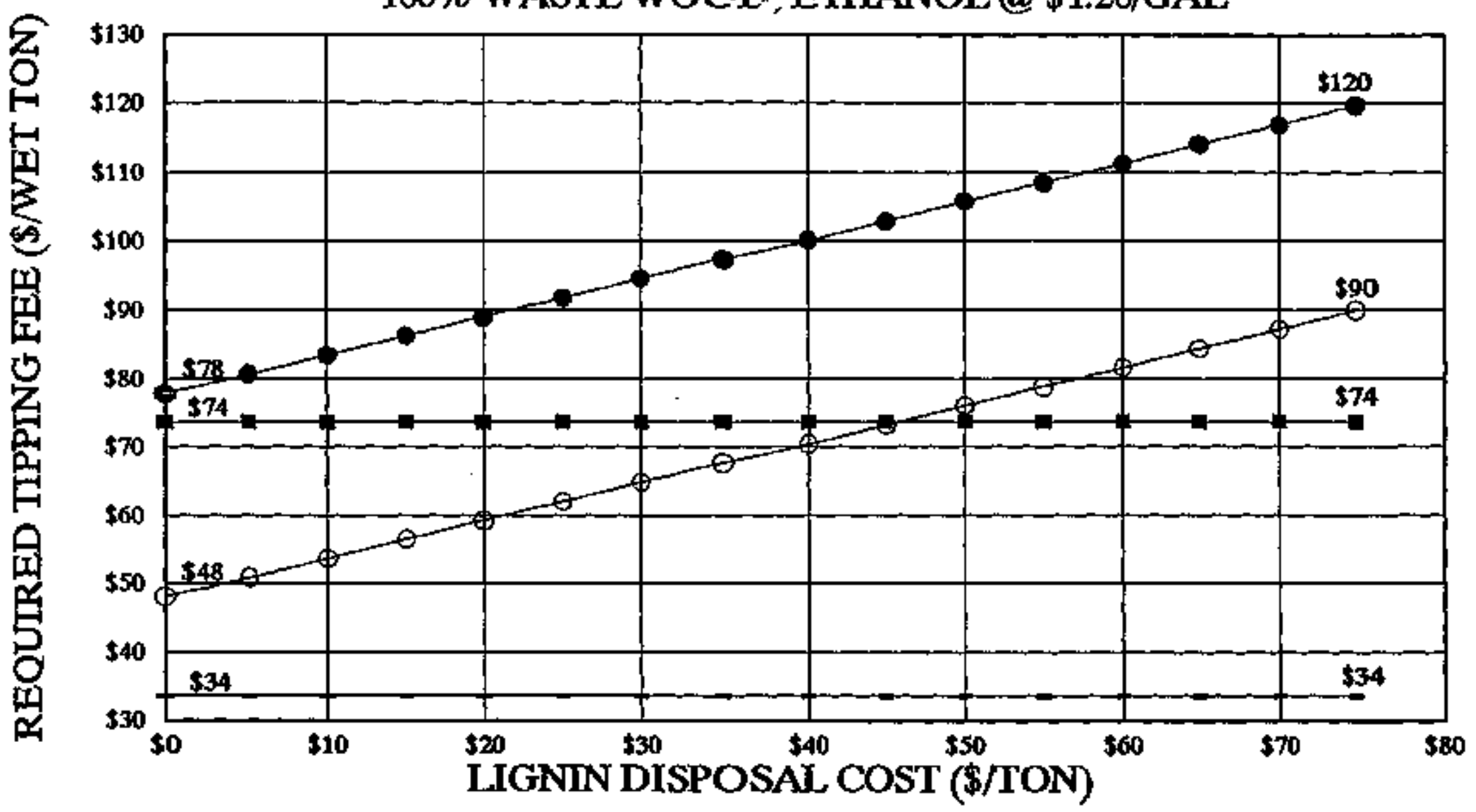

-1-OPT.13: 500 TPD, GASIFICATION OPT.19: 1000 TPD, GASIFICATION
-— OPT.14: 500 TPD, WTTHOUT GASIFICATION ๑ OPT.20: 1000 TPD, WITHOUT GASIFICATION STONE \& WEBSTER DEVELOPMENT CORPORATION

FIGURE 49 
REQUIRED TIPPING FEE vs. LIGNIN DISPOSAL COST 100\% WASTE WOOD; ETHANOL @ \$1.50/GAL

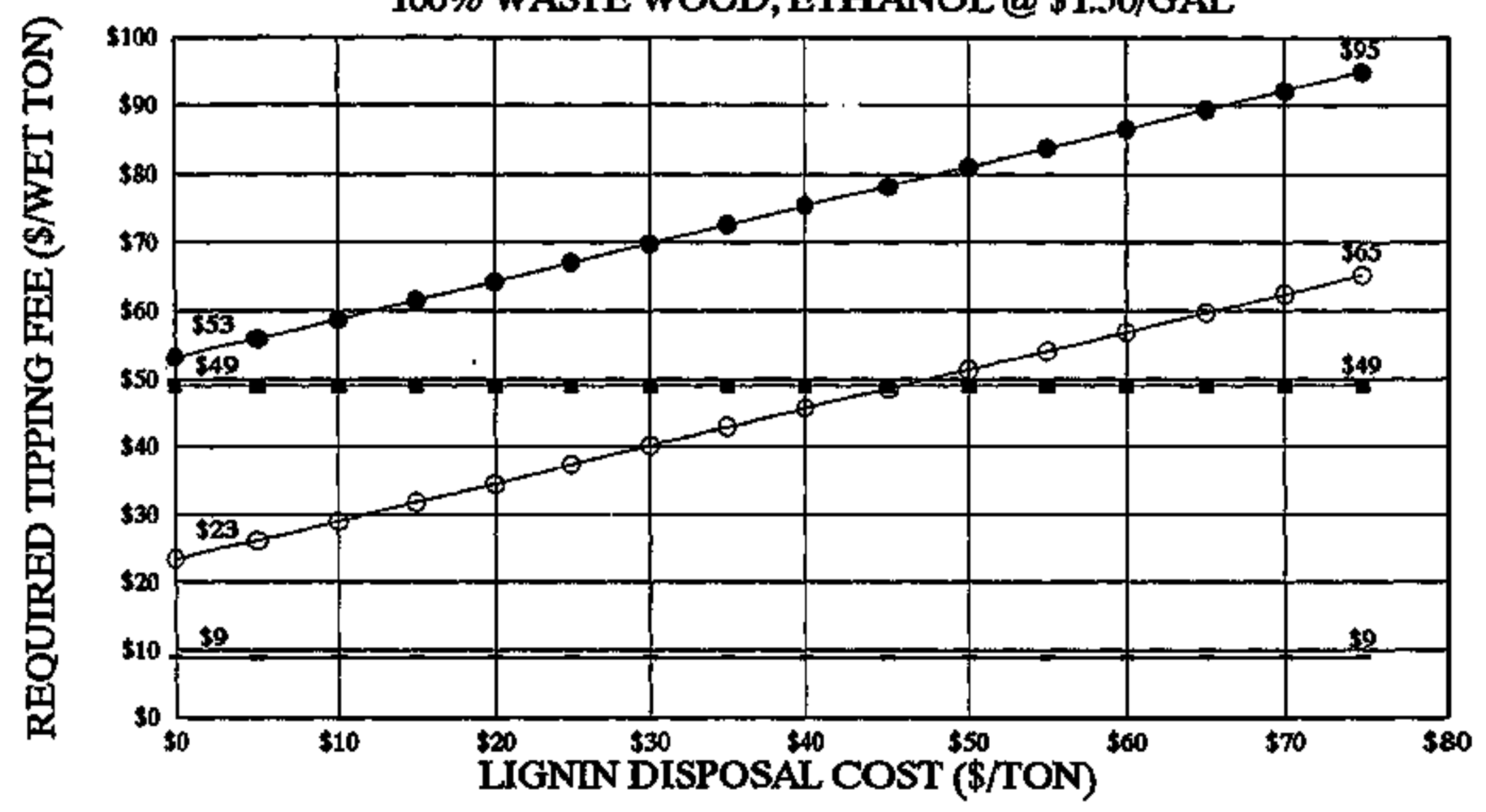

$\rightarrow$ OPT.15: 500 TPD, GASIFICATION

__ OPT21: 1000 TPD, GASIFICATION
- OPT.16: 500 TPD, WITHOUT GASIFICATION

- OPT-22: 1000 TPD, WITHOUT GASIFICATION STONE \& WEESTER DEVELOPMRNT CORPORATION

FIGURE 4-10 


\section{REQUIRED TIPPING FEE vs. CHEMICAL COST}

蔡

100\% WASTE WOOD; ETHANOL @ \$1.20/GAL

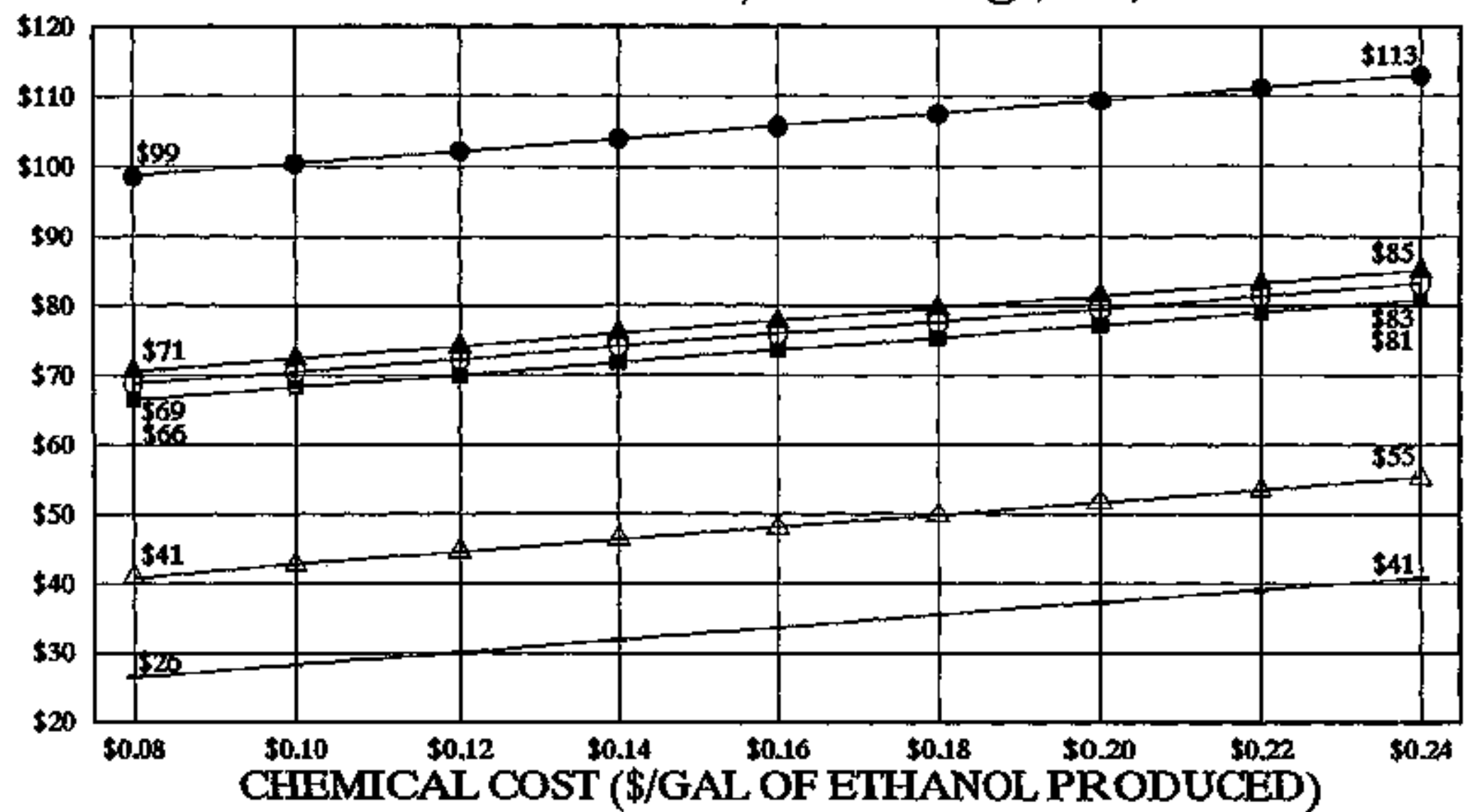

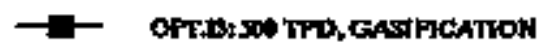

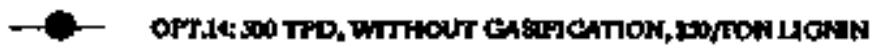

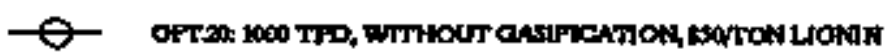

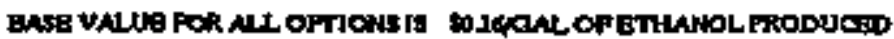

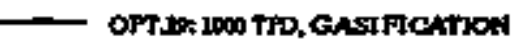

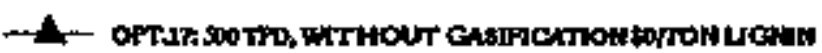

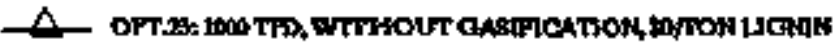


REQUIRED TIPPING FEE vs. CHEMICAL COST

点 100\% WASTE WOOD; ETHANOL @ \$1.50/GAL

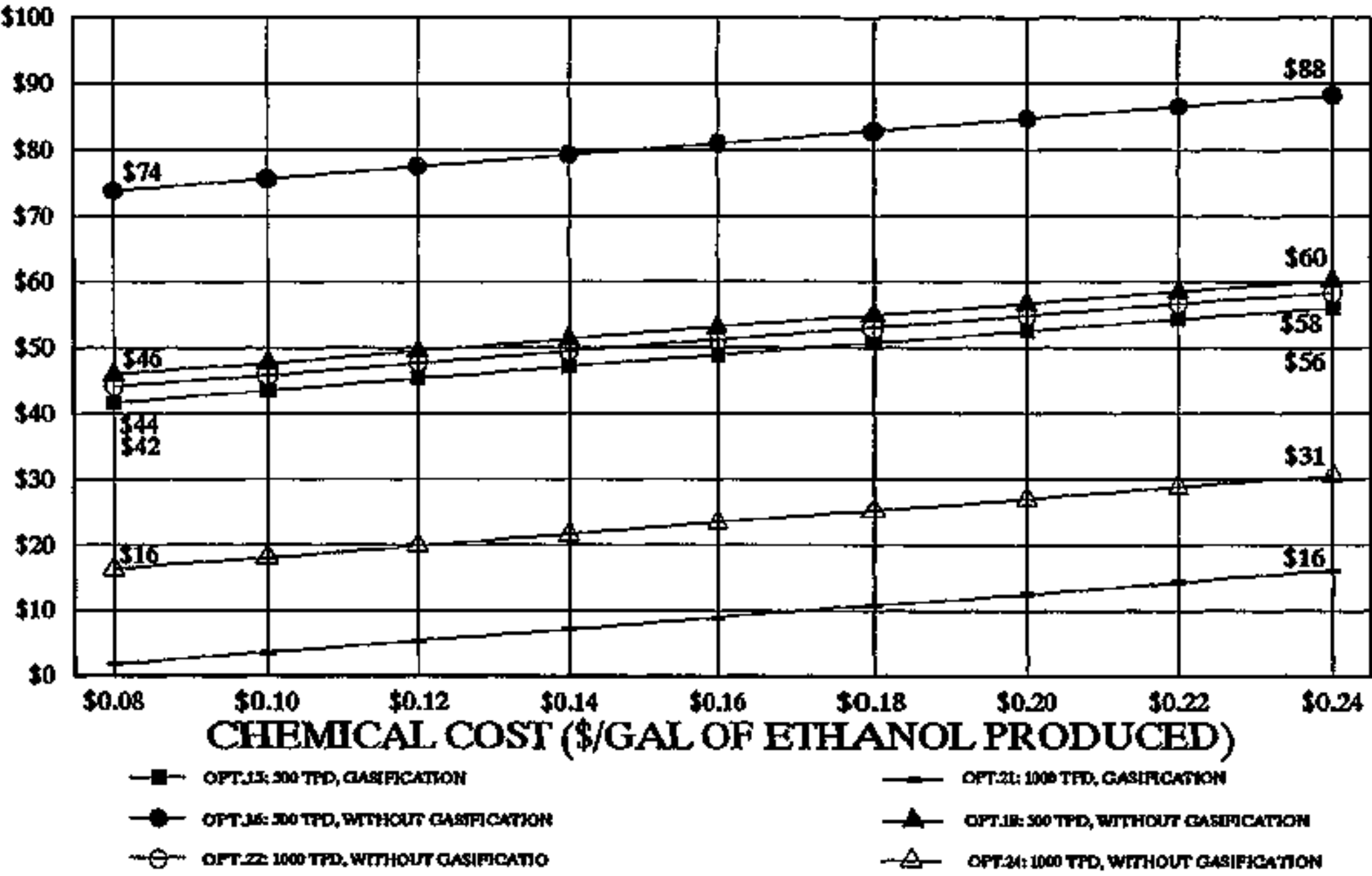

BAST VALUE FOR ALL OPTOAT IS 


\section{REQUIRED ETHANOL PRICE vs. CAPITAL COST AS A PERCENTAGE OF ESTIMATED COST}

$100 \%$ WASTE WOOD; WOOD COST @ \$20/TON

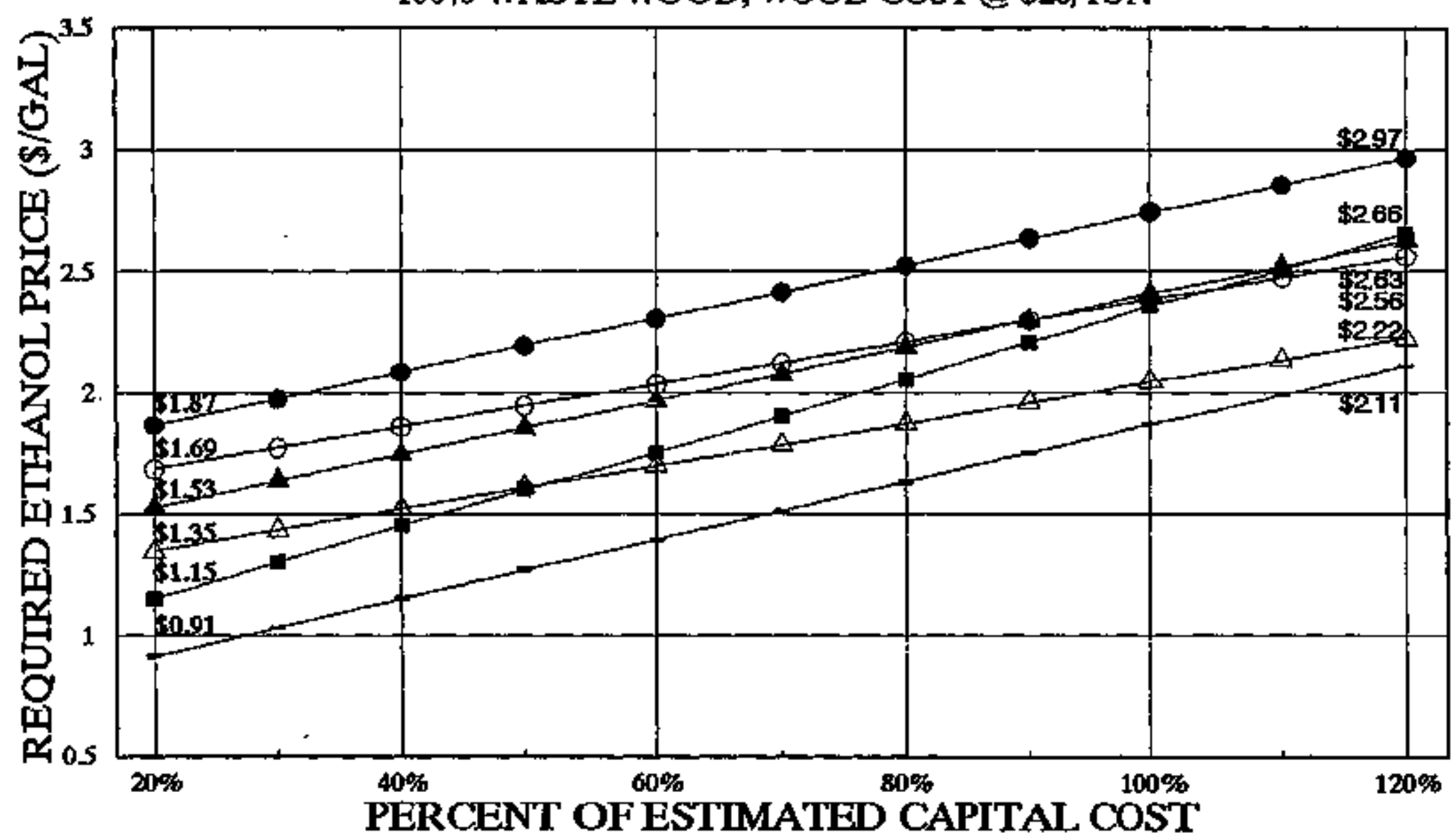

-

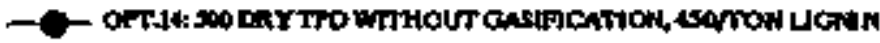

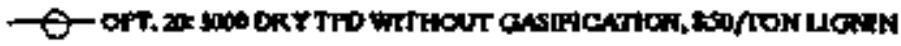

CAPITAL COST FCA OPT.19 = \$179,890,000: FOA CPT.14 17 = \$1 08,200,000

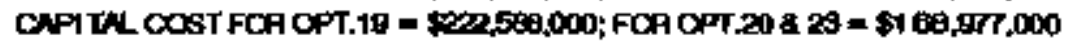

- OPT. 19, j000 DRT TPD WITH QNASIFCATTON

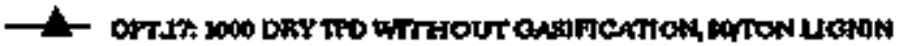

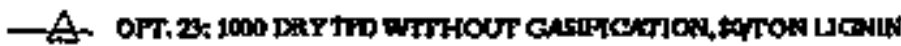

STONE \& WESTER DEVETOPAEtTCORPORATCN

FIGUAE 4-13 


\section{REQUIRED ETHANOL PRICE v. CAPITAL COST AS A PERCENTAGE OF ESTIMATED COST}

100\% WASTE WOOD; WOOD COST @ \$40/TON

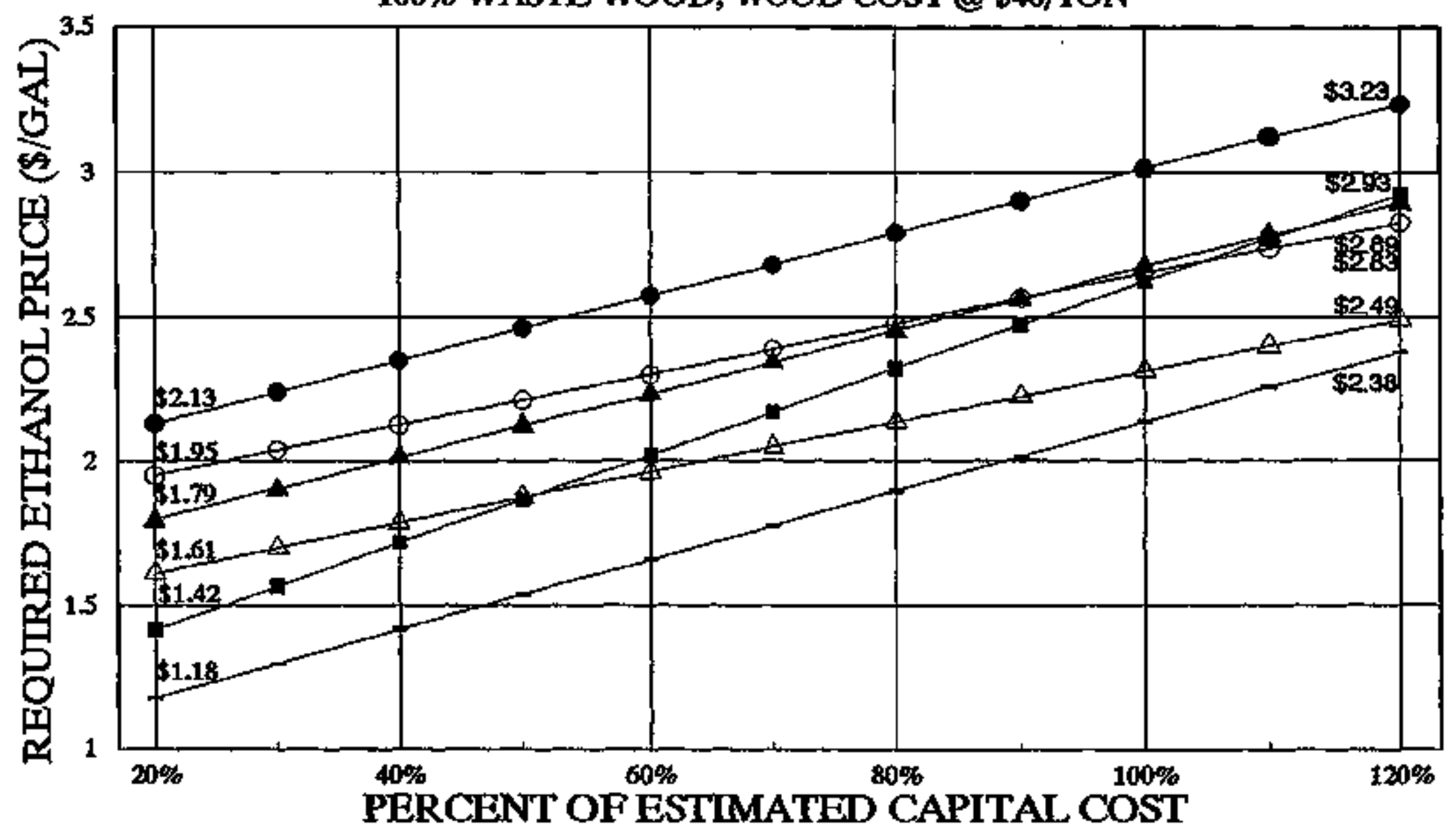

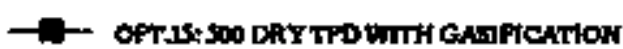

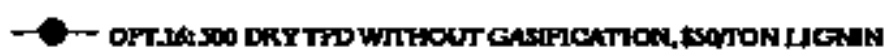

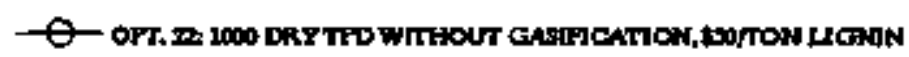

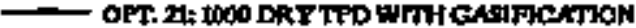

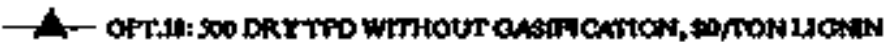

$\triangle \cdot$ OPT. 3T 1000 DRY TFD WTHOUT GASWFCATICN, DORTONLIGNIN STONE \& WEESTER DEVELOPMENT CCRPORATION

FIGURE 4-14 


\section{REQUIRED ETHANOL PRICE vs. O\&M COST 100\% WASTE WOOD; WOOD COST @ \$20/TON}

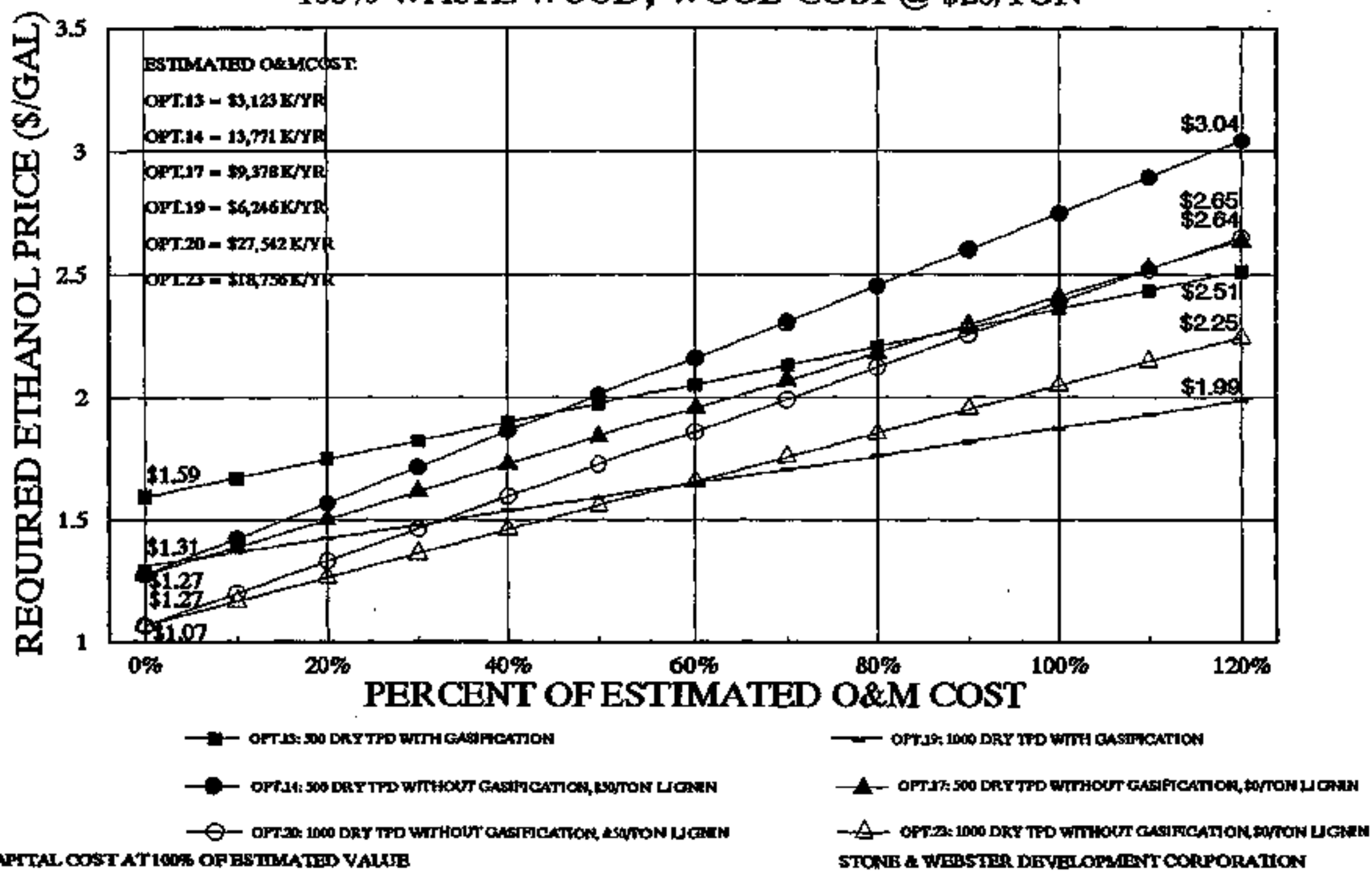

FIGURE 4-15 


\section{O\&M COST vs. REQUIRED ETHANOL PRICE 100\% WASTE WOOD; WOOD COST @ \$40/TON}

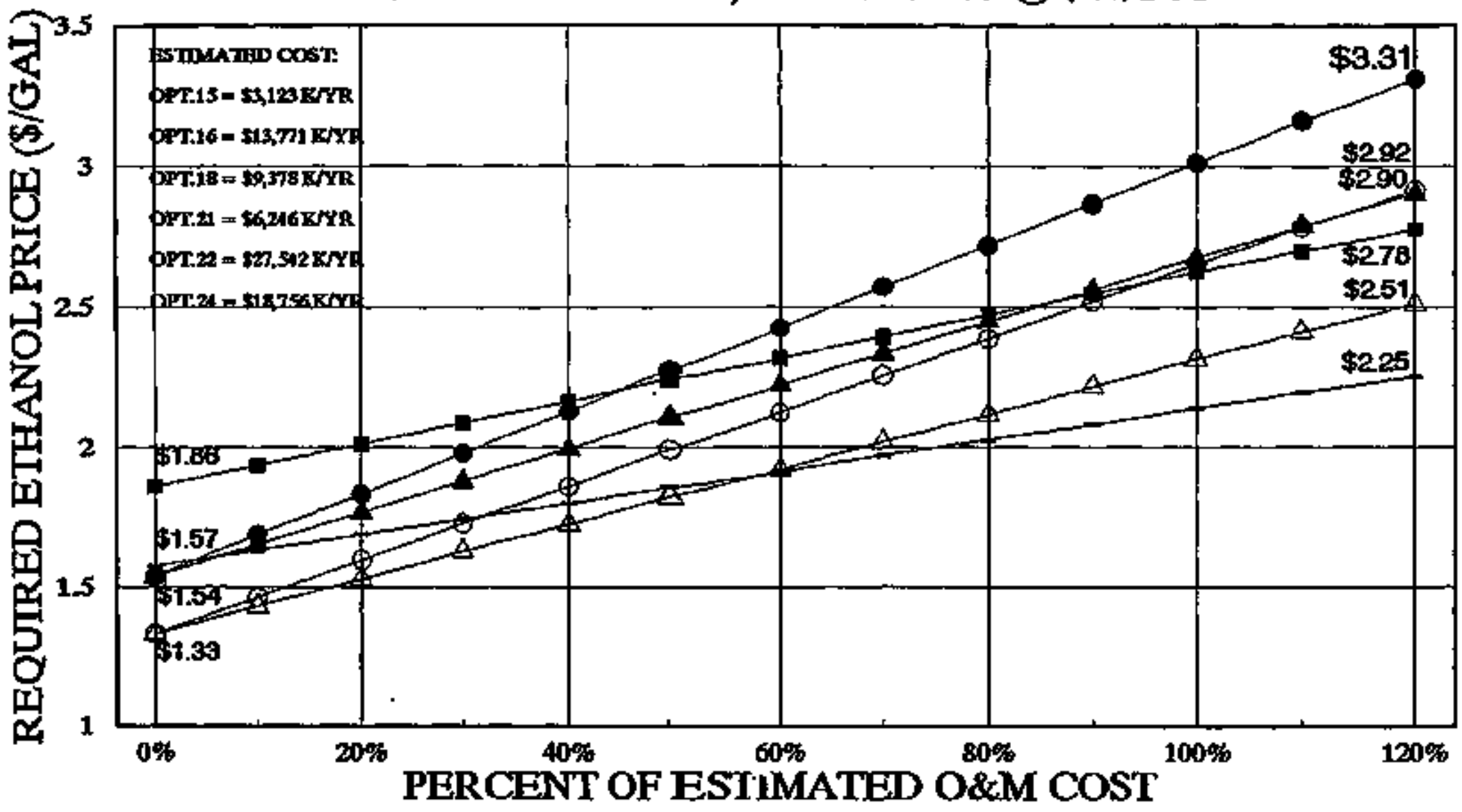

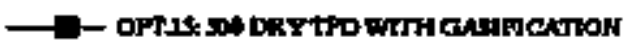

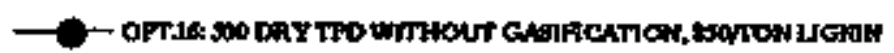

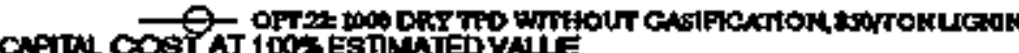
CST AT 100\% EsTAMIEDVALE

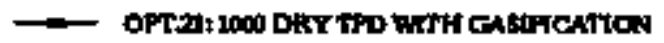

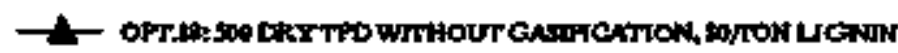

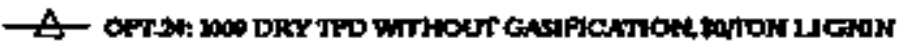
STONE \& WEISTER DEVELOPLIENTCORPORATCN

FGURE 4-16 
ATTACHMENT 4-2

RECYCLED WOOD PROCESSORS

\section{NAMEIOCATION}

$\mathrm{BQE}$ Services

Brooklyn, NY

ATTN: Joe Ciulla

Recycled Wood Products

P.O. Box 414

Sewell, NJ 08080

(609) 989-1501

Star Recycling 221 Verrick Ave.

New York, NY

(718) 387-2794 (Fran)

ATTN: Bob Pratt
BOTENTIAL SUDPPLY

300-400 tons/week woodchips

$375-625$ tons/week wondchips

$\$ 24-28 /$ ton

1989 PRICES EOB

$\$ 10 /$ ton

Source

$500-800$ tons/week woodchips

$\$ 16 /$ ton

NYC*

Source

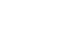




\section{ATTACHMENT 6-1}

Municipal Solid Waste by Amount and Location:

Raper Products, Yard Waste, and Construction and Demolltion Wood 
GENERATTON OF PAPER WASTE

In Upstate New York

\begin{tabular}{|c|c|c|c|}
\hline AGENCYTלTY & REEGKN & $\begin{array}{l}\text { UNir QF } \\
\text { WhStE }\end{array}$ & $\begin{array}{l}\text { MHOUNT QF } \\
\text { PAFET WASTE }\end{array}$ \\
\hline Aloany Ca/Albair & 4 & Tonstrear & 127,765 \\
\hline Calumbla $\mathrm{Ca}_{1}$ & 4 & Tong/raar & 14,794 \\
\hline Deldinte Co. & 4 & Tonsy at & 11,500 \\
\hline Ertant Co. & 4 & Tons Your & $11,7 / 5$ \\
\hline homigomary ca. & 4 & Tomsfrear & 13.483 \\
\hline Olsopese & 4 & Thiss Yod & 16.522 \\
\hline Scholptio & 4 & Tonstring & 5998 \\
\hline Rannsolatar Ca. & 4 & Tons rear & 6, 8.6 \\
\hline Schenecindy Co. & $4:$ & Tonstryor & $46,5,5$ \\
\hline $\begin{array}{l}\text { Todal for Reglon } 4 \\
\text { Total tor Regiton } 4\end{array}$ & & $\begin{array}{l}\text { Tons Year } \\
\text { Tonsidoay }\end{array}$ & $\begin{array}{r}255.971 \\
701\end{array}$ \\
\hline Cinton ca. & 5 & Tons Yerr & 21.687 \\
\hline Extax Co. & 5 & Tonerryer & 9,952 \\
\hline Frandin Sufin & 5 & Tonș Yoar. & 10,764 \\
\hline Fution $\mathrm{Ca}$. & $\$$ & Tonsfear & $\$ 5,257$ \\
\hline Hämiluen Co. & $\mathbf{S}$ & Tonsfrear & 826. \\
\hline Surnorin Colsaraleca spings & 5 & Tororereir & 44,812 \\
\hline Warrọn Co. & $\mathbf{5}$ & Jons roir & 24,144 \\
\hline Wratilaton Ca. & 5 & Tonstrar & 10,$6 ; 0$ \\
\hline $\begin{array}{l}\text { Total lor Raglon } 5 \\
\text { Totat for Aogon } 5\end{array}$ & & $\begin{array}{l}\text { Tonsfrear } \\
\text { Tonstogy }\end{array}$ & $\begin{array}{r}135.977 \\
370\end{array}$ \\
\hline Jetterson $\mathrm{Co}$ & 6 & Tonsyrat & 27.475 \\
\hline Lentis Ca & 8 & Tonsragr & 5,118 \\
\hline Onnida & 6 & Tonesypar & $74,6,5$ \\
\hline Horkelingr & $\mathbf{B}$ & Toneryout & 15,113 \\
\hline SI. Lamance SWOA & $\theta$ & TonsYaer & 29,674 \\
\hline Total lor Region 6 & & Tongrow & 151,393 \\
\hline Todal lor Raglon 6 & & Toniday & 415 \\
\hline Oroons Ca. & 7 & Tonstroar & 70,134 \\
\hline Crysen Co. & 7 & Tonșrạr & 18,809 \\
\hline Chenango ca & 7 & TongYgar & $11.0 \mathrm{ge}$ \\
\hline Conland Ca. & 7 & Tans'rex & 14,8 \\
\hline Haclon Co. & 7 & Tanstot & 16.398 \\
\hline Onandaga Co. ARAystazuse & 7 & Tonsmesp & tQ4.589. \\
\hline Oswato Co. & 7 & Tonifrytar & 24,232 \\
\hline Floga Co. & 7 & Tonstrea & 9.918 \\
\hline Tomplinis Ca & 7 & Tonstrar & 30,980 \\
\hline 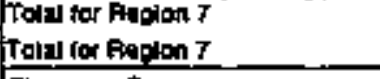 & & $\begin{array}{l}\text { Tonsiryar } \\
\text { Jonsivar }\end{array}$ & $\begin{array}{r}\text { 358,782 } \\
703 \\
\end{array}$ \\
\hline Chamung Ca. & 量 & Tons?ry & $12,5,41$ \\
\hline Sanesea & 8 & Tongrear & 16.257 \\
\hline Lhinnsion & $\mathbf{B}$ & Tonstyer & 12907 \\
\hline Oreans & 8 & Tongroar & Q. \\
\hline Mortos Codfifochnoter & 8 & Tong $Y$ Per & 247,107 \\
\hline Onlarin Ca. & 且 & Fonșrear & 30.203 \\
\hline Beguryin ca. & $\mathbf{B}$ & Tonertar & 3,472 \\
\hline someca & 6 & Torosinear & 6.090 \\
\hline Warne & 8 & Tonsfrex & 16,899 \\
\hline 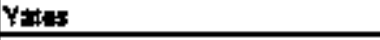 & 8 & Tanshyagr & 4,127 \\
\hline Sibuban Co. & g & Tonstoryear & $45,64 t$ \\
\hline Nlopany Co. & 9 & Tonsryop & 12,065 \\
\hline Colliarauges $\mathrm{Ca}$ & 9 & Tonsyerr & 23.717 \\
\hline Chedousqua & 9 & Tonsfrar & 39,642 \\
\hline Erlo CarBullals & 9 & Torty Yatr & 337,607 \\
\hline Nilagata Co. & 9 & Tonșraar & 67.928 \\
\hline Wrominto & 9 & Tonoryear & 8,590 \\
\hline Tolal tor Reglon 9 & & Torestrat & 409557 \\
\hline folda bor Radon 9 & & Totseray & 1,341 \\
\hline
\end{tabular}


RECYCLING OF PAPER WASTE

In Upsiate Naw York

\begin{tabular}{|c|c|c|c|}
\hline ABENCYFETK & AECAON & $\begin{array}{l}\text { UNIT OF } \\
\text { WASTE }\end{array}$ & $\begin{array}{r}\text { ANOUNT OF PAPEA } \\
\text { AEOYCLOO }\end{array}$ \\
\hline Aloany Co JAbary & 4 & Tonserrear & 20,426 \\
\hline Colurabia Co. & 4 & Tonshear & $1,9.99$ \\
\hline Dolaware Ca. & 4 & Tenervor & 7,795 \\
\hline Frange Co. & 4 & Tons Yas & 1,396 \\
\hline MOSA SMm Authar & 4 & Tonsorex & 4,092 \\
\hline Pennselian Co. & 4 & Tonstripar & 2,340 \\
\hline Sctienectedr Co. & 4 & Tonsprear & 7,193 \\
\hline Total for Aeplont 4 & & Tonsyitif & 45,161 \\
\hline 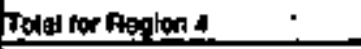 & & Ton:Dy & $\$ 24$ \\
\hline Cinken Co. & $\mathbf{5}$ & Tons Y Y & 2.035 \\
\hline Es:ax Co & 5 & Tomstrar & 546 \\
\hline Frantaln sim & 5 & Tonstrar & 633 \\
\hline Fullon Ca & 5 & Torishray & 1,464 \\
\hline Hamlion ca & 5 & Tonerer & 454 \\
\hline 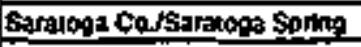 & 5 & Tonsfyes & 45,973 \\
\hline Warten Co. & 5 & Tonsryear & 1.69 \\
\hline Wretilingen Co. & 5 & Tonseryegr & 2582 \\
\hline Toted lox Region 5 & & Tom:Yur & $\$ 5.534$ \\
\hline Total Iox Rogion 5 & & Tonspar & 152 \\
\hline $\mathrm{Jun}$ etsen Co & 8 & Tonsycrer & 41 \\
\hline Lonks Ca. & 6 & Tonstryeer & 630 \\
\hline Onadde-Herkimet & 6 & Tonstrat & 19.827 \\
\hline 9. Lawrencel Simba & E & Tonsyem & $9,7\{B$ \\
\hline Tolal tor Rogken 6 & & Tonsoryar & 35,172 \\
\hline Total lar fopolons & & Tatis:0和 & 86 \\
\hline Broomm Co. & 7 & Torthyor & 0 \\
\hline Cryuga co. & 7 & Tonstron & 3,587 \\
\hline Chanango $\mathrm{CO}$. & 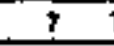 & Tons'roer & 2.508 \\
\hline Cordand Co. & 7 & Tonstroar & 2,238 \\
\hline Aadlogen Ca. & 7 & Tonoryerr & 4,699 \\
\hline Onondaga Co. RPAarsyracues & 7 & Tonervar & $\$ 2.070$ \\
\hline Oswerpo ca. & 7 & Tonsivar & 6,342 \\
\hline Thop Co. & 7 & Tonstrear & 2,280 \\
\hline Tampodins Co. & 7 & Tantrerer & 5,595 \\
\hline $\begin{array}{l}\text { Total lor Aegion } 7 \\
\text { Total lor Paftan } 7\end{array}$ & & $\begin{array}{l}\text { Tonsonfeds } \\
\text { Tonepay }\end{array}$ & $\begin{array}{r}139,276 \\
527\end{array}$ \\
\hline Chemung $\mathrm{Ce}$ & 8 & Teneryerr & 6.939 \\
\hline Gow Region swad & 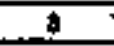 & Tpnteryear & 3,560 \\
\hline Honroe Covachesser & e & Ton:fYar & 45,268 \\
\hline Omario Ca. & $\theta$ & Tonefyer & $4,3,35$ \\
\hline Fchuvier ca. & 6 & Tans? & 205 \\
\hline W. Finger Lakes & $B$ & Tonsryear & 5.247 \\
\hline Stevuben co. & 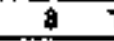 & Tonteryed & 2918 \\
\hline Forad tar Hotion 6 & & Fonterfoes & 68,478 \\
\hline Torat tot Pepolon 6 & & Tonsony & 182 \\
\hline Allegany $\mathrm{Co}$. & 9 & Tonsforat & 1,222 \\
\hline Collarauous ca. & 9 & Tonstrar & $4,045$. \\
\hline Chalaruara co. & $\mathbf{s}$ & Torbyear & B.313 \\
\hline 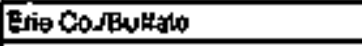 & 8 & Tonshyear & 31,303 \\
\hline Nagara Ca & 9 & Tons/Year & 11,605 \\
\hline $\begin{array}{l}\text { Total lor Aepion } 9 \\
\text { Tutal lor Region } 9\end{array}$ & & $\begin{array}{l}\text { Tonstrates } \\
\text { Tonstory }\end{array}$ & $\begin{array}{r}56.488 \\
155\end{array}$ \\
\hline
\end{tabular}

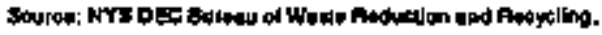

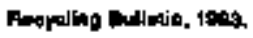


GENEAATION OF WOOL IN CONSTRUCTION \& DEMOLITEN WASTE In Upstate New York

\begin{tabular}{|c|c|c|c|}
\hline AHENGKCTY & FEGIC & $\begin{array}{l}\text { UNTS OF } \\
\text { WASTE }\end{array}$ & $\begin{array}{r}\text { AHCUNT OF WOOD } \\
\text { WASTE }\end{array}$ \\
\hline Aluny Costherny & 4 & Tonaryaar & 19,457 \\
\hline Coluntek Ca & 4 & Tinsplor & 012 \\
\hline Delenwars co. & 4 & Tonis Yoar & 987 \\
\hline Sreang Ca & 4 & Tonstiver & $\$ 21$ \\
\hline Mosa STM Arithor. - & 4 & Tentryor & 3,199 \\
\hline E. Rennselar CStum & 4 & Tonstetr & 500 \\
\hline Sachengeizor Ca & 4 & Tonsfyour & 9,887 \\
\hline $\begin{array}{l}\text { Total lor Region } 4 \\
\text { Total lor Feojon } 4\end{array}$ & & $\begin{array}{l}\text { Tonoryear } \\
\text { Toneroy }\end{array}$ & $\begin{array}{r}35.141 \\
\$ \$ 5\end{array}$ \\
\hline Clinton co. & 5 & Tonw Year & 3,95 \\
\hline Essanct con & $\underline{s}$ & Toneffey & 12,935 \\
\hline Franknn SWM & 5 & Toneryagr & 1,747 \\
\hline Futing & 5 & ToneNer & 1,000 \\
\hline Haindion Ca. & $\$$ & Tonspryat & $\sqrt{20}$ \\
\hline 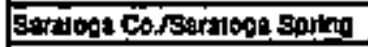 & 5 & Tone'Nar & 3.739 \\
\hline Wherten of Whesingtan $\mathrm{Co}$. & $\$$ & Toissing & 1.560 \\
\hline 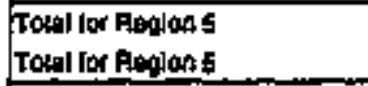 & & $\begin{array}{l}\text { Tonstrar } \\
\text { Tonstopar }\end{array}$ & $\begin{array}{r}25.097 \\
69 \\
\end{array}$ \\
\hline Jallerson Co. & 6 & Tonstrear & 2,80 \\
\hline Lomis Ca. & 6 & Tontryerr & 2 \\
\hline 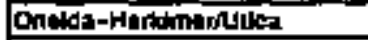 & 6 & Tortryer & 24,757 \\
\hline S1. tewrence SWDA & 6 & Tonerrour & 7,219 \\
\hline Tolal Ior Riglon 6 & & TonerYatr & 29,092 \\
\hline Totax ter Roglon 6 & & Topisolay & 60 \\
\hline Broone Ca. & 7 & Tons Yoar & 8,87 \\
\hline cryenco & 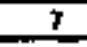 & Tonstryar & $4,4,6$ \\
\hline Chenango Co. & $i$ & Tonsprogr & 1,151 \\
\hline Corliand ce & 7 & ToneNopr & 3,346 \\
\hline Andsan Ca. & 7 & Toneroar & \$14 \\
\hline 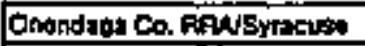 & 7 & Tontwray & 20,250 \\
\hline Oswopoca & 7 & Tonstryear & 7,394 \\
\hline Tloga Ca & 7 & TafsNaar & $\pi$ \\
\hline Tomakins $\mathrm{Ca}$ & 7 & Tonsmay & 2,201 \\
\hline $\begin{array}{l}\text { Tolal for ferpion } 7 \\
\text { Total tor Peopion } 7\end{array}$ & & $\begin{array}{l}\text { Tonsforar } \\
\text { Torrobay }\end{array}$ & $\begin{array}{r}5.369 \\
145 \\
\end{array}$ \\
\hline Chaming $\mathrm{Co}$ & 1) & Tonstriest & 3,429 \\
\hline GLOW Roglan SWN & 8 & Tongryour & 4,945 \\
\hline Monow Co.jpocheser & 8 & Tonstraar & 61,742 \\
\hline Seniuyter Ca. & 8 & Tontryour & 372 \\
\hline W. Flancr Lutas & 8 & Toptsylyew & 3,700 \\
\hline Simpor $\mathrm{Co}_{0}$. & $\theta$ & Tonsfres & $\$, 279$ \\
\hline Tolal lor fregion & & Tonsfing & 7,414 \\
\hline Tater lox Pegdion 8 & & Tonspoy & 212 \\
\hline Anteorny $\mathrm{Ca}$ & 8 & Tons Yalar & 1,220 \\
\hline Caluarautut Co. & 5 & Tonsfrear & 2,479 \\
\hline Chatauqua $\mathrm{Co}$. & 9 & Tonfypor & 3,405 \\
\hline Ela CoJ Evillato & 9 & Tont/Yar & 35,065 \\
\hline Nlapara ca & 9 & Tonsfytr & 7,722 \\
\hline $\begin{array}{l}\text { Folal for Rooion } g \\
\text { Tolal lox fleptong } 9\end{array}$ & & $\begin{array}{l}\text { Tentrear } \\
\text { TansiDay }\end{array}$ & $\begin{array}{r}19, \sqrt{102} \\
137\end{array}$ \\
\hline
\end{tabular}

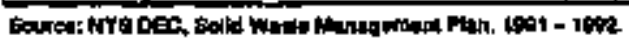


GENERATION OF YARD WASTE

in Upsiate New Yoik

\begin{tabular}{|c|c|c|c|}
\hline AOENCYICTYY & FEFon & $\begin{array}{l}\text { UNT OF } \\
\text { WhSTE }\end{array}$ & $\begin{array}{l}\text { hWOWT Of } \\
\text { rafio WASTE }\end{array}$ \\
\hline 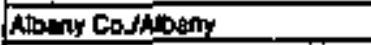 & 4 & TGterrear & 27,972 \\
\hline Columbin Co. & 4 & Tonishor & $2+169$ \\
\hline Detsurare Con. & 4 & Tonviras & 3,208 \\
\hline Ereone Da & 4 & Tonstroar. & 2,268 \\
\hline Wosk SWh tunthor. & 4 & Tonovear & 9,614 \\
\hline E Ricnnsulaer Cswain & 4 & -Ton: & 2409 \\
\hline Selinoctady $\mathrm{Co}$ & 4 & Tons rex & 11, 㛃然 \\
\hline Topal for Reglon 4 & & TonsYry & 58,584 \\
\hline Todnl kor Rad on 4 & & Torstpar & 160 \\
\hline Cilunom co. & 5 & Tonstrear & 4851 \\
\hline Essex Co. & 5 & Fonstrout & 7,856 \\
\hline Frangidln SWA & 5 & Tonshrear & 2,807 \\
\hline$\overline{F u l l o n} \overline{c o}$. & 5 & Tonstrapr & 3,857 \\
\hline Hermilion $\mathrm{Ca}$. & $\mathbf{5}$ & Tringryed & $\$ 19$ \\
\hline 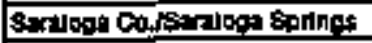 & 5 & Toneryear & 20,470 \\
\hline Wanen, Washlaquon Ca. & 5 & Tonsherar & 11,463 \\
\hline Total her fiagdon 5 & & Toneryter & 47,176 \\
\hline Total tor fiedon 5 & & Tonstoy & 129 \\
\hline Jallempont Co. & 5 & Tonstrear & 9.840 \\
\hline Cowls Ca. & $\mathbf{B}$ & Tonerrear & 1,091 \\
\hline Ordodn-Harkinger & 6 & Tonstragr & 17,111 \\
\hline Si Lomence Sthon. & 6 & Tohtspear & 7,023 \\
\hline Tokat lor Alapion 6 & & Tonfyear & 35,005 \\
\hline Tod tor Reden th & & Toxsty & 98 \\
\hline Groome Co. & 7 & Tondyear & $21,21 \theta$ \\
\hline 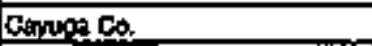 & 7 & Topgryat & 7.341 \\
\hline Chandivos Co & 7 & Toneryer & 2,469 \\
\hline Cortand Co. & 7 & 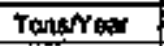 & 2.242 \\
\hline A dadsen Ca. & 7 & Tonefreri & 4546 \\
\hline 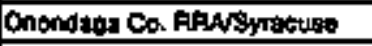 & 7 & Tonstresr & $69,45+\dot{B}$ \\
\hline Oswotedoch & $y$ & Tenstrear & 10.510 \\
\hline Tago $\mathrm{Co}$ & 7 & Ton:Pryar & 2,195 \\
\hline Jompdns Ca & 7 & Tonstryear & 6,210 \\
\hline Tokal lor Paglon 7 & & Tontefrear & 125,423 \\
\hline Tolaj lor Pugion 7 & & Tonstoy & 34 \\
\hline Chameno to & 早 & Tons repar & 6.026 \\
\hline GLOW Feplon SWH & $\theta$ & Fontryenr & 13,553 \\
\hline Honos Corfoche:ter & 是 & Tonspear & 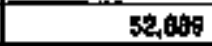 \\
\hline Sonwhur Ca. & 8 & Tors Near & B666 \\
\hline W. Flnet Laked & $\mathbf{g}$ & Toneryear & 11565 \\
\hline Stevbenco. & i) & Tons'rear & 4,002 \\
\hline $\begin{array}{l}\text { Tolal lor Fiagion } 8 \\
\text { T억 lor Fealio } 8\end{array}$ & & $\begin{array}{l}\text { Jonoriat } \\
\text { Toneroy }\end{array}$ & $\begin{array}{r}69.501 \\
245\end{array}$ \\
\hline Alvgany Co. & $\bar{\theta}$ & Tongryar & 2,430 \\
\hline Contauput Co. & 5 & Toneryos & 4.528 \\
\hline Chataluqua Co. & 9 & ToneNos & 9.660 \\
\hline Ela CojBullalo & 9 & Tons Yax & g1, 0292 \\
\hline Nllagrac Ca. & 9 & Tonsfryar & 18,930 \\
\hline $\begin{array}{l}\text { Todal lor Pegtion \$ } \\
\text { Tody lor Region } 9\end{array}$ & & $\begin{array}{l}\text { Tonsirear } \\
\text { Tonsory }\end{array}$ & $\begin{array}{r}127.647 \\
350\end{array}$ \\
\hline
\end{tabular}

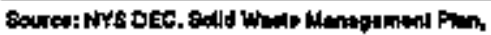

1 int - 140: 
RECYCLING OF WOOO IN CONSTRUCTION \& DEMOLTION WASTE in Upotale New York

\begin{tabular}{|c|c|c|c|}
\hline ASENCYNCITY & AESTH & $\begin{array}{l}\text { UNT OF } \\
\text { whste }\end{array}$ & $\begin{array}{r}\text { AHOUWT OFWOOD } \\
\text { FECYCLED }\end{array}$ \\
\hline Nhany Co.RAthany & 4 & Tonshoper. & 0 \\
\hline Codumbla co. & 4 & Tons/rated & $\underline{\mathbf{0}}$ \\
\hline Oedzware ca. & 4 & Tonsfrear & $\underline{\mathbf{0}}$ \\
\hline Greane Ca. & 4 & Tonspryear & 足 \\
\hline heosis Sind Author. & 4 & Tonsfyear & D \\
\hline Fonsolder Co, & 4 & Toneryey & 0 \\
\hline Schepectady Co. & 4 & Tonprar & 1,345 \\
\hline $\begin{array}{l}\text { Toxal Ior Reglon } 4 \\
\text { Tolel Ior Feolon } 4\end{array}$ & & 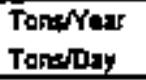 & $\begin{array}{r}1,345 \\
4 \\
\end{array}$ \\
\hline Cithlan Co. & 5 & Tonetyar & $\cdot$ \\
\hline Essex con & 5 & Tonelver & 0 \\
\hline Frandin Sinn & 5 & Tonsivear & 0 \\
\hline Fullon Ca & 5 & Tanexear & 0 \\
\hline Hemilton Co. & 5 & Tonsryear & 214 \\
\hline 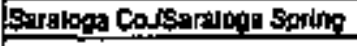 & 5 & Tonerxin & 121 \\
\hline Warran Co. & 5 & Tonshyear & 0 \\
\hline Waghingonon Co. & 5 & Tenopyogr & 10 \\
\hline Total for Roglen 5 & & Tonsfreer & 355 \\
\hline Tolalal lor Peglo5 & & Tonsogy & 1 \\
\hline Jellersin co. & 6 & Tonstrer. & 0 \\
\hline Lems Co. & 6 & Tongryter & 0 \\
\hline Ortalda-Heridragriduter & 6 & Tonsorrar & 0 \\
\hline St. Lawence SWDA & 6 & Tonssyear & 0 \\
\hline Tolal for Fegion 6 & & Tons/reat & 0 \\
\hline Totat lor Reglon 6 & & Tont & 0 \\
\hline Erosing Ca & 7 & Tersis/régr & 4.844 \\
\hline Coyura Ca & 7 & Tongryper & 8,432 \\
\hline Chenanoo Co. & 7. & Tontryon & 0 \\
\hline Cordind $\mathrm{Ca}$. & 7 & Tonstert & 107 \\
\hline Marison Co. & 7 & Tonsyryor & 9 \\
\hline Onondapa Ca FRusyracusa & $?$ & Tons'year & 3,002 \\
\hline Orwapaca & 7 & Tons Yoar & $\bar{D}$ \\
\hline Tlope Ca & 7 & Tonefrom & 0 \\
\hline Tompkins Co & 7 & Toneryar & 0 \\
\hline Tolal tor Rapon 7 & & Tonstrar & 16.394 \\
\hline Todal lor Rieplon 7 & & Tonsionay & 49 \\
\hline Chaming Ca. & 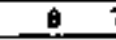 & Tonstreaf & \$19 \\
\hline BLOW Aaglon SWed & 8 & Tons Year & o \\
\hline Monfos Cospachesiar & 8 & Tong Yoar & 2,412 \\
\hline Dirtatio Co. & 8 & Tons'ret: & 0 \\
\hline Schuy & 8 & Tonis Y par & 0 \\
\hline W, Finow Lakes & 8 & Tonstrad & 0 \\
\hline Sillubin Ca & $a$ & Tons/rat & 0 \\
\hline Total tot Aedon $\theta$ & & Tonspryar & 2.72 \\
\hline Total lor Region 0 & & Tons: Day & 7 \\
\hline Allogany Co. & $\overline{9}$ & Tons/Yegr. & 0 \\
\hline Canarapupas Co. & 8 & Tonsryet & 0 \\
\hline Chalaupud Co. & 9 & Tonssyear & 662. \\
\hline Eris Co.raurang & 9 & Tonefyear & 258 \\
\hline Napare Ca & 9 & Tonswear & 5.735 \\
\hline $\begin{array}{l}\text { Total lor Rapion } 9 \\
\text { Total tor Region } 9\end{array}$ & & $\begin{array}{l}\text { Torts Yay } \\
\text { Tons/Day }\end{array}$ & $\begin{array}{r}6065 \\
19 \\
\end{array}$ \\
\hline
\end{tabular}

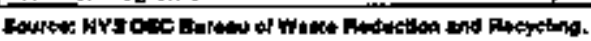

Anereling Hullem, 100. 
RECYCLING OF YARD WASTE

In Upotabe New York

\begin{tabular}{|c|c|c|c|}
\hline NGENCYTICTY & REOKFN & $\begin{array}{l}\text { UNIT OF } \\
\text { WASTE }\end{array}$ & $\left\{\begin{array}{l}\text { ANOUNT OF YARD } \\
\text { WASTE AECYCLED }\end{array}\right.$ \\
\hline Aoumy Codsham & 4 & Tonervitipi & 8,425 \\
\hline Columbla ca. & 4 & Tonshyer & 0 \\
\hline Delawarla Co. & 4 & Torasyest & 0 \\
\hline Greang Ca & 4 & Tonsfrepr & $\mathbf{0}$ \\
\hline AOSk Shin huther. & 4 & Tonstrai & 0 \\
\hline Pannsplan Co. & 4 & Tonstray & 700 \\
\hline Schenefolady Corrsehenecied & 4 & Tons:ryegr & 17,453 \\
\hline $\begin{array}{l}\text { Total lor Fogolon } 4 \\
\text { Total ker Region } 4\end{array}$ & & $\begin{array}{l}\text { Tonstrept } \\
\text { Tonopay }\end{array}$ & $\begin{array}{r}26,574 \\
73 \\
\end{array}$ \\
\hline Cintion Co. & 5 & Tompror & $\mathbf{0}$ \\
\hline Ftancon & 5 & Tonsyer & $\underline{0}$ \\
\hline Franitin sin & 5 & Tongyar & 57 \\
\hline Fungon Co. & 5 & Tonsyear & 800 \\
\hline Hamilum Co. & \pm & Tonstrear & t的 \\
\hline 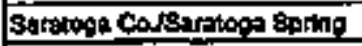 & 5 & Tons $\mathrm{Nes}$ & 7,500 \\
\hline Warren Co. & 5 & Tonsy ther & 0 \\
\hline Washinvion Co. & 5 & Totserear & 675 \\
\hline $\begin{array}{l}\text { Tolol lor Pegtan } 5 \\
\text { Tatal for Aletan } 5\end{array}$ & & $\begin{array}{l}\text { Tens } \\
\text { Tonsory }\end{array}$ & $\begin{array}{r}9,312 \\
26 \\
\end{array}$ \\
\hline jellaron Co & $\dot{\theta}$ & Tontrogr & 3,720 \\
\hline Lowis Cat. & $\theta$ & Tonspryent & 699 \\
\hline Greba-Heridanor & 6 & Tonstear. & 0 \\
\hline St Lamenso SMad & 6 & Toxurear & 0 \\
\hline $\begin{array}{l}\text { Tral lor Peqlon } 6 \\
\text { Iotat lox Apalon } 6\end{array}$ & & $\begin{array}{l}\text { Tonsyer } \\
\text { Tonstury }\end{array}$ & $\begin{array}{r}4,625 \\
13 \\
\end{array}$ \\
\hline Eroomen Caringhamion & 7 & Tontsfrear & 25,534 \\
\hline Corused Co. & 7 & Tonsyer & $4,0,097$ \\
\hline Chenango Co. & 7 & Tongrroder & 300 \\
\hline Conlland Co. & 7 & Tonstrear & 们 \\
\hline Mrotison Co. & 7 & Tonorrear & 2 安 \\
\hline 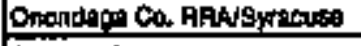 & 7 & Tonstreir & 3.75 \\
\hline Osiogo Co. & 7 & Tonefred & 3,806 \\
\hline Fonaca & 7 & Tonstrear & 0 \\
\hline Tompkins Ca & 7 & Tonarvas: & 50 \\
\hline Total lor Aeglon 7 & & Tonsryour & 39,008 \\
\hline Total bor Plostan 7 & & Tonolotay & 104 \\
\hline Chemung $\mathrm{Ca}$ & $\mathbf{B}$ & Tontryar & 1,600 \\
\hline CLLOW Aepion SWA & 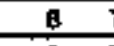 & Tonervar & 423 \\
\hline 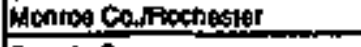 & $\theta$ & Tonstrar & 7,722 \\
\hline Omeanoco. & $\theta$ & Tonerap & 0 \\
\hline Sentyrer Co. & 臭 & Fonstrat & 1,231 \\
\hline W. Finger Laks & 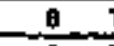 & Tonsoryour & 0 \\
\hline Sinden Co. & 8 & Tonsfymer & t7 \\
\hline 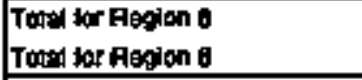 & & $\begin{array}{l}\text { Tonsfroor } \\
\text { Tonsuov }\end{array}$ & $\begin{array}{r}11,078 \\
\quad 30 \\
\end{array}$ \\
\hline Alvagany $\mathrm{CO}$. & $\theta$ & Tongr'roogr & 0 \\
\hline Gaturaupous Co. & 9 & Tonshow & 1.656 \\
\hline Chorivua Co. & 9 & Tonstrater & 4.401 \\
\hline Erto Coslayllak & 9 & Tonsiyear & 36,685 \\
\hline Nieqara Ga & B & Tons $\mathrm{Yan}$ & 1,000 \\
\hline $\begin{array}{l}\text { Tolal lor Roglan } 9 \\
\text { Tolal lor Rogitan } 9\end{array}$ & & $\begin{array}{l}\text { Tonefrast } \\
\text { Tonertury }\end{array}$ & $\begin{array}{r}43,945 \\
120\end{array}$ \\
\hline
\end{tabular}

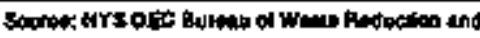

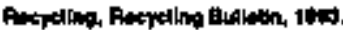




\section{ATTACHMENT 6-2}

Industrial Waste by Amount, Location, and Tipping Fees:

Lumber By-Products and Paper Sludge 
GENERATION OF PAPEF SLUDGE

in Upotate Naw York

\begin{tabular}{|c|c|c|c|}
\hline MCDACYRtir & FHEIOH & $\begin{array}{l}\text { UNif of } \\
\text { WhSTE }\end{array}$ & $\begin{array}{l}\text { ANOOWT OF } \\
\text { PAPEA STUDGE }\end{array}$ \\
\hline Albany Coldttbuny & 4 & Tonsfran & 0 \\
\hline Colunida Ca. & 4 & Tonsheprir & $\underline{0}$ \\
\hline Deknore fe. & 4 & Tontryar & 0 \\
\hline Ercene Ca. & 4 & TonteYyour & 0 \\
\hline mosh shin kuthor. & 4 & Tonstitit & 0 \\
\hline 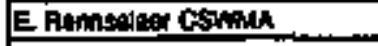 & 4 & Tons $\mathrm{Yaer}$ & 35 \\
\hline Senerectaof $\mathrm{Co}$. & $4 \cdot$ & TonsNuar & D \\
\hline Toter lox feglon 4 & & Tonstrar & 365 \\
\hline Toped lor Rention 4 & & Tondsy & 1 \\
\hline Clelon Ca & $\mathbf{5}$ & Tonsryut & B,125 \\
\hline $\operatorname{Esin} C_{0}$ & 5 & Tonsyeak & .0 .125 \\
\hline Frankla SMN & 5 & Toptryar & 0 \\
\hline Fúlon Co. & $\mathbf{B}$ & Tonsryear & 0 \\
\hline Bamlion Ca. & $\underline{B}$ & Torshoar & 0 \\
\hline 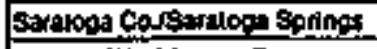 & $\underline{5}$ & Tons & $31,0 \overline{3}$ \\
\hline Varren, Whingrion Co. & 5 & Torterfutis & 40,150 \\
\hline Tolt ker Roton 6 & & Tonaryear & 89,425 \\
\hline Tolal lar Aaplon 5 & & TonsDar & 245 \\
\hline Jatlersen Ca & 6 & Thasfear & 23,580 \\
\hline Lonitg Ca. & 6 & Tons Yroar & 2,890 \\
\hline Onalda-thempingr. & 5 & Tonsryear & 0 \\
\hline St Lomirence stroh & 6 & Tonstrear & 1,055 \\
\hline Toted for Restion 6 & & Tons'Ygat & 27,375 \\
\hline Joer for fterion 6 & & Touspay & 75 \\
\hline Eroomin Ca & 7 & Tenaryay. & $\underline{0}$ \\
\hline Cayoa Ca & 7 & Tonervaat & 0 \\
\hline Gilenange Co. & 7 & Toraryear & 0 \\
\hline Conland Ca & $\mathbf{7}$ & Tensrrear & 0 \\
\hline Ladksin Ca. & 7 & Toneryen & 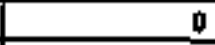 \\
\hline 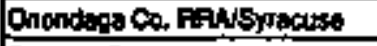 & 7 & TonsNear & 0 \\
\hline Oswe o Ca. & 7 & Tonstrour & 3로:5 \\
\hline Tlogt: Co. & 7 & Tongryar & 0 \\
\hline Tomptins C. & 7 & Tonstren & 0 \\
\hline $\begin{array}{l}\text { Tord for fieglon } 7 \\
\text { Todnd for Replon } 7\end{array}$ & & 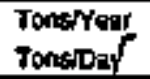 & $\begin{array}{r}3,285 \\
9\end{array}$ \\
\hline Chomung Ca. & $\mathbf{B}$ & Tonsyes: & 0 \\
\hline gLow Per lon SWA & $B$ & Tonerrear & 965 \\
\hline Pronroo Coffrochester & 8 & Tongryor & $\overline{0}$ \\
\hline Senumiar $\mathrm{Ca}$ & 8 & Tans reat & 0 \\
\hline W. Flnom LீKes & 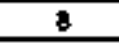 & Tonstral & a \\
\hline Steduen Co. & 8 & Tonsfyedr & 0 \\
\hline Toted for Reolon 8 & & Tonshyer & 385 \\
\hline 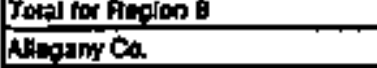 & $\theta$ & $\frac{\text { TonitDay }}{\text { Tensyor }}$ & $-\frac{1}{0}$ \\
\hline Ertentaveus $\mathrm{Co}$. & 9 & Tengrerar & 0 \\
\hline 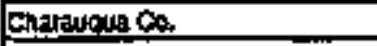 & 9 & Tonsyrear & 0 \\
\hline Eria Cortantido & $\theta$ & Torsh $\mathrm{Yag}$ & 0 \\
\hline Nimgra $\mathrm{CO}_{2}$ & 9 & Tonstrear & 4,380 \\
\hline To익 lor Reglon & & Tonshear & 4,340 \\
\hline Total bor Reglon 1 & & Tonsidy & 12 \\
\hline
\end{tabular}


SAMPLING OF SOURCES OF WOOD WASTE PAODUCTS

Including Wood Chips, sawdust, Shavings, and Bark

In Upstake New York

\begin{tabular}{|c|c|c|}
\hline Nama/Location & Fotemlal Supply & Picto \\
\hline $\begin{array}{l}\text { Adirondack Forest Industrins } \\
\text { Gatway }\end{array}$ & $\begin{array}{l}14 \text { tonyday wood thips } \\
3 \text { tonsfday saudut }\end{array}$ & $\begin{array}{l}\text { scoiton } \\
\text { staton }\end{array}$ \\
\hline $\begin{array}{l}\text { Harris Fallet } \mathrm{CO} \text {. } \\
\text { Albamy }\end{array}$ & 6 tonsyday wood chlps & s2siton \\
\hline $\begin{array}{l}\text { S } \mathrm{J} \text { Log Lumber Co. } \\
\text { Dover Ptains }\end{array}$ & $\begin{array}{l}43 \text { tonsiday wood chips } \\
86 \text { tonsiday sawdus }\end{array}$ & $\begin{array}{l}\text { \$33non } \\
\text { section }\end{array}$ \\
\hline $\begin{array}{l}\text { Johirston } 4 \text { Rhodes Gluestone } \\
\text { East Branch }\end{array}$ & 3 tonslday wood thips & stesion \\
\hline $\begin{array}{l}\text { Mallery Lumber } \\
\text { Hancock }\end{array}$ & $\begin{array}{l}37 \text { lonsiday wood chips } \\
14 \text { tons/day samdust } \\
14 \text { toruskday berk }\end{array}$ & $\begin{array}{l}\text { \$15iton } \\
\text { \$anton } \\
\text { \$15hton }\end{array}$ \\
\hline $\begin{array}{l}\text { McGiraw Lumber } \\
\text { Woodboume }\end{array}$ & $\begin{array}{l}\text { 3 - \$ tonsiday wood chips } \\
\text { a - } 11 \text { tonsiday bark }\end{array}$ & $\begin{array}{l}\text { solton } \\
\text { s28ion }\end{array}$ \\
\hline $\begin{array}{l}\text { Phillips Lumber } \\
\text { Hartwick }\end{array}$ & $\begin{array}{l}9 \text { - } 11 \text { tonsiday wood chipa } \\
6 \text { tonskday bark }\end{array}$ & $\begin{array}{l}\text { S15ton } \\
\text { sopton }\end{array}$ \\
\hline $\begin{array}{l}\text { Pomeroy Lumber } \\
\text { Ninevah }\end{array}$ & $\begin{array}{l}14 \text { tonsiday wood chips } \\
10 \text { torsifitay sawdust }\end{array}$ & \\
\hline $\begin{array}{l}\text { Sism Lumber } \\
\text { Otego }\end{array}$ & $\begin{array}{l}3 \text { tonsiday wood chips } \\
3 \text { - } 6 \text { tons/day bark }\end{array}$ & 523 - 29rton \\
\hline $\begin{array}{l}\text { Webb } 8 \text { Sons } \\
\text { Now Bertin }\end{array}$ & $\begin{array}{l}\text { 11 - } 21 \text { tonsiday wood chips } \\
7 \text { - } 14 \text { tonsiday sondus }\end{array}$ & \\
\hline
\end{tabular}


ATTACHMENT 6-3

Compilation of Municipal and Industrial Solid Waste 
COMPILATION OF WASTE WN ETHANUL FACILITY SITING ZONES In Upstate Now York

\begin{tabular}{|c|c|c|c|c|c|c|c|}
\hline \multirow[t]{2}{*}{ AGENCYCITY } & \multirow[t]{2}{*}{ UNIT OF WASTE } & \multicolumn{6}{|c|}{ MHOLNT OF WASTE } \\
\hline & & PAPEA & $\begin{array}{l}\text { OONSTFUCTION AHO } \\
\text { DEMDUTLON WOOO }\end{array}$ & YAFD & $\begin{array}{r}\text { PAPEA } \\
\text { SLUDCE }\end{array}$ & LuMBef & $\begin{array}{l}\text { TOTAL } \\
\text { WASTE }\end{array}$ \\
\hline \multicolumn{8}{|l|}{ Albany Zone } \\
\hline Albany Co.labany & Tonshear & 34,675 & 9,658 & $19,54 ?$ & 0 & & 64,077 \\
\hline Columbla Co. & Tonshyear & 4,439 & 156 & 2.169 & 0 & & 6,782 \\
\hline Greene $\mathrm{CO}$ & Tons/Year & 3,495 & 210 & 2,266 & $\mathbf{0}$ & & 5,971 \\
\hline E. Rannselagr CSWhth & Tonsyear & 239 & 450 & 1,709 & 365 & & 2,783 \\
\hline Schenectady Co. & Tonshrear & 13,595 & 3,650 & $(5,525)$ & 0 & & 11,720 \\
\hline Baraloga Co./Saratoga springs & TonsYyear & $(26,767)$ & 1,757 & 12.970 & 31,025 & & 18,965 \\
\hline Warren, Washinglon Co. & Tonsryear & 10,509 & 670 & 11,288 & 40,150 & & 62,617 \\
\hline Total for Albany Zone & Tonerrear & 40,184 & 16.748 & 44,423 & 71,540 & 171,400 & \$4, 295 \\
\hline Total for Abany Zono & Tonsiday & 110 & 46 & 122 & 198 & 470 & 943 \\
\hline \multicolumn{2}{|l|}{ Syracuse Zone } & & & & & \multicolumn{2}{|c|}{ 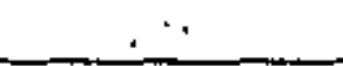 } \\
\hline Cayupa Ca. & Tonelfear & 4,465 & $(6,179)$ & 3,304 & 0 & & 1,590 \\
\hline Madison Co. & ToneNvear & 2,205 & 407 & 3,290 & $\mathbf{0}$ & & 5,992 \\
\hline Onondaga Co. FFAfsyracuse & Tonstraar & $(21,170)$ & 0,030 & 6,695 & 0 & & 52,745 \\
\hline Osmego Co. & TonsNen & 4,119 & 3,692 & 6,644 & 3,285 & & 17,640 \\
\hline Tolal tor Syracuse Zone & Tonsfyar & (10,291) & 5,950 & 79,020 & 3,285 & 164,000 & 241,967 \\
\hline Total lor \$yracuse Zone & Tons:lDay & (2) & 10 & 217 & 9 & 449 & $68 \mathrm{~s}$ \\
\hline \multicolumn{8}{|l|}{ frochesher zone } \\
\hline Monroe Ca,rRochaster & TonsiYear & 61,695 & 28,470 & 45,167 & 0 & 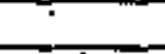 & 135,322 \\
\hline W. Flnger Lakes & Tons Yoer & 14,905 & 1,654 & 11,565 & 0 & & 20,324 \\
\hline Total for Aochester Zone & Tonsryear & 76,590 & 30,324 & 56,732 & 0 & 205,0010 & 360,646 \\
\hline Totel for Poche:sler Zons & TonsiDay & 210 & 89 & 155 & 0 & seg & 1,010 \\
\hline \multicolumn{8}{|l|}{ Butitalo Zone } \\
\hline Erte Co./zuffato & TonsYear. & 114,610 & 17,155 & 55.407 & 0 & & 197,072 \\
\hline Nlagara Co. & TonşYyear & 17,663 & $(1,053)$ & 17,980 & 4,380 & & 38,120 \\
\hline GLOW feglon swm & Tonsryear & 3,664 & 2,472 & 13,190 & 365 & & $19,6 \times 1$ \\
\hline Totat lor Bulfak Zons & TonsNyear & 135,887 & 17,774 & 86,267 & 4,745 & 365,000 & 609,8E9 \\
\hline Total for Bullalo Zone & Torsiday & 372 & 49 & 237 & 13 & 1,000 & 1,671 \\
\hline
\end{tabular}

Eouroe: NYS OEC; NYS DEO; Einnt Wobntor, Chemplos; EloO yoto. 
ADDITIONAL ETHANOL FACILITY SITING ZONES

In Upstate New York

\begin{tabular}{|c|c|c|c|c|c|c|c|}
\hline \multirow[t]{2}{*}{ AGENCYCITY } & \multirow[t]{2}{*}{ UAAT OF WASTE } & \multicolumn{6}{|c|}{ AHOUNT OF WASTE } \\
\hline & & PAPER & $\begin{array}{l}\text { CONSTPUICTION ANO } \\
\text { DEMOLITKON WOOD }\end{array}$ & YARD & $\begin{array}{r}\text { PAPER } \\
\text { SLUDGE } \\
\end{array}$ & LUMAEA & $\begin{array}{l}\text { TOTAL } \\
\text { WASTE }\end{array}$ \\
\hline \multicolumn{8}{|l|}{ Platsburgh Zone } \\
\hline Cimon $\mathrm{CO}$. & Tonsivear & 7,356 & $t, 970$ & 4,631 & 9,125 & & 23,920 \\
\hline Essex Co. & Tonsyyear & 3,312 & 6,467 & 2,816 & 9,125 & & 21,720 \\
\hline Total tor Platisburgh Zono & Toritrear & 10,668 & 6,445 & 7,677 & 18,250 & & 45,040 \\
\hline Tolal lor Platlshurgh Zone & Tons/Nay & 29 & 23 & 21. & son & & 120 \\
\hline \multicolumn{8}{|l|}{ Walertown Zone } \\
\hline Jefterson Co. & Tonsryear & 7,143 & $t, 435$ & 6,114 & 28,360 & & 38,052 \\
\hline Lewis $\overline{C o}$. & TonsNear & 1,585 & 128 & 132 & 2,920 & & 4,768 \\
\hline S1. Laurrence Co. & Tons/Year & 2.907 & 609 & 7,023 & 1,095 & & 11,634 \\
\hline Total for Wateriown Zone & Tonsryear & 11,635 & 2,170 & 13,269 & 27,375 & & 54,449 \\
\hline Total for Waienlown Zane & Tonsy & 32 & 6 & 36 & 76 & & 149 \\
\hline \multicolumn{8}{|l|}{ Bhathamton Zone } \\
\hline Delawaro $\mathrm{Co}$ & Tonstrear & $(2,760$ & 493 & 3,226 & 0 & & 859 \\
\hline Eroone Co. & Tonsriegi & 70,060 & 12,410 & $(4,3,0)$ & 0 & & 78,110 \\
\hline Chanango Co. & Tons rear & 2,329 & 575 & 2,199 & 0 & & 5,093 \\
\hline Total for Binghamton Zone & Tonservear & 69,648 & 13,478 & 1,035 & 0 & . & 84,162 \\
\hline Toxal for Blnghamton Zome & Tons/Day & 191 & 37 & 3 & 0 & & 231 \\
\hline
\end{tabular}

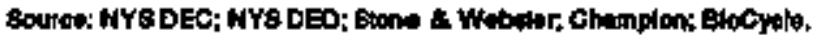




\section{ATTACHMENT 6-4}

\section{Existing Waste-to-Energy Facilities}

by Feedstock Amount, Location, and Tipping Fees 
EXISTING AND ADVANCED PLANNIING WASTE-TO-ENERGY FACHUITIES In Upstate New York

\begin{tabular}{|c|c|c|c|c|c|c|c|}
\hline Project Name \& Localion & $\begin{array}{l}\text { Design } \\
\text { Capacity } \\
\text { TonstDay }\end{array}$ & $\begin{array}{l}\text { Aclual } \\
\text { Capechy } \\
\text { Tonerbay }\end{array}$ & $\begin{array}{l}\text { Ownerf } \\
\text { Operator }\end{array}$ & $\begin{array}{l}\text { Feedstock } \\
\text { Area }\end{array}$ & $\begin{array}{l}\text { Tppling } \\
\text { Fer } \\
\text { STon }\end{array}$ & $\begin{array}{l}\text { Currenth } \\
\text { Operallonal }\end{array}$ & $\begin{array}{l}\text { Scheduled } \\
\text { Operation }\end{array}$ \\
\hline ANSWERS Project, Abany & 800 & 612 & $\begin{array}{r}\text { Pubtital } \\
\text { EAC Oparation }\end{array}$ & $\begin{array}{l}\text { Abeny County (except } \\
\text { tor Town ol Colonle). } \\
\text { Chy of Schenectady, } \\
\text { Cuy of Fennsetaer }\end{array}$ & 56 & $Y_{05}$ & \\
\hline $\begin{array}{l}\text { Albany Steam Generaltig } \\
\text { Facilliy, Abany }\end{array}$ & 600 & 340 & $\begin{array}{l}\text { Putallof } \\
\text { Pubilc }\end{array}$ & $\begin{array}{l}\text { Abany Couny (except } \\
\text { for Town of Cotonle). } \\
\text { City of Scheneciedy. } \\
\text { Clly of Pannselas }\end{array}$ & NA & . & \\
\hline Ereane island, Graene Istand & 1500 & 1370 & $\begin{array}{l}\text { Am. Pal-Fuotf } \\
\text { Am. Ral-Fuel }\end{array}$ & 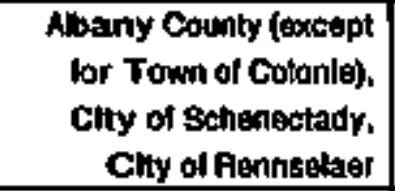 & $\begin{array}{r}75 \\
. \quad \cdot\end{array}$ & No & $6 / 96$ \\
\hline $\begin{array}{l}\text { Aditondack Rasource flecovery } \\
\text { Facilliy, Hustson Falls }\end{array}$ & 400 & 410 & $\begin{array}{l}\text { Adirondack R.R./ } \\
\text { Adliondack R.R. }\end{array}$ & $\begin{array}{l}\text { Washinglon and Warren } \\
\text { Counulas, some of } \\
\text { Sarakopa County }\end{array}$ & 75 & Yes & \\
\hline $\begin{array}{l}\text { Kodak Rasoluce Racowery } \\
\text { Facillay, Rochesler }\end{array}$ & 150 & 119 & $\begin{array}{l}\text { Eastman Kodałt } \\
\text { Eastman Kodak }\end{array}$ & NA & NA & $Y_{\theta S}$ & \\
\hline $\begin{array}{l}\text { Ameilcan Rel-Fuel of Nlagara, } \\
\text { Negara Falls }\end{array}$ & 2000 & 1600 & $\begin{array}{l}\text { Am. Pet-Fugli } \\
\text { Am. Rei-Fugl }\end{array}$ & $\begin{array}{r}\text { Erle and Nlagara } \\
\text { Counttes, Canada, } \\
\text { Ponusyivanla } \\
\end{array}$ & 60 & Yes & \\
\hline $\begin{array}{l}\text { Onondage Counly Fesource } \\
\text { Hecovery Faclihy, Syracuse }\end{array}$ & 890 & $\overrightarrow{808}$ & $\begin{array}{l}\text { Ogden Marln } \\
\text { Ogden Martin }\end{array}$ & Oncndaba County & $\operatorname{tas}$ & No & 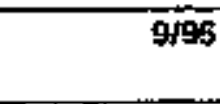 \\
\hline $\begin{array}{l}\text { Onelda Cournly Eneroy Recovery } \\
\text { Faclity. Rome }\end{array}$ & 200 & 160 & $\begin{array}{l}\text { Publict } \\
\text { Putilu }\end{array}$ & $\begin{array}{r}\text { Onelda and Herkimex } \\
\text { Counkles }\end{array}$ & 75 & Yes & \\
\hline $\begin{array}{l}\text { Oswego County Eneroy fecovery } \\
\text { Facilly. Votney }\end{array}$ & 200 & 183 & $\begin{array}{l}\text { Publlel } \\
\text { Pubilit }\end{array}$ & Oswero County & 50 & Yes & \\
\hline
\end{tabular}


ATTACHMENT 6-5

Additional Figures 
EENEAALIZED FEEOSTOCK ZONES OY MA JOA URBAN CEENTEAS

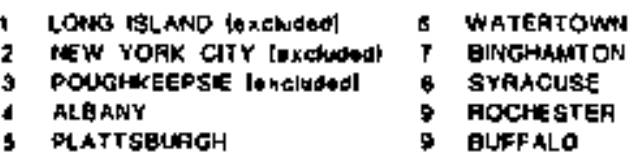

WATEATOWN

BINGHAHTON

SYAACUSE

- GLFFALO

\section{MOTE.}

OENEAALIZE FEEDSTOCK ZONES FOLLOW COMNTY OUTCUMES

TO SIMPLIFY GALCULATIONS OF FEEDSTOCK SLPPLY

FOR THE FACILITY SITHE ZOMES.
DETALED ETHANOL FACUITY SITING ZONES within New York State

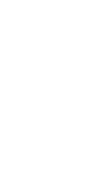

$+2$

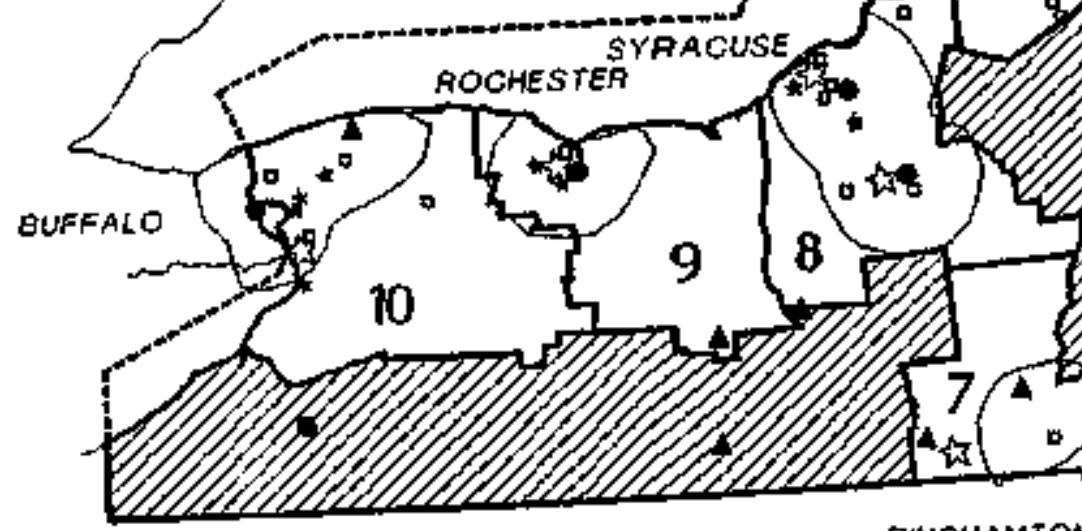

BNGHAMTON
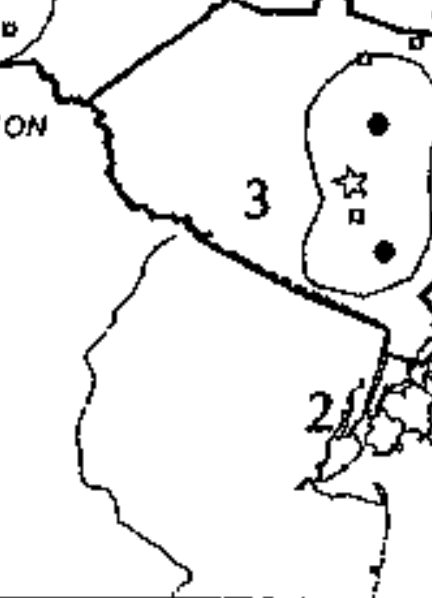

POUGHKETSE
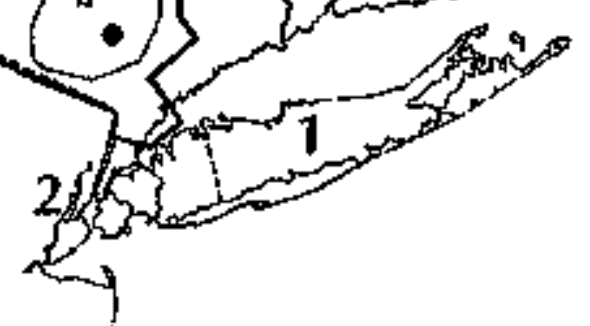
GERSERALIZEO FEEOSTOCK ZONES GY MHAJOR UABAN CENTEAS.

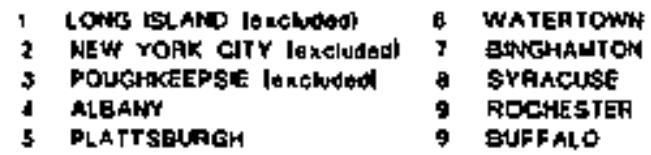

5 plattseyagh

9 BUFFALO

MYSDEC WASTE MANACEMENT REGON GY OFFCE LOCATION
1 LONG ISLAND
TEW YORK CITY
3 WESTCMESTEA
ALBANY
6 UTCA
SYARCUSE
- RUFFALO
PLATtSGURISH

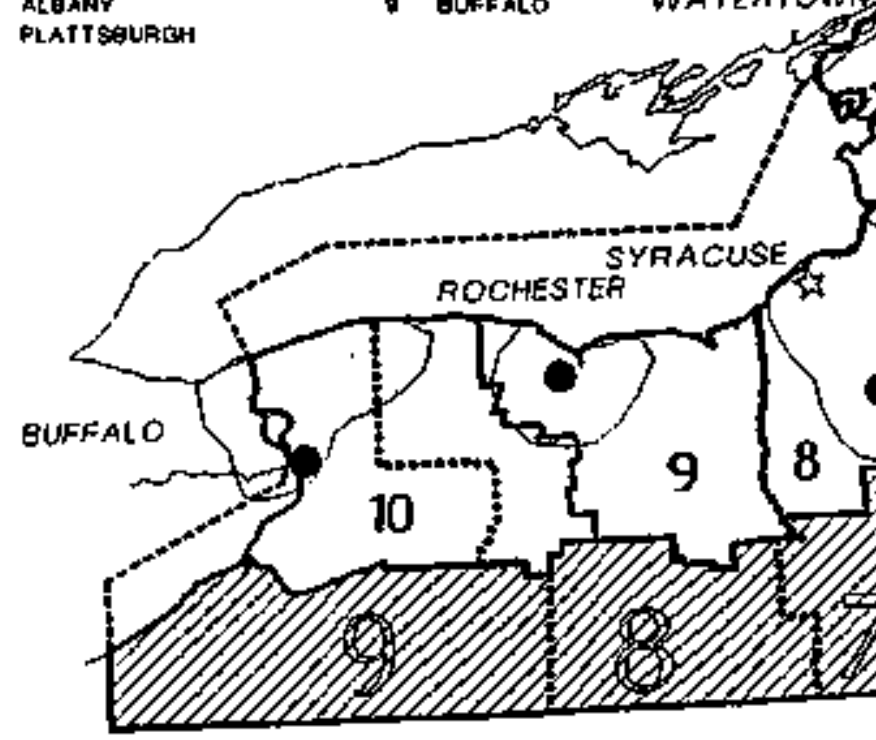

NOTE

GENERMLIZED FEEDSTOCK 2ONES FOLLOW COUNTY OUTLINES

TO SMDUFY CALCU ATIONE OF FEEDSTOCK SUPPLY

FOR THE FACILITY SITIMB ZOHES

THE DIFFERENCE GET WEEN FEEDSTOCK ZONES A NYSOEC REGONS

is DUE TO difFerent FeEDSTOCK \& ACCesS LOGSTIGS

FOR EXAMPLE, W NYSOEC ZONE Q, TWE MULTICOUNTY

- GLOW- WASTE MAMAGEMENT DATA COLLCCTION ODOY

CROSEES NYSDEC COROERS, AHD WAS THEAEFOAE

ASSHONEO TO FEEOSTOCK 2ONE WO TO AYOD SMITTING DATA
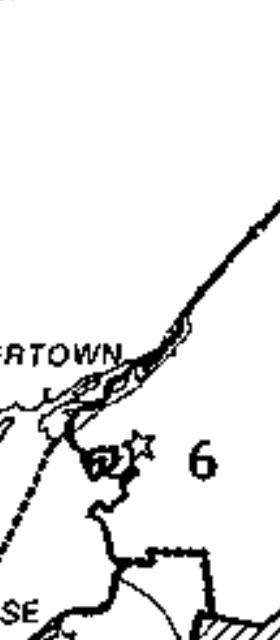

(

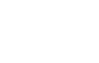

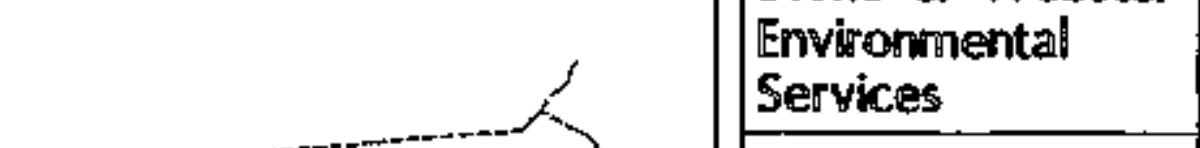

(

FACLITY \$ITIMG ZONE

2O MINES TO EOTH A WASTE

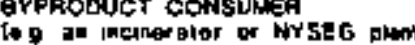

to

is o. onodralli!

27 MAJOA UAGAM CENTEA

35

GENARALIZEED

FEEDSTOCK ZONE

עy

COUNTY AREAS EXCLUDEO OECAUSE OF 1 OW FEEOSTOCK

3

GOADER OP TEGONAL OfFICES OF SOLID WASTE EBINIEES

OF SOLID

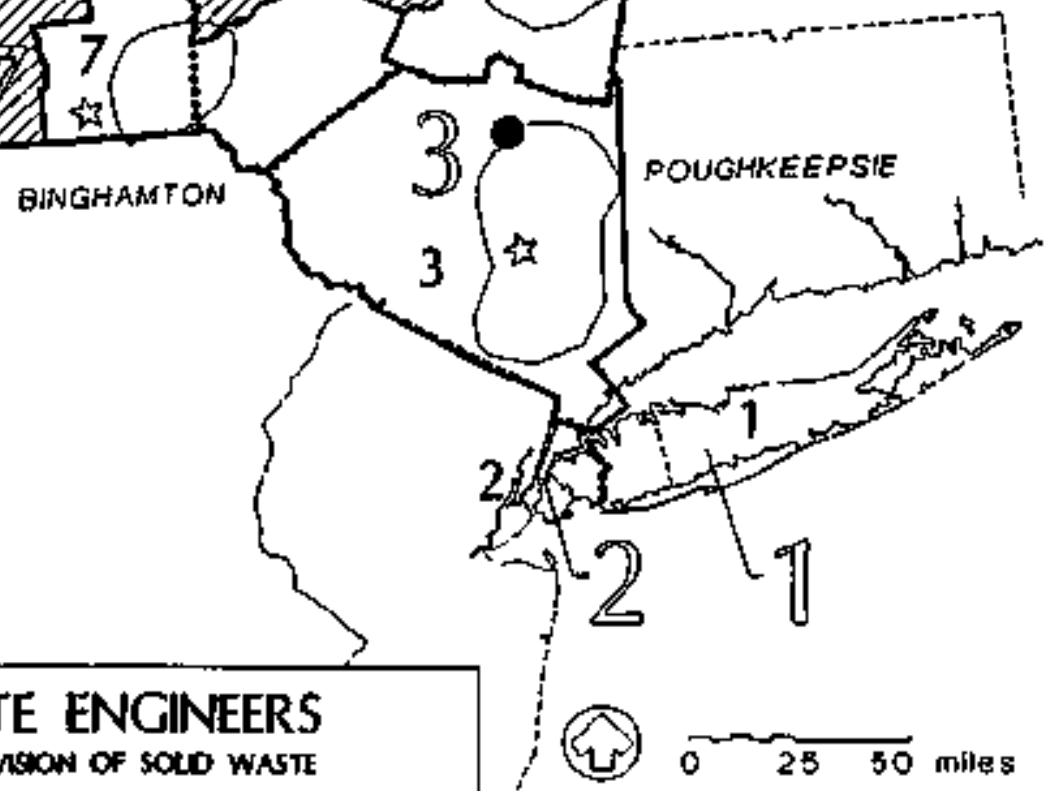

WYSOEC WASTE ENGIMEERS NYSOEC WASTE

BIOMASS to ETHANOI

PLANT SITNG STLDY

FIGURE A-3
REGIONAL OFFICES / SOLID WASTE ENGINEERS

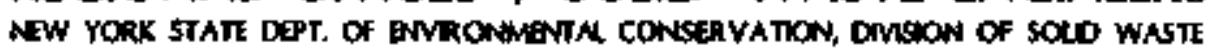




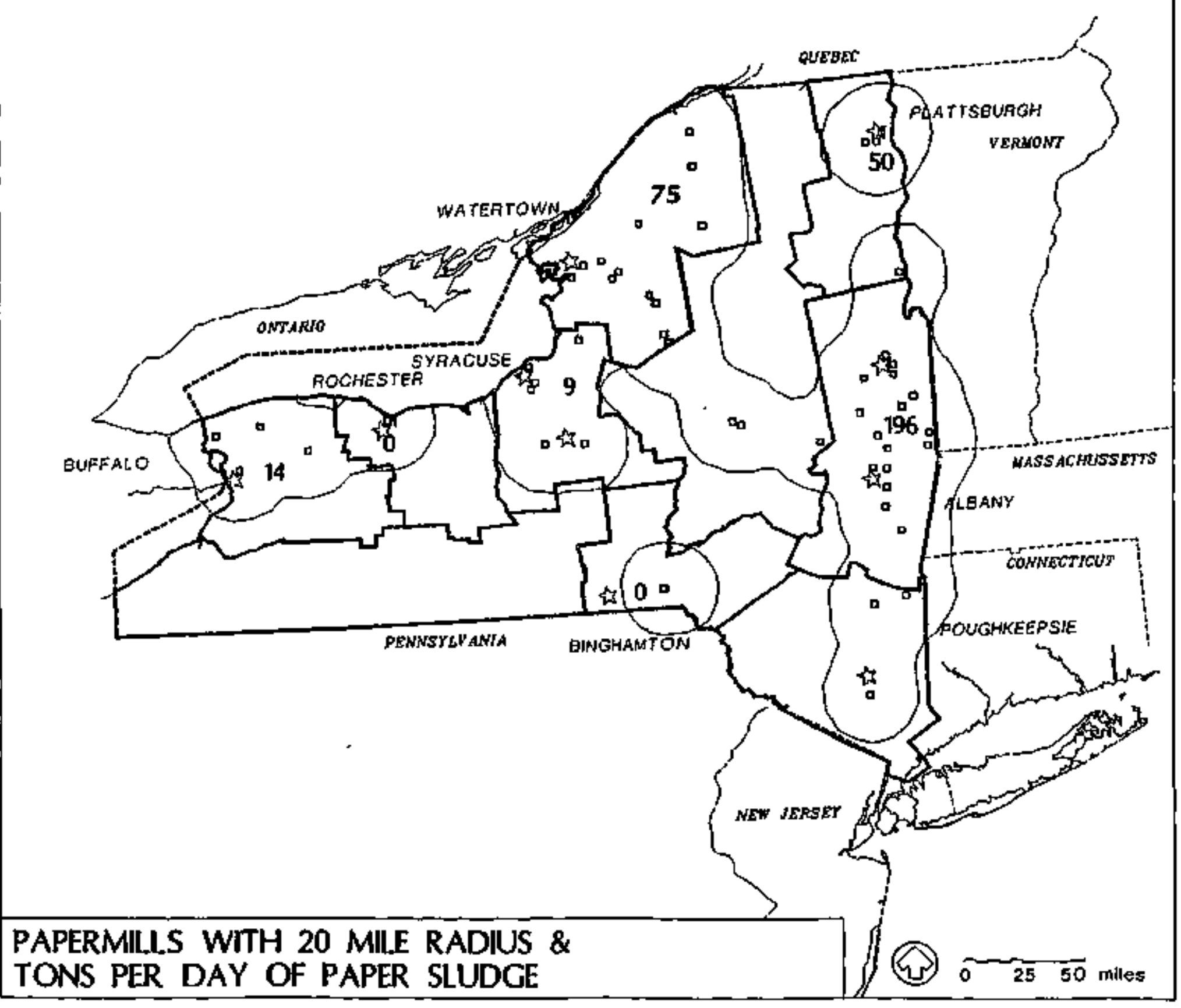

Stone Webster

Environmental

Services

C) 20 MILES TO A PAPEA Mal

0 Oay lons pla bay of paper

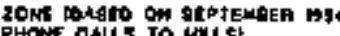

- papeanil locathon

ZA mavor uaban center

BIOMASS to ETHANOL

PLANT SITNG STUDY

FIGURE $\mathrm{A}-4$ 


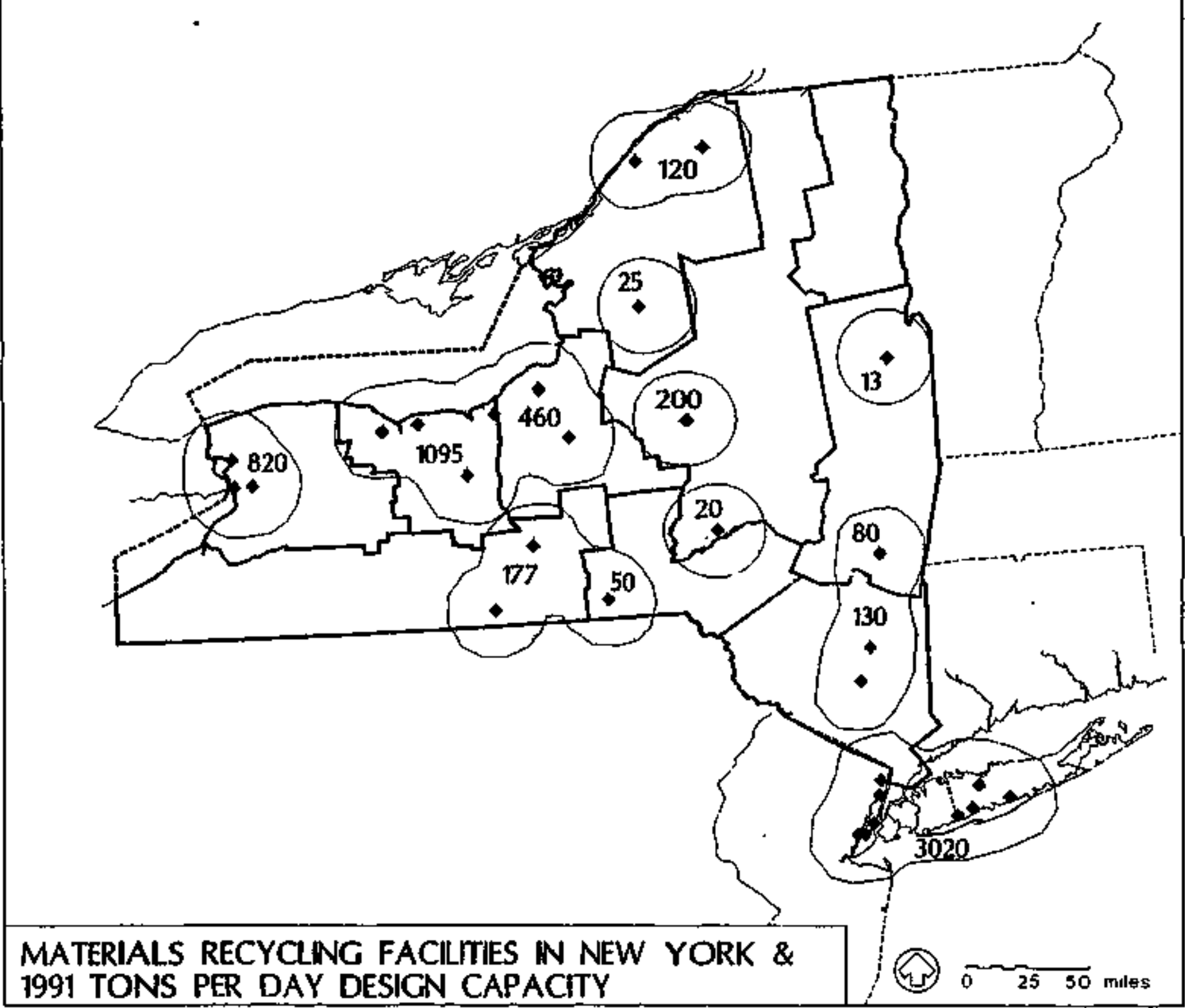

Stone \& Webster Environmental

Services

O

20 MLES ACGESS RADUS IO A MATERIALS FEOYLUE FACRITY

$+$

MATEFAALS RECYCLING FACLITY

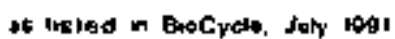

13

TONS PEF OAY CAPACITY OY FEEOSTOCK ZONE

BIOMASS to ETHANOL PLANT SITING STUDY

FIGURE A-5 
NOTE

MONADE EALE ONANOAGA

COUNTIES SEEW TO HAVE THE

GAEATEST WNATE GENEARTION

POTENTIML PER SOMUARE MHE

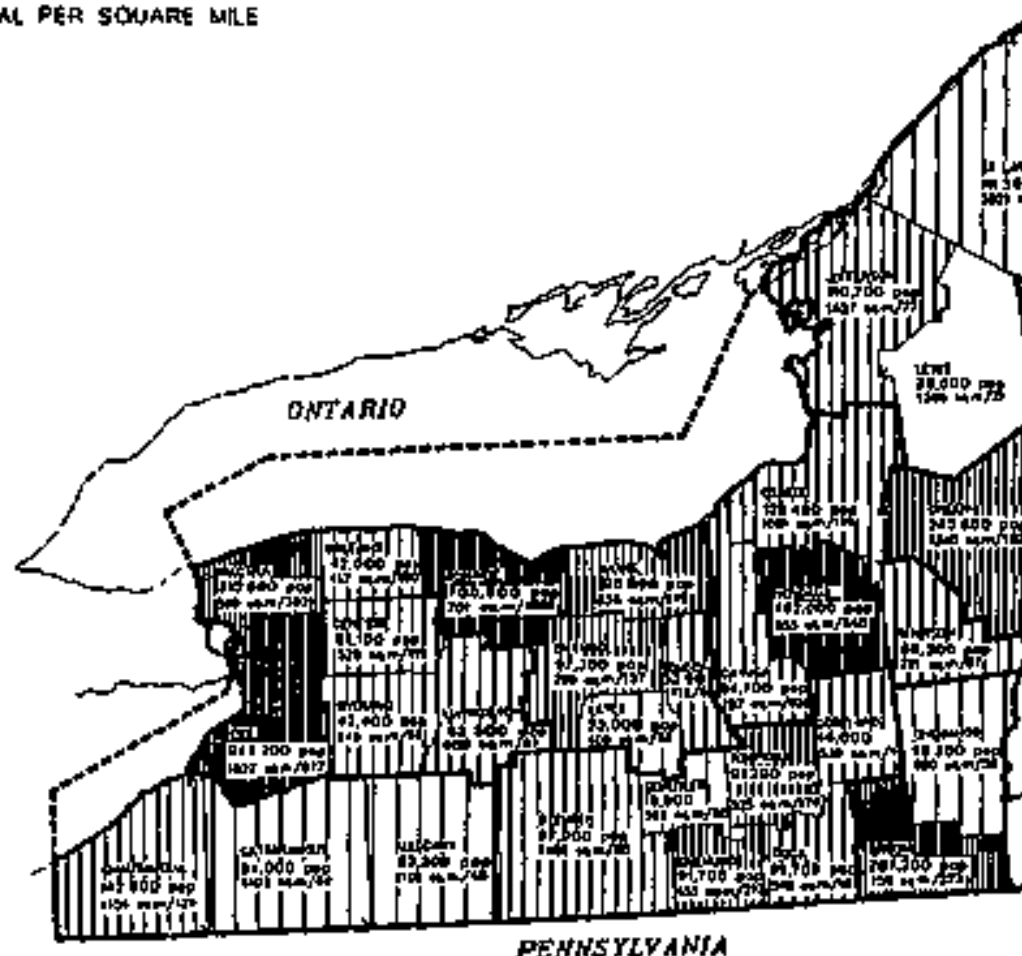

PENHSYLYANA

FANING OF UPSIATE WEW YOAK STATE COUNTES EY POPUATION DENSTYY

1. MUEROE COUNTY a 1006 peopht par soparo mils

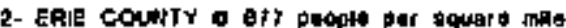

5. SCMEMCTABY COUNTY a 607 papolo per squaro min

1. ONANDAOA COUNTY O 510 o.ople per square mils

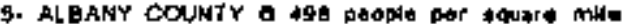

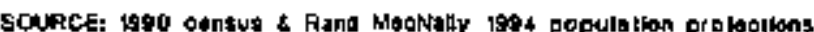

1994 POPULATION DENSITY FOR NEW YORK STATE COUNTIES

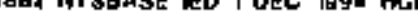
( 


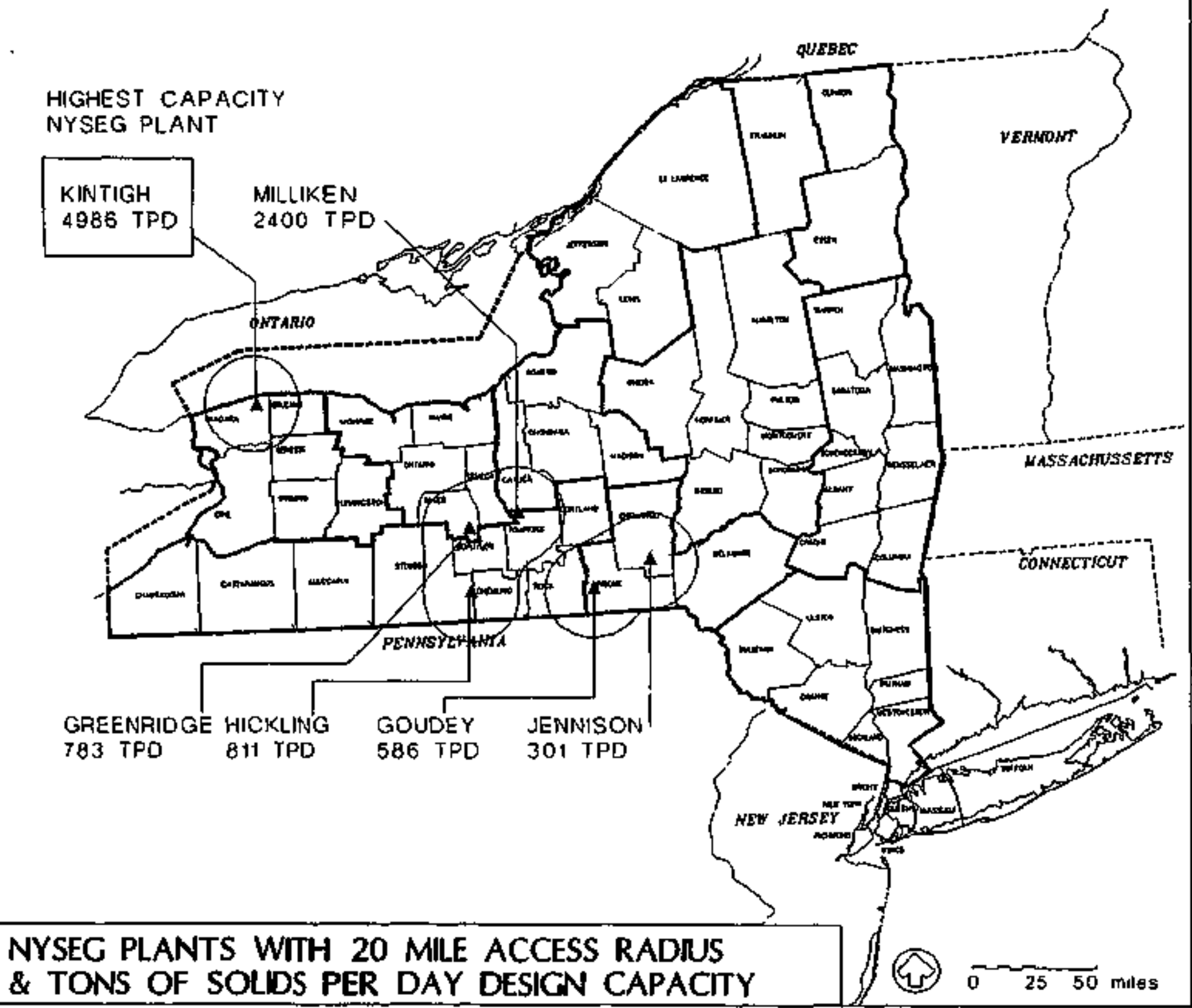

Stone \& Webster
Environmental
Services

Services

$+\quad$ MYSEG

D20 NILES ACCESS RAONS

BIOMASS to ETHANOL

PLANT SITING STUDY

FIGURE A-7 


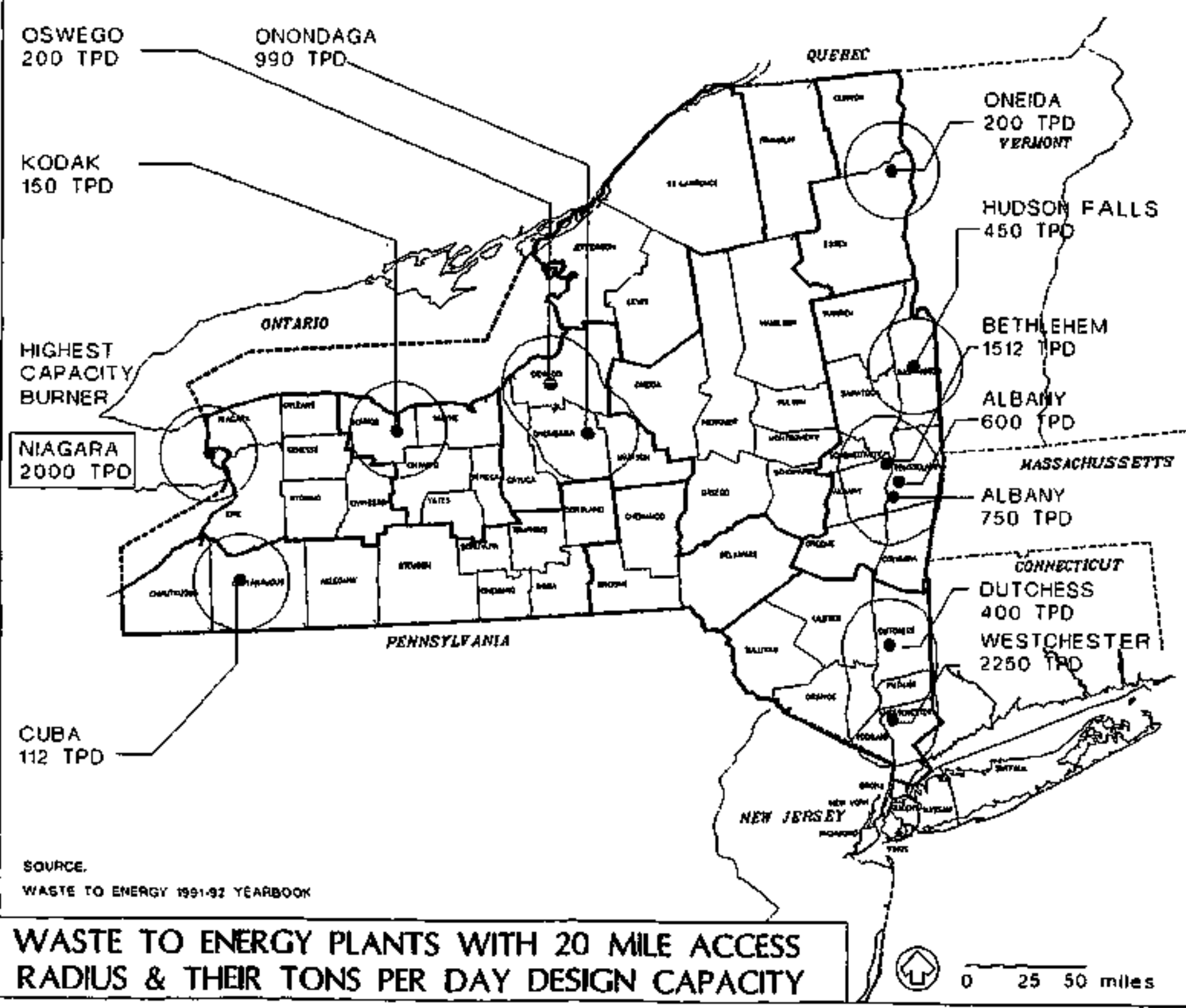

Stone \& Webster
Ervironmental
Services

- hoste buanga plant LOCATION

() 20 mine

BIOMASS to ETHANOL PLANT SITNG STUDY

FIGURE $\cdot \mathrm{A}-\mathbf{8}$ 
ATTACHMENT 6-6

\section{LIST OF CONTACTS}


PEOPLE OR PLACES THAT WERE CONTACTED:

Jim Redmond

Albany Economic Development Office

(518) 434-5133

Albany Public Works Recycling, Program

(518)427-7480

Garrett Dolan

New York State Deparment of Economic Development (DED)

Office of Recycling Market Development

(716)325-1944

Arnie Wills

DED

Albany Region

$(518) 432-2697$

Bill Graper

DED

Regional Economic Development

$(518) 474-2217$

Jeff Janiszewsks

DED

Regional Economic Development

(518)474-2217

Richard Forgea

New York State Department of Environmental Conservation (DEC)

Region 4 Ofince

(518)382-0680.

Dave Blackman

DEC

Central Office

(518) $485-5855$ 
Cheryl Webster

DEC

Region 9 Office

(716)851-7220

Bud Colden

DEC

Burean of Waste Reduction and Recycling

(518)457-7337

Dave Willur

DEC

Division of Solid Waste

Facility Management

(518)485-5856

Mike Urban

Department of Lands and Forests

(518)457-7370

Ken Casperzak

Departient of Lands and Forests (716)372-0645

Dan Parent

Department of Lands and Forests (607)776-2165

Frank Park

Departiment of Lands and Forests (607)652-7364

Lisa Kavanaugh

Galesi Group

(518)356-4445

Tony Riccio

Galesi Group Developers

(212)755-3700

Jefi Petersen

NYSERDA

(518)465-625i 
Michael Treadwell

Operation Oswego County, Inc.

(314)343-1546

\section{Daniel! Bartett}

Partnership for Economic Development in Sullivan County (914)794-3000

Syracuse Public Works Recycling Program (315) 448.2489 
ATTACHMENT 6-7

BIBLLOGRAPHY

: 


\section{BIBILOGRAPHY}

New York State Deparment of Economic Development Office of Recycling Market Development, "Commercial and Industrial Solid Waste Generation in New York State," 1991.

New York State Department of Environmental Conservation Division of Solid Waste, "New York State Solld Waste Management Plan 1991 - 1992 Update," 1993.

New York State Department of Environmental Conservation Division of Solid Waste, "New York State Solid Waste Management Plan 1993 - 1994 Update," 1994.

New York State Department of Environmental Conservation Division of Solid Waste, "The Recycling Bulletin," 1993.

Spencer, Robert, Taking Control of C \& D Debris," BioCucle, 32, 7:65-68, 1991. 


\section{ATTACHMENT 7 - 1}

\section{BASES OF ESTMATES}

\section{BUDGET ESTMMATE FOR BHOMASS TO ETHANOL FACILITY WITH ON-SITE OXIDATION}

\section{PART 2 - NY STATE PRONECT EVALUATION - BUFFALO}

1. Process Istand Cast adjusted to Buffalo Cost.

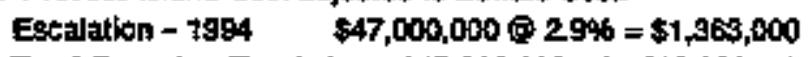

Tolad Cost atter Escalation $-\$ 47,000,000+\$ 1,343,000=\$ 48,333,000$

Asstume Labor @ onpthird of Total Cost - \$49,363,00013 = \$16,121,000

Labor Factor betweeten Buffalo \& Houston = 27.0a/18.45 a 1.47

Total Labor Cost Adustiment =\$16,121,000 \$ 1.47 = \$23,698,000

Therefiore Tetal adjusment $=\$ 29,698,000-\$ 16,12 t, 000=\$ 7,577,000$

Total Adjussed N.Y.S. Cost $=\$ 48,3 \$ 3,0000+\$ 7,57,000=\$ 55,940,000$

2.

BALANCE OF PLANT

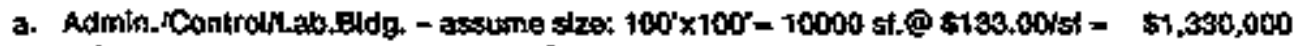
Concrate Foundation $-10,000$ st $10 \$ 10.53 / 5 t=$

Tokal

5105,300

$\$ 1,435,300$

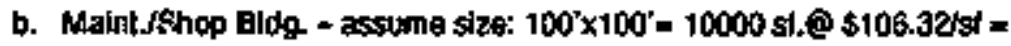

Tools and Equtpment =

Concrete Foundation $-10,000$ st fo $\$ 10.59 / 9 \mathrm{l}=$

Total

$\$ 1,069,300$

$\$ 135,000$

5105,300

$\$ 1,302,400$

c. Plant Air Supply:

Two (2) Comprossots =

$\$ 115,200$

Ats Dryars =

Plping assume o 5,000'\$44.30it -

Totals

$\$ 17,700$

5021,500

$\$ 254,400$

d. Aerobic Watertreationent:

Assume @ 150,000 gaLiday @is \$8.89/gal. =

$\$ 1,329,000$

e. ChilfediCoollng Water Supphy.

Includes: Caoling Towar, Fdn., Piping @250' =

$\$ 920,200$

1. Aollar Feedwater (Demin.) allow:

$\$ 55,000$

g. Sofid OxidetionPower \& Disiribution:

$\$ 25,580,000$

n. Boiler Package:

$\$ 886,000$

i. Steam Tumine:

$\$ 221,500$ 
BASES OF ESTIMATES

PUDEET ESTMATE FOR EMOMASS TO ETHANOL. FACILTY WITH ON-SITE OXIDATION

PAFT 2 - NY \$TATE PROIECT EVALIJATION - BLFFLO

J. STE IMAPOVENENI

1. Clearing \& Grubbing:

544.500

2 serwer and Draispoge:

Assurie; 5s00tu'g plpe 9 \$135.ponf Manhotest.B. - to ea. 9 \$1D,570

Total

sros,000

$\$ 105,700$

3. Access Roadways \& Parking:

$\$ \$ 16,700$

$\left(4900^{\circ} \times 2 \sigma^{\prime}+20,000\right)=106,000$ of $\$ \$ 6.70=$

$\$ 708,600$

4. Filing assume whote silte:

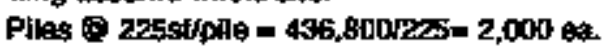

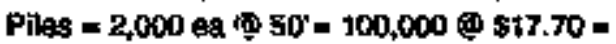

$\$ 1,772,200$

5. Dematering allowance for excration:

543,000

6. Make-up \& Pokabtw Waler:

Piping o 5,300 \&

$\$ 471,600$

Metering 8 Hook-ups

Total

$\$ 60,000$

$\$ 531,600$

7. Sewer Hook-ups for Bathroom, atc.allow.

$\$ 98,1900$

8. Fire Water : 5300 If 0 \$221,50il =

Hydrant \& Misc items =

Total

$\$ 1,175,000$

$\$ 65,000$

$\$ 1,240,000$

9. Sprinikler System whole site plus Standplpe 200,000 of in $\$ 5.001$ का

$\$ 686,000$

10. Fention:

$\$ 62,000$

10. Gas Pipeline (allow):

$\$ 132,000$

11. Site Lghing:

436990 st $\$ 1.04=$

$\$ 452,000$

k. MAFK-UPS:

Start-up Cost $=1.5 \%$

Engineering = 10\%

Contingency $=20 \%$ 
ATTACHMENT 7 - 2

BASES OF ESTMATES

\section{BUDGET ESTMMATE FOR BIOMASS TO ETHANOL FACIITY WITH ON-SITE OXIDATION}

\section{PARI 2 - NY STATE PAOJECT EVALUATION - ROCLESTER}

1. Process Istand Cost adjusted to Fochester Cost.

Escalation - $1994 \quad \$ 47,000,000 @ 29 \%=\$ 1,36 \%, 000$

Todal Cost atter Escalation - \$47,000,000 +\$1,\$63,000 =\$48,96\$,000

Assunte Labor @ onetithird of Tolad Cost - $\$ 48,363,000 / 3=\$ 16,121,000$

Labor Factor between Fochester \& Houstor a 28.02h18.45 - 1.41

Total Labar Cost Adjusiment $=\$ 16,121,000 \Leftrightarrow 1,41=\$ 22,730,600$

Thereiore Total adjusment $=\mathbf{\$ \$ 2 , 7 3 0 , 6 0 0 - \$ 1 6 , 1 2 1 , 0 0 0 = \$ 6 , 6 0 9 , 6 0 0}$

Total Adjusted N.Y.S. Cost $=\$ 48,363,000+\$ 6,609,600=\$ 54,972,600$

2.

BALANCE OF PLANT

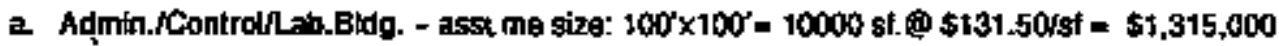
concrete Foundation - 10,000 af $\$ \$ 10.601$ si $=$

Total

$\$ 105,000$

$\$ 1,421,000$

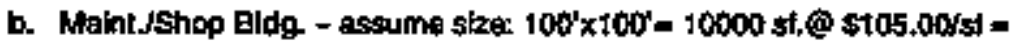
Tools and Equipment -

Concrete Foundation $-10,000$ st $\& \$ 10,50$ ist $=$

Total

$\$ 1,050,000$

$\$ 135,0 \mathrm{pO}$

$\$ 105,060$

$\$ 1,289,000$

c. Plant Atr Supply:

Two (2) Compressars -

$\$ 115,000$

Atr Dryers :-

Piping assume (1,000'@ \$45.50月t =

$\$ 8,800$

$\$ 227,100$

Tokal

$\$ 30,900$

d. Aerobic Watertreatment:

Assume @ 150,060 qal.jday @ \$10.00jgal. =

$\$ 1,315,900$

e. Chilled/Cooling Water Supply.

Includes : Coolino Tower, Fodn., Piping @250" =

$\$ 614,000$

1. Bottar Feedwater (Demin.) allow:

$\$ \$ 50,900$

0. Sold Oxodabion:Power \& Distribution:

$\$ 26,319,000$

h. Boilar Packaga:

$\$ 877,000$

i. Steam Turbine:

$\$ 219,300$ 
PAPT 2 - NY STATE PADJECT EVALUATION - ROCHESTEA

\section{j. SIE IMPAOVEMEN}

1. Claaring 8 Crubbing :

2 Sewer and Droinage:

Asstume: s300'uth pipe (ii) \$131.50hi $\$ 697,000$ Masholesic.1日. - 10 fa, $\$ \$ 10,500$

Todal

3. Accoss Roadwriys \& Pafking :

$\$ 602.900$

$\left(4300^{\prime} \times 20^{\prime}+20,000\right)=106,000$ 여 $0 \$ 6,60$ r

$\$ 70+, 900$

4. Pillng asoume whole sito:

Piles (i) 225stipile = 436,600/225-2,000 ca.

Piles = 2,000 ea $\$ 50^{\circ}=100,000$ (1) $\$ 17.55=$

$\$ 1,755,000$

5. Oewatering allowance tor excenation:

$\$ 458,700$

6. Makt-up a Potable Waler:

Piging @ 5,300' s87.70月f

Metering \& Hook-ups

$\$ 465,000$

$\$ 51,000$

Totar

$\$ 526,010$

7. Sower Hook-ups for Bathroom, Btc.allow.

$\$ 87,700$

8. Fire Waker : 5300 if $\$$ \$2z0.00in -

Hydrant \& Miss. thems =

$\$ 1,163,000$

$\$ 85,000$

Totol

$\$ 1,20 \overline{8}, 00 \overline{0}$

9. Sprinter System whole sthe phus Standpipe 200,000 sf $C \$ 4,40 s t=$

$\$ 9 / 7,000$

10. Fencing :

$\$ \$ 1,000$

10. Gas Pipedina (alkw)

$\$ 130,000$

11. Sike Lighting :

435000 st $5 \mathrm{st} .02=$

5447,090

k. MAF*-UPS:

stant-up Cost $=1.5 \%$

Engingering = 10\%

Contingency $=20 \%$ 
ATTACHMENT 7 - 3

BASES OF ESTMATES

\section{BUDGET ESTIMATE POR BIOMASS TO ETHANOL FACILITY WTTH ON-SITE OXIDATION}

PART 2 - NY STATE PRONECT EVALUATION - SYRACUSE

1. Process istand Cost adjusted 10 Syracusa Cost.

Escalailon-1994 \$7,0010,000 @ $2,9 \%=\$ 1,364,000$

Total Cost after Escalation $-\$ 47,000,000+\$ 1,36 \$, 000=\$ 48,363,000$

Assurma Labor @ one/hird of Total Cost $-\$ 19,363,00013=\$ 16,121,000$

Labor Factor between Syracuse \& Housion $=23.97 / 18,45=1.30$

Tolad Labor Cost Adjustment $\pm \$ 16,121,000 @ 1.30=\$ 20,957,300$

Therelore Total adjusmem $=\$ 2,0,9 \$ 7,300-\$ 16,121,000-\$ 4,836,300$

Total Adjusted N.Y.S. Cast $=\$ 48,369,000+\$ 1,836,3190=\$ 53,199,300$

2.

BALANCE OF PLANT

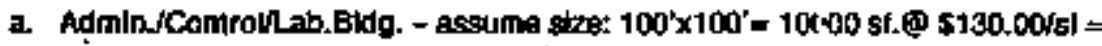
Concrete Foundation - 10,000 st $59.60 / \mathrm{si}=$

Total

$\$ 1,300,000$

$\$ 95,000$

$\$ 1,394,500$

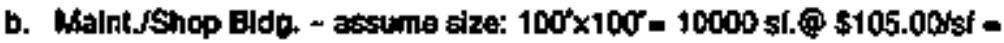

$\$ 1,050,000$

Tods and Equipment -

$\$ 110,000$

Concrete Foundation - t0,000 st $@$ \$10.50/s $=$

Total

$\$ 1,265,000$

c. Plant Air Suppty.

Two (2) Compressors -

$\$ 115,000$

Alr Dryers .

Piping assums 9,000 '@ sas.oct =

$\$ 15,000$

5214,000

Total

$\$ 344,000$

d. Aorobic Watertreatmen:

Assume @150,000 gal.,day (1) \$B.60/9al. =

$\$ 1,291,000$

b. Chilmed/Eooling Water Supply;

Includes: Cooting Towert, Fdn., Plping @250" =

$\$ 602,500$

I. Bdler Feedwater (Demin.) alkow:

$\$ \$ 44,000$

g. Solid OxidatkniPowg \& Distribution;

$\$ 25,820,000$

i. Boilter Packaga:

5860,000

d. Steam Turting:

$\$ 215,000$ 
BASES OF ESTIMATES

BUDGET ESTMAATE FOR BOMASS TO ETHANOL FACIUTY

WITH ON-SIE OXDATION

PART 2 - NY STATE PROJECT EVALUATION - SYRACUSE

j. STE MPROVEMENT

1. Clearing \& Grubbing:

\$43,000

2. Sewer and Drainage:

Assume: 5300'wg pdpe @ \$t30.00Af Manholos/CB. - 10 ea. 9 \$12,0140

5685,600

$\$ 108,000$

Total

$\$ 767,600$

3. Access Foadways \& ParkIng :

$\left(4300^{\prime} \times 20^{\circ}+20,000\right)=106,000$ st $0 \$ 5.50=$

$\$ 688,000$

4. Filing assume whole site:

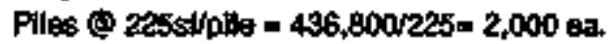

Piles = 2,000 at $@ 50^{+}=100,000$ o $\$ 17.25=$

$\$ 1,722,000$

5. Dowatering allowente for excorvation:

$\$ 430,000$

6. Makgup \& Polable Water:

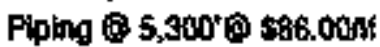

Mataring \& Hook-ups

Total

$\$ 456,000$

$\$ 60,500$

$\$ 516,500$

7. Sown Hook-ups for Bathroom,atc,alkow.

$\$ 86,000$

8. Fre Water : 5400 if $9 \$ 21500 \mathrm{MH}=$

Hyetrant 4 tisc. items = Total

$\$ 1,145,000$

$\$ 90,000$

$\$ 1,205,000$

9. Spritkles Systern whole sile ptus Standpipe 200,000 sf a $\$ 4.30$ ts $=$

$\$ 860,000$

10. Fencing :

50,000

to. Gas Plpeline (atow):

$\$ 130000$

11. Ste Lighting :

436800 혀 $9 \$ 1.00=$

$\$ 440,000$

k. MARK-LPS:

Stan-up Cost $=1.5 \%$

Enginegring = $10 \%$

Contlngency = 20\%6 
ATTACHMENT 7 - 4

BASES OF ESTIMATES

\section{BUDGET ESTIMATE FOR BIOMASS TO ETHANOL FACILITY WITH ON-SITE OXIDATION}

\section{PART 2 - NY STATE PROIECT EVALUATION - ALBANY}

1. Process istand Cost adjusted to Abany Cost.

Escalation - $1994 \quad \$ 47,000,000 @ 29 \%=\$ 1,363,000$

Total Cost after Escalation $-\$ 47,000,000+\$ 1,363,000=\$ 46,363,000$

Assume Labor $@$ oneilhird of Total Cost - $\$ 48,363,00013=\$ 16,121,010$

Labor Factor between Albeny \& Houston = 24.57/18.45 = 1,33

Total Labor Cost Adjustment = \$16,121,000 $@ 1,33=\$ 21,441,000$

Thereioce Total adjusment $=\$ 21,441,000-\$ 16,121,000-\$ 5,320,000$

Total Adiusted N.Y.S. Cost $=\$ 48,363,060+\$ 5,320,000=\$ 53,683,000$

2.

BALANCE OF PLANT

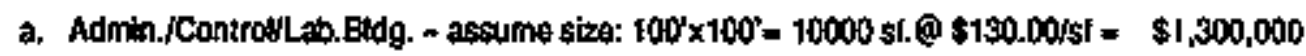

Concrele Foundalton $-10,000$ if $\$ \$ 10.12$ st $=$

Tota

$\$ 101,800$

$\$ 1,401,800$

b. Maint.JShop Eddg. - assume size: 100' $\times 100$ - 10000 8t,@ \$104.00/st -

Tools and Equipment =

Conerete Foundation - 10,000 st @ \$10.25/st -

$\$ 1040,300$ $\$ 130,000$

Total

$\$ 102,500$

$\$ 1,272,500$

c. Plant Air Supply.

Two (2) Compresors -

$\$ 112,500$

Air Dryers =

$\$ 8,600$

Ptpino assume @ 5,000'@ \$45.00Mf -

525,100

d. Aerobic Watertreatment:

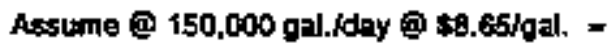

$\$ 1,298,000$

A. Chllled/Cooling Water Supply:

Includes : Cooling Tomer, Fon, Piping e250" -

$\$ 605,900$

l. Boller Feectwater (Demin.) atlow:

$\$ 346,200$

o. Solld OxidalloniPomer \& Distribution;

$\$ 25,971,000$

b. Botter Packege:

$\$ 965,300$

I. Srean Tubina:

$\$ 2+6,400$ 


\section{QASES OF ESTIMATES}

BUDGET ESTIMATE FOA BIOMASS TO ETHAHOL FACILITY WTH ON-SITE OXIDATIOA

PAFT 2 - NY STATE PRONECT EVALUATION - ALBANY

\section{J. STE MMPROVENENT}

1. Claaring 8 Grubbing :

$\$ 43,300$

2 sewer and Drainage:

Assume: $5300^{\prime} \mathrm{utg}$ pipa $\$ \$ 130.009 \mathrm{~F}$

588,400

Manhotesft., $-10 \%$. $\$ 10,400$

Total

$\$ 104,000$

$\$ 792,400$

3. Access Roadways \& Parking:

$\$ 692,800$

4. Piting assume whole site:

PHies \& 225stipile = 436,800/2ns $=2,000$ ea.

Ples = 2,000 вa 6i 50' 100,009 Q $\$ 17.30=$

$\$ 1,732,000$

5. Dewatering allowance for excavation:

$\$ 433,000$

6. Make-up \& Potable Water:

Piping o 5,300' 690.0 sir

S477,000

Metering 8 Hook-lips

$\$ 42,600$

Total

$\$ 519, \overline{600}$

7. Sewer Hook-ups for Eaihroom,olc.allow:

$\$ 96,600$

B. Fire Water : 5300 If $(\$ 216.504 t=$

Hyarant \& Misc- ftems =

Tota:

$\$ \tau, 147,400$

ses, $00 \mathrm{CO}$

$\$ 1,212,400$

9. Sprinklar Systern whole site plus Standpipe

200,000 sf (i) $35.001 \mathrm{~s} 1=$

$\$ 868,000$

10. Fençing:

$\$ 60,000$

10. Gas Pipetine (alkow):

$\$ 130,000$

11. Exe Lonting:

496800 혀 붕 $\$ 1.01$.

$\$ 441,600$

K. MAPK-UPS:

start-up Cost $=1.5 \%$

Engineering - $10 \%$

cantingency = 20\% 


\title{
ATTACHMENT 7 - 5
}

\author{
BASES OF ESTIMATES
}

\section{BUDGET ESTTMATE FOR BIOMASS TO EIHANOL FACHITYY WITHOUT OXIDATION}

\author{
PAFT 2 - NY STATE PRONECT EVALUATION - BUFFALO
}

1. Process lsland Cost adjusied to Buffato Cost. .

Escalallox - $1994 \quad \$ 47,000,000$ @ 29\% - \$7,393,000

Total Cost after Excalation - \$47,040,0100 + \$1,369,000 = \$48,\$63,060

Assume Labor $\odot$ one/third of Total Cost $-\$ 46,369,000 / 3=\$ 16,121,000$

Labor Factor between Gulfale \& Houston = 27.0818.45 - 1.47

Tctal Labor Cost Ad]ustment = \$16,121,000 \& 1,47 =\$28,699,000

Therelore Total ad usment = $\$ 23,698,000-\$ 16,121,000=\$ 7,57,000$

Total Adjusted N.Y.S. Cast $=\$ 49,363,000+\$ 7,577,000=\$ 55,940,000$

2.

BALANCE OF PLANT

a. Admin./Controllab.B1dg. - assume size: $100^{\prime} \times 100^{\prime}=10000$ st.@ $\$ 133.00 /$ sf $=\$ 1,330,090$

Concrele Foundaltion - 10,000 st ia $\$ 10.53$ raf =

Tutal

$\$ 105,300$

$\$ 1,435,390$

b. Maint./Shop Bidg. - assume size: 100'x100'= 10000 sf. $\$ 106.32 / s I$ -

Toods and Equlpment -

$\$ 1,063,300$

$\$ 135,000$

Concrete Fotmdation $-10,000$ st @ $\$ 10.53 /$ sf $=$

$\$ 105,300$

Total

$\$ 1,312,400$

c. Plam Alr Supply.

Two (2) Compressitora a

$\$ 115,200$

Air Dryers =

Piping asseme @ 5,000'@ \$44.30\% =

$\$ 17,700$

$\$ 221,500$

Total

$\$ 354,400$

d. Aerobic Watertreatment

Assume (i) 150,000 gal./day @ $\$ 8.86 / g a l . ~=$

$\$ 1,329,000$

e. Chilledrcooling Waler Supply:

Inctudes : Cooling Jower, Fdn., Piping @250" =

$\$ 620,200$

4. Bother Feodwater (Demin.) altow.

$\$ 354,000$

g. Power \& Distritultion:

$\$ 4,4 \$ 0,400$

h. Boiler Package:

$\$ 966,000$

I. Stangm Tubina:

$\$ 221,500$ 
BASES OF ESTDMATES

\section{BUDGET ESTIMATE FOR BIOMASS TO ETHANOL FACILITY WITHOUT OXIDATION}

PART 2 - WY STATE PFOJECT EVALIATION - BUFFALO

j. STE IMAFFONEMENT

1. Clearing \& Giubing:

$\$ 44,300$

2. Sewer and Dralnaga :

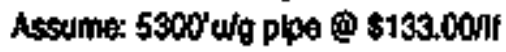

$\$ 705,040$

Manholesic.B. $=10$ oa. @ $\$ 10,570$

$\$ 105,700$

Total

$\$ 810,700$

3. Access Foadways a Parking :

$\left(4300^{\circ} \times 20^{\prime}+20,000\right)=106,000$ sf $856.70=$

5708,800

4. Piling assume whole site:

Ples to 225siphe = 435,800/205-2,000 6a.

Piles = 2,000 $9 \mathrm{a} @ 50^{\circ}=100,000$ @ $\$ 17.70=$

$\$ 1,772,200$

5. Dowatering allowarnce for texcavition:

$\$ 443,000$

6. Make-up a Potabla Waler:

Piping 5,300! \$a9.001:

$\$ 471,600$

Metering \& Hook-ups

Tote:

$\$ 60,000$

$\$ 591,600$

7. Senwer hook-ups for Bathroom,etc.allow.

589,600

8. Fre Water : 5300 if 05221.50 if =

Hyutant 8 Misc. ïlens =

Total

$\$ 1,175,000$

$\$ 65,000$

$\$ 1,240,000$

9. Sprinkler System whole site phus Standpipe 200,00051 is \$5.00\%st =

$\$ 886,000$

10. Fencing:

$\$ 62,000$

10. Gas Pipeline (atow):

$\$ 132000$

11. Sin toghting:

434900 st $9 \$ 1.04=$

$\$ 452,100$

k. MAPK-UPS:

Slatt-up Cost $=0.5 \%$

Englneering = 1046

Cortingency = 20\% 


\section{ATTACHMENT 7 - 6}

\section{BASES OF ESTMMATES}

\section{BUDGET ESTMMATE FOR BIOMASS TO ETHANOL FACILITY WITHOUT OXIDATION}

\section{PAAT 2 - NY STATE PAOJECT EVALUATION - ROCHESTER}

1. Process Island Cost axjusied to Rochester Cost.
Escalation - 1994
$\$ 47,000,000) 2.996=\$ 1,369,0010$
Total Cost after Escatation - \$47,004,040 + \$1,369,000 = \$49,363,000
Assumn Labor $\Theta$ onethird of Total Cost - \$46,363,00019 $=\$ 16,121,000$
Lator Facter between Fochester \& Houston = 26.02/18,45 = 1.41
Total Labor Cost Adjustmand $=\$ 16,121,000$ @ $1.4 t=\$ 22,730,600$
Theretore Tatal adjyusment $=\$ 22,730,600-\$ 16,121,000=\$ 6,609,600$
Tetal Adjusted K., Y.S. Cost $=\$ 48,363,000+\$ 8,609,600=\$ 54, \$ 72,600$

2.

BALANCE OF PLANT

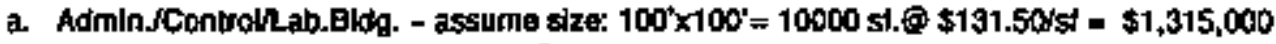
Concrete Foundation - 10,000 st $\odot \$ 10.60 \mathrm{st}$ -

Tolal

$\$ 106,000$ $\$ 1,421,000$

b. Malnt.Shop Bldg. - assume size: to0' $\times 100 '=10080$ st.@ \$105.00/sf = Toois and Equipment Concrets Foundation - 10,000 st 9 \$10.50/st =

$\$ 1,050,000$ $\$ 135,000$ Total $\frac{\$ 105,000}{\$ 1,269,000}$

c. Plant Alr Supply:

Two (2) Compressors =

$\$ 115,000$ Air Dryars =

Piping assume @ 5,000'@ 545_50hlf =

$\$ 2 / 27,100$

Total $\$ 550,900$

d. Aerobic Watertreatment: Assume @ 150,000 gal./day @ s10.00\}pal. =

$\$ 1,315,900$

e. Chilladrecoling Water Supply: Inctudes : Coolting Tower, Fdn., Piping @250' =

$\$ 614,000$

I. Boller Foedwater (Demin.) allow:

$\$ 350,000$

a Power \& Distriburtion:

$\$ 4,386,600$

h. Boller Package:

597,000

f. Steam Tutitag:

$\$ 219,300$ 


\section{BUDDGET ESTTMATE FOR BIOMASS TO ETHANOL FACLITY WITHOUT OXIDATION}

PAFT 2 - WY STATE PAOJECT EVALUATION - AOCHESTER

\section{J. SITE LMFFOVEMENT}

1. Clearing 8 Gnubing :

$\$ 43,800$

2. Sower and Dralmape :

Assumer 5300'ưg plpe@ \$131.50Mt

Manholes/C.8. - 10 ag, $\$ \$ 10,500$

$\$ 697,000$

$\$ 105,000$

$\$ 802,800$

3. Access Roadways \& Parking:

Tokal

$\$ 701,900$

4. Plling assume whole sitet

Piles if 225stpile $=436,8000225=2,000$ a

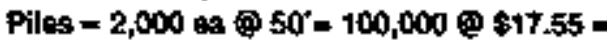

$\$ 1,755,000$

5. Dewalering allowance for excogration:

$\$ 438,700$

6. Make-up \& Potable Water:

Piping \& 5,300'\$ \$87.701f

$\$ 465,000$

Metering \& Hook-ups

$\$ 61,000$

Tolal

$\$ 526,000$

7. Sewer Hook-ups for Bathroom,etc.alkew:

$\$ 87,700$

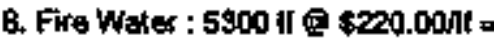

\section{Hydran 8 Alsc. Herns =} Total

$\$ 1,163,000$

$\$ 65,400$

$\$ 1,228,000$

9. Sprinklar System whole sile plus Stanchipe 200,000 st is \$4,40tst =

$\$ 877,000$

10. Fencing:

$\$ 61,000$

10. Gas Pipetine (allow)

$\$ 130,0100$

11. Stke Lighing :

$\$ 447,000$

k. MAAK-UPS:

Stert-up $\operatorname{Cos}=1.5 \%$

Engineering $=10 \%$

Cortingency = $20 \%$ 
ATTACHMENT 7 - 7

BASES OF ESTIMATES

\section{BUDGET ESTMMATE FOR BIOMASS TO ETHANOL FACIEITY WITHOUT OXIDATION}

\section{PAAT 2 - NY STATE PAONECT EVALUATION - SYAACUS:}

1. Process Istand Cost adjusted to Syracuse Cost.

Escalation - $1994 \quad \$ 47,000,000$ \% $2.9 \%=81,363,000$

Total Cast after Escatation - \$47,000,000 + \$1,363,000 = \$48,353,000

Assume Lobor \& onelthird of Total Cost - \$49,363,00001 = \$16,121,000

Labor Factor between Syracuse \& Houston $=23.97112 .45=1.30$

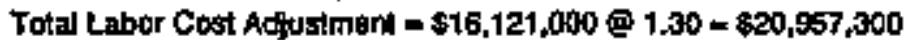

Theretore Tatal adjusmemt =\$20,957,\$00 - \$16,121,000 = \$4,636,300

Total Adjusted N.Y.S. Cost - \$48,3\$3,000 + \$4,836,300 - \$53,199,300

2.

BALANCE OF PLANT

a. Admin.fControll..ab.Eldo - assume size: 100'x100' - 10000 \$1.@ \$130.00\%s = Concrete Foundation - 10,000 sf to $59.50 \mathrm{st}$ -

Total

0,000

$\$ 95,020$

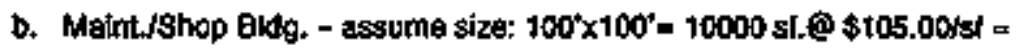
Toaks and Equipment =

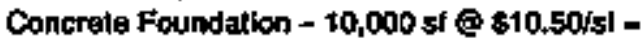

Total

$\$ 1,394,500$

$\$ 1,050,000$

$\$ 1+0,000$

$\$ 105,000$

$\$ 1,265,000$

G. Pianl Air Supply:

Two (2) Compressors -

$\$ 115,000$

Air Dyyers =

$\$ 15,000$

Pipting assume o 5,000\% \$49.001 =

Toled

5214,000

$\$ 344,000$

d. Aerobic Watertreatment:

Assume @i 150,000 gaL/day @ \$6,60/gal. -

$\$ 1,2 \$ 1,000$

a. CallledrCooling Water Supply.

includes : Cooting Tow

$\$ 602,500$

t. Boliar Feedwaler (Demin.) allow.

$\$ 344,000$

b. Power \& Disitribution;

$\$ 4,300,00 \%$

h. Boller Packaga:

$\$ B 60,000$

1. Steam Turbine:

$\$ 215,000$ 
BUDGET ESTIMATE FOR BIOMASS TO ETHANOL FACA.TTY WITHOUT OXIDATION

PAFT 2 - NY STATE PAQIECT EVALUATION - SYAACUSE j. STE IMPRONENENT

1. Clearing \& Grubbing : $\$ 4 \$, 100$

2 Sower and Crainage: Assumex 5soo'wo pipe o \$130.00it Manholes'c. $\$ 895,600$

Access Foadways \& Parking :

$\$ 102,000$

$\left(4300^{\circ} \times 20^{\prime}+20,000\right)=106,000$ 녀 $@ 58,50=$

$\$ 797,600$

4. Pीing assume whole sitex

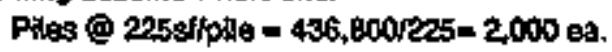

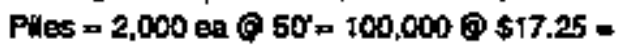

$\$ 1,722,000$

5. Dewatering allowance for excavation:

$\$ 430,000$

6. Make-up \& Potabla Waler:

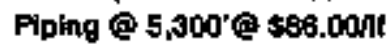

$\$ 688,000$

Metering \& Hook-ups

$\$ 456,000$

$\$ 60,500$

Total

क516,500

7. Sewer Hook-tups for Bathroom,elc-allow.

$\$ 86,000$

g. Fore water : 5300 of (B) \$215.00Mr =

Hytrant \& Hisc. items = Totat

$\$ 1,145,000$

$\$ 60,000$

$\$ 1,205,000$

9. Sprintler System whole stte plus Standpipe 200,000 st 6 \$. motsi =

$\$ 860,000$

10. Fencing:

$\$ 60,000$

10. Gas Plpefrio (allow):

$\$ 1 \$ 0,400$

1t. sho Llghing:

436900 at @ $\$ 1.00=$

$\$ 440,000$

k. MARK-UPS:

Start-up Cost $-1.5 \%$

Ençina

Contingency $=20 \%$ 


\section{ATTACHMENT 7 - B}

BASES OF ESTEMATES

\section{BUDGET ESTIMATE POR BIOMASS TO ETHANOL FACILITY WTTHOUT OXIDATION}

\section{PART 2 - NY STATE PROJECT EVALIUATION - ALEANY}

1. Process island Cost adjusted to Albany Cost.

$$
\text { Escalation - } 1994 \quad \$ 47,000,000 \text { @ 29\% - } \$ 1,363,000
$$

- Total Cost after Escalakion - $\$ 47,000,000+\$ 1,363,000-\$ 49,343,000$

Assume Labor @ onefthird of Total Cost - \$48,363,00\$s = \$16,121,000

Labor Factor between Abany \& Housten $=24.57 / 18.45=1.33$

Total Letor Cost Ad]ustment = \$16,121,000 우 1.33 = \$21,441,000)

Theretere Total ac;usment $=\$ 21,441,000-\$ 16,121,000=\$ 5,320,000$

Tot:I Adjusted N.Y.S. Cost $=\$ 48,363,000+\$ 5,320,000=\$ 53,683,000$

2

\section{BALANCE OF PLANT}

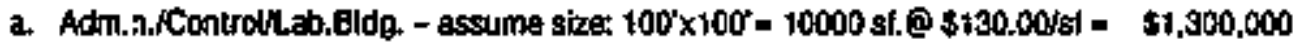
Concrete Foundation - 10,000 si @ \$10.19/gi Total $\$ 101,800$ $\$ 1,401,800$

b. Maint Jshop 버g. - assume size: 100' $\times 100^{\circ}$ - 10000 st.@ \$104.00\%st Tous and Equipment $\$ 1,040,300$ Concrete Foundation - 10,000 sf @ $\$ 10,25 / 8 f$ t $\$ 130,000$

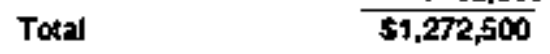

c. Plant Air Supply;

Two (2) Compressors = Air Dryers =

Pipind assume @ 5,000 \$45.00:4 -

Total

$\$ 112,500$

$\$ 6,600$

$\$ 225,100$

5346,200

4. Aerobic Watertreatmant:

Assumm @ 150,400 galjday @ \$8.65/gal. =

$\$ 1,293,000$

e. ChillediCoollng Water Supply: lecludes: Cooting Tower, fdn, Piping @250" -

$\$ 605,900$

f. Boiler Fegowater (Demin) alkow:

$\$ \$ 46,200$

b. Power \& Distritoulion:

$\$ 4,326,500$

h. Bolier Package:

$\$ 960,300$

i. Seam Turbine: 
BUDGET ESTMMATE FOR BIOMASS TO ETHANOL FACПLITY WITHOUT OXIDATION

PART 2 - NY STATE PAQJECT EVALUATION - ALAANY

f. STE MPFOVEMENT

1. Clearing \& Grubbing : $\$ 49,300$

2. Sower and Drainate:

Assume: 5300'wo pipe Q \$150.00iff 5669,400

Manholestc. B. - 10 62. $\$ \$ 10,400$

Total

$\$ 104,000$

3. Accoss Roadways \& Parking:

$\left(4300^{\prime} \times 20^{\circ}+20,000\right)=106,000$ sf $6 \$ 6.54=$

$\$ 792,400$

4. Piling assume whole site;

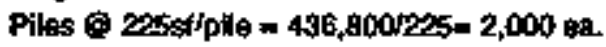

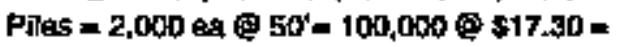

$\$ 692,600$

5. Dewatering allowance tor extravation:

$\$ 1,722,900$

$\$ 433,000$

6. Make-up \& Potate Water.

Piping \& 5,300 g s90.0041

$\$ 477,000$

Melering 8 Hook-ups

$\$ 42,600$

Total

$\$ 19,600$

7. Sower Hook-ups for Bathroom, etc.allow:

$\$ 86,900$

8. Fire water : 5300 if 0 \$216.509f =

Hyotrant \& Misc items =

Total

$\$ 1,147,400$

S\$S, 000

$\$ 7,212,400$

9. 8 prinklar System whole site plus standpipe 200,000 st 1 s5.00isf =

$\$ a 66,000$

10. Fencing:

$\$ 60,000$

10. Gas Pipeline fallow)

$\$ 130,000$

11. Sita Lighting:

436800 sf $9 \$ 1.01=$

$\$ 41,600$

k. MAFK-UPS:

Start-up Cost $=1.5 \%$

Enginearing $=10 \%$

Conlingency $=20 \%$ 
ATTACFMENT 8-1

TABLES 8-5 TO 8-19

FIGURES 8-1 TO \&-28

RRO FORMA ANALYSIS 


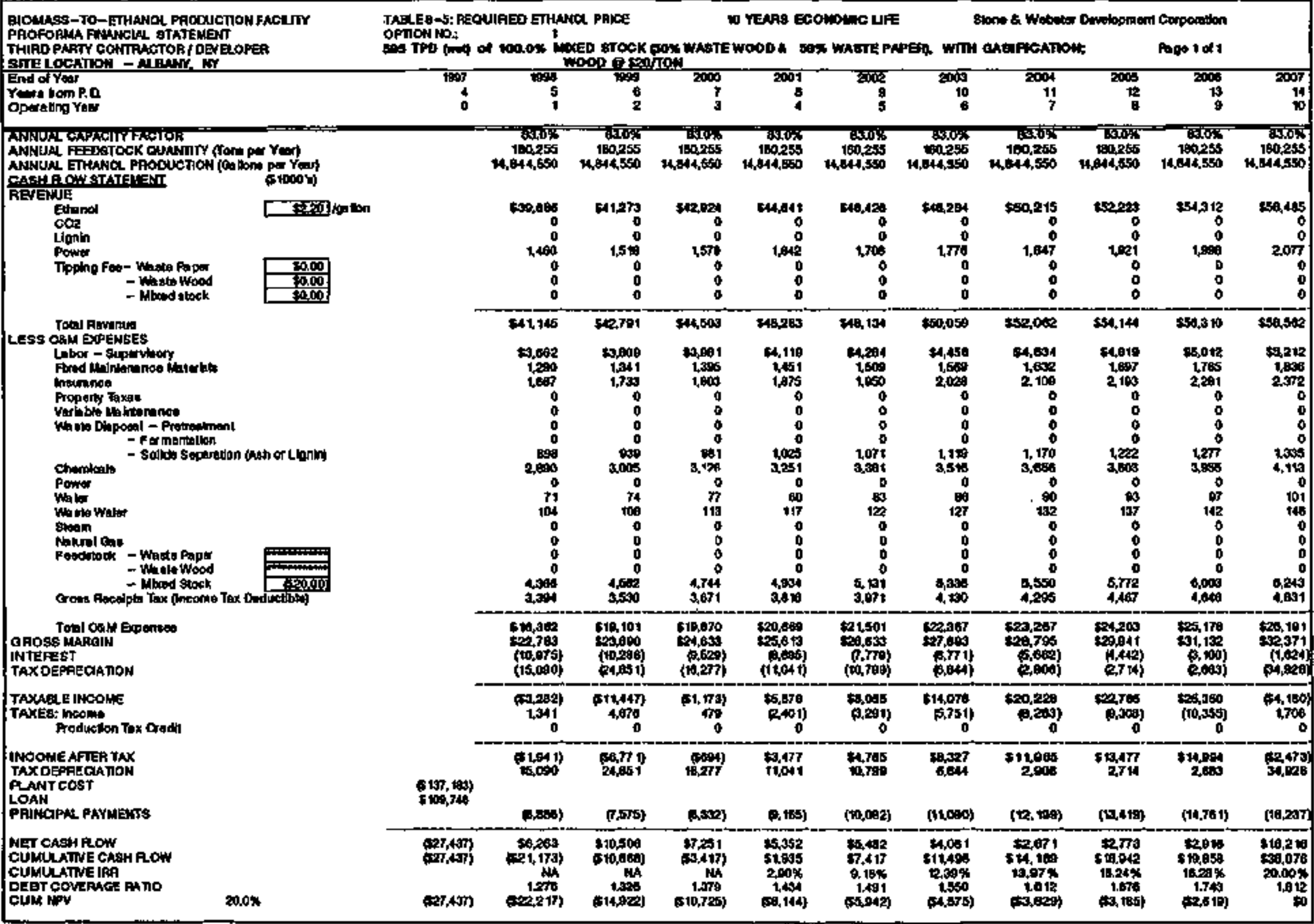




\begin{tabular}{|c|c|c|c|c|c|c|c|c|c|c|c|}
\hline \multirow{2}{*}{ 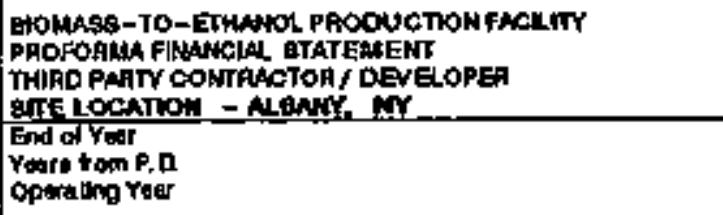 } & \multicolumn{3}{|c|}{ 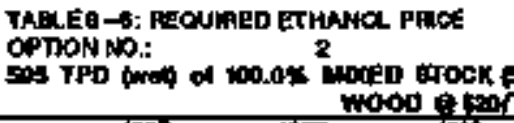 } & \multicolumn{3}{|c|}{ 10 VENPS EDONONO LRE } & togt & \multicolumn{3}{|c|}{ 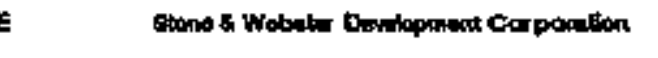 } & \\
\hline & 167 & $\begin{array}{r}5 \\
5 \\
1\end{array}$ & & $\begin{array}{r}7000 \\
7 \\
3\end{array}$ & 200 & $\begin{array}{r}2006 \\
3\end{array}$ & $\begin{array}{c}2003 \\
t 0 \\
6\end{array}$ & $\begin{array}{r}304 \\
71 \\
7\end{array}$ & $\begin{array}{r}2005 \\
12 \\
0\end{array}$ & 2006 & 2017 \\
\hline 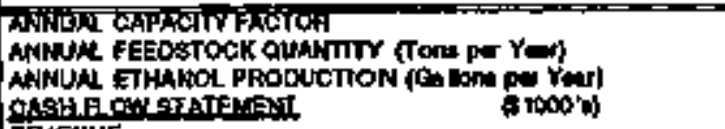 & • & $\begin{array}{r}100,255 \\
1,044,540\end{array}$ & 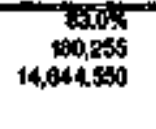 & 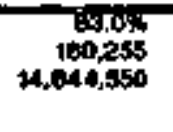 & $\begin{array}{r}80,036 \\
100,255 \\
14,044050\end{array}$ & $\begin{array}{r}1820 \% \\
100,255 \\
404450\end{array}$ & 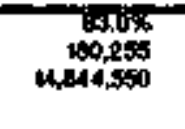 & 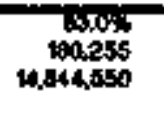 & 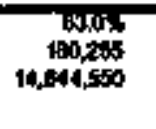 & 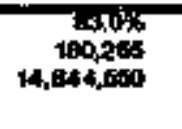 & 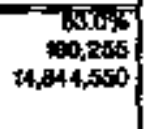 \\
\hline 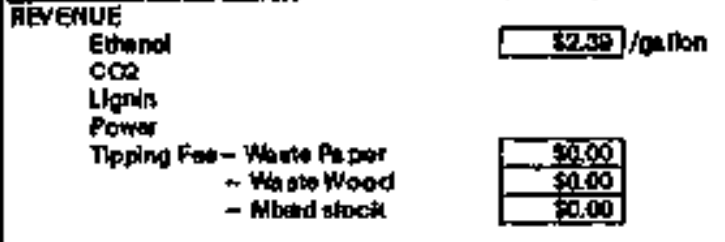 & & $\begin{array}{r}443,160 \\
0 \\
0 \\
0 \\
0 \\
0 \\
0 \\
\end{array}$ & $\begin{array}{r}\$ 400 \\
0 \\
0 \\
0 \\
0 \\
0\end{array}$ & $\begin{array}{r}\$ 46,681 \\
0 \\
0 \\
0 \\
0 \\
0 \\
0\end{array}$ & $\begin{array}{r}549.54 \\
0 \\
0 \\
0 \\
0 \\
0 \\
0\end{array}$ & $\begin{array}{r}40,400 \\
0 \\
0 \\
0\end{array}$ & $\begin{array}{r}69,810 \\
0 \\
0 \\
0 \\
0 \\
0 \\
0\end{array}$ & $\begin{array}{r}5010 \\
0 \\
0 \\
0 \\
0 \\
0 \\
0\end{array}$ & $\begin{array}{r}565, r 9 \\
0 \\
0 \\
0 \\
0 \\
0 \\
0\end{array}$ & $\begin{array}{r}\text { CESOad } \\
0 \\
0 \\
0 \\
0 \\
0\end{array}$ & $\left.\begin{array}{r}301,420 \\
0 \\
0 \\
0 \\
0 \\
0 \\
0\end{array}\right]$ \\
\hline 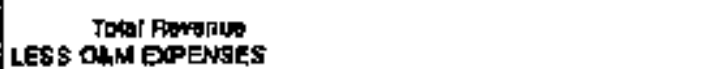 & & & 44tans & Fit,est & $\$ 10,840$ & $\$ 00,400$ & 50,610 & 55,0 to & $\$ 00,704$ & 150,048 & 181,420 \\
\hline 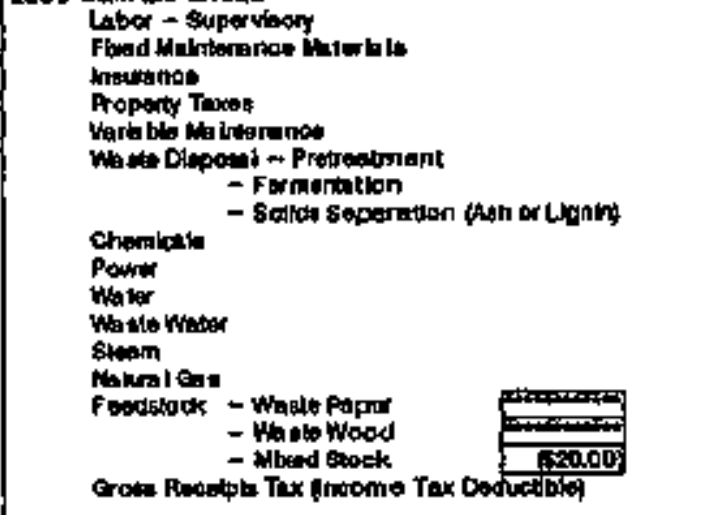 & & 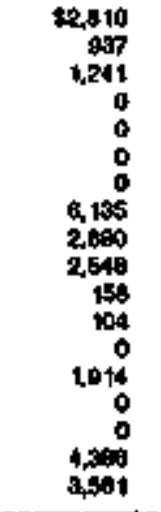 & 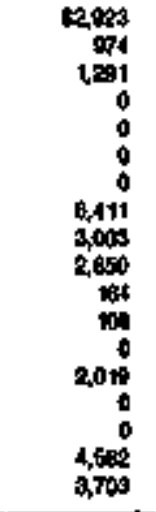 & 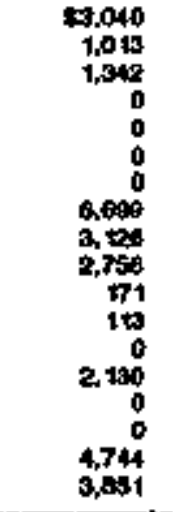 & $\begin{array}{r}5,101 \\
1,004 \\
1,308 \\
0 \\
0 \\
0 \\
0 \\
7,001 \\
3,251 \\
2,600 \\
170 \\
17 \\
0 \\
2,247 \\
0 \\
0 \\
4,034 \\
4,000\end{array}$ & 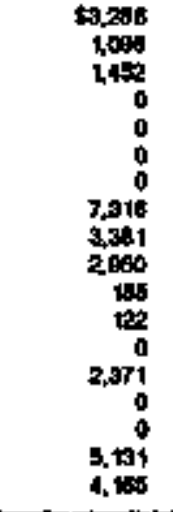 & 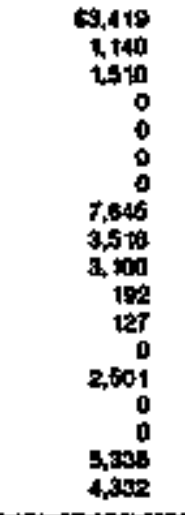 & 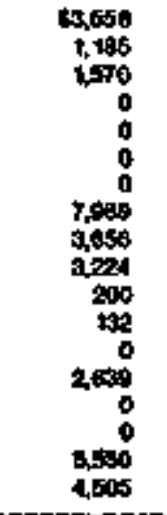 & 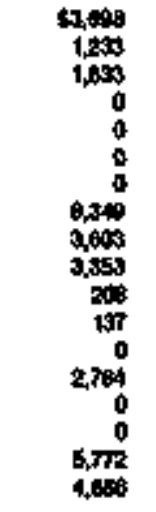 & 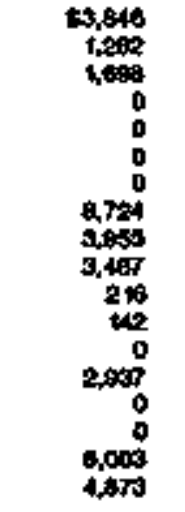 & 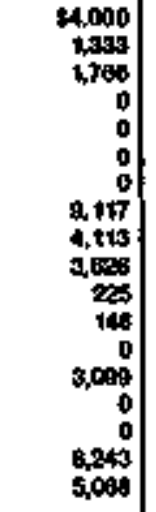 \\
\hline 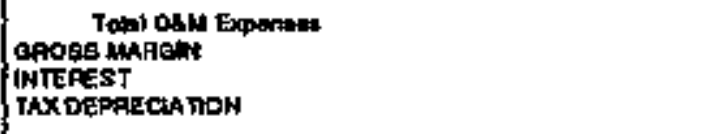 & & 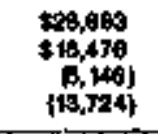 & 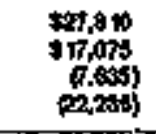 & $\begin{array}{r}+0,005 \\
17,090 \\
(7,072) \\
(13,007)\end{array}$ & 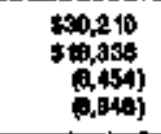 & $\begin{array}{l}101,467 \\
6+2,003 \\
(5,774) \\
(3,7 \% 5)\end{array}$ & 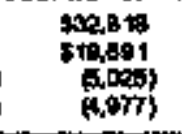 & $\begin{array}{l}24,207 \\
\sin , 400 \\
(1,202) \\
(1,12)\end{array}$ & 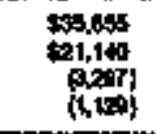 & $\begin{array}{l}\sin , 194 \\
\infty 1,002 \\
0,301) \\
(1,1+9)\end{array}$ & 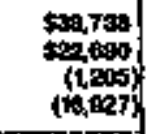 \\
\hline 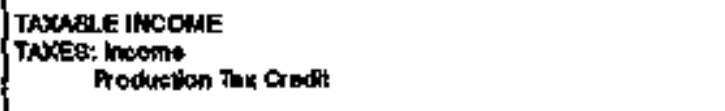 & & $\begin{array}{c}0 ., 3941 \\
2,203 \\
0\end{array}$ & $\begin{array}{r}\$ 1,700] \\
5,200 \\
0\end{array}$ & $\begin{array}{r}0.2833) \\
1.353 \\
0\end{array}$ & $\begin{array}{c}\$ 9,000 \\
0.2 * 1) \\
0\end{array}$ & $\begin{array}{c}+4,46) \\
(t, 023) \\
0\end{array}$ & $\begin{array}{c}\operatorname{sacse} \\
0008 \\
0\end{array}$ & $\begin{array}{c}\$ 5,009 \\
0.131\} \\
0\end{array}$ & 50 & $\begin{array}{c}518,400 \\
0500 \\
0\end{array}$ & 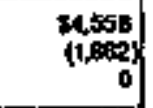 \\
\hline $\begin{array}{l}\text { INCOANE AFTEA TAX } \\
\text { TAXDEPAECAATION } \\
\text { P.ANT COST } \\
\text { LOAN } \\
\text { PAWHCAPAL PAVMENTS }\end{array}$ & $\sin _{\sin (1,400}$ & 0,181 & Fi, & $\begin{array}{l}61,030) \\
\text { ta,897 }\end{array}$ & 51,797 & $=0,640$ & is, rat & 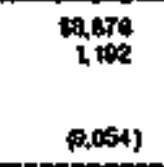 & itos & $\$+10,032$ & $\begin{array}{l}\$ 2,806 \\
10,027\end{array}$ \\
\hline $\begin{array}{l}\text { NET CASH FLOW } \\
\text { CUWHLTWE OAOH RLW } \\
\text { CUWULATWE JAR } \\
\text { DEBT CONERAE FA TO } \\
\text { CUH NPV }\end{array}$ & 500,3 (3) & 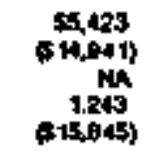 & 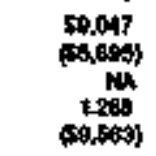 & 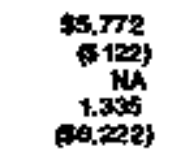 & 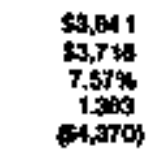 & $\begin{array}{l}\$ 1,029 \\
0,042 \\
0,015 \\
1,439 \\
0,7934\end{array}$ & 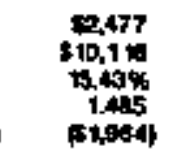 & $\begin{array}{r}\$ 1,018 \\
\$ 11,194 \\
1,20 \% \\
t, 580 \\
\$ 1,000\end{array}$ & 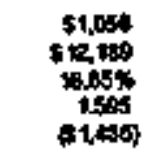 & $\begin{array}{l}51,005 \\
+10,285 \\
17,39 \% \\
1,052 \\
(1,2020)\end{array}$ & $\begin{array}{r}57,572 \\
200,056 \\
20,004 \\
1.74 \\
50\end{array}$ \\
\hline
\end{tabular}




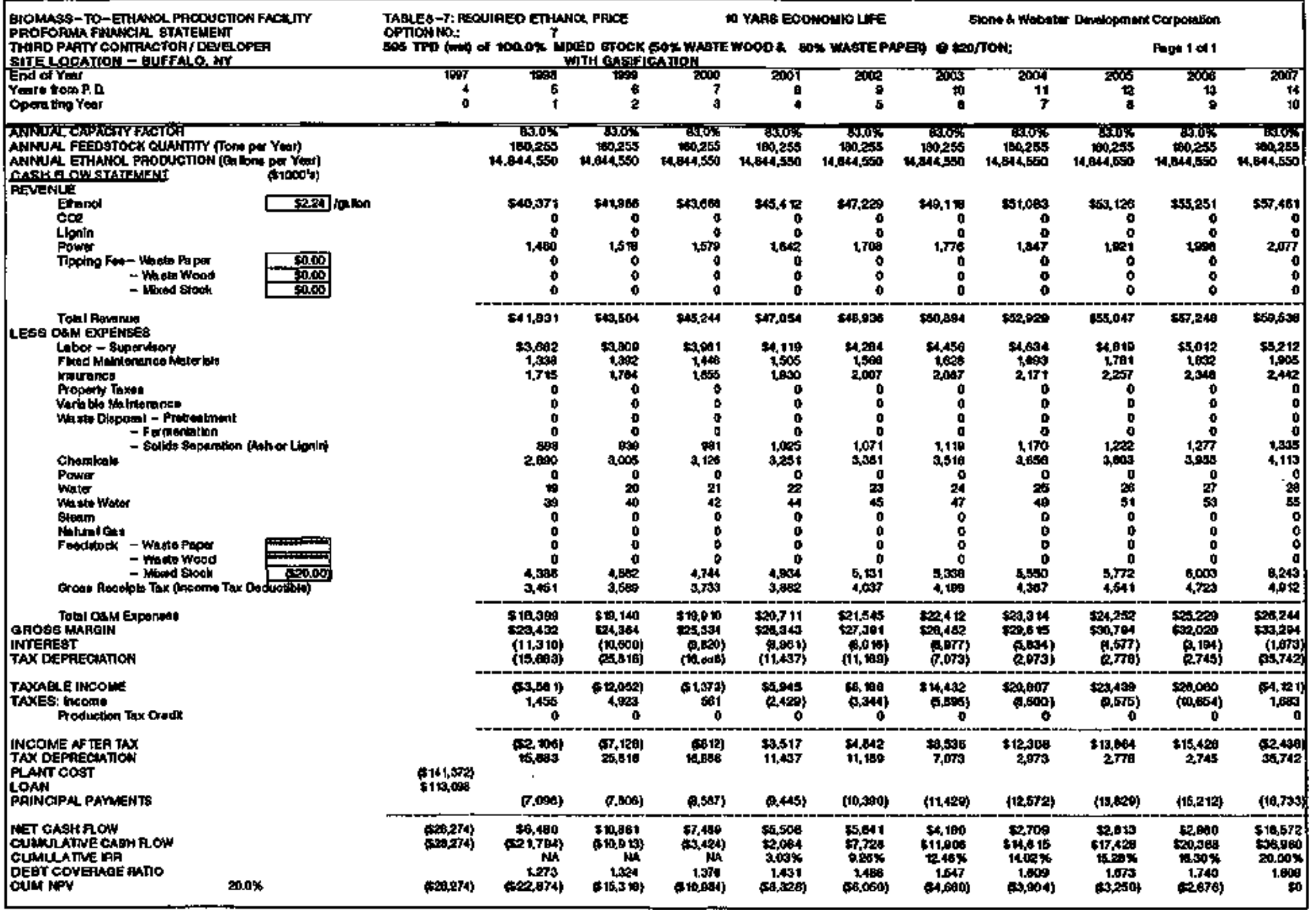




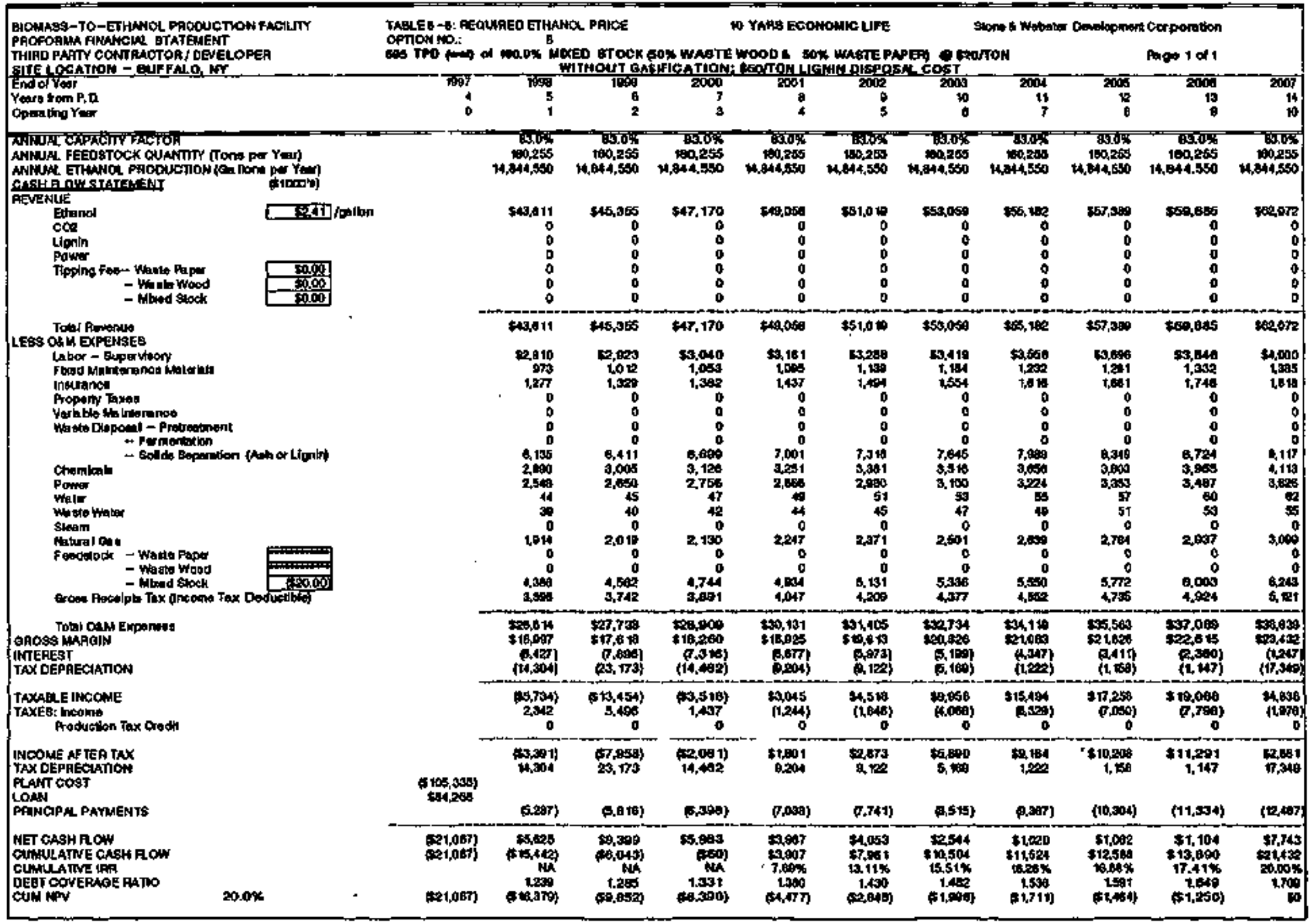




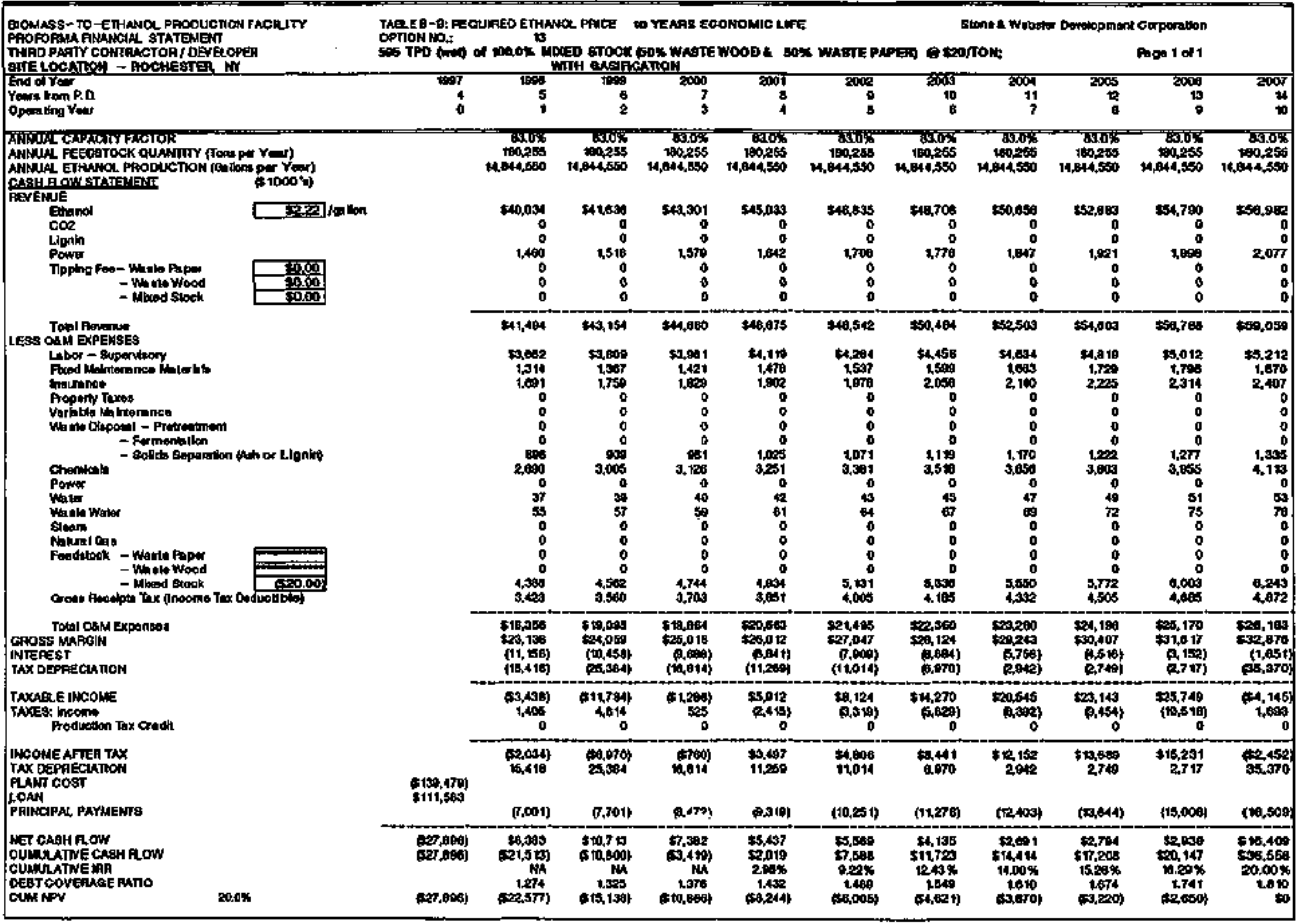




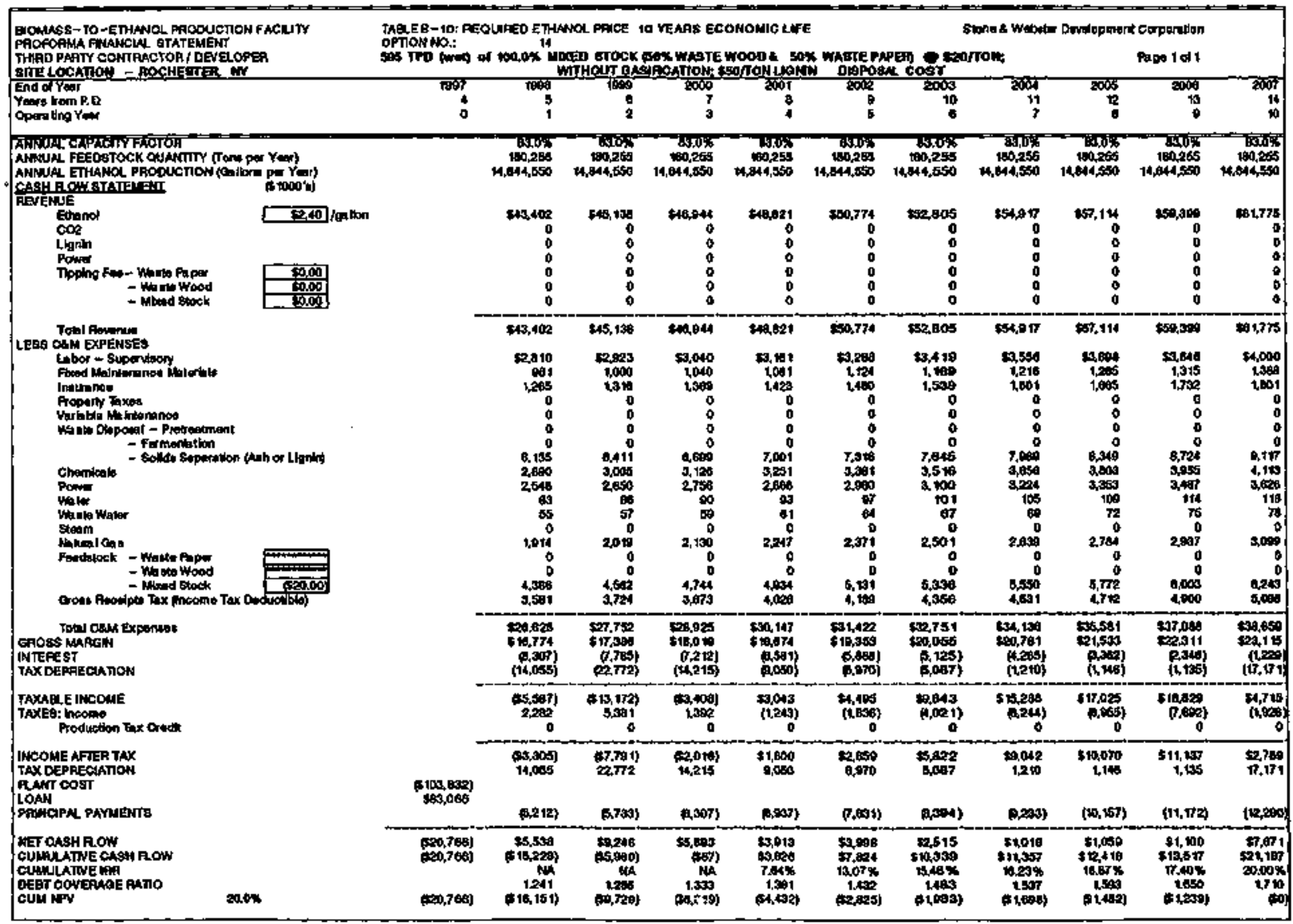




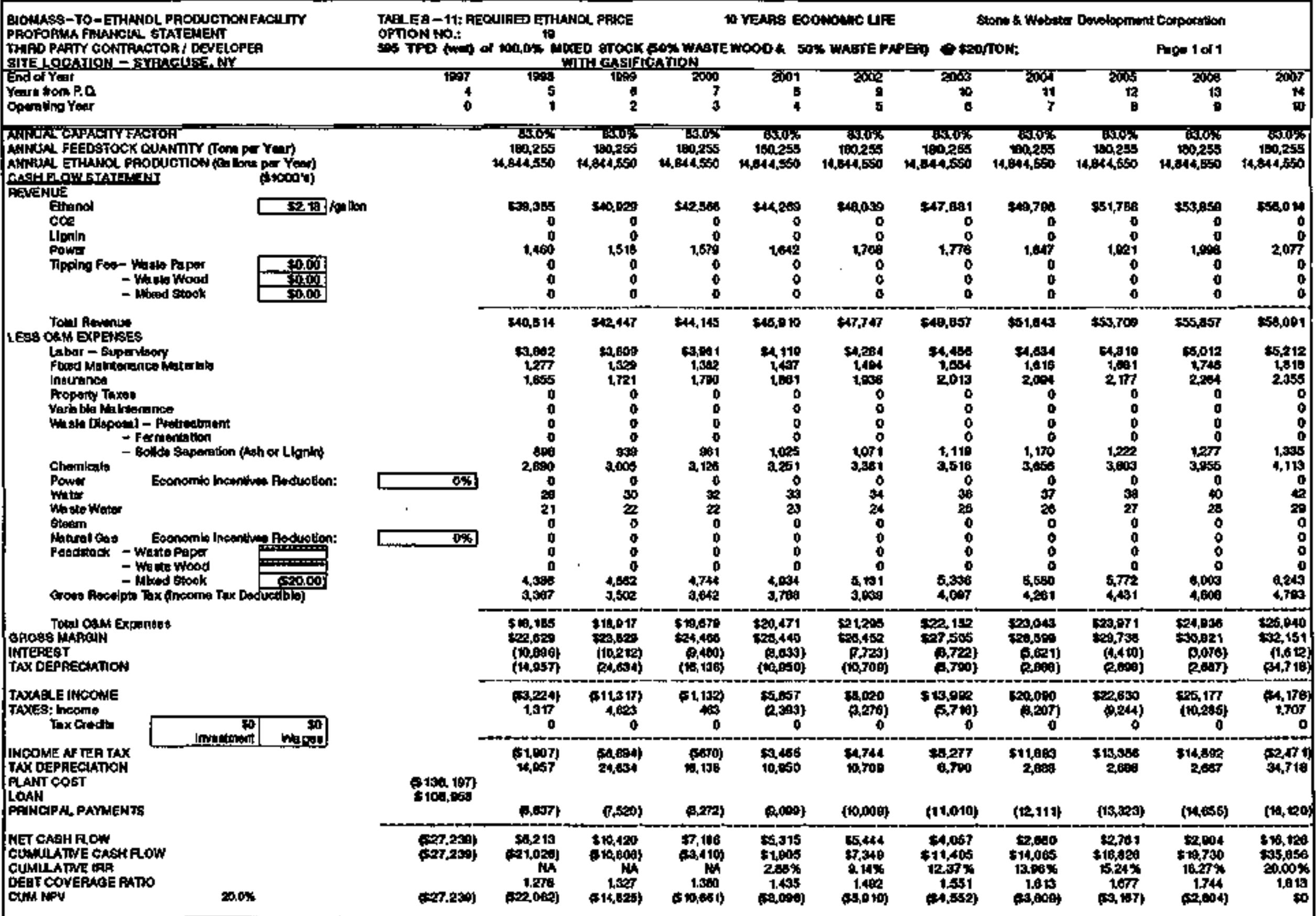




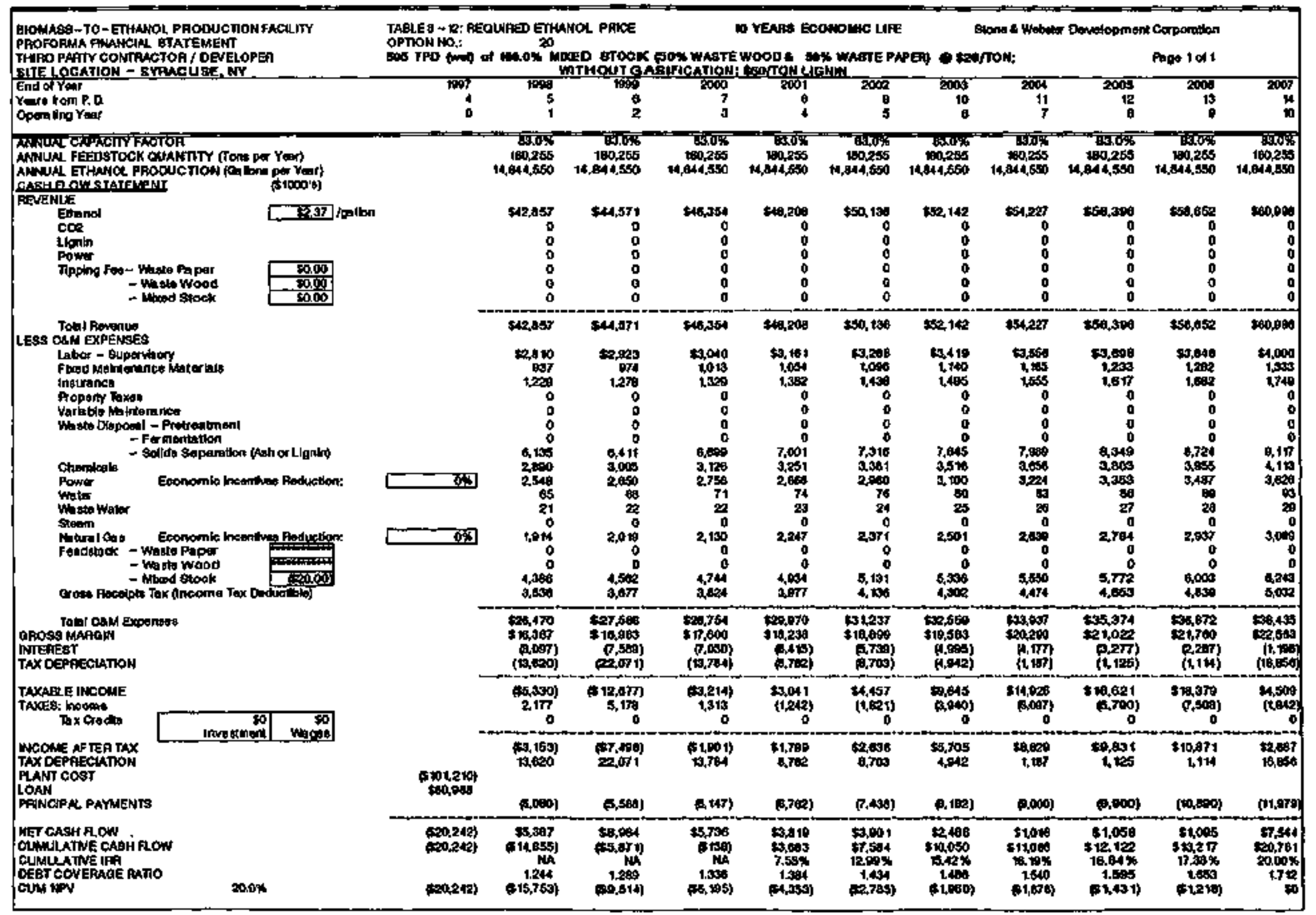




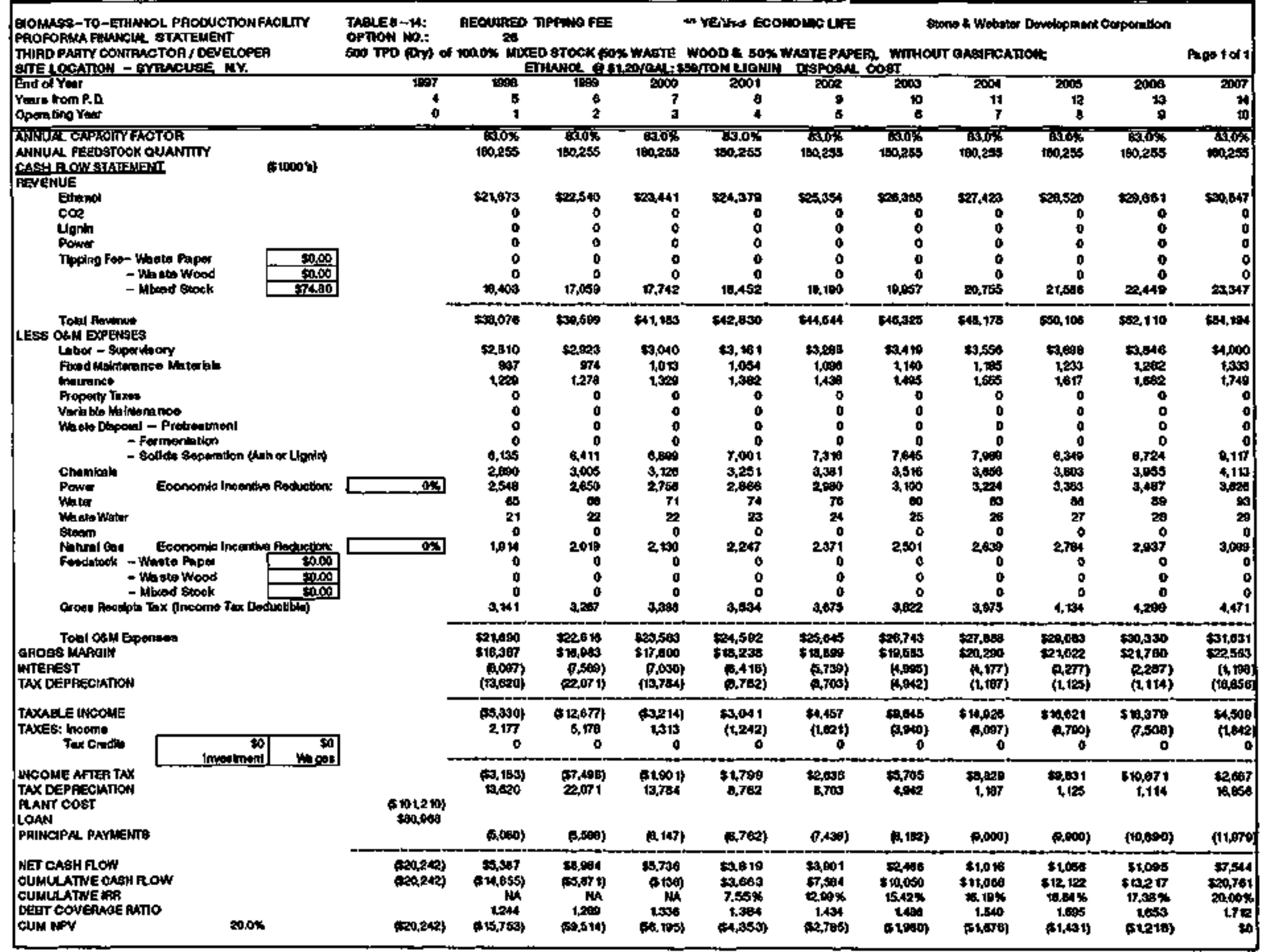




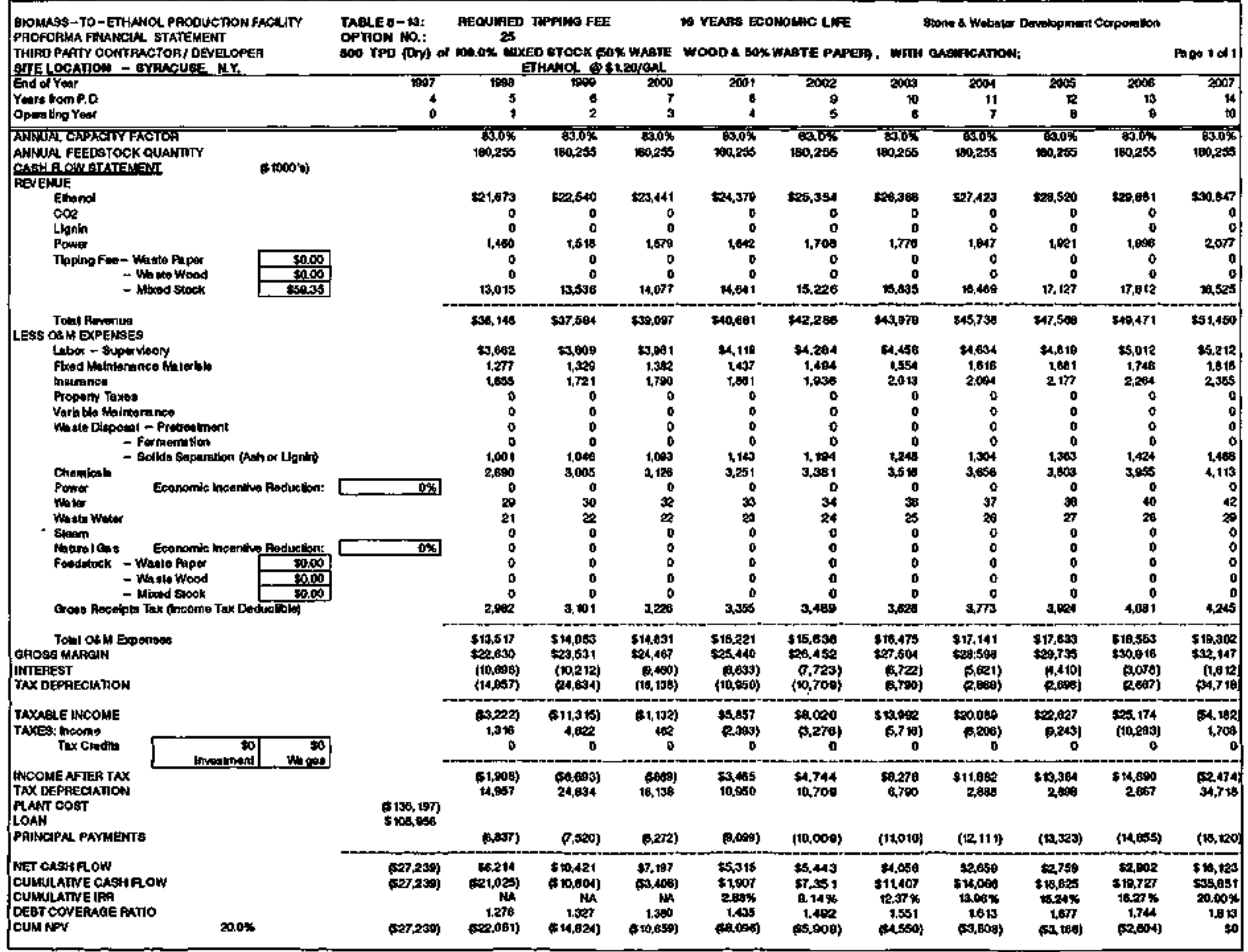




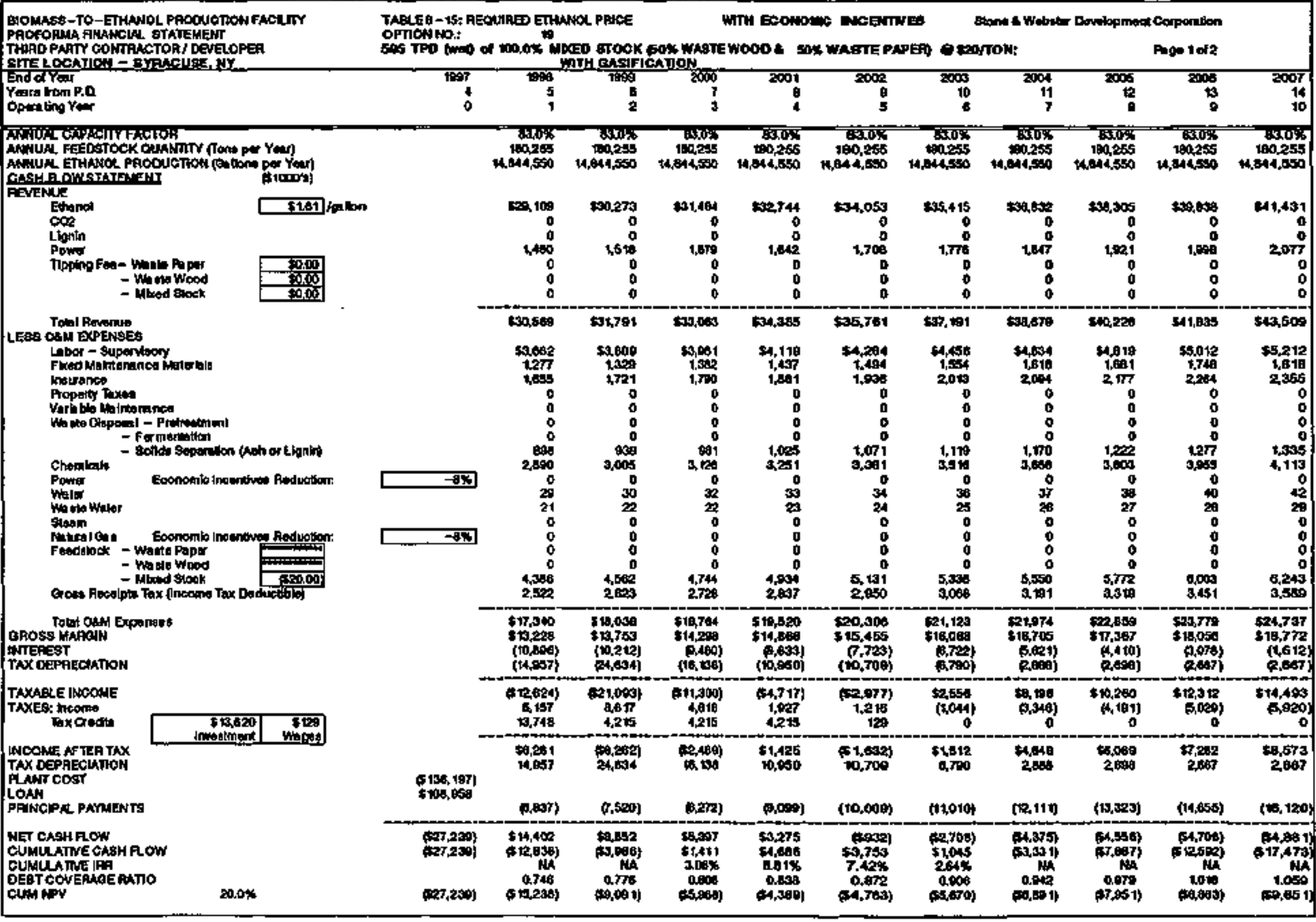




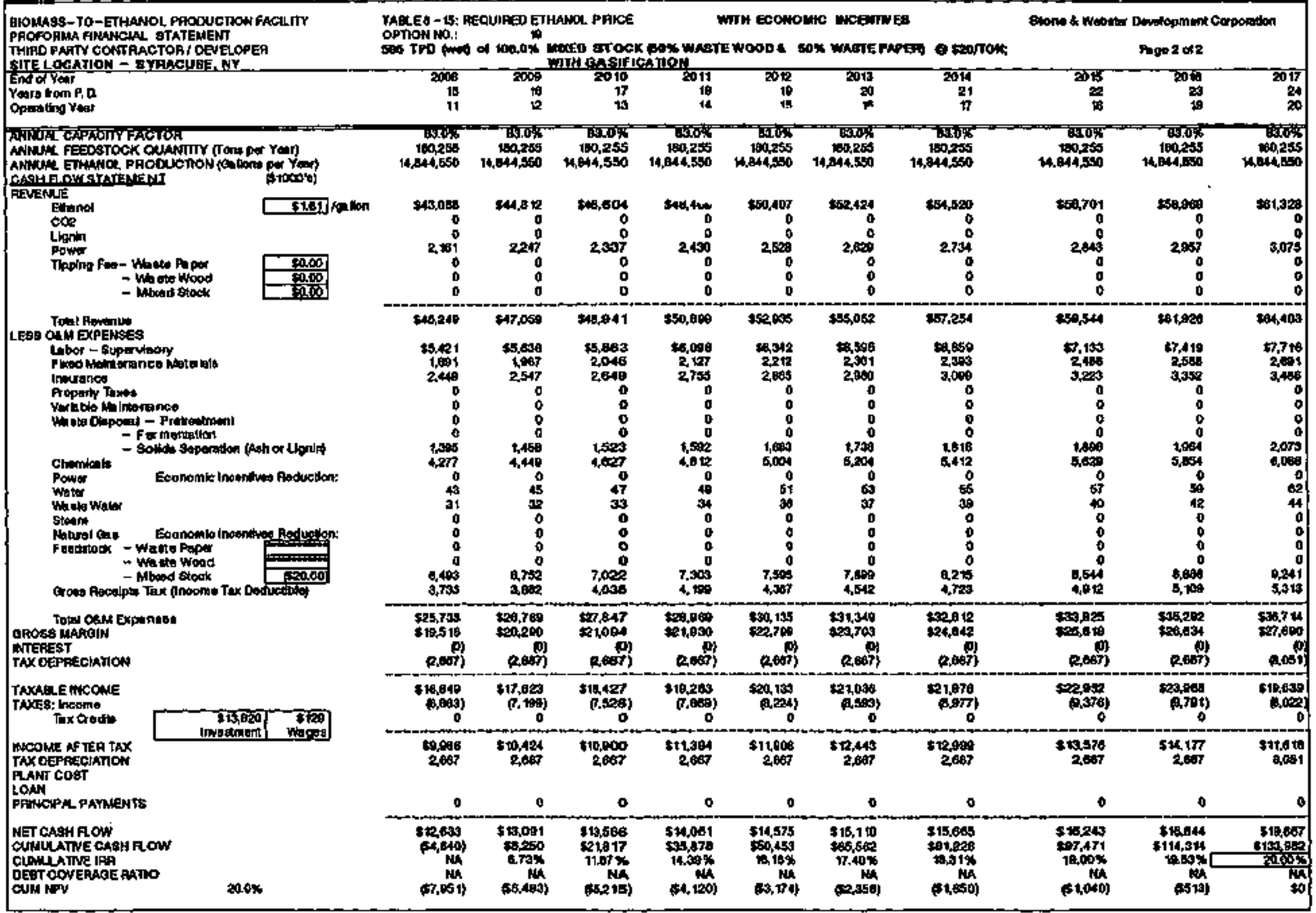




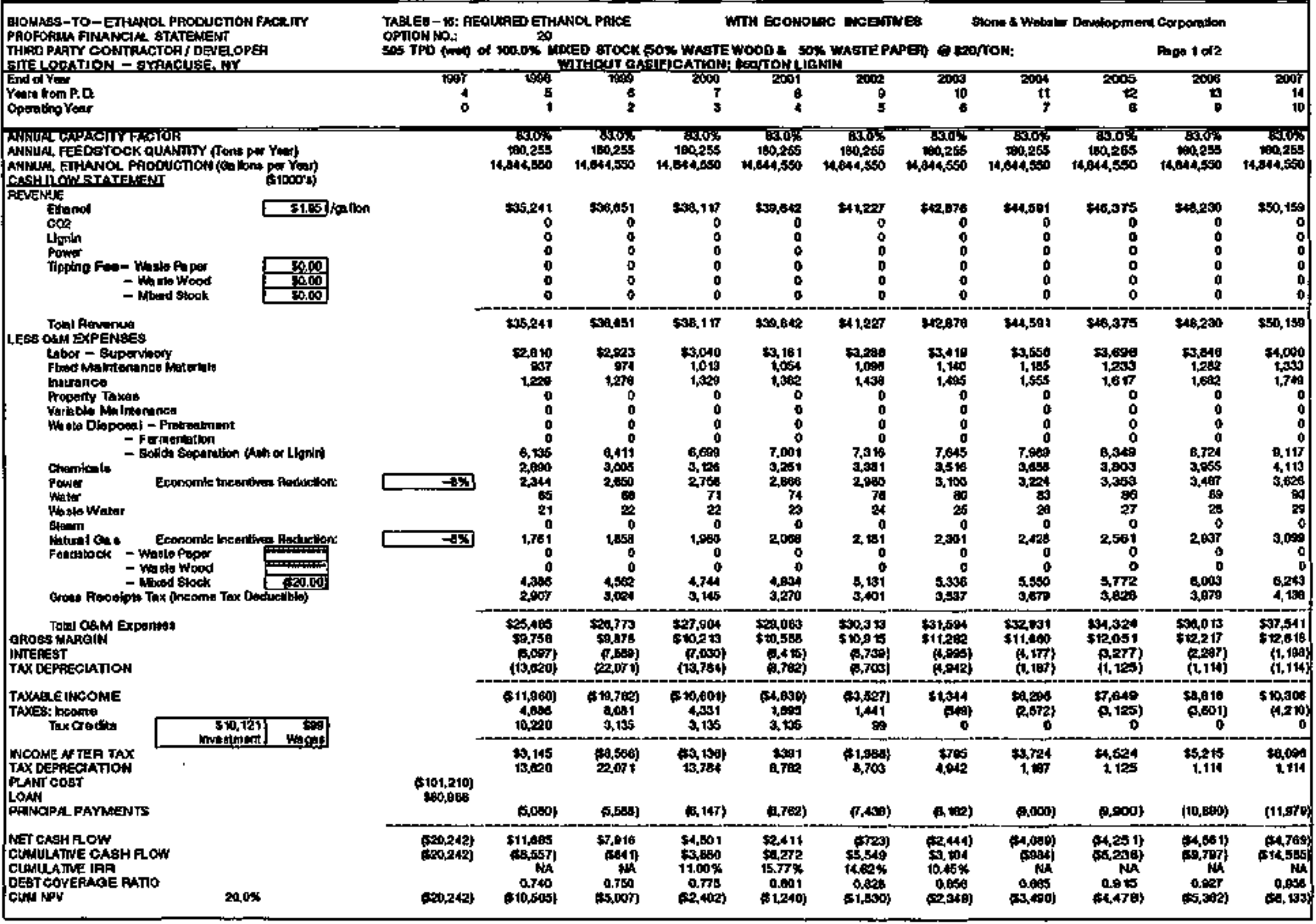




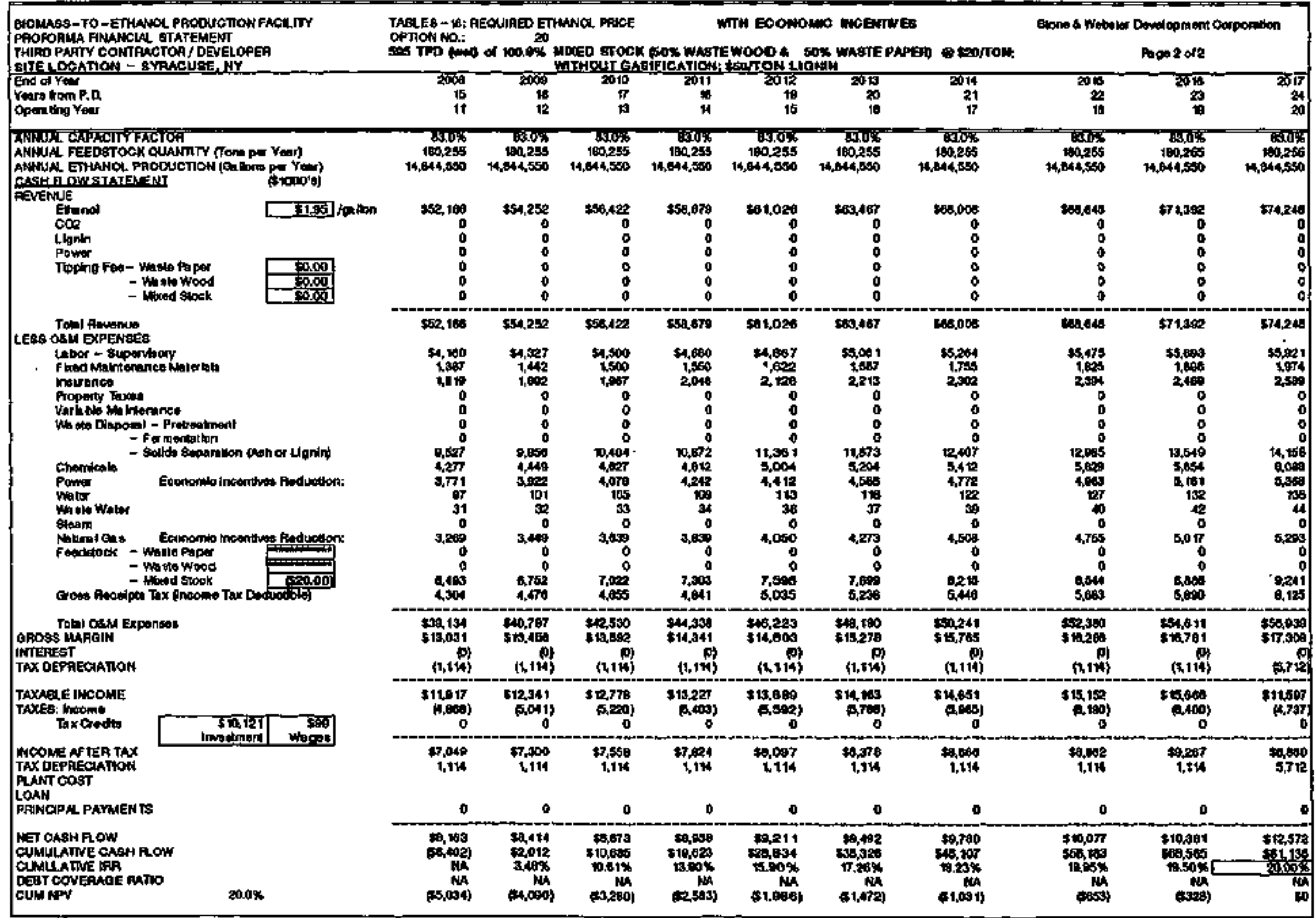

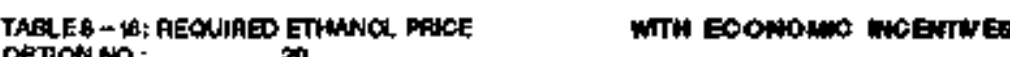

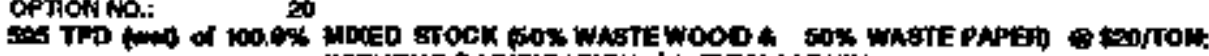
THIRO PAITY CON 20,

10

promol

20

550

H DOW SIATE, $\underbrace{}_{N}$

Expon

Llonk

Toring Fen - Viate Paper

- Wh in wood

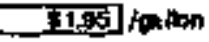

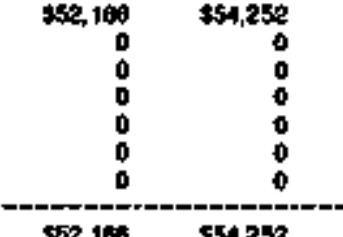

Tow I Amence

Iebor - supentuor

insurence

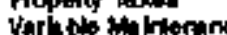

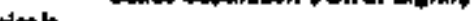

Pamp-

Wh wh Wato

*an

- Whillo Paper

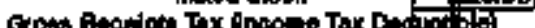

B: nowomt.

MWOLE AFTERTAX

\$1, wo 


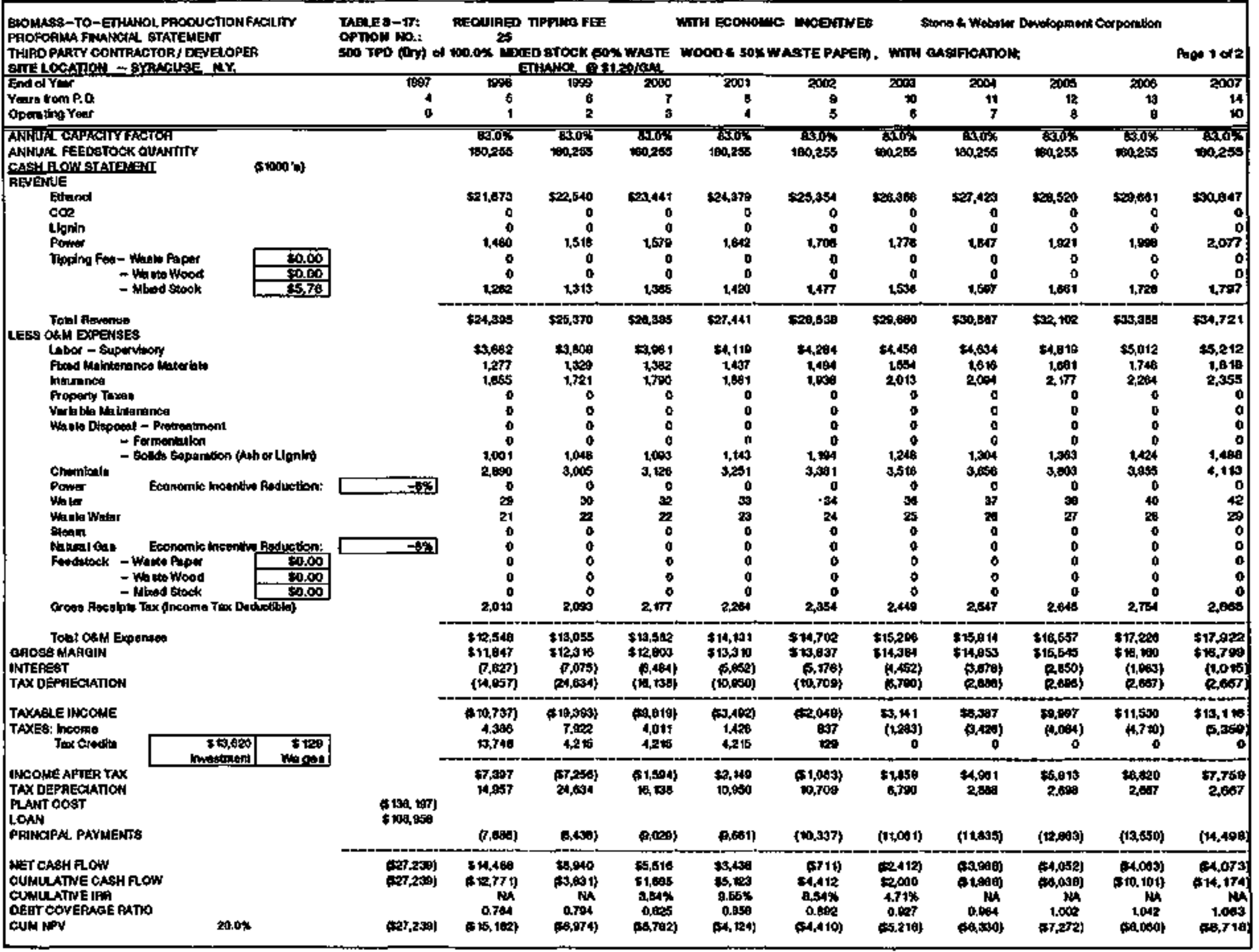




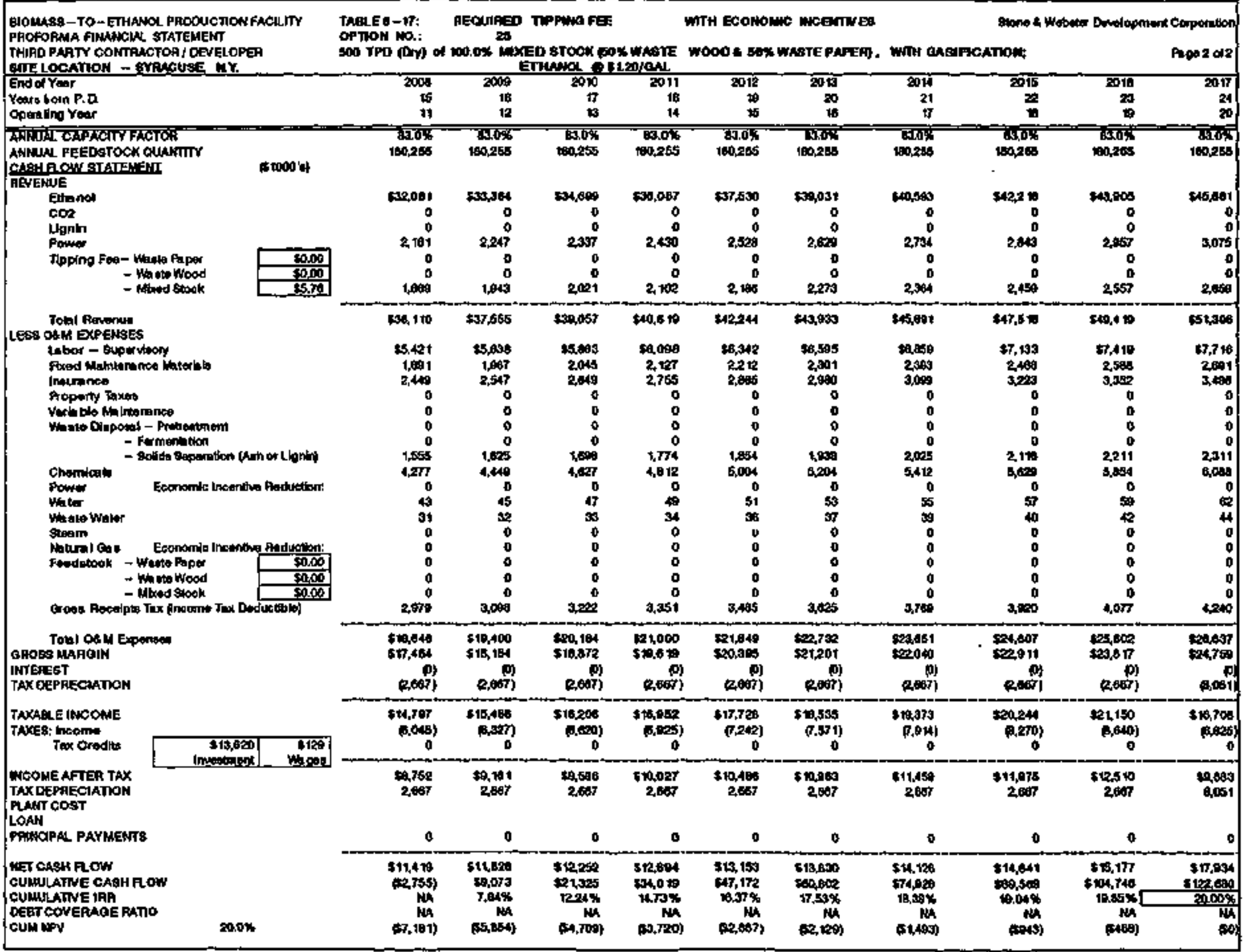




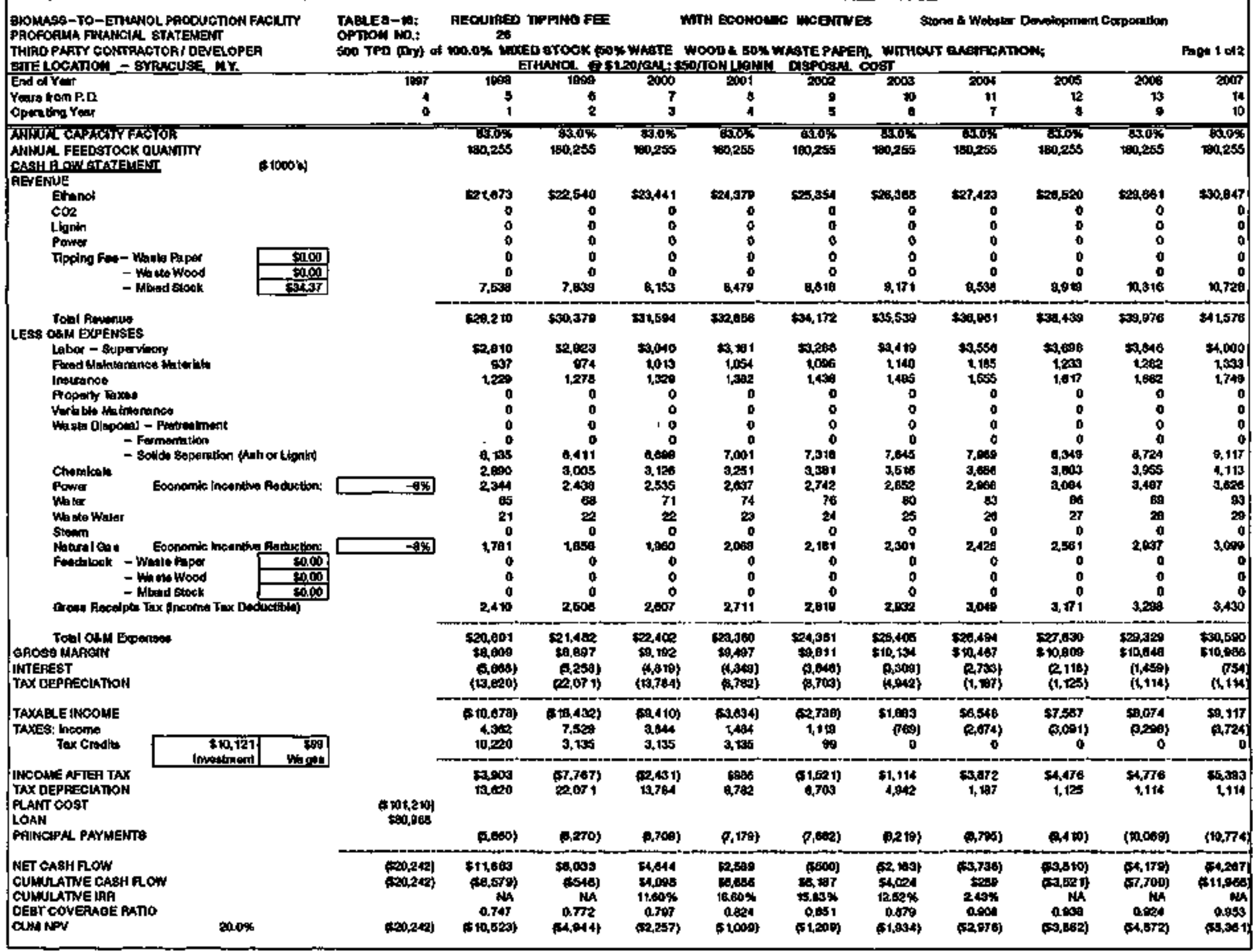




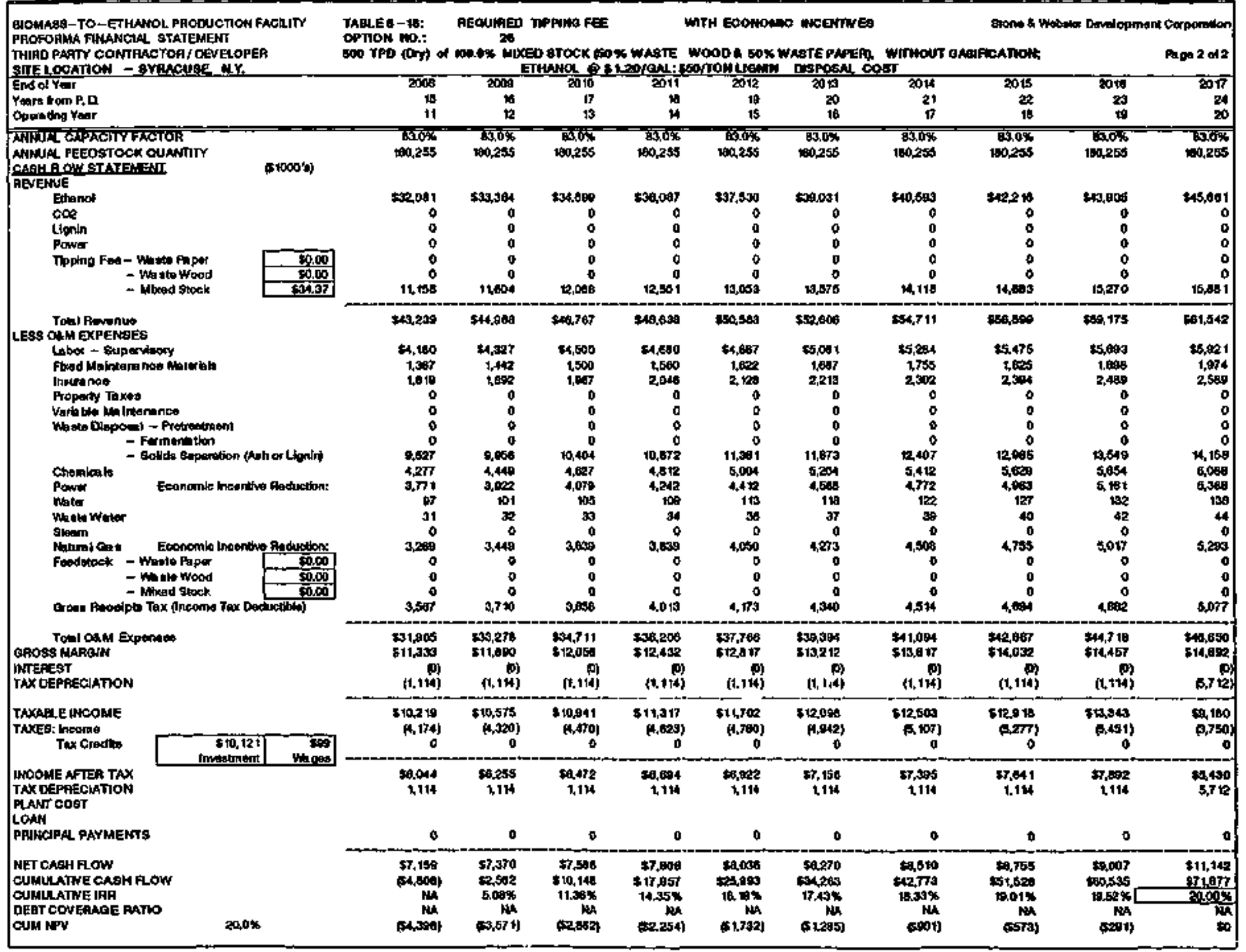


TABLE 6-19

ECONOMIC

INCENTIVES TABLE

\begin{tabular}{|c|c|c|c|c|c|c|c|}
\hline \multicolumn{2}{|c|}{ 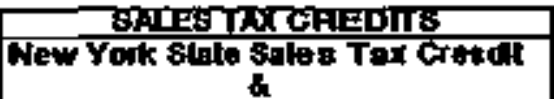 } & \multirow{2}{*}{ LABOR } & \multirow{2}{*}{ FINANCING } & \multirow{2}{*}{$\underset{\text { COST }}{\text { CAPINA }}$} & \multicolumn{3}{|c|}{ TAXES } \\
\hline 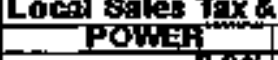 & Enerper crodit & & & & & & \\
\hline $\begin{array}{r}\text { Aectuction lor: } \\
\text { yeas: } \\
\end{array}$ & $\begin{array}{r}\text { Peduction tor: } \\
\text { reas: } \\
\end{array}$ & 0 & intereal rafe & D & 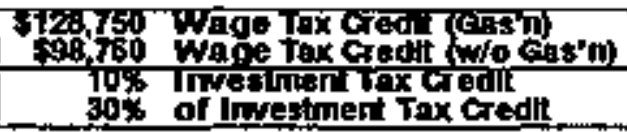 & $\begin{array}{l}\text { SYr. } \\
\text { 5Yrs. } \\
\text { JYrs: } \\
\text { 3Yrs: }\end{array}$ & 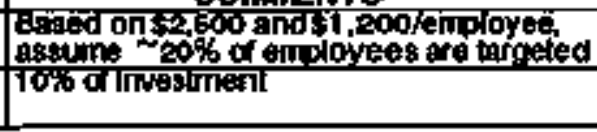 \\
\hline
\end{tabular}

slong \& Wetbster Devalopmeri Caparation 


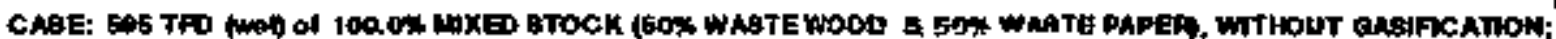

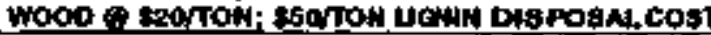

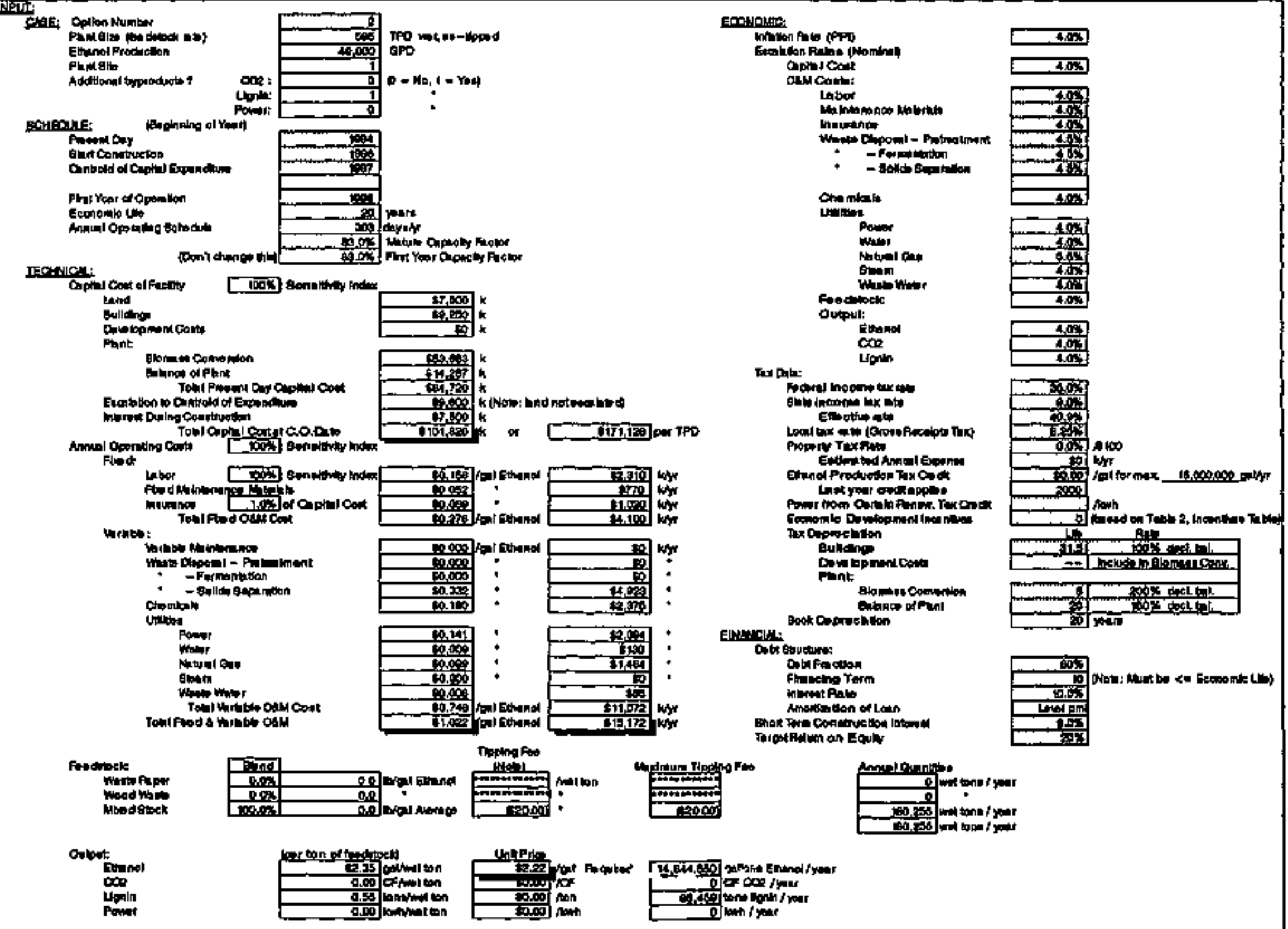

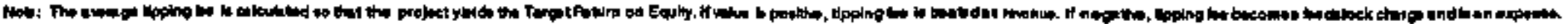




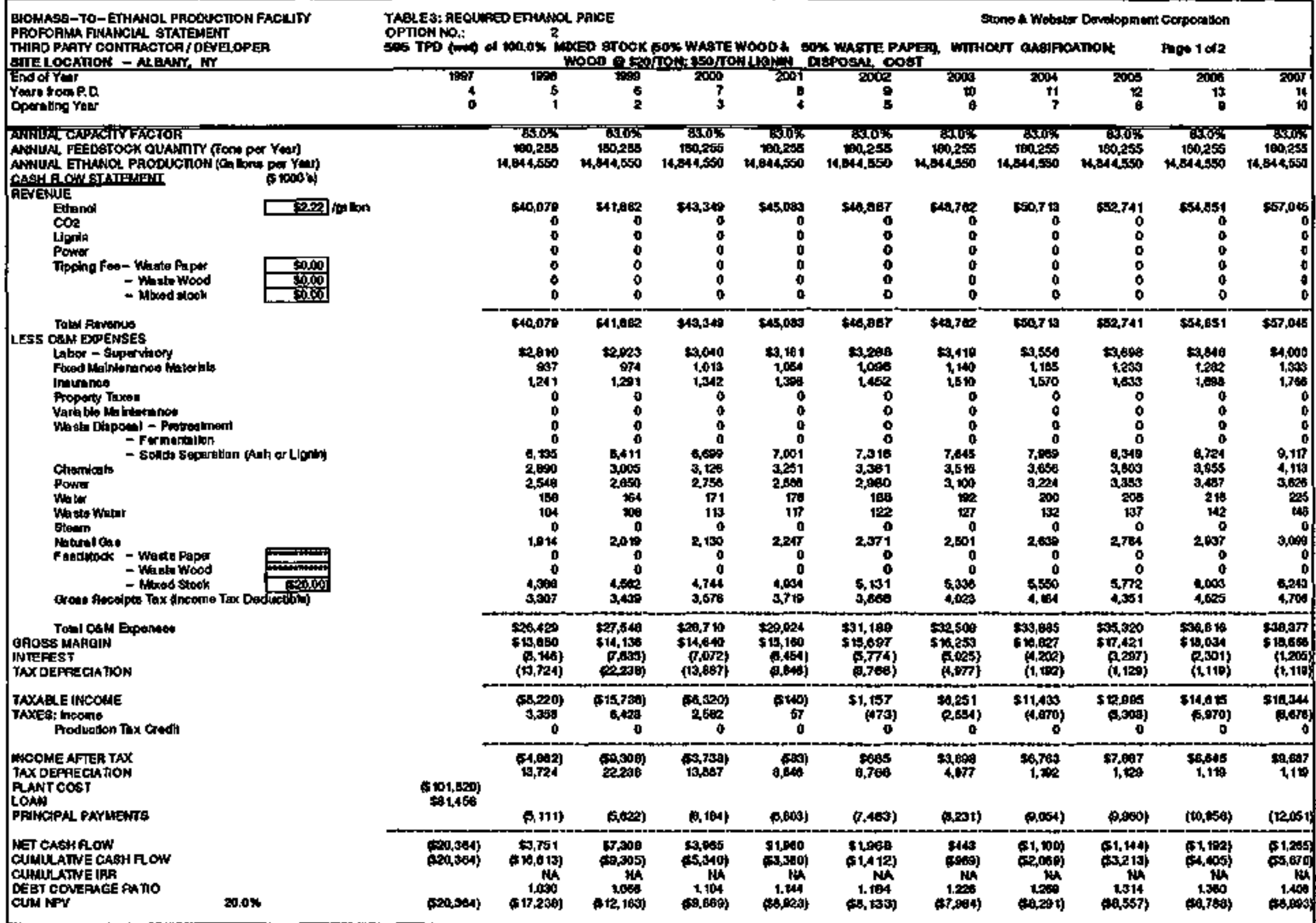




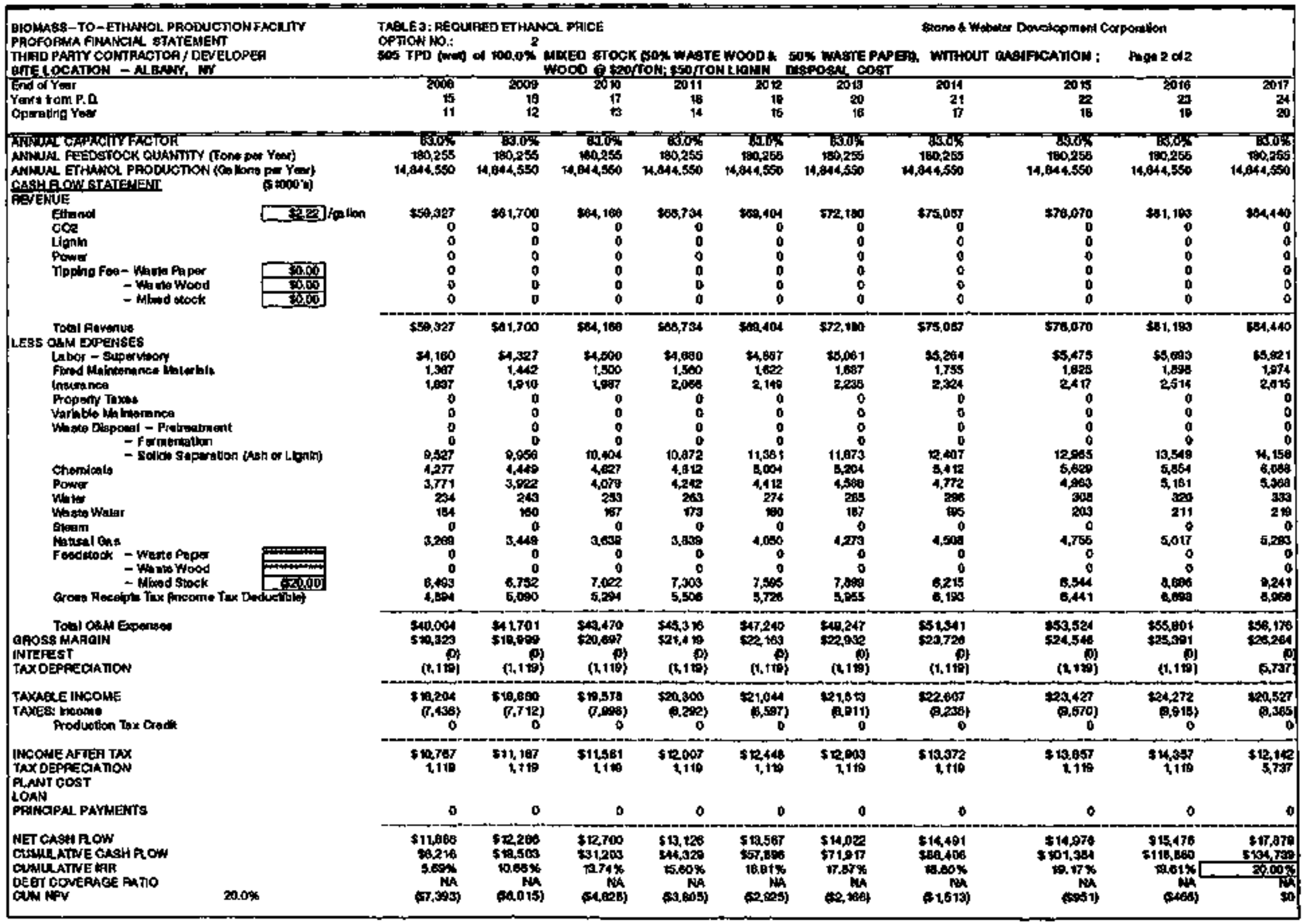



MOOD R SAOTH

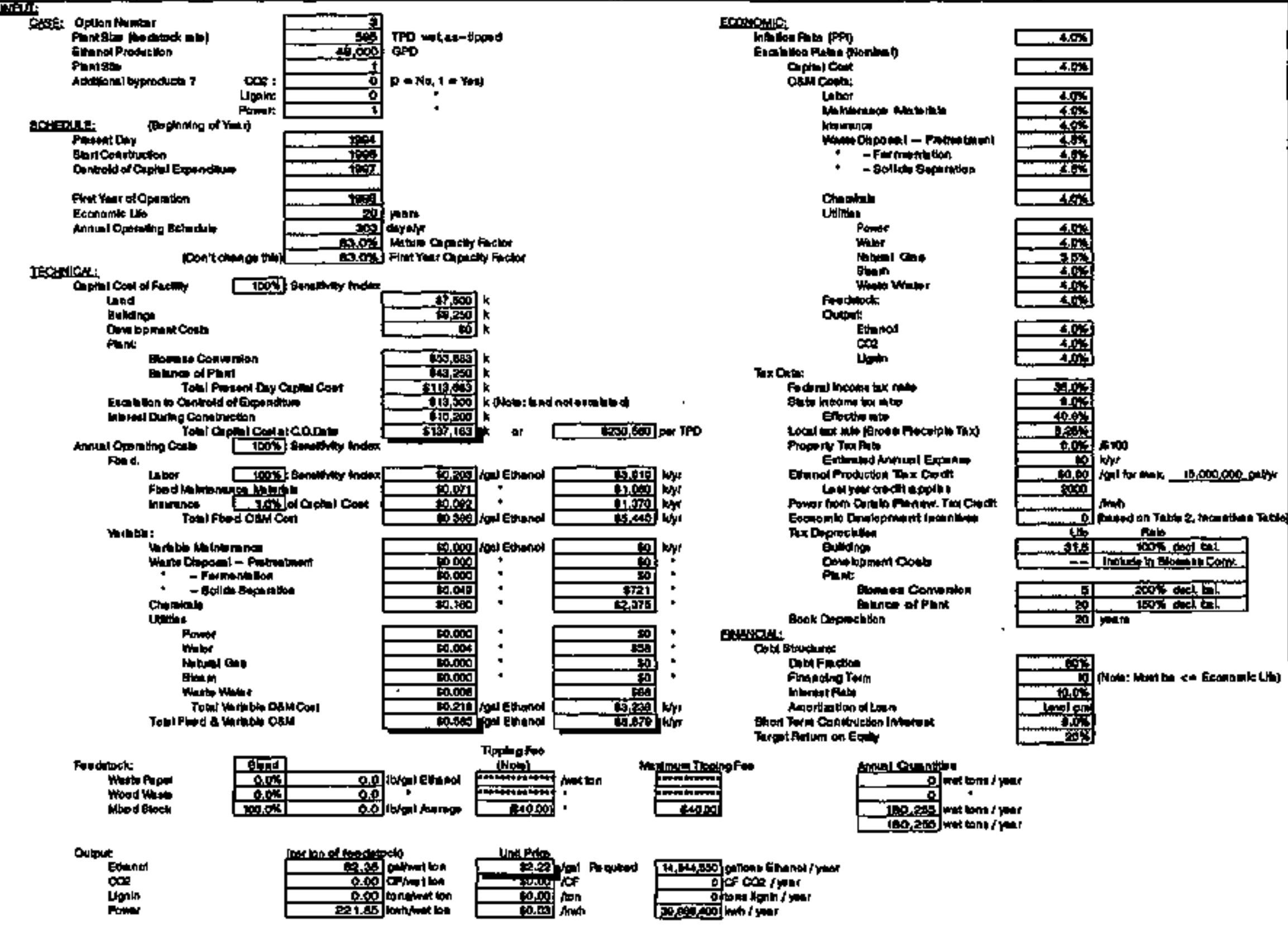

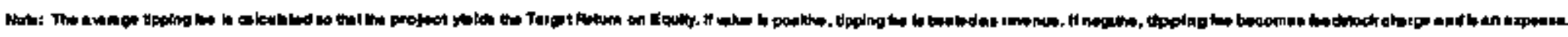




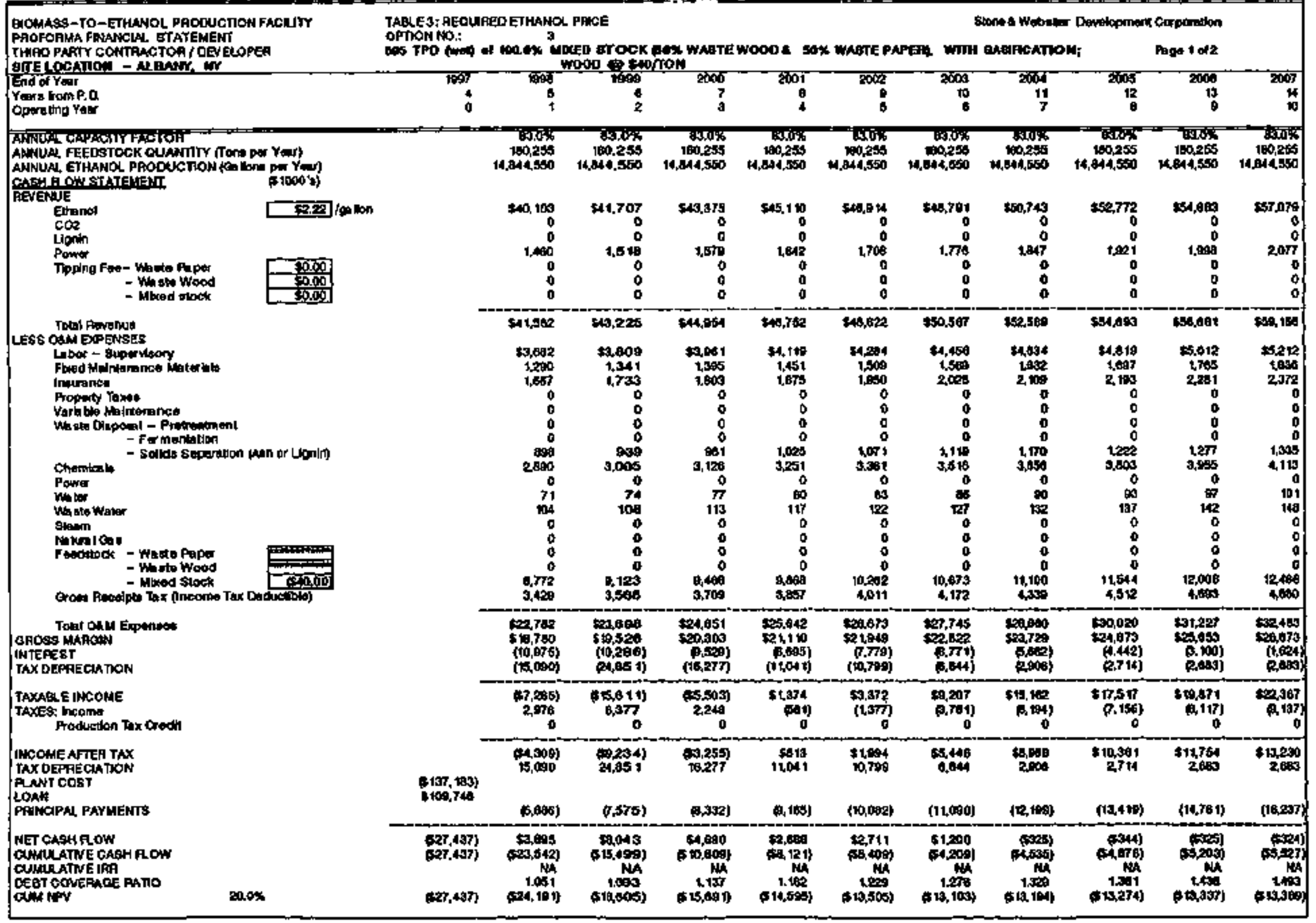




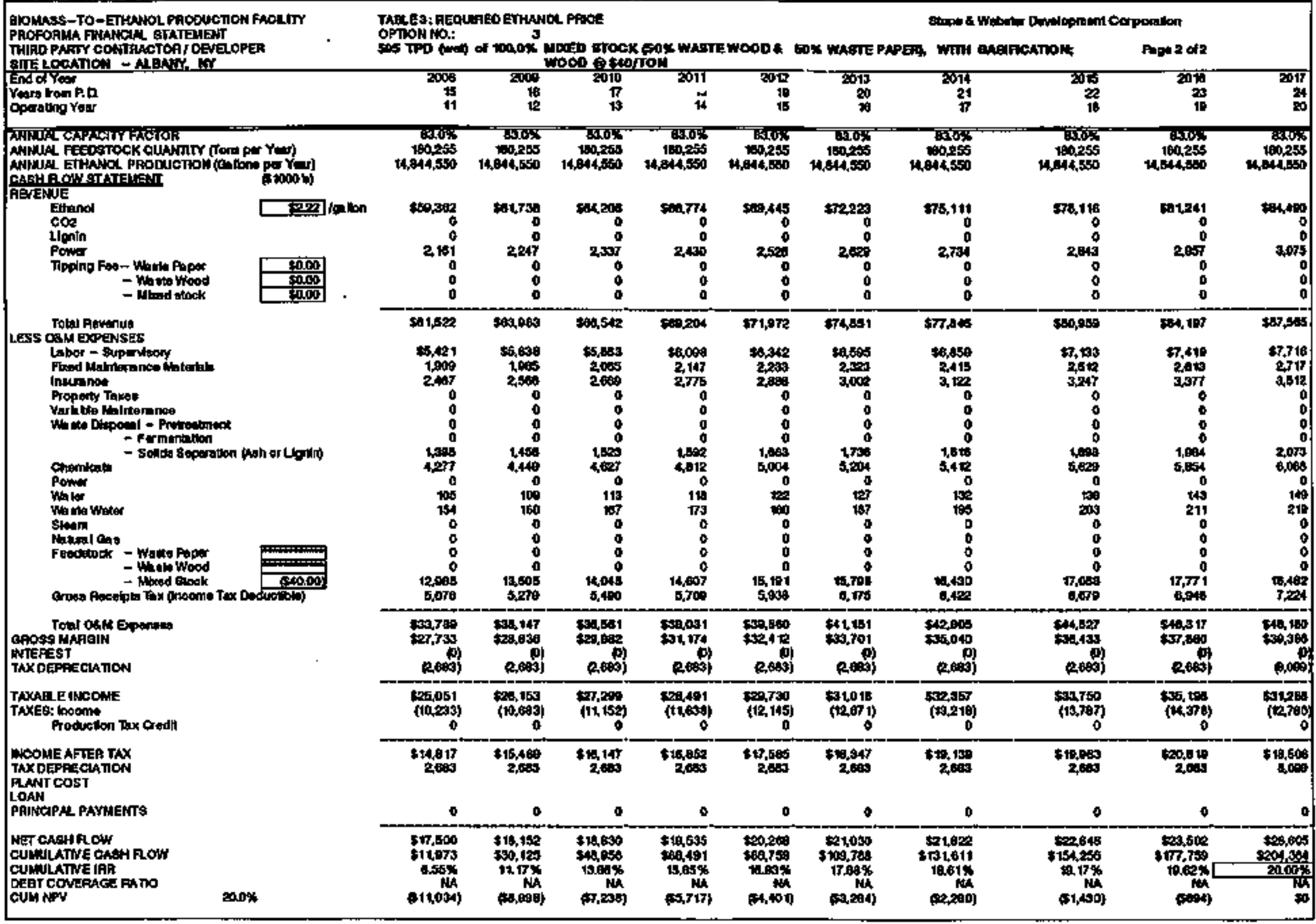




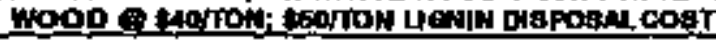

exoming

inhition Fue (PF)

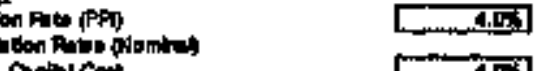

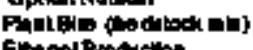

Emenol Producilon

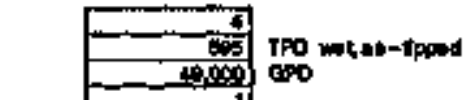

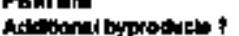

$\cos$

Ploing

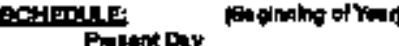

senconous

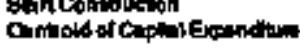

Fin Xur ofopmenes

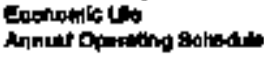

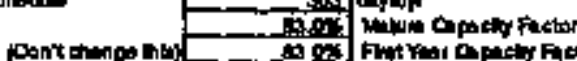

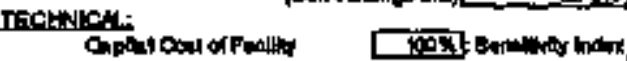

Gapalional

Buldion

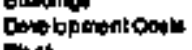

nint.

Bromenoconnention

Cont

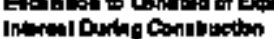

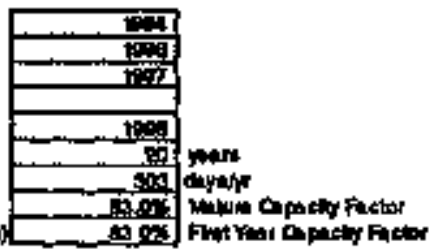

creas:

Malnoming Nunte

manow

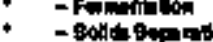

chenver

nomir

Nones

Whetwe Wher

Andioste

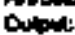

Bimed

$\operatorname{lom}_{\text {Lion }}^{\infty}$

$\tan \cos$

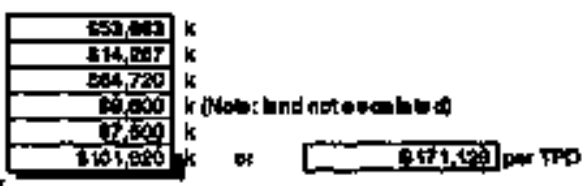

or [-

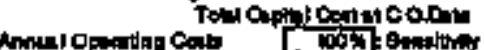

Froed:

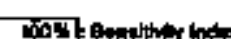

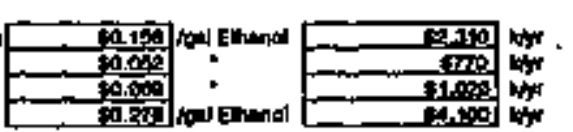
Wribst

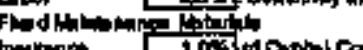

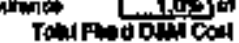

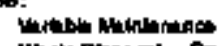

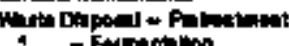

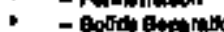

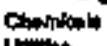

Uaw:

miner

morelen

gines.

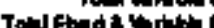
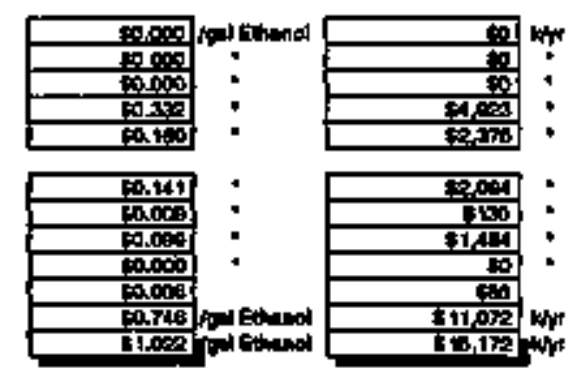

ind :

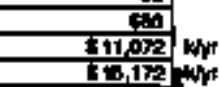

Inpping fie

15172

Fondibek

Werto fuper

Wood thincer

wiod atock

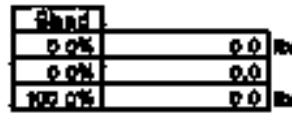

mot bon

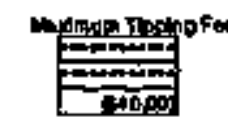

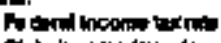

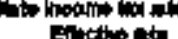

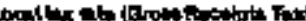

Propotity Tor prate

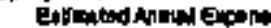

Etunal prodiction hay ced

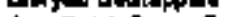

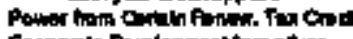

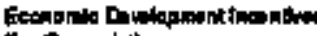

ax Enevereston

euting corts

Phate

glocena Comanton

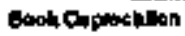

Eutercy

Invinam:

Ood Fastos

Finneng The

Tatexelfrim

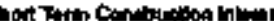

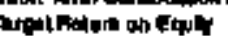

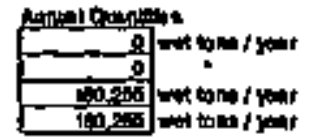

oupuc

romel
Pon
Poinh

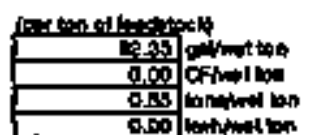

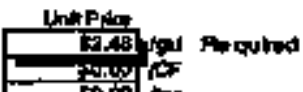

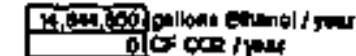

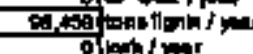

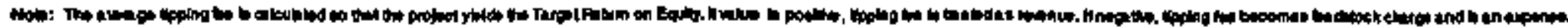




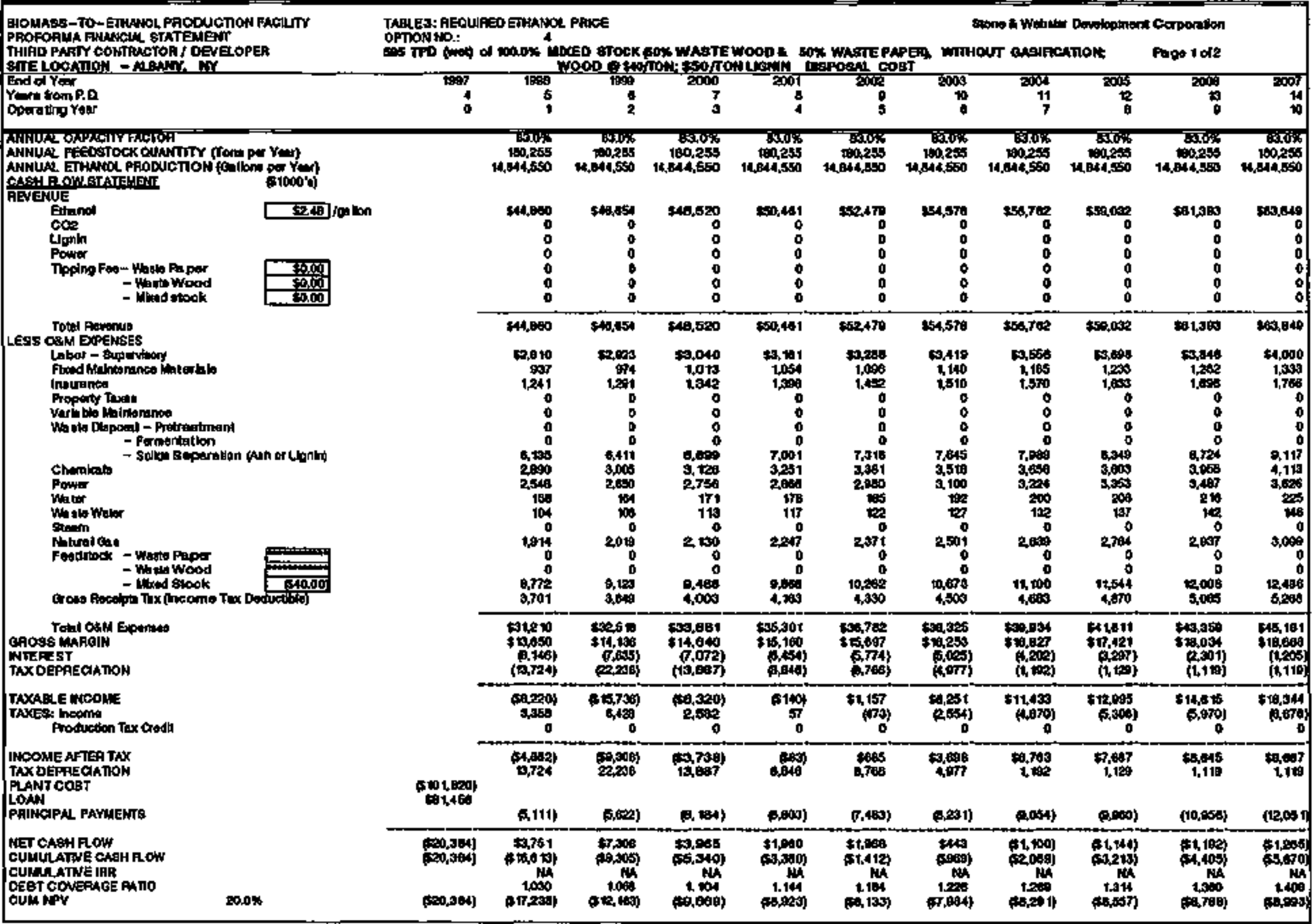




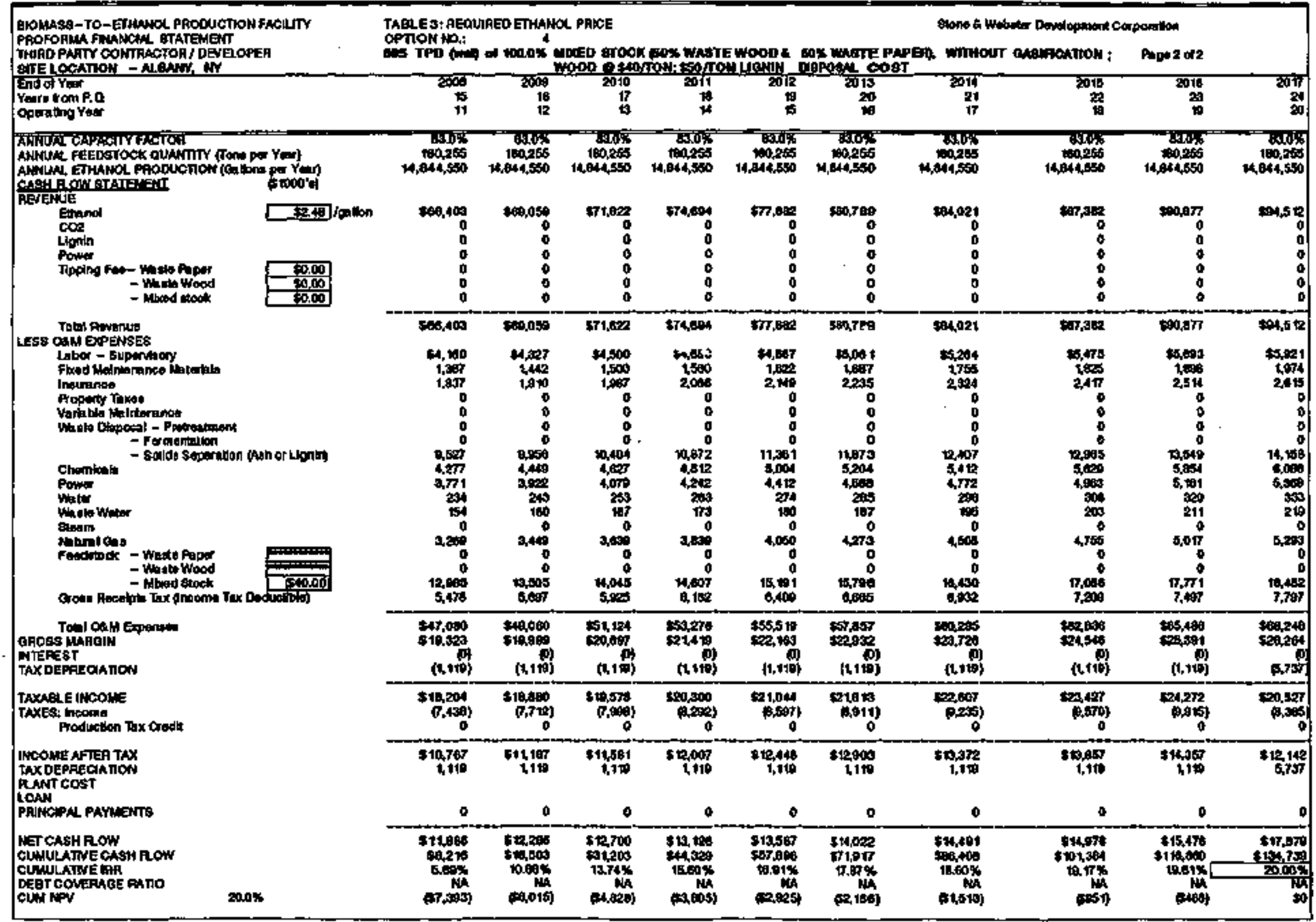

coras.

$\hat{s}$ 


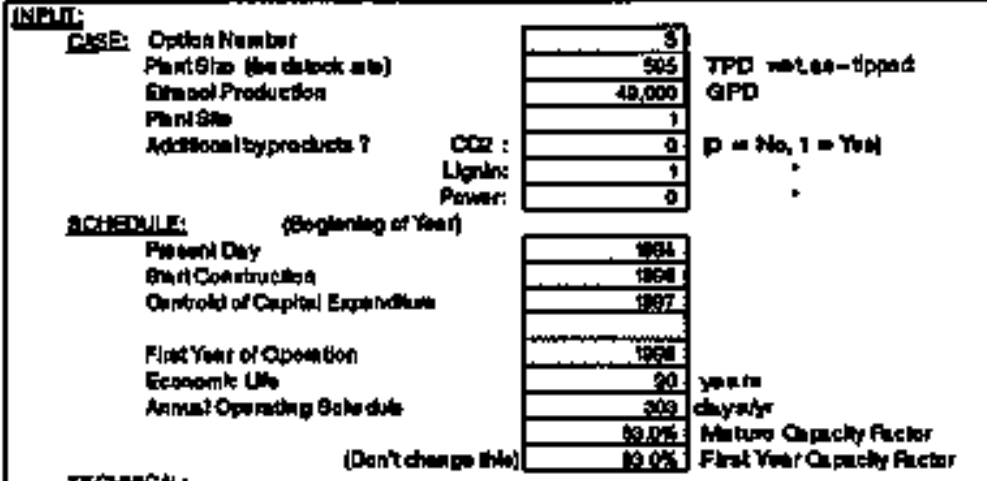

Irstancin:

Dent denoph the

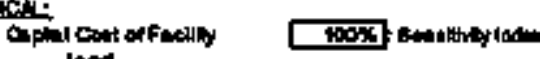

Ind

Bullinon

Puat

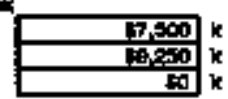

Blomsil canarion

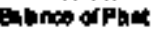

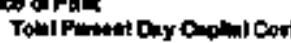

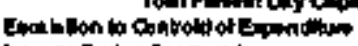

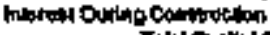

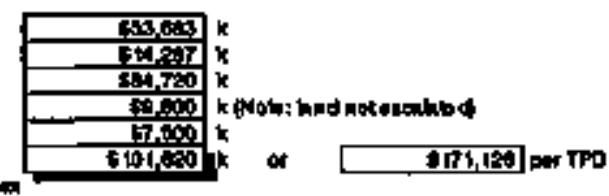

EConitanc:

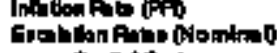
optacicox

asul cots:

Whithene Motula

inumeree

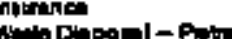

- Femmenimion

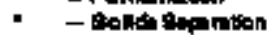

Cronkons

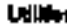

$$
\text { Powar }
$$

Hencito.

Then

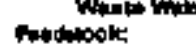

Outert:

$$
\text { Eximent }
$$

cos

Thy CK2:-

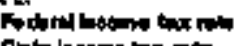

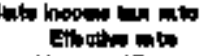

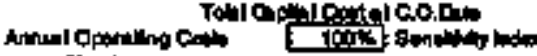

Plis

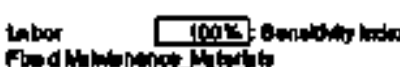

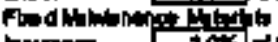

wilubl:

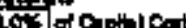

Whath thlowines

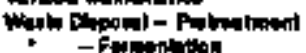

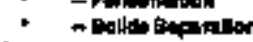

cumbin

Utitume

Poum

Wiming

thimal bien

whenterwer

orencots

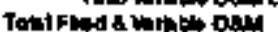

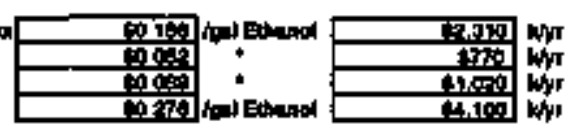

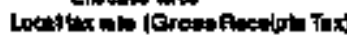

Propont Ter Rinte

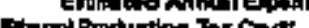

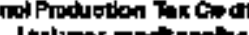

1

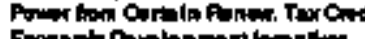

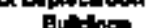

Dumbonent Coses

Bapman Comatip took topmetion
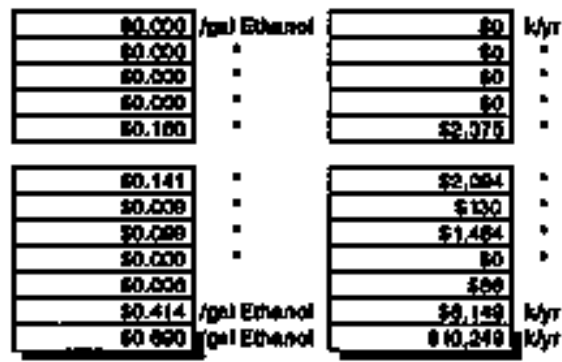

Denmols:

Aook thepent

hance orplant
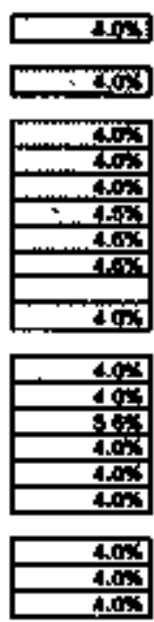

Dabe Fincion

Frenoly Itom

mintions

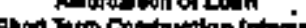

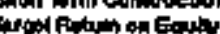

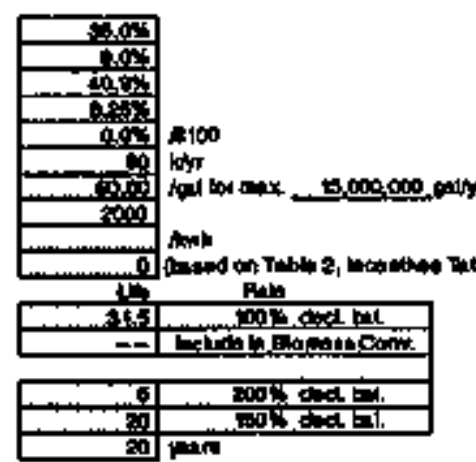

Futubek

Wan Fupe

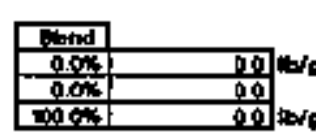

Tlophong Fon

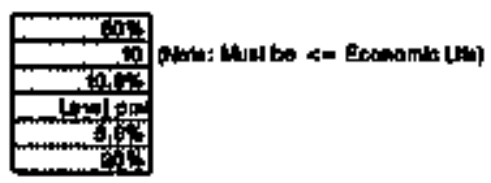

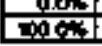

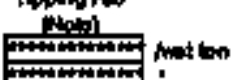

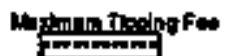

Fin

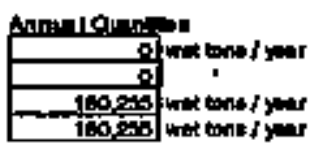

Ontout

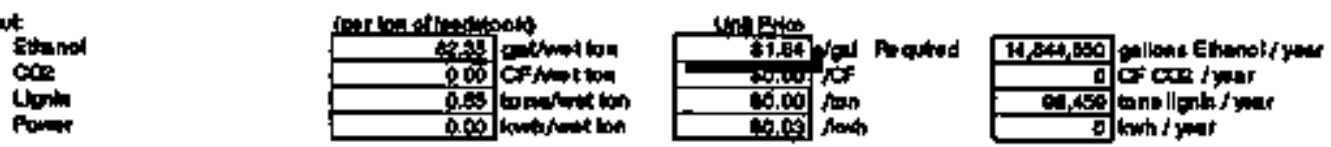

form

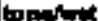

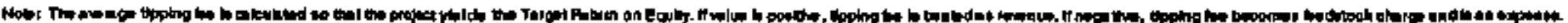




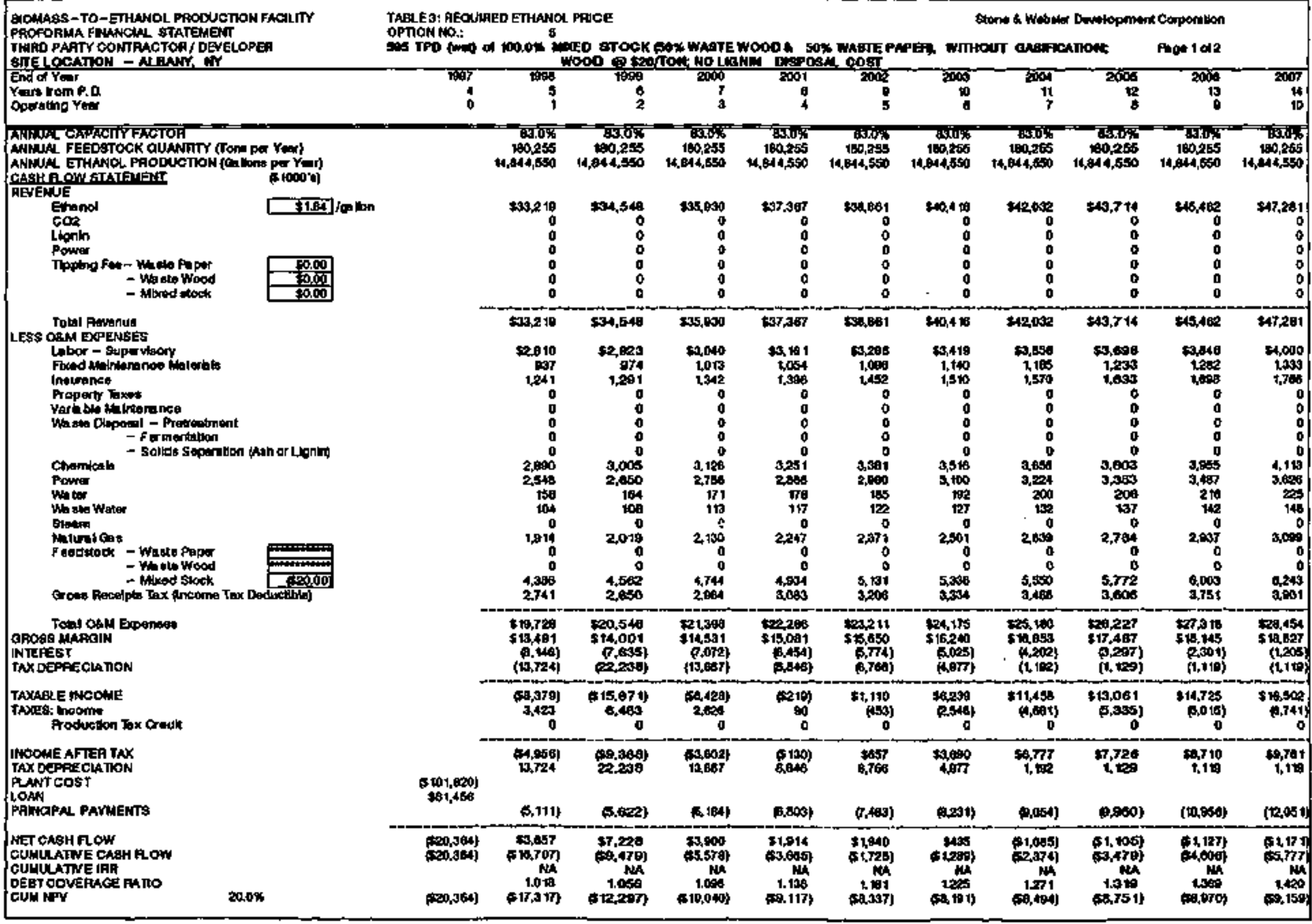




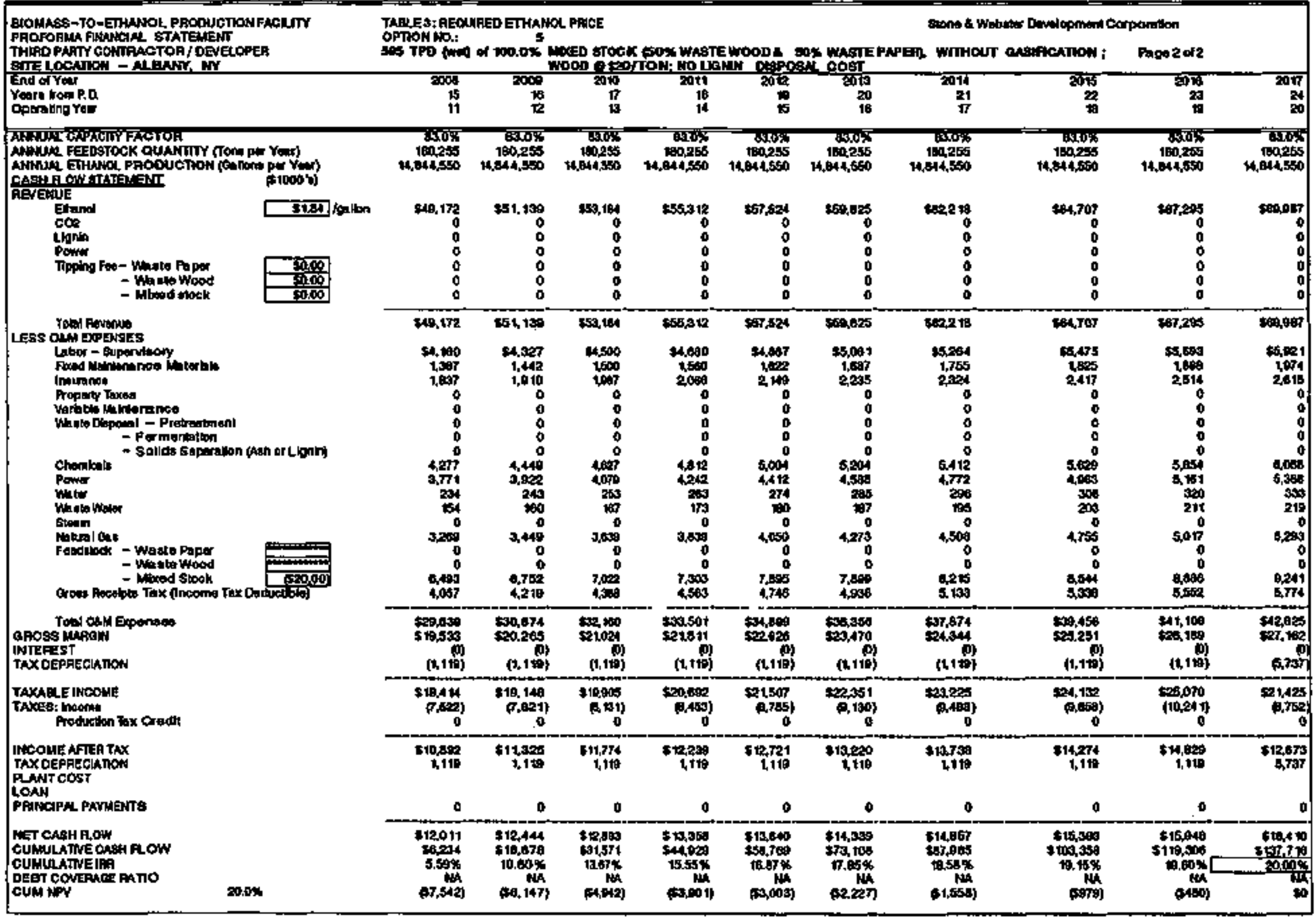




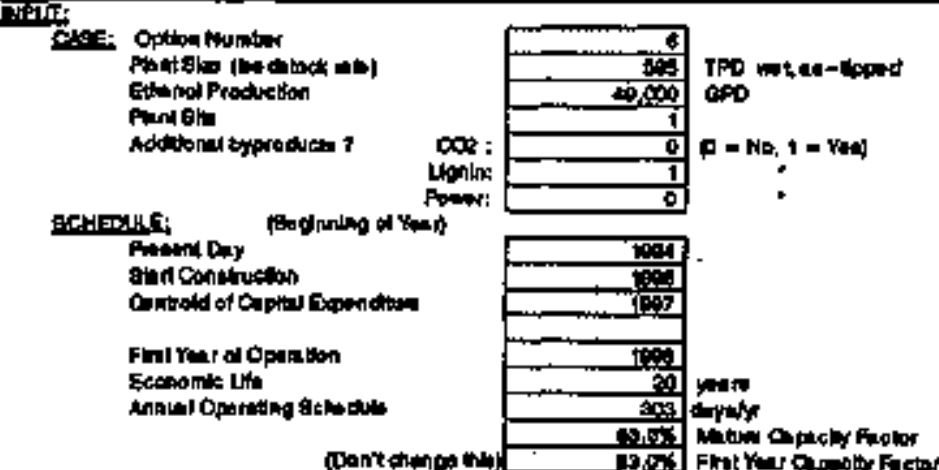

Tequiled

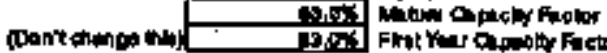
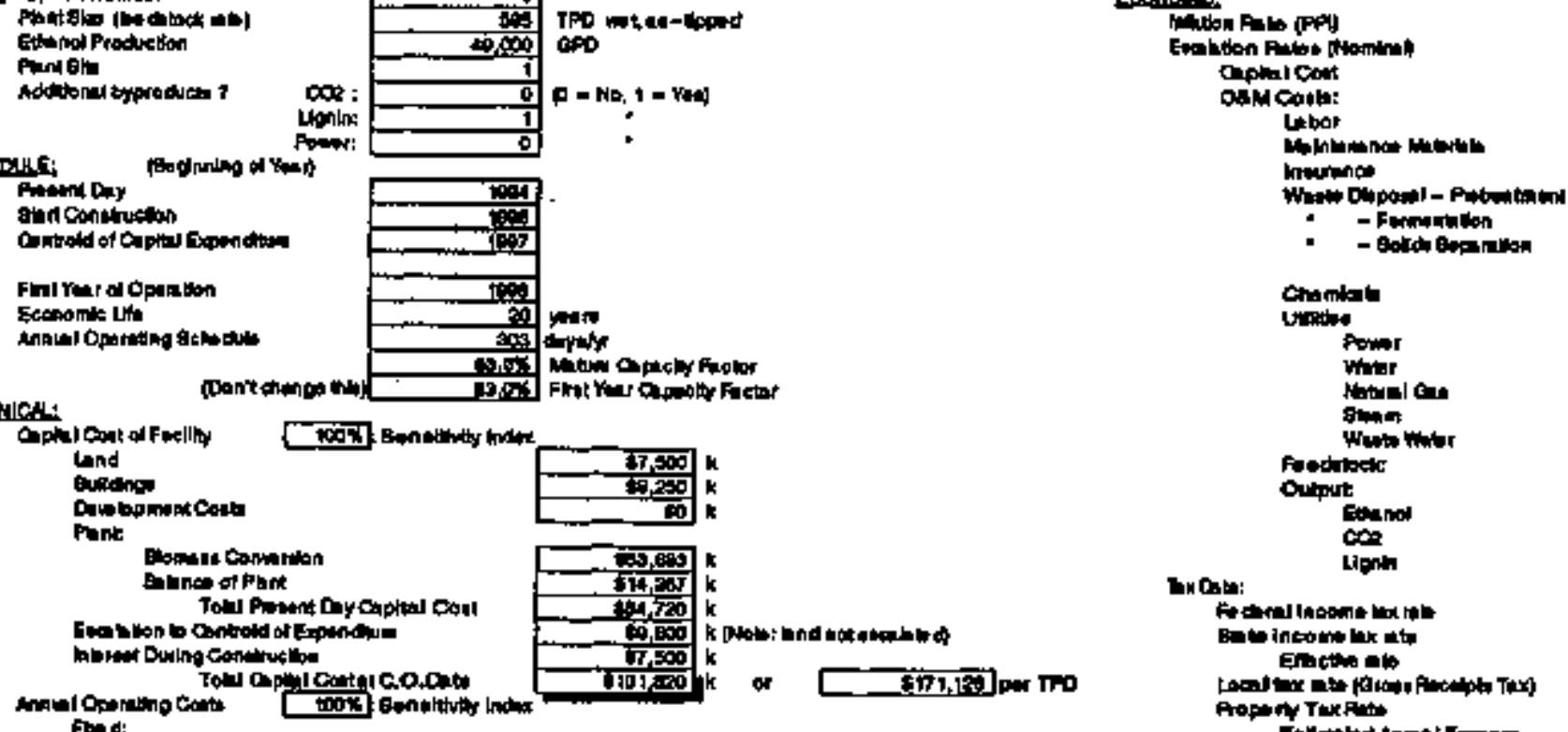
book Copnetuon

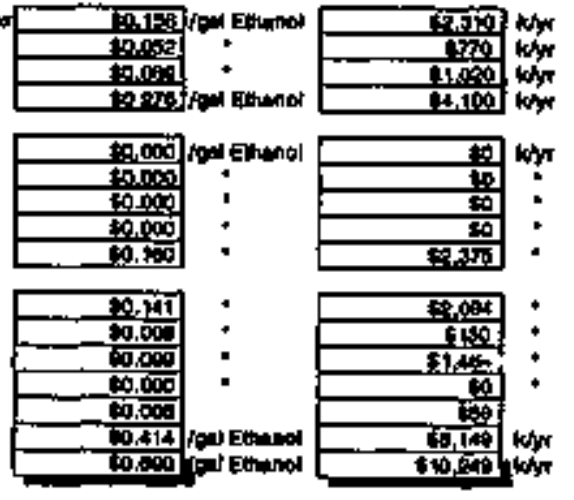

E...ments:

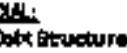

Dit Fration finenetng Torn

Nacritation of to

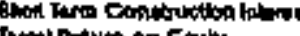

turpiliadim on Equly

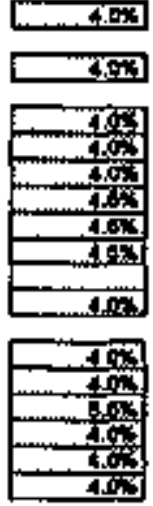

Thoping Fis
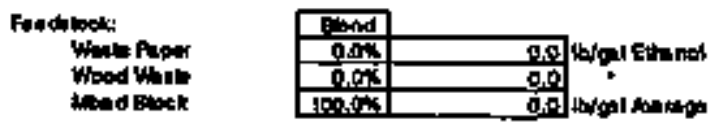

Fint ton
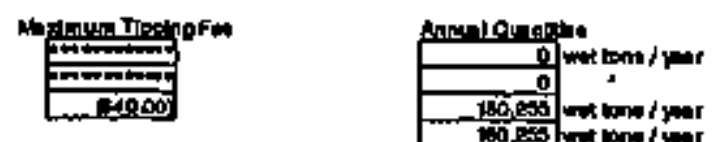

dortr
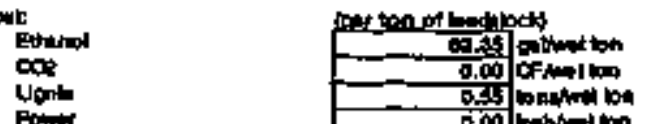

- T.
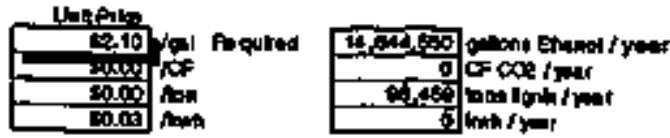

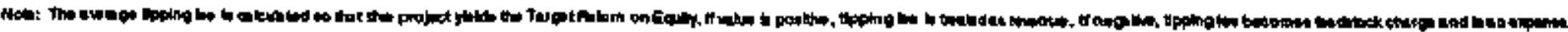




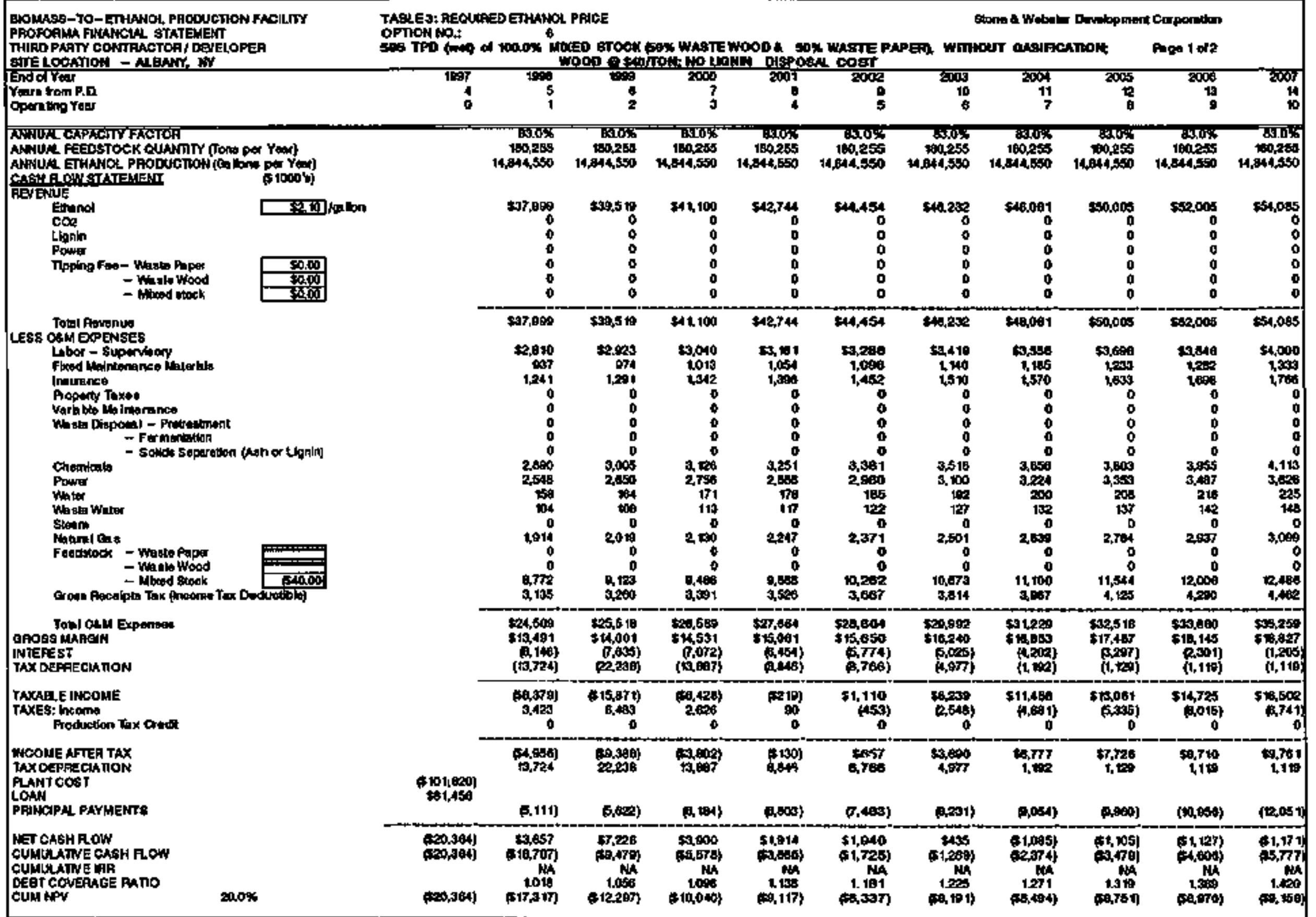




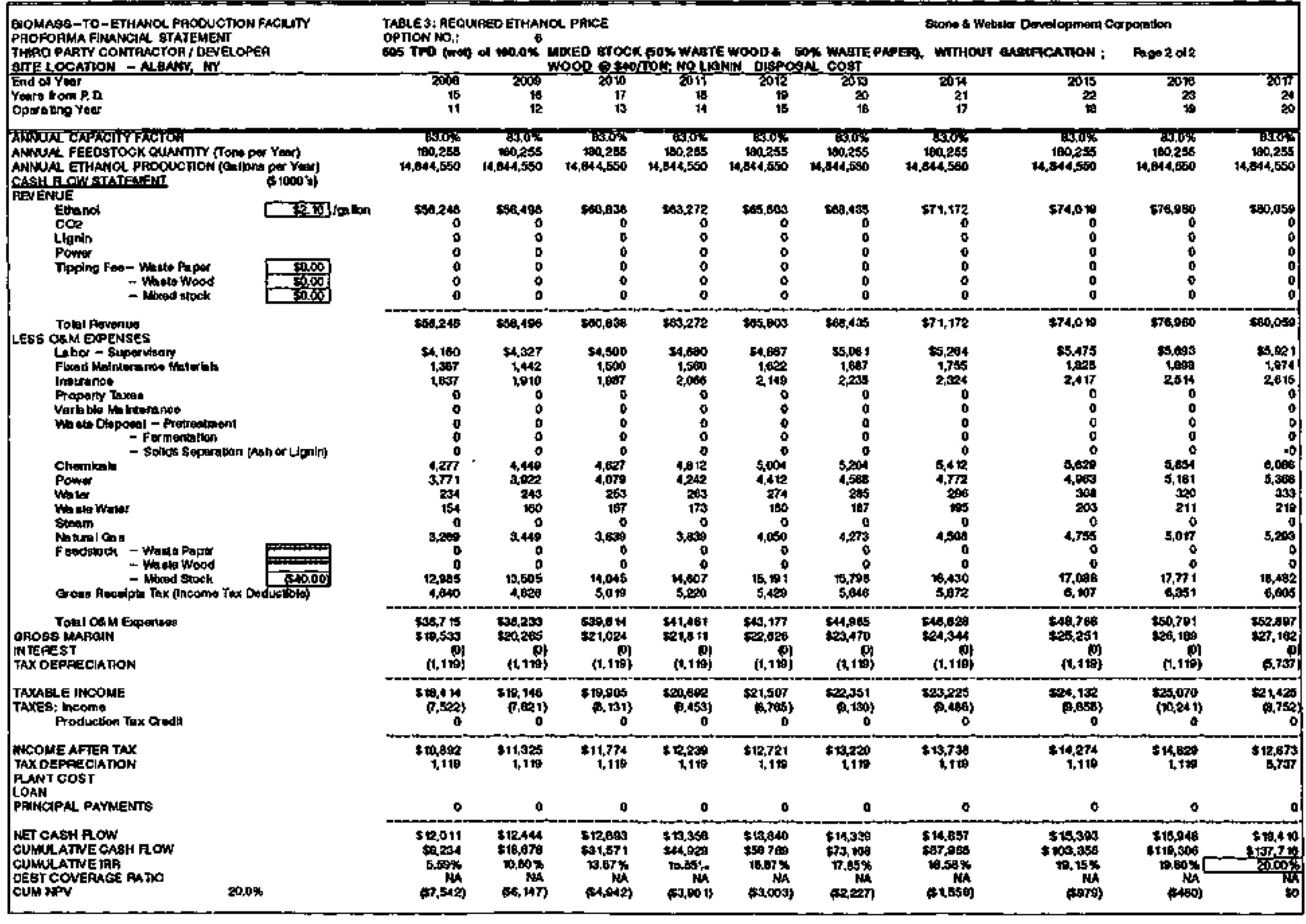

03/3095 


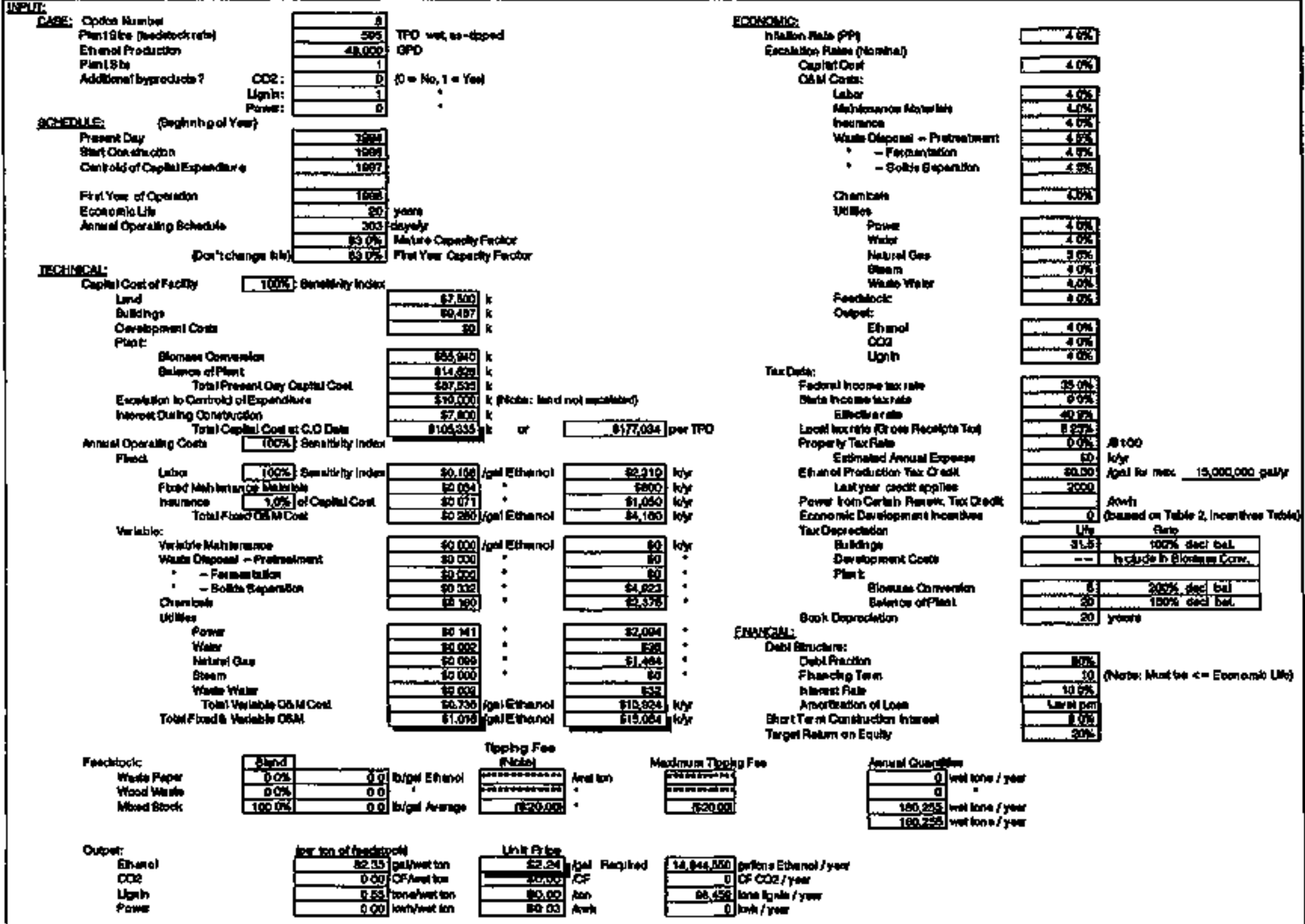

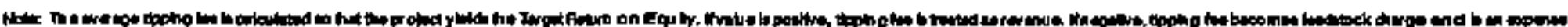




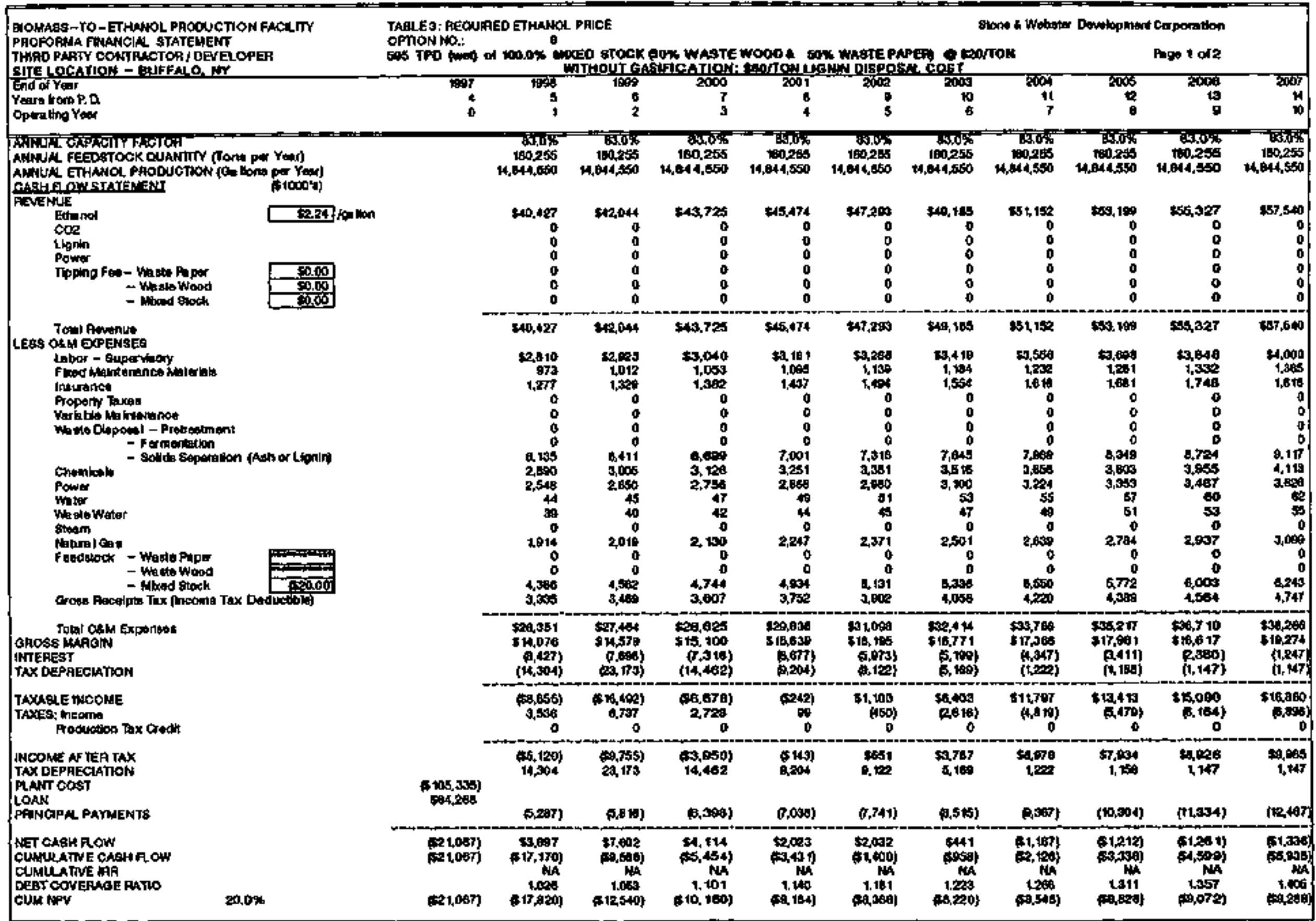




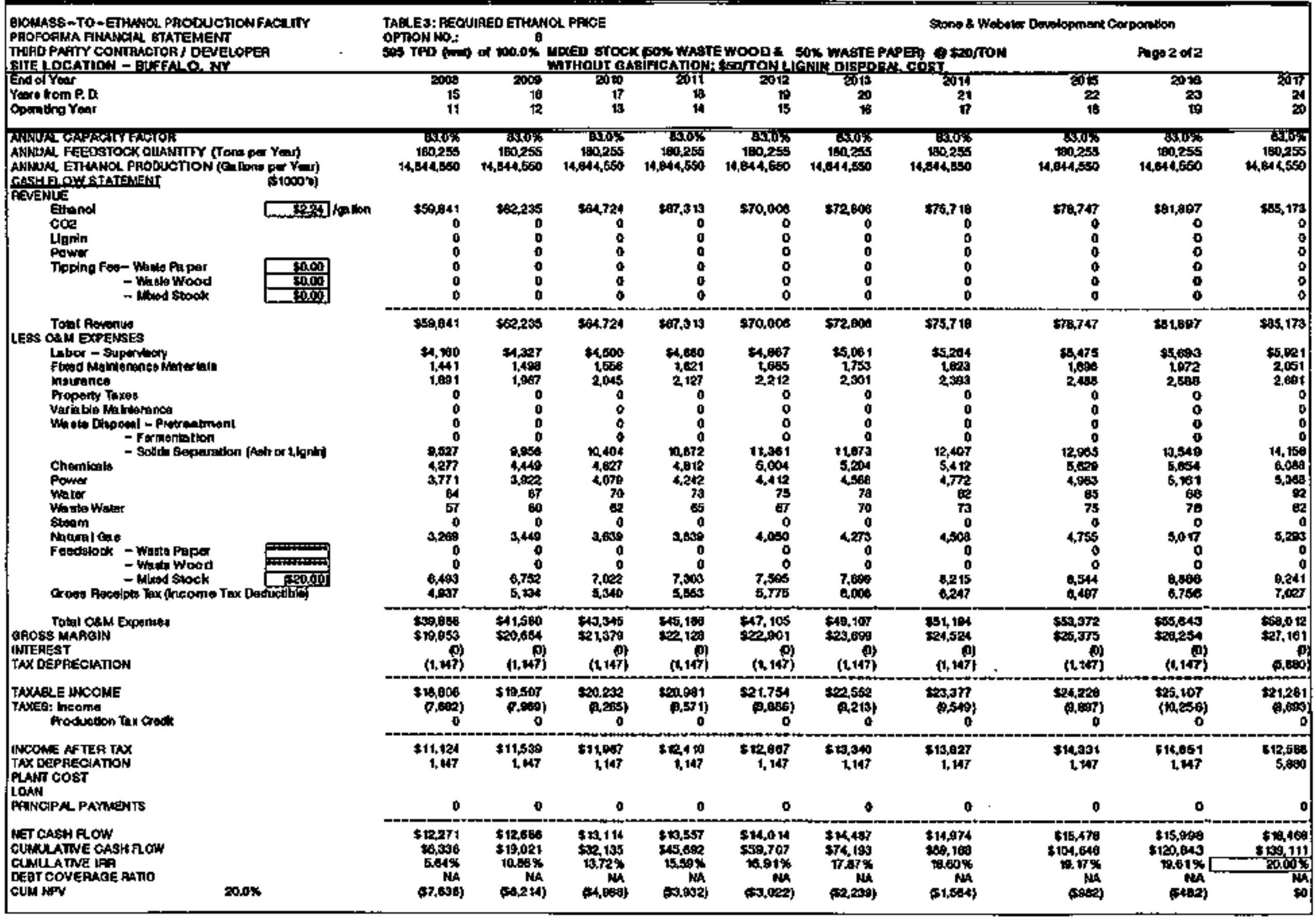




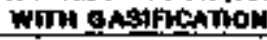

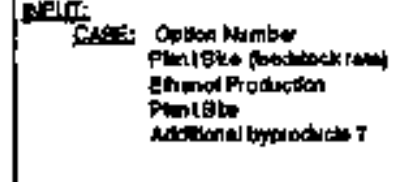

ecasaine: Lonin
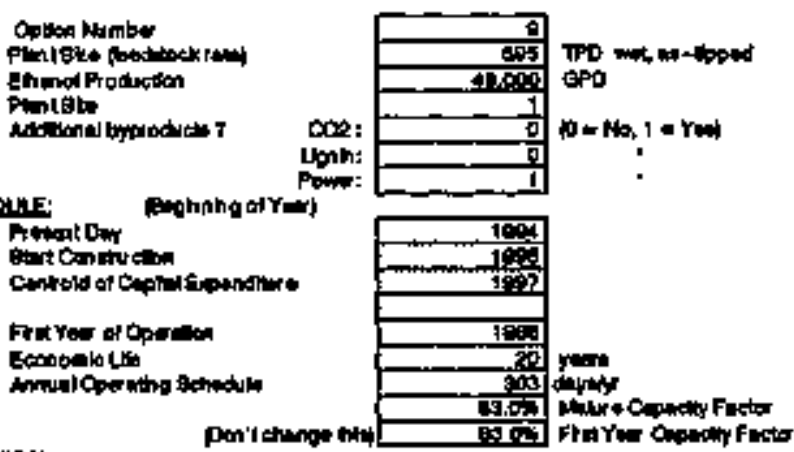

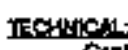

Pen'letenge thit

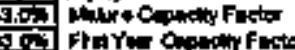

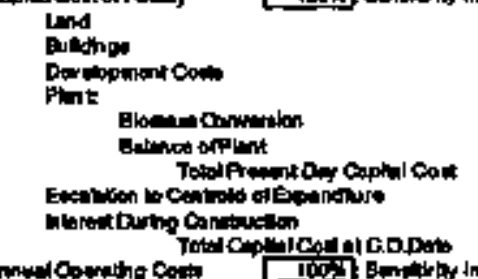

Torbomponititan
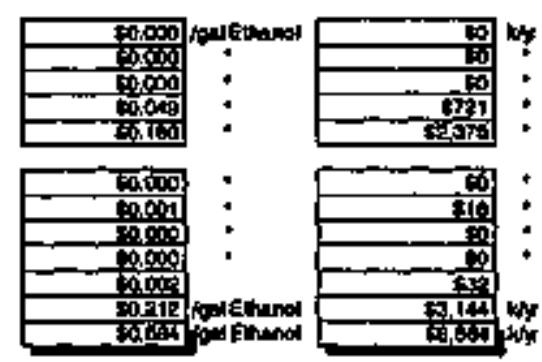

oniment cont

Plats

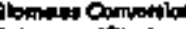

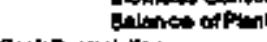

Eviniting

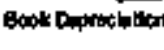

Oesbstantur:

Dablifution

howotions

Aroctiation of Loen

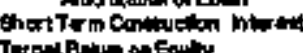

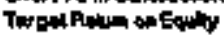

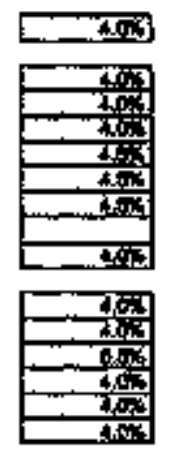

Tophig Fo.
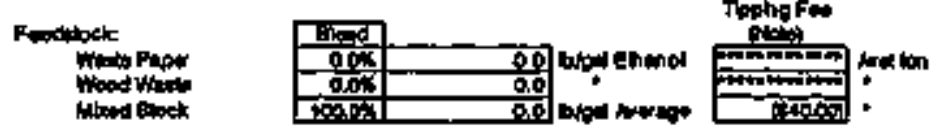

Nom
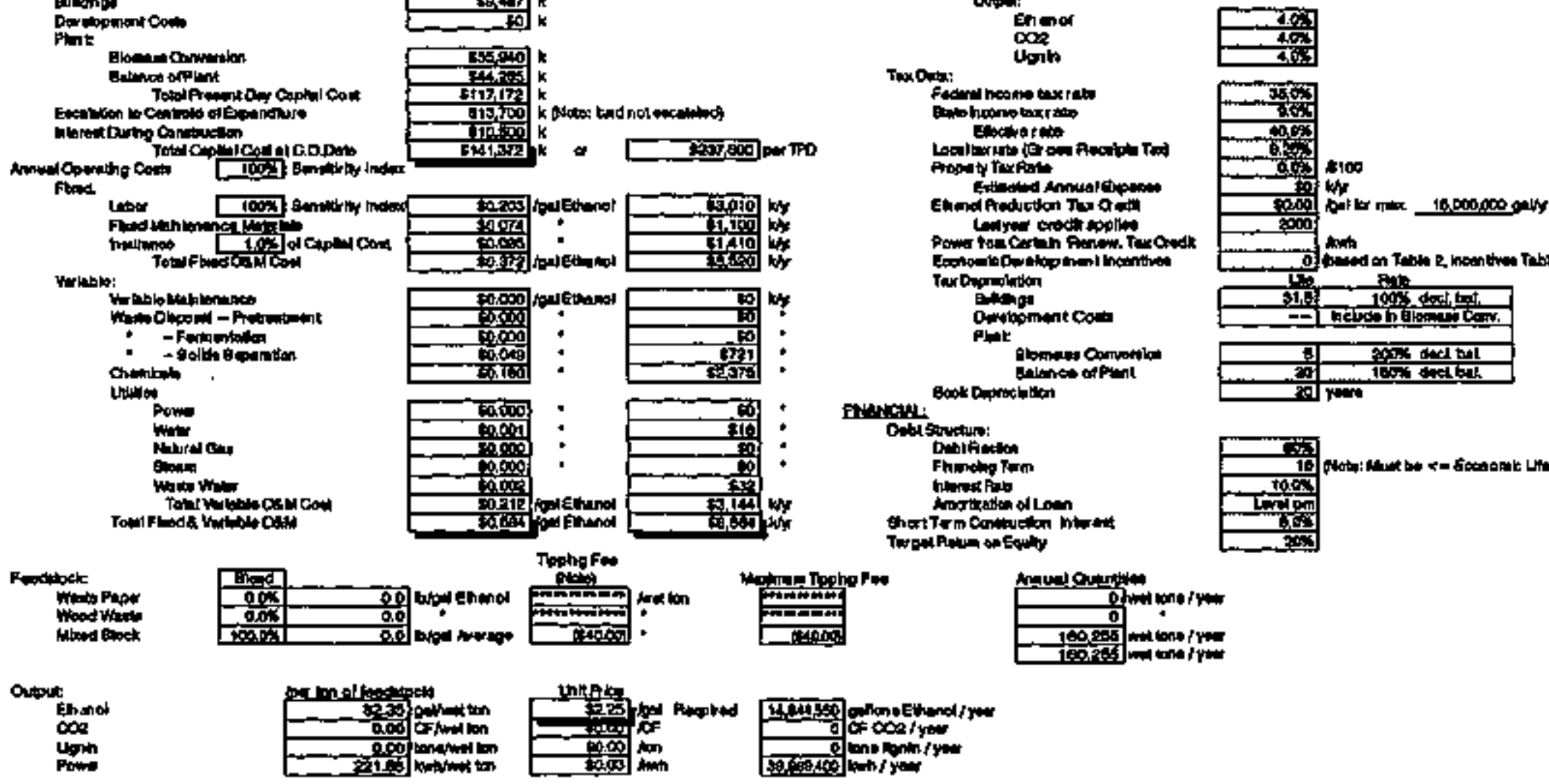

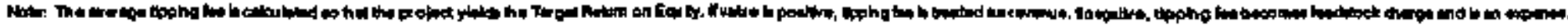




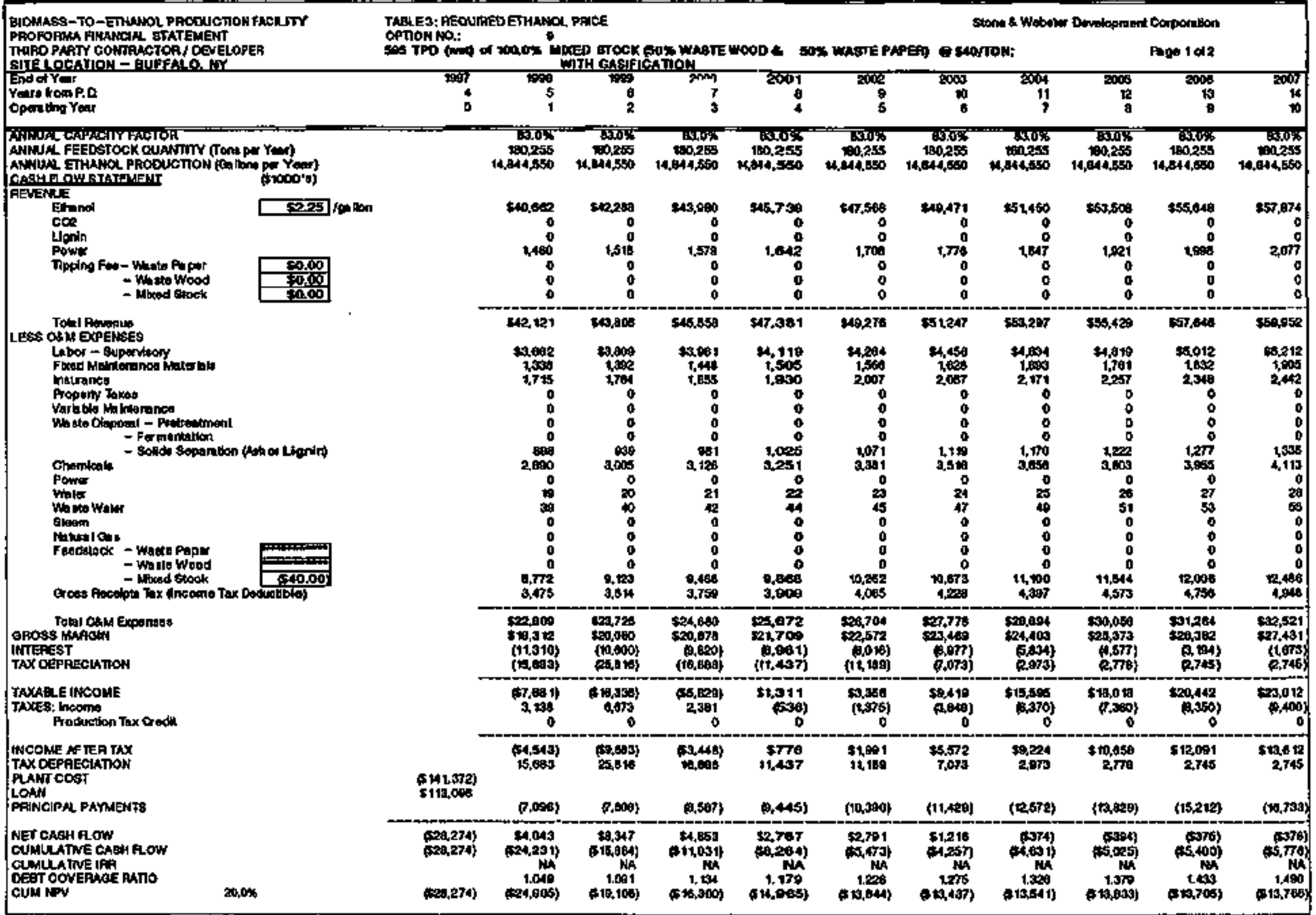




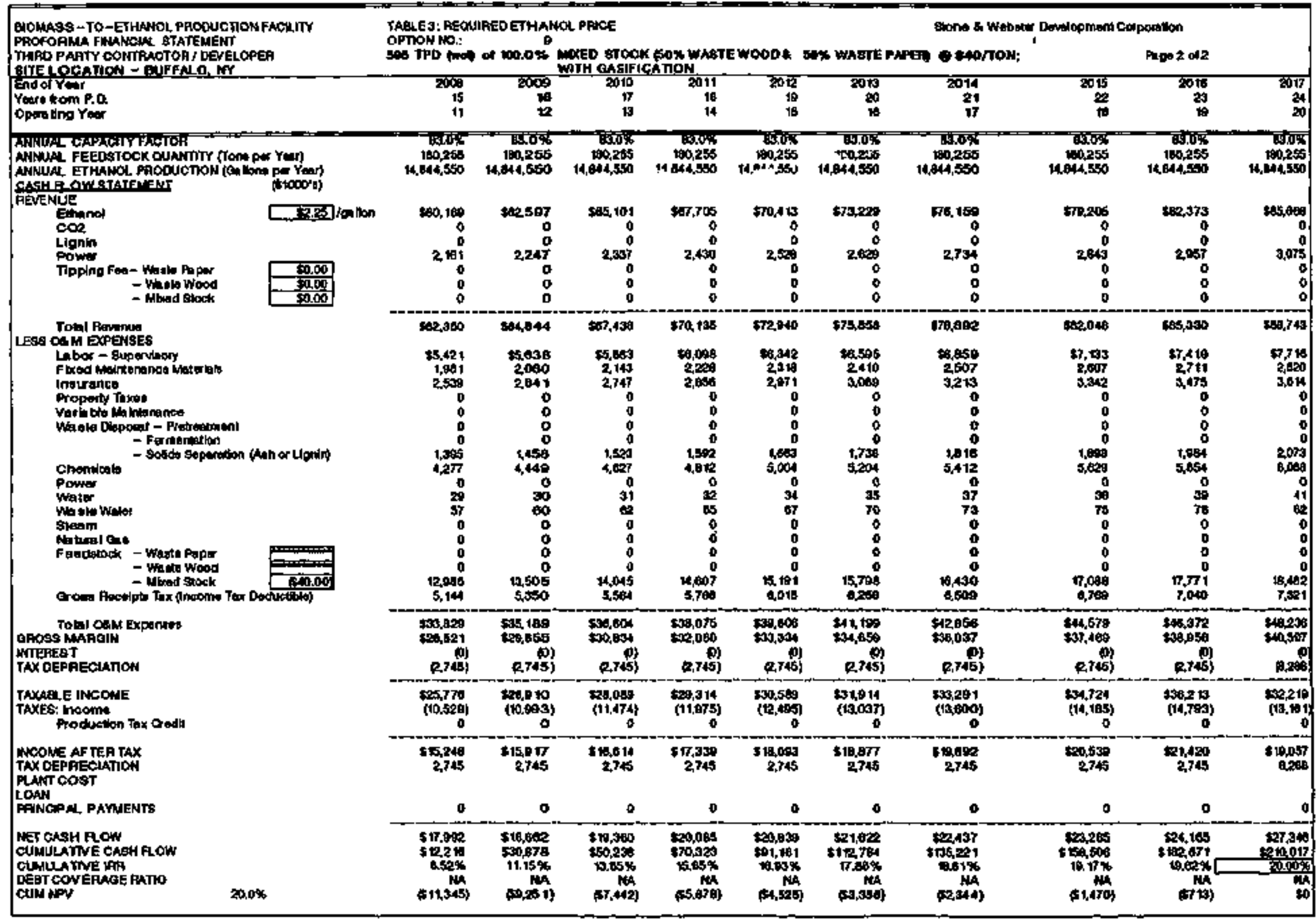




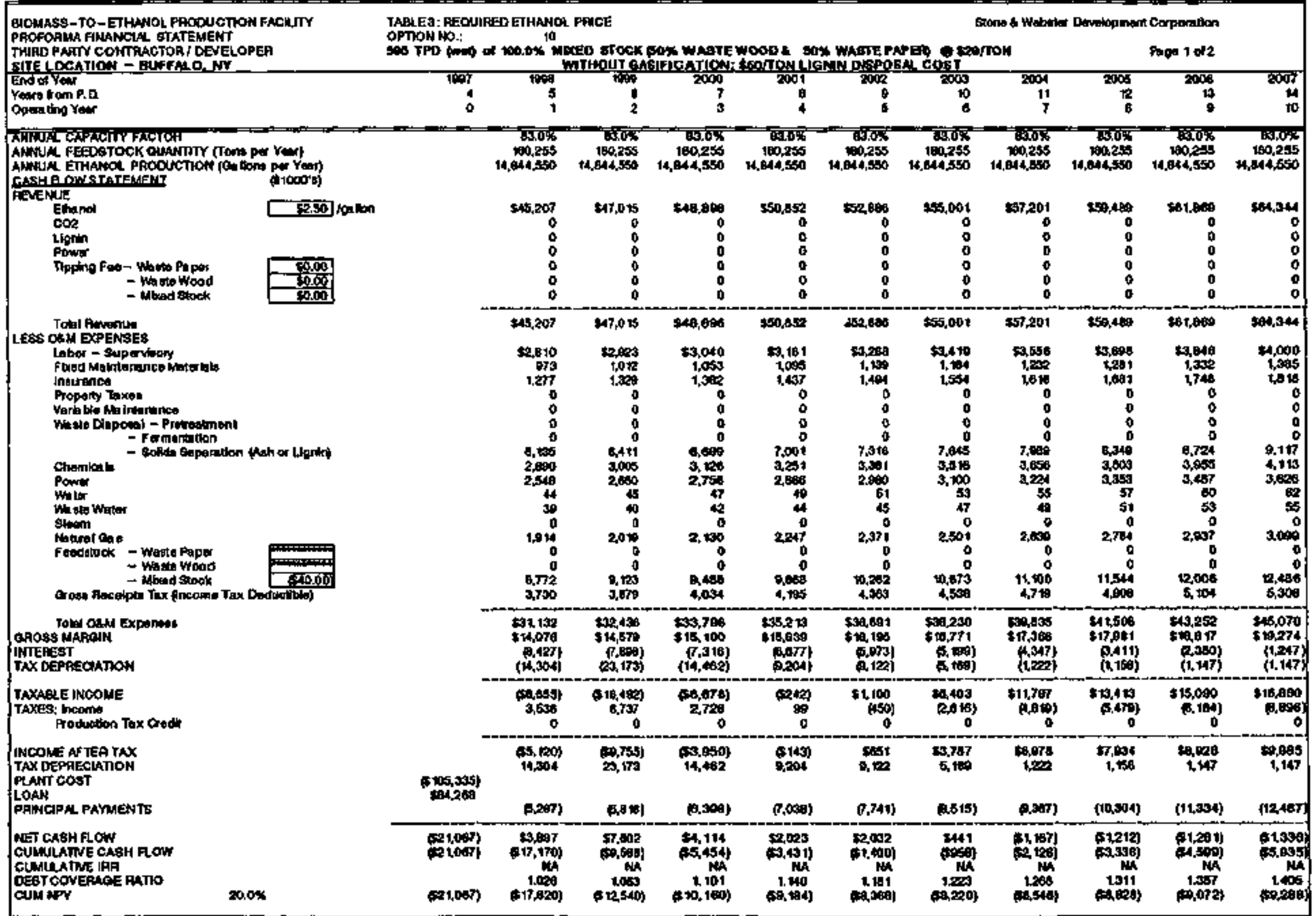




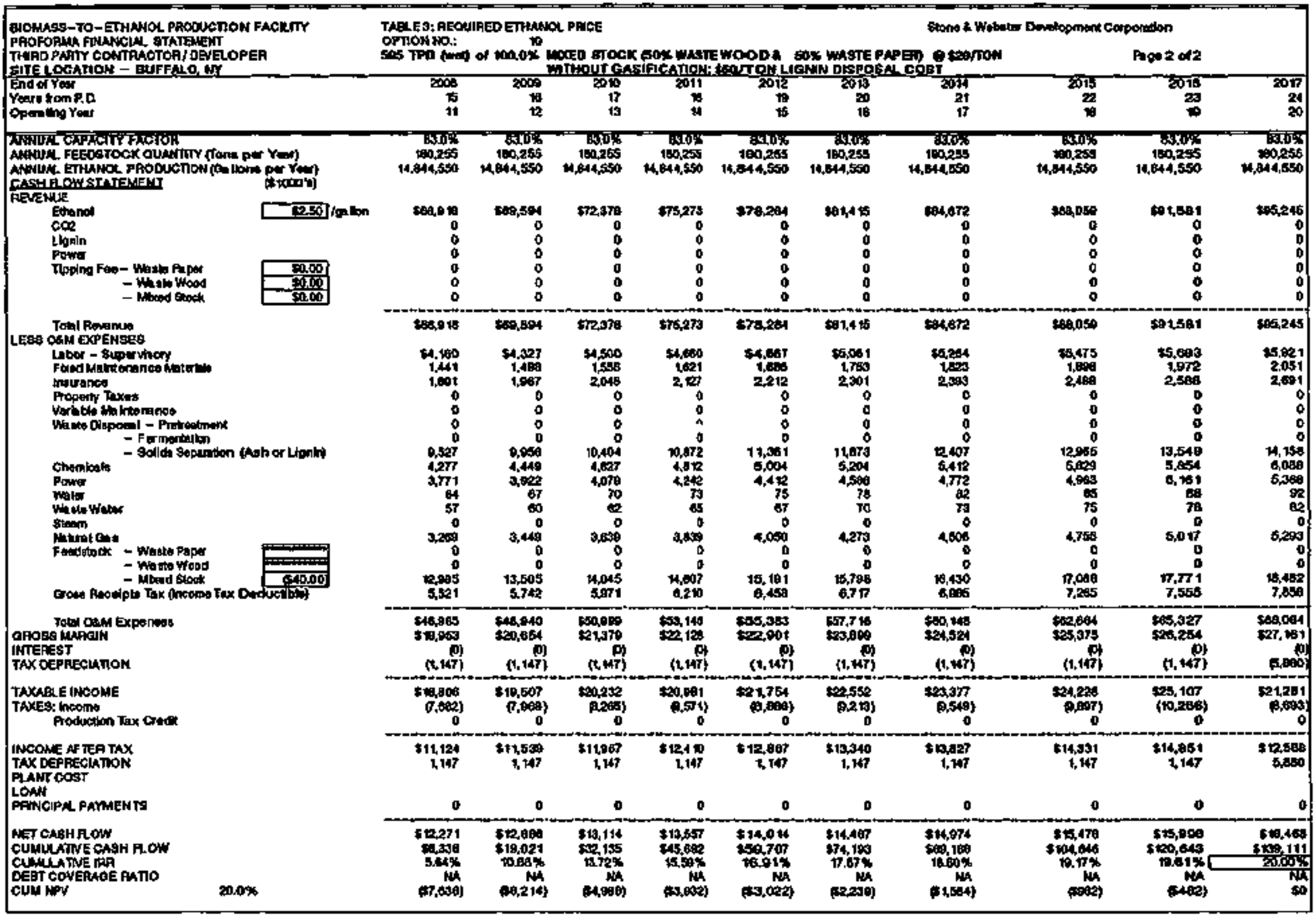

aspops

$n$ 


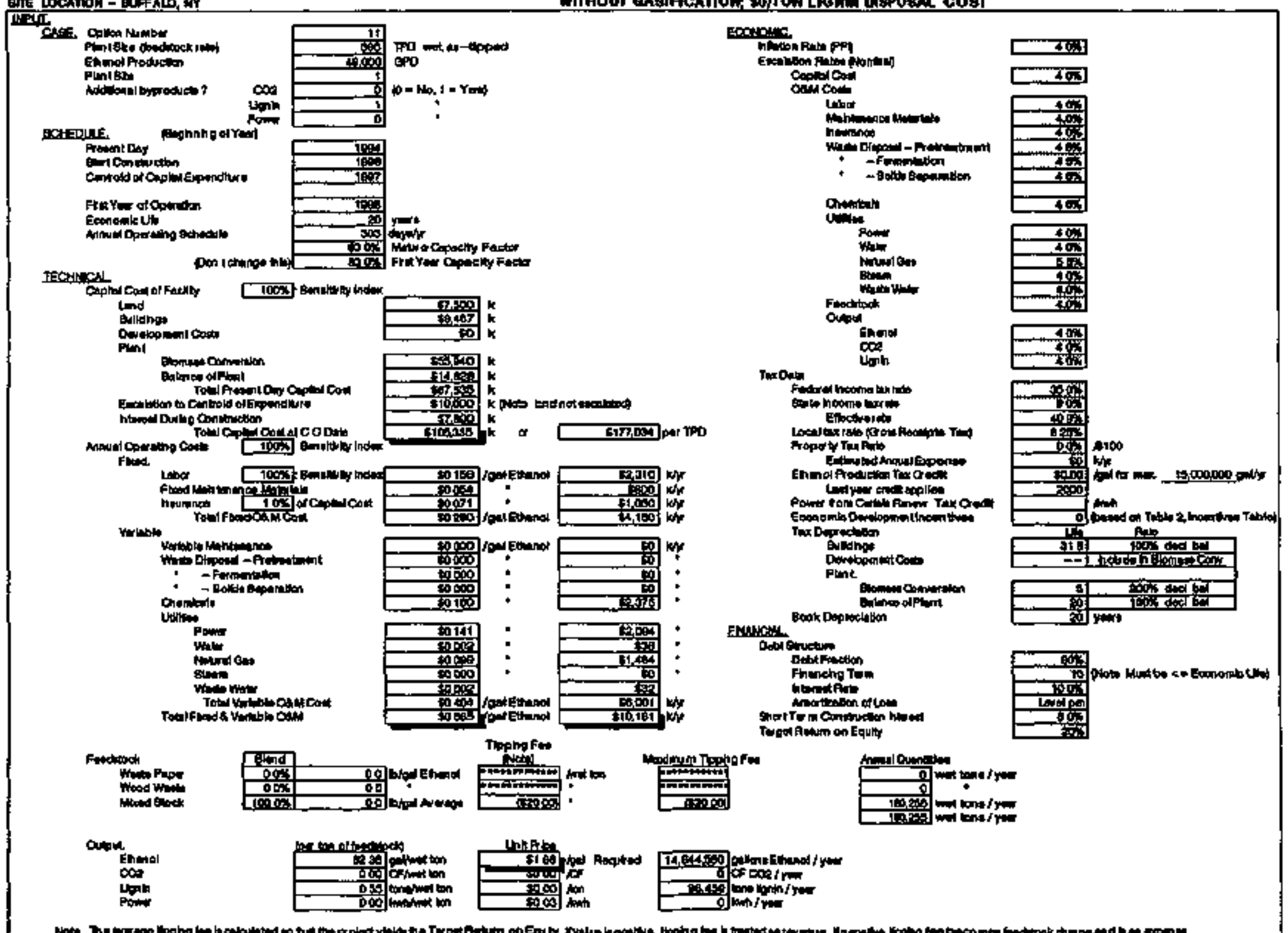

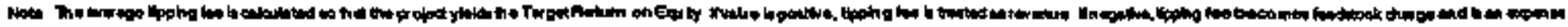




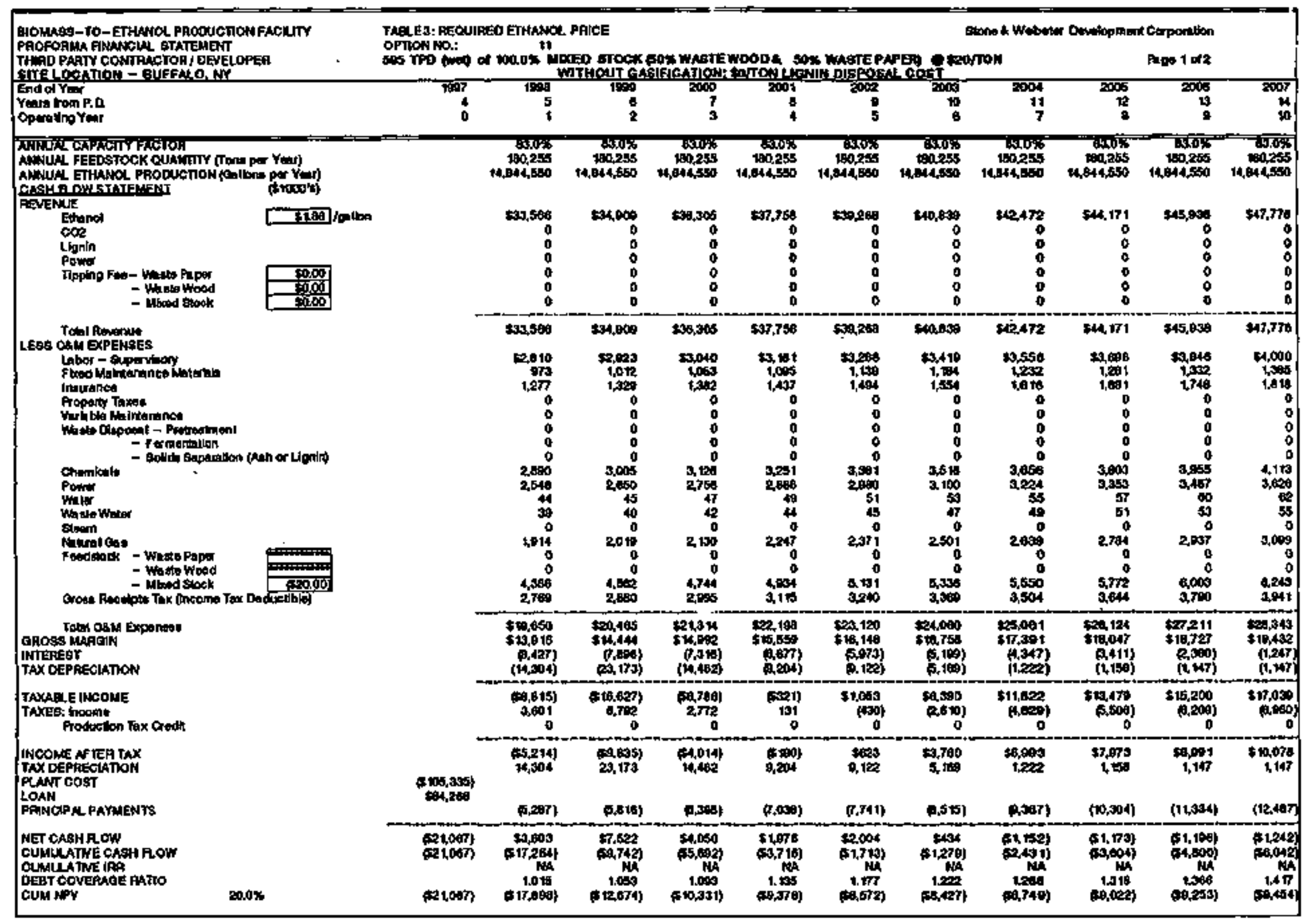




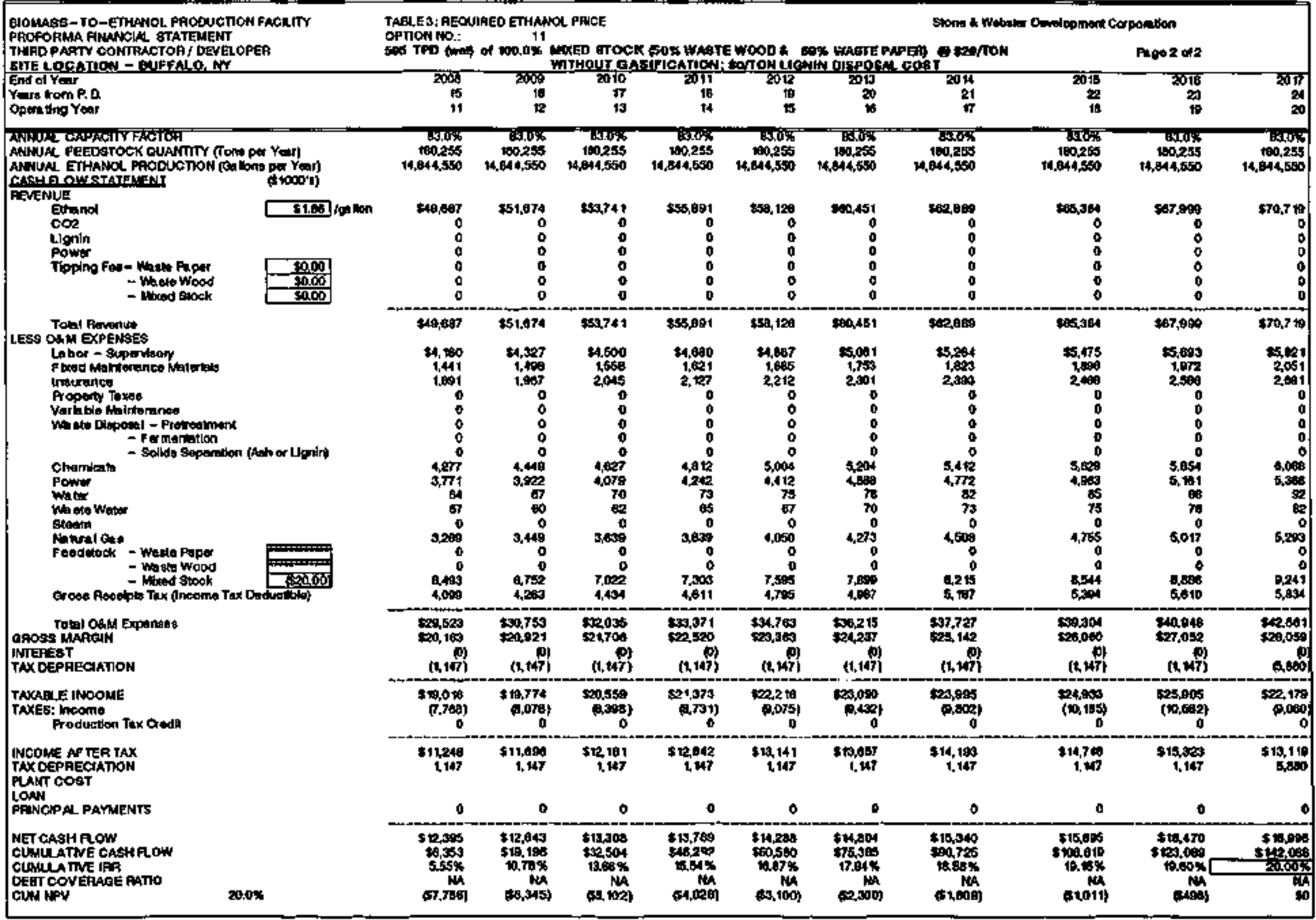




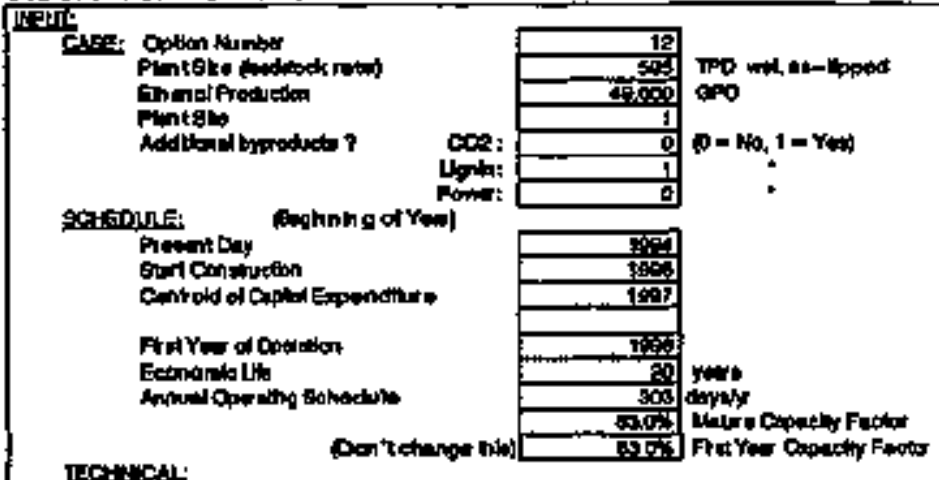

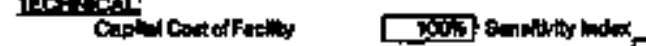

$$
\text { Ind }
$$

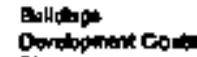

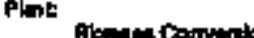

olbonim Cominatikn

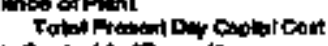

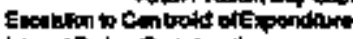

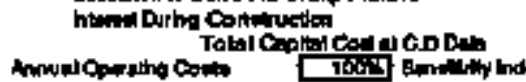

Fhom

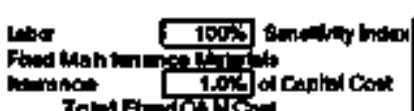

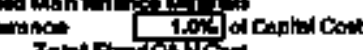

then
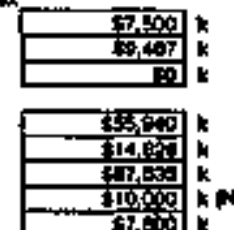

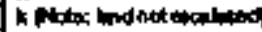

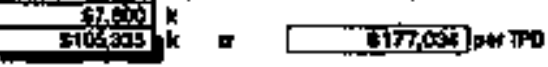

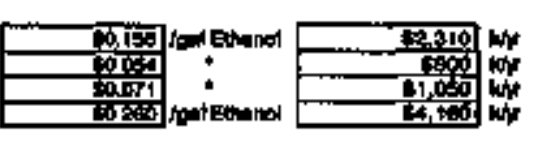

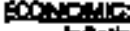

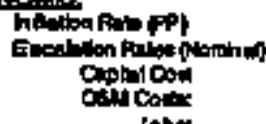

labor

minines

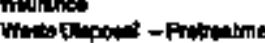

- -Fothumbition

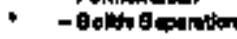

Ondmbet

bitilin:

pant

thiontan

Him

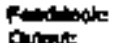

Ouput:

Exer

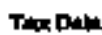

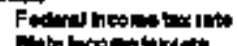

(and

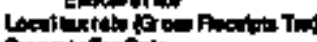

Papary Texpose

The

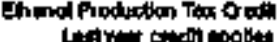

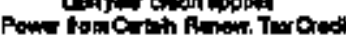

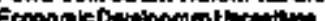

Tuxpopicimiten

entump

Denotomint Cot

Put

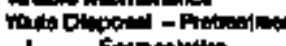

: - Fernemition

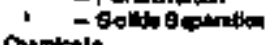
Onimeato

Colon

Pomplit

Naterot tom

waste wate

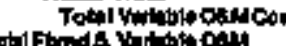

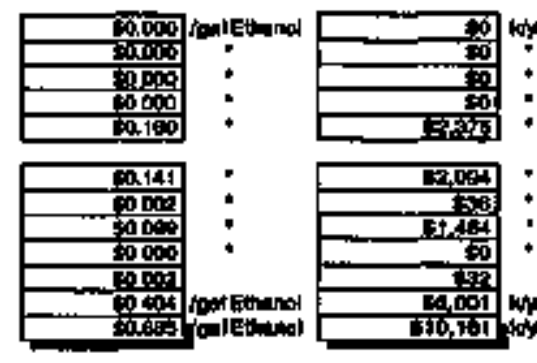

Exaction:

Book Doponotitor

IFII PA

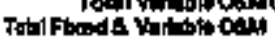

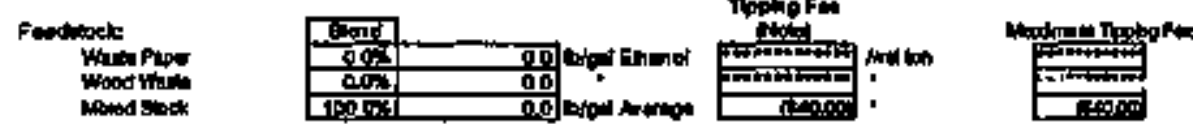

Dething

Fhenchint

homenters

Amatiention of Lom

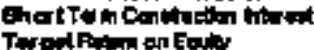

Twodpinon an Exhy
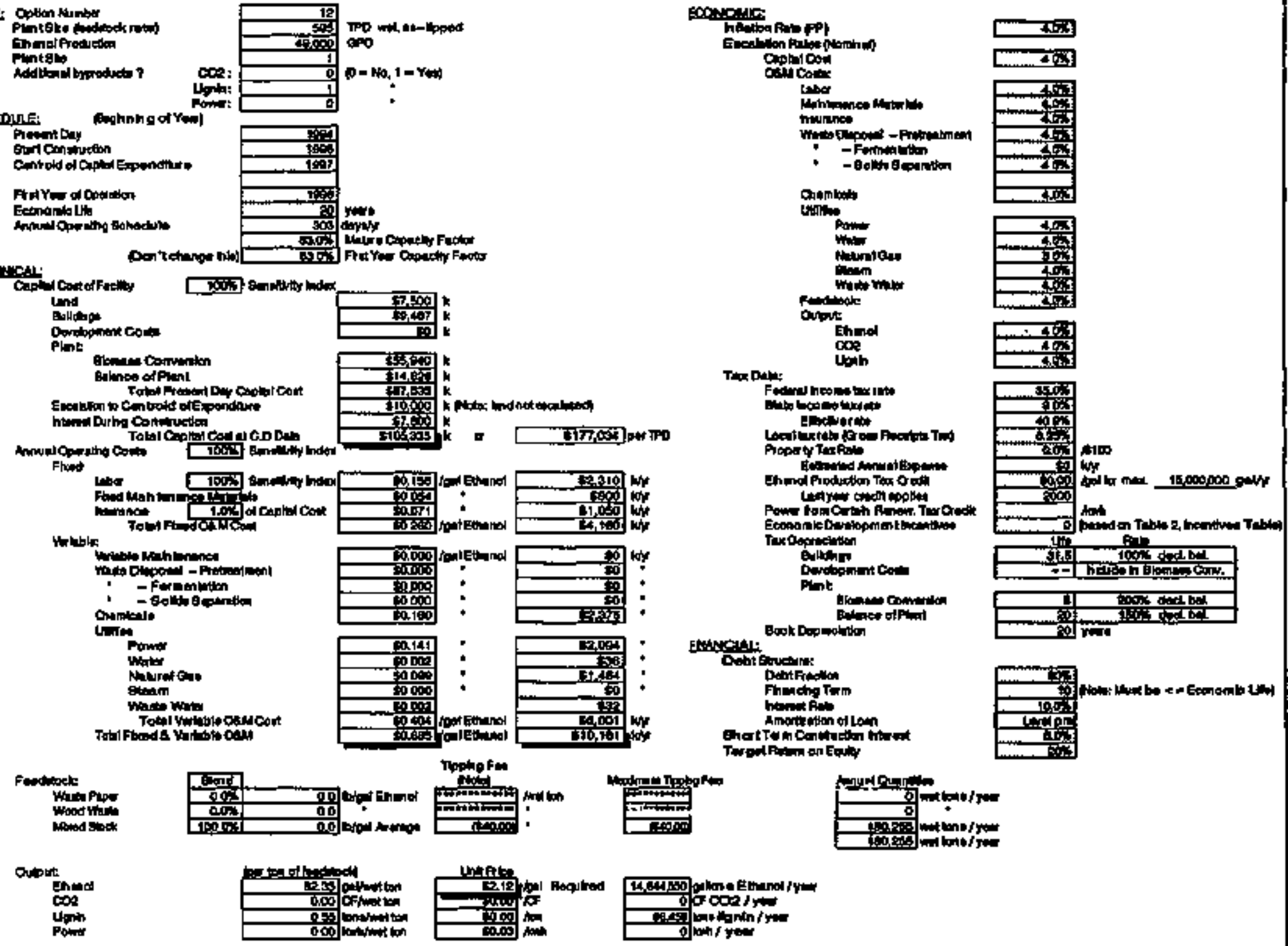

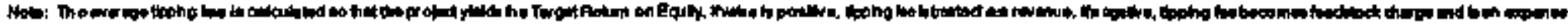

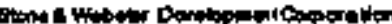




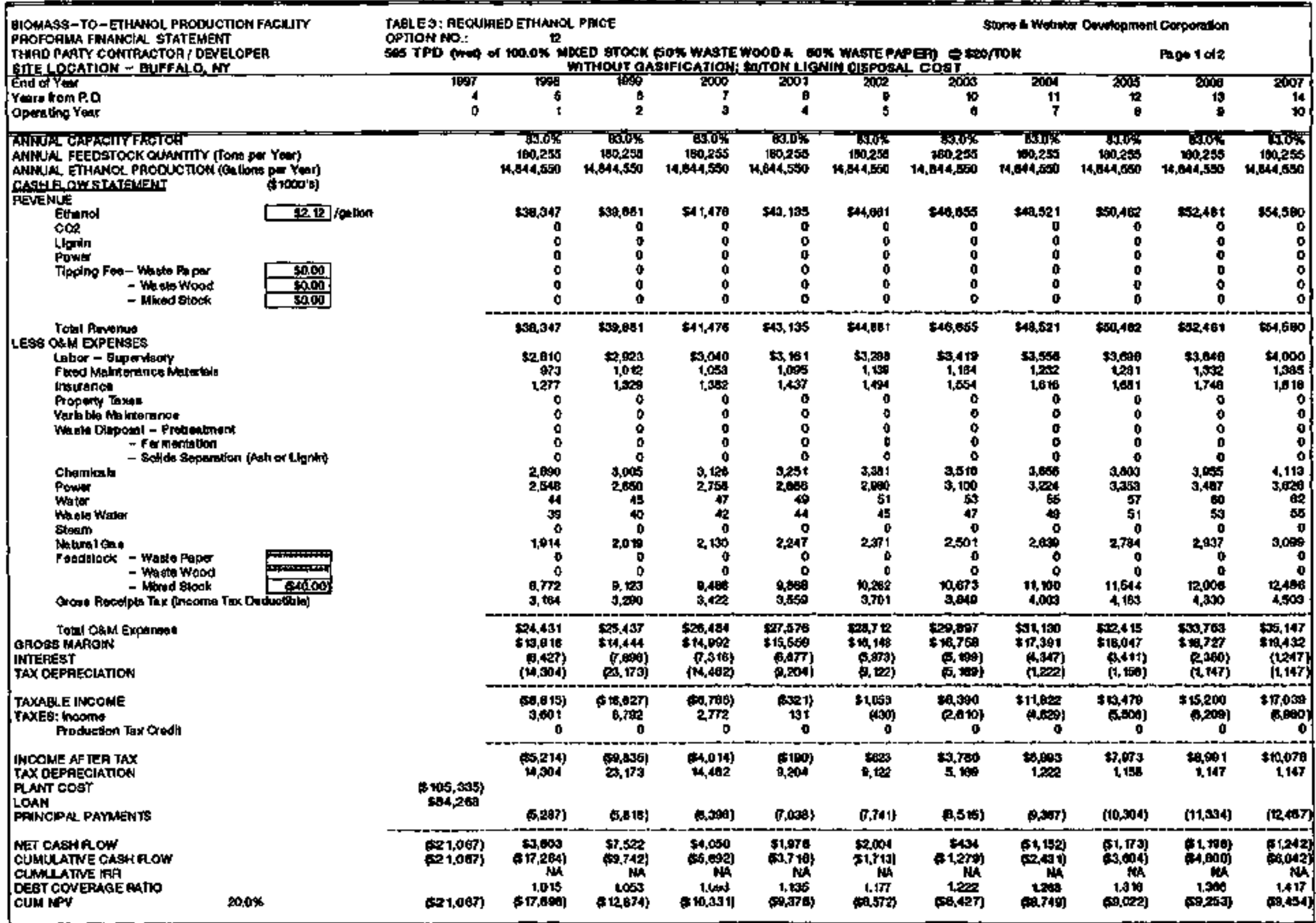




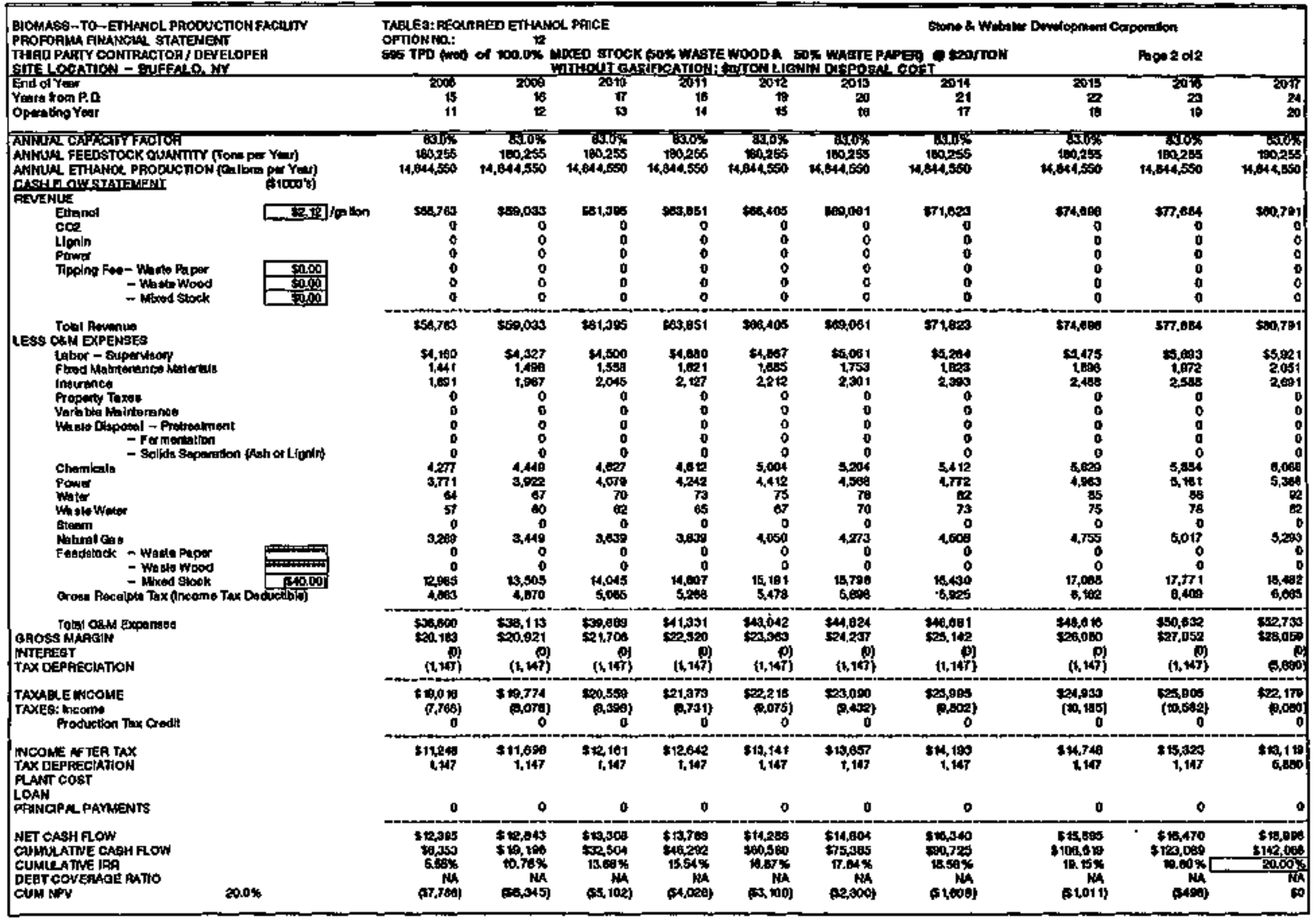




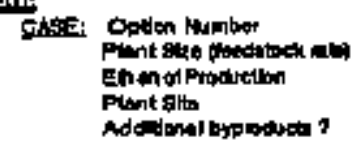

Ehise: Ootion Mumber

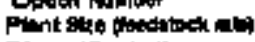
Enmingripatelion Plent alto

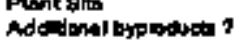

Softenulet:

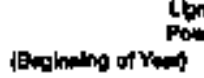

$$
\text { Prosant Ony }
$$

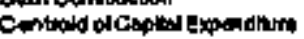

Fint Yow 어 Opention

Exompante Lito

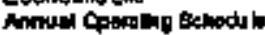

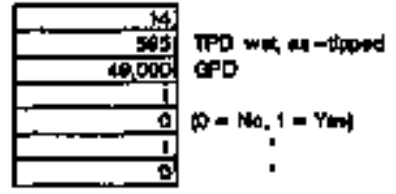

yeaty

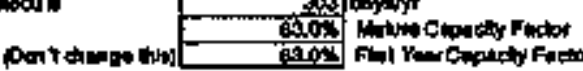

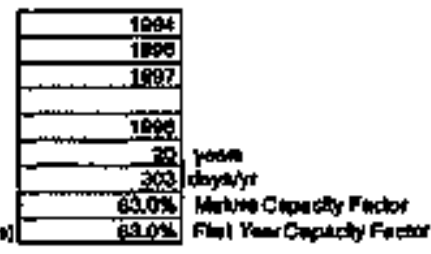

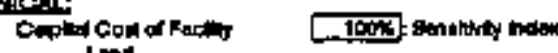

Euminos

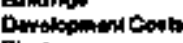

Plent

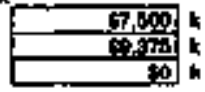

$$
\text { mones of Punl }
$$

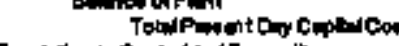

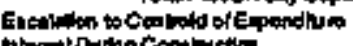

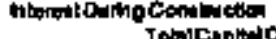

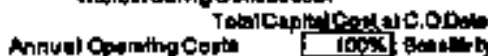

Frant

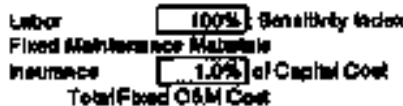

Vortion:

作

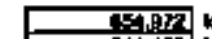

S14.155

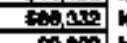

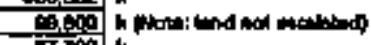

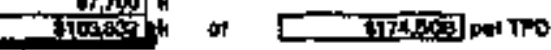

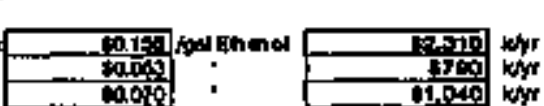

0.00 .

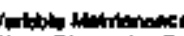

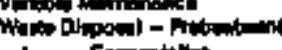

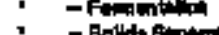

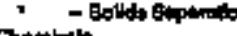

Conting

Pom

Nentalen

Dawer

Want woter

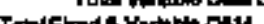
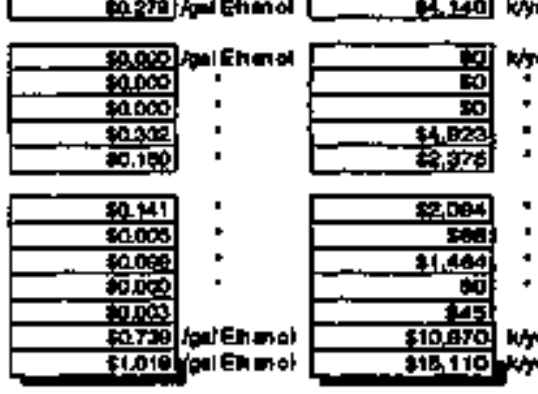

\section{Inolog Fon}

Fondibate

Wortum Paper

Hood Sinction
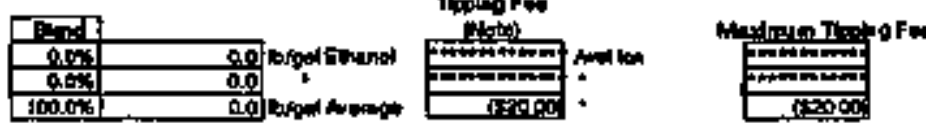

Exons:yri:

hrotor fien opt

Con Fum omanis

Cophacowis:

Lon

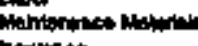

ramimation

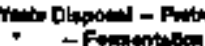

- Bolda Bepentor

Chatinkate

Utulition

Poner

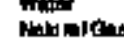

Givern

Fondibet:

Dript

Ehes

tonim

Taxcola

Fedend heome bx a

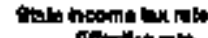

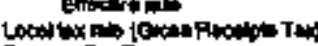

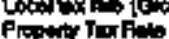

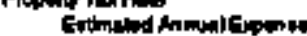

作

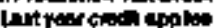

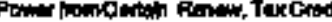

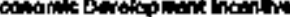

Torominowition

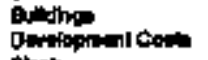

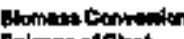

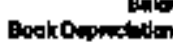

Guandist:

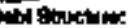

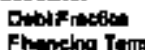

Mnciations

Amortinaten of ton

mitin or For

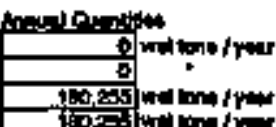

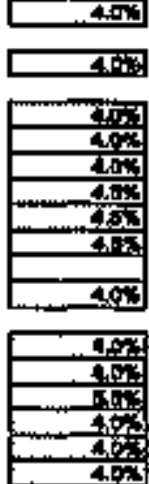
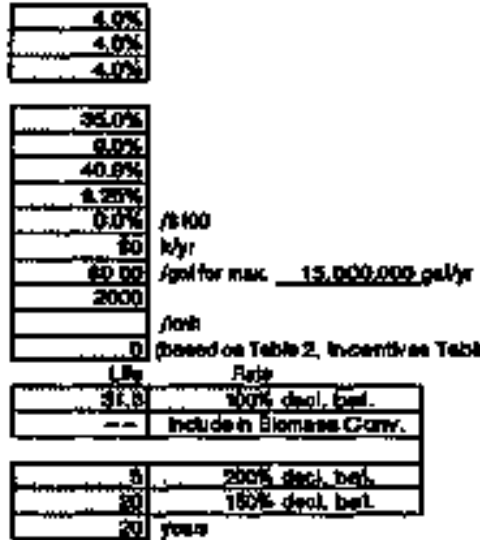

齐保

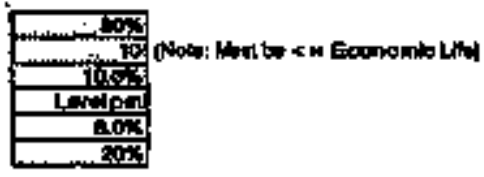

Outout En

$\cos 2$

Cor
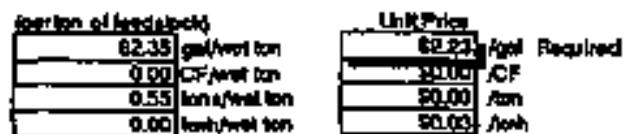

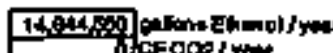

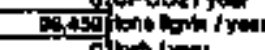

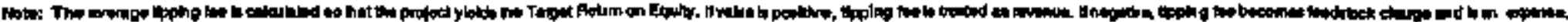




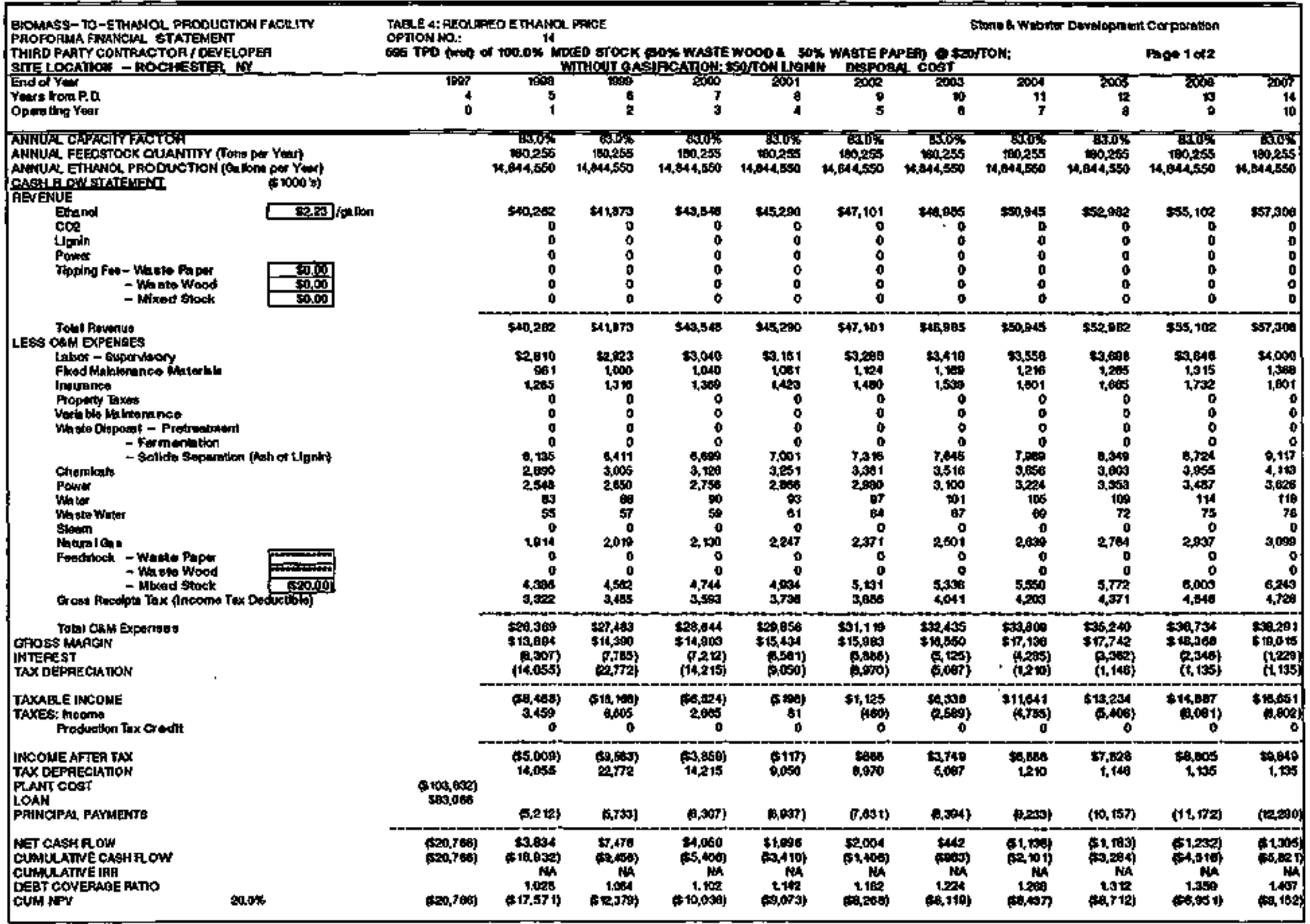




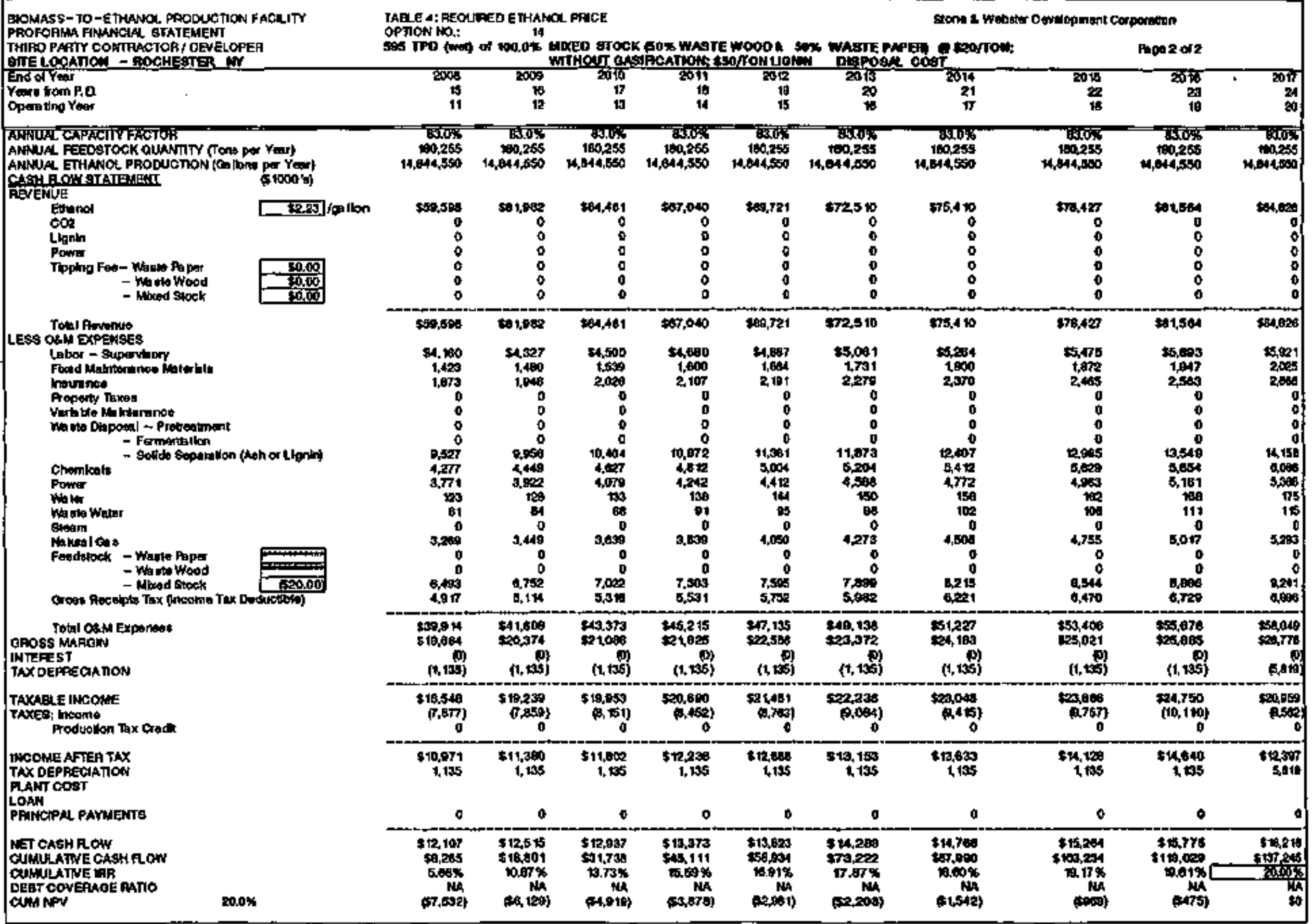

araos 


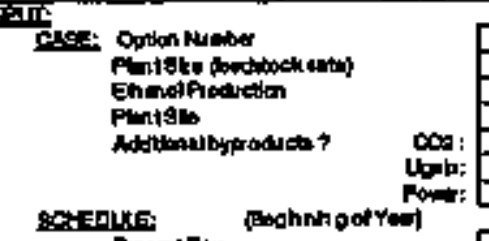

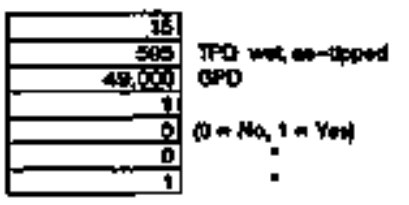

Promtdoy

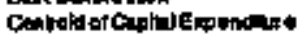

Fiprear oroptration

Teenomiculo

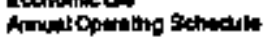

Ifectarial:

Ron'tangentive

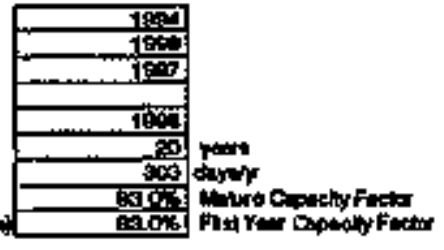

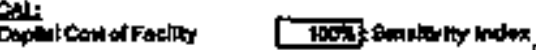

Inilong.

Ownopint con

Plani:

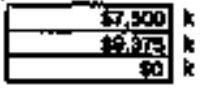

Bombia Comminion

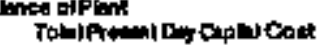

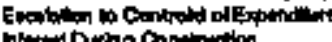

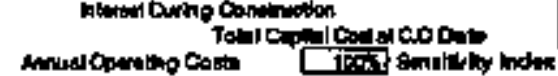
Find

$$
\text { fober }
$$

valublo:

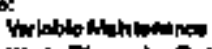

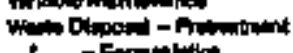

: -Forminton

antomal.

Un:

Pond

Nabralans

Etwort

wast min

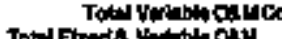

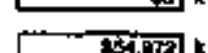

- M115

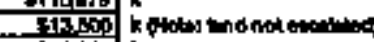

-

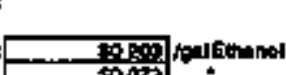

en
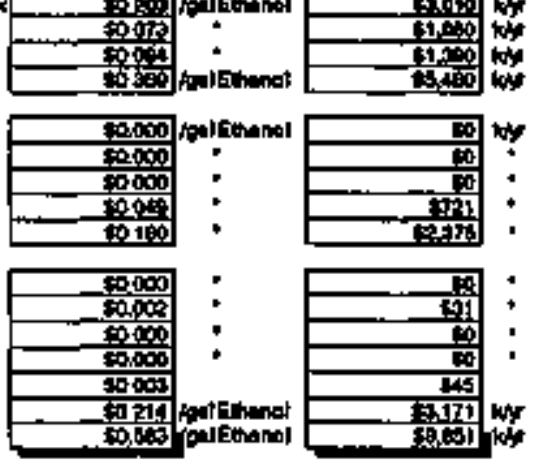

\section{Tophg fen}

Finimitions

Wood When

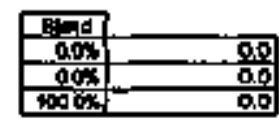

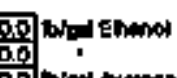

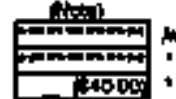

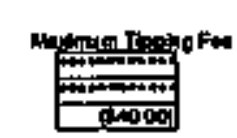

Econsonis:

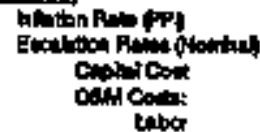

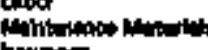

himinam

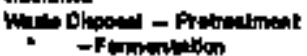

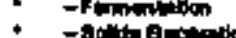

Onembert

Vition

Fon

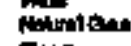

a.e.

Fencols

Ouput

thand

TorDan:

Ion

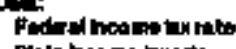

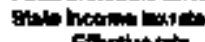

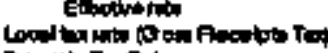

Prow ty Tox fill

landyen tow The

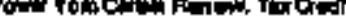

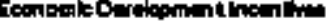

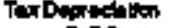

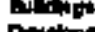

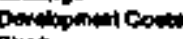

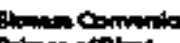

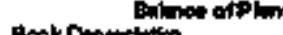

Evenchas:

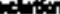

Dototonchm:

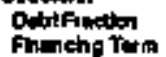

hrementimb

Ancountion of lom

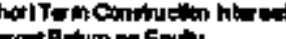
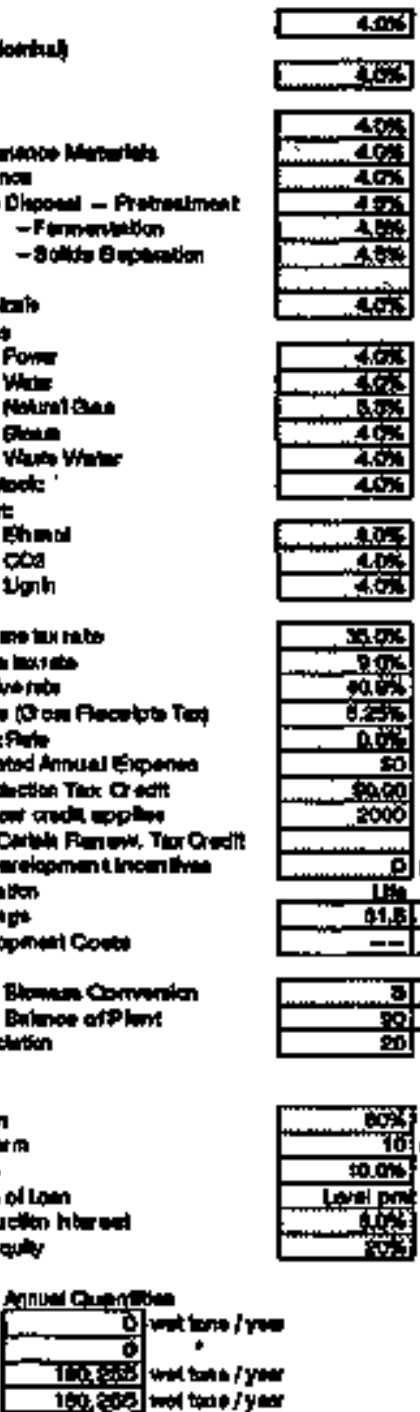

$\operatorname{lom}_{\ln }$

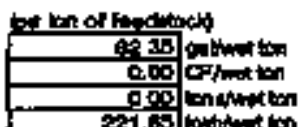

Inthing

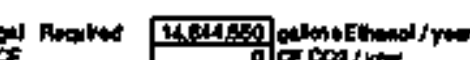

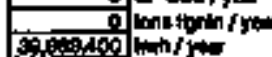

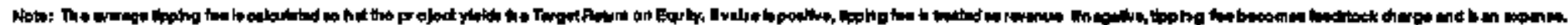




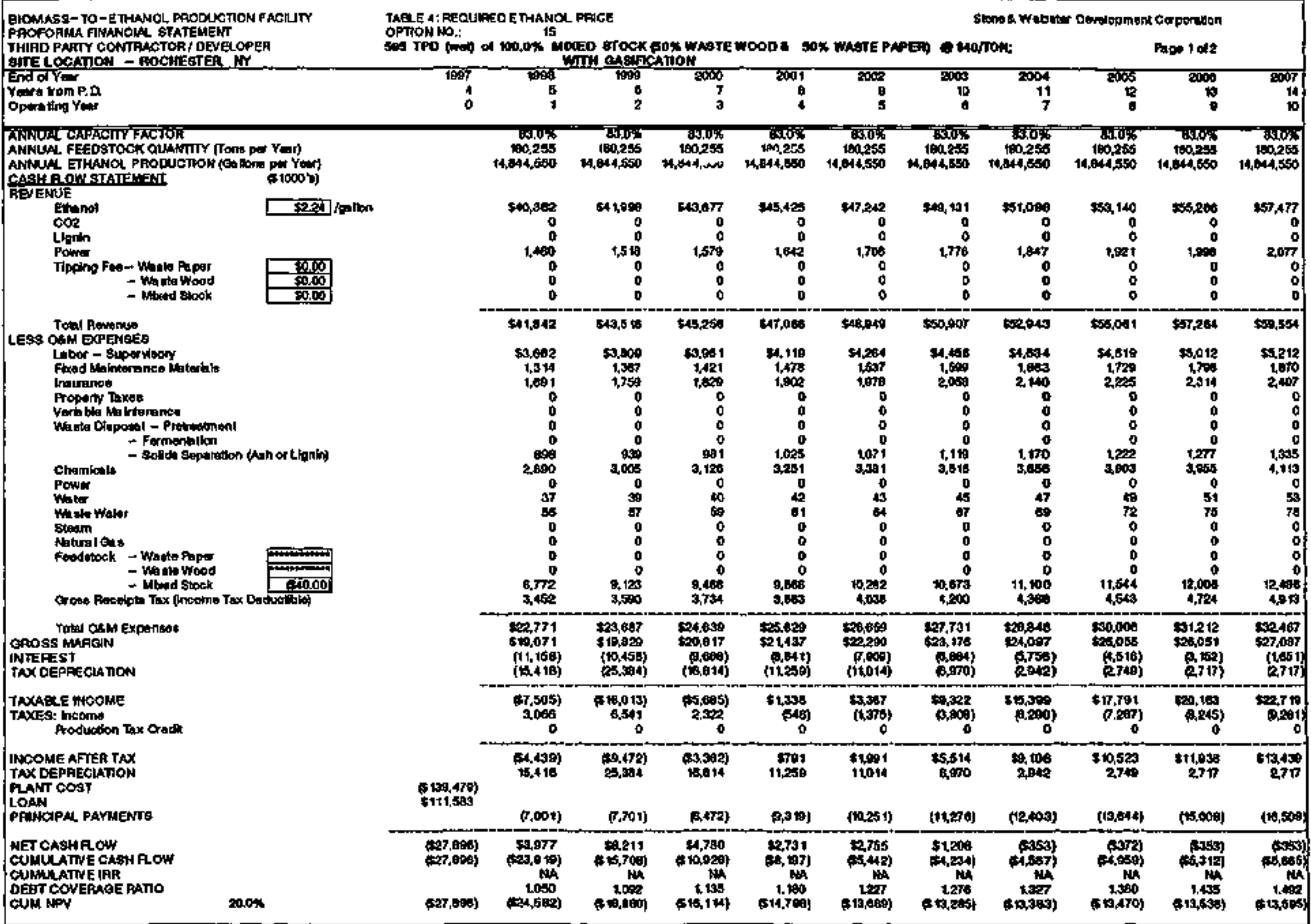




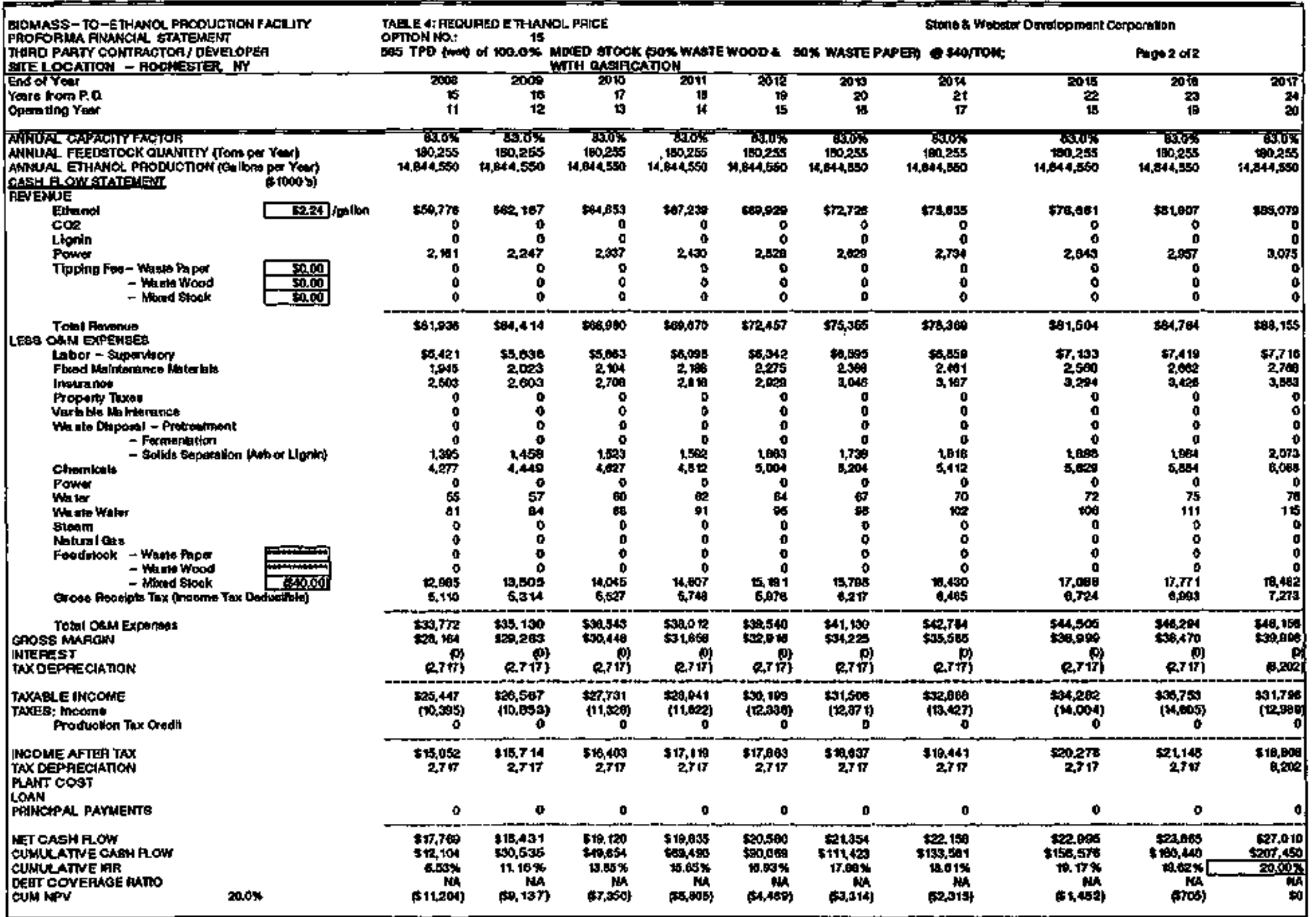




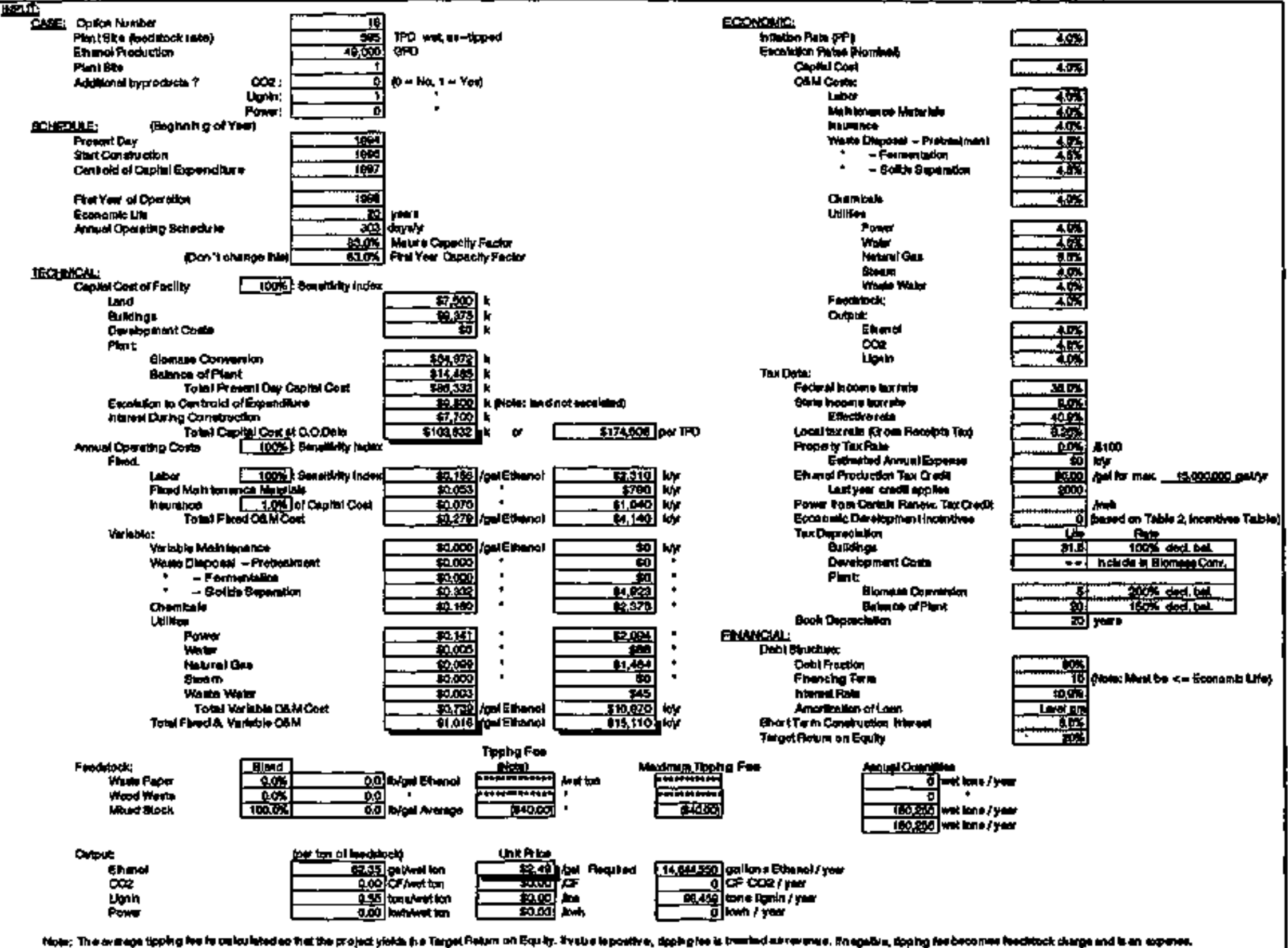




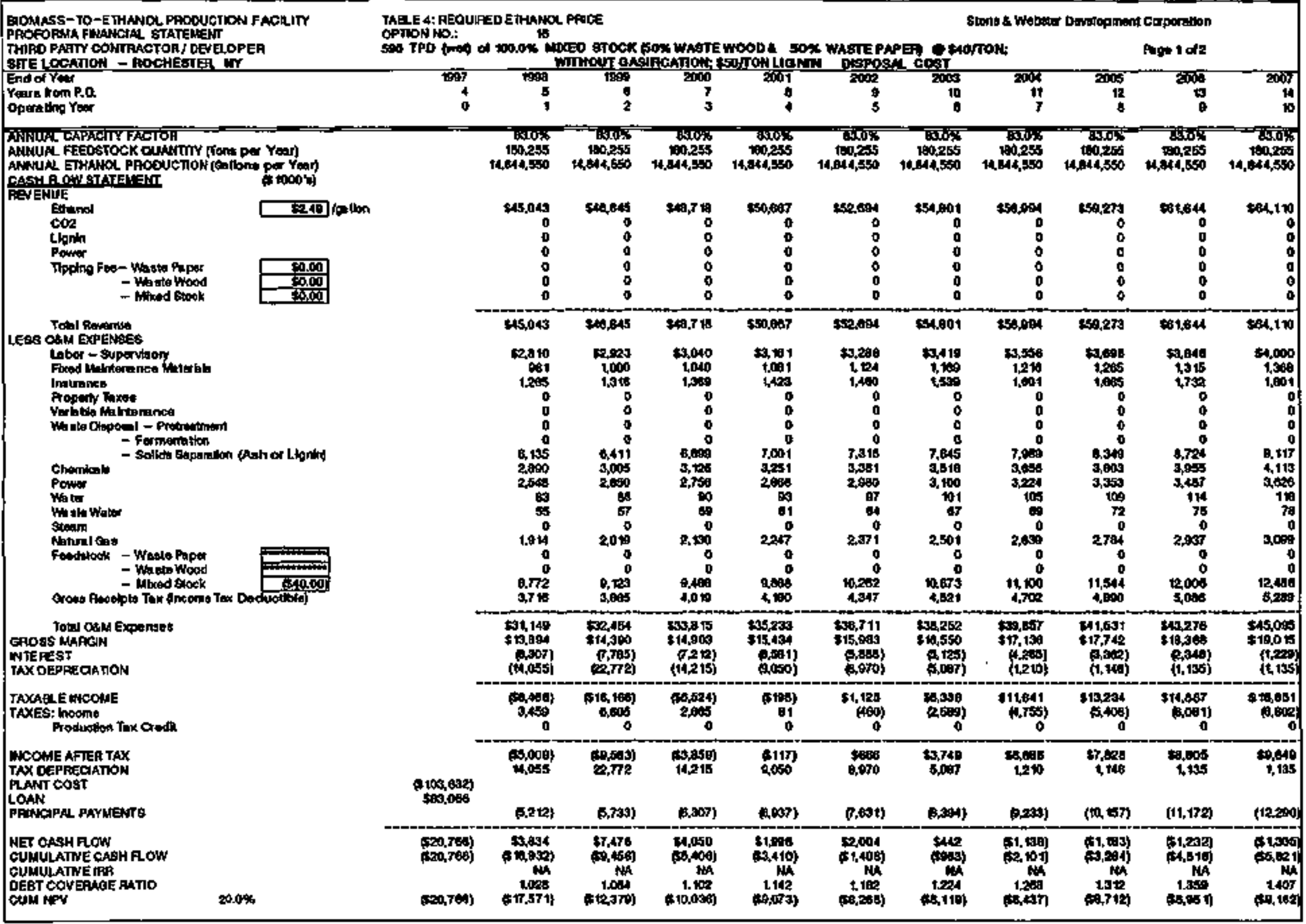




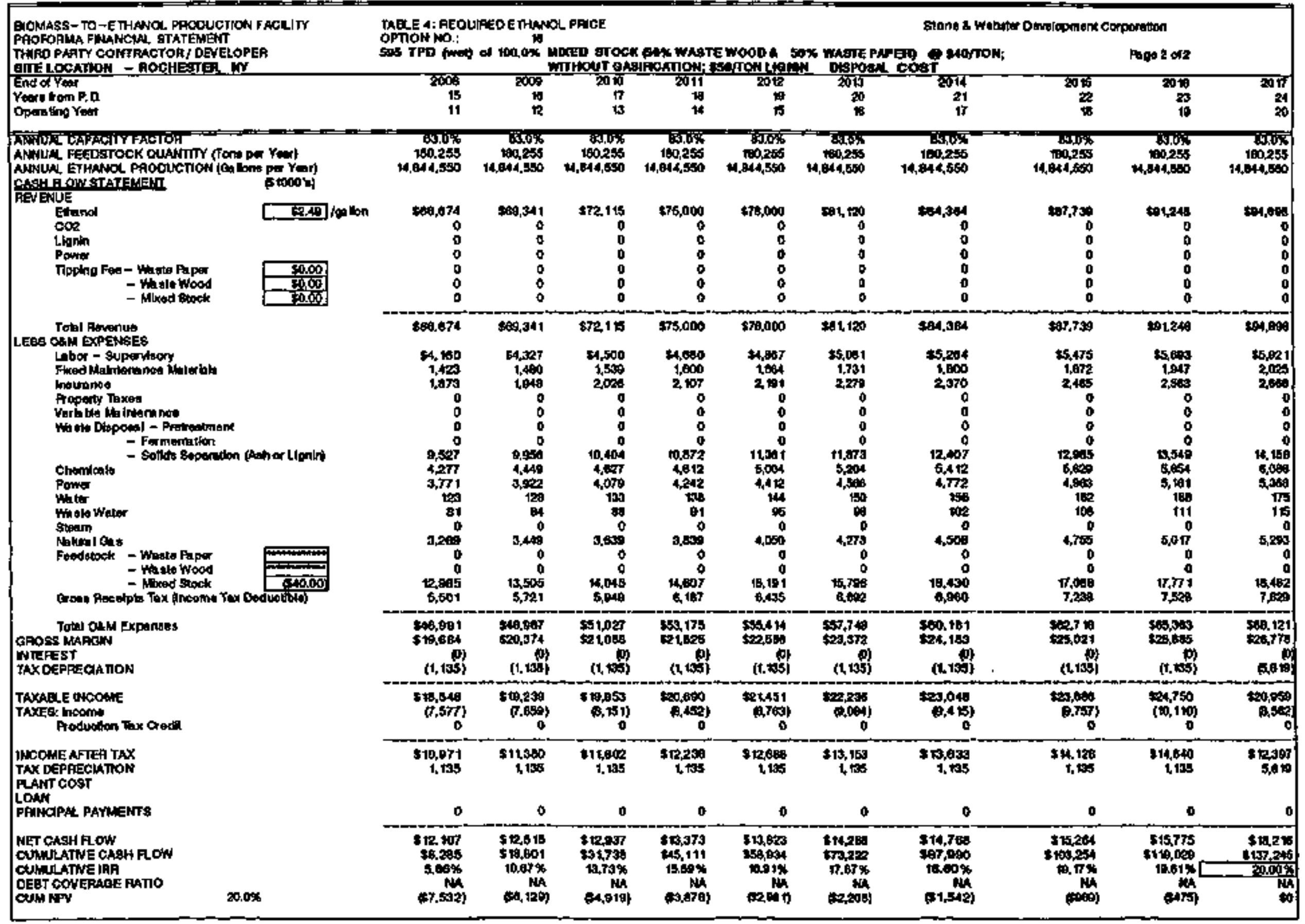

03aps: 
SIE LOCANOH - AOCHESTEA, NT

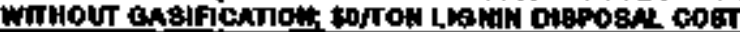

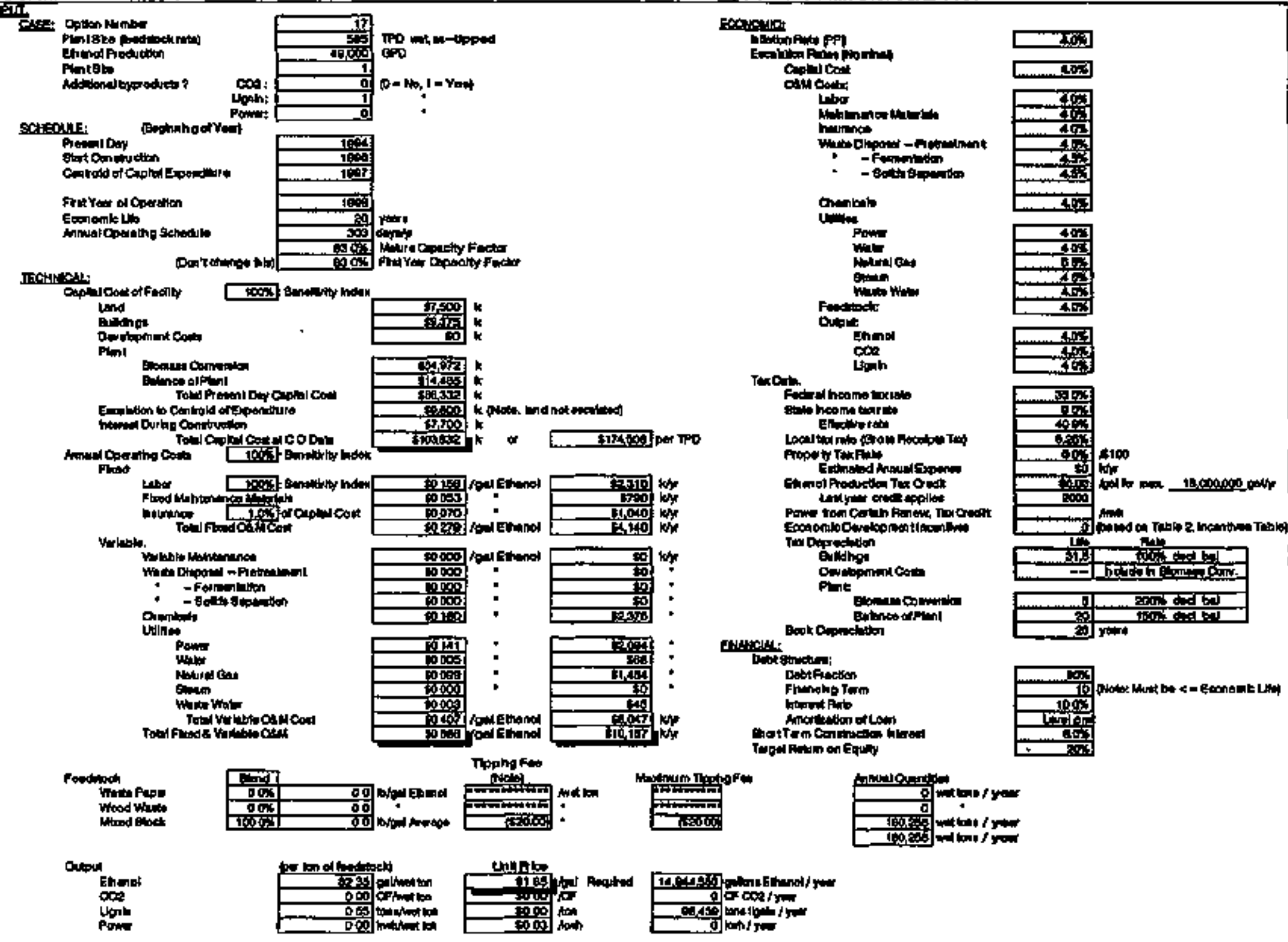

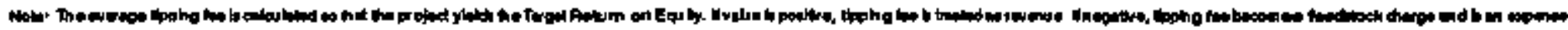




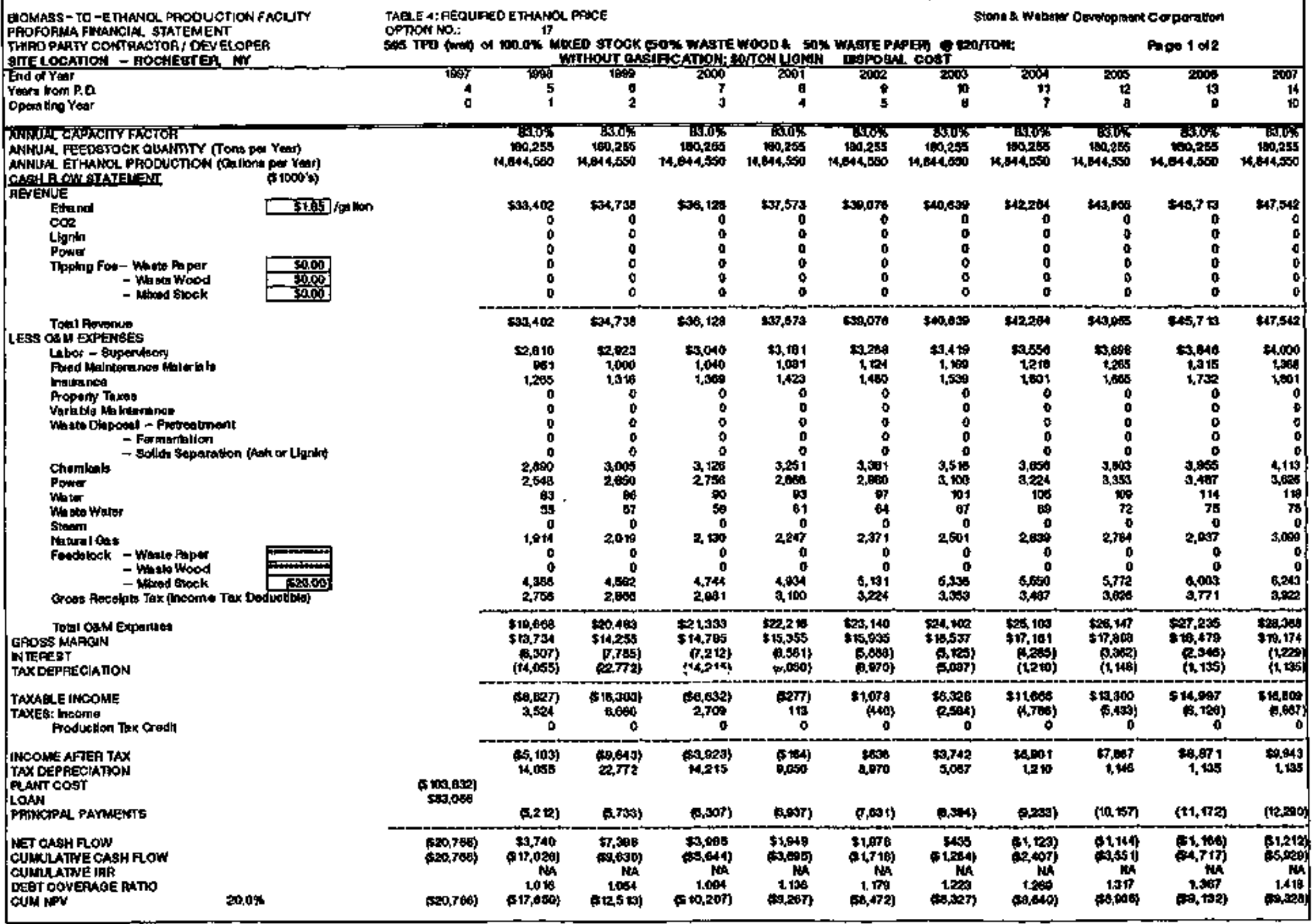




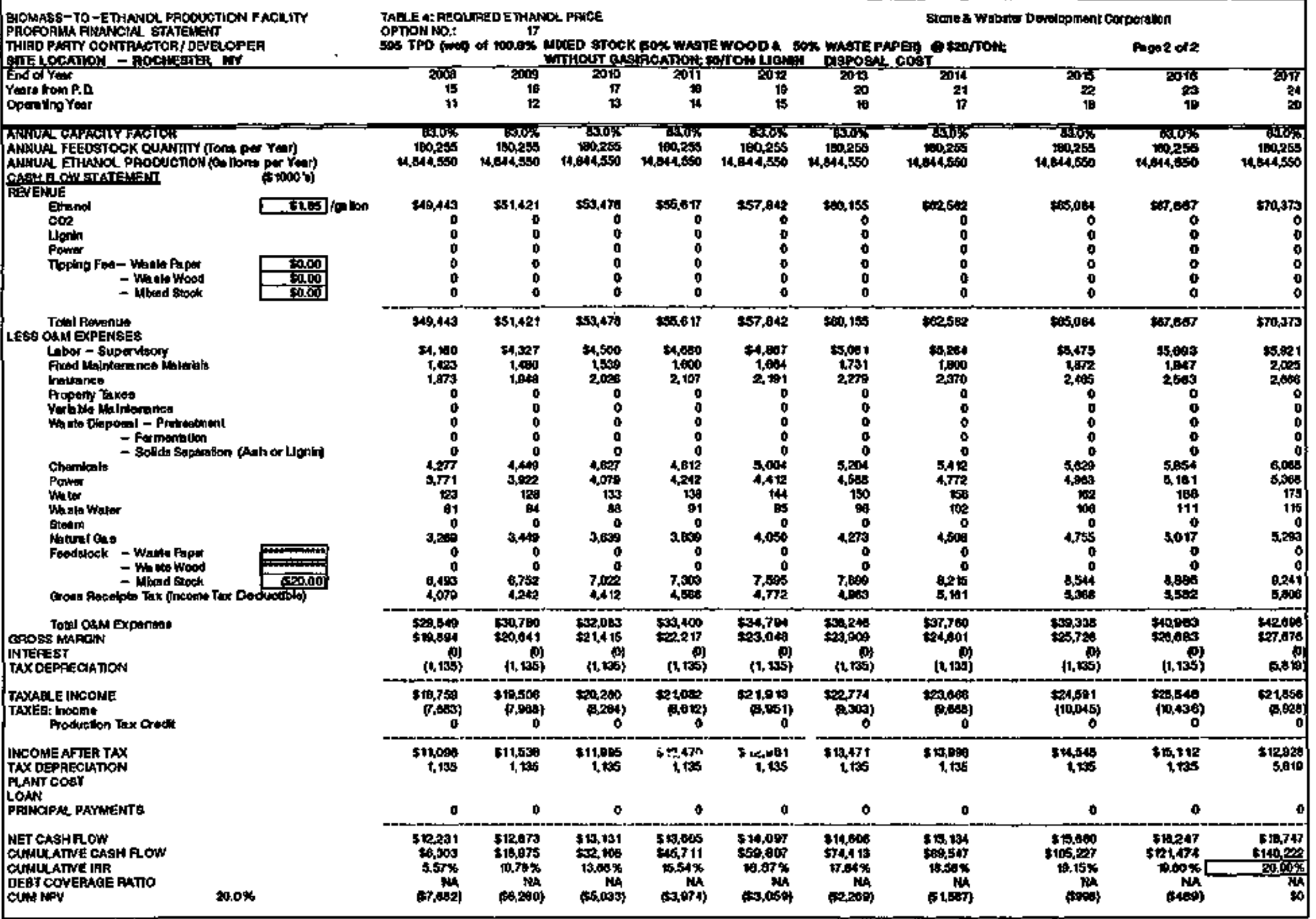


GITE LOCATON - FOCHEETER, WY

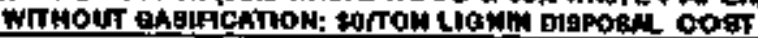

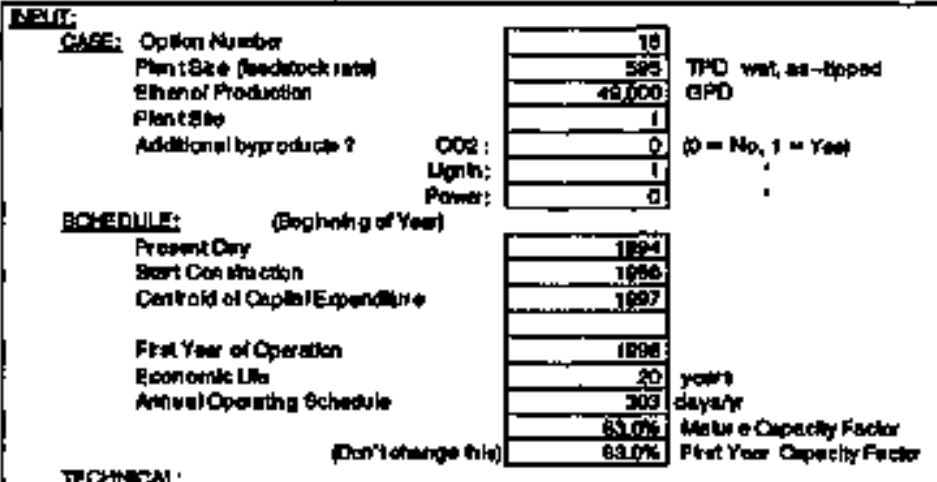

Incan

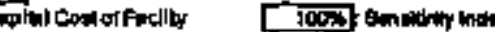

Led

Cullowes

Penl:

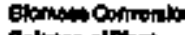

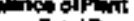

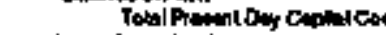

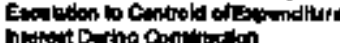

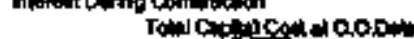

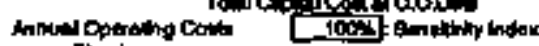

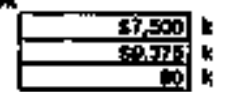

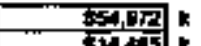

-

- $100000 \%$ a

Faroes Ther Vorlebles:
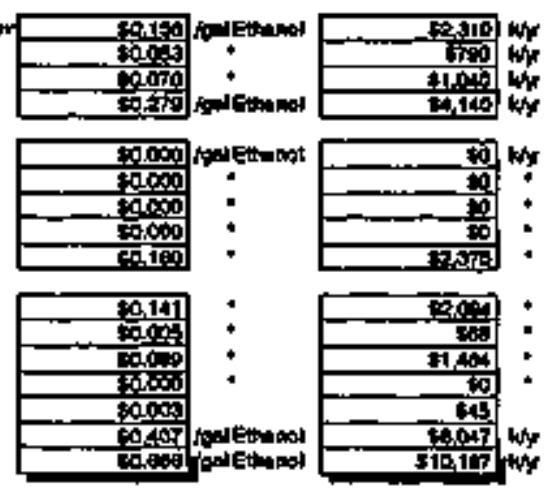

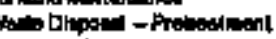
- Fremontition

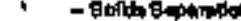
Chentom Pow

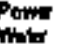

Noternat on

Sancerting

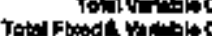
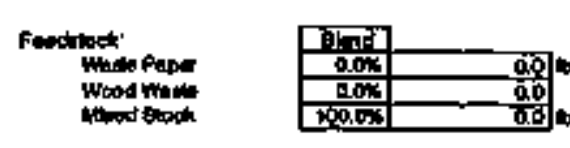

Tlpoph Fis
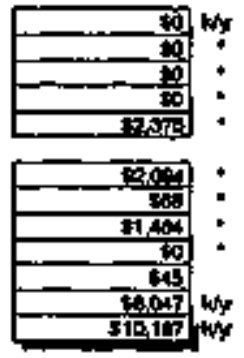

型

:

Fon

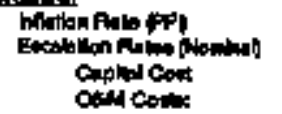

-

laber

hrivenes

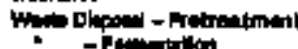

- - Bobsamant

Cienowath

Unther

Pown

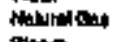

glosen

Fundoets

Oupute

Gan

Wor

TakDowe

Fichal hoow wath

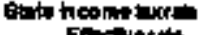

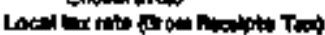

Property Texpen

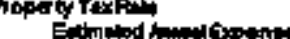

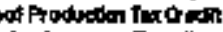

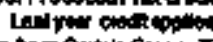

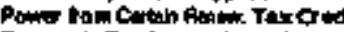

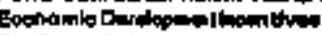

TaM Doprotion

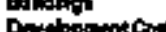

Dorsto

Blomancominnon

Buncos offitint

Every.t.

Cosk Dopontion

Ditit Bindutio:

Dotrt Eriotion

Fhenchy Iom

incotemition od tow

Ghal Tern controotion hio

Torgarinem of Equhy

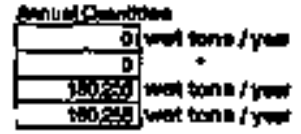

1100

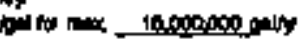

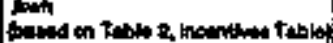

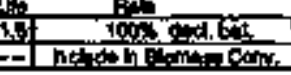

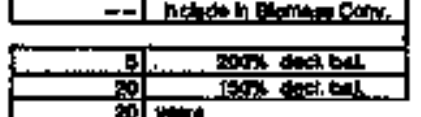
sin

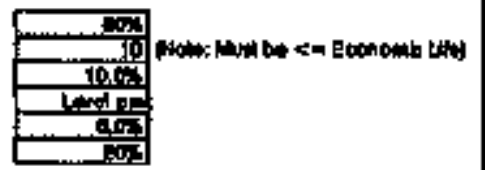

Oroput:

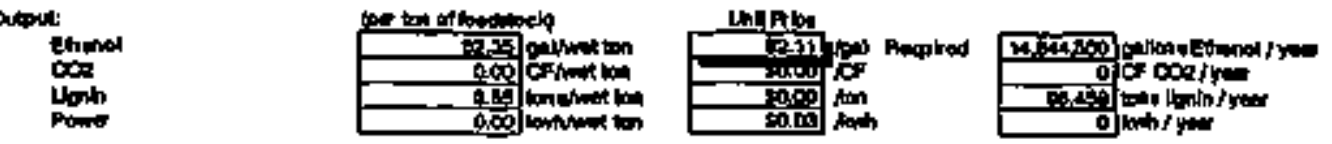

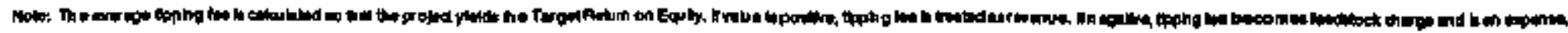




\section{A PALE}

Poge 1 ok

AIIELOCATION - AOCHESTER WY

Find of Year $\mathrm{P}$.

Operithing Yeer

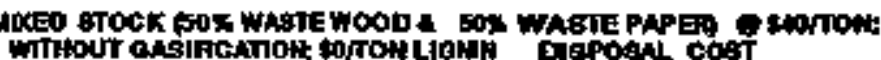

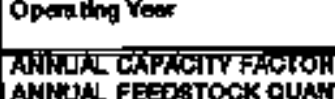

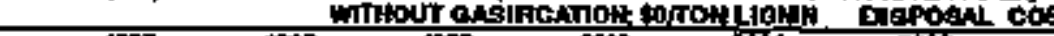

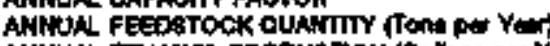

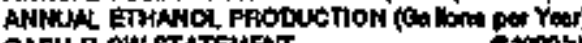

GAEH.BLOWLTATTE!.1ENT

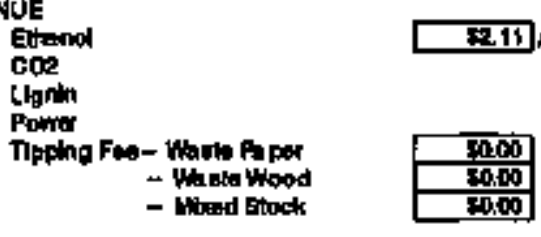

Totol favenus

LESS QWH EXPENSES

Labor - suptinony

Flod Malnten

Pepenty Takes.

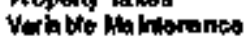

Whata Dieporal - Pretreatument

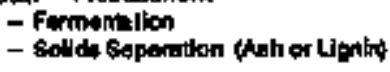

Themingls

Dowim

Water woter

Siliam

Nhand cas

Peodstock - wate Paper

- Whelo hood

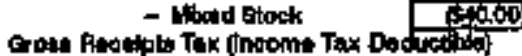

Tolal osm Exponsos

GAOSS MAPGN

INTEREST

TAX DEPFECIATION

TAXABEF INCOH

TAXES: neoms

Production Tax Otalt

INCONE AFTER TAX

TAX DEPAECWKTIOH

PLANT COST

LOAN

PFAlHEPAL PAYWENTS

MET CABH FLOW

CUNWLAIVE CASH FL OW

CUHULATME IRA

OEgT COYERAOE RATO

cum Nov

$20.0 \times$

\begin{tabular}{|c|c|c|c|c|c|c|c|c|c|}
\hline $\begin{array}{r}+\% \\
5 \\
1\end{array}$ & $\begin{array}{r}1 \% \\
2 \\
2\end{array}$ & $\begin{array}{r}7000 \\
7 \\
3\end{array}$ & $\begin{array}{r}2001 \\
4\end{array}$ & $\begin{array}{r}700 \\
9 \\
5\end{array}$ & $\begin{array}{r}6006 \\
6 \\
6\end{array}$ & $\frac{110}{7}$ & 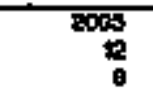 & $\begin{array}{r}5000 \\
13 \\
0\end{array}$ & \begin{tabular}{r|}
5007 \\
14 \\
10
\end{tabular} \\
\hline $\begin{array}{r}24.0 \% \\
280.255 \\
14,644,560\end{array}$ & $\begin{array}{r}0.0 \% \\
100.245 \\
14,944500\end{array}$ & $\begin{array}{r}6300 \% \\
160,2005 \\
+4,64,550\end{array}$ & 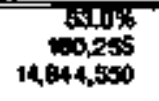 & $\begin{array}{r}160,254 \\
4,044,050\end{array}$ & 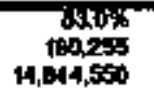 & $\begin{array}{r}180,265 \\
14,044,500\end{array}$ & $\begin{array}{r}1, k 10 \% \\
1400,255 \\
140,550\end{array}$ & $\begin{array}{r}16,0 \% \\
160,253 \\
14,644,60\end{array}$ & $\begin{array}{r}65,50 \% 1 \\
+00,255 \\
14,244,250\end{array}$ \\
\hline $\begin{array}{r}\text { sta } 183 \\
0 \\
0 \\
0 \\
0 \\
0 \\
0\end{array}$ & $\begin{array}{r}\$ * 9,7 \\
0 \\
0 \\
0 \\
0 \\
0 \\
0 \\
0\end{array}$ & $\begin{array}{r}* 1,280 \\
0 \\
0 \\
0 \\
0 \\
0 \\
0\end{array}$ & $\begin{array}{r}5.900 \\
0 \\
0 \\
0 \\
0 \\
0 \\
0\end{array}$ & $\begin{array}{r}-44,0 \\
0 \\
0 \\
0 \\
0 \\
0 \\
0\end{array}$ & $\begin{array}{r}40,450 \\
0 \\
0 \\
0 \\
0 \\
0 \\
0\end{array}$ & $\begin{array}{r}40,2 \text {. } \\
0 \\
0 \\
0 \\
0 \\
0 \\
0\end{array}$ & $\begin{array}{r}0,24: \\
0 \\
0 \\
0 \\
0 \\
0 \\
0\end{array}$ & $\begin{array}{r}0 \\
0 \\
0 \\
0 \\
0 \\
0 \\
0\end{array}$ & $\begin{array}{r}5 \text { s }, 340 \\
0 \\
0 \\
0 \\
0 \\
0 \\
0\end{array}$ \\
\hline 500,403 & 20.70 & $\$ 1,2 \infty$ & t+20,0 & *4,669 & 560,455 & 49.319 & $\$ 50,240$ & $35,25 a$ & 954,346 \\
\hline 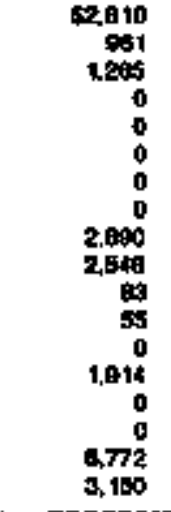 & 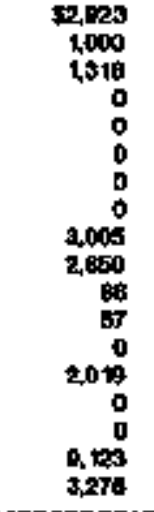 & 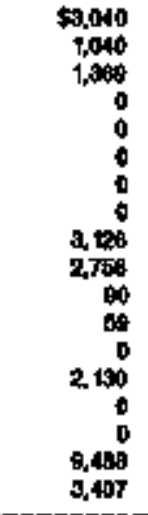 & 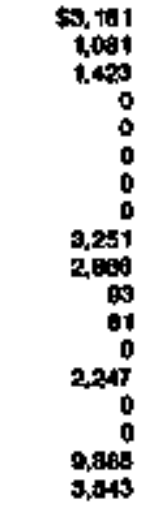 & $\begin{array}{r}+3,298 \\
1,04 \\
1,400 \\
0 \\
0 \\
0 \\
0 \\
0 \\
3,391 \\
2,900 \\
97 \\
64 \\
0 \\
2,371 \\
0 \\
0 \\
0,208 \\
3,606\end{array}$ & 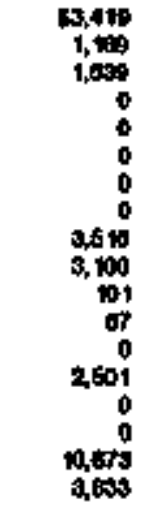 & 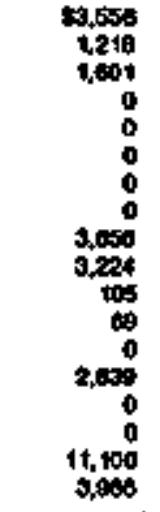 & 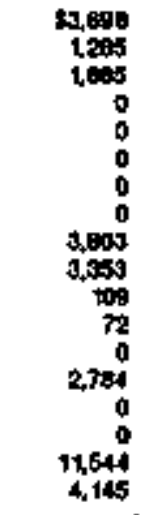 & 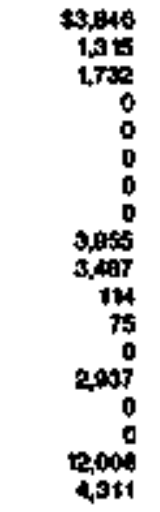 & $\begin{array}{r}4,000 \\
1,306 \\
1,001 \\
0 \\
0 \\
0 \\
0 \\
0 \\
4,113 \\
3,625 \\
1 \% 9 \\
79 \\
0 \\
3,099 \\
0 \\
0 \\
12,406 \\
4,404\end{array}$ \\
\hline $\begin{array}{l}=24,449 \\
\$ 14,734 \\
(0,307) \\
(14,05,6)\end{array}$ & $\begin{array}{c}5,455 \\
+1,25 \% \\
(7,705) \\
0,772)\end{array}$ & $\begin{array}{l}50,500 \\
514,7 \% 5 \\
f, 212) \\
(14,215)\end{array}$ & $\begin{array}{l}27,590 \\
615,350 \\
4,5811 \\
9,0001\end{array}$ & 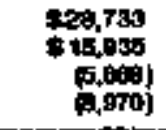 & 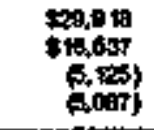 & $\begin{array}{c}531,150 \\
517,461 \\
4,205) \\
(1,210)\end{array}$ & 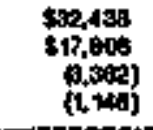 & $\begin{array}{c}\$ 45,771 \\
\$ 16,470 \\
(7,346) \\
(1,165)\end{array}$ & $\begin{array}{r}\$ 25,172 \\
\$ 10,174 \\
(1,220 \\
(1,135)\end{array}$ \\
\hline $\begin{array}{r}0.097 \\
2.804 \\
0\end{array}$ & $\begin{array}{r}\text { pt8,300 } \\
6600 \\
0\end{array}$ & $\begin{array}{r}2,704 \\
0\end{array}$ & $\begin{array}{r}17 \% \\
113 \\
0\end{array}$ & $\begin{array}{r}\$ 1,078 \\
+400 \\
0\end{array}$ & 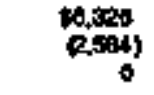 & $\begin{array}{c}\$ 11,609 \\
0,769) \\
0\end{array}$ & $\begin{array}{r}19,300 \\
(0,453) \\
0\end{array}$ & $\begin{array}{c}\$ 14,099 \\
5,120) \\
0\end{array}$ & $\begin{array}{r}\$ 16,000 \\
\beta, 607) \\
0\end{array}$ \\
\hline $\begin{array}{l}45,1033 \\
14,055\end{array}$ & $\underset{2,772}{0,94}$ & $\begin{array}{l}03,023) \\
14,215\end{array}$ & $\begin{array}{l}\$ 18 d) \\
9,0 \infty 0\end{array}$ & toma & $\begin{array}{l}\$ 3,742 \\
\text { B,007 }\end{array}$ & $\begin{array}{l}\operatorname{sac}_{1,001} \\
1,2 \text { to }\end{array}$ & $\begin{array}{r}57,067 \\
t, 146\end{array}$ & 1961 & $\begin{array}{l}\operatorname{mons} \\
1,26\end{array}$ \\
\hline (5.212) & (6.70) & $(6,607)$ & 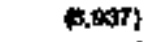 & $(7,001)$ & Bras) & 9203) & $(10,157)$ & $(11, \pi 7\rangle$ & $(2,200)$ \\
\hline $\begin{array}{c}\$ 2,740 \\
\$ 17,026) \\
\mathrm{NA} \\
1,006 \\
\$ 17,050)\end{array}$ & 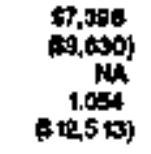 & 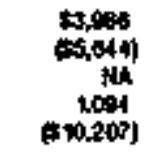 & 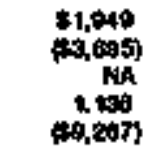 & 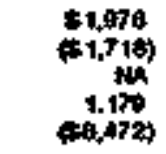 & 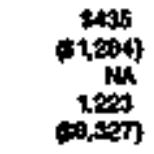 & 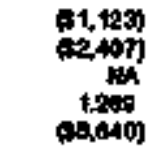 & 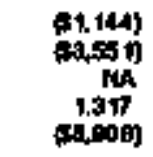 & 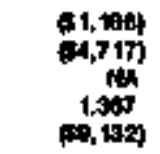 & 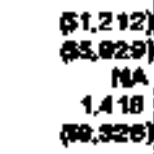 \\
\hline
\end{tabular}




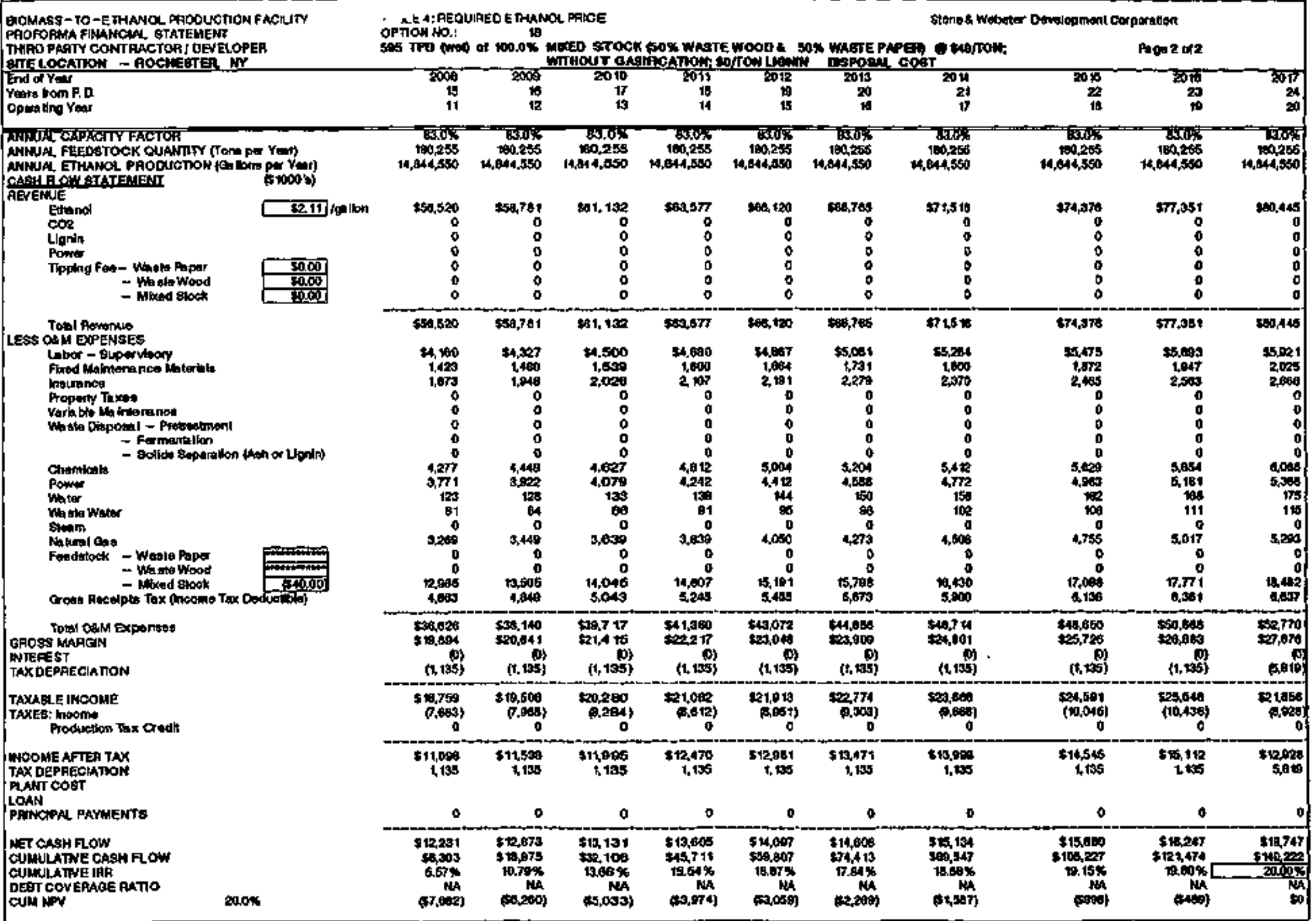




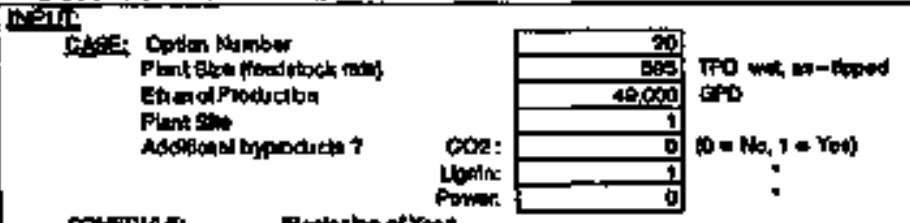

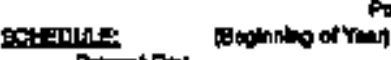

Pition

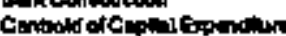

Fial Yeer of copanition

Teononk Lth

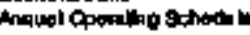

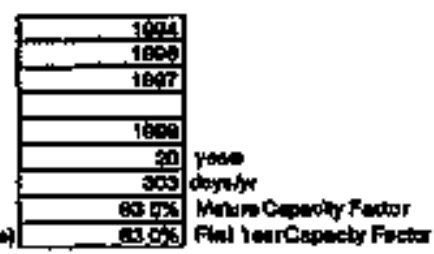

IEAtrick

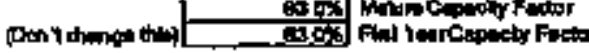

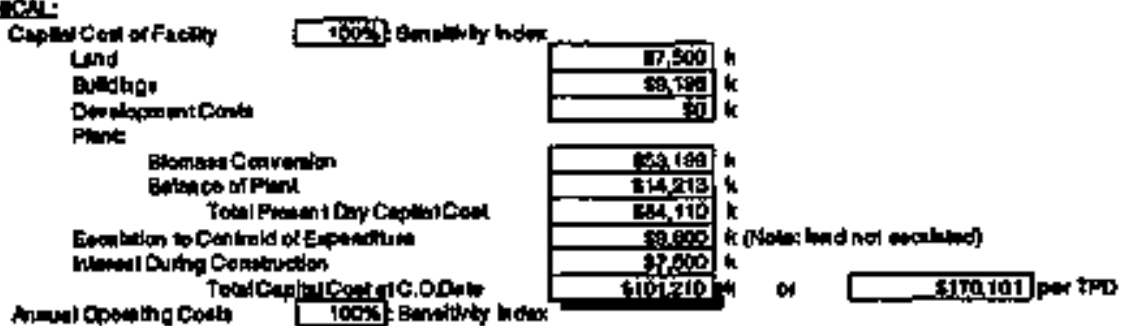

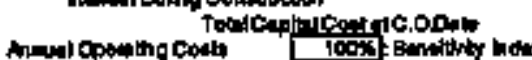

Finct

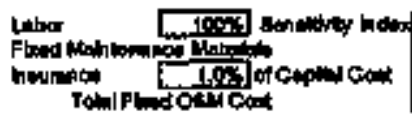

Whables:

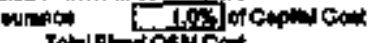

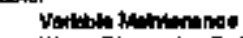

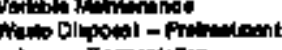

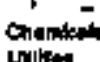

Pown

Wain

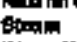

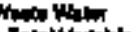

Tonction

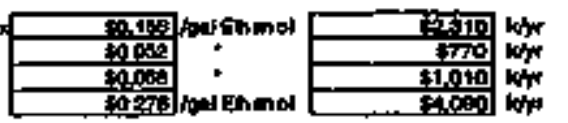

Thoon

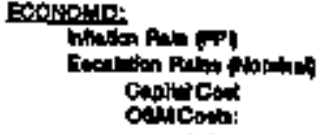

Lentror

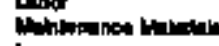

hangenos

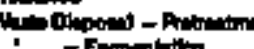

. -

Chentorts

unite

Pown

Villow

Bum

Fundrocin

Orout

COn-

unin

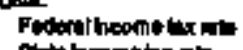

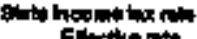

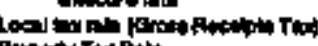

Thaty Tex

Exthrotod Anmual 5 mence

Chmorifroduction TarCark

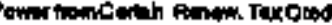

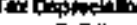

allinges

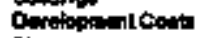

Donerit Ganwanion

Book Oopnestition

as나디나:

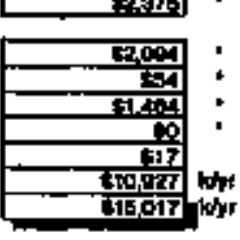

Dott flumenow

Phat Fincten

nowion

Anottanton of ton

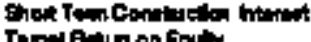

Torifund $n$

Thong foe.

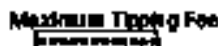

Finter

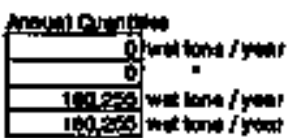

수련

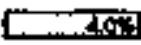

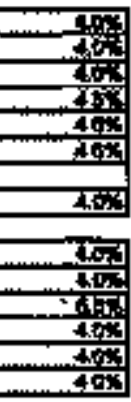

Fandisente

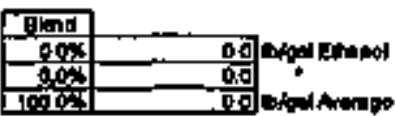

Poter

isones.

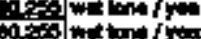

Outout:

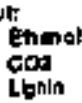

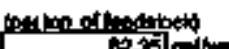

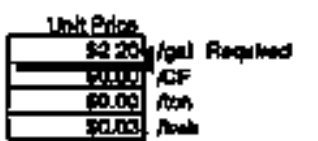

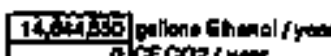

S.che

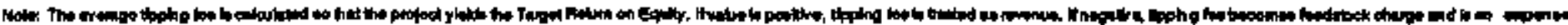




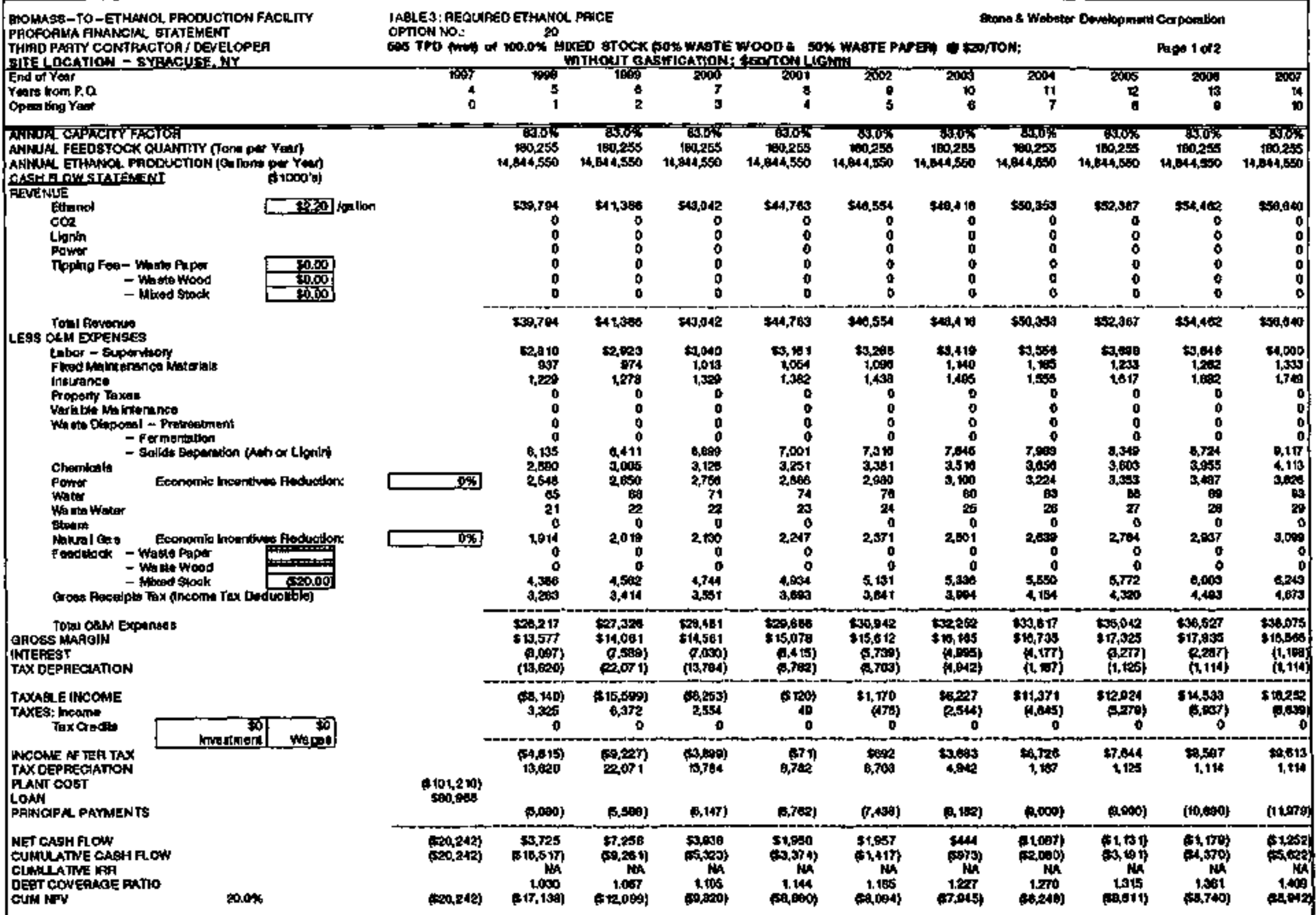


BIOMASS-TO-ETHWOL PHOCUCOIDNFACHLTY PHOFOFIUA FIMALCAL STATEMENT THIAD PAATY CONIPACTOA / DEVELOPEA SIIE LPGATION - \$TRMCUSE, HY Find \&ी Yoit Oparating Year

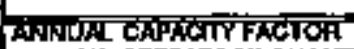

ANNUA FEEDSTOCK QUANMTY (TONE Pa Yant

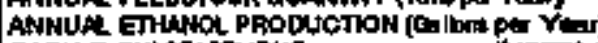
GASHE OWLSTAFGMENT FEVEN

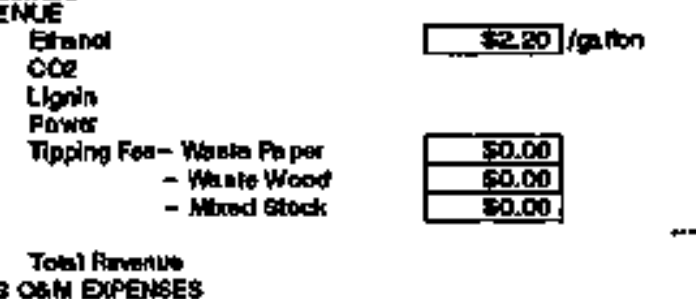

LESS OH DXPGHSES

lobor - supuntory

imarencice

Wopont Taxos

Varbelo

* Formenpotion

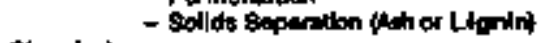

Phombals Eoonomito inconimes Reduction:

Pomer

Whito waw

sioen

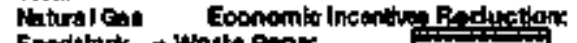

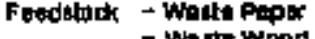

- Wa kis wood

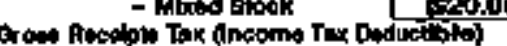

Tabl oris Enporis:

GAOSS MAAG:

INTEAEST

TAX OEPAECLTIOK

TAXABLE INCONE

TAXES: hoome

INCOME AF TER TAX

TAX DEPAECHTION

PLANT

PFINICIPA PAMUENTS

NET CASH RLOH

CULALATINE CAEH FLOW

CUhut a mve IRP

DEAT COVERAOE FATO

CUIN WFV

$\mathbf{2 0 . 9 8}$
JAG Ea: RECUARE ETHANOL PRACE

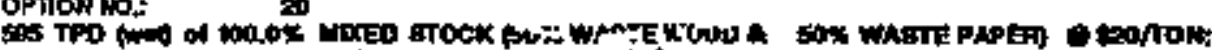

gaves Wabutar Development Corporation करण

2007
11
11

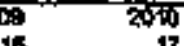

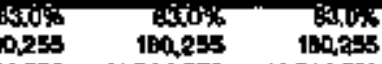

13

18

10,040

(5)

2014
10

21

$20+5$

Pupozatz

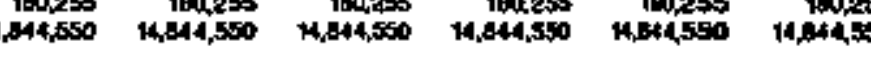

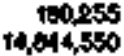

-

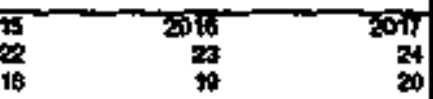

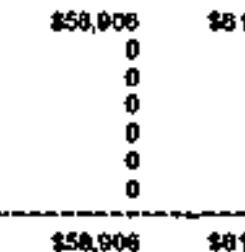

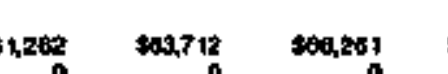

\begin{tabular}{|c|c|c|c|c|c|c|c|c|c|}
\hline $\begin{array}{r}r 5000 \\
0 \\
0 \\
0 \\
0 \\
0 \\
0\end{array}$ & $\begin{array}{r}\boldsymbol{s}, 26 \\
0 \\
0 \\
0 \\
0 \\
0 \\
0\end{array}$ & $\begin{array}{r}100712 \\
0 \\
0 \\
0 \\
0 \\
0 \\
0\end{array}$ & $\begin{array}{r}50,251 \\
0 \\
0 \\
0 \\
0 \\
0 \\
0\end{array}$ & $\begin{array}{r}\operatorname{sen} 11 \\
0 \\
0 \\
0 \\
0 \\
0 \\
0\end{array}$ & $\begin{array}{r}\boldsymbol{H t}, \mathbf{0 6} \\
0 \\
0 \\
0 \\
0 \\
0 \\
0 \\
0\end{array}$ & $\begin{array}{r}574,45 \\
0 \\
0 \\
0 \\
0 \\
0 \\
0\end{array}$ & $\begin{array}{r}5 \text { ro } 16 \\
0 \\
0 \\
0 \\
0 \\
0 \\
0\end{array}$ & $\begin{array}{r}* 0,6 \\
0 \\
0 \\
0 \\
0 \\
0 \\
0\end{array}$ & \begin{tabular}{r|}
503,041 \\
0 \\
0 \\
0 \\
0 \\
0 \\
0
\end{tabular} \\
\hline 550,000 & 物1,202 & $\operatorname{sis} 3,7$ t & $\operatorname{sen} 201$ & stage 11 & 371,666 & $\mathbf{9} 4, \mathbf{s} \mathbf{4}$ & \$ั7,510 & $\operatorname{sen} 646$ & $503,84+$ \\
\hline 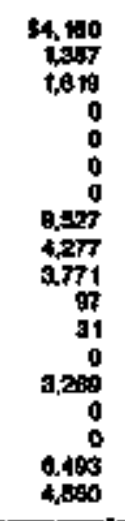 & 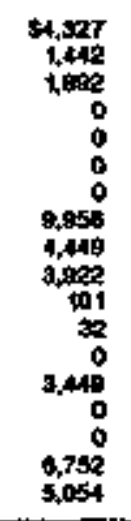 & 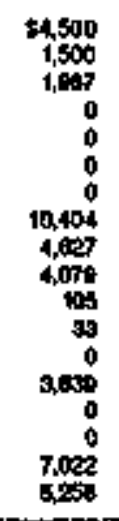 & 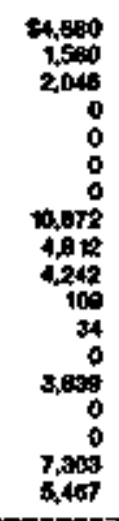 & $\begin{array}{r}4,097 \\
1,07 \\
2120 \\
0 \\
0 \\
0 \\
0 \\
21,361 \\
5,004 \\
4,412 \\
143 \\
30 \\
0 \\
4,050 \\
0 \\
0 \\
7,08 \\
6,095\end{array}$ & 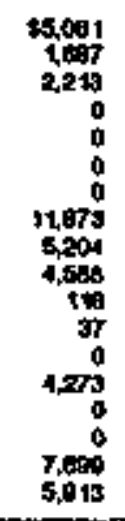 & 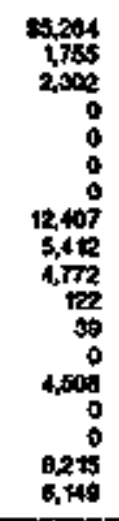 & 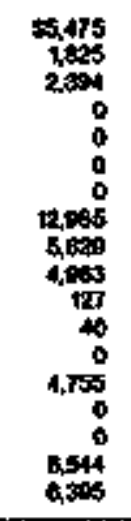 & 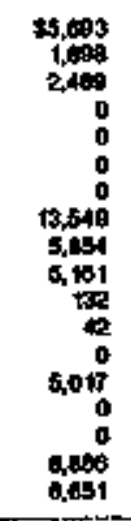 & 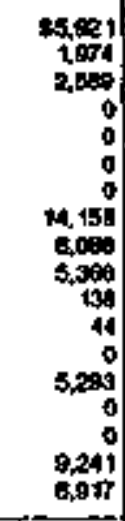 \\
\hline $\begin{array}{r}59691 \\
+6,215 \\
0 \% \\
(1,114)\end{array}$ & $\begin{array}{r}141,375 \\
\$ 19,087 \\
\text { p) } \\
(1.114)\end{array}$ & $\begin{array}{r}+4,131 \\
00,5181 \\
p+ \\
(1,1+4)\end{array}$ & $\begin{array}{r}540,905 \\
521,290 \\
(1,119)\end{array}$ & $\begin{array}{c}50,074 \\
02,049) \\
(0,110)\end{array}$ & $\begin{array}{r}14000 \\
901 \\
(1,114)\end{array}$ & $\begin{array}{r}\sin 9044 \\
+20,400 \\
(0,114)\end{array}$ & $\begin{array}{r}0+112 \\
04,404 \\
9) \\
(1,144)\end{array}$ & 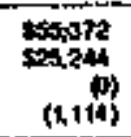 & $\begin{array}{r}\$ 57,700 \\
\$ 25.111 \\
0.712\end{array}$ \\
\hline $\begin{array}{c}* 01 \\
0.301 \\
0\end{array}$ & $\begin{array}{c}\$ 19,773 \\
(7,009) \\
0\end{array}$ & $\begin{array}{r}90,467 \\
(7,052) \\
6\end{array}$ & $\begin{array}{r}\$ 0,163 \\
\$, 245) \\
0\end{array}$ & $\begin{array}{c}00,023 \\
0475 \\
0\end{array}$ & $\begin{array}{r}21,607 \\
0,059) \\
0\end{array}$ & $\begin{array}{r}+2,470 \\
0,181) \\
0\end{array}$ & $\underset{0}{0.514)}$ & $\begin{array}{r}20,+130 \\
0,007) \\
0\end{array}$ & $\begin{array}{r}500,396 \\
0,300 \\
0\end{array}$ \\
\hline $\begin{array}{r}50,707 \\
1,114\end{array}$ & $\begin{array}{r}511,104 \\
1,114\end{array}$ & $\begin{array}{l}111,54 \\
1114\end{array}$ & $\begin{array}{r}\$ 11,999 \\
1,114\end{array}$ & $\begin{array}{r}\$ 1297 \% \\
1.114\end{array}$ & $\begin{array}{r}712,86 t \\
1,114\end{array}$ & $\begin{array}{r}19,294 \\
1,114\end{array}$ & $\begin{array}{r}513,770 \\
1,114\end{array}$ & $\begin{array}{r}34,270 \\
t, 116\end{array}$ & $\begin{array}{r}\$+2,069 \\
5,712\end{array}$ \\
\hline 0 & 0 & 0 & 0 & 0 & 0 & 0 & 0 & 0 & - \\
\hline $\begin{array}{r}\$ 11,021 \\
\$ 0,290 \\
5.70 \% \\
\mathrm{NA} \\
\$ 7,351)\end{array}$ & 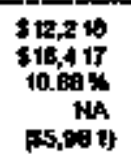 & $\begin{array}{r}\$ 2,62 \% \\
\$ 1,046 \\
13,74 \% \\
\mathrm{k4} \\
\$ 4,800)\end{array}$ & 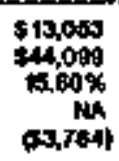 & 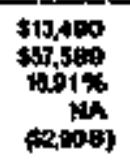 & $\begin{array}{r}\$ 15,902 \\
\$ 7,502 \\
7,07 \% \\
1,4 \\
72,154]\end{array}$ & $\begin{array}{r}\$ 14,400 \\
105940 \\
100 \% \\
51,505)\end{array}$ & 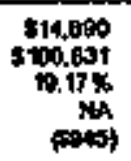 & 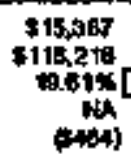 & 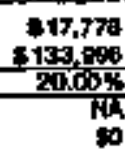 \\
\hline
\end{tabular}

$\$ 50,000$
54,100
1,07
$t, 019$

\$43,7

\begin{tabular}{|c|c|c|c|c|c|c|c|c|c|}
\hline $\begin{array}{l}0 \\
0 \\
0 \\
0 \\
0\end{array}$ & $\begin{array}{l}0 \\
0 \\
0 \\
0 \\
0\end{array}$ & $\begin{array}{l}0 \\
0 \\
0 \\
0 \\
0\end{array}$ & $\begin{array}{l}0 \\
0 \\
0 \\
0 \\
0\end{array}$ & $\begin{array}{l}0 \\
0 \\
0 \\
0 \\
0\end{array}$ & $\begin{array}{l}0 \\
0 \\
0 \\
0 \\
0\end{array}$ & $\begin{array}{l}0 \\
0 \\
0 \\
0 \\
0\end{array}$ & $\begin{array}{l}0 \\
0 \\
0 \\
0 \\
0\end{array}$ & $\begin{array}{l}0 \\
0 \\
0 \\
0 \\
0\end{array}$ & $\begin{array}{l}0 \\
0 \\
0 \\
0 \\
0\end{array}$ \\
\hline ssepos & *1,202 & $\sin , 70$ & $\operatorname{san} 241$ & shas oft & 371,696 & 574,534 & $\$ 7,516$ & $\sec , 646$ & $500,84+$ \\
\hline 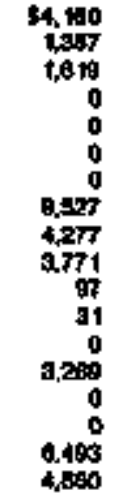 & 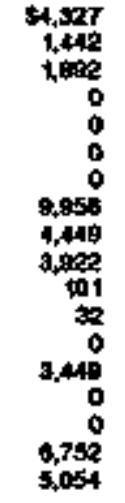 & 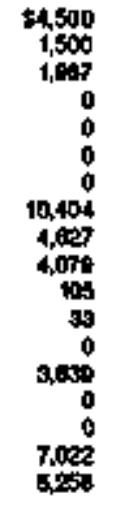 & 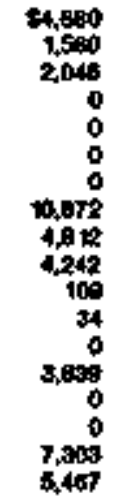 & 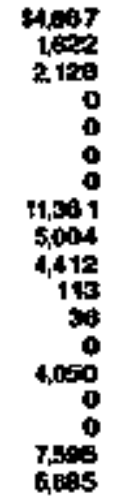 & 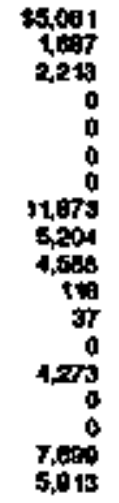 & $\begin{array}{r}25,204 \\
1,755 \\
2,302 \\
0 \\
0 \\
0 \\
0 \\
12,407 \\
5,4+2 \\
1,772 \\
t 27 \\
30 \\
0 \\
4,500 \\
0 \\
0 \\
0,2+5 \\
0,140\end{array}$ & 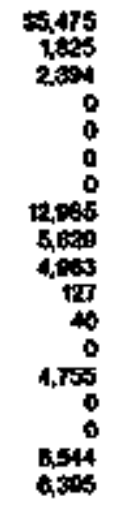 & 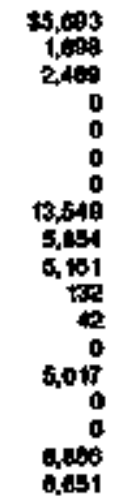 & 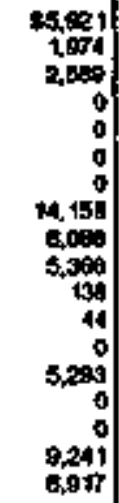 \\
\hline $\begin{array}{r}50901 \\
\$ 4,215 \\
\text { os } \\
(1,114)\end{array}$ & $\begin{array}{r}11,375 \\
\$ 19,007 \\
\text { p) } \\
(1.114)\end{array}$ & $\begin{array}{r}+43,131 \\
50,561 \\
p+ \\
(1,1+4)\end{array}$ & $\begin{array}{r}540,905 \\
521,290 \\
(1,119)\end{array}$ & $\begin{array}{r}50,074 \\
00,049 \\
(0,10)\end{array}$ & $\begin{array}{r}1+0,001 \\
(1,114)\end{array}$ & $\begin{array}{r}50,944 \\
\$ 20,400 \\
00,114]\end{array}$ & $\begin{array}{r}04,112 \\
04,404 \\
(1,10)\end{array}$ & 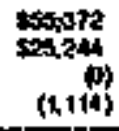 & $\begin{array}{r}157,734 \\
\$ 28,111 \\
0.712\end{array}$ \\
\hline $\begin{array}{c}* 101 \\
0,304 \\
0\end{array}$ & $\begin{array}{c}51 a_{,}, 73 \\
(7,009) \\
0\end{array}$ & $\begin{array}{c}969,467 \\
(7,852) \\
6\end{array}$ & $\begin{array}{r}50,163 \\
\$ 3,245) \\
0\end{array}$ & $\begin{array}{c}50,023 \\
0475 \\
0\end{array}$ & $\begin{array}{r}1,007 \\
0,050) \\
0\end{array}$ & $\begin{array}{c}\$ 2.470 \\
\text { Q.151) } \\
0\end{array}$ & $\underset{0,514)}{0}$ & $\begin{array}{r}50,1300 \\
0,007) \\
0\end{array}$ & $\begin{array}{r}5,396 \\
0,3030 \\
0\end{array}$ \\
\hline $\begin{array}{r}\$ 10,707 \\
1+14\end{array}$ & $\begin{array}{r}511,104 \\
1,114\end{array}$ & \$11, 114 & $\$ 1 t, 909$ & $\begin{array}{r}\$ 12,37 \% \\
1.144\end{array}$ & $\begin{array}{r}312,564 \\
1,114\end{array}$ & $\begin{array}{r}913,294 \\
1,114\end{array}$ & $\begin{array}{r}513,779 \\
1,114\end{array}$ & $\begin{array}{r}34,270 \\
t, 114\end{array}$ & $\begin{array}{r}\$ \text { te, } \\
5,71200\end{array}$ \\
\hline 0 & 0 & 0 & 0 & 0 & 0 & 0 & 0 & 0 & 0 \\
\hline $\begin{array}{r}\$ 11,021 \\
\$ 0,190 \\
5.70 \% \\
N A \\
\$ 7,351)\end{array}$ & 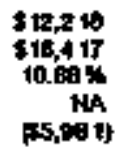 & $\begin{array}{r}\$ 12,020 \\
\$ 1,046 \\
13,74 \% \\
144 \\
94,800)\end{array}$ & $\begin{array}{r}\$ 13,000 \\
\$ 4,099 \\
1,00 \% \\
\text { mak }\end{array}$ & 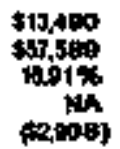 & 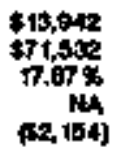 & 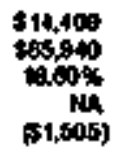 & 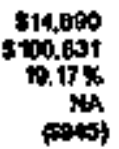 & 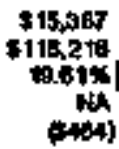 & $\begin{array}{r}\quad 17,779 \\
5133,908 \\
21,065 \\
14 \\
\infty\end{array}$ \\
\hline
\end{tabular}

11 \& the

\begin{tabular}{|c|c|c|c|c|c|c|c|c|c|}
\hline $\begin{array}{r}\mathbf{r} 5900 \\
0 \\
0 \\
0 \\
0 \\
0 \\
0\end{array}$ & $\begin{array}{r}0,26 \\
0 \\
0 \\
0 \\
0 \\
0 \\
0\end{array}$ & $\begin{array}{r}503712 \\
0 \\
0 \\
0 \\
0 \\
0 \\
0\end{array}$ & $\begin{array}{r}500,251 \\
0 \\
0 \\
0 \\
0 \\
0 \\
0\end{array}$ & $\begin{array}{r}11 \\
0 \\
0 \\
0 \\
0 \\
0 \\
0\end{array}$ & 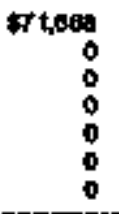 & $\begin{array}{r}574,034 \\
0 \\
0 \\
0 \\
0 \\
0 \\
0\end{array}$ & $\begin{array}{r}10 \\
0 \\
0 \\
0 \\
0 \\
0 \\
0\end{array}$ & $\begin{array}{r}* 0,6 \% \\
0 \\
0 \\
0 \\
0 \\
0 \\
0\end{array}$ & \begin{tabular}{r|r}
503,041 \\
0 \\
0 \\
0 \\
0 \\
0 \\
0
\end{tabular} \\
\hline s5e,000 & 钟,200 & 403,7 证 & sac,201 & sterg 14 & 971,086 & 94,534 & $\$ 77,510$ & $\operatorname{sen}, 646$ & 503.844 \\
\hline 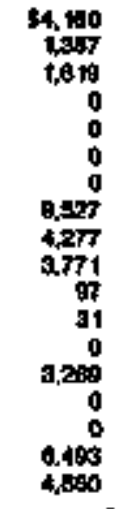 & 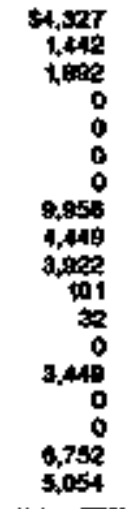 & 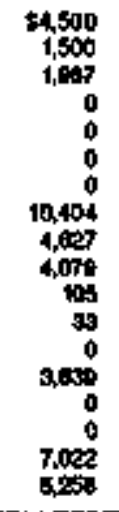 & 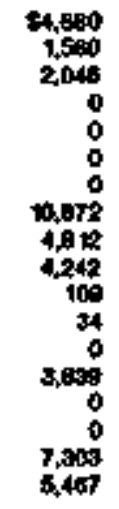 & $\begin{array}{r}4,97 \\
1,602 \\
2120 \\
0 \\
0 \\
0 \\
0 \\
71,361 \\
5,004 \\
4,412 \\
143 \\
50 \\
0 \\
4,050 \\
0 \\
0 \\
7,58 \\
6,805\end{array}$ & 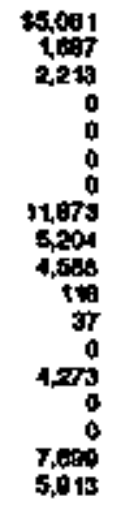 & 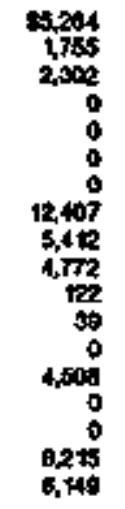 & 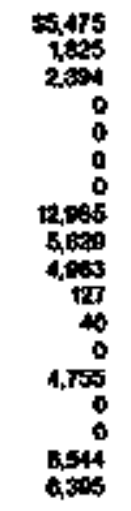 & 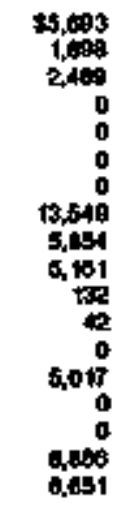 & 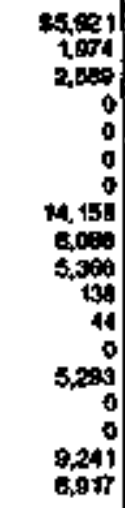 \\
\hline $\begin{array}{r}59,601 \\
+6,215 \\
(1,114)\end{array}$ & $\begin{array}{r}\$ 1,375 \\
\$ 19,087 \\
\text { p) } \\
(1.114)\end{array}$ & 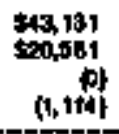 & $\begin{array}{r}540,905 \\
521,290 \\
(1,119)\end{array}$ & $\begin{array}{r}50,074 \\
00,049 \\
(0,10)\end{array}$ & $\begin{array}{r}1+0,001 \\
(1,114)\end{array}$ & $\begin{array}{r}50,944 \\
\$ 20,800 \\
(0,114]\end{array}$ & $\begin{array}{r}04,112 \\
04,404 \\
(1) \\
(1,114)\end{array}$ & $\begin{array}{r}\text { sor,3re } \\
\text { (1,114) }\end{array}$ & $\begin{array}{r}\$ 57,700 \\
\$ 0.111 \\
0.712\end{array}$ \\
\hline $\begin{array}{c}* 01 \\
0.301 \\
0\end{array}$ & $\begin{array}{c}51,773 \\
(7,009) \\
0\end{array}$ & $\begin{array}{c}96,467 \\
(7,052) \\
0\end{array}$ & $\begin{array}{r}50,163 \\
\$, 245) \\
0\end{array}$ & $\begin{array}{c}20,023 \\
0,77 \\
0\end{array}$ & $\begin{array}{r}21,007 \\
0,859 \\
6\end{array}$ & $\begin{array}{c}52.470 \\
0.1811 \\
0\end{array}$ & 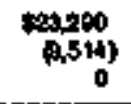 & $\begin{array}{r}020,130 \\
0,007) \\
0\end{array}$ & $\begin{array}{r}5=0,396 \\
0\end{array}$ \\
\hline $\begin{array}{r}50,707 \\
1,+14\end{array}$ & $\begin{array}{r}511,104 \\
1,114\end{array}$ & $\begin{array}{l}* 11,514 \\
114\end{array}$ & $\begin{array}{r}\$ 1 \text { 1t,994 } \\
1,114\end{array}$ & $\begin{array}{l}\$ 12.97 \% \\
1,144\end{array}$ & $\begin{array}{r}12,596 \\
1,114\end{array}$ & $\begin{array}{r}13,294 \\
1,114\end{array}$ & $\begin{array}{r}513,770 \\
1,114\end{array}$ & $\begin{array}{r}\$ 4,273 \\
\$, 114\end{array}$ & $\begin{array}{r}\$ \text { te.,060 } \\
5,712\end{array}$ \\
\hline 0 & 0 & 0 & 0 & $\mathbf{0}$ & 0 & 0 & 0 & 0 & 0 \\
\hline $\begin{array}{r}\$ 11,021 \\
\$ 0,290 \\
5.70 \% \\
N A \\
\$ 5,351)\end{array}$ & 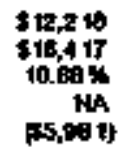 & $\begin{array}{r}\$ 12,62 \% \\
\$ 1,046 \\
13,74 \% \\
14 \\
\$ 4,800)\end{array}$ & 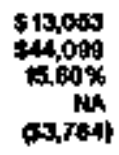 & 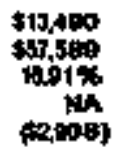 & $\begin{array}{r}\$ 15,012 \\
\$ 7, \$ 42 \\
7,07 \% \\
14, \\
72,154]\end{array}$ & $\begin{array}{c}\$ 1,400 \\
15 \$ 940 \\
100 \% \\
\text { Na } \\
51,505)\end{array}$ & 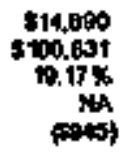 & 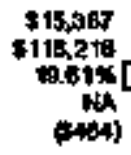 & 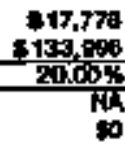 \\
\hline
\end{tabular}

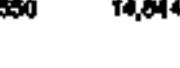

\begin{tabular}{|c|c|c|c|c|c|c|c|c|c|}
\hline $\begin{array}{l}0 \\
0 \\
0 \\
0 \\
0\end{array}$ & $\begin{array}{l}0 \\
0 \\
0 \\
0 \\
0\end{array}$ & $\begin{array}{l}0 \\
0 \\
0 \\
0 \\
0\end{array}$ & $\begin{array}{l}0 \\
0 \\
0 \\
0 \\
0\end{array}$ & $\begin{array}{l}0 \\
0 \\
0 \\
0 \\
0\end{array}$ & $\begin{array}{l}0 \\
0 \\
0 \\
0 \\
0\end{array}$ & $\begin{array}{l}0 \\
0 \\
0 \\
0 \\
0\end{array}$ & $\begin{array}{l}0 \\
0 \\
0 \\
0 \\
0\end{array}$ & $\begin{array}{l}0 \\
0 \\
0 \\
0 \\
0\end{array}$ & $\begin{array}{l}0 \\
0 \\
0 \\
0 \\
0\end{array}$ \\
\hline ssepos & *1,202 & $\sin , 70$ & $\operatorname{san} 241$ & shas oft & 371,696 & 574,534 & $\$ 7,516$ & $\sec , 646$ & $500,84+$ \\
\hline 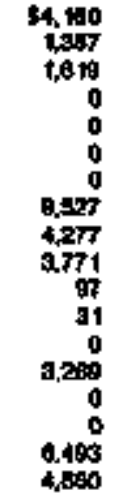 & 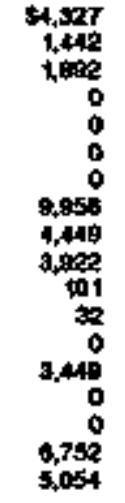 & 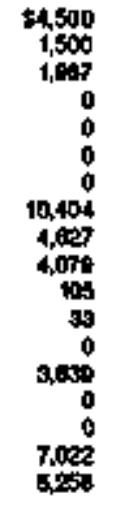 & 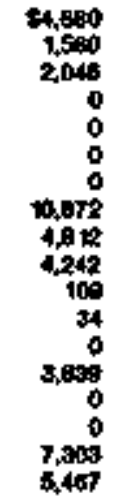 & 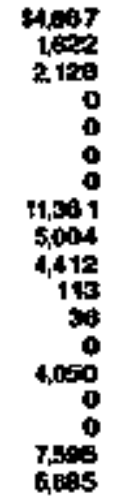 & 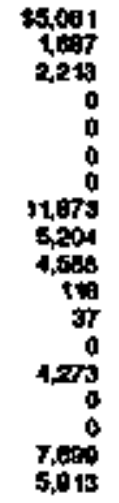 & $\begin{array}{r}25,204 \\
1,755 \\
2,302 \\
0 \\
0 \\
0 \\
0 \\
12,407 \\
5,4+2 \\
1,772 \\
t 27 \\
30 \\
0 \\
4,500 \\
0 \\
0 \\
0,2+5 \\
0,140\end{array}$ & 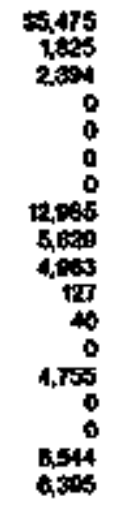 & 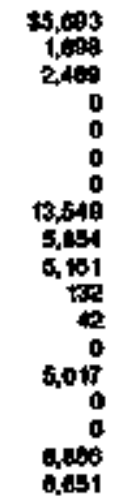 & 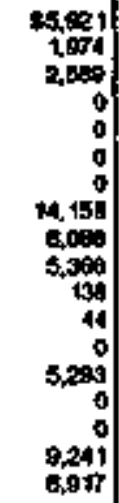 \\
\hline $\begin{array}{r}50901 \\
\$ 4,215 \\
\text { os } \\
(1,114)\end{array}$ & $\begin{array}{r}11,375 \\
\$ 19,007 \\
\text { p) } \\
(1.114)\end{array}$ & $\begin{array}{r}+43,131 \\
50,561 \\
p+ \\
(1,1+4)\end{array}$ & $\begin{array}{r}540,905 \\
521,290 \\
(1,119)\end{array}$ & $\begin{array}{r}50,074 \\
00,049 \\
(0,10)\end{array}$ & $\begin{array}{r}1+0,001 \\
(1,114)\end{array}$ & $\begin{array}{r}50,944 \\
\$ 20,400 \\
00,114]\end{array}$ & $\begin{array}{r}04,112 \\
04,404 \\
(1,10)\end{array}$ & 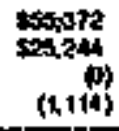 & $\begin{array}{r}157,734 \\
\$ 28,111 \\
0.712\end{array}$ \\
\hline $\begin{array}{c}* 101 \\
0,304 \\
0\end{array}$ & $\begin{array}{c}51 a_{,}, 73 \\
(7,009) \\
0\end{array}$ & $\begin{array}{c}969,467 \\
(7,852) \\
6\end{array}$ & $\begin{array}{r}50,163 \\
\$ 3,245) \\
0\end{array}$ & $\begin{array}{c}50,023 \\
0475 \\
0\end{array}$ & $\begin{array}{r}1,007 \\
0,050) \\
0\end{array}$ & $\begin{array}{c}\$ 2.470 \\
\text { Q.151) } \\
0\end{array}$ & $\underset{0,514)}{0}$ & $\begin{array}{r}50,1300 \\
0,007) \\
0\end{array}$ & $\begin{array}{r}5,396 \\
0,3030 \\
0\end{array}$ \\
\hline $\begin{array}{r}\$ 10,707 \\
1+14\end{array}$ & $\begin{array}{r}511,104 \\
1,114\end{array}$ & \$11, 114 & $\$ 1 t, 909$ & $\begin{array}{r}\$ 12,37 \% \\
1.144\end{array}$ & $\begin{array}{r}312,564 \\
1,114\end{array}$ & $\begin{array}{r}913,294 \\
1,114\end{array}$ & $\begin{array}{r}513,779 \\
1,114\end{array}$ & $\begin{array}{r}34,270 \\
t, 114\end{array}$ & $\begin{array}{r}\$ \text { te, } \\
5,71200\end{array}$ \\
\hline 0 & 0 & 0 & 0 & 0 & 0 & 0 & 0 & 0 & 0 \\
\hline $\begin{array}{r}\$ 11,021 \\
\$ 0,190 \\
5.70 \% \\
N A \\
\$ 7,351)\end{array}$ & 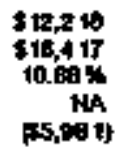 & $\begin{array}{r}\$ 12,020 \\
\$ 1,046 \\
13,74 \% \\
144 \\
94,800)\end{array}$ & $\begin{array}{r}\$ 13,000 \\
\$ 4,099 \\
1,00 \% \\
\text { mak }\end{array}$ & 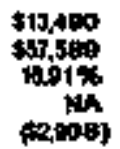 & 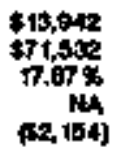 & 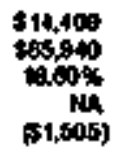 & 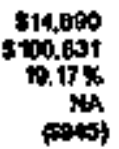 & 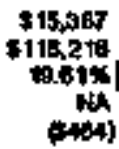 & $\begin{array}{r}\quad 17,779 \\
5133,908 \\
21,065 \\
14 \\
\infty\end{array}$ \\
\hline
\end{tabular}

19,

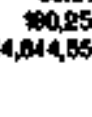

\begin{tabular}{|c|}
\hline $\begin{array}{r}30,250 \\
14,044,050\end{array}$ \\
\hline
\end{tabular}

\begin{tabular}{|c|c|c|c|c|c|c|c|c|c|}
\hline $\begin{array}{r}\mathbf{r} 50,000 \\
0 \\
0 \\
0 \\
0 \\
0 \\
0\end{array}$ & $\begin{array}{r}\Delta, 20 \\
0 \\
0 \\
0 \\
0 \\
0 \\
0\end{array}$ & $\begin{array}{r}\operatorname{ser} 712 \\
0 \\
0 \\
0 \\
0 \\
0 \\
0\end{array}$ & $\begin{array}{r}50,251 \\
0 \\
0 \\
0 \\
0 \\
0 \\
0\end{array}$ & $\begin{array}{r}\operatorname{sep} 11 \\
0 \\
0 \\
0 \\
0 \\
0 \\
0\end{array}$ & $\begin{array}{r}\forall+60: \\
0 \\
0 \\
0 \\
0 \\
0 \\
0\end{array}$ & $\begin{array}{r}574,054 \\
0 \\
0 \\
0 \\
0 \\
0 \\
0\end{array}$ & $\begin{array}{r}7+516 \\
0 \\
0 \\
0 \\
0 \\
0 \\
0\end{array}$ & $\begin{array}{r}* 0,6 \% \\
0 \\
0 \\
0 \\
0 \\
0 \\
0\end{array}$ & \begin{tabular}{r|}
503,041 \\
0 \\
0 \\
0 \\
0 \\
0 \\
0
\end{tabular} \\
\hline s5epos & st1,262 & 403,7 th & $\operatorname{sen} 201$ & stag It & 8T1,06a & 974,534 & sT,510 & $\sec , 646$ & 503,844 \\
\hline 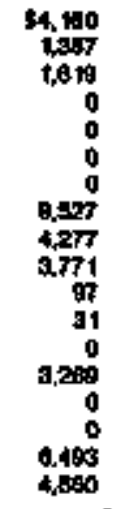 & 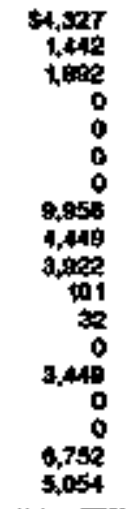 & 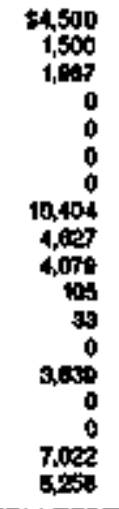 & 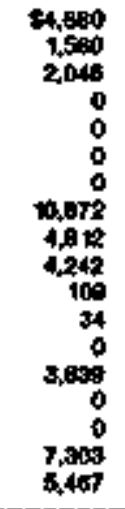 & 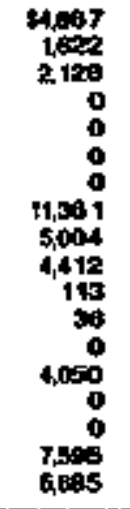 & 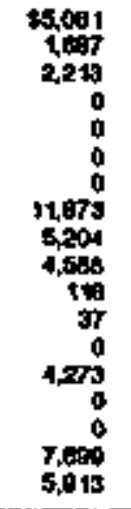 & 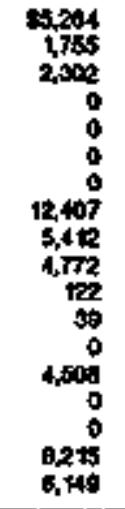 & 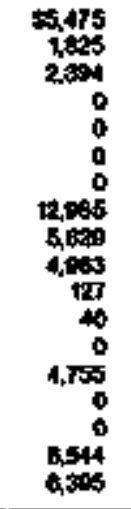 & 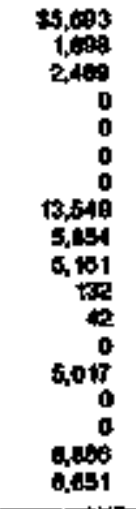 & 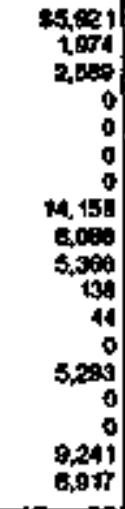 \\
\hline $\begin{array}{r}50901 \\
\$ 40,215 \\
\text { os } \\
(1,114)\end{array}$ & $\begin{array}{r}\$ 11,375 \\
\$ 19,087 \\
(1.114)\end{array}$ & $\begin{array}{r}+43,131 \\
50,581 \\
p \\
(1,1+4)\end{array}$ & $\begin{array}{r}540,905 \\
\$ 21,290 \\
(1,119)\end{array}$ & $\begin{array}{r}50,074 \\
00,048 \\
(0,10)\end{array}$ & $\begin{array}{r}140,060 \\
1,119)\end{array}$ & $\begin{array}{r}50,944 \\
\$ 20,400 \\
00 \\
(0,114]\end{array}$ & $\begin{array}{r}04,112 \\
24,404 \\
019 \\
(1,014)\end{array}$ & 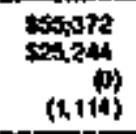 & $\begin{array}{r}157,730 \\
+20,111 \\
6,7 \mathrm{t})\end{array}$ \\
\hline $\begin{array}{c}+101 \\
0,304 \\
0\end{array}$ & $\begin{array}{c}\$ 18,773 \\
(7,009) \\
0\end{array}$ & $\begin{array}{c}910,467 \\
(7,852) \\
0\end{array}$ & $\begin{array}{r}510,163 \\
\$, 245) \\
0\end{array}$ & $\begin{array}{c}=0,025 \\
0473 \\
0\end{array}$ & $\begin{array}{r}21,007 \\
0,59 \\
0\end{array}$ & $\begin{array}{c}52.470 \\
0.181 \\
0\end{array}$ & $\begin{array}{c}\text { (20,500 } \\
0,514) \\
0\end{array}$ & $\begin{array}{r}50,+30 \\
0,007) \\
0\end{array}$ & $\begin{array}{r}5 \geq 0,396 \\
0,300 \\
0\end{array}$ \\
\hline $\begin{array}{r}510,707 \\
1+14\end{array}$ & $\begin{array}{r}511,104 \\
1,114\end{array}$ & $\begin{array}{l}* 11,514 \\
1114\end{array}$ & $\begin{array}{r}\$ 1 \text { 1, } 894 \\
\text { t, 114 }\end{array}$ & $\begin{array}{r}\$ 1297 \$ \\
1.114\end{array}$ & $\begin{array}{r}312,896 \\
1,114\end{array}$ & 919,294 & $\begin{array}{r}513,770 \\
1,114\end{array}$ & $\begin{array}{r}34,270 \\
t, 114\end{array}$ & $\begin{array}{r}\$ 20,060 \\
5,712\end{array}$ \\
\hline 0 & 0 & 0 & 0 & 0 & 0 & 0 & 0 & 0 & 0 \\
\hline $\begin{array}{r}\$ 11,021 \\
\$ 0,099 \\
5.70 \% \\
N A \\
\$ 7,351)\end{array}$ & 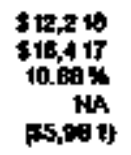 & 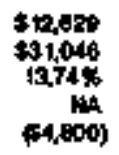 & $\begin{array}{r}\$ 13,060 \\
\$ 4,009 \\
1,0 \% \\
m, 764\end{array}$ & 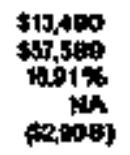 & $\begin{array}{r}\$ 15,012 \\
\$ 7,502 \\
7,07 \% \\
\text { 14, } \\
72,154)\end{array}$ & $\begin{array}{r}\$ 14,400 \\
\$ 65940 \\
100 \% \\
\text { Na } \\
51,505)\end{array}$ & 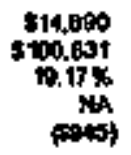 & 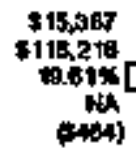 & 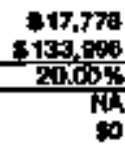 \\
\hline
\end{tabular}

64.

0002095 


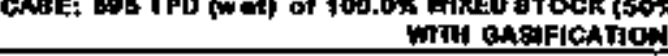

आRE LOOATKON - SRACLLE. NY
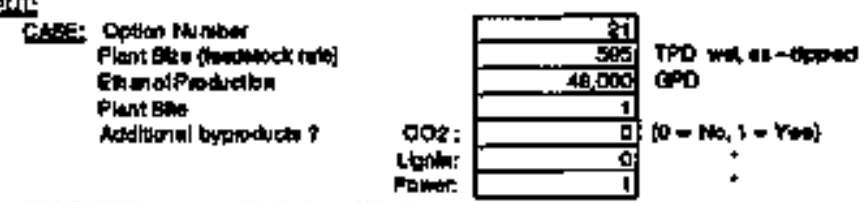

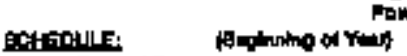
Pinintion

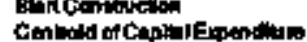

Fint Year of Oponiton Toononele Lite

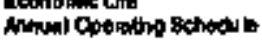

Iectulecta
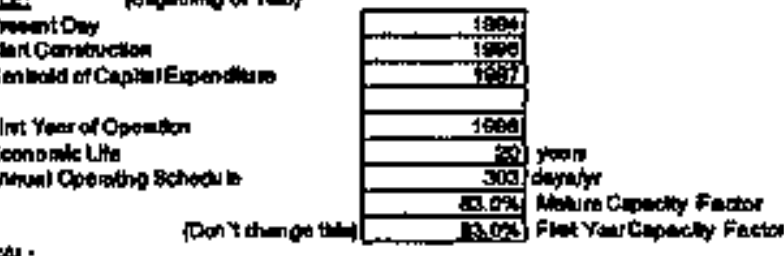

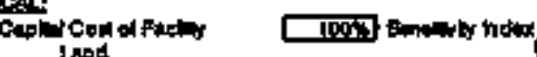
Inat Ditangen

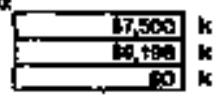

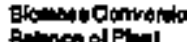

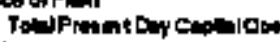

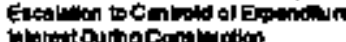

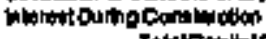

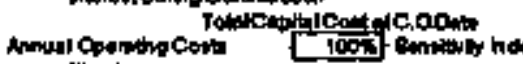
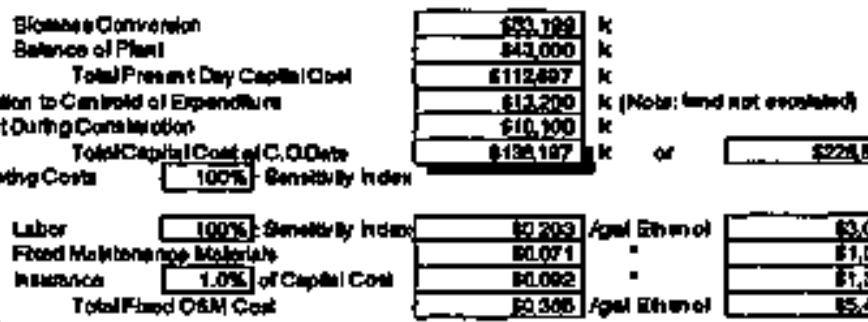

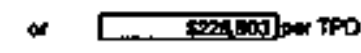
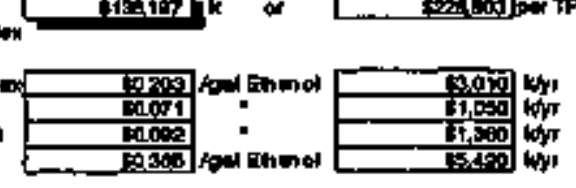

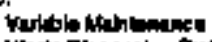

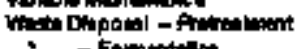
: Formmation

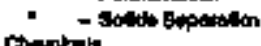
cininbatian

Crition Pouns

Powar

Natron an

Stwalit

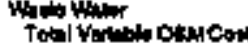

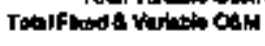
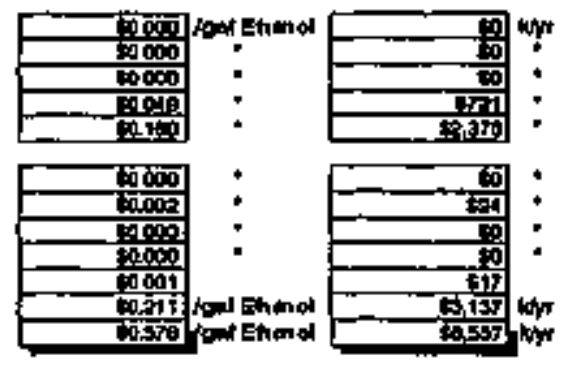

roping Fes
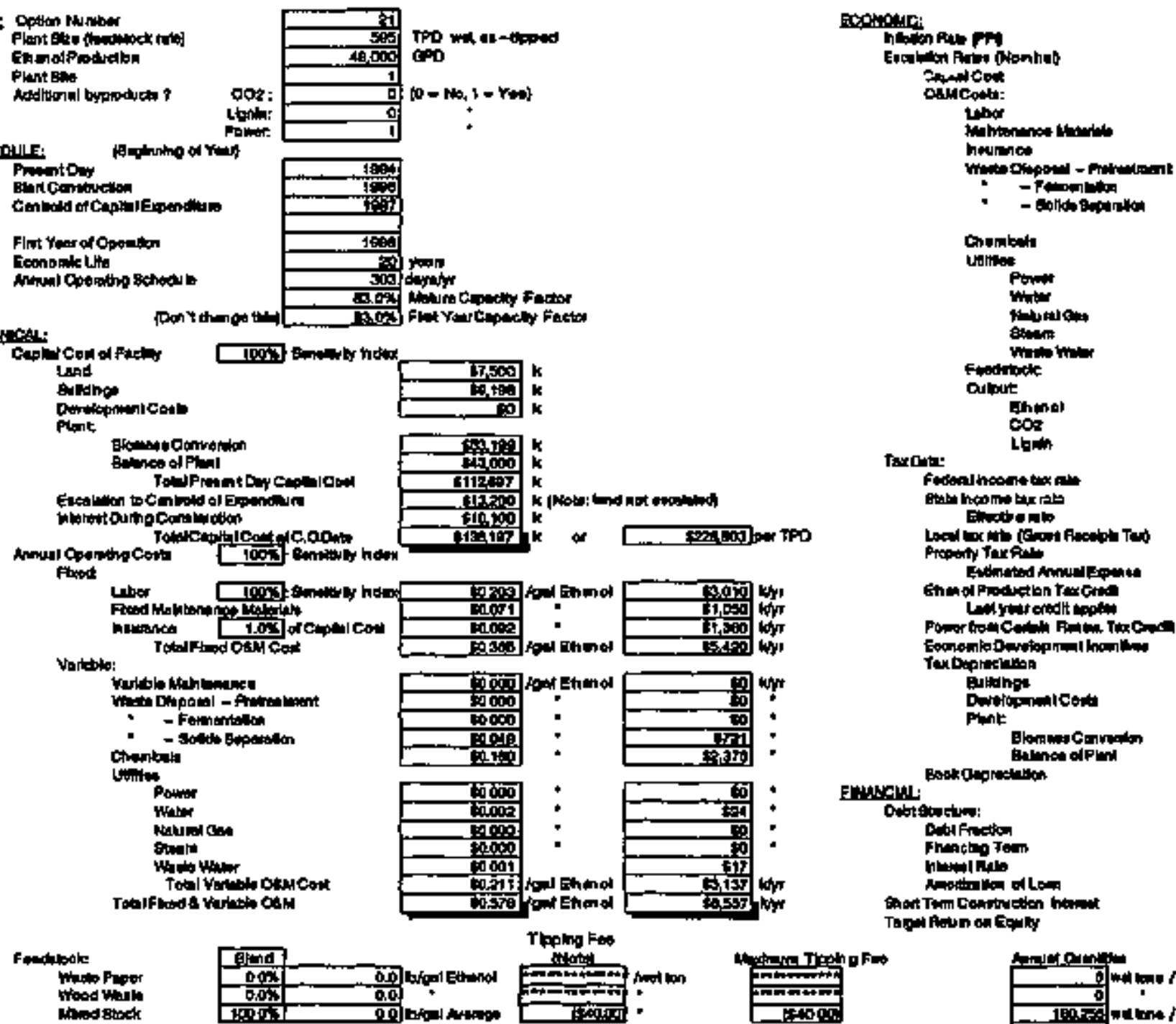

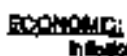

ntion fus mis

Osucout:

that

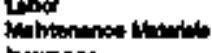

nounces

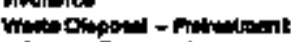

: - 7monlon

Conombati

Uulitio:

Ponm

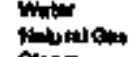

armin

Finditiocte

Oulput

Thinel
cos
tom

Paxping:

foroal hoonetox an

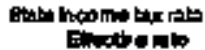

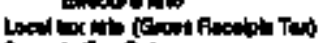

Popenty Tarsin.

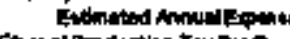

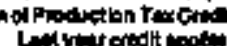

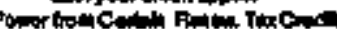

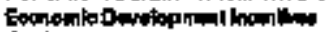

Terbepietures

Didition

Elomingacenvadon

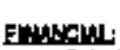

Boskangrectabon

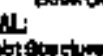
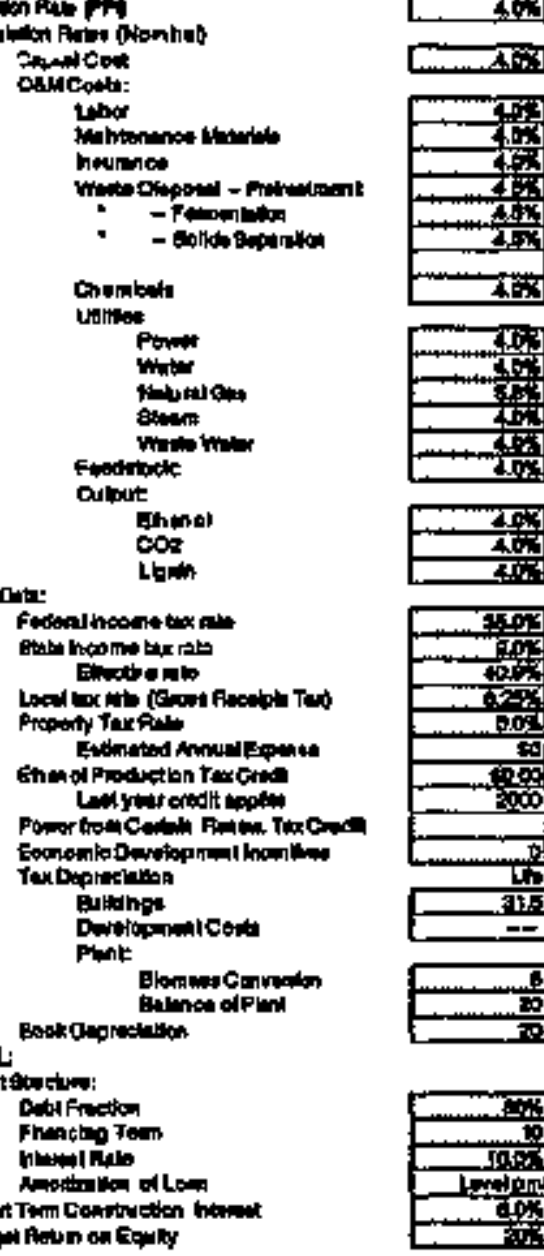

An
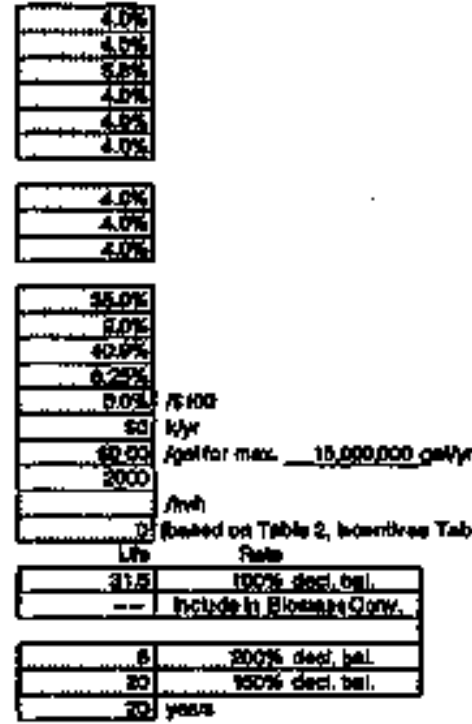

जि

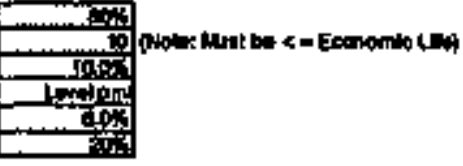

Tapow heun on Eatery

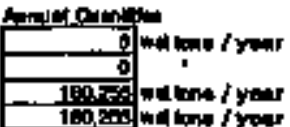

Outwo

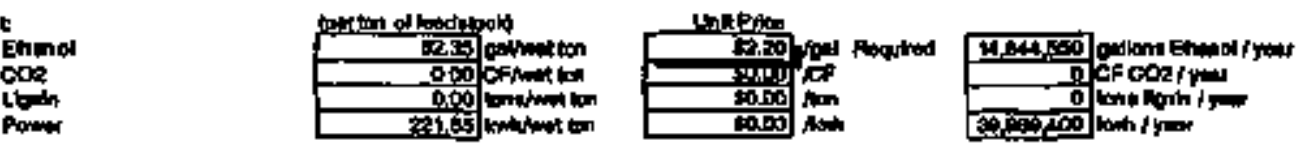

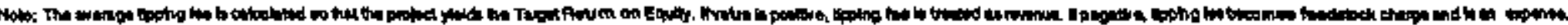




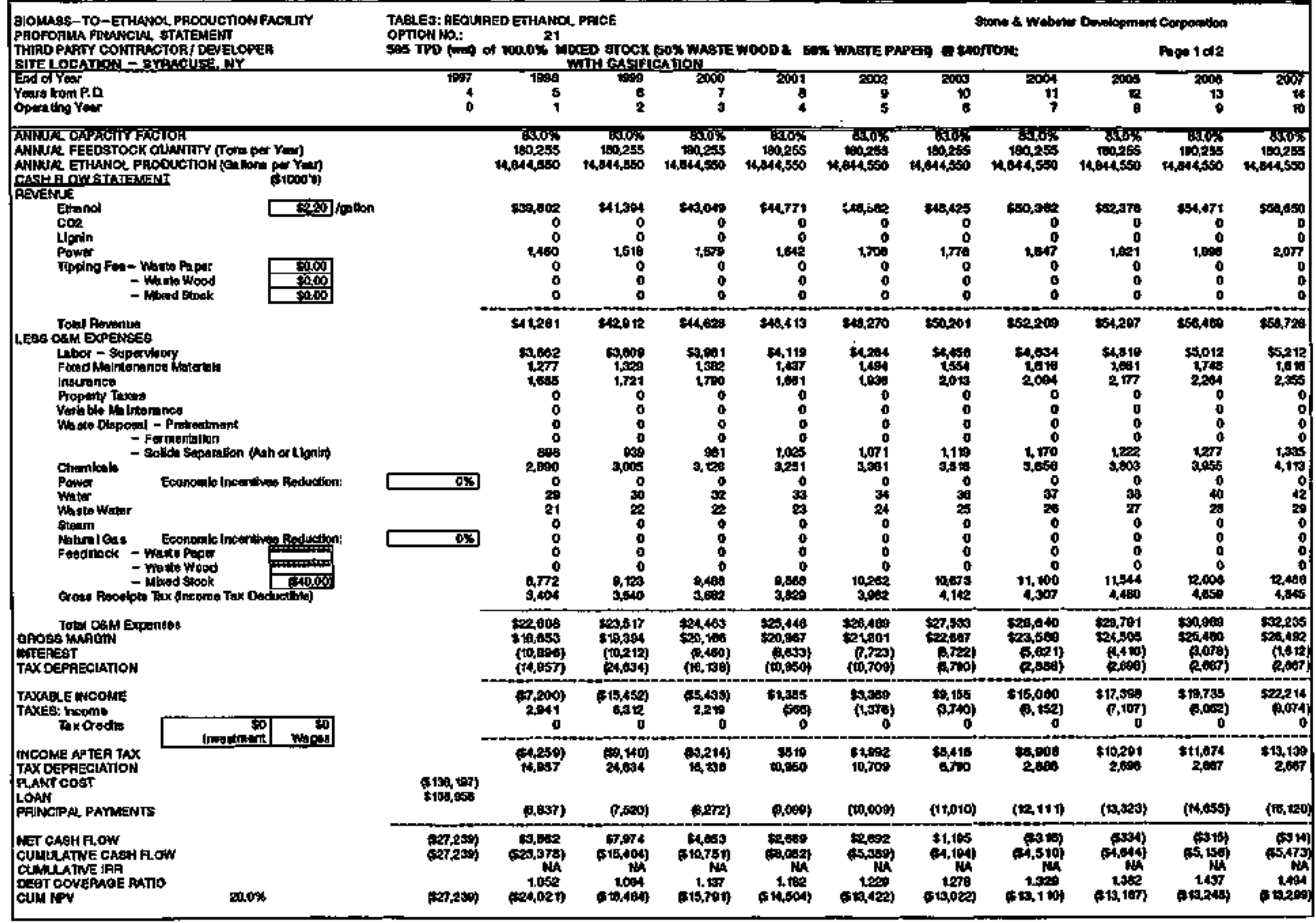




\begin{tabular}{|c|c|c|c|c|c|c|c|c|c|c|}
\hline \multirow{2}{*}{ 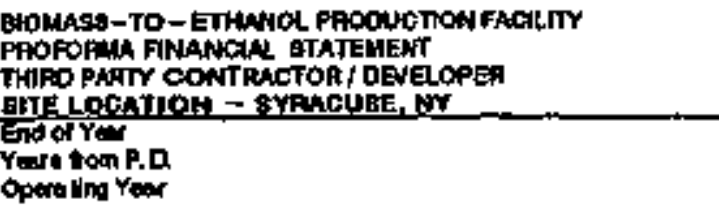 } & \multicolumn{5}{|c|}{ 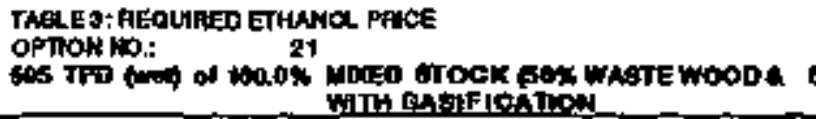 } & \multicolumn{2}{|c|}{ 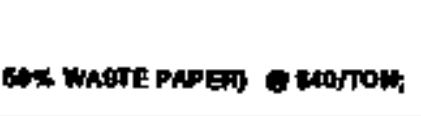 } & \multicolumn{3}{|c|}{ 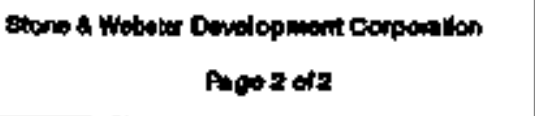 } \\
\hline & $\begin{array}{r}2000 \\
6 \\
11\end{array}$ & tod & $\begin{array}{r}2010 \\
17 \\
10\end{array}$ & $\begin{array}{r}2011 \\
18 \\
14\end{array}$ & $\begin{array}{r}2012 \\
15 \\
15\end{array}$ & 20 & $\begin{array}{r}204 \\
21 \\
17\end{array}$ & $\begin{array}{l}7 \sqrt{5} \\
22 \\
16\end{array}$ & 20 & \begin{tabular}{r|}
2017 \\
24 \\
20
\end{tabular} \\
\hline 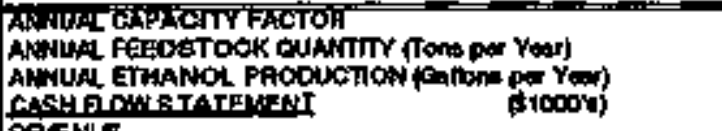 & 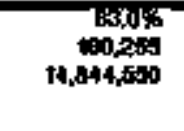 & $\begin{array}{r}0.0 \% \\
100,25 \% \\
m, 844,5 \% 0\end{array}$ & $\begin{array}{r}13 \times 0 \% \\
100,255 \\
10,044,550\end{array}$ & $\begin{array}{r}\text { ajkyin } \\
180,255 \\
4,644,560\end{array}$ & 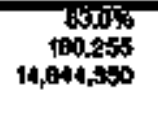 & 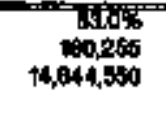 & 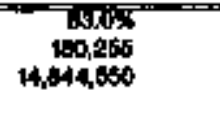 & 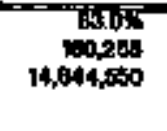 & $\begin{array}{r}\text { Exisy } \\
100,280 \\
14,044,500\end{array}$ & 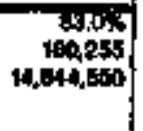 \\
\hline 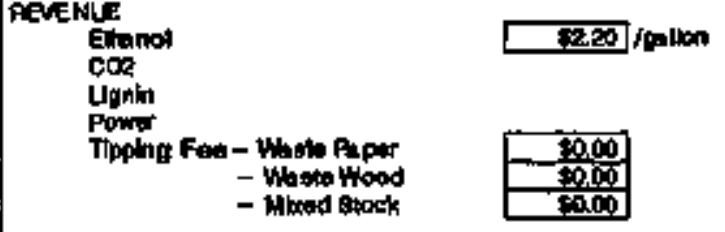 & $\begin{array}{r}250 \% \\
0 \\
2.181 \\
0 \\
0 \\
0\end{array}$ & $\begin{array}{r}62573 \\
0 \\
2,247 \\
0 \\
0 \\
0 \\
\end{array}$ & $\begin{array}{r}10.724 \\
0 \\
0 \\
2 \times 7 \\
0 \\
0 \\
0\end{array}$ & $\begin{array}{r}06,275 \\
0 \\
0 \\
2,400 \\
0 \\
0 \\
0 \\
0\end{array}$ & $\begin{array}{r}09 \\
0 \\
2520 \\
0 \\
0 \\
0\end{array}$ & $\begin{array}{r}\$ 7081 \\
0 \\
2000 \\
0 \\
0 \\
3\end{array}$ & $\begin{array}{r}574,549 \\
0 \\
2,794 \\
0 \\
0 \\
0\end{array}$ & $\begin{array}{r}4 \pi 950 \\
0 \\
0 \\
2,93 \\
0 \\
0 \\
0\end{array}$ & $\begin{array}{r}\sin 001 \\
0 \\
0 \\
2,067 \\
0 \\
0 \\
0\end{array}$ & $\begin{array}{r}500,050 \\
0 \\
0 \\
3,050 \\
0 \\
0 \\
0\end{array}$ \\
\hline 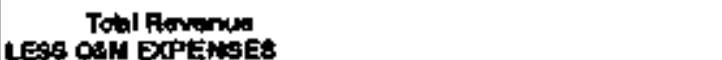 & & $\sin 68$ & $20,0 \% 1$ & 196,70, & $\$ 7,451$ & 974,340 & 977,290 & $\div 0,373$ & $\operatorname{ses} 509$ & 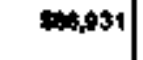 \\
\hline 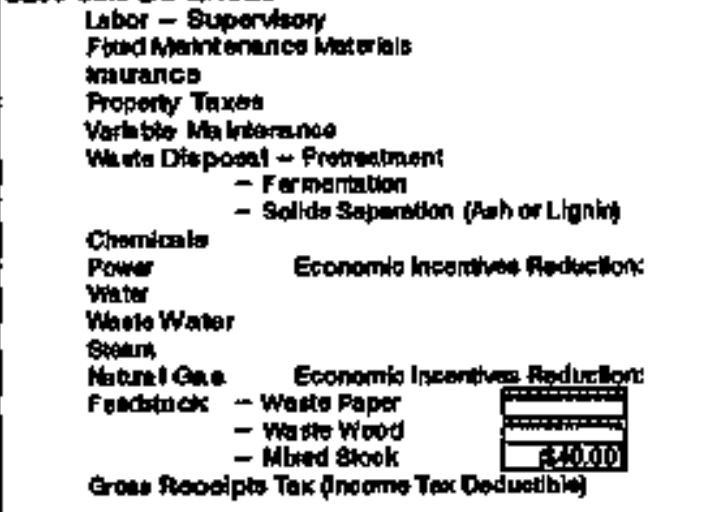 & 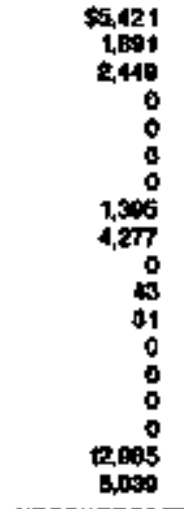 & 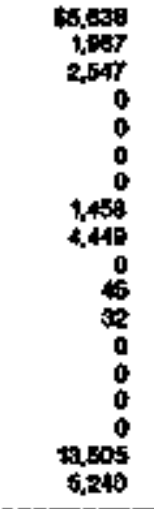 & 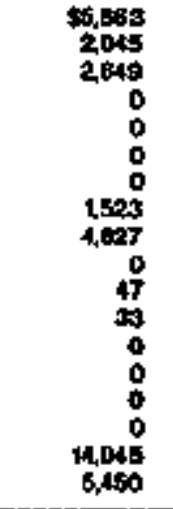 & 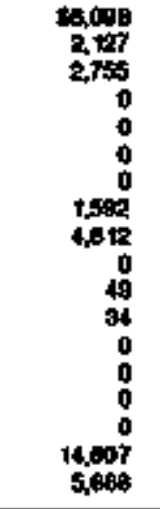 & $\begin{array}{r}5,342 \\
2,212 \\
2,665 \\
0 \\
0 \\
0 \\
0 \\
1,008 \\
6,000 \\
0 \\
51 \\
36 \\
0 \\
0 \\
0 \\
0 \\
15,01 \\
5,695\end{array}$ & 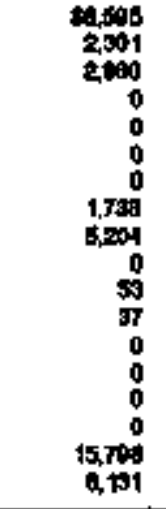 & $\begin{array}{r}2,050 \\
2,390 \\
3,009 \\
0 \\
0 \\
0 \\
0 \\
1,014 \\
8,412 \\
0 \\
5 \\
30 \\
0 \\
0 \\
0 \\
18,00 \\
0,370\end{array}$ & $\begin{array}{r}5,193 \\
2,160 \\
3,200 \\
0 \\
0 \\
0 \\
0 \\
1,680 \\
5,020 \\
0 \\
57 \\
40 \\
0 \\
0 \\
0 \\
0 \\
17000 \\
0.091\end{array}$ & 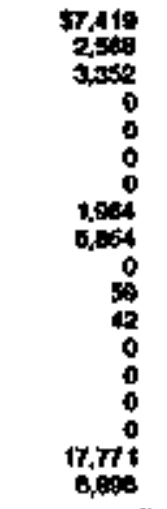 & 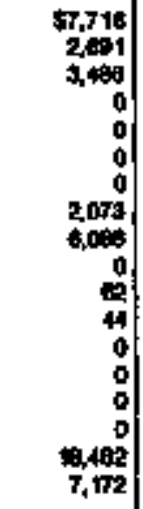 \\
\hline 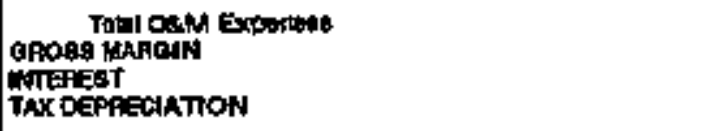 & 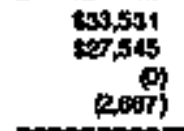 & $\begin{array}{l}\$ 4,060 \\
\$ 20,60 \\
0,607)\end{array}$ & 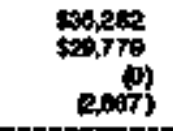 & 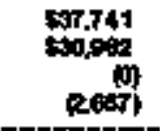 & simation & $\begin{array}{l}50,057 \\
30,472 \\
90,9\end{array}$ & parion & 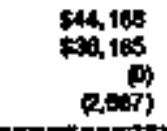 & 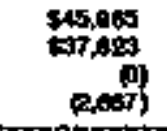 & 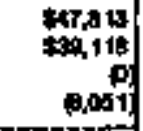 \\
\hline 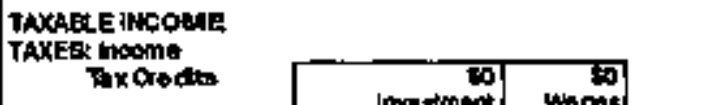 & $\begin{array}{c}24,070 \\
(20,163) \\
0\end{array}$ & $\begin{array}{c}525,074 \\
(10,610) \\
0\end{array}$ & $\begin{array}{c}527,112 \\
(+1,075) \\
6\end{array}$ & $\begin{array}{c}50205 \\
(12,559) \\
0\end{array}$ & 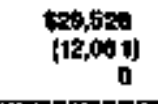 & $\begin{array}{c}+50,006 \\
(12,591) \\
0\end{array}$ & $\begin{array}{c}60,136 \\
(13,127) \\
0\end{array}$ & 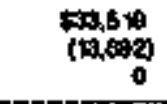 & $(14,200)$ & 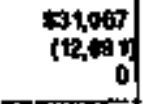 \\
\hline $\begin{array}{l}\text { WWOOHE AFTEF TAK } \\
\text { TAX DEPAECANTON } \\
\text { LANT COST } \\
\text { LOWN } \\
\text { PFWCIPAL PAMMENTS }\end{array}$ & $\begin{array}{r}814,7 \\
2.007\end{array}$ & $\begin{array}{r}\$+1,304 \\
2,606\end{array}$ & $\begin{array}{r}516,037 \\
2,667\end{array}$ & $\begin{array}{l}+\operatorname{ta} 737 \\
2667\end{array}$ & $\begin{array}{l}17,400 \\
2,067\end{array}$ & $\begin{array}{l}\$ 10,221 \\
2007\end{array}$ & $\begin{array}{r}\$ 19,000 \\
2,607\end{array}$ & $\sin _{2006}$ & $\begin{array}{l}\operatorname{seg} 6 \pi \\
2007\end{array}$ & $\begin{array}{r}\$ 10,370 \\
0,051\end{array}$ \\
\hline 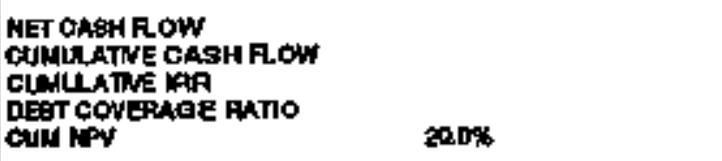 & 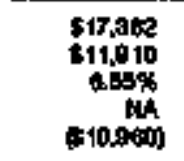 & 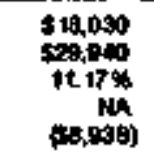 & 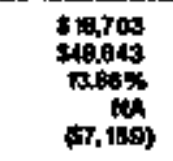 & 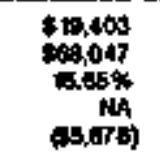 & 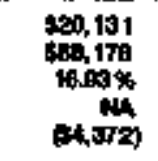 & 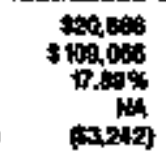 & $\begin{array}{r}21,675 \\
\sin , 741 \\
10814 \\
\text { Ma } \\
0.205)\end{array}$ & 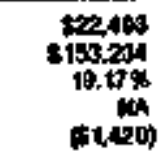 & 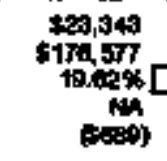 & 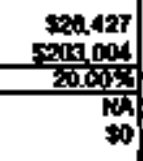 \\
\hline
\end{tabular}

praps 


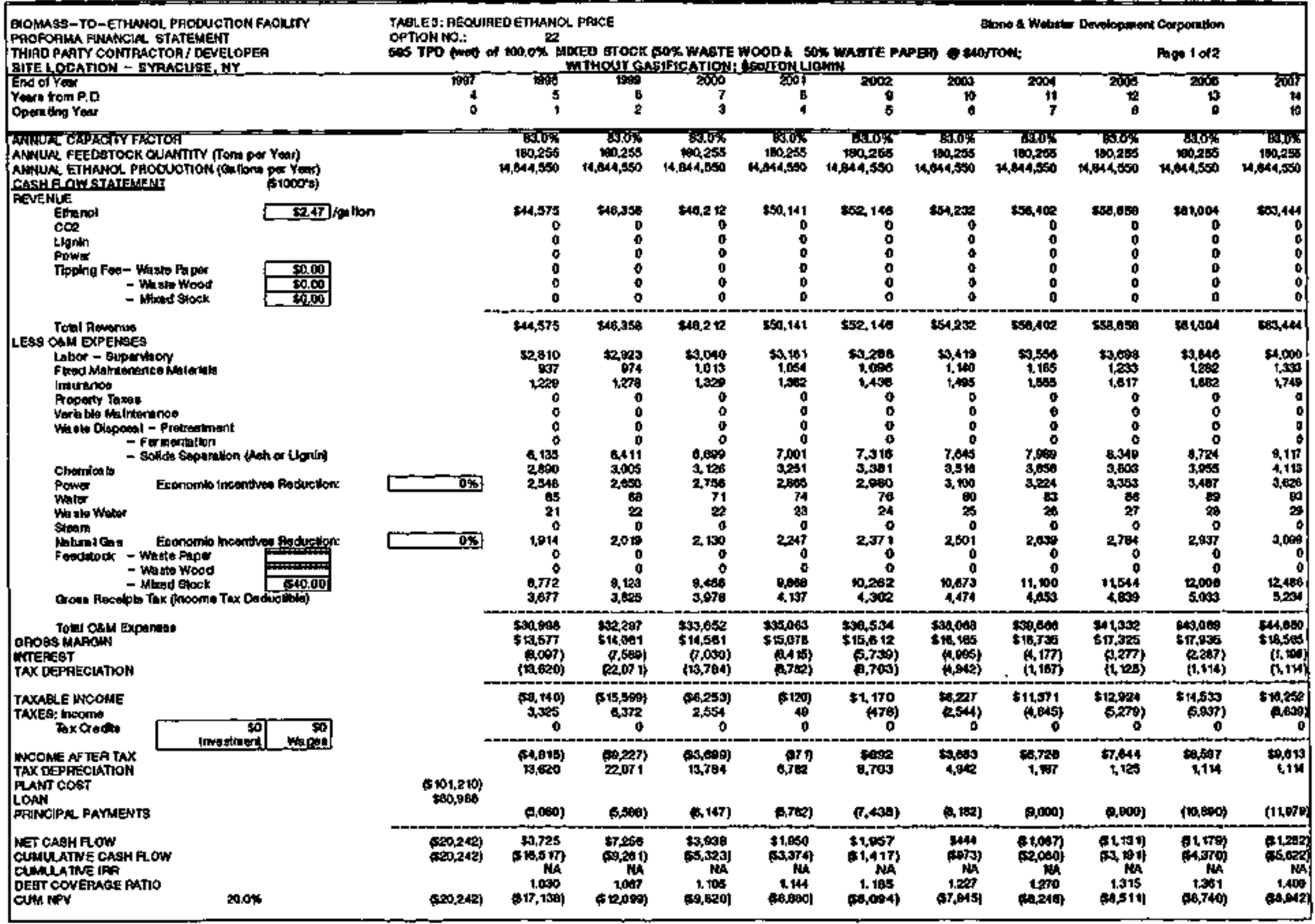




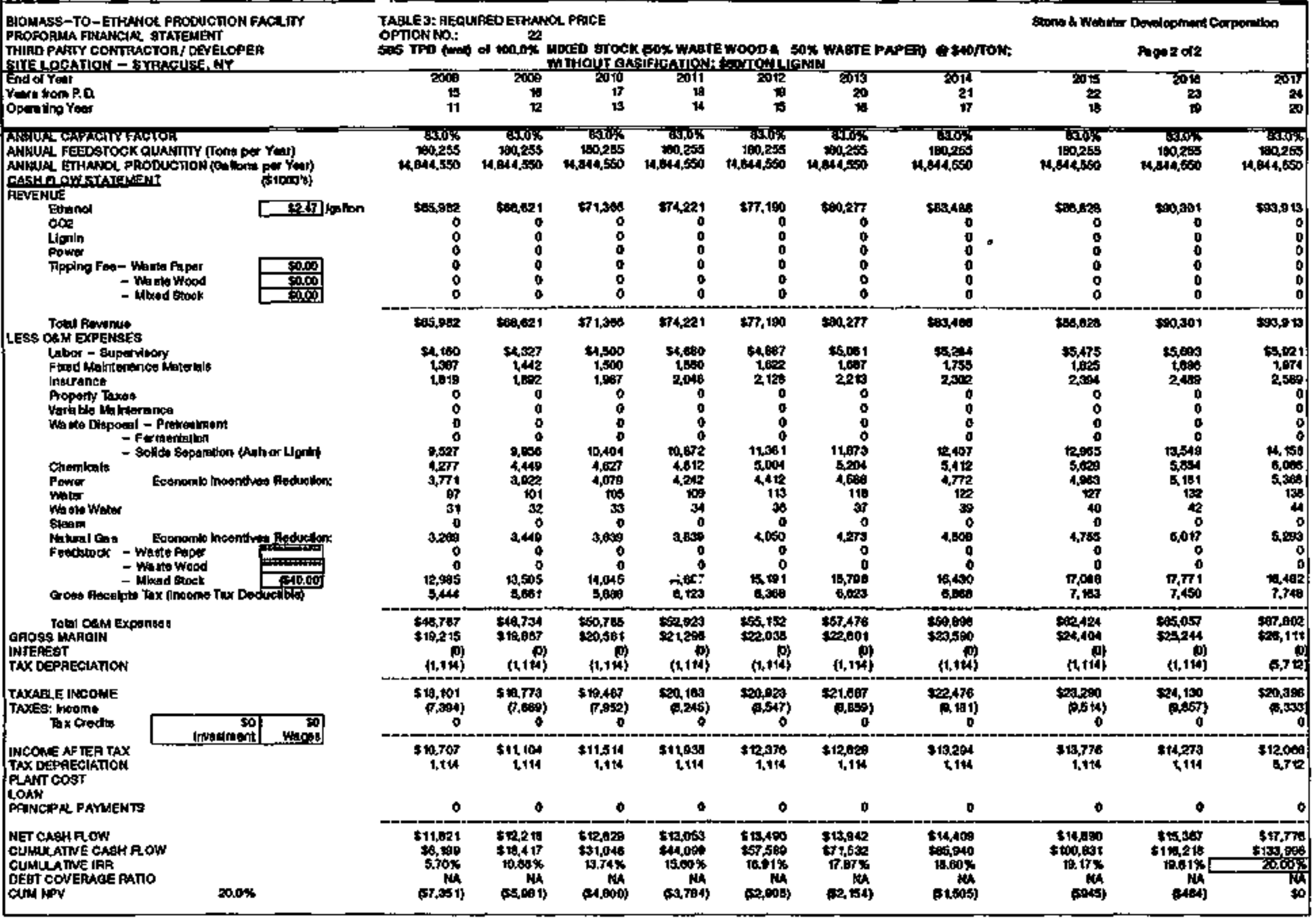




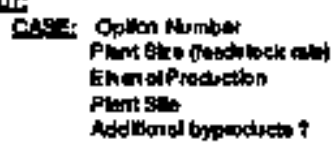

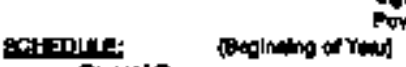

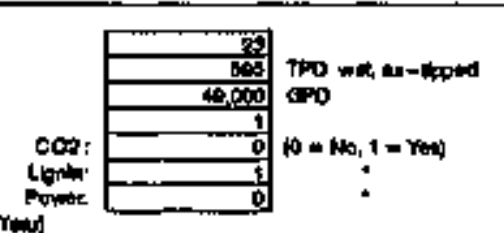

Pintingor

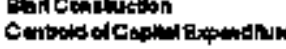
Eesnomie Ln

IEAHMich

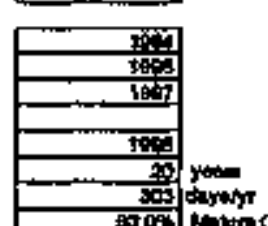

yowit
Flat Yot ol Opesetion

econsalto:

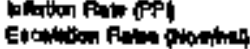
Coplestist

sugho:

Cobor

mions

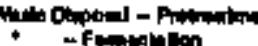

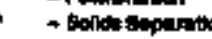

Conpatoms

Utlition:

Pim

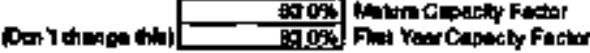

Cupluy con of Foenty

$$
\text { lind }
$$

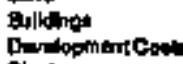

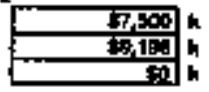

Plemt

Gomingenvemican

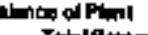

Concuplacent inold of Exinthy monew Quing conemuction

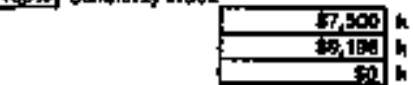

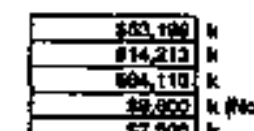

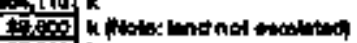

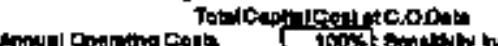
Futert

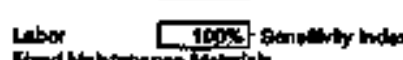

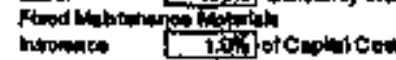
valus: Totratodosticon

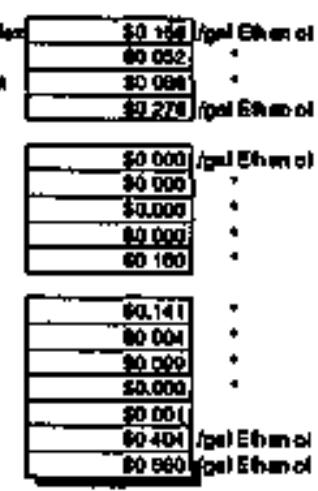

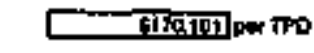

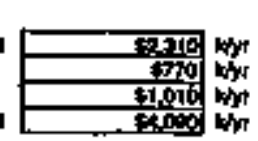

Vimblo Nahlomente.

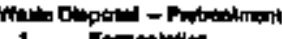

- - Foldatantion

Chambers

Intorion

Pond

Nativentom

Gimen

Tom the Tom f Frod at vaming coust

Punduati:

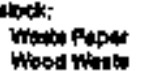

Wood niont

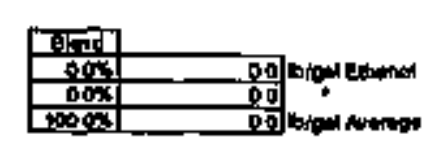

Itoping roo
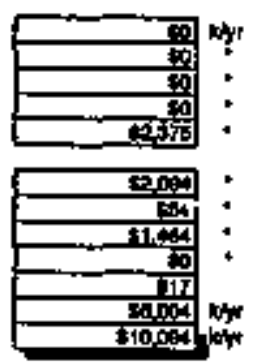

Ellentitit

joctis.

mised and

Funceris?

Gha

cos

$\operatorname{Tax}$ Drot

Lit

Fodecidheome tax note

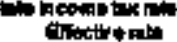

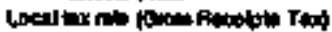

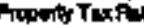

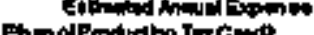

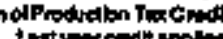

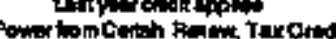

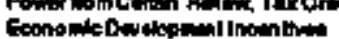

Dopioditum

alling

Pint

Boneconverion

onoh Depumationt

Antion:

Datifingen

mimatis

Thench

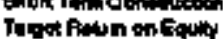
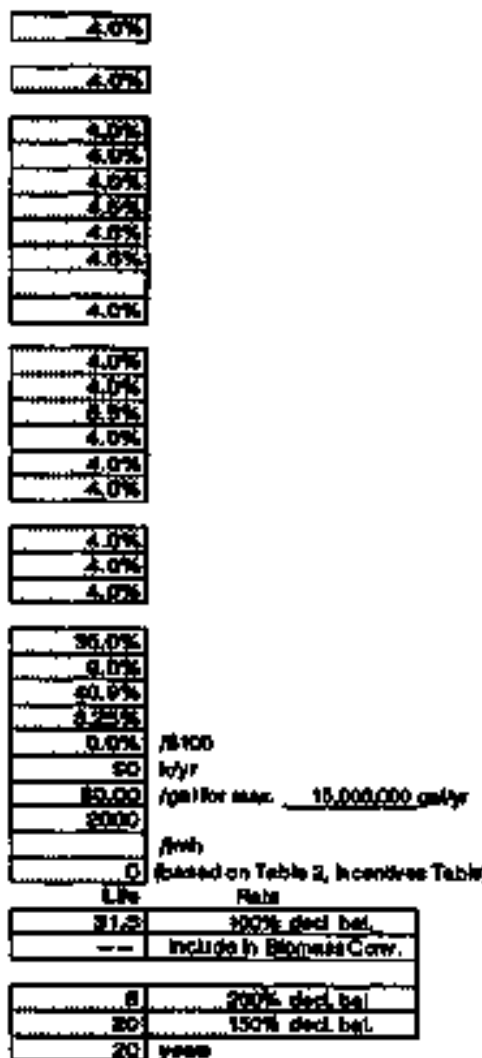

त讨

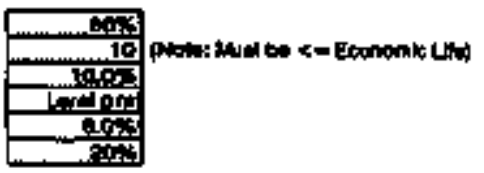

Orpule

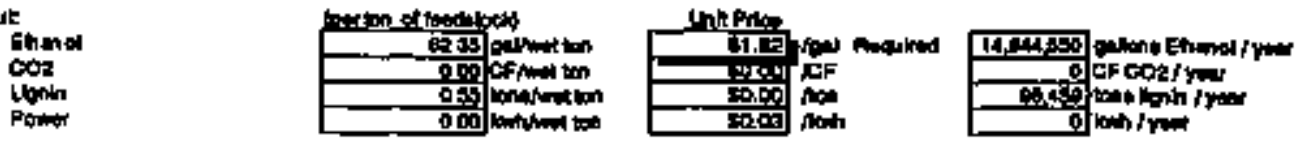

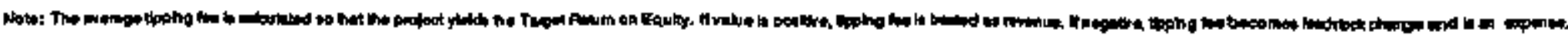




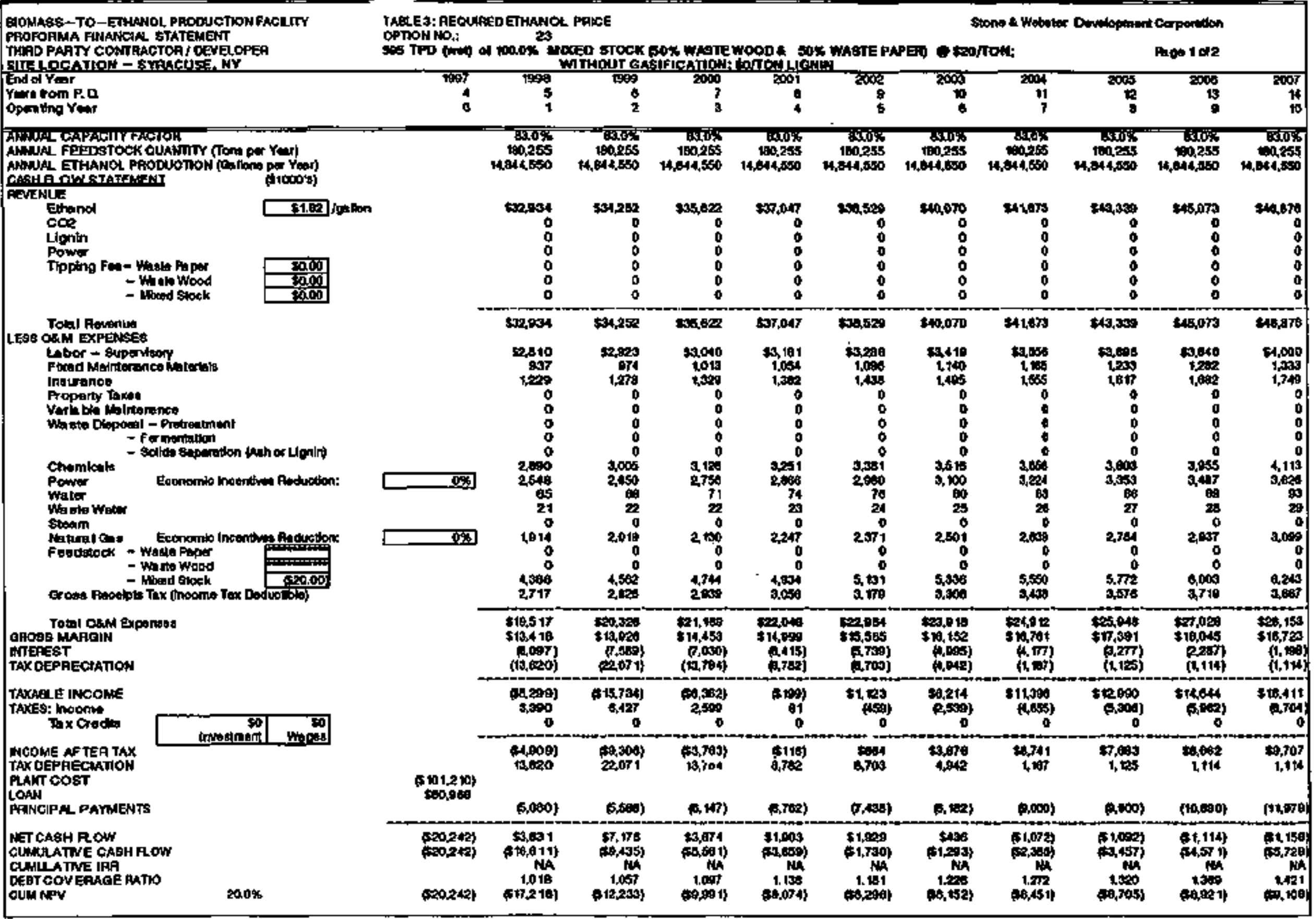




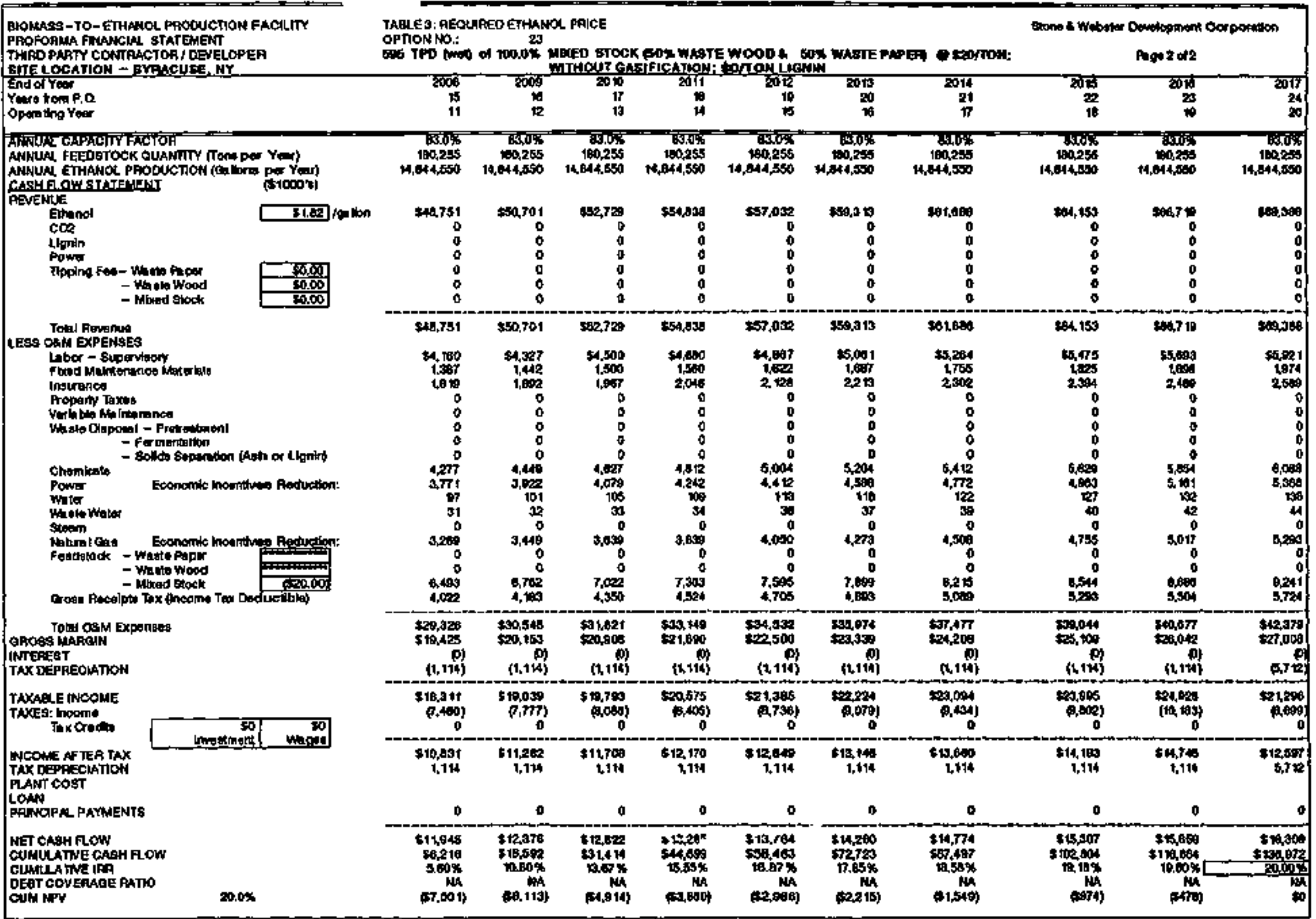




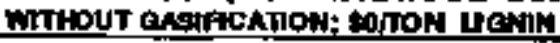

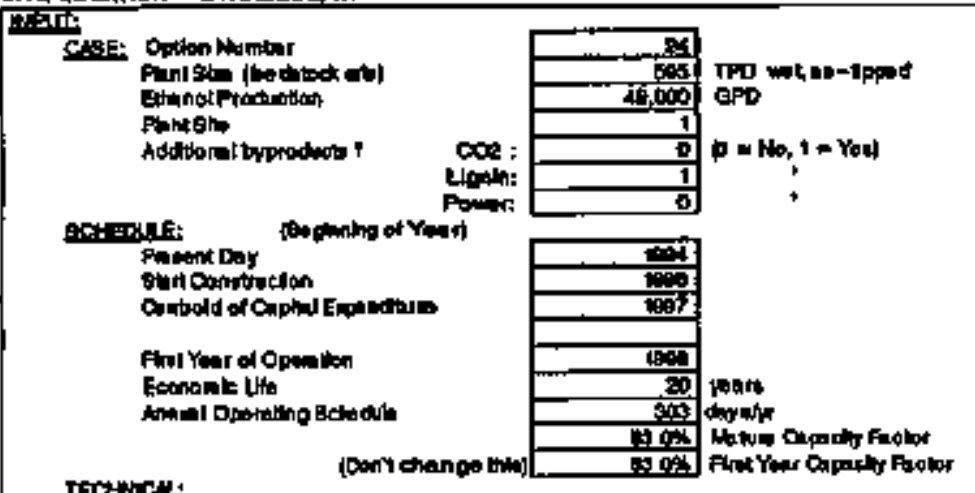

Ifaction.

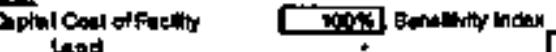

lead

Dewoborencint

Pant

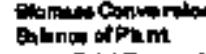

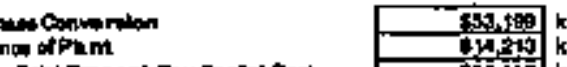

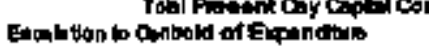

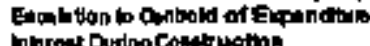

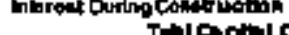

Anmol O

Fint- to

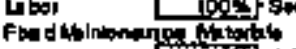

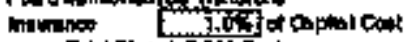

Warbel:

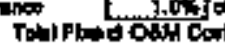

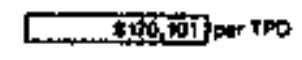

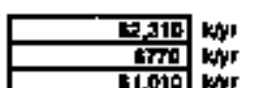
Ii, 심

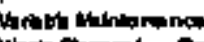

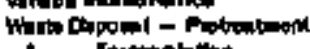
- - Fordionbution

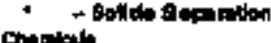
Constion

Powar Ningente. simim What Wate

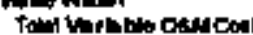

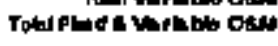
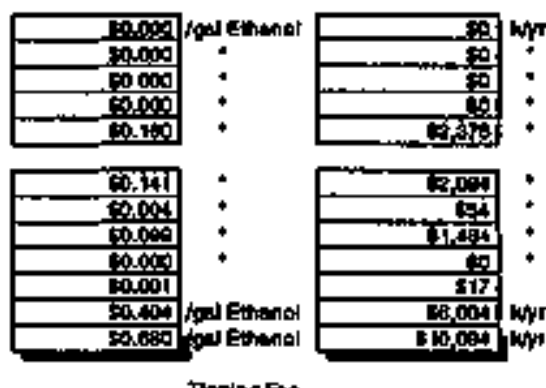
Theping Fon

Fodutiols

Whod Poper

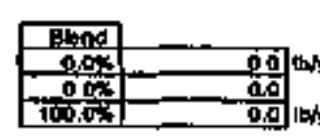

管

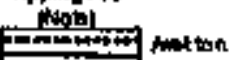

.

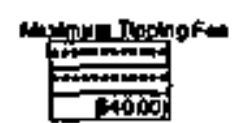

Ink Dat:

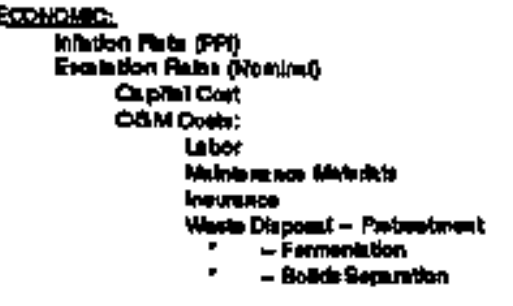

- - Boldeserpmiton

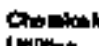

(untom:

Fom

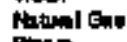

when

Pondrack:

Emanol

uom

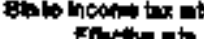

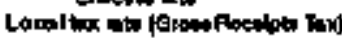

Propary Tux Ru

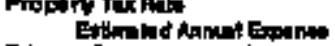

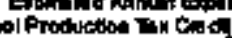

Lal your trod appose.

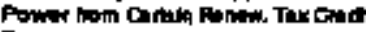

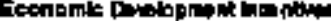

Tox Deprocivion

Gulctipe

Dosobpencenter

Elomen Comprotor

Boot Dopenchiton

Existortis.

Det otritom

Doti fundon

Finnolno Twm

mantintion of tom

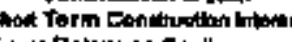

Tupot inetum on Edoly
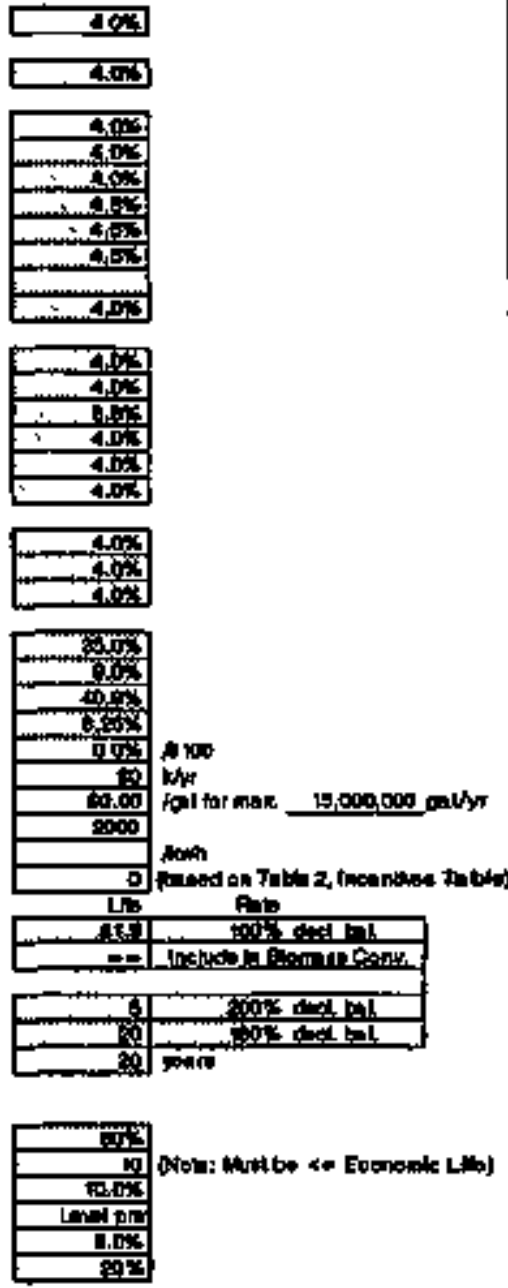

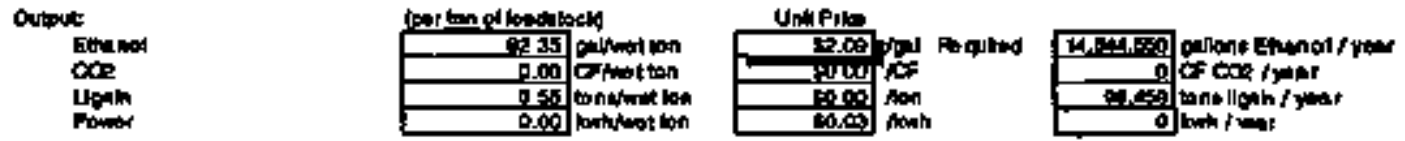

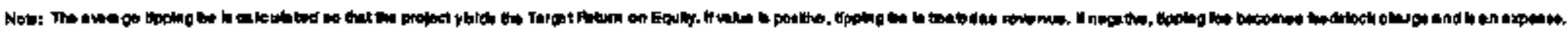




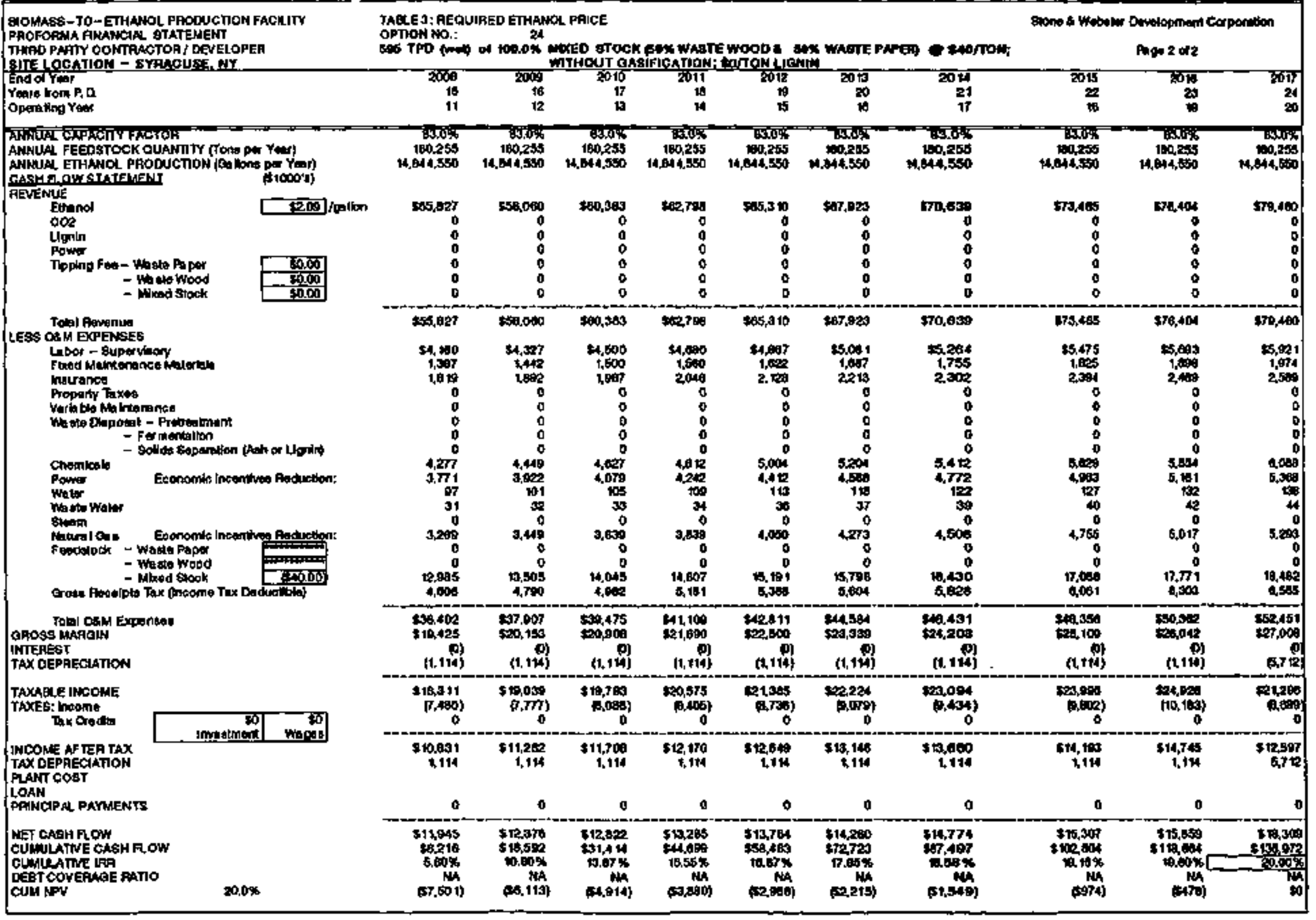




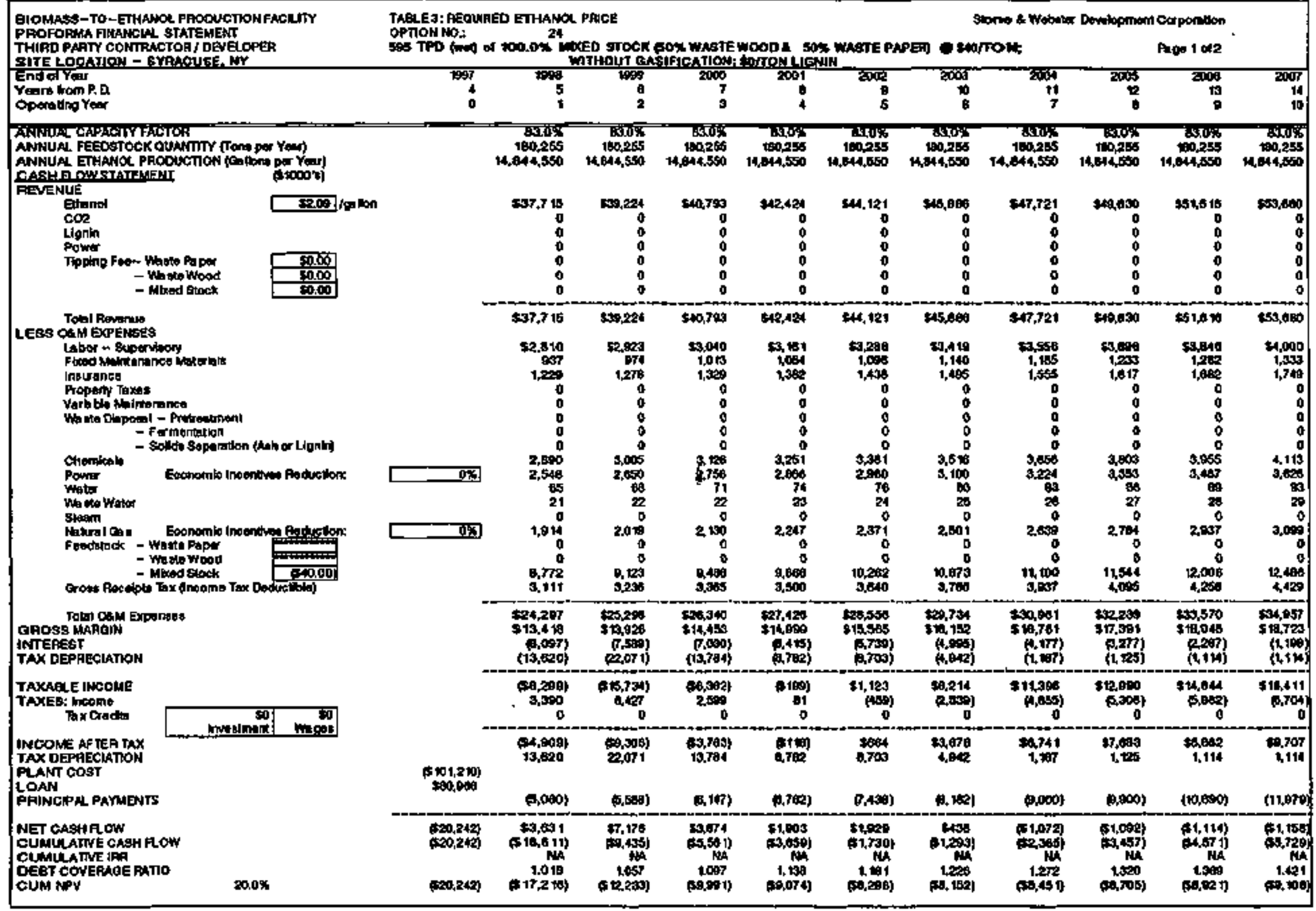




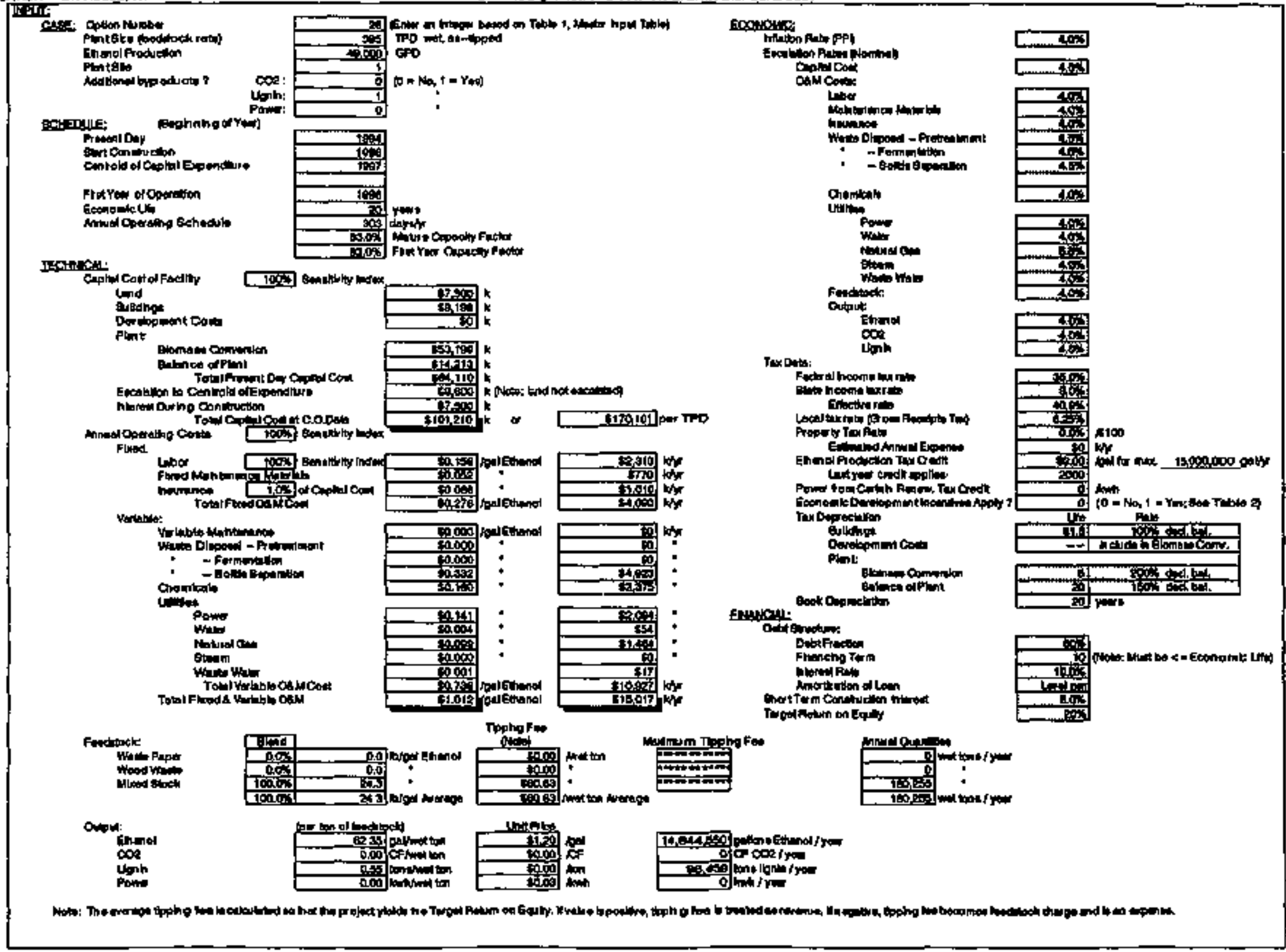

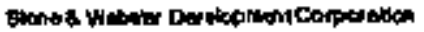




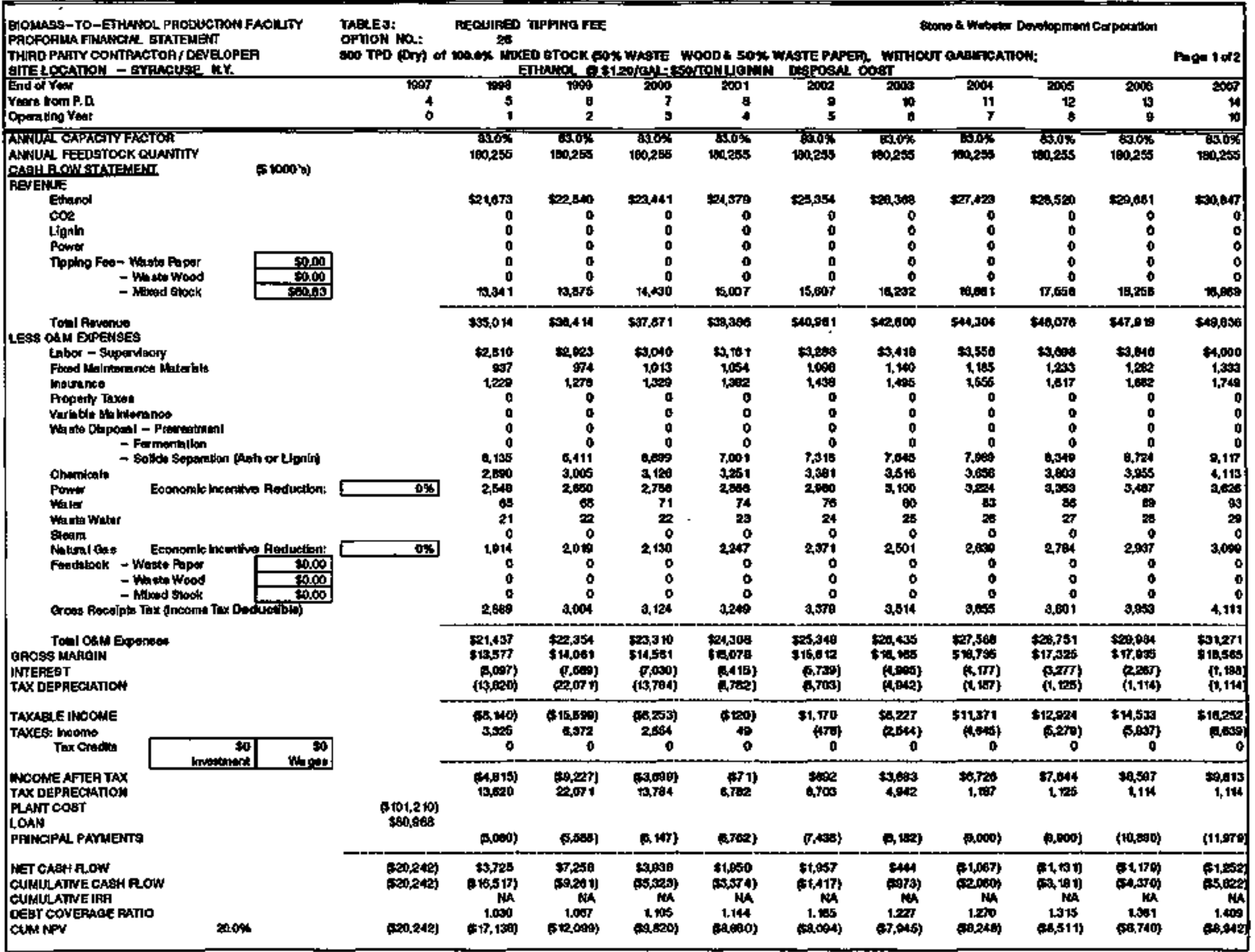




\begin{tabular}{|c|c|c|c|c|c|c|c|c|c|c|}
\hline 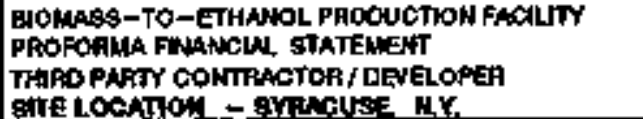 & $\begin{array}{l}\text { TABLES: } \\
\text { OPTOM No.: } \\
\text { Dos TPD (0ny) of }\end{array}$ & 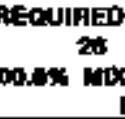 & 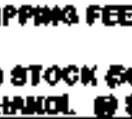 & whote & 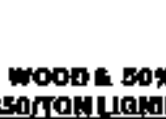 & 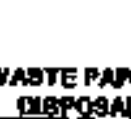 & $\pi$ & Bionest & I D D D & Pagez or \\
\hline $\begin{array}{l}\text { End ol You } \\
\text { Yeare tom P. D } \\
\text { Optenting Year }\end{array}$ & $\begin{array}{r}2008 \\
\$ 5 \\
11\end{array}$ & $\begin{array}{r}2009 \\
10 \\
12\end{array}$ & $\begin{array}{r}2010 \\
7 \\
17\end{array}$ & 2011 & $\begin{array}{r}2012 \\
75 \\
45\end{array}$ & $\begin{array}{r}2013 \\
30 \\
16\end{array}$ & $\begin{array}{r}2014 \\
21 \\
17\end{array}$ & $\begin{array}{r}200 \\
2 \\
10\end{array}$ & $\begin{array}{r}2010 \\
23 \\
20\end{array}$ & $\begin{array}{r}2017 \\
24 \\
20\end{array}$ \\
\hline 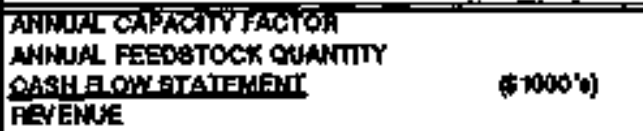 & 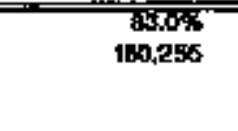 & 18:0,265 & $\begin{array}{r}6.0 \% \\
160,256\end{array}$ & $\begin{array}{l}760 \% \\
\text { mans }\end{array}$ & $\begin{aligned}+330 \% \\
180,256\end{aligned}$ & $\begin{array}{r}04,0 \% \\
160,250\end{array}$ & 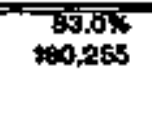 & $\underset{00,255}{1050}$ & $\begin{array}{l}\mathrm{bank} \\
100,256\end{array}$ & $\begin{array}{r}650 \% \\
100,265\end{array}$ \\
\hline 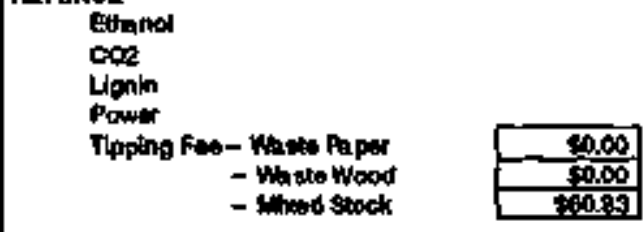 & $\begin{array}{r}\$ 32,001 \\
0 \\
0 \\
0 \\
0 \\
0 \\
+2,746\end{array}$ & $\begin{array}{r}233,364 \\
0 \\
0 \\
0 \\
0 \\
0 \\
20,538\end{array}$ & $\begin{array}{r}\$ 4,000 \\
0 \\
0 \\
0 \\
0 \\
0 \\
21,300\end{array}$ & $\begin{array}{r}\$ 9,007 \\
0 \\
0 \\
0 \\
0 \\
0 \\
22,214\end{array}$ & $\begin{array}{r}37,530 \\
0 \\
0 \\
0 \\
0 \\
0 \\
23,509\end{array}$ & $\begin{array}{r}\$ 30,091 \\
0 \\
0 \\
0 \\
0 \\
0 \\
24,025\end{array}$ & $\begin{array}{r}40,503 \\
0 \\
0 \\
0 \\
0 \\
0 \\
24,80 .\end{array}$ & $\begin{array}{r}2+2,10 \\
0 \\
0 \\
0 \\
0 \\
0 \\
0\end{array}$ & $\begin{array}{r}43,000 \\
0 \\
0 \\
0 \\
0 \\
0 \\
27.007\end{array}$ & \begin{tabular}{r|r} 
Cos, 061 \\
0 \\
0 \\
0 \\
0 \\
0 \\
20,109
\end{tabular} \\
\hline 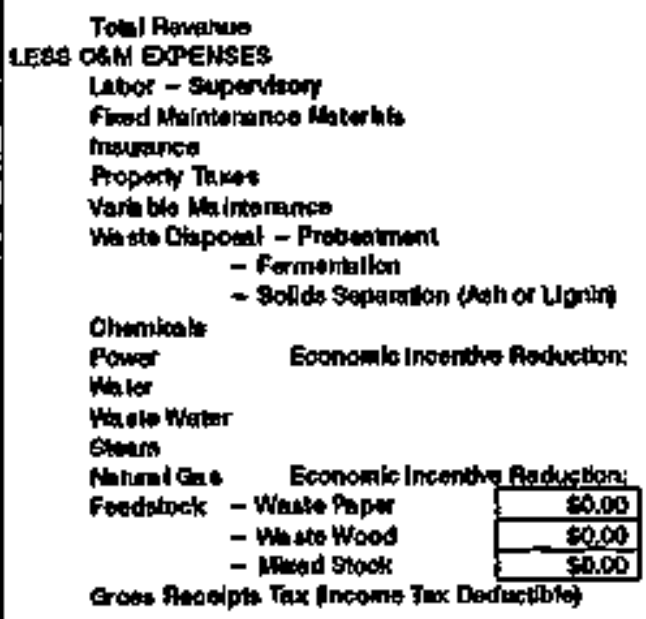 & 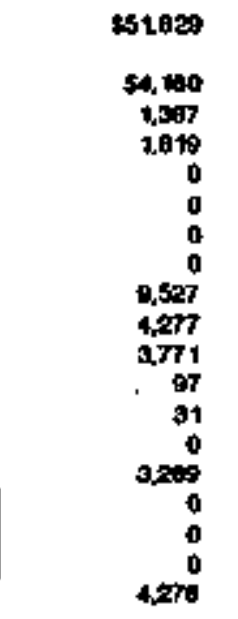 & 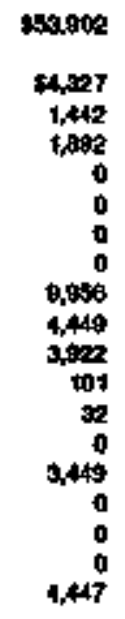 & 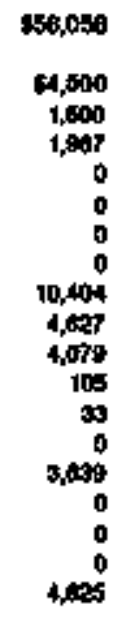 & 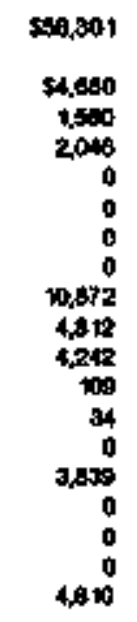 & $\begin{array}{r}300,639 \\
4,007 \\
1,602 \\
2,120 \\
0 \\
0 \\
0 \\
0 \\
11,381 \\
5,004 \\
4,412 \\
113 \\
30 \\
0 \\
4,060 \\
0 \\
0 \\
0 \\
5,002\end{array}$ & $\begin{array}{r}509,050 \\
53,001 \\
1,607 \\
2,219 \\
0 \\
0 \\
0 \\
0 \\
11,073 \\
5,204 \\
4,900 \\
716 \\
97 \\
0 \\
4,273 \\
0 \\
0 \\
5,200\end{array}$ & $\begin{array}{r}305,500 \\
55,254 \\
1,755 \\
2,302 \\
0 \\
0 \\
0 \\
0 \\
12,407 \\
5,402 \\
4,7 \pi 2 \\
122 \\
30 \\
0 \\
4506 \\
0 \\
0 \\
0 \\
5,410\end{array}$ & 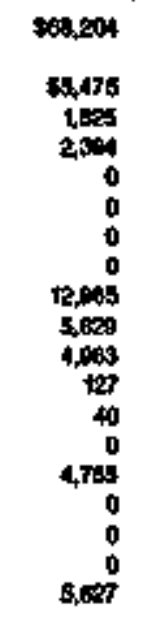 & $\begin{array}{r}50,982 \\
50,493 \\
+, 090 \\
2,409 \\
0 \\
0 \\
0 \\
0 \\
15,349 \\
5,054 \\
5,101 \\
132 \\
42 \\
0 \\
5,017 \\
0 \\
0 \\
0 \\
5,058\end{array}$ & 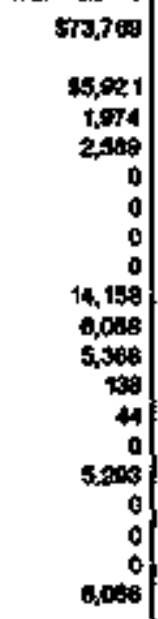 \\
\hline 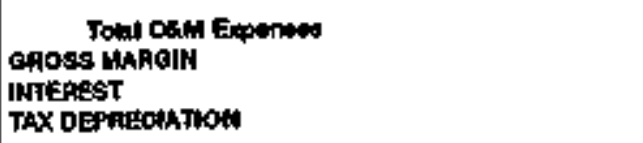 & $\begin{array}{r}\$ 2,614 \\
\$ 10,215 \\
0\} \\
(1,1,14)\end{array}$ & $\begin{array}{c}24,016 \\
610,907 \\
(1,114)\end{array}$ & $\begin{array}{r}1+4,470 \\
0,501 \\
(0) \\
(1,114)\end{array}$ & $\begin{array}{r}\$ 21,000 \\
\$ 90 \\
(1,114)\end{array}$ & 50,58s & $\begin{array}{r}50,257 \\
+2,001 \\
0) \\
(1,114)\end{array}$ & $\begin{array}{c}41,900 \\
\tan , 500 \\
(1,14)\end{array}$ & $\begin{array}{l}\text { sas,owa } \\
\text { set,404 } \\
(1,14)\end{array}$ & $\begin{array}{c}\$ 15,060 \\
+2,244 \\
(1,1+4)\end{array}$ & $\begin{array}{r}\$ 1,65 a \\
0 \times 11 \\
0,7+2)\end{array}$ \\
\hline 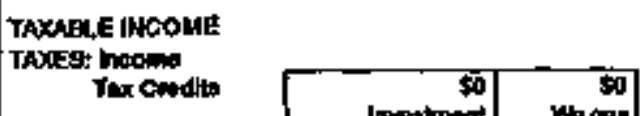 & $\begin{array}{c}\$ 18,01 \\
0,504) \\
0\end{array}$ & $\begin{array}{c}\$ 10,779 \\
(7, \infty 68) \\
0\end{array}$ & 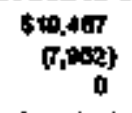 & $\begin{array}{c}50,139 \\
0.245) \\
0\end{array}$ & $\underset{0}{200928}$ & $\begin{array}{c}\$ 21,007 \\
0,059) \\
0\end{array}$ & $\begin{array}{c}20,40 \\
0\end{array}$ & $\begin{array}{r}20 \times 200 \\
0,540 \\
0\end{array}$ & $\begin{array}{r}04,130 \\
0105 \% 1) \\
0\end{array}$ & $\begin{array}{r}=0,396 \\
0,340] \\
0\end{array}$ \\
\hline 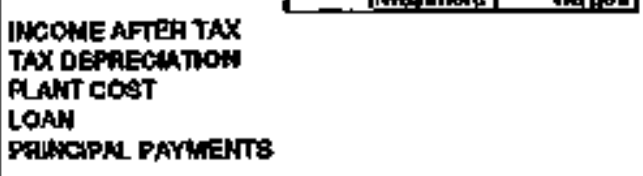 & $\begin{array}{r}\$ 10,707 \\
1,114\end{array}$ & $\begin{array}{r}512,104 \\
4,114\end{array}$ & $\begin{array}{r}\mathbf{s 1 t}, 54 \\
x, 114\end{array}$ & $\begin{array}{r}\$ 11,839 \\
1,114\end{array}$ & $\begin{array}{r}5+2,376 \\
1,114\end{array}$ & $\begin{array}{r}512,826 \\
1,114\end{array}$ & $\begin{array}{r}5 \times 284 \\
1,114\end{array}$ & $\begin{array}{r}+13,776 \\
1,114\end{array}$ & $\begin{array}{r}\$ 14,273 \\
1,114\end{array}$ & $\begin{array}{r}5+20000 \\
0,7+2\end{array}$ \\
\hline 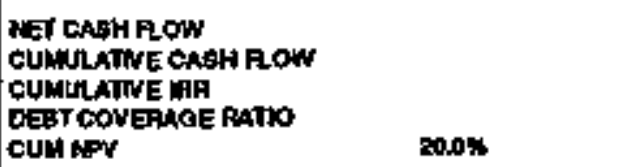 & $\begin{array}{l}51,621 \\
50,198 \\
5.70 \% \\
104 \\
07,3511\end{array}$ & 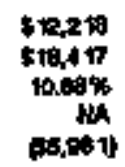 & 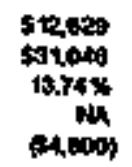 & 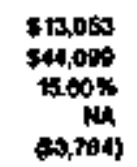 & 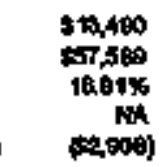 & 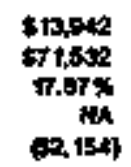 & 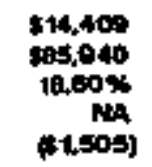 & 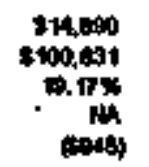 & 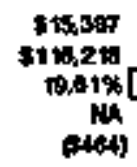 & 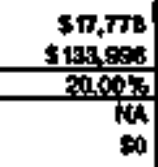 \\
\hline
\end{tabular}




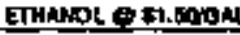

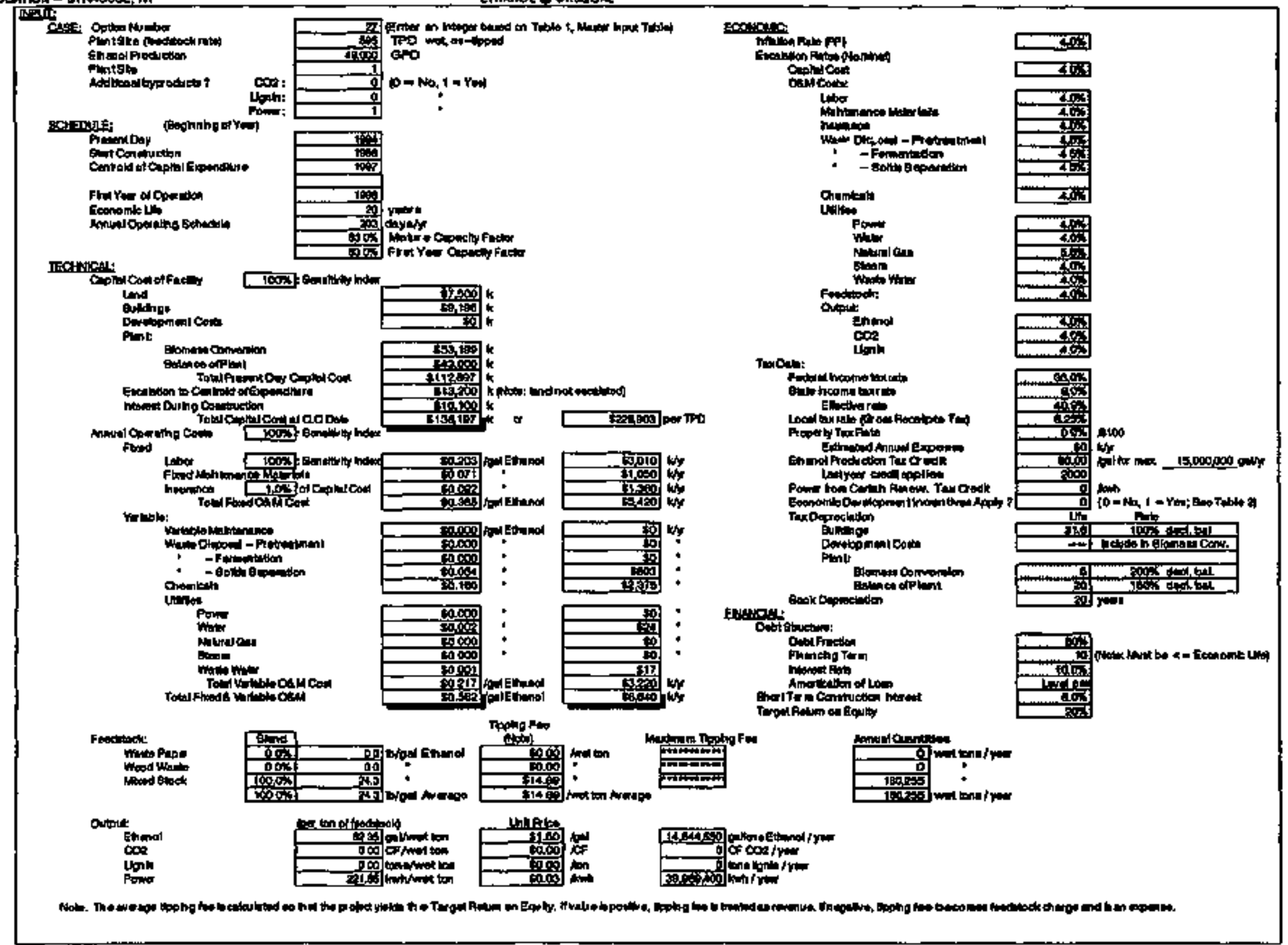




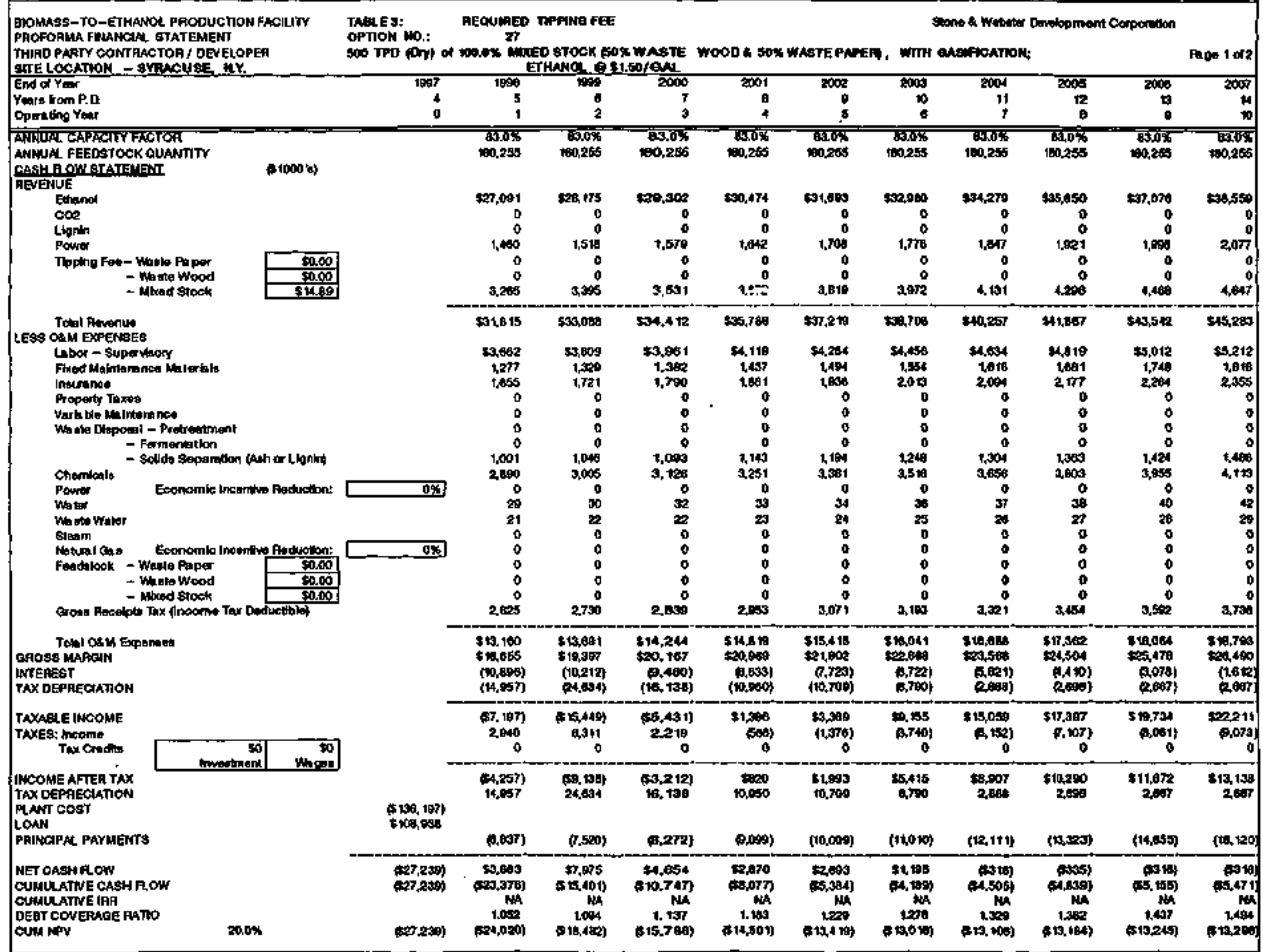




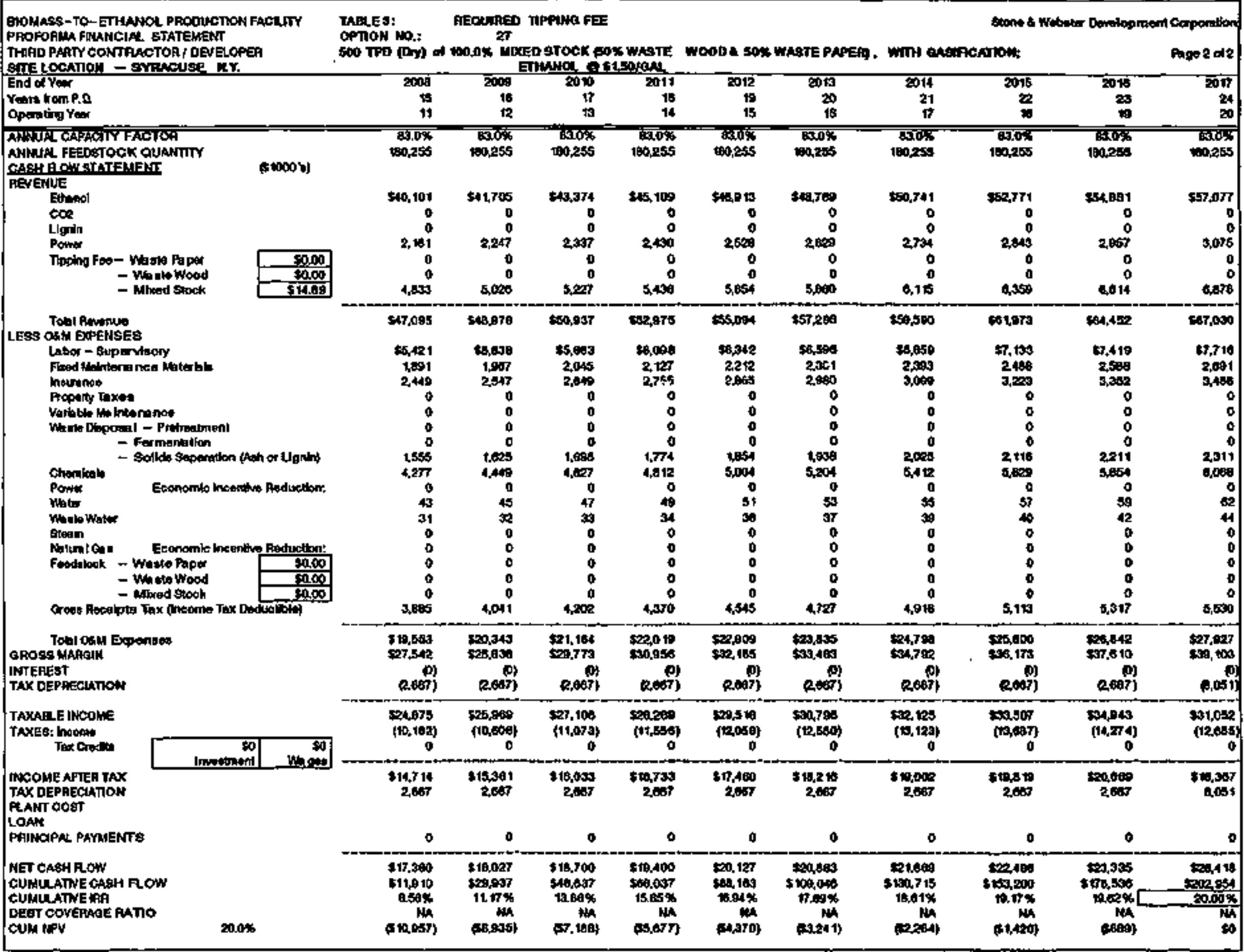




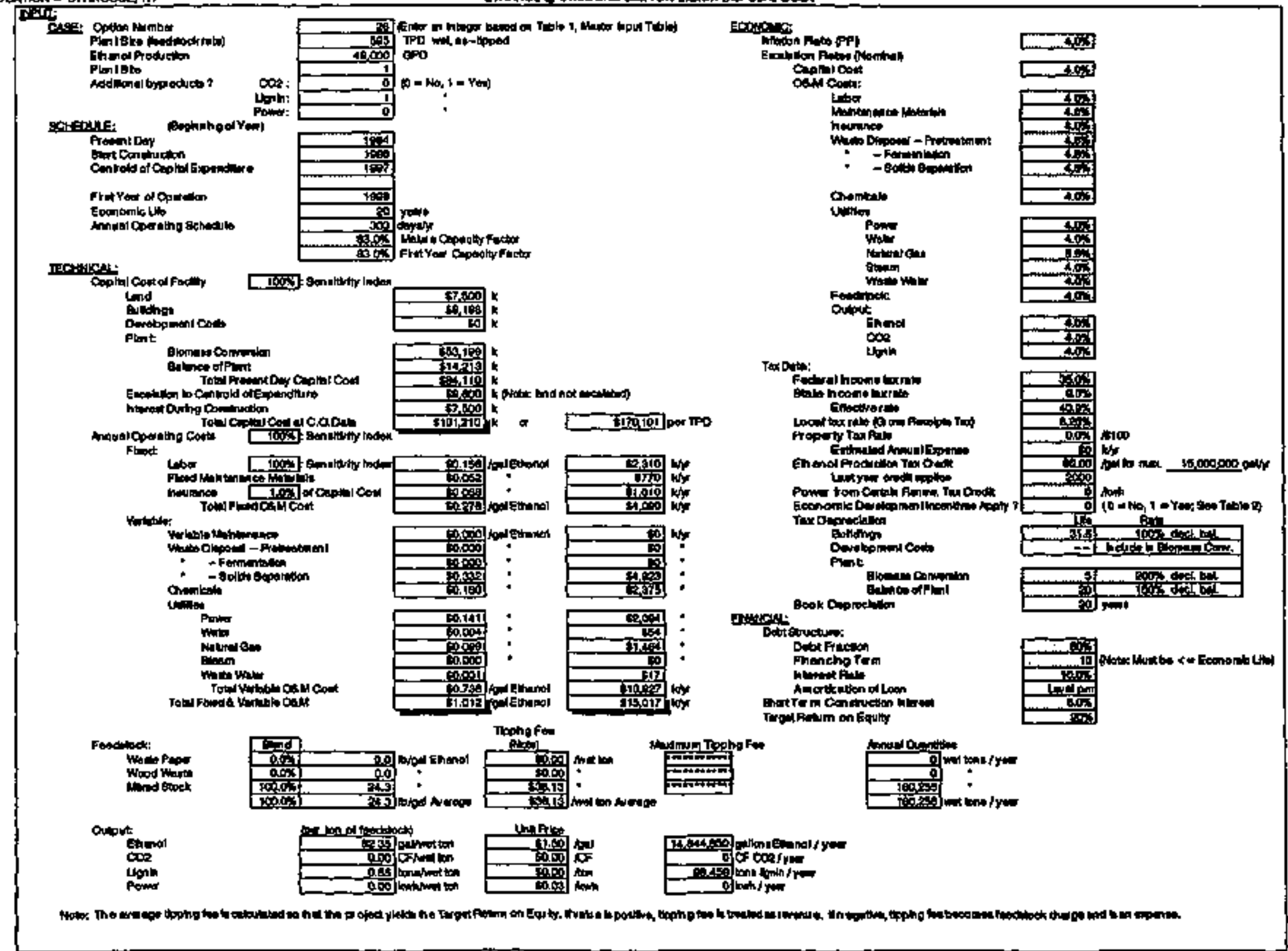

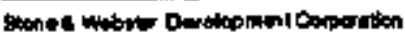




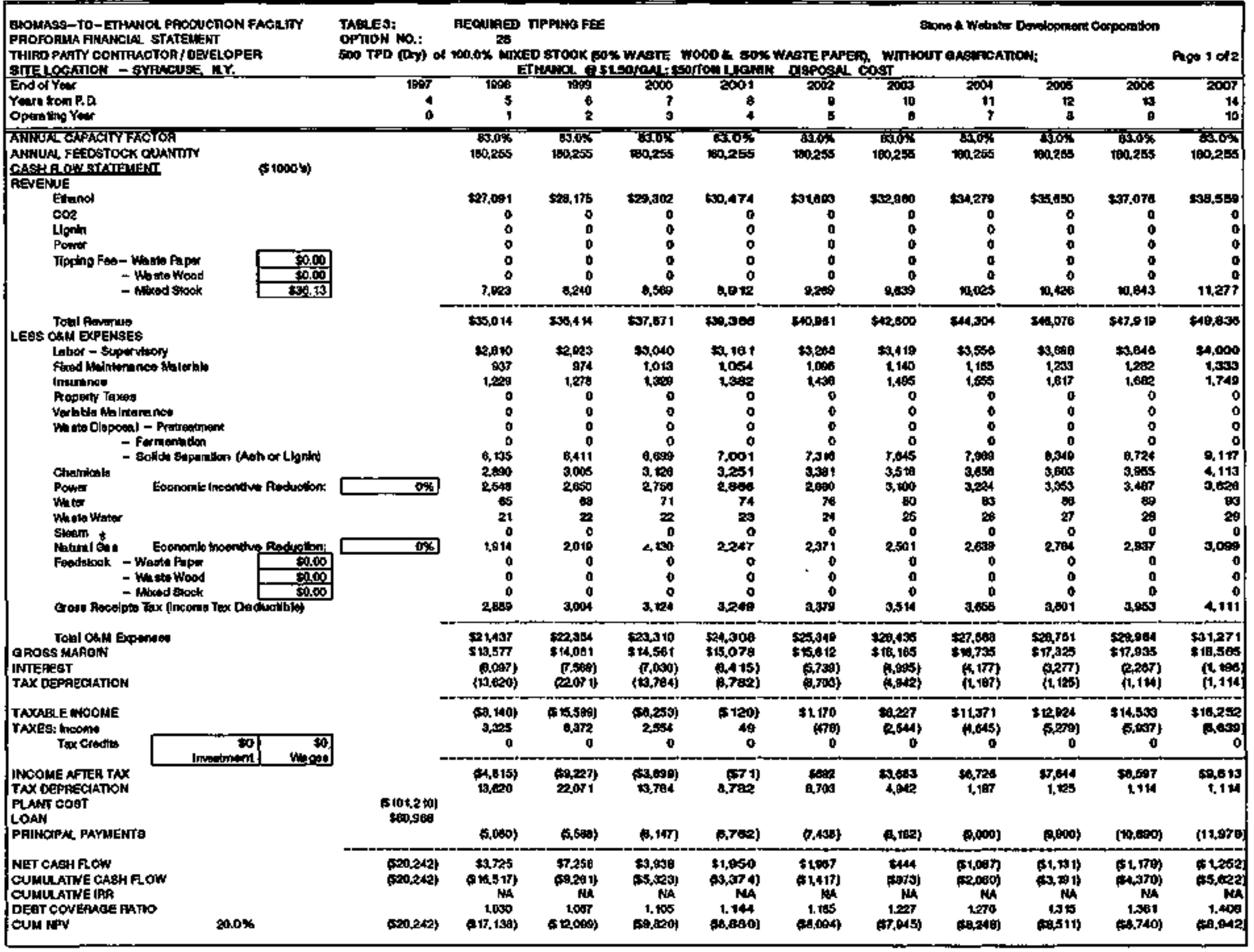




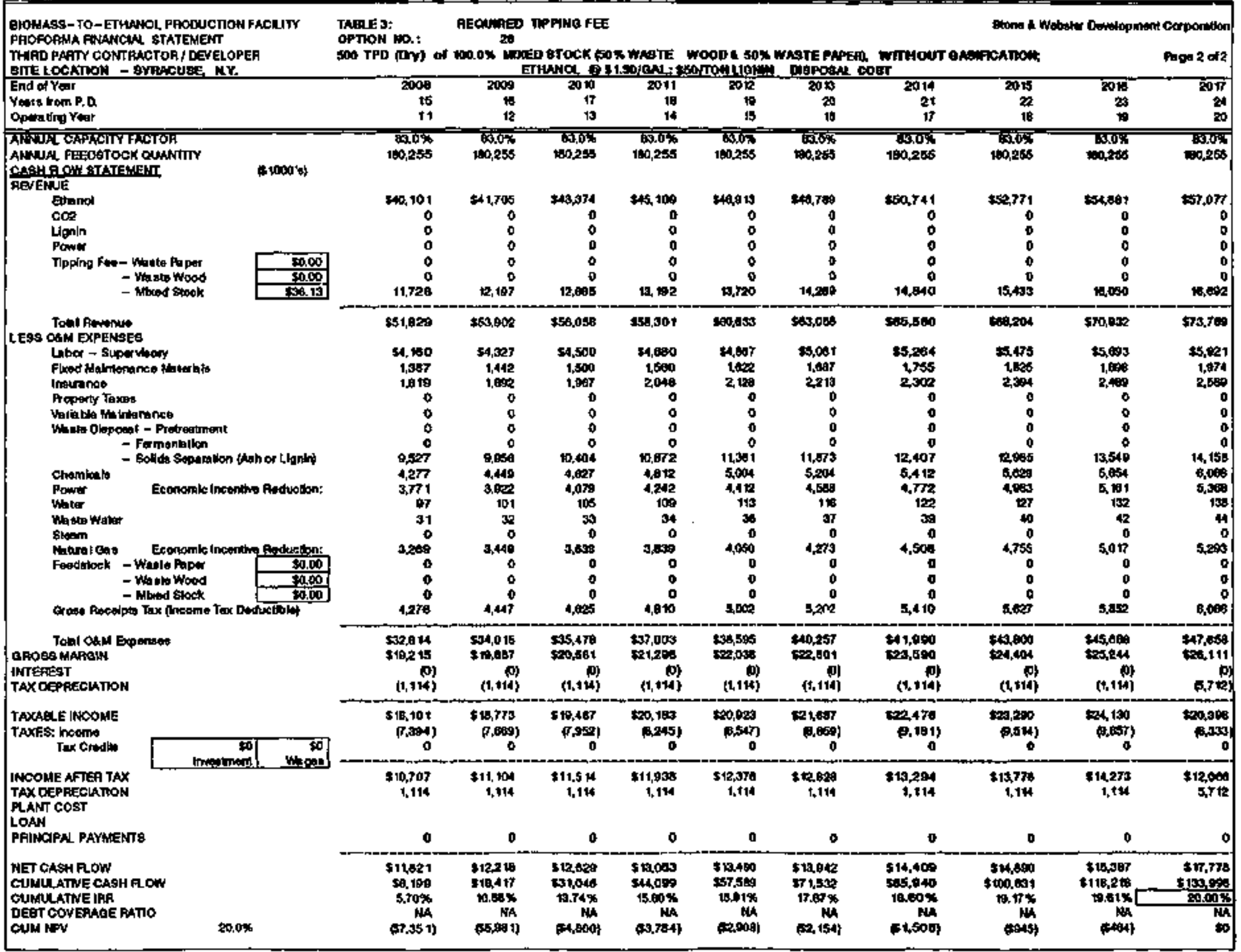




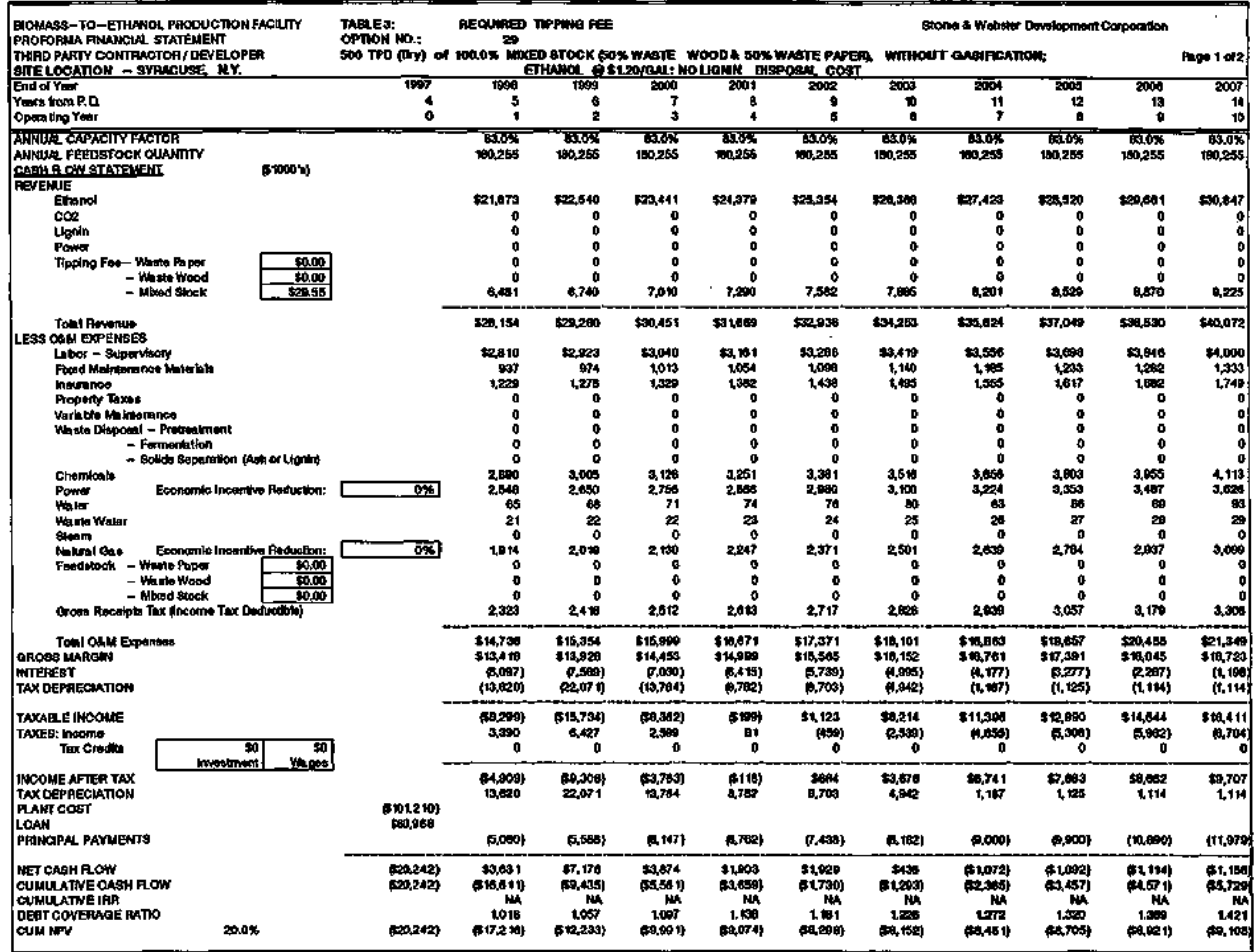

$\therefore$ 


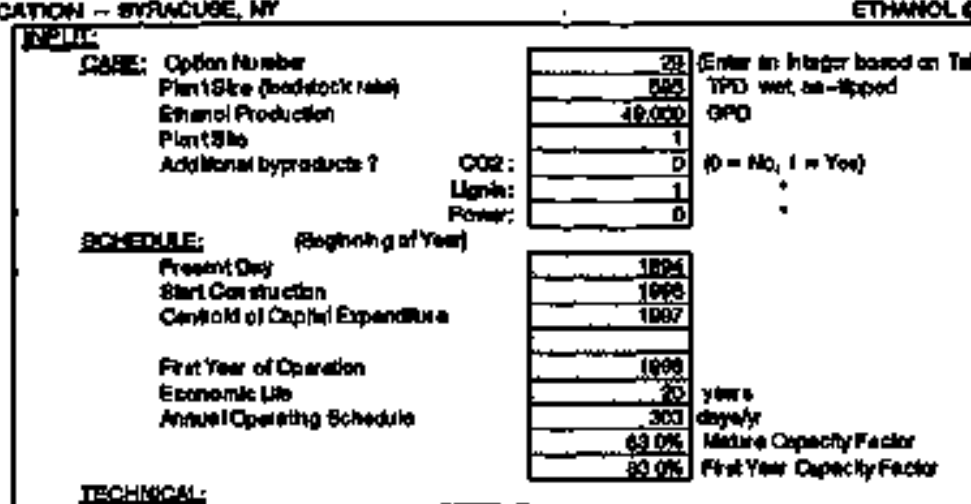

Frestisps:
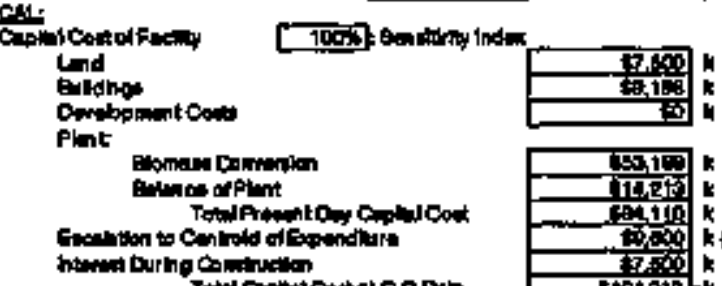

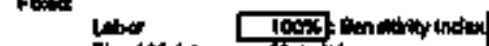

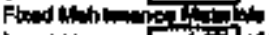

wables.

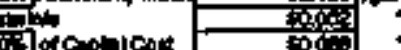

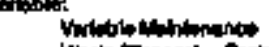

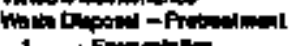
: t Foth Chemitein Chemitis

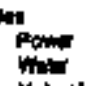

Nituraten

sach

Tomiveringinom

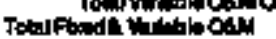

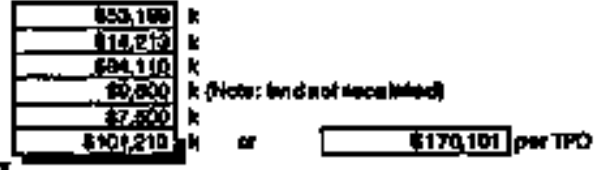

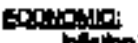

Gocion Fing fipt

Oaphidow

ofitcotix

Itida

mevement

10 -

-

Orembats

U⿴囗十⺝木

Pons

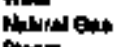
mations

Fonstionse

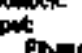

chent

Tuxplat:

unh

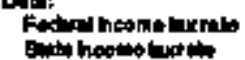

Enotherats

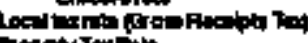

row rime

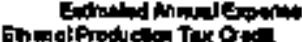

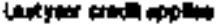

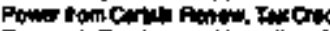

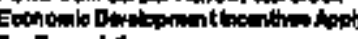

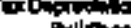

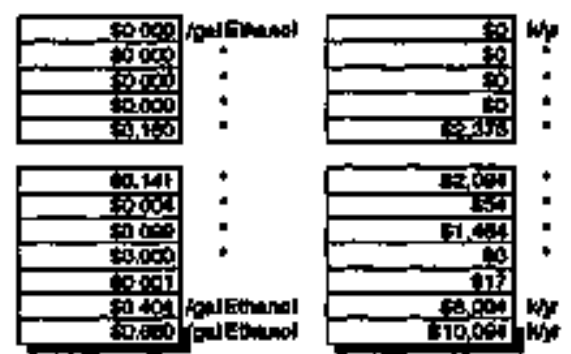

Owingonimi cont

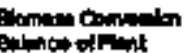

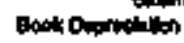

Firiscin:

I. Enven:

Opoxfinition

Fhiming Tor

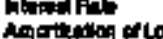

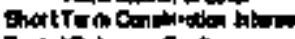

Tomiffintum on
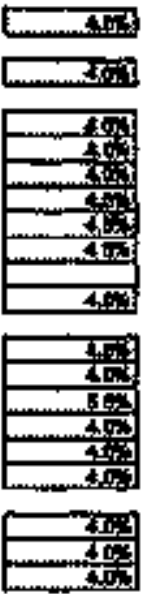

Thengen
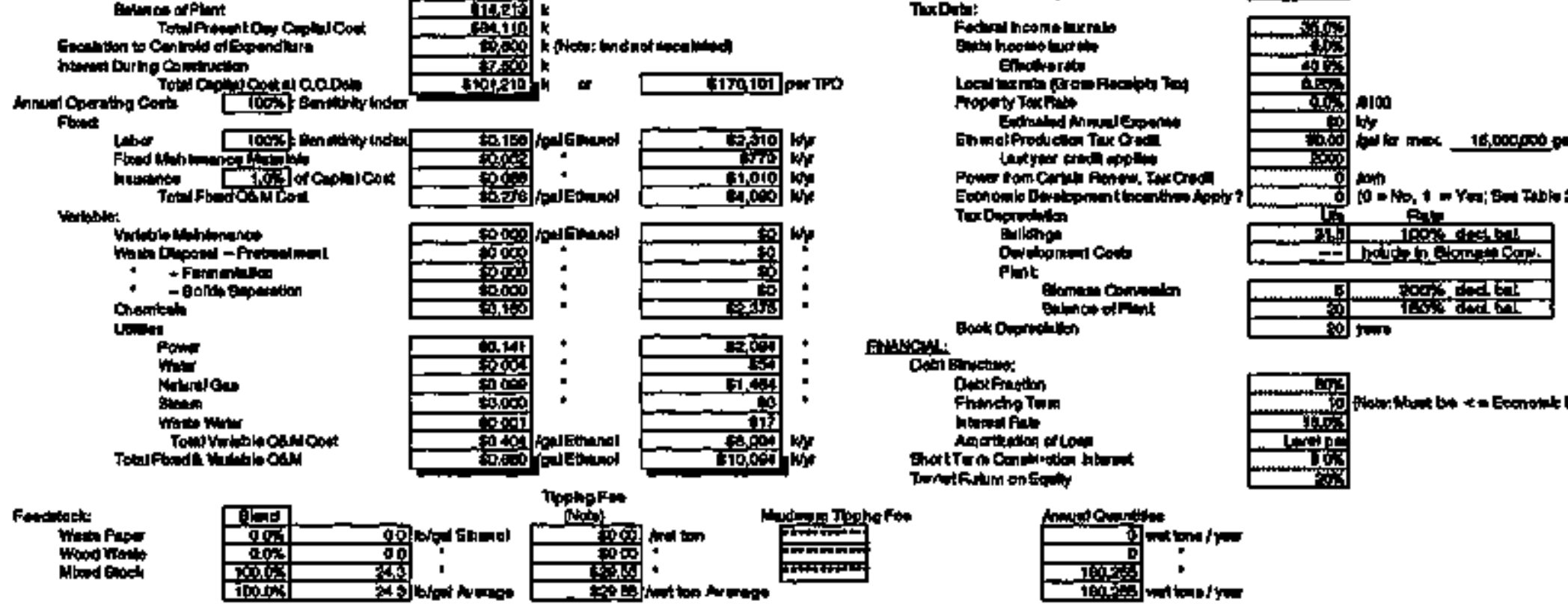

iñon

4 )

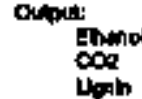
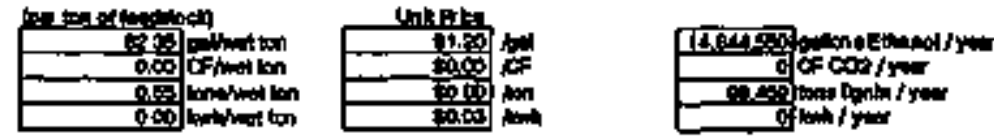

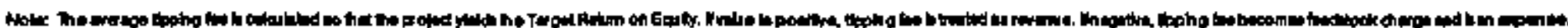




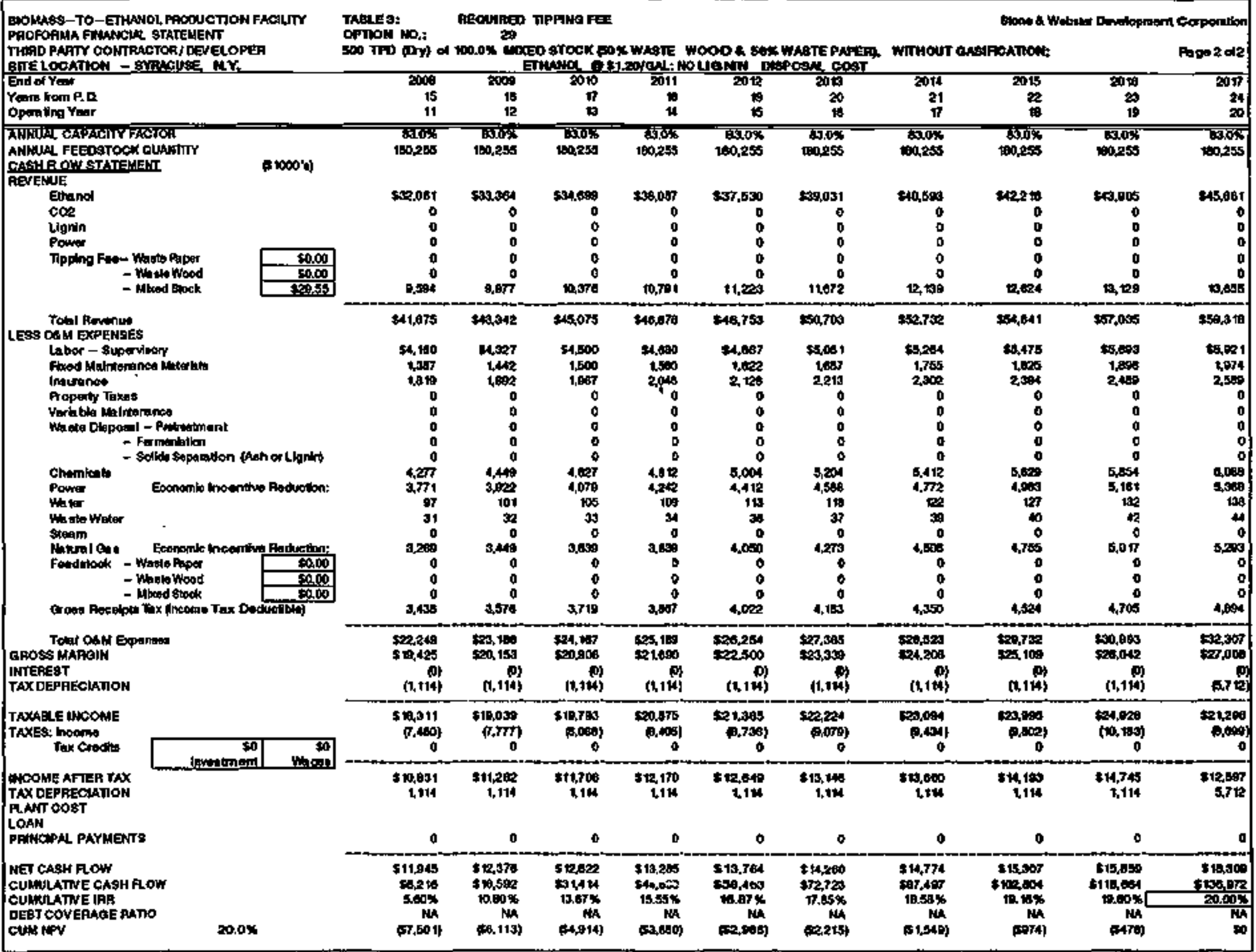




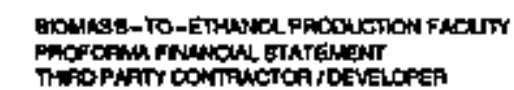
GIIE WOATON - GMPUCukE, MP IIIE wOanON-
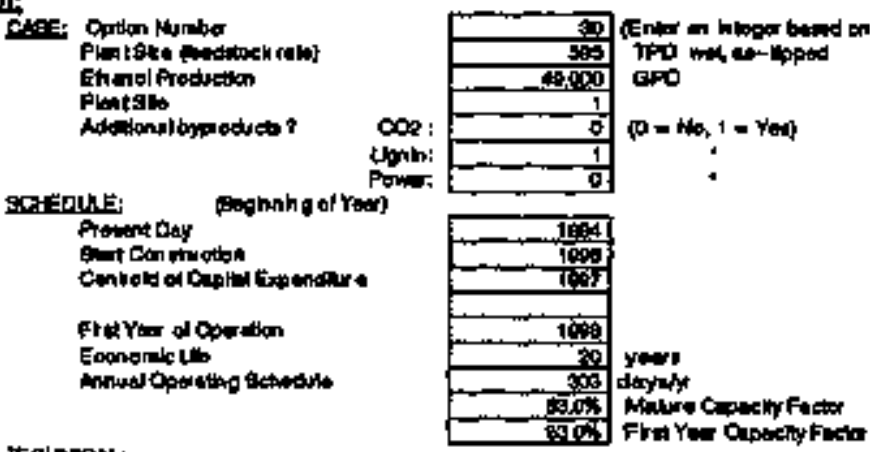

TAETE 2 :

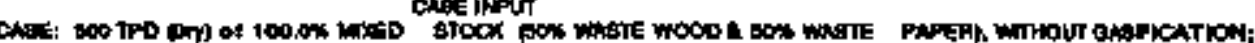

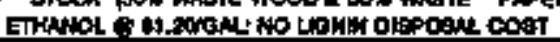

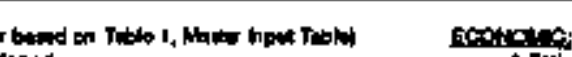

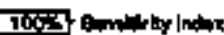
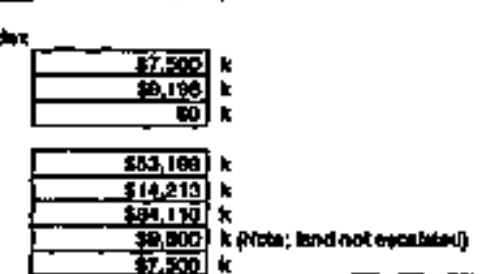

-
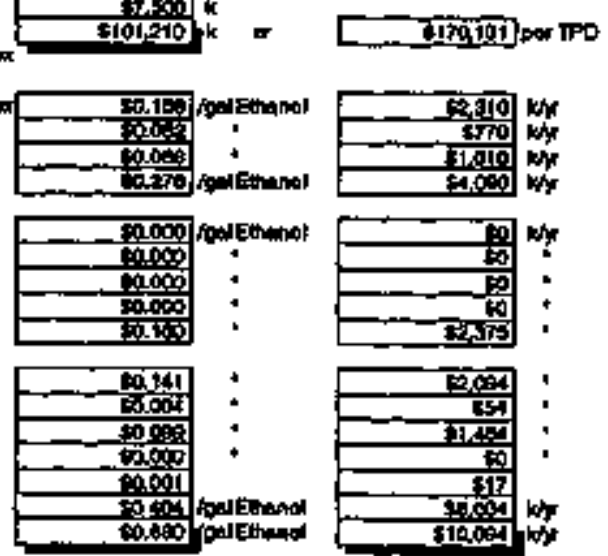

Emencist.

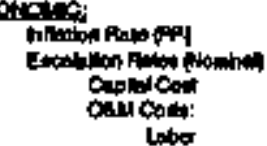

icen:

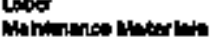

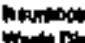

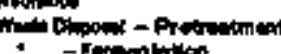

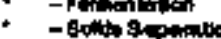

Oanoming

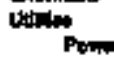

Pow

simengen

fontolet

$\cos _{1}$

$\infty$

That Delat

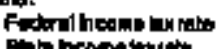

encinedanowition

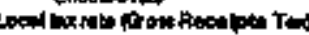

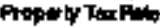

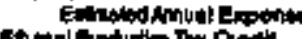

1 hodution Im a oth

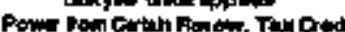

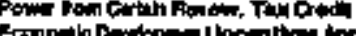

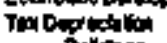

âlingit

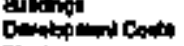

Alomencominemator

Geok nepescitiven

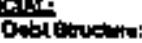

DobtFicetion

Themeth

Amorates oflow

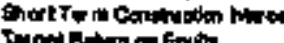

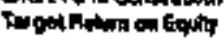

$\cos 20$

427

\section{Tohofes}
Fopdineck:
Hoed Hois
Wrod ancek

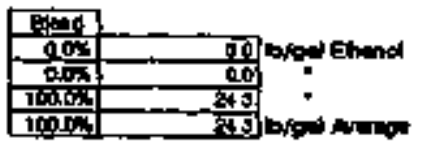

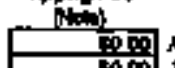

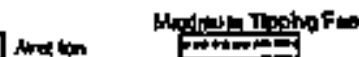

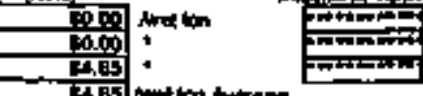

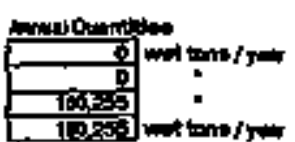

Outent:

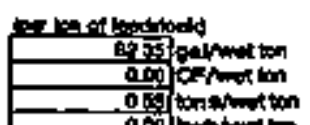

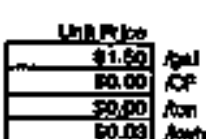

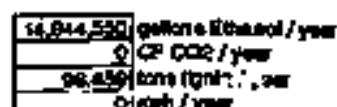

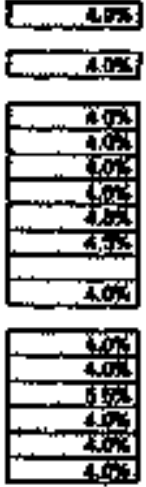
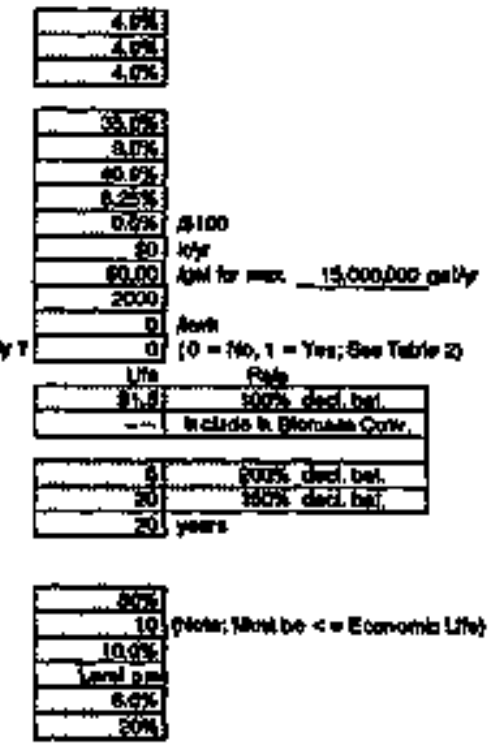

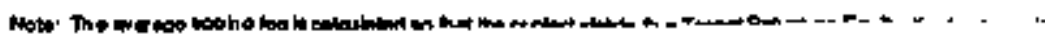




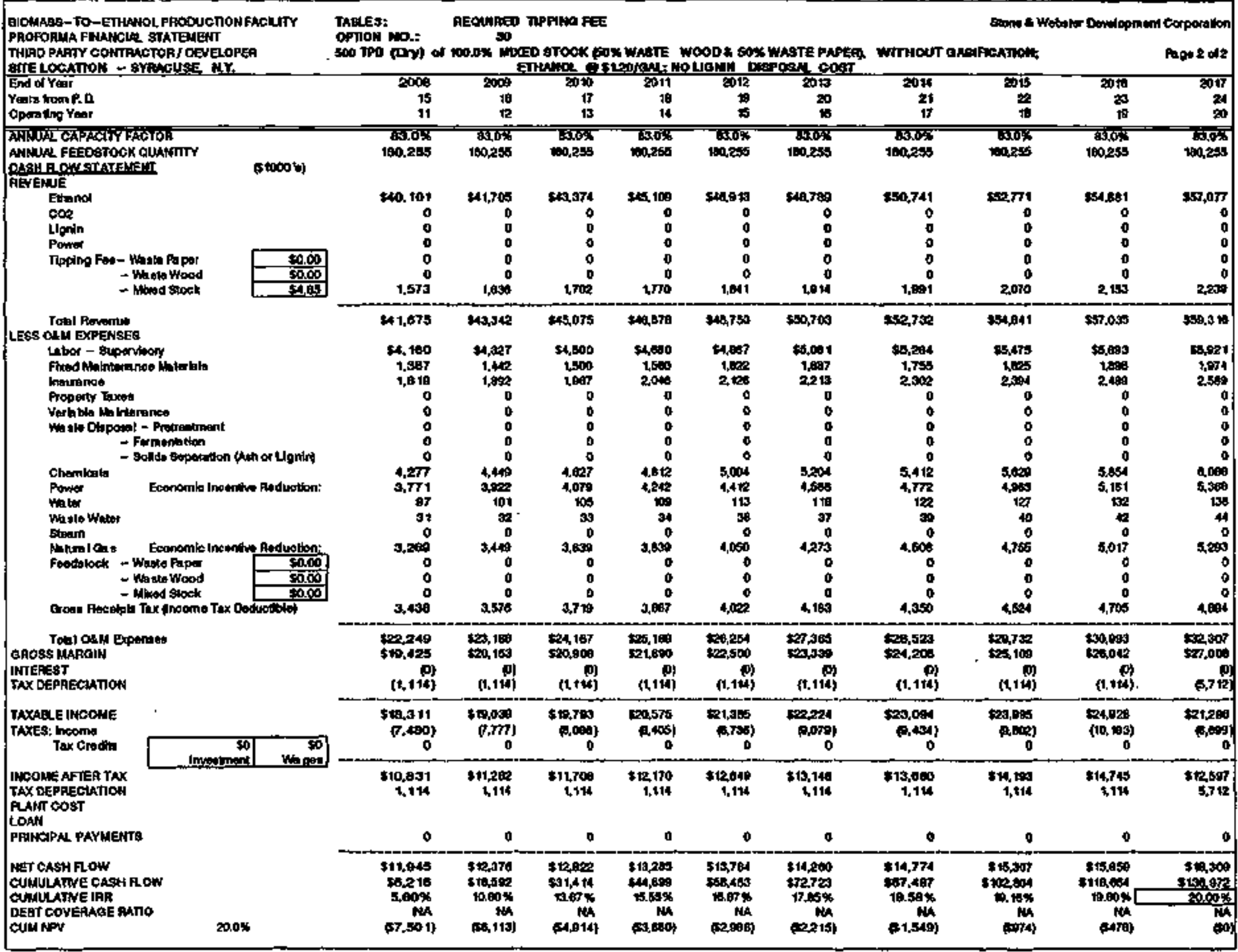




\section{REQUIRED ETHANOL PRICE vs. CAPTTAL COST AS A PERCENTAGE OF ESTTMATED COST}

$100 \%$ MIXED STOCK@\$20TON

SITE LOCATION - ALBANY, NY

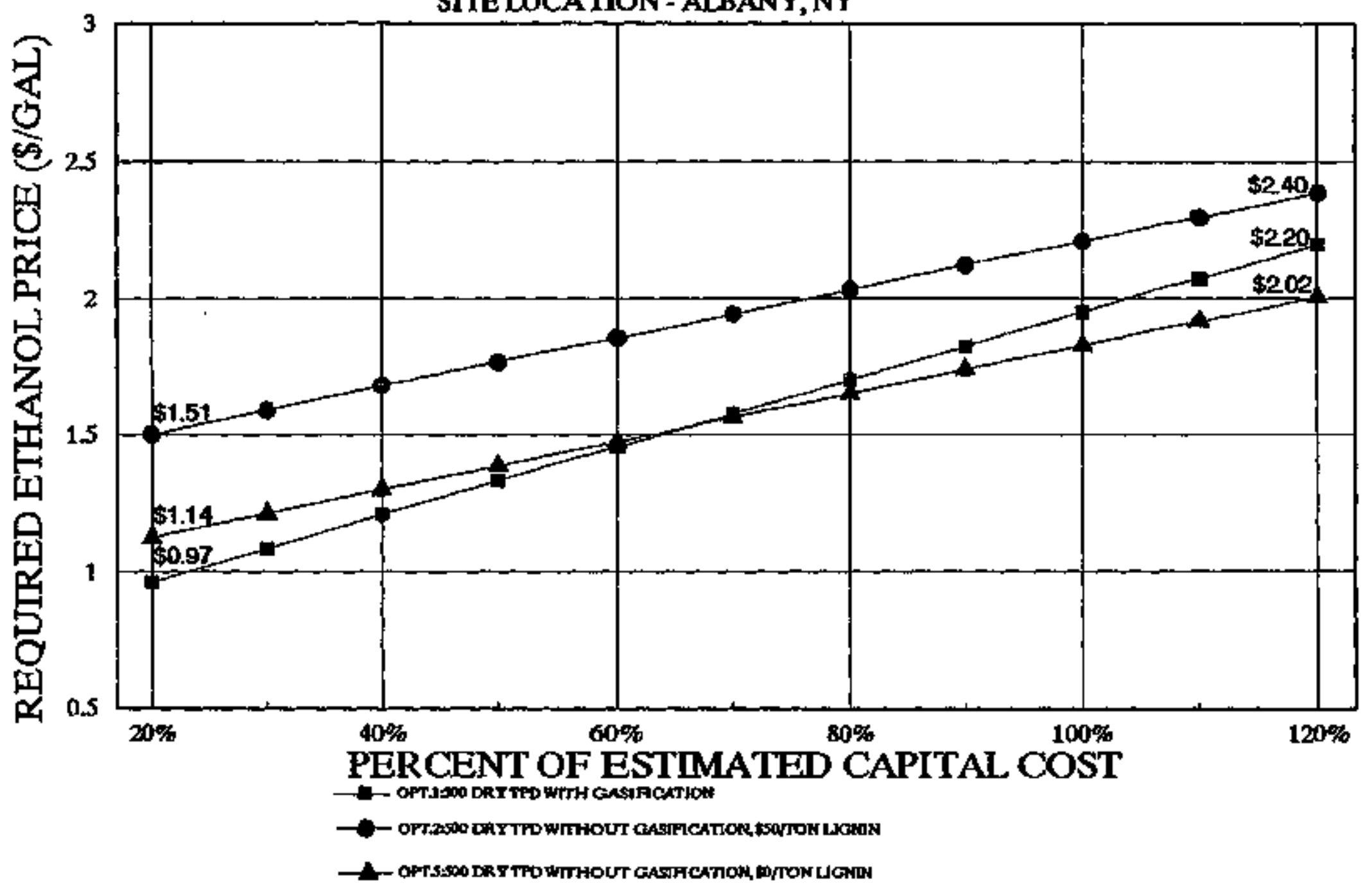

CAPYTAL COSTFOR CPT1 $=\$ 113,683,600$

CAPITAL COSTROR CPT2 \& 5 - $2 \$ 4,220,000$

STONH \& WEBSTER DEVELOPMFNT CORPOHATION

Figure 8-1 
REQUIRED ETHANOL PRICE vs. CAPTTAL COST AS A PERCENTAGE OF ESTIMATED COST 100\% MIXED \$TOCK $@ \$ 40 / T O N$

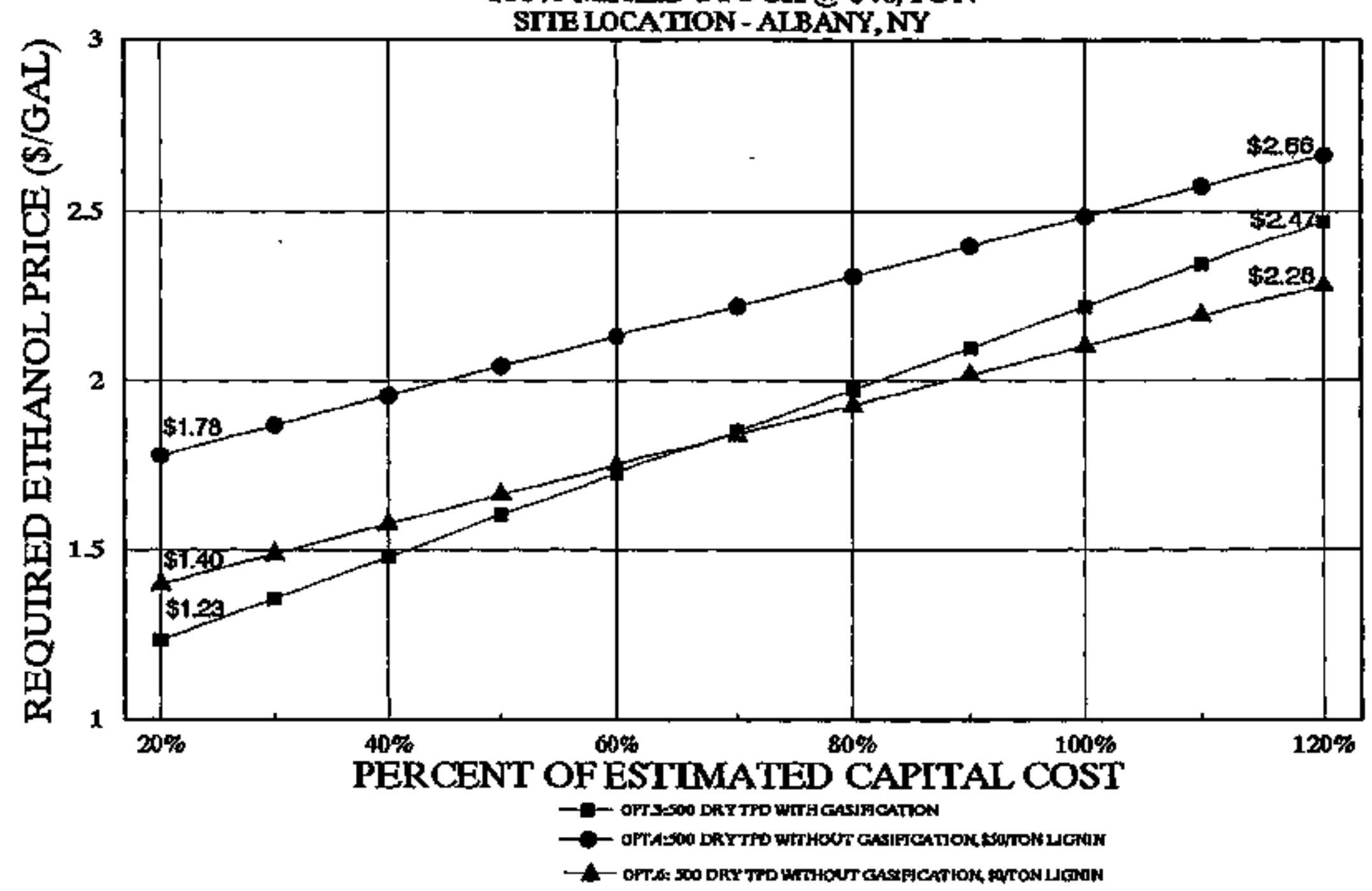

CAPTIAL COSTFOR OPT $3=313,064,000$ CAFITNL COSTFOR OPTA \& 6 - \$3,72Q,000

STONR \& WESTIER DEVEIOPBIGNTCORPORATION

Figure 8-2 
REQUIRED ETHANOL PRICE vs.O\&M CNST AS A PERLENTAGE OF ESTIMATED COST $100 \%$ MDXED STUCK @ \$20/TON

SITE LOCATION - AIBANY, NY

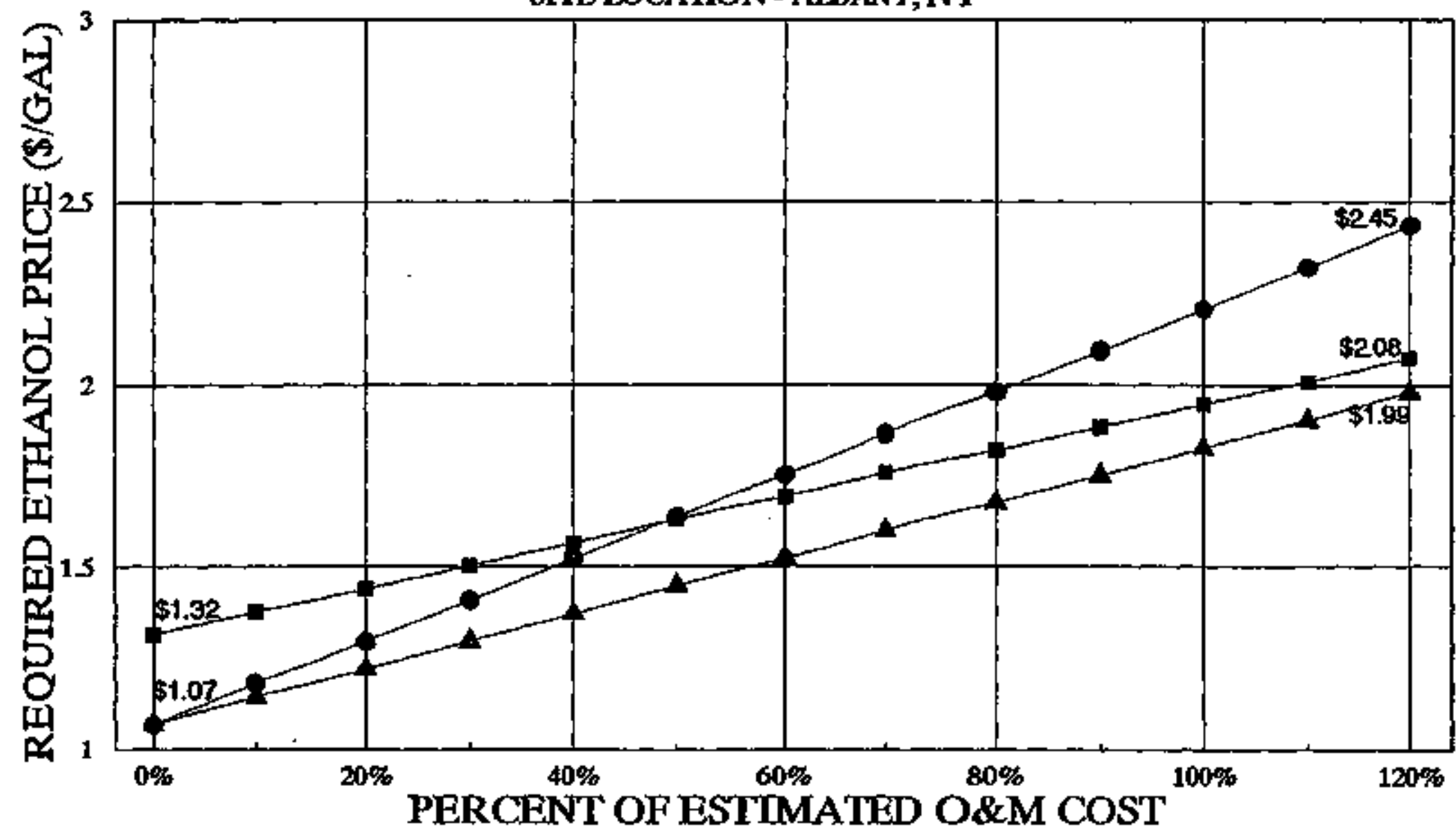

ESTTMATED O\&M COSTS:

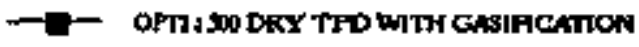

OPT.1 = $\$ 3,209 \mathrm{~K} / \mathrm{YR}$

OrT:2 = \$t1, m KryR

OPT.5 $=\$ 6,149 \mathrm{Kr} / \mathrm{R}$

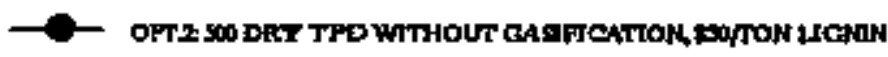

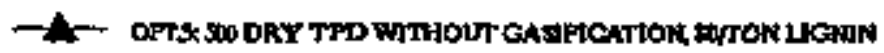
STCWE \& WEBSTER DEVILOHANTCORPORATION

FIGURE 8-3 
REQUIRED ETHANOL PRICE vS.O\&M COST AS A PERCENTAGE OF ESTIMATED COST $100 \%$ MTXED STOCK @ \$40/TON

SITE LOCATION - AIBANY, NY

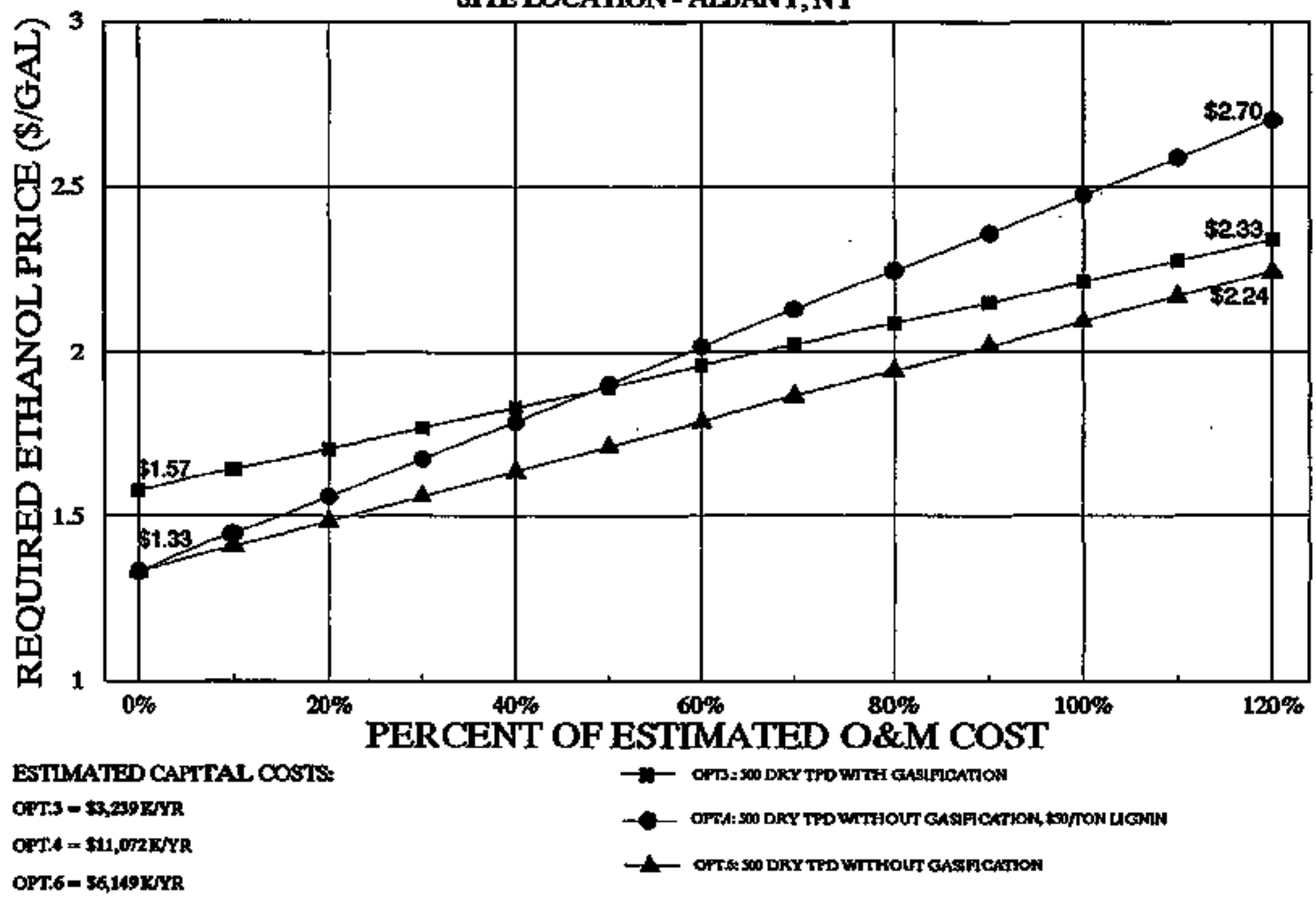

STCNE \& WERSTER DEVELOPMIENT CORPORATION

FIGURE 8-4 


\section{REQUIRED ETHANOL PRICE vs. CAPITAL COST AS A PERCENTAGE OF ESTIMATED COST $100 \%$ MLXD STOCK @ \$20/TON}

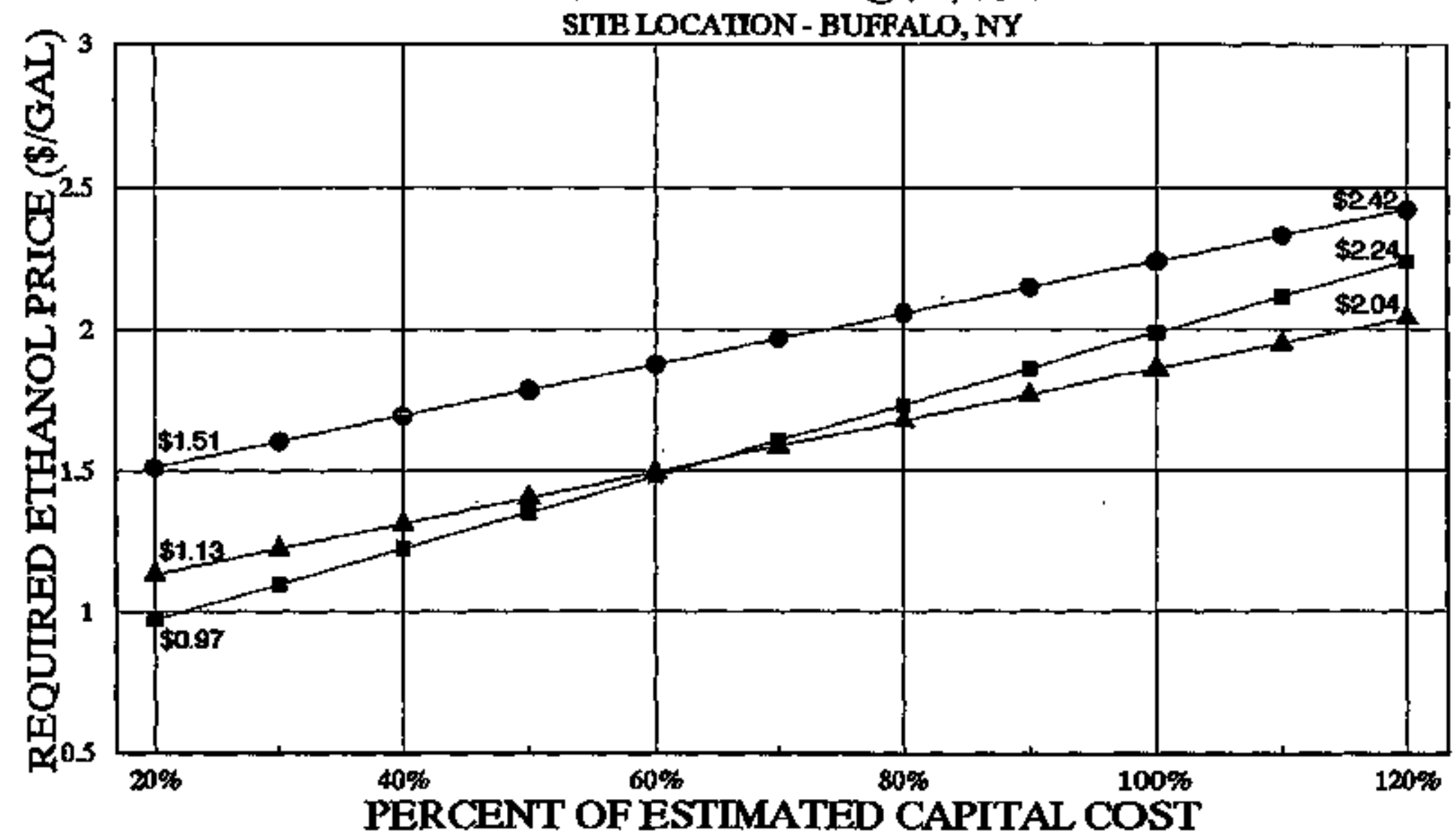

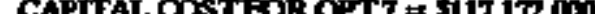
CAPTYA, COSTKOR OPTs a 11 - \$7,505,009

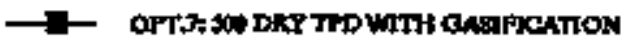

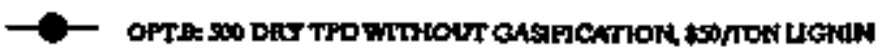

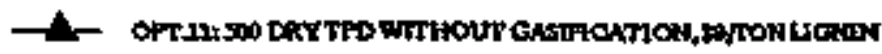
STONE * WEESHER DEVHLOPMENT CORPORATION

Figure $8-5$ 


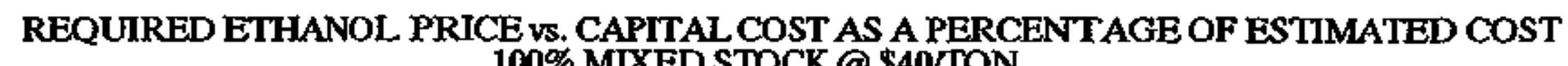
$100 \%$ MIXED STOCK @ \$40/TON

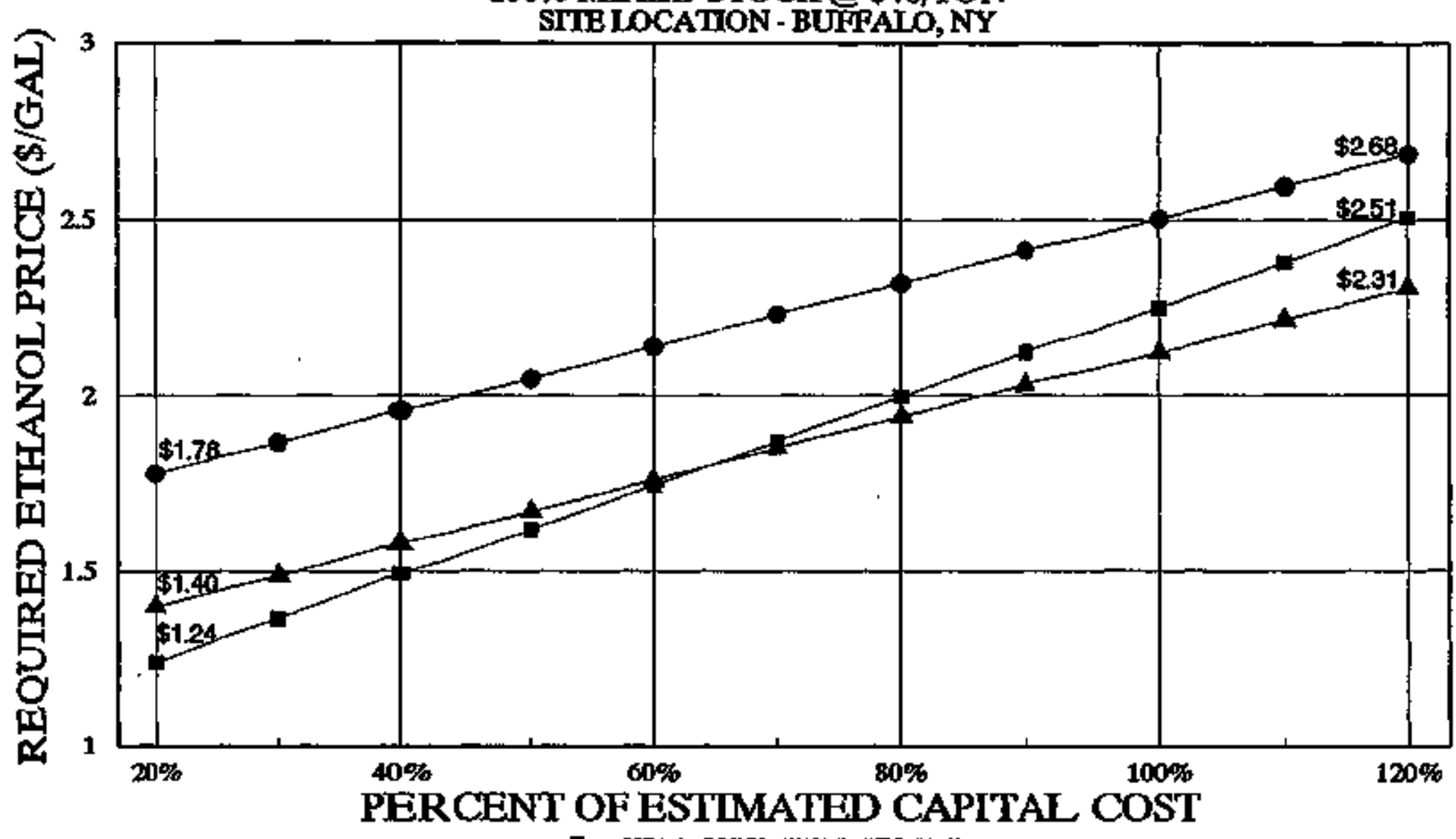

- OFT

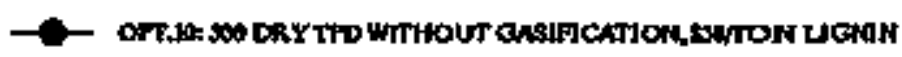

CAITIA COSTrOR OPT' = \$2T2,17,000

CAPTCAL COSTFOR OPT10 \& 12 = \$21,55,000

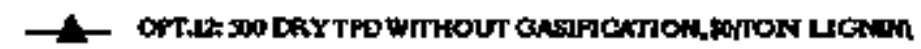

STONE \& WEASTER DEVRLOPMIENTCORPORATON

Figure 86 
REQUIRED ETHANOL. PRICE VS,O\&M COST AS A PERCENTAGE OF ESTIMATED COS? $100 \%$ MLXED STOCK @ \$20/TON

SI'TE LOCA'TON - BUFFAIO, NY

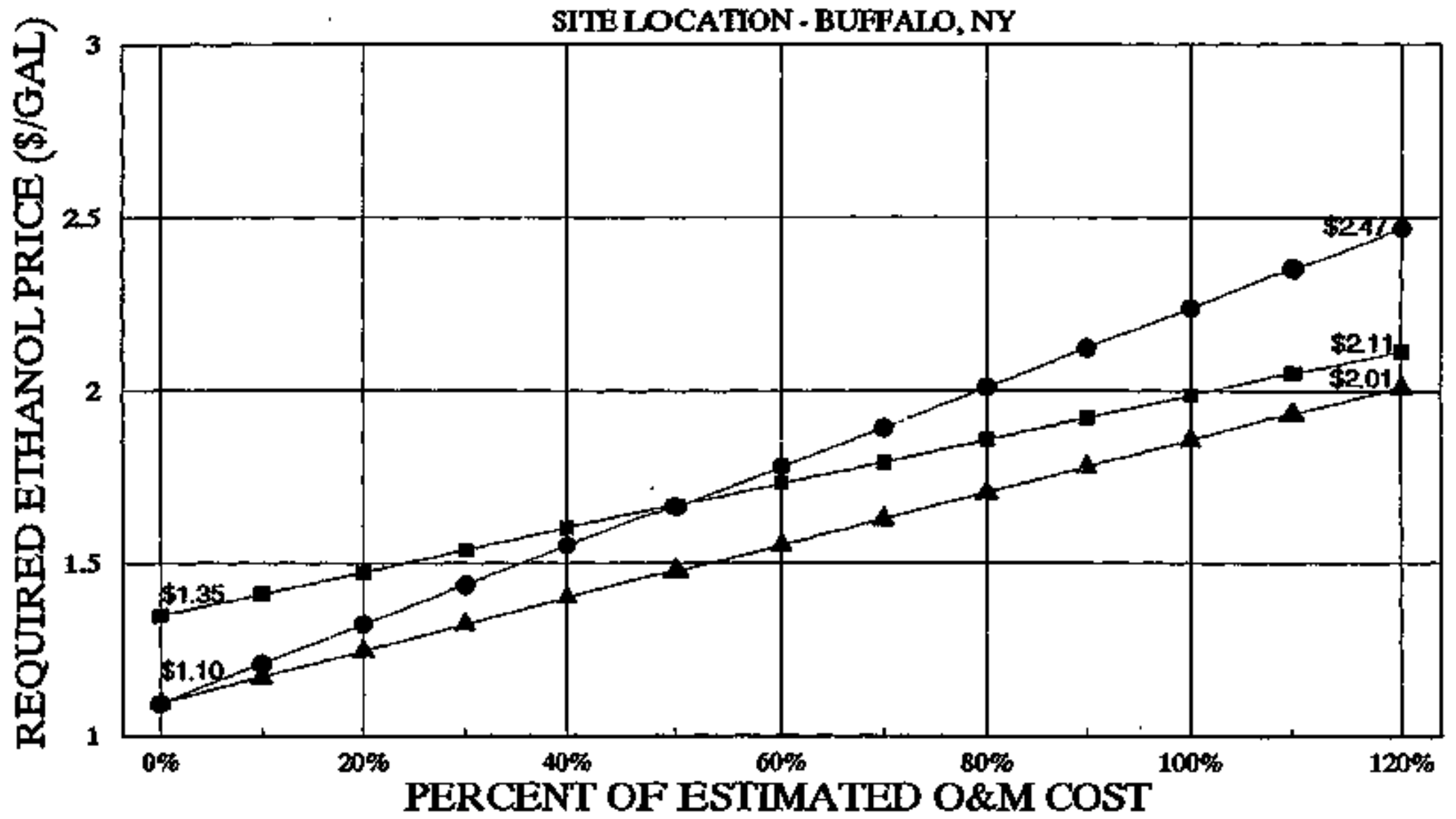

ESTMMATED O\&MCOSTS:

OPT:7 = \$3.144 K/YRR

OPT.8 = \$10,924 K/YR

-2 - OFT:K 500 DEY TPO WTTH GASAPCATAN

OFT.11 = 36,001 R/NR

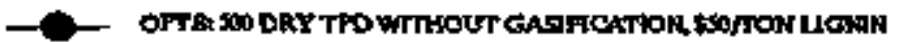

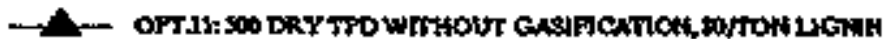

STONE A WESTER DEVELOPAENT CORPORATION

Figure 8-7 

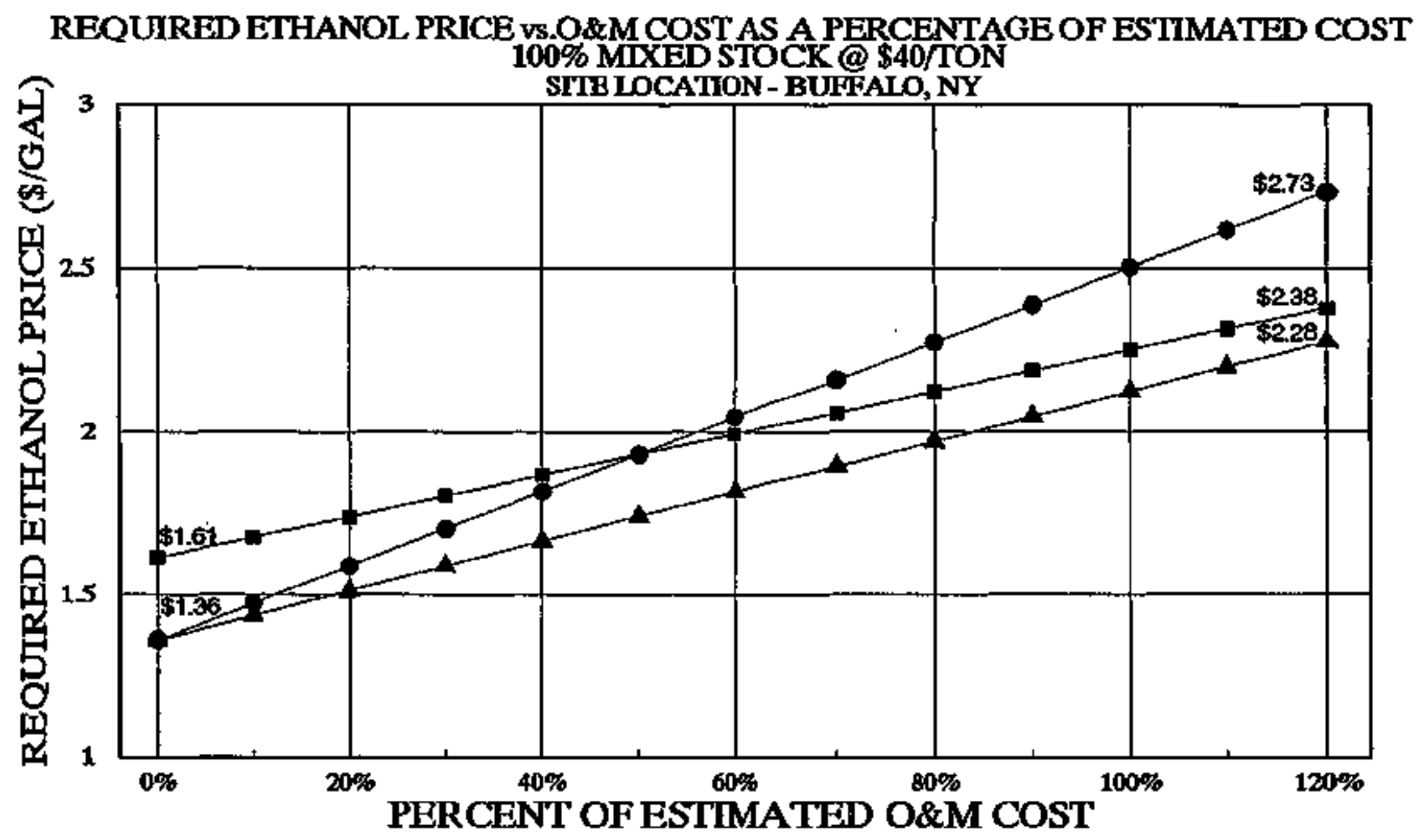

ESTMMATED O\&MOOSTS:

OPT.9 = \$\$.14 $\mathrm{E} / \mathrm{TR}$

OFT10 $=\$ 19924$ K/YR

OrT:12 = $56,001 \mathrm{~K} / \mathrm{YR}$

$\rightarrow$ OFT 9 900 DRY TFOWITH GASIFYATHON

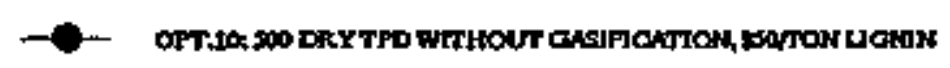

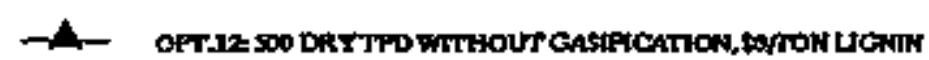

STONE \& WEBSTER DEVELOPMENTCORPORATHON

Figure 8-8 


\section{REQUIRED ETHANOL PRICE v. CAPTTAL COST AS A PERCENTAGE OF ESTTMATED COST $100 \%$ MIXED \$TOCK@ \$20/TON}

SITE LOCATION - ROCHESTER, NY

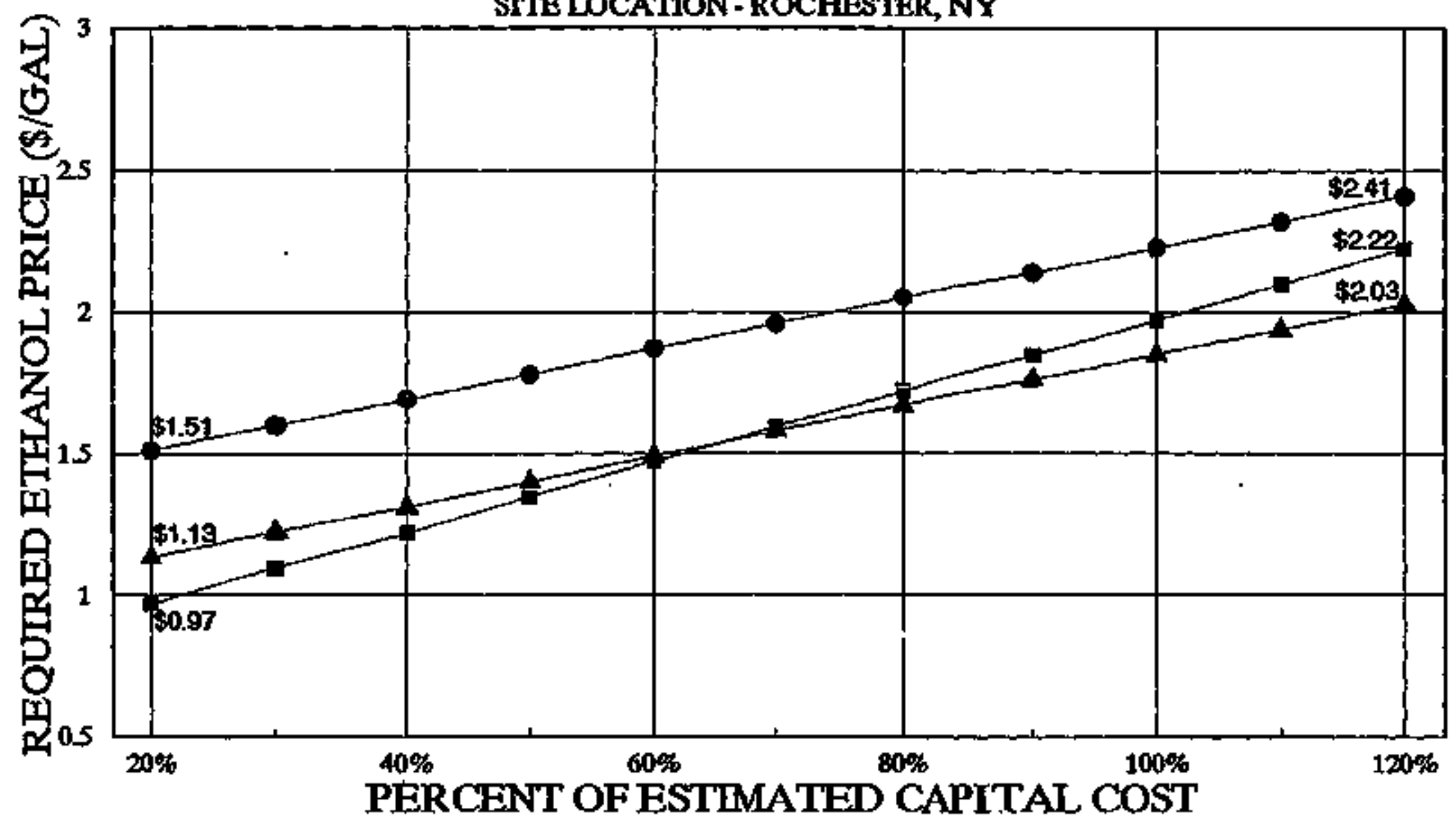

-D- OPT.L:500 DKYTRO WITH GASTMCATION

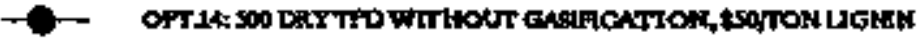

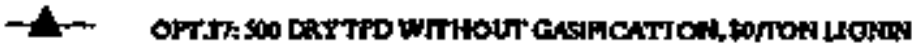


REQUIRED ETHANOL PRICE vS. CAPITAL COST AS A PERCENTAGE OF ESTIMATED COST $100 \%$ MDXED STOCK @ \$40/TON-

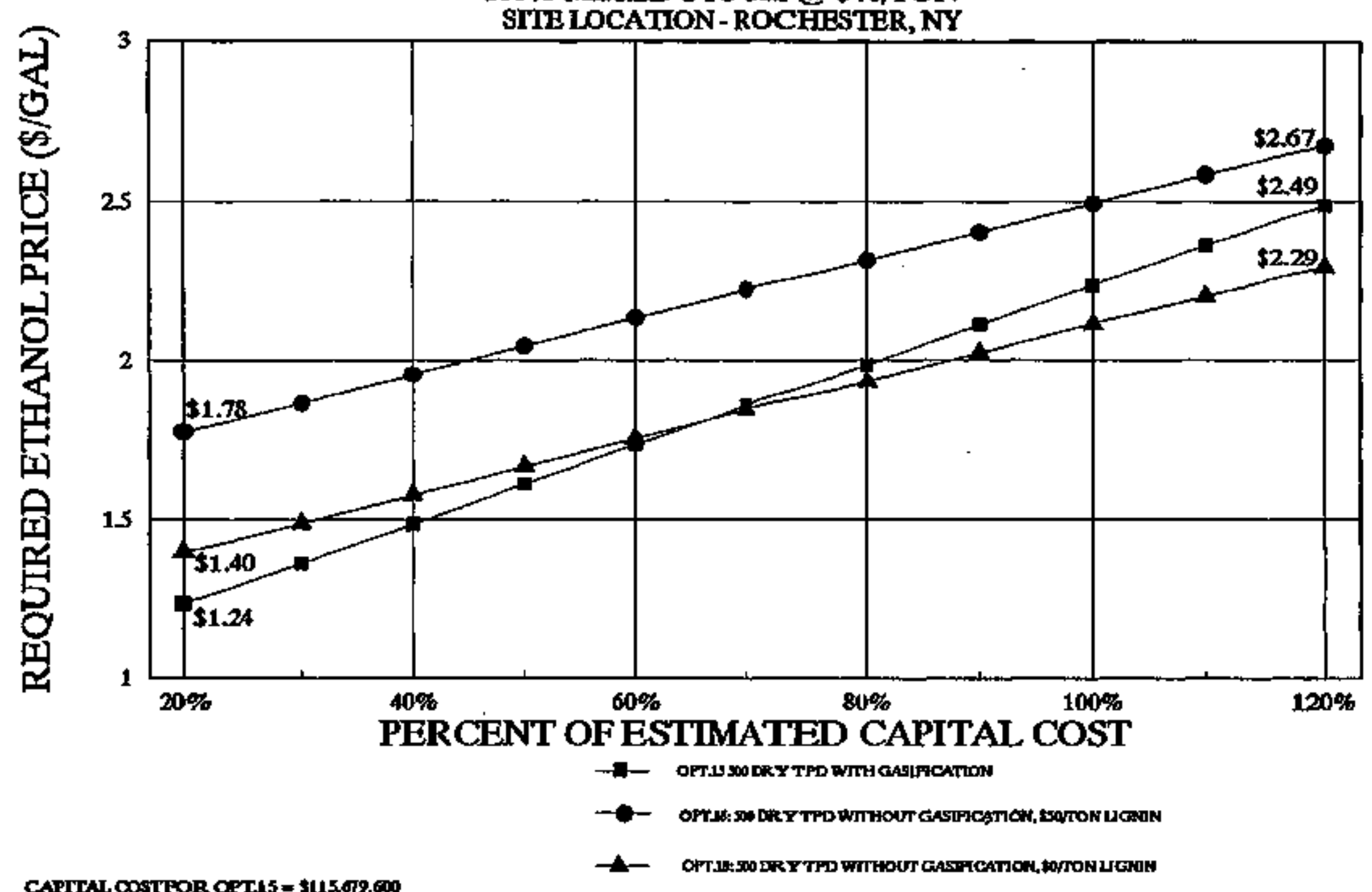

CAPITAL COSTROR OFTJ6 \& 18 - 396333,300

STONE \& WEESTER DEVELOPMANT CORPORATION

FIGURE 8-10 
REQUIRED ETHANOL PRICE vS. O\&M COST AS A PERCENTAGE OF ESTTMATED COST $100 \%$ MIXED STOCK @ \$20/TON

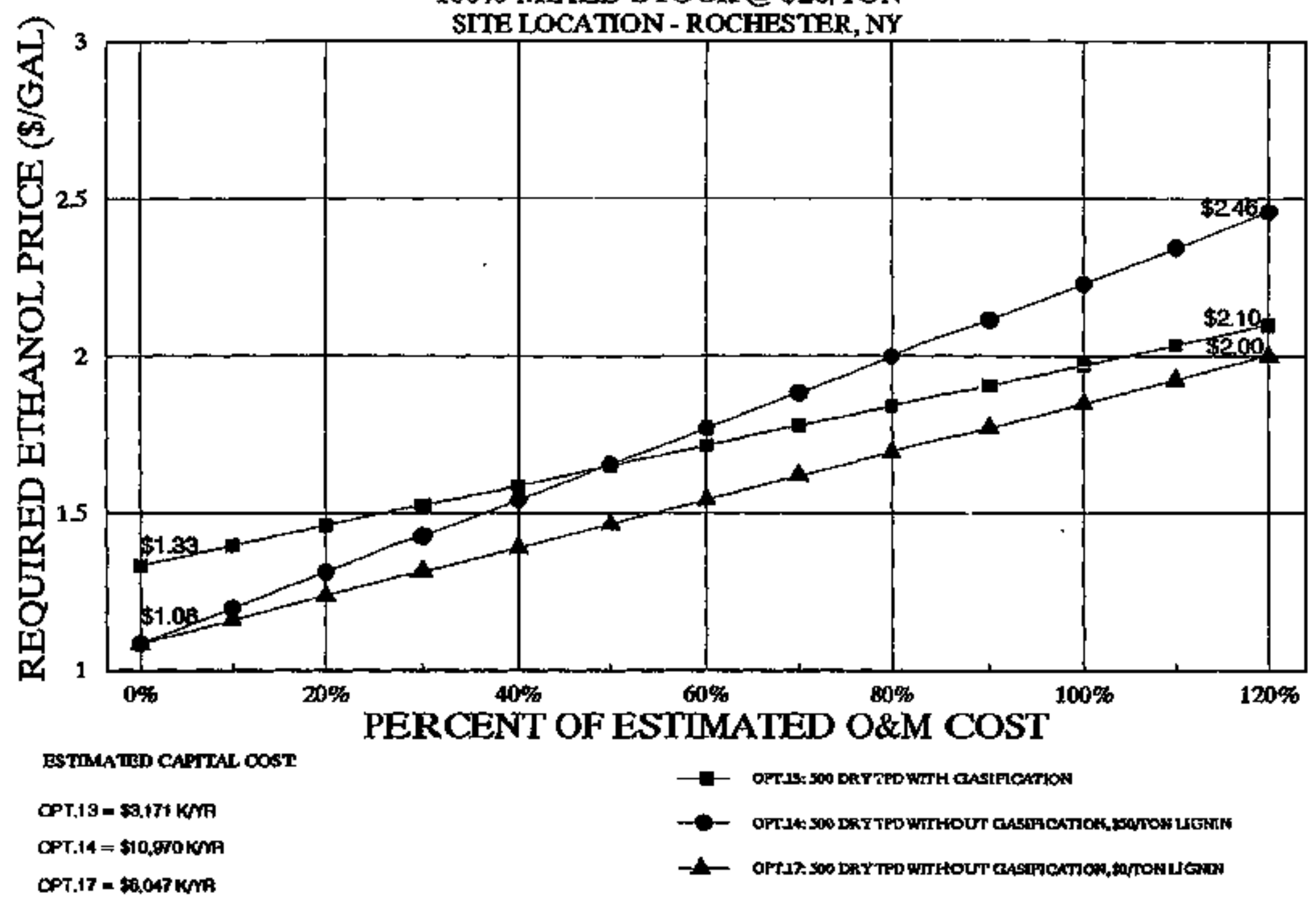

STONE \& WEBSTER DEVEIOPAFINTCORPORATIOA

Figure $8-11$ 


\section{REQUIRED ETHANOL PRICE vs, O\&M COST AS A PERCENTAGE OF ESTIMATED COST} 100\% MIXED STOCK@ \$40TON

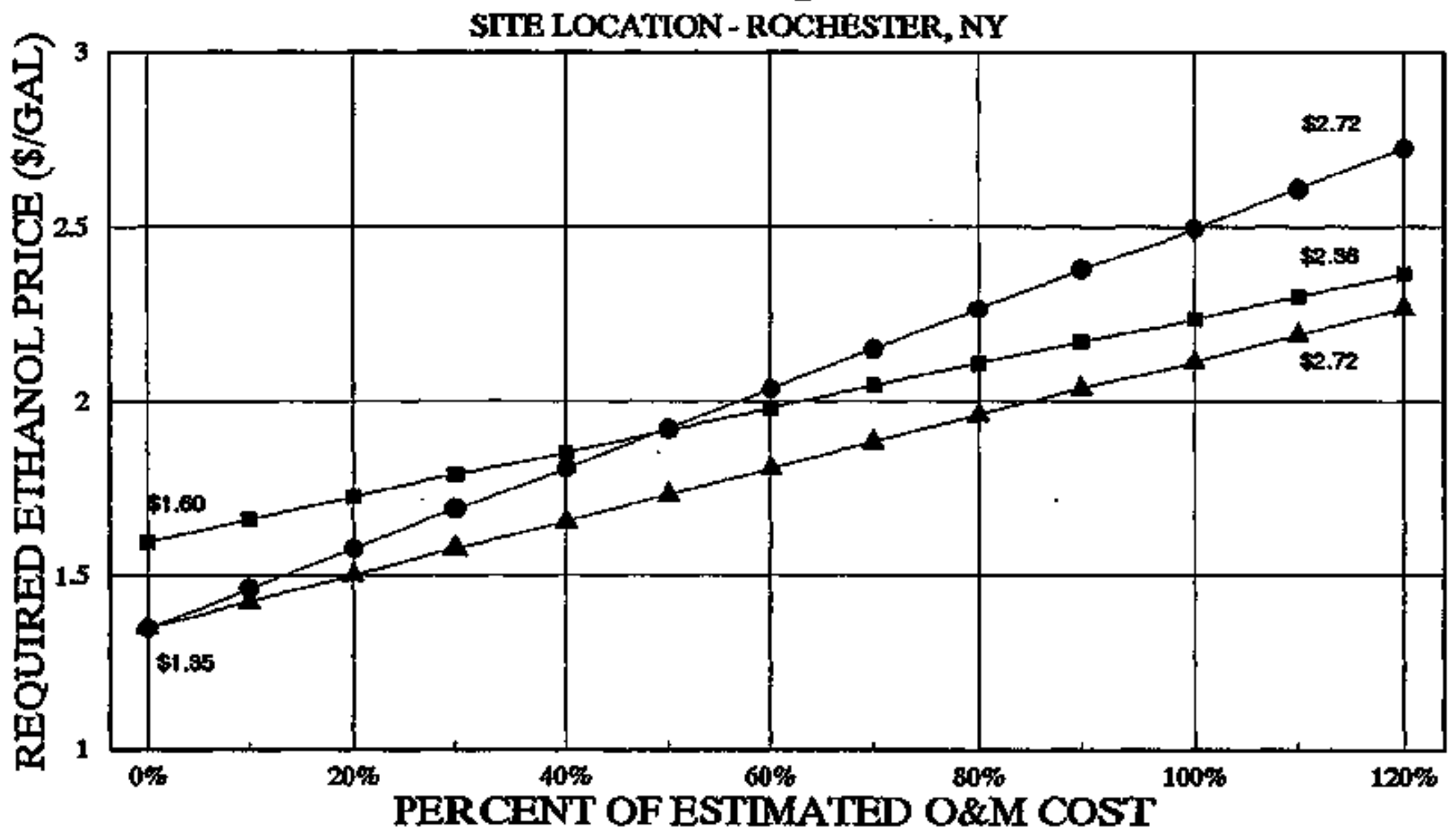

\section{ESTMMATED O\&M COSTS:}

$$
\begin{aligned}
& \text { OPT.15 }=\$ 3,17 \mathrm{~K} / \mathrm{YR} \\
& \text { OPT.16 }=\$ 10,970 \mathrm{~K} / \mathrm{YR} \\
& \text { OPT.18 }=\$ 6,077 \mathrm{~K} / \mathrm{YR}
\end{aligned}
$$

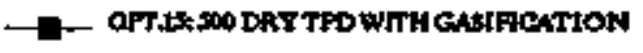

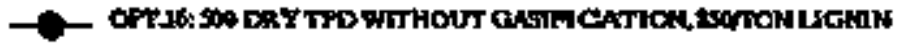

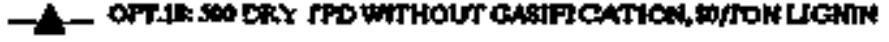

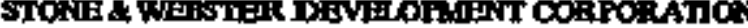

FIGURE 8-12 
REQUTRED ETHANOL PRICE vs. CAPITAL COST AS A PERCENTAGE OF ESTIMATED COST $100 \%$ MLXED STOCK@ \$20/TON

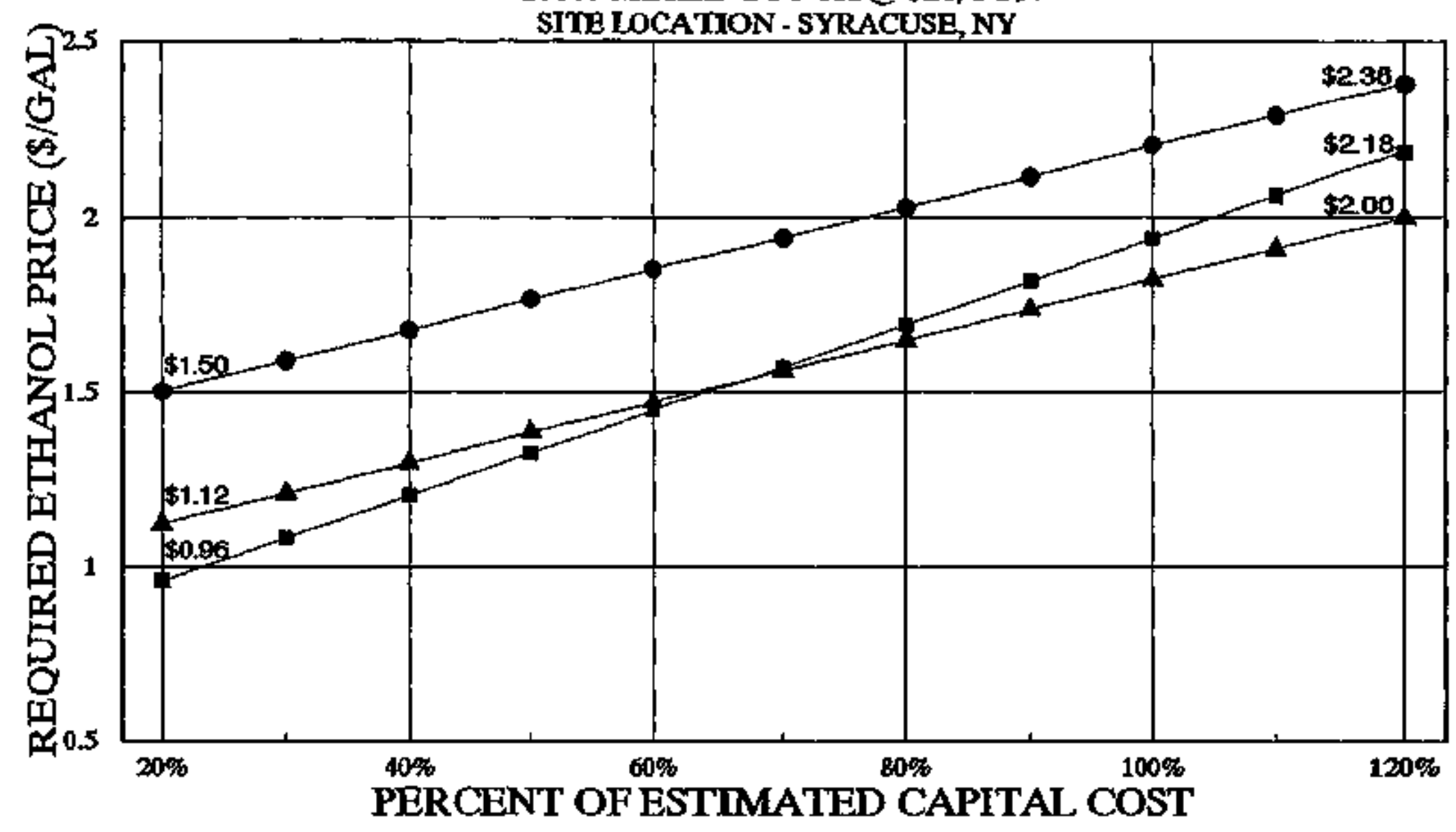

$\rightarrow$ OPT.19:500 DRY TED WTH GNSIFICATICN

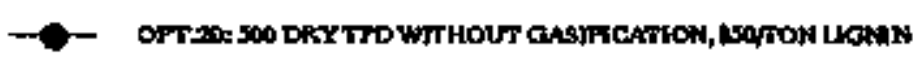

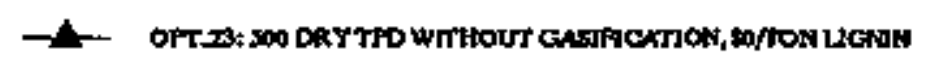


REQUIRED ETHANOL PRICE vs. CAPTALCOST AS A PERCENTAGE OF ESTIMATED COST 100\% MLXED STOCK @ \$40/TON

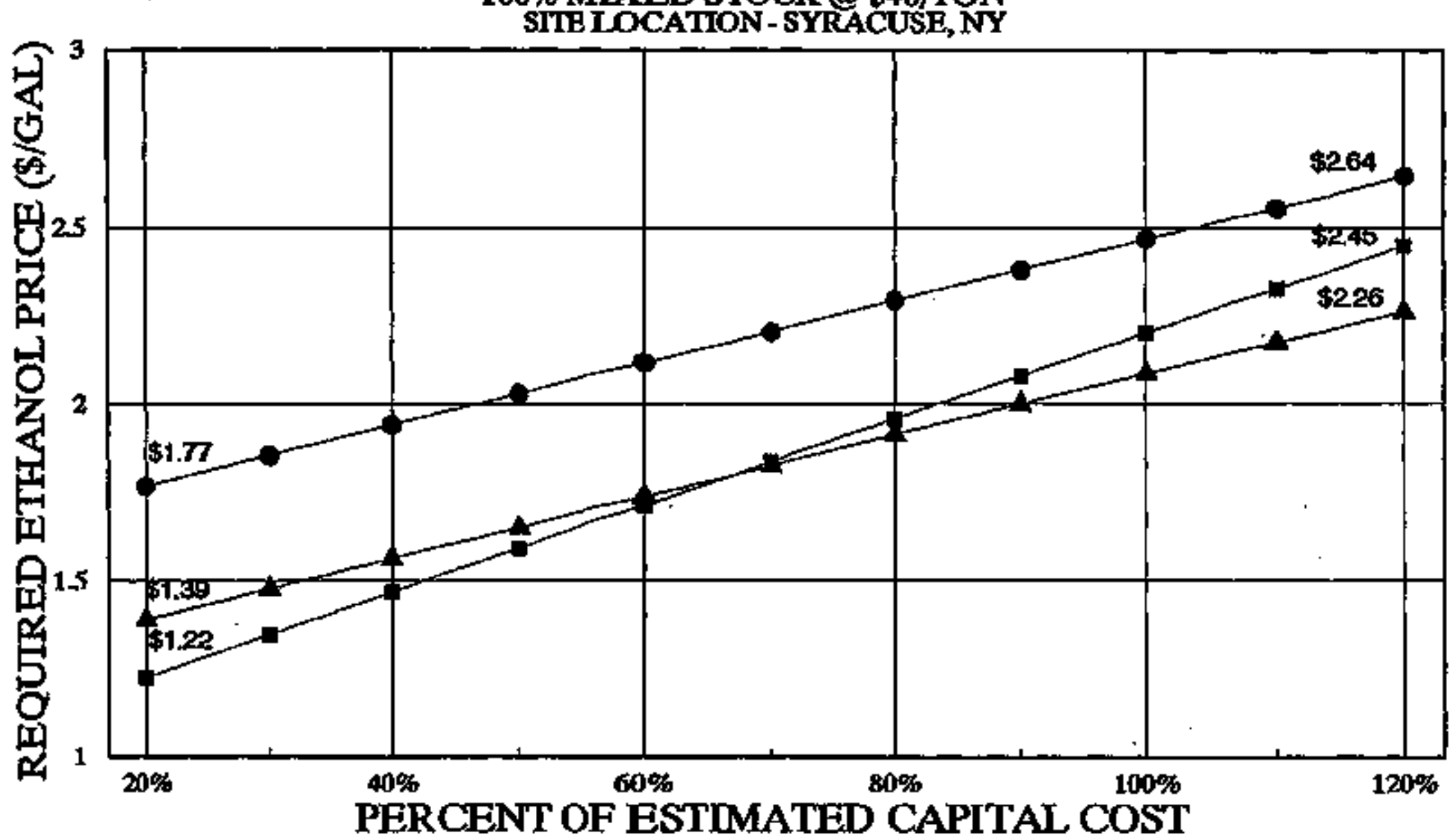

$\rightarrow$ OPT:21SOO DRY TTD WhTA OABIFICATTON

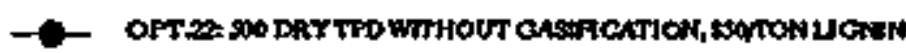

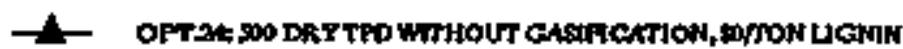

STONA \& WESTER DEVEIOPMIRNTCORPORATHON

FIGUAE 8-14 
REQUTRED ETHANOL PRICE vs. O\&M COST AS A PERCENTAGE OF ESTIMATED COST $100 \%$ MLXED STOCK @ \$20/TON

STIE LOCATION - SYRACUSE, NY

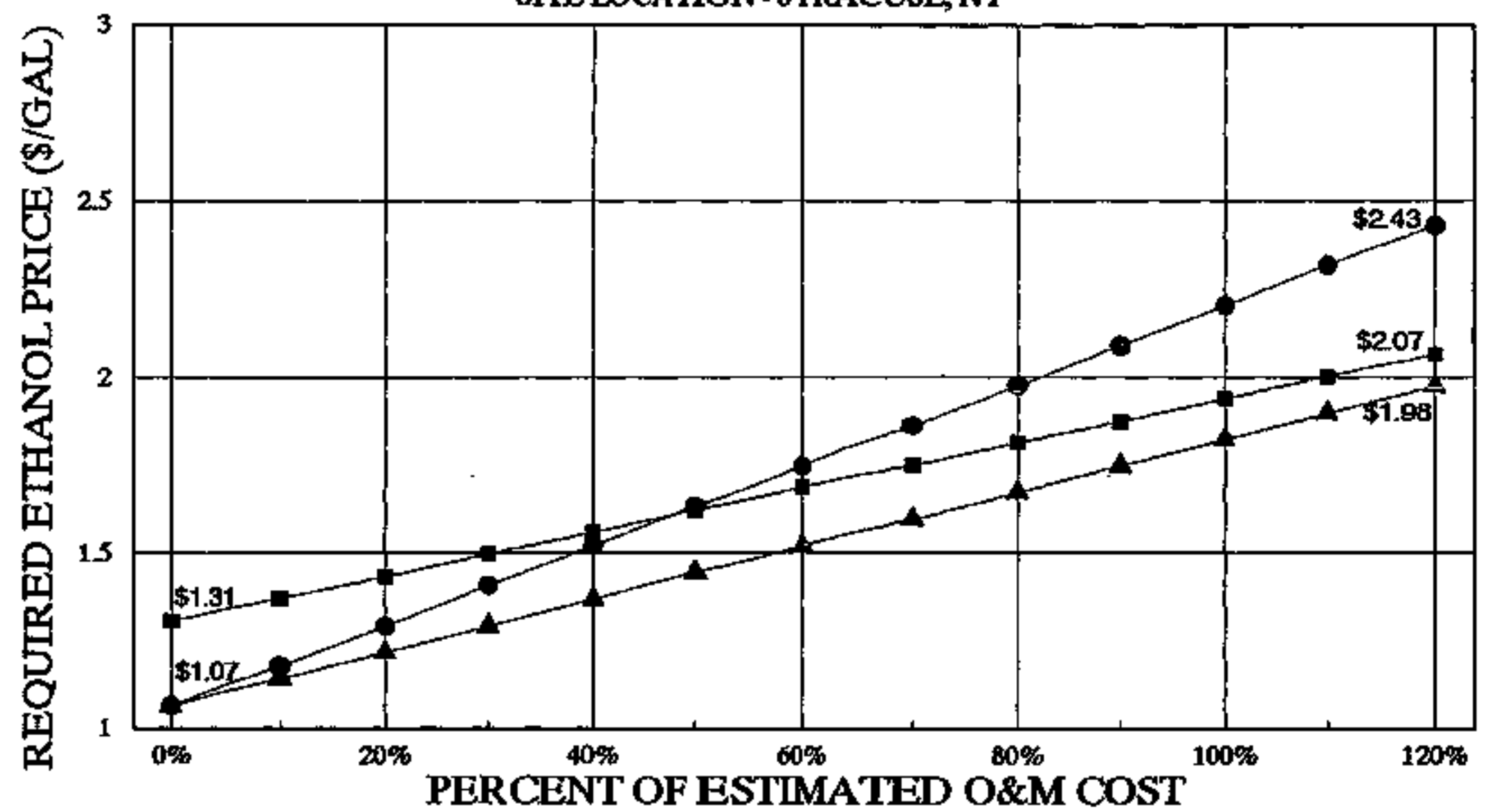

ESTMATED OAM OOSIS:

OPT.19 = 33,137 XYR

OFT.20 = \$4927 EYR

OPT:Xs = \$6,004 E/YR

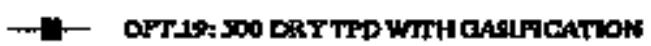

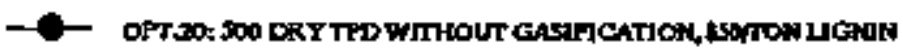

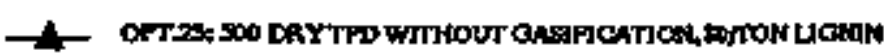

STONE \& WESSTEA DEVETOMMENTCORPORATCW

Figure 8-15 
REQUIRED ETHANOL PRICE vs. O\&M COST AS A PERCENTAGE OF ESTMMATED COST $100 \%$ MIXED STOCK@ @ 40/TON

SIIE LOCATION - SYRACUSE, NY

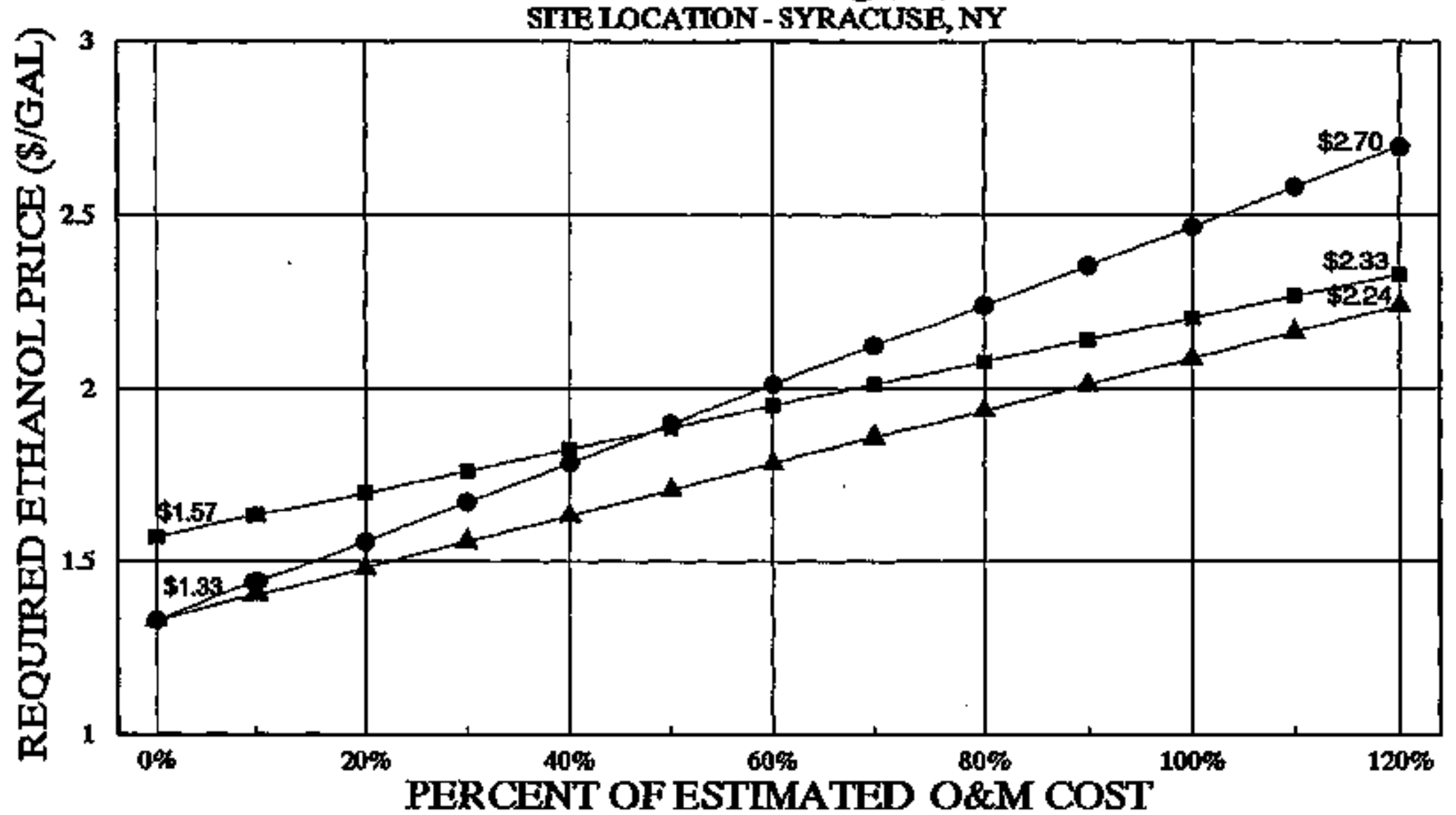

ESTIMATED OAM COSTS:

OPL21 $\Rightarrow \$ 3,137 / \mathrm{K} / \mathrm{KR}$

OTT.z2 - \$10,927K/YX

ORT:2A = SGOONETR

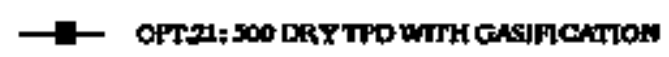

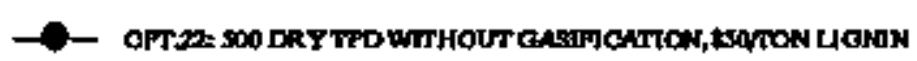

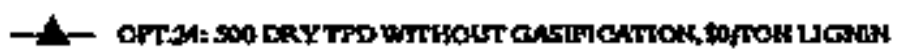

STOAE \& WESTER DEVELOPMIANT CORPORATON

Figure 8-16 


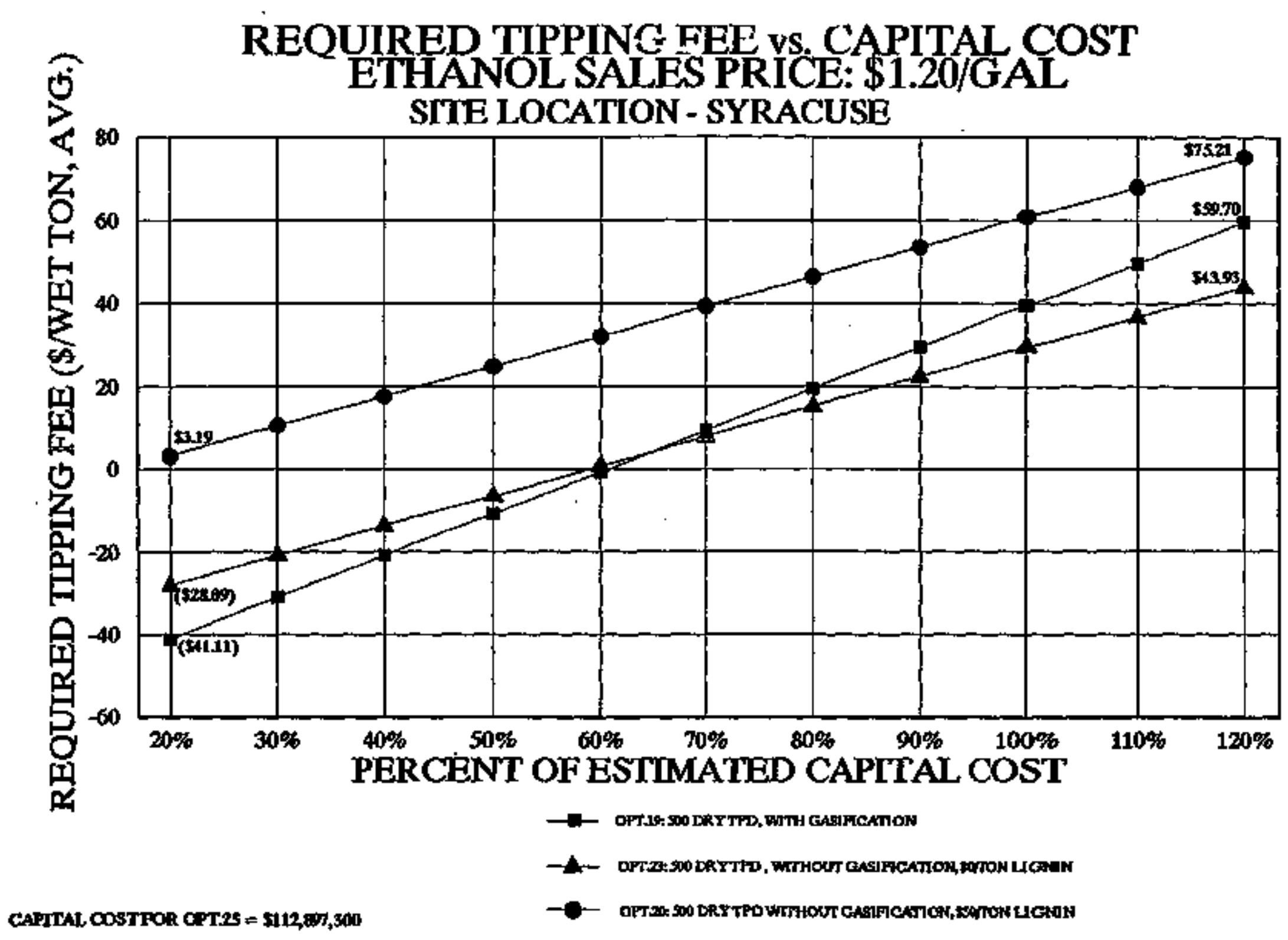

CAPIEN, COSTYOE OPT'26 \& 20 = \$81,114,300

STCAE \& WESTER DEVETOFMENTCORPORATION

FIGURE 8-17 


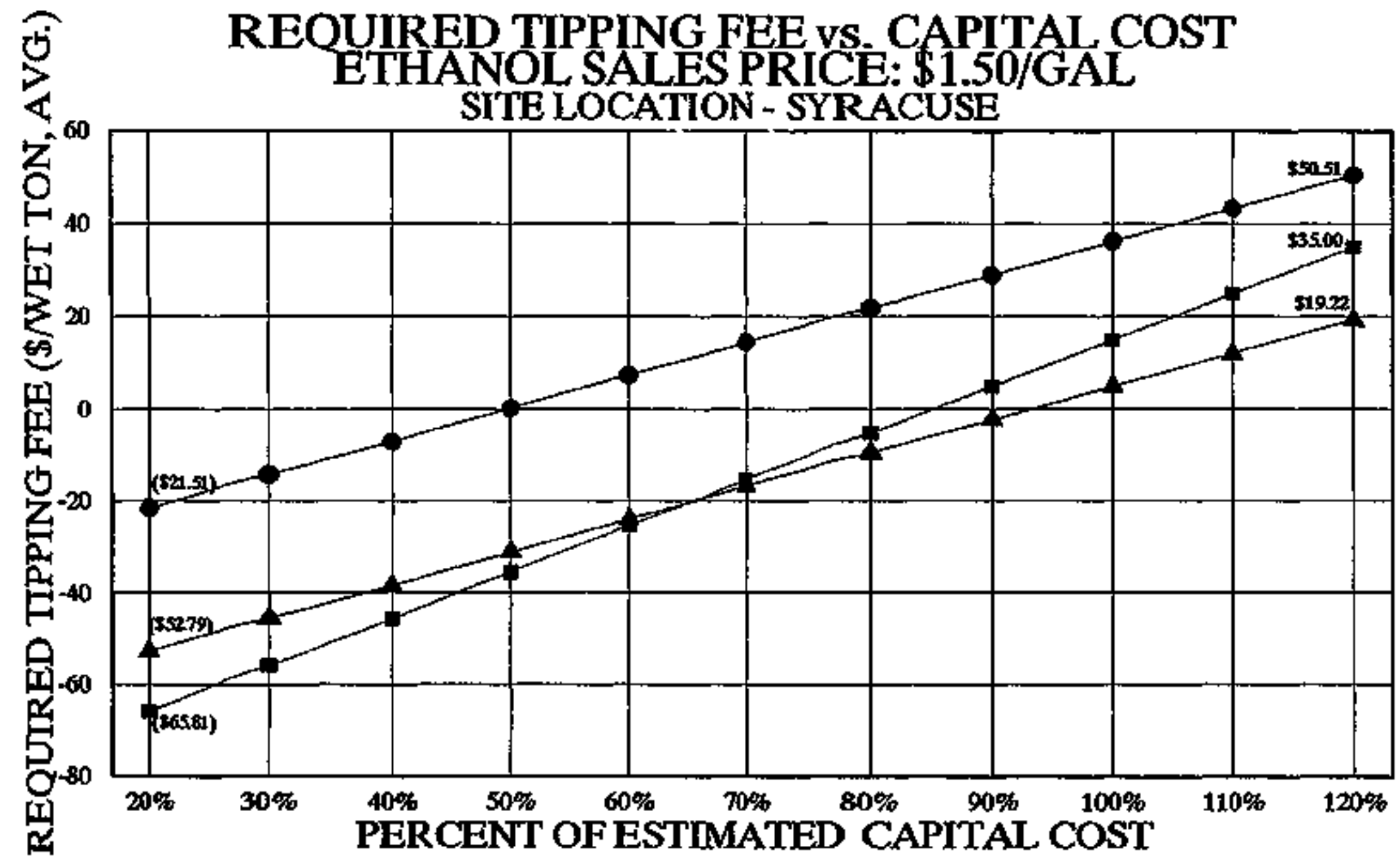

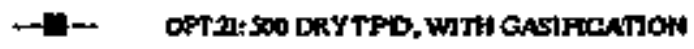

-

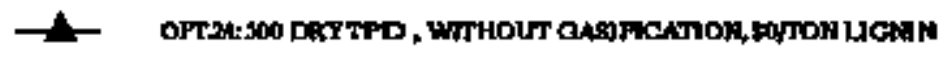




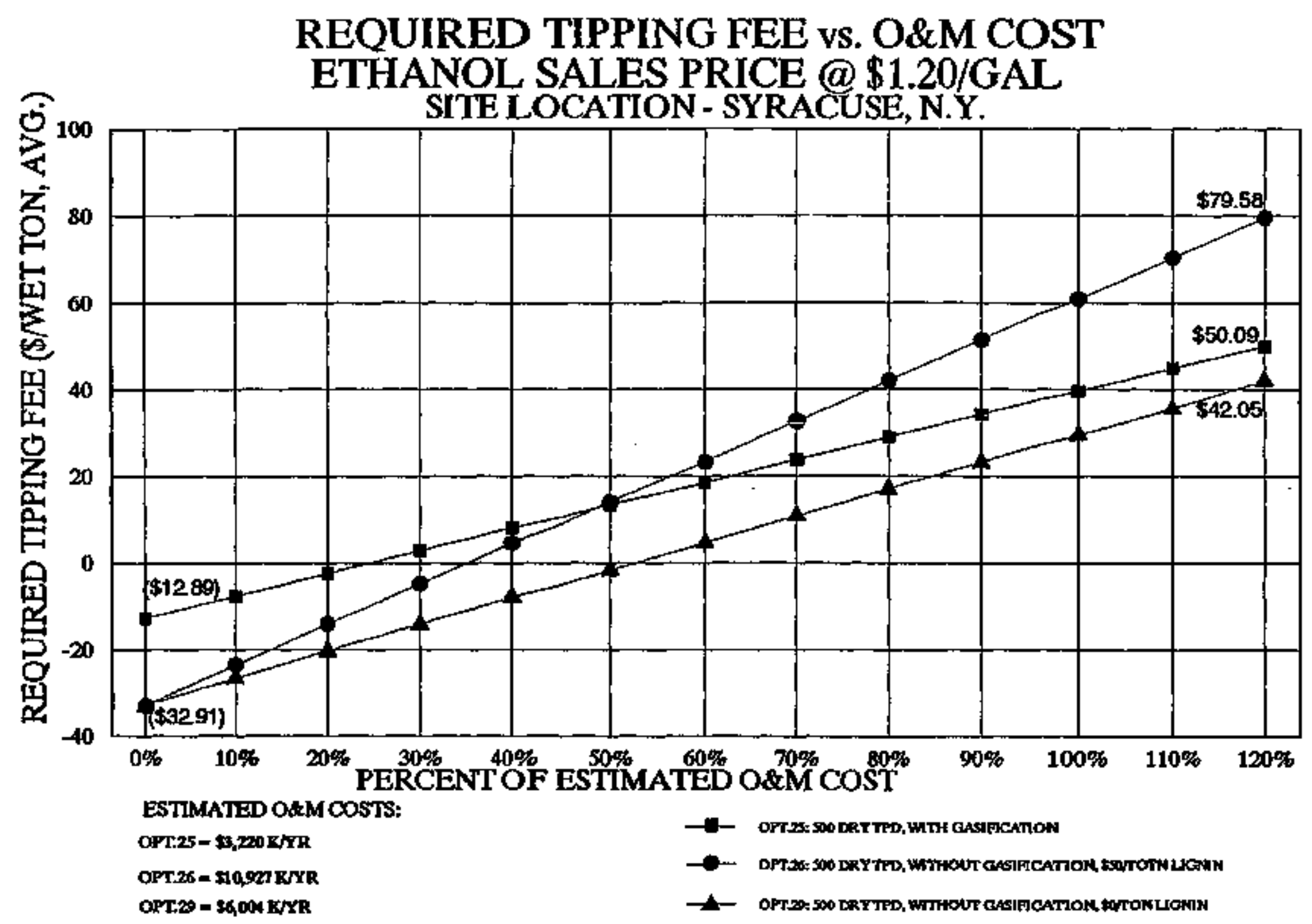

STONE \& WESTER DHVELOPMENTCORPORATION

FIGURE 8-19 


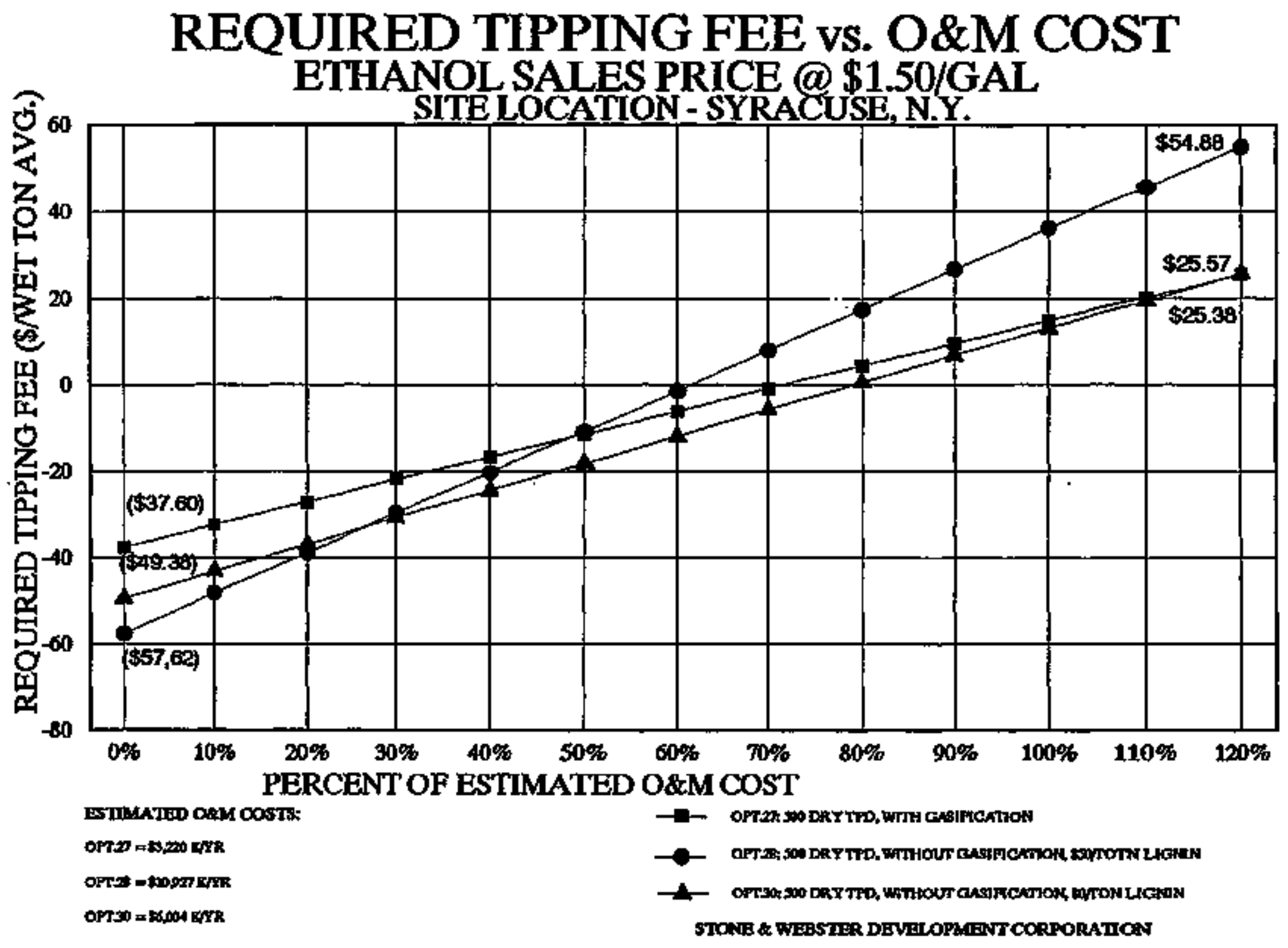

FIGURE 8-20 


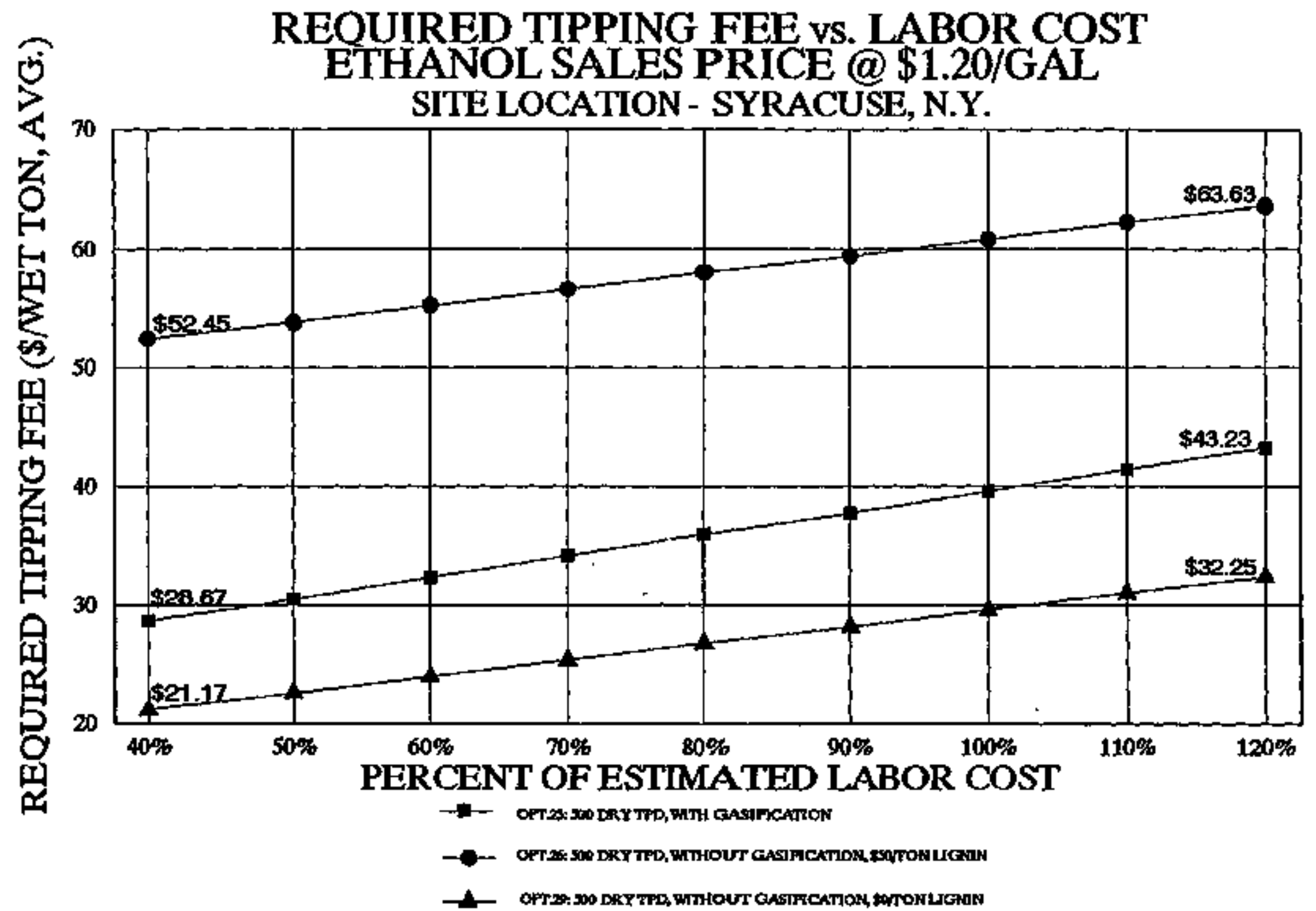

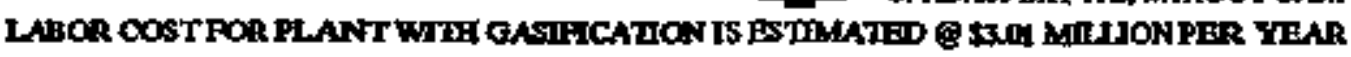

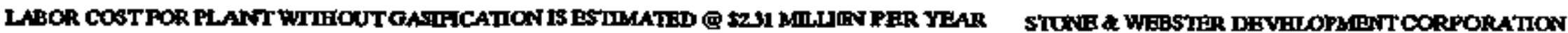

FIGURE 8-21 


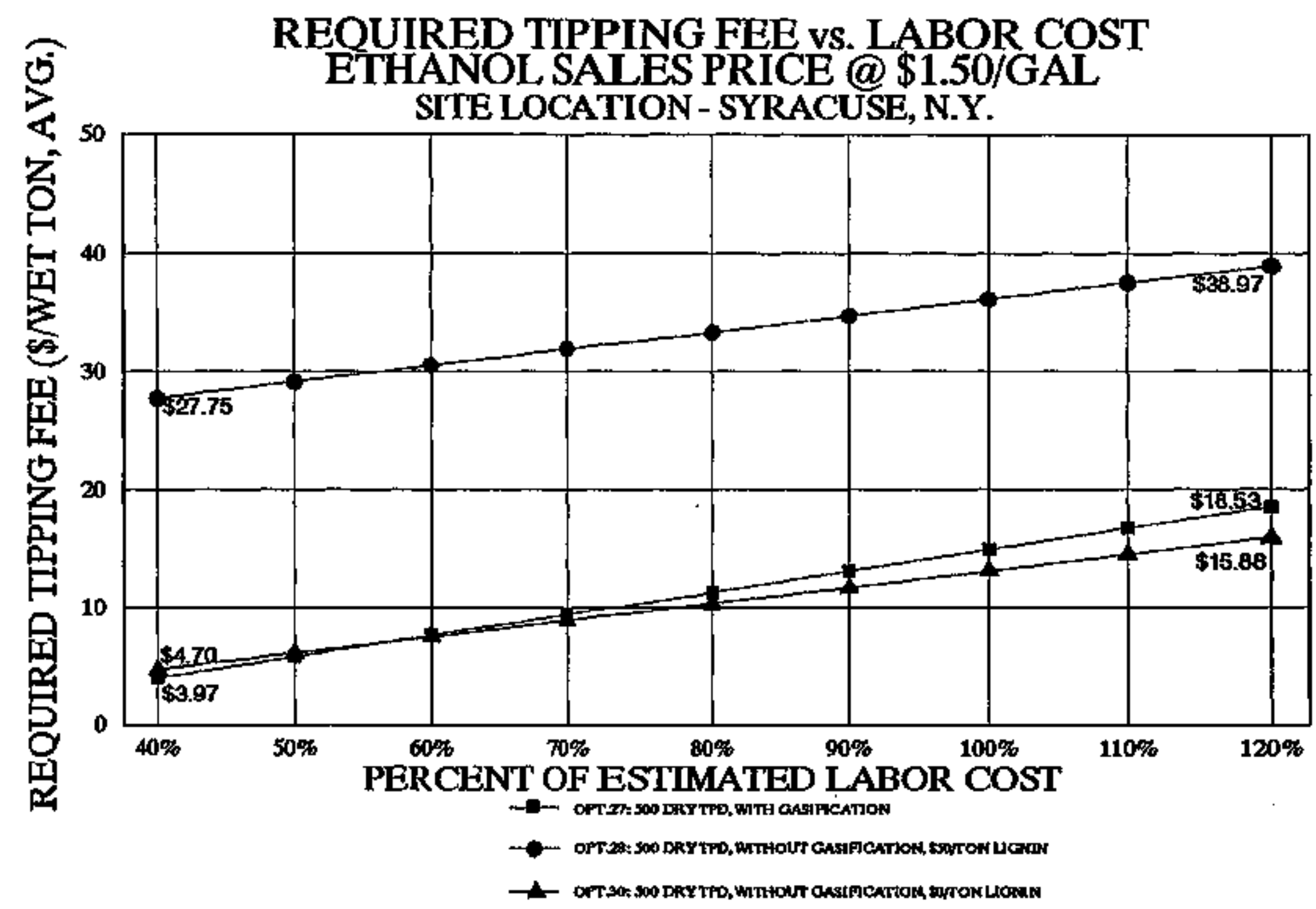

LABOR COST FOR PLANT WITH GASIFCATION ESTMMATED @ W.0U MILLION PER YEAR LABCR COST WITHOUT OASIFACADCN IS ESTMATED @ \$231 MILUION PER YEAR

STONE \& WEBSTER DEVHLOPLFNTCORPORATION

FIGURE 8-22 


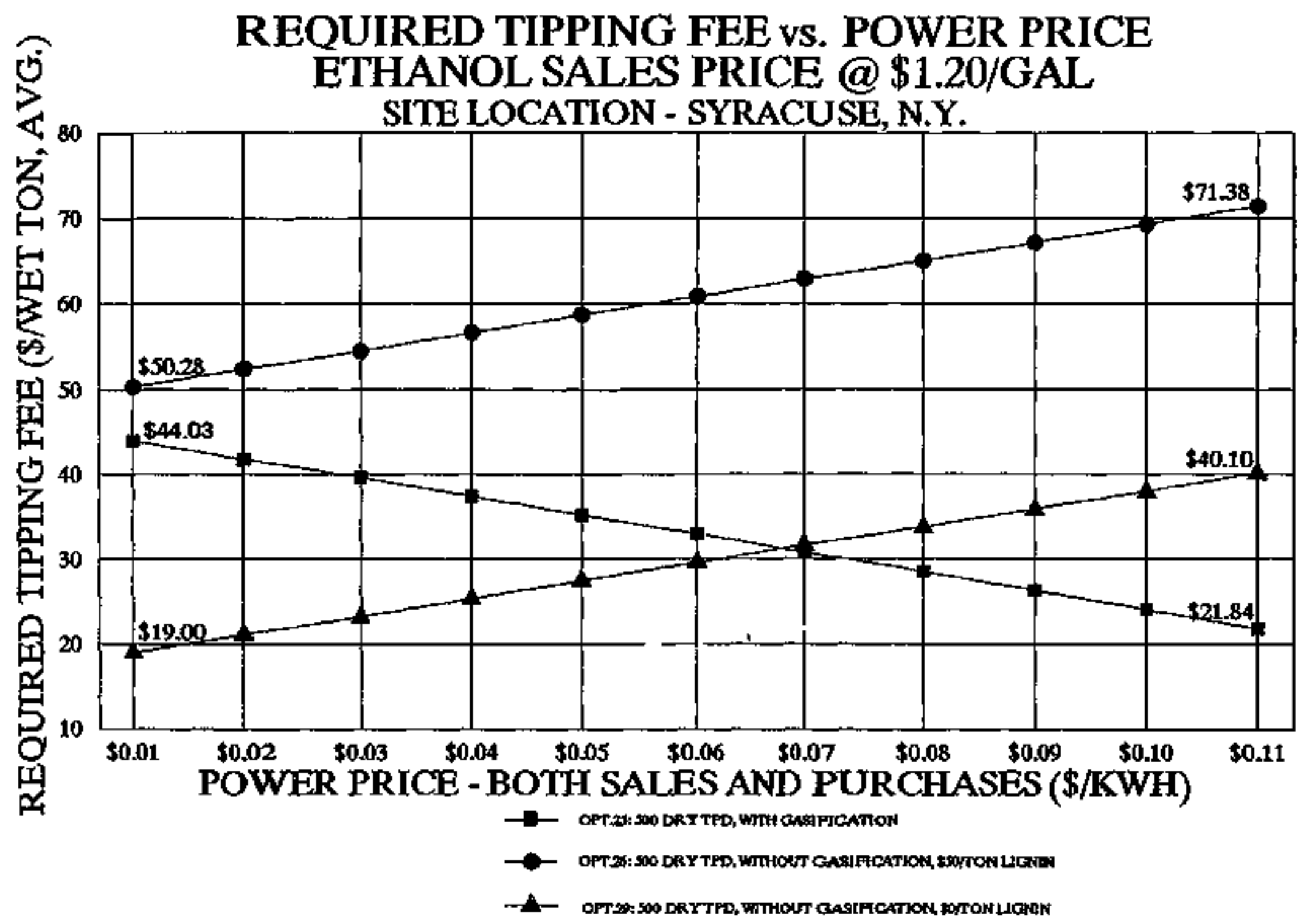




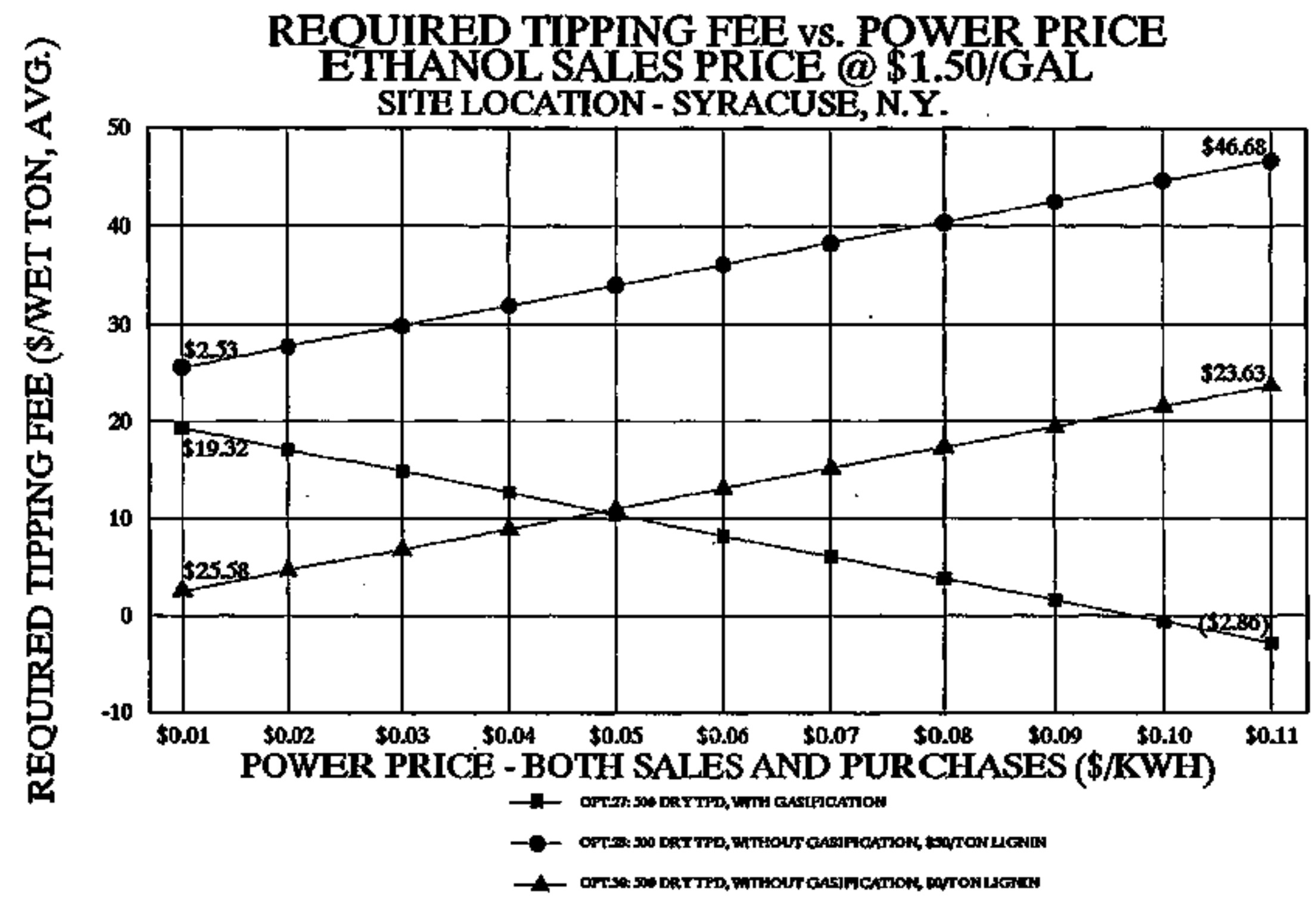




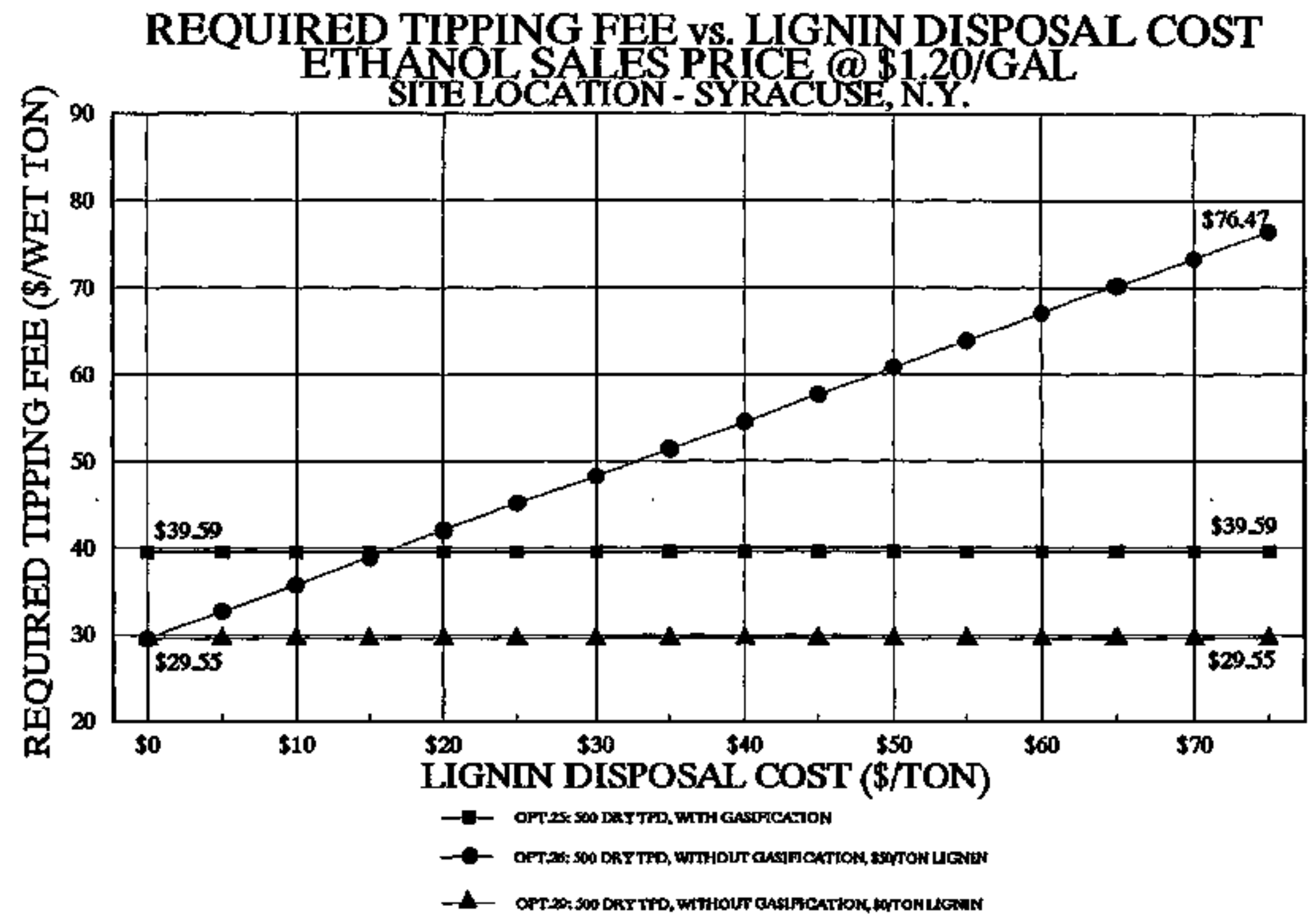

STONE \& WFESTER DEVELOPMENT OCRPORATION

FIGURE 8-25 


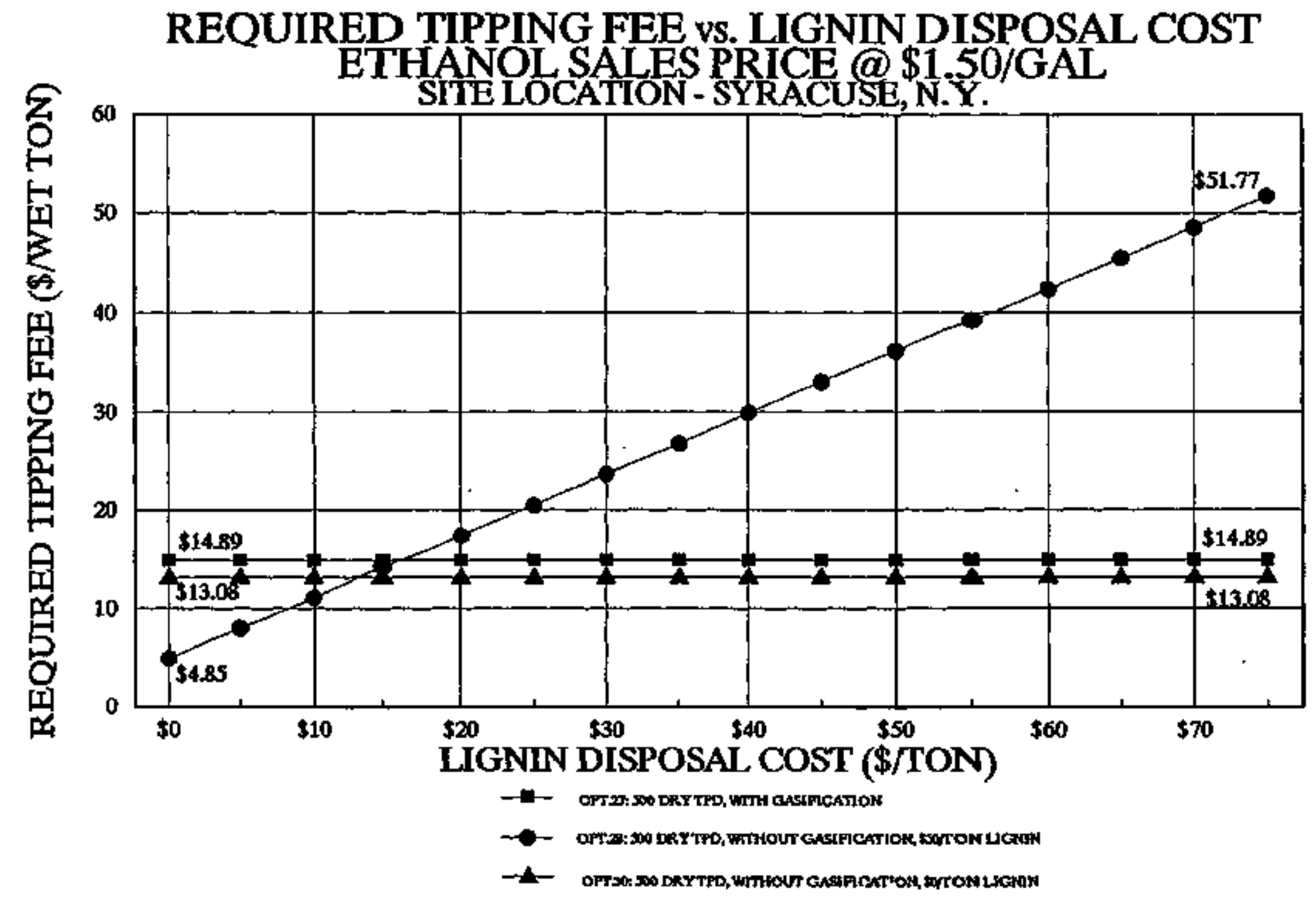

STONE \& WEASTER DEVELOPMENT CORPORATION FIGURE 8-26 


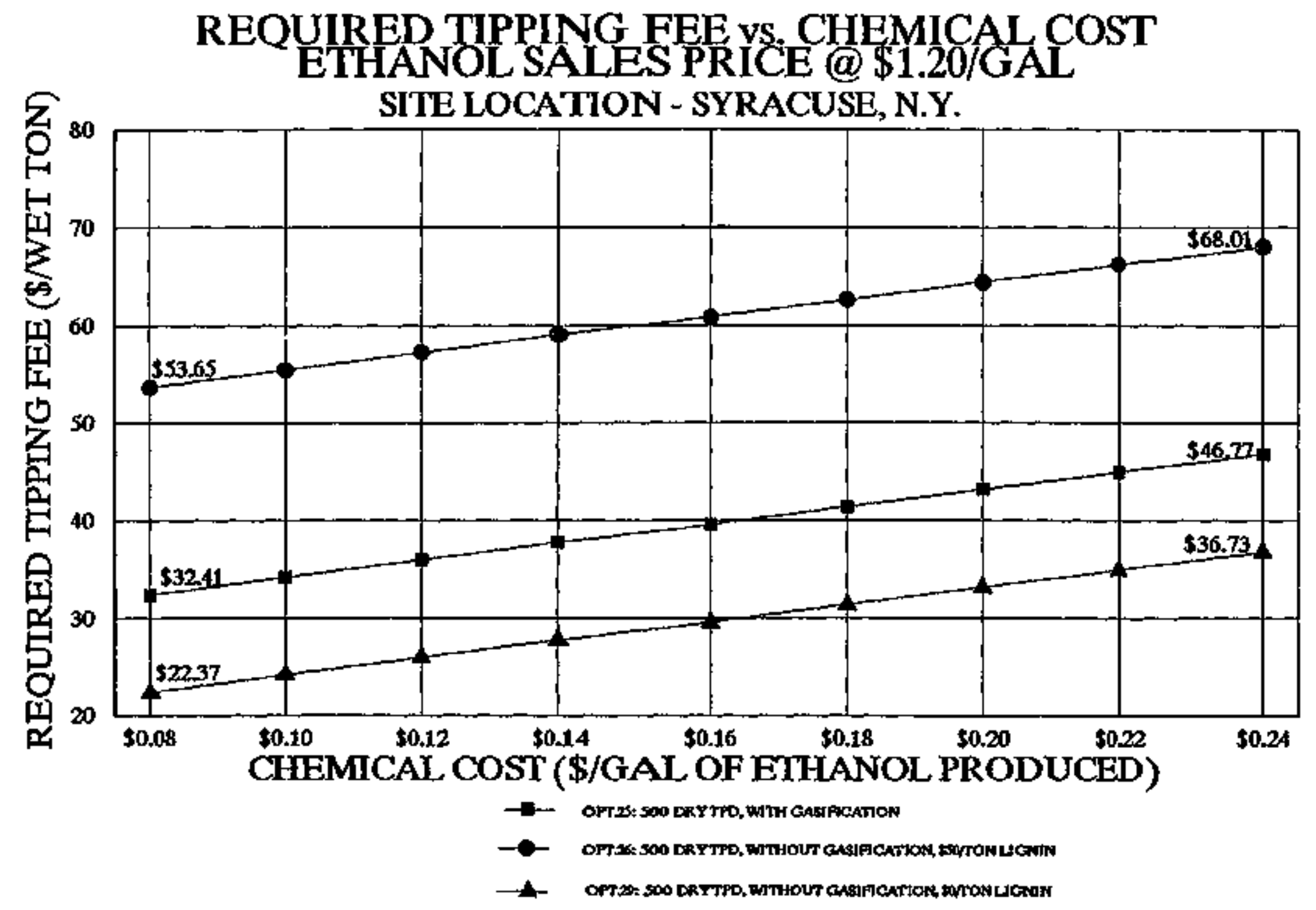




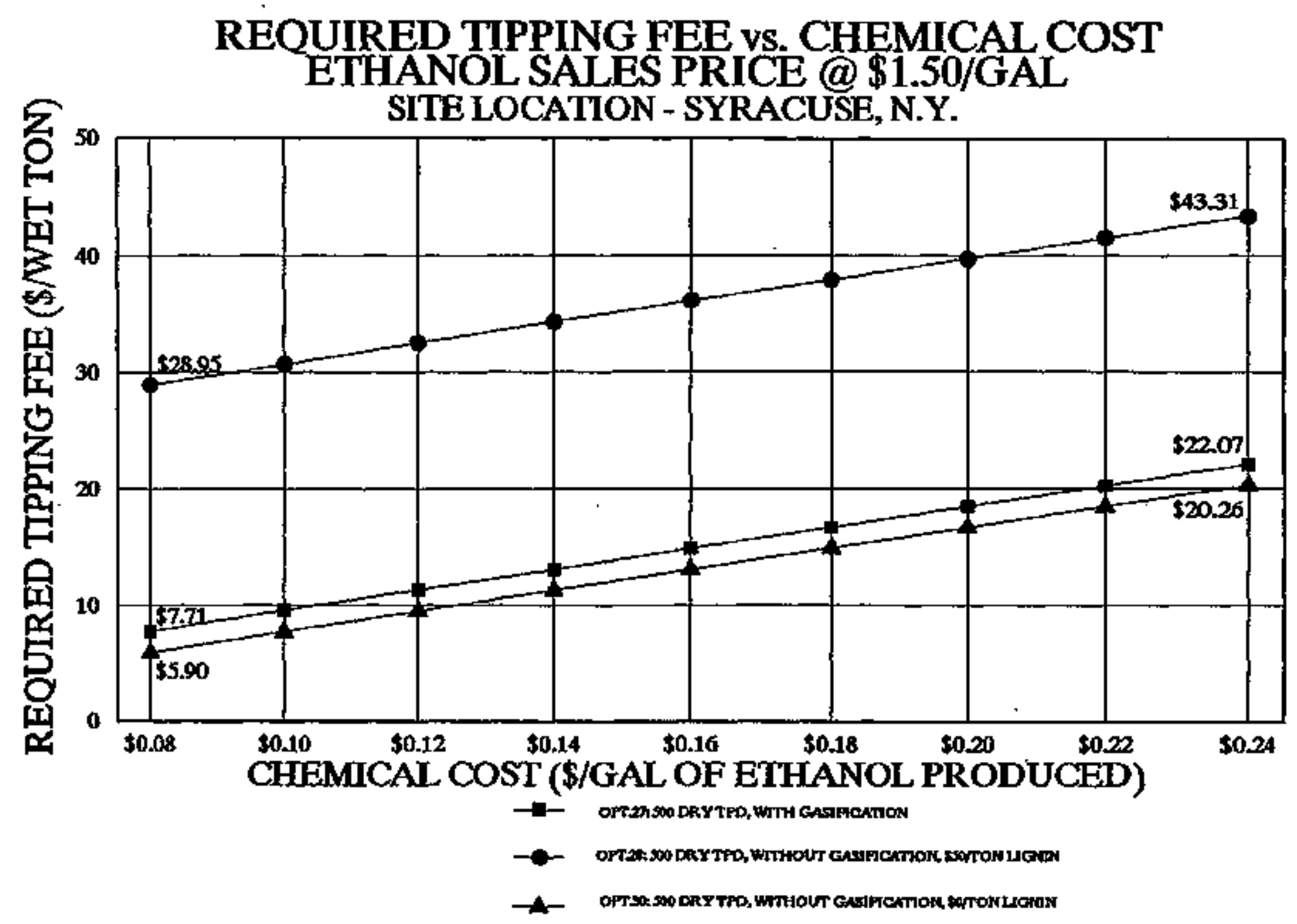

BASE VALUR POR AHL OPTICNS IS \$1160AL, OF ETHANOL, PRODUCED

STONE \& WEISTER DEVELOPMENT CORPORATION FIGURE 8-28 
POLICY RESEARCH CENTER, INC

ANNE D. STUBE

Exeeurive Directc

(200) 624-845

August 31, 1994

Dixon Brandt

Senior Principal Euviroumental

Engineer

Stone and Webster

246 Summer Street

Boston, MA 02107

Dear Mr. Brand:

We invite your attendance and participation at our Wednesday October' I2, 1944 torm; "Siting an Ethanol Plant in the Northeast", to be held at New England Air Museum, Bradley Field, Hartford, Connecticut from 9:30 AM to 5 PM. The goal of the forum is the identification of barriers and prospective strategies for the siting of one or more biomass-to-ethanol plants in the region during the next eighteen months. The forum's cosponsors, the National Renewable Energy Laboratory and the Northeast Regional Biomass Program, are coinmitted to bringing together all of the prospective parties which can make the construction of an ethanol plant happen in the Northeast.

The target aidience for the forum is developers. However representatives of important stakeholder groups such as: waste haulers, landfill operators, pulp and paper companies, air and solid waste regulators, słate economic development and energy offictals, researchers, public fleet managers, auto company representatives, oil companies, and investors will be present and provide input to the process. We anticipate between 75 and 85 attendees.

Several Eactors have prompted the cosponsors to convene the forum at this cime: increasing costs associated with the disysal of biomass wastes: environmental and energy regulations encouraging refornulated gasoline, fuel extenders, oxygenated fuels and other altemative transportation fueis; the potential economic benefits for the Northeast arising from the development of indigenous-renewable fueis: and the desirability of providing additional fuel choices for alternatively fueled vehicles.

The Northeast Regional Biomass Program's release of a new report, "A Resource Assessment for Ethanol in the Northeast, ${ }^{*}$ provides an initial foews for the forum.

As the attached agenda reflects, the forum considers the following issues:

* the candidate feedstocks, their short and long term availability and costs-among these include uban woody wastes, waste papen, paper sludge, food wastes, cheese whey, and forest product residues; 
* the Clean Air Act Amendments, Energy Policy: Act of 1992, and other environmental regulations which provide economic incentives for altemative fuels. including biomass-toethanol:

* the salient environmental and energy regulations and their relationship with market development and with the siting of an ethanol plant in the Northeasten states;

* the potential for an economic win win scenario for developers of biomass-to-ethanol facilities and the Norheast states;

* an update on conversion technologies: and

* potential markets for ethanol in the region.

The concluding panel will consider any other issues participants raise which may affect the successful siting of an ethanol plant. The forum will also be charged with generating an agenda for the Northeast Regional Biomass Program and NREL to consider for ailocating their resources in the months atead.

There will be no fees for participants at the Fonum and lunch will be provided. Please retum the attached reservation by maij or FAX. We look forward to seeing you on October 12th.

Sincerely yours,

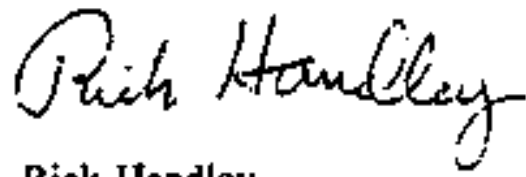

Rick Handley

Program Manager. NRBP

Attackment 
Attracting an Ethanol Plant to the Northeast:

\section{A Liquid Fuels Forum}

Bradley Field, Harford Ct.

Wednesd2y, October 12, 1994

\section{DRAET_AGENDA}

\section{8:30-9:30 Registration and Continental Breakfast}

9:30 -9:45 Welcome:

Rick Handiey, Northeast Regional Biomess Program

David Lavine, Connecticut Office of Policy and Management

John Ferrell, U.S. Department of Energy

9:45.10:00 The Day's Agenda:

Barry Lawson, Forum Moderator

10:00-10:45 Resource Assessment - Available Feedstocks

Christine Donovan, C. T. Donovan Associates

10:45-11:00 Break

11:00-12:00 Harnessing the Waste Stream - Short and Long-term Availability and Cost:

Urban wood wastes: Richard Wills, Stapleton Recycling (invited)

Woad residues: State Utilization Forester, TBD

Impact of Recycling laws: State Recycling Coordinator, TBD

Paper sludge: AFPA Spokesman, (invited)

Impact on landfills: Landfill Operator, TBD

12:00-1:00 Eunch (provided)

1:00-1:30 Technology Update/Economies of Biomass-to-Ethanol:

Norm Hinman, National Renewable Energy Lab

1:30-2:00 Eeonomic Impacts - The "Win Win" Potential for Development:

Joel Gordes, Connecticut Office of Policy and Management (invited)

2:00-2:45 Markets - The Potential for Ethanol in the Northeast:

A panel discussion of the potential markets for ethanol in the

Northeast.

2:45: $\cdot 3: 00$ Break

3:45-5:00 Where do we go from here: Facilitated Discussion

Devejopment of an atrion plan for the Northeast 


\section{ATTACHMENT \&-3}

September 29, 1994

Mr. Henry Gastwirth

Stone \& Webster Engineering Corp.

P. O. $90 \times 2703$

New York, NY 10116

Dear Mr. Gastwirth:

\section{Re: AMOCO BIOMASS-TO-ETHANOL SITING STUDY}

New York State Electric \& Gas Corporation (NYSEG) woutd like to participate in your efforts to identify and evaluate sites in upstate New York that can accommodate a biomass-to-ethanol facility.

NYSEG is interested in participating for a number of reasons:

1) NYSEG is involved in promoting economic development in its service territory. Your ultimate objective of long-term operatlon of a financially attractive and environmentally sound biomass-to-ethanol facility is certainly in synch with our economic development goals.

2) NYSEG has power plants at sites that could be developed to accommodate a biomass-to-ethanol plant. Proximity to an existing power plant offers a number of benefits. For example: nearby electric and steam supply, accessible water and sanitary services, existing landfill facilttes, established truck and/or rail access with weighing capabilities, and existing environmental monitoring and control programs. Some of our sites have gas supply available as well. In addition, since our plents are existing industrial sites, licensing new facilities would most likely be easier and less impact than at a "greenfield" site.

3) Waste lignin or other waste products from your facility may be acceptable alternalive fuel at certain NYSEG power plants. Thus, we may be able to help address your waste handling needs. We would expect to be reimbursed for any processing or drying required to transform waste products to acceptable fuel, but when such costs are compared to disposal costs, a waste-to-fuel partnership could signiticantly help project economics. 
4) NYSEG believes biomass-to-energy projects are among the most promising renewable energy options for upstate New York. Consequently, we are interested in advancing economically viable biomass-to-energy initiatives.

I look forward to working with you on this project.

Sincerely,

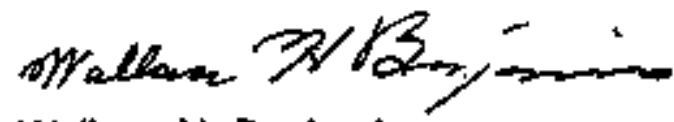

Wallace H. Benjamin

Technical Specialist

cc: P. G. Carnoy/M. R. Testa

M. J. Garner

J. W. Rettberg

T. M. Turner.C. Reilly/M. Richardson

D. E. Wickham/J. C. Kobuskie 
ATTACHMENT 8-4

NYSEG SITES 


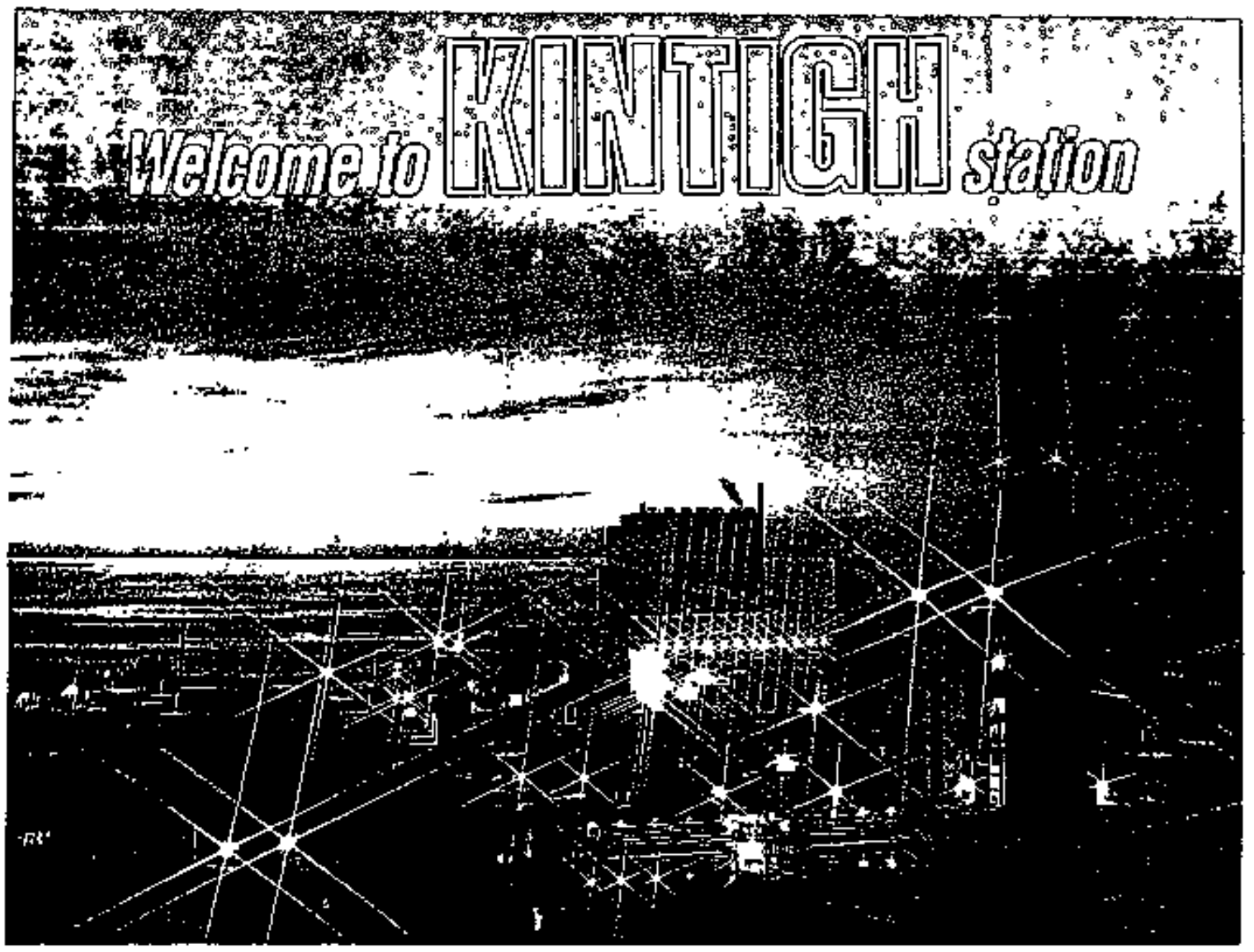

Located on the southem shore of Lake Ontario is NYSEG's newest and largest coakfired generating station in New York State. Completed in 1984

One of eight genetating stations owned or c0-0umed by New York State Electric $\&$ Gas Copporation (NYSEE) after more than three years of construction, Kintigh Station generates about 688,000 kilowatts ( 688 million watts) of electricity. This accounts for about 25 percent of NYSEG's total generating capacity and helps guarantee our custorners an adequate supply of etectsicity well into the $1990 \mathrm{~s}$.

In its first years of operation, Kintigh has proved to be one of the most efficient and reliable generating stations in the United States. Moreover, because it was engineered with the environment in mind, it's one of the cleanest. These are same of the reasons why the Electric Power Research Institute (EPRI) chose Kintigh as the site for its High Sulfur Tesi Center.

For the Niagara region, Kintigh Station means tax revenue, more business and jobs. And because it's a coalfired plant, Kintigh hetps reduce our state's dependence on foreign oil.

At NYSEG, we realize how vital electricity is to our lives and to New York's economy. We also recognize the importance of producing electricity in an efficient and envirommentally acceptable manner. We welcome you to Kintigh Station and imvite you to learn more about how NYSEG uses coal to produce electricity. 
KINTIGH STATION

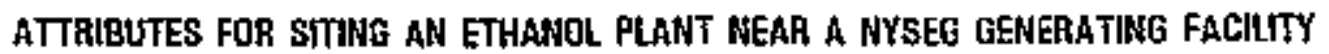

\begin{tabular}{|c|c|}
\hline Land availabillty & $3000+$ acres \\
\hline Towm & Somerset \\
\hline County & Nisyara \\
\hline Zoning & Industrial \\
\hline Zoning - offsite & Agyitultural \\
\hline Access & Reil, Truck \\
\hline Weigh tacitities & Reil, Truck \\
\hline Water f100GPM Min\} & Potable \& Incustrivel Grade \\
\hline Steant & Avaiflable for all requifirgatents \\
\hline Gas & Not Availablg \\
\hline
\end{tabular}




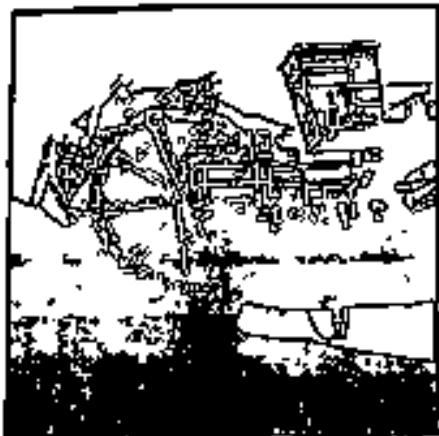

The huge reclamet scoops coal trom the slorage pile ando conveyors leadung to Ine plani

\begin{tabular}{|l|c|}
\hline COAL TO QUINKEF & S,400 TONSHOAY \\
\hline COAL N STORAGE & UD TO 500,000 TONS \\
\hline CDAL SUPPLY (APPROOX & 90 DaYS \\
\hline & \\
\hline & \\
\hline & \\
\hline & \\
\hline
\end{tabular}

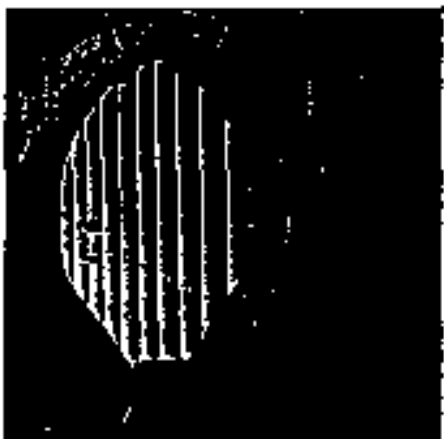

Putrerized coal s blown into the bailer where it bums. Waler in lubes anng the boiler is lumed usto sleam.

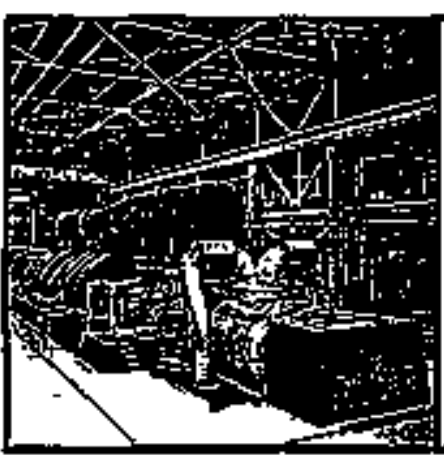

The iutine-generator is the heat of the generating station. Energy from the steam spins the whtule which turns the rolor of the generator, producting elesetricity.

\begin{tabular}{|c|c|}
\hline STEAM TEMFERATURE & $1,005 \cdot F$ \\
\hline STEAM PAESSURE & 2.505 FSIC \\
\hline STEAhHAOUR & $4,500,000$ Lbs \\
\hline COAL BUANEDHOUH & 225 Tons \\
\hline TYPE OF BUFNIEA & Pulverizod: \\
\hline BOALFA EULN' HEGHT & $256^{\prime}$ \\
\hline 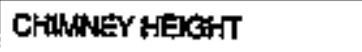 & $625^{2}$ \\
\hline
\end{tabular}

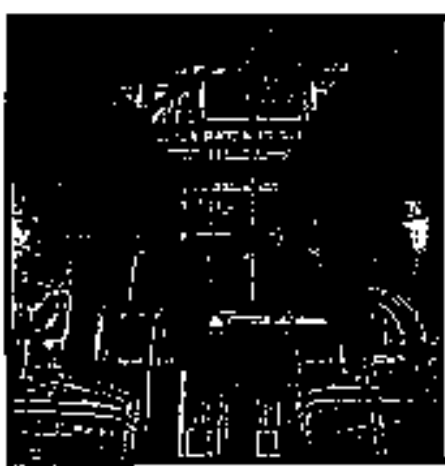

Lake water is drawn inlo the condenser la cool steam beck inlo water so that il can be rewsed in the boiler.

\begin{tabular}{|c|c|}
\hline 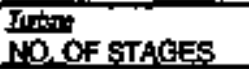 & 1 \\
\hline 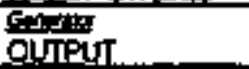 & $689,000 \mathrm{kw}$ \\
\hline WOLTAGE & 24,600 \\
\hline SPEEO & $3,600 \mathrm{RPM}$ \\
\hline CODUNG & Hydropen \\
\hline MANUFACTUAER & General Electric \\
\hline
\end{tabular}

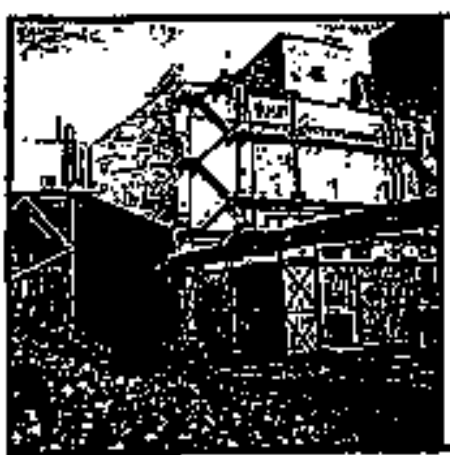

Electronlatic precipitators remove line perticles of thy ash from the the gases.

\begin{tabular}{|l|c|}
\hline CAPACTIY & 165,000 Galihin \\
\hline COOLNG SUAFACE & 365,000 Sq. Ft. \\
\hline TEMP. DEFERENTIAL & $35 \circ \mathrm{F}$ (Max) \\
\hline & \\
\hline & \\
\hline & \\
\hline
\end{tabular}

Stelle-othe-art equipment is used to controt the flue gas desulfurization (FGD) process, where a sturny of inteslane and waler is used to remove sulfur compounds trom the gases.

\begin{tabular}{|l|c|}
\hline DALY ASH LOAD & 400 TONG \\
\hline DALY ELECTRIGAL USE & $8,400 \mathrm{kWh}$ \\
\hline EFFKCIENCY & $99.86 \%$ \\
\hline & \\
\hline & \\
\hline & \\
\hline & \\
\hline
\end{tabular}

\begin{tabular}{|l|c|}
\hline DALY SO, RENOWED & 230 Tons \\
\hline DALY ELECTRICAL USE & $144,000 \mathrm{kwh}$ \\
\hline DALY LMESTONE USE & 340 Tons \\
\hline EFFICHENCY & $70.80 \%$ \\
\hline & \\
\hline
\end{tabular}



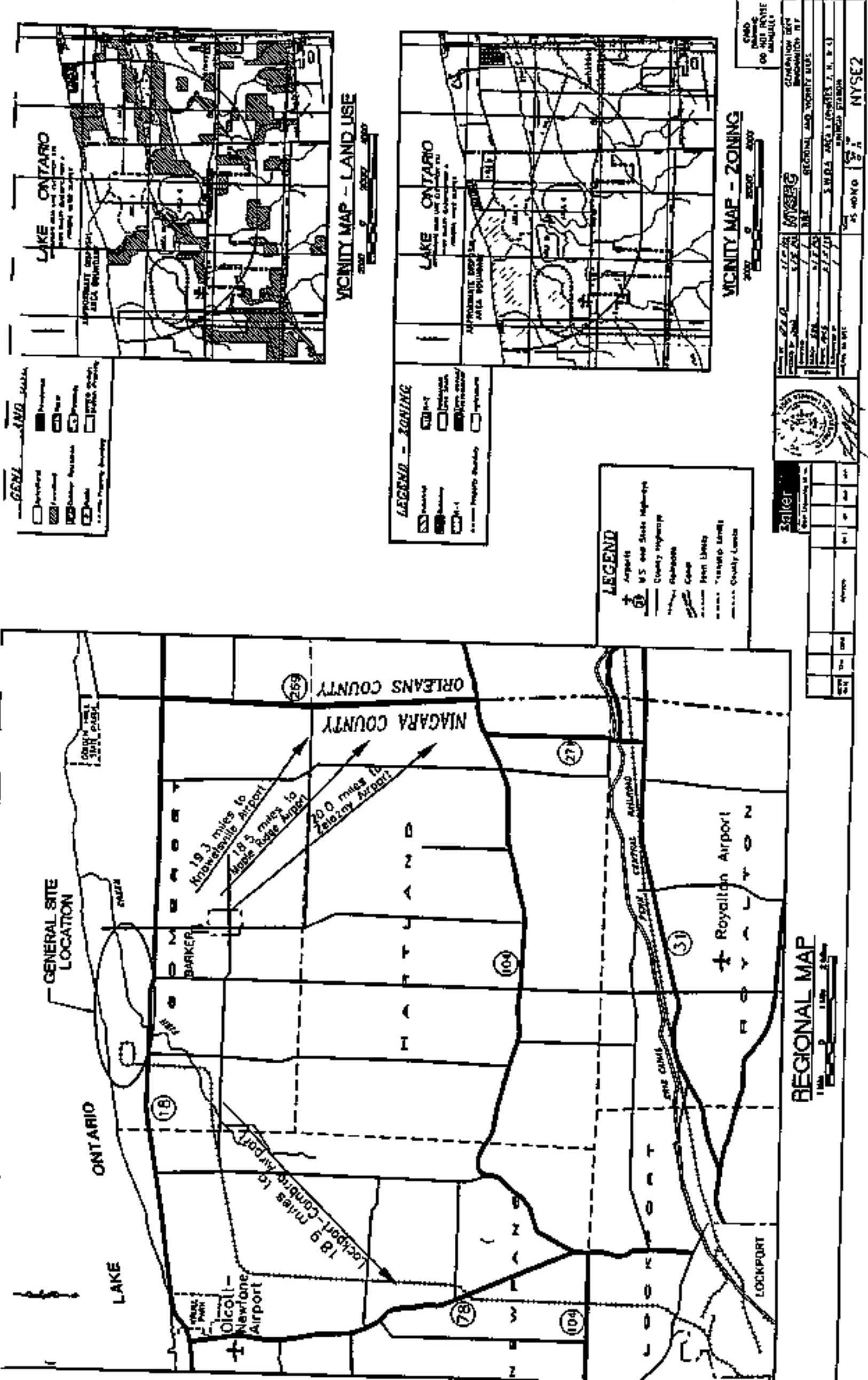


\section{MLLLIKEN STATION}

ATTRIBLTES FOR STTING AN ETHANOL PLANT NEAR A MYSEg gENERATING FACILTY

\begin{tabular}{|c|c|}
\hline Land avalïability & $50+$ acres \\
\hline Town & Lansing \\
\hline County & Tompkins \\
\hline Zontang & Industrial \\
\hline Zotung - offorite & Agricultural \\
\hline Accass & Roil, Truck \\
\hline Weigh facilitios & Rail Avallable Now, Truck in 1995 \\
\hline Water [100GPM Min] & Potable \& Industrial Grade \\
\hline Stanm & Avallable for all requirements \\
\hline Gas & Not Ayjilatle \\
\hline
\end{tabular}




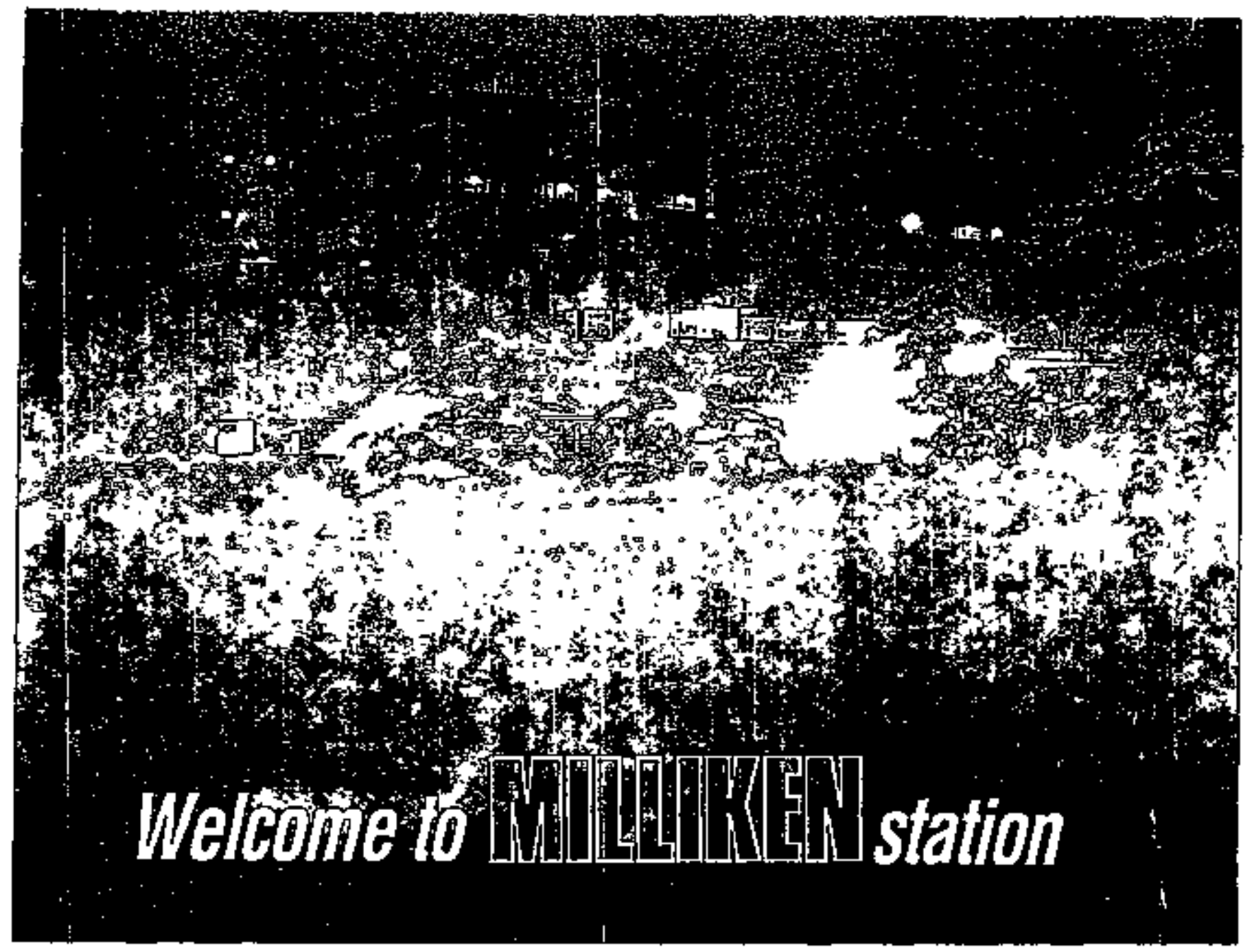

Miliken Generating Station is one of seven coal-fired stations in the NYSEG generating system. It first provided electricity to NYSEG

One of seven coal-fired generating stations owned and operated by New York State Electric \& Gas Corporation customers in 1955, and a second generating unit was added in 1958 to increase the plant's generating capacity to 320 megawatts.

In part because Milliken continues to be one of the nation's most efficient generators, it has received $\$ 45$ million from the U.S. Department of Energy's Clean Coal Technology Program toward the instaliation of a $\$ 135$ million air pollution control system. The system, whuch will begin operating in early-1995, will demonstrate one way to use coal, an abundant domestic resource, in an environmentallyresponsible manner.

Electrostatic precipitators that remove 99 percent of the fly ash from tlue gases are also being refurbished as part of the air pollution control project.

Milliken Station burns approximately 800,000 tons of bituminous (soit) coal each year. The fuel is delivered to the plant by train from Pennsylvania. Ohio or Kentucky

At NYSEG, we realize how vital electricity is to our ines and to New York's econorny We also recognize the importance of producing electricity in an efficient and environmentally-responsible manner.

We welcome you to Milliken Statron and invite you to learn more about the Miliken Clean Coal Demonstration Project and how NYSEG uses coal to generate electricity 


\section{MILLIKEN STATION . . . FACTS AND FIGURES}

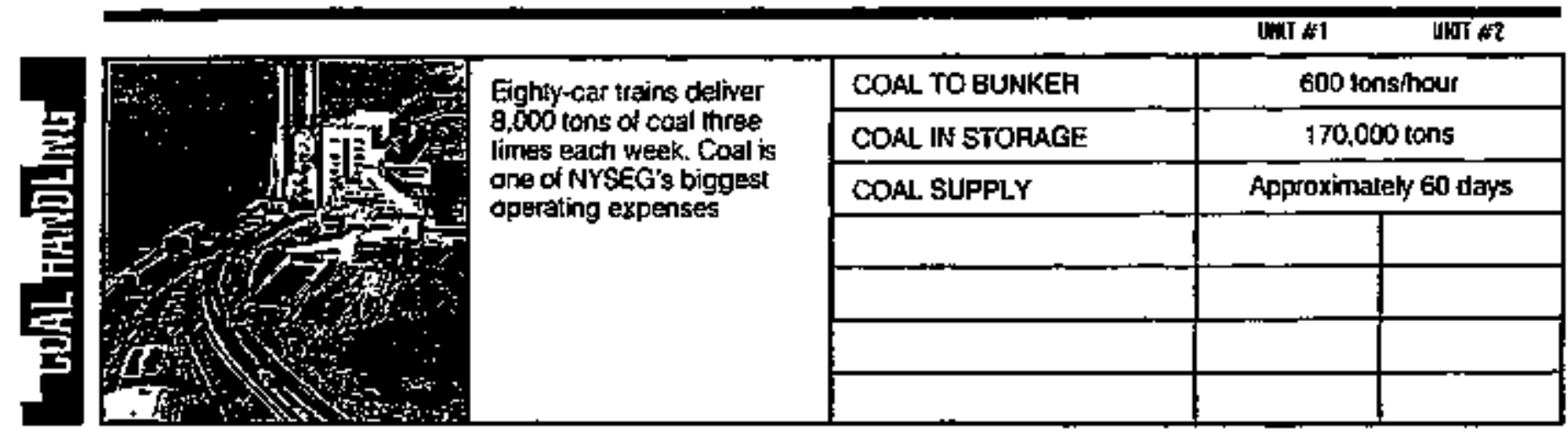

Putverized coal is blown into the boiler where il burns. Water in lubes lining the boiler then lurns into stearn.

\begin{tabular}{|c|c|c|}
\hline STEAM TEMPERATURE & $1,000^{\circ} \mathrm{F}$ & $1,000^{\circ} \mathrm{F}$ \\
\hline STEAM PRESSURE & 1,800 psig & 1,850 psig \\
\hline \$TEAMHOUR & I neillon pountsos & 1 milltan pounds \\
\hline COAL BUANEOHOUR & 50 tons & 50 tons \\
\hline TYPE OF BURNER & \multicolumn{2}{|c|}{ 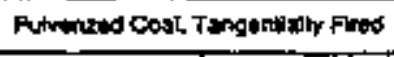 } \\
\hline BOALEA BUIDING HEJGHT & 140 teet & 140 feet \\
\hline CHINNEY HEIGHS & 250 fees & 250 teet \\
\hline
\end{tabular}

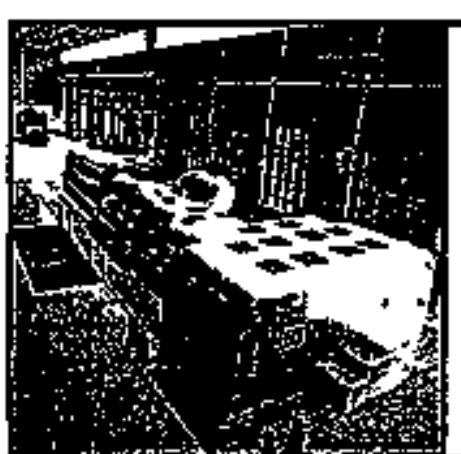

The lurbine-generator is the hean ol the generating station. Energy from the steam spins the turbone which tums the rolor ol the genergior, producing electricily.

\begin{tabular}{|l|l|l|}
\hline NUWWEER OF STAGES & \multicolumn{1}{|c|}{45} & \multicolumn{1}{|c|}{21} \\
\hline & & \\
\hline OUTPUT & $148,000 \mathrm{kw}$ & $14 \mathrm{~B}, 000 \mathrm{kw}$ \\
\hline VOLTAGE & 13,000 & 18,000 \\
\hline SPEEO & $3,600 \mathrm{fpm}$ & $3,600 \mathrm{tpm}$ \\
\hline COOLING & Hydrogen & Hydrogen \\
\hline MANUFACTUREA & Wesinghouss: & Gen. Electric \\
\hline
\end{tabular}

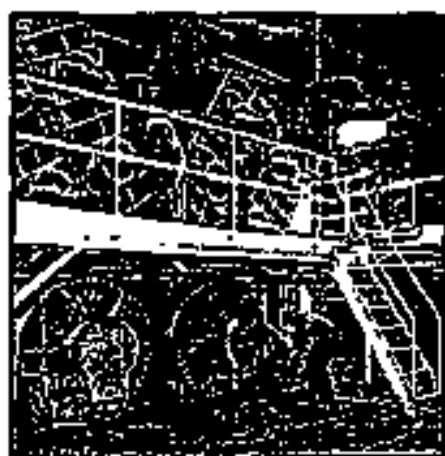

Lake water is drawn into the condenser to cool sleam back into water so that it can be reused in the boiler.

\begin{tabular}{|c|c|c|}
\hline CAPACITY & 84,500 gatson & 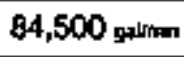 \\
\hline COOLUNG SURFACE & 70,000 n & $70,000 \mathrm{nn}$ \\
\hline TEMP. DIFFERENTTAL & $16^{\circ} \mathrm{F}$ & $16^{*} \mathrm{~F}$ \\
\hline & & \\
\hline & & \\
\hline & & \\
\hline & & \\
\hline
\end{tabular}

Electrostatic precelpitators remove fine particles of thy ash trom the flue gases.

\begin{tabular}{|l|c|c|}
\hline DAILY ASH LOAD & \multicolumn{2}{|c|}{$\$ 60$ IOns } \\
\hline DAILY ELECTRICAL USE & $12,200 \mathrm{kwh}$ & $12,200 \mathrm{kwh}$ \\
\hline EFFICIENCY & $99 \%$ & $99 \%$ \\
\hline & & \\
\hline & & \\
\hline & & \\
\hline
\end{tabular}




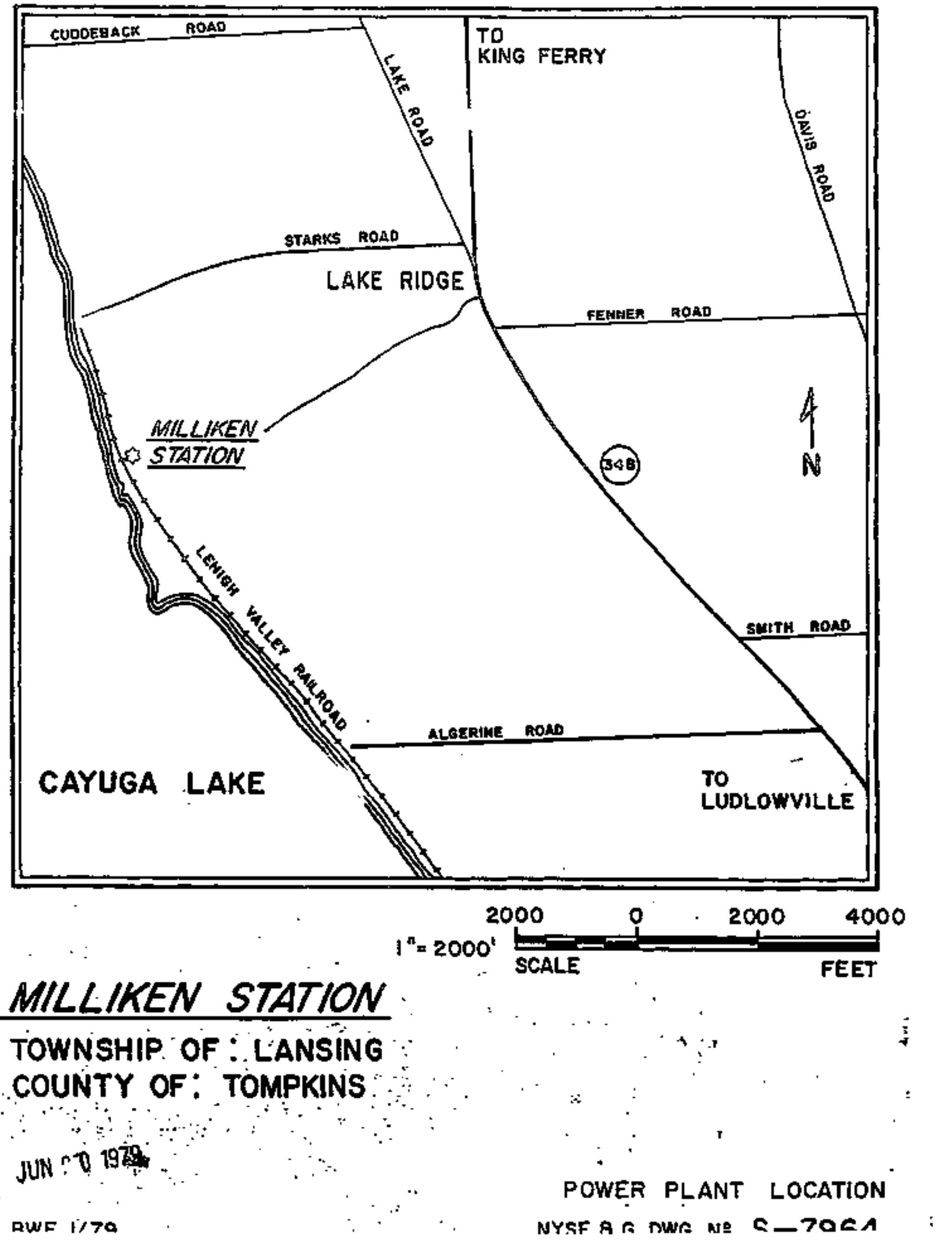


GREENIDGE STATION

ATtRIBUTES for SITLNG an ETHANOL PLANT NEAR A NYSEg bENERATING FACILITY

\begin{tabular}{|c|c|}
\hline Land availability & 30+ actres on Site, $20+$ acres adjoining \\
\hline Town & Tary \\
\hline County & Yates \\
\hline Zoning & Industrial \\
\hline Zoning · offsite & Agrituttured \& Indestrial \\
\hline Accoss & Raid, Truck \\
\hline Weigh factilitios & Bail, Truak \\
\hline Water (100EPAS Min\} & Potable \& Industrited Grade \\
\hline Steam & Avelabble for all requirements \\
\hline Gas & Yes \\
\hline
\end{tabular}




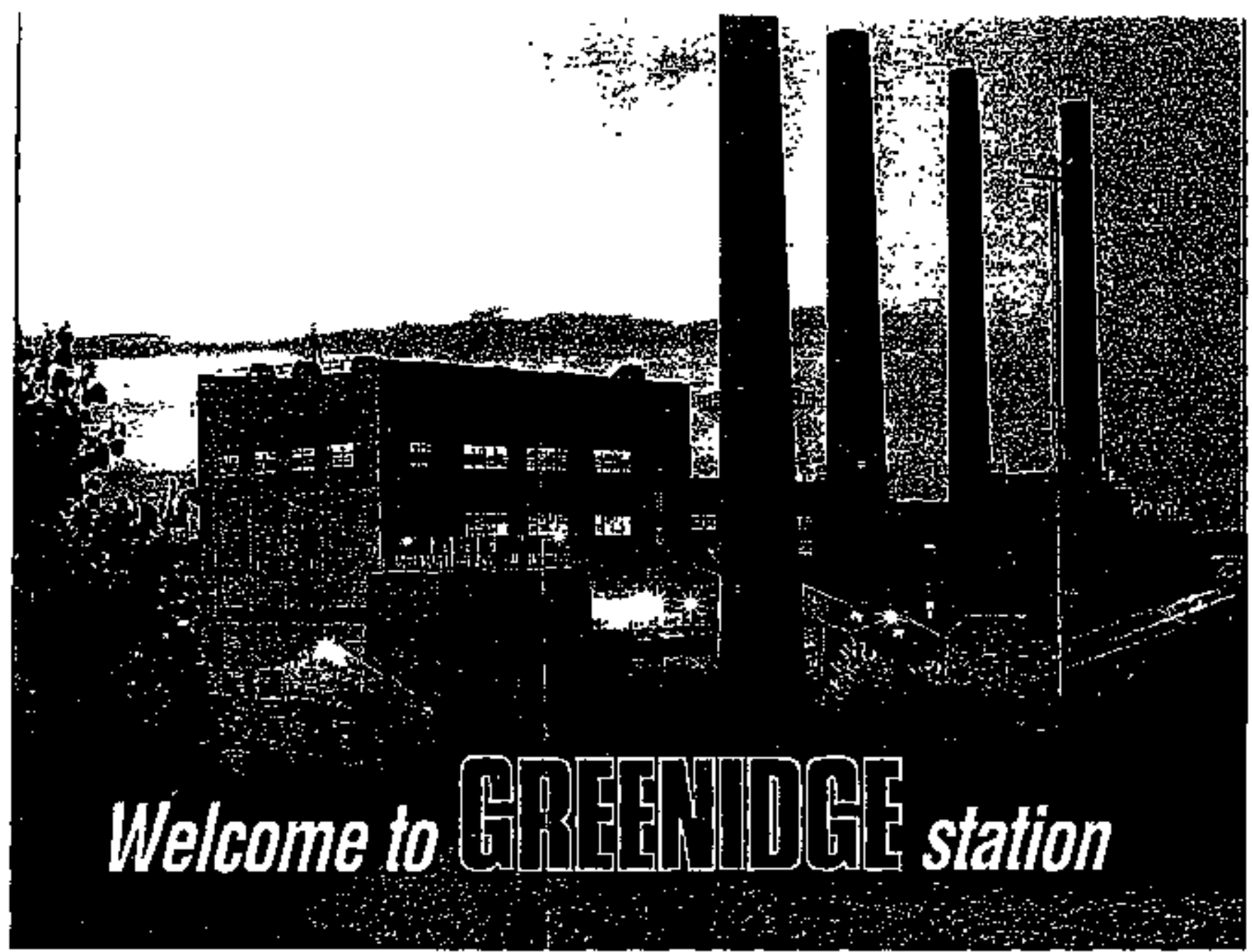

Located on the western shore of Seneca Lake in Dresden, NY, Greenidge Station's ijrst generator went into service in 1938. Additions in 1942, 1950 and

One of seven coal-fired generating stations owned and operated by New York State Electric $\&$ Gas Corporation 1953 brought the plant to its present size. The original generating unit and the unit added in 1942 were relired in 1985 . The plant's mexurnum generaing capacity is now 163,000 kilowatis (163 miltron watts).

Greenıdge was named in honor of Charles A. Greenıdge, an industry pioneer who supervised major construction projects for NYSEG and selected the site for the plant.

Greenidge bums bituminous (soft) coal which is mined in Pernsylvania and delivered to the plant by truck and tran. Each year, the plant bums approximately 400,000 tons of low-sulliur coal The nation's concern for the environment in the earhy 1970's inspired passage of the Clean Aur Act, and in 1975, NYSEG installed a new pollution control system al Greenidge Station to comply with clean air standards.

At NYSEG, we realize how vital electrichly is to our lives and to Now York's economy We also recognize the inportance of producing electricity in an efficient and erwironmentally acceptable manner. We welcome you to Greenidge Station and irvte you to learn more about how NYSEG uses coal to produce electriclty. 


\section{GREENIDGE STATION . . . FACTS AND FIGURES}

\begin{tabular}{|c|c|c|c|c|}
\hline & \multirow{5}{*}{$\begin{array}{l}\text { Up to roo trucks. each } \\
\text { carfyng about } 22 \text { tons ol } \\
\text { coal, arrive daly The cosl } \\
\text { of coal represents one ol } \\
\text { NYSEG's largesl operating } \\
\text { expenses. }\end{array}$} & COAL TO BUNKEF & 0 & shour \\
\hline & & COAL IN STORAGE & \multicolumn{2}{|c|}{$70-100,000$ Tons } \\
\hline & & COAL SUPPLY (APPROX) & \multicolumn{2}{|c|}{60 Days } \\
\hline & & & & \\
\hline & & & & \\
\hline & \multirow{7}{*}{$\begin{array}{l}\text { Pulverized coal is blown } \\
\text { into the boiler where It } \\
\text { bums. Water in tubes } \\
\text { lining the boller inen turns } \\
\text { into stearn. }\end{array}$} & STEAM TEMPERATURE & Dines.s & $1005^{\circ} \mathrm{F}$ \\
\hline \multirow{6}{*}{ 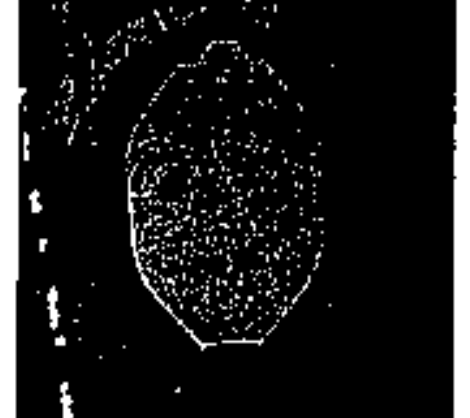 } & & STEAM PRESSURE & enteria & 1465 PSIG \\
\hline & & STEAMHOUR & 600000464 & 780,000 Lbs \\
\hline & & COAL EURNEOHOUR & 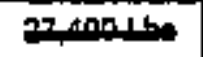 & a4,400 பbs \\
\hline & & TYPE OF BURNER & Eronlafirads & Tangenıl \\
\hline & & BOILEA BUDDING HEICHT & mo & $132^{\prime}$ \\
\hline & & CHIHANEY HEIGHT & $=\pi$ & $227^{\prime}$ \\
\hline \multirow{6}{*}{ 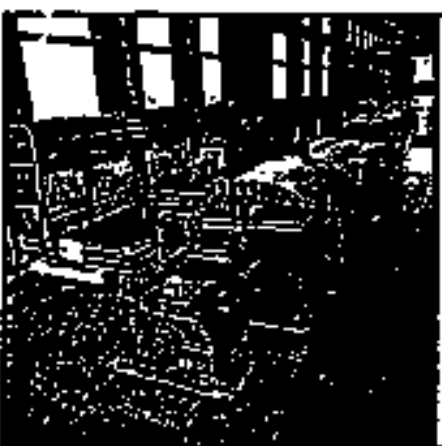 } & \multirow{6}{*}{$\begin{array}{l}\text { The turbine-generator is } \\
\text { the hear of the generating } \\
\text { station. Energy from the } \\
\text { steam spins the turting } \\
\text { which turns the rotor ol the } \\
\text { generator, producing } \\
\text { electricly. }\end{array}$} & NO.OF STAGES & $\Rightarrow$ & 23 \\
\hline & & $\begin{array}{l}\text { Gonniloy } \\
\text { OUTPUT }\end{array}$ & 40 & $106,000 \mathrm{kw}$ \\
\hline & & VOLTAGE & Haven & 13,890 \\
\hline & & SPEED & 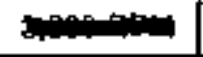 & 3,600 APM \\
\hline & & COOLING & Aningon & Hydrogen \\
\hline & & MANUFACTURER & General & Electric \\
\hline
\end{tabular}

Lake waler is drawn inlo the condenser to $c 00$ steam back into water so Inat li can be reused in the boiler.

\begin{tabular}{|c|c|c|}
\hline CAPACITY & (3) & 59,600 G $\mathrm{mm}$ \\
\hline COOLANG SUFFACE & 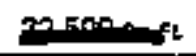 & $50,000 \mathrm{sq} \mathrm{Ft}$ \\
\hline TEMP. DIFFERENTIAL & (1) & $14^{\circ} \mathrm{F}$ Max. \\
\hline & & \\
\hline & & \\
\hline & & \\
\hline & & \\
\hline
\end{tabular}

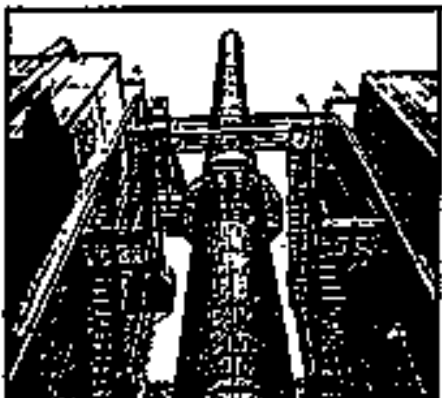

Elec|rostatic precipitators semove fine particles al fly ash from the the gases.

\begin{tabular}{|l|c|c|}
\hline OAILY ASH LOAD & & 30 Tons \\
\hline OAILY ELECTHICAL USE & & \\
\hline EFFICIENCY & & $97.5 \%$ \\
\hline & & \\
\hline & & \\
\hline & & \\
\hline & & \\
\hline
\end{tabular}



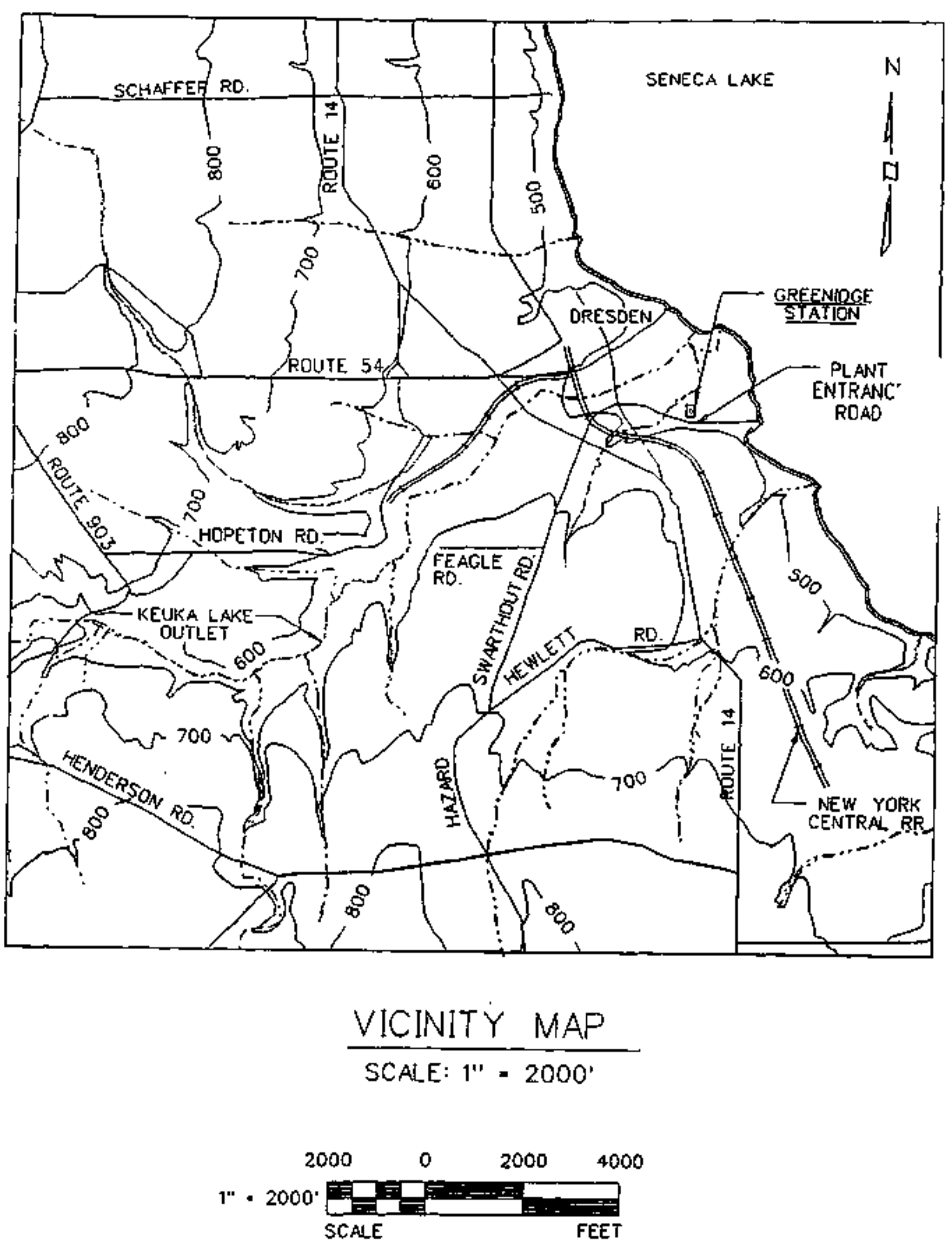


\section{JENNISON STATION}

ATTRIBUTES FOR SITHG an ETHANOL PLANT NEAR A NYSEg GENERATING FACILITY

\begin{tabular}{|c|c|}
\hline Land availabiity & 11 scres on site, Other adjoining land available \\
\hline Town & Bainbridge \\
\hline Courty & Chenango \\
\hline Zoning & Iradustrial \\
\hline Zoning - offisite & Agricuttural \& Industrial \\
\hline Access & Raì, Truck \\
\hline Weigh factitias & Rat, Truck \\
\hline Water \{100GPM Min\} & Patable \& Industrial Grate \\
\hline Steam & Available for all requirements \\
\hline Gas & Nat Avaliabla \\
\hline
\end{tabular}




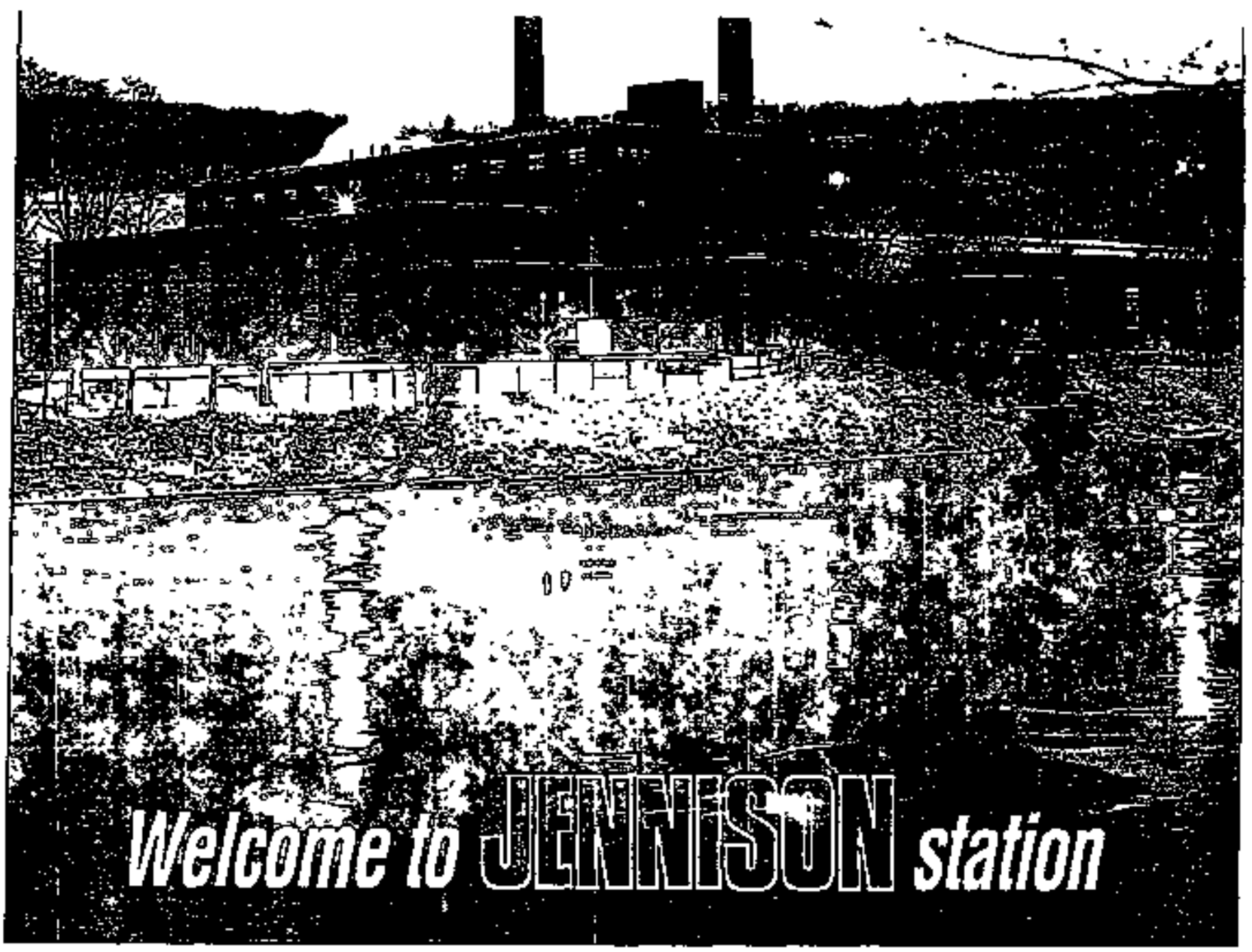

Located alongside the Susquehanna River in Bainbridge, NY, Jennison

One of seven coal-fired generating stations owned and operated by New York State Electric \& Gas Corporation Station began generating electricity in 1945. A second generating unit was added to the plant in 1950. The total maximum generating capacity of the piant is 71,000 kitowatts ( 71 million watts).

Jernison Station is named in honor of Ralph Jennison, NYSEG president from 1941 to 1949 and board chairman from 1949-1955. It was one of the largest projects, in terms of money and effort, undertaken during the Company's ambitious expansion of its generation, transmission and distribution tacilities. in 1975, NYSEG installed a new pollution control system at Jennison to comply with Clean Air Act standards.

Jennison Station burns up to 300,000 tons of low-Sulfur, biturninous (soft) coal annually; it is mined in Pennsylvania and delivered to the plant by truck, In 1992, after over a year of successful research and test burns at Jersnison, NYSEG became the first utility in Now York State licensed to bum scrap tires regularly to generate electicity. The plant can burn the equivalent of $4 \psi_{2}$ million tires $(45,000$ tons) perr year in a blend of 25 percent tire chips with 75 percent coal, saving roughly 65,000 tons of coal per year.

At NYSEG, we realize how vital electricily is to out lives and to New York's econorny. We also recognize the importance of producing electricity in an efficient and environmentally acceptabte manner. We wetcome you to Jennison Station and irvite you to leam more about how NYSEG uses coal and tire chips to produce electricity. 


\section{JENNISON STATION . . . FACTS AND FIGURES}

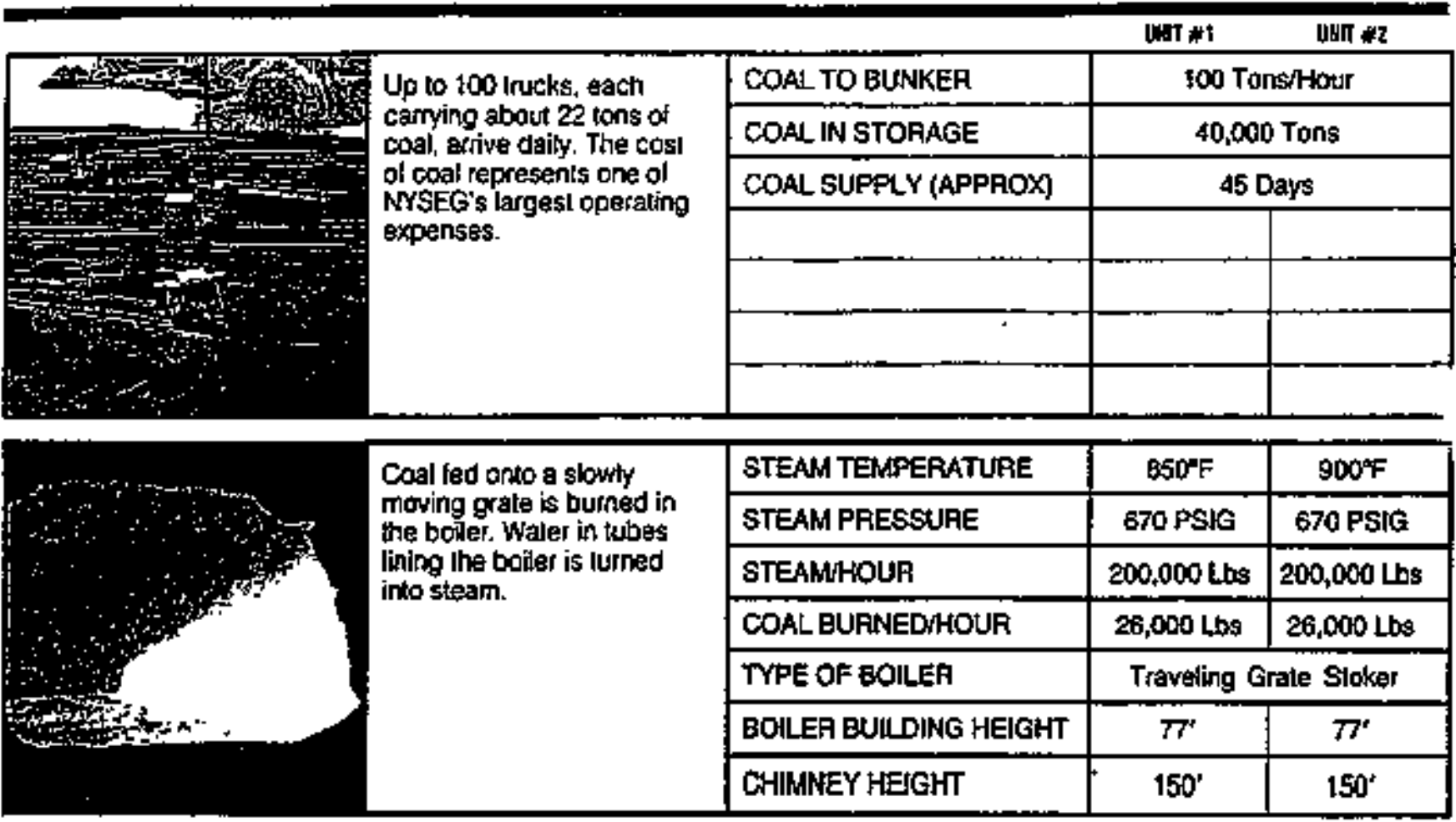

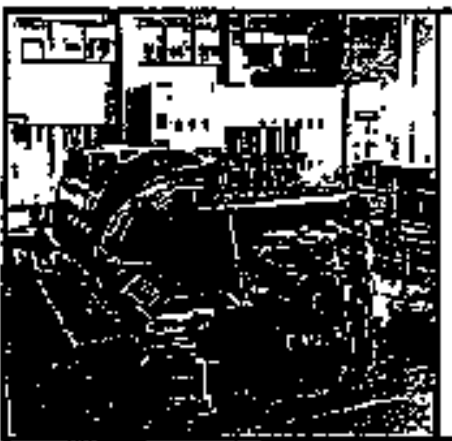

The turbine-generater is the heart of the generating siation. Energy from the steam spins the turbine which lums the rotor of the generater, producing aleciricily.

\begin{tabular}{|c|c|c|}
\hline THOSOS OF STAGES & 18 & 18 \\
\hline $\begin{array}{l}\text { Fogonthy } \\
\text { OtगTPU }\end{array}$ & $33,000 \mathrm{kw}$ & $38,000 \mathrm{~km}$ \\
\hline VOLTAGE & 13,800 & 13,800 \\
\hline SPEED & $3,600 \mathrm{APM}$ & $3,600 \mathrm{RPM}$ \\
\hline COOLNG & Hydrogen & Hyctrogen \\
\hline MANUFACTURER & \multicolumn{2}{|c|}{ General Electric } \\
\hline
\end{tabular}

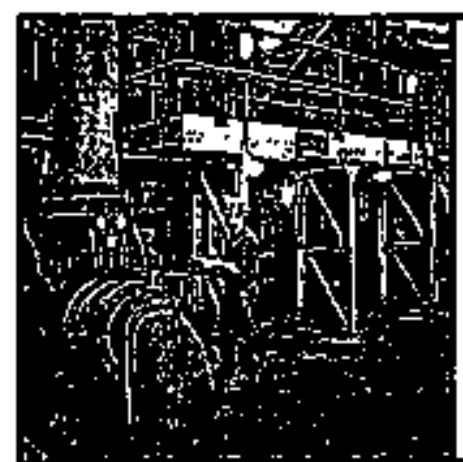

Fiver water is drawn into the condenser to cood steam back into waler so that it can be reused in the botler.

\begin{tabular}{|c|c|c|}
\hline CAPACTY & 22,650 atikn & 22,650 ands \\
\hline COOLING SURFACE & $23,000 \mathrm{s4} \mathrm{r}$ & $25,000 \approx n$ \\
\hline TEKAP. DHFFERENTIAL & $15 \cdot 20^{\circ} \mathrm{F}$ & $\$ 5 \cdot 20^{\circ} \mathrm{F}$ \\
\hline & & \\
\hline & & \\
\hline & & \\
\hline & & \\
\hline
\end{tabular}

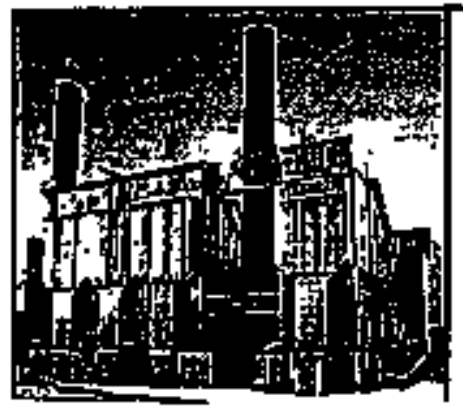

Elecirostatic precipilators and mecharical precipitators remove line particles ol fly ash irom the flue gases.

\begin{tabular}{|l|c|c|}
\hline DAILY ASH LOAO & \multicolumn{2}{|c|}{20 Tons } \\
\hline OAILY ELECTRICAL USE & $1,800 \mathrm{kwh}$ & $\mathbf{1 , 8 0 0 \mathrm { kwh }}$ \\
\hline EFFKCIENCY & $99 \%+$ & $99 \%+$ \\
\hline & & \\
\hline & & \\
\hline & & \\
\hline & &
\end{tabular}




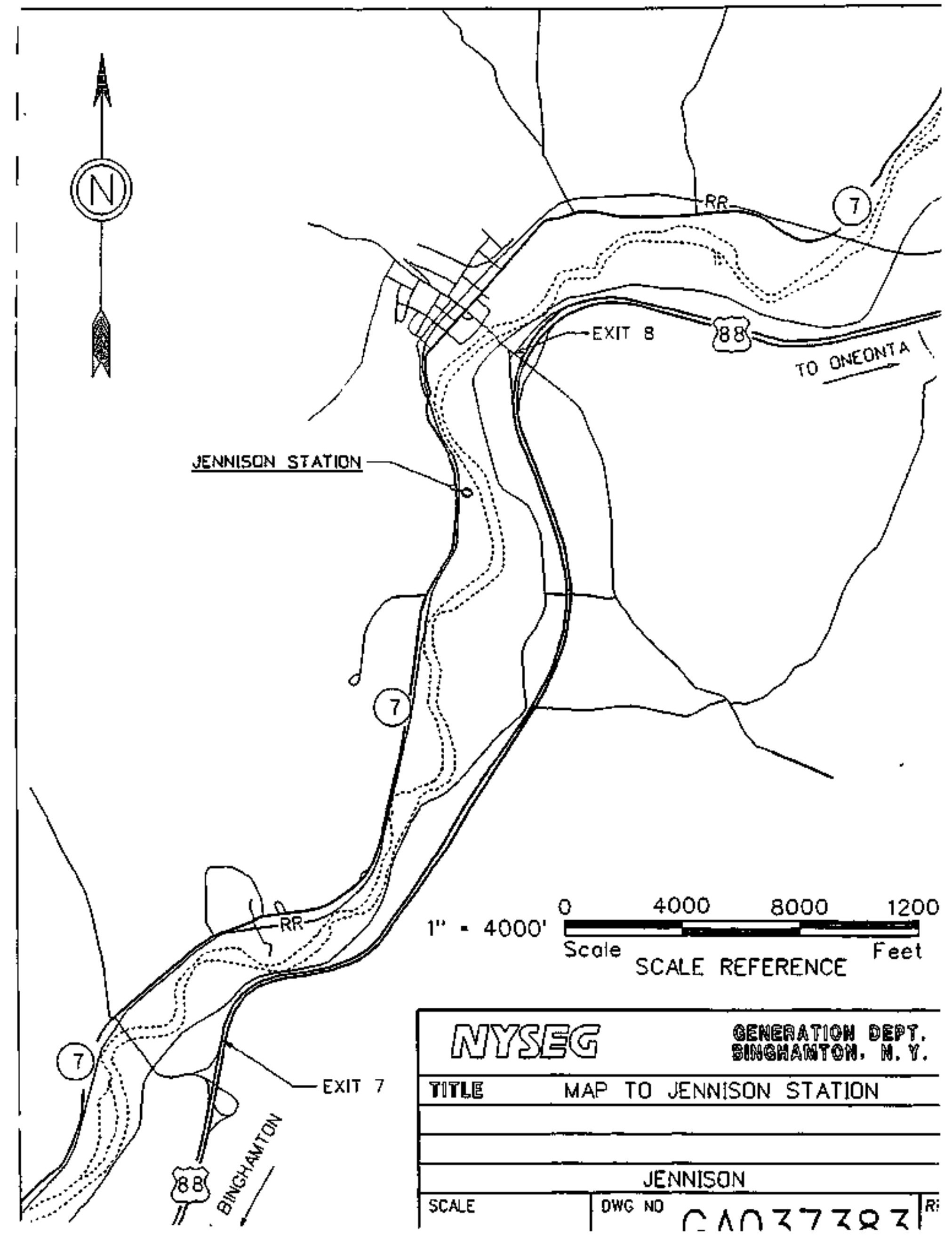


December 13, 1994

\section{Ms. Suzarne Low}

Stone \& Webster

One Penn Plaza

250 West 34th Street, 31st Ficor

New York, NY 10019

\section{Dear Suzanne:}

Plense find enclosed the recently pased kgistation that brings the former Miller Brewery factitity into the New York State designated Fulton Econtomic Development Zone.

Becoming a certified business within the Economic Devehopmen Zone (EDZ) has many benefits. The State Inyestment Tax Credits would amount to $19 \%$ over a period of 4 years $(10 \%, 3 \%$, $3 \%, 3 \%$ ) on the original investment of the purchase and equipging of a manufacuring facility in the BDZ. The kcal utility, Niagara Mohawk Power Corporation, can offer $\mathbf{3}$ tate of around $\$ .02-.03 / K W H$ for electricity to a large user in the EDZ. '10 years of discointed electric rates)

The State also offers up to $\$ 7,500.00$ in wage lax credits for each new employee hired within the EDZ by an EDZ certified business. .

This 19 year oid facility has afany valtuable assets. I trave highlighted a few of the these assets below and atrached a fact sheen prepared by the Robert A. Hood Real Estale Company.

This site is located on the State Barge Canal system (3 locks south of Lake Ontario) that can access the St. Lawrence Seaway via lake and ocean going vessels.

- This site has tho ows rail sidings off of a main line Conrail track and tracks itto the buildings at varions points, 20 minates to Exit 39 of the New York State Throway (I-90), 20 minutes to I-8I.

- The plant has coal fired boiters and a 6 MGW generator. A large water cupecity 7 MGD with more available from the Metropolitan Water Board Line (62 MGD) of from the Oswego River. It has its onn sewheg treatment plant (5 MGD +) and also a large munber (hundreds) of staidiless steel tanks for processing and storage that were used in the beer brewing process. 
Ms. Suzanne Low

December 13, 1994

Page 2

"Presently, the briilding is equipped to produce 3-4,000,000 barrels of beer per year, in the past. it previous'y ras capable of $9,000,000$ barrals/year.

It is not poussible for me to determine whether any of the equipment could be uctilized in protucing methanol or ettanol, but 1 an certain that Sione and Webster could make that determination.

In aditition to the immediate factory and grounds, there is as adritional 300 acres of undeveloped land avaỉable in this $\mathbf{4 5 0}$ acre site that coutd easily be developed.

Please share these facts with your client. As I arn sure you already know from your survey, there is a great deal of siudge being developed at the International Paper plant in Oswego, the Schoeller Paper milil in Pulaski, the Southem Container Plant in Solvey/Syracuse, and the Artastrong International plant in Futton. There is also soon to be butilt a new linerboand plant in Oswego, New York near the pew 1040 megawatt gas fired cogeneration plant in Scriba/Oswego, New York. All of these paper making facilities are within 45 inisutes of the Miller facility and are expected to genkerate about 64,000 tons of paper sludge per year. There are also many other sources of raw material that could be feedstock nearby at canning plants. Municipal Sewage Treatment Plants, etc., nearby.

If you feel that the Miller Brewing plant could be a viable caodidate s a mamufacturing facility for your client, Opcrarion Oswego County, Inc. and the Connty of Oswego Indastrial Developmeat Agency stand ready to assist your client with the financing of firther enginetring feasibility sturfies. Please contact us with any questions yoc may have.

Sincerely yours,

SWR/m

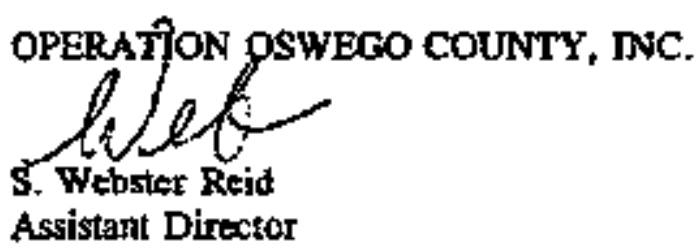

Attachment 


\section{Milue nawne faclutr supdMany of sallant FACTs}

\section{lochtrolst}

LNo Anst

zoNimes

TOTAL gulibing AREs

Aos of etrolkdy

Constatlatol:

Gisung sutit:

INAT!

WATER SHordr;

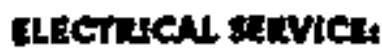

Trtex LoApiners

Nart llowt $\mathbf{5 7}$ and Owan Roud, Volney

Oawep Councy Now Yort.

423 Acrs

Induttid

$1,375,633$ Square Fot

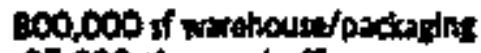

$\$ 3,000$ of general oflet wat

236,000 sf production

246,633 of gentersl purposu/limited use

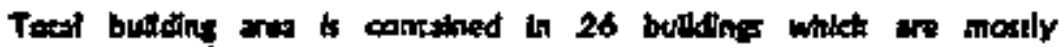
Interomsnected

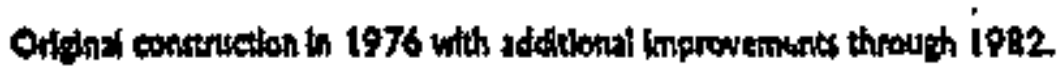

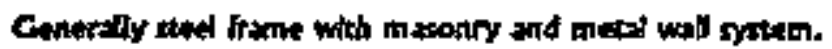

26'-30' genesd selling helghts throuthoue

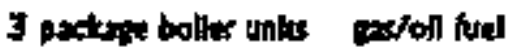

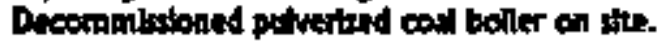

$7,000,000$ g:tlons per asy capacty via a $60^{\circ}$ maln and $20^{\circ}$ submaln.

$20,000 \mathrm{~kW}$ epody

40 callegue looding dickst.

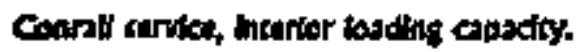

CONTACT RORERT L. HOOD, SHOR.

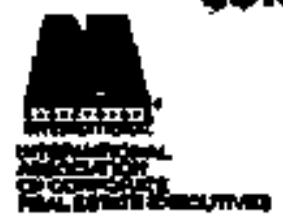

(315) 423-9200 


\section{ATTACHMENT 9-1}

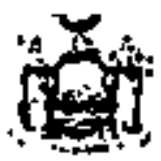

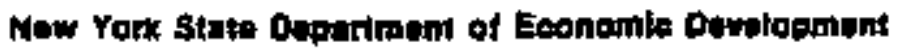

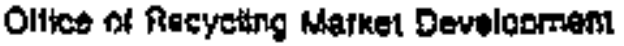

if It East Avanue, Suhe 220

Rochester, Now Yark 1400s

71E 325.1044

FAX: 718325 -

July 21, 2994

Rich Weber

Champion International corp.

Anderson Ave.

Deferiet, New York İ628

Dear Mr. Weber:

You may recal1, last month sooke on the phethe where I invited you to participate on a project wrti fit York state pepartment of teonomic Development - office of Recycling Harket Development (ORMD) to find beneficial uses for your mili'g sludge.

I an pletsed to report that over the lagt month 25 milis in the state have expressed an interest in working with oplD. Additionelly, the New York Stat: Energy Research and Development Authority (MYSERDA) has agreed to pirthar with us in support of this inftiative. Joinely we axe preparing to selense a Reguest For Proposals (RFE) to hire a qualified consultant to conduct research that will strategically inform our economie and environmental agsistance programs in the evture.

I have included a copy of the "Praject Description" which describeg the goals, objectives, and expo frames of our work. please note, we are only seaking a censultant to complete pheses I and II. Phase III technical assigtance neads will be determinod at a leter time.

We have also invited the New York state Department of Environmental conservation to advise the project where appropriate.

In order for the project to move thad as expeditiotusy as poseible, we reguest your full cooperation in wotking with tha gelected congultant. In fectu ug areplanning on inviting you to

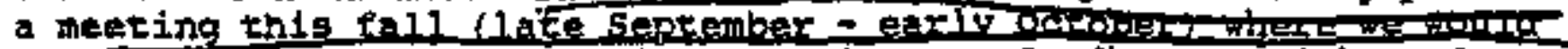
oritine the project in detail, bolicit your feedback, and introduce कhe constrtant.

If you would like copy of the RFP or have any recommendiations as to qualified consultants that could bid on the project, flease feel tree to contact we at the above address. 
We look forward to working with you to flnd benefteial uses for your sludge and zecovering the economic benel1to that are likely to restrlt.

SLrearejy,

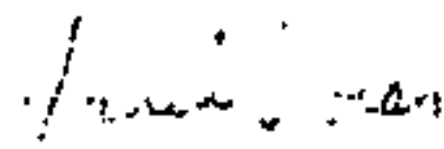

Garrett Dolan

Recycling Harket Development gpectaligt

e: NYSTRDA 
PROJECY DEECRIFHIOH

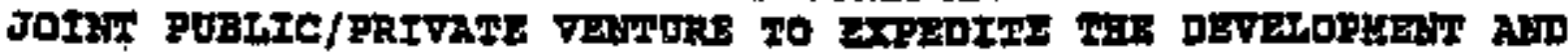

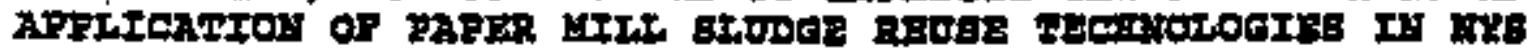

Gasl atatement - to develop/tacilitate the moverent of olndge from at least three papor mills currentily roliant upon disposal capacity to beneficial use epplications.

\section{OEJECSIVES}

- To maximize the racovary and relupe of some or all components of paper mill sludge genntrated by milis

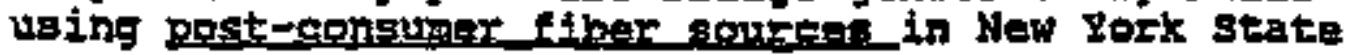

- To minimize mill reliance on salid warte disposal cepacity for mill Eliuges

- To improve the eost eftectivenose of will slutge menagament practices

Thete will be tour major components to this venture: research, information dissemination, and inplewentation needs assessmant, and implententation.

\section{DHASE I EESERRCA}

Dosorlption: - Phase I reseazch will be conducted to foeus and guide the work for the remaintng components of the project. Whe abjective of this portion of the projest in to brostion each venture partner's uraesstanasng of sudge management in key York state, and techrology sources nationuide.

Research to be ampletad will tnclude a murey of milis to learn more about sludge generation, managenent, characteristies, and costs on a mill poeticic and statewide basis, Benetictal use technologies will be jontifled end agsesged in terms of corrent development status (1.9. commereial, pre-commercial), and potantial for Iull staie compreialization. A Ifnel preduet of

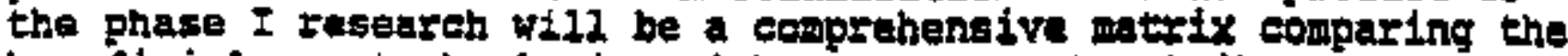
benefieial use teabnelogies with the relevant attributes of sludge genezated by Naw York mills.

The work for this phase would be completed by $1 / 22 / 94$.

costas Costs for this phase could be shared between Dot and MYSEROA. Partieipating aijls will contribute to this phase by providing the nocassary information aboit their aludge costs, characteristics, and masigement practions. 
Deroriptiont The purpose of Fhase II would be to disactinate the Elndinge trom Rhase I. A workthop would be organized and held before the end of rebruary, 1995 featuring presentationg Irom the selected consultant, representatives of Bludge rause technology cotures, and state represantatives. Bpacifiealiy, three aqtivities wil1 take place: participating companises would be mate aware of the benefteikf 100 teshnologies/wakket applioatiens that have been identiflad as appropriate for their aludga; New yoxk state Depertinent of Economie Development - offiec of Rocyeling

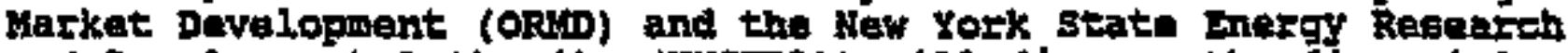
and Development Authority (KYSERDA) will discrgs the Einanclal and techifical adgistance programs available to mills that wish to pursw. tho beneticinl une technologies/ancket applieation:

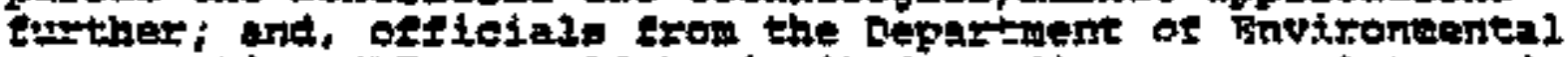

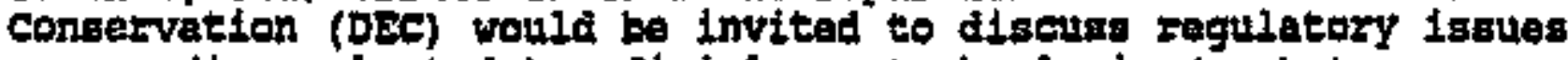
arzounding selected benefleial uge technologies/market apgileations.

costsi costs for this phase would be shured between DED and WY SERDA.

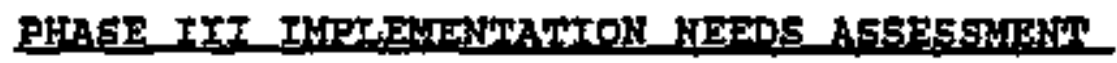

Desertption: Phage III woula invelve greater partiolpation by pil1. interested in woving torward with the information gathered In pheses I and II. Emphasin wiII be given to explering oppertunsties for mills to roxk ooporatively to manage the $x$ siudges through the use of a partscular teehrelogy. Intarested mills, working alone or in cooperation with other wills, would identify the technieal confultant of their cholce to undertake an evaltuation of the technical and economic feasibility at utilizing ar acressing one az severel of the shudge reuse technologief pzeviously identified an being uultable.

A second component at this phase weuld ba a caco by cage specifle sagesmmant of what is naeded to begin moving mill sludges to eertain beneftelal use technology gources. This phase colald 1nclude arzanging meatings between state officiais, technology soureas, and mili offloikis to litentify implemantation tesuas and the resources avaliable to ovarcome unos barriers auch as: capital tinancing, aighting acsistence, training, fogulatory

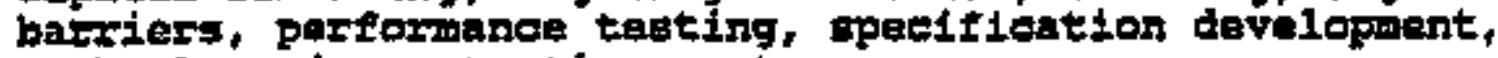
technology demonstrations, ate.

The teasibllity analyses would be completed before the and of Tune, 1995 .

coats: costs for the feastbility analyses could be offset by DED, and NYSEROA, but tha participating mills would have a much larger financlal postition than in the earlier Phases. 


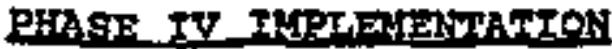

Daneription: Phase Iv vil1 toous exelueively on tmplementing technologies and/or market applications. This process will be an ongaing effort by ORtib and NYSEROA, and our limited inancial resources w11 epent on a tirje-come firgt-aerve besis. costsi costs tor tmplamentation gould be born by the following groups: DFD (e.g. spesification tevelopment, eapital flnancing); WYSBRDA (e.g. Innovattve technology demonstration grant(s)), BENEFICIAL USE TECHNOLOGIES/COLDANIES (0.9. cost sharing far business development; and, uIIIS (e.g. espital tinancing). 


\section{ATTACHMENT 9-2}

'ow York State Department of Environmental Conserratlon .J Wolf Road, Albany, New York, 12233

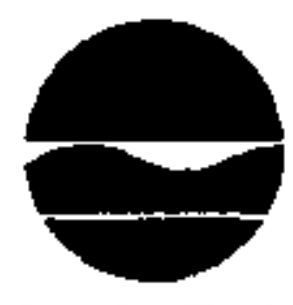

Lengulen Manth Commiteloner

Aite 51994

Mr. Joseph Masin

Amoco Corporation

Amoco Research Center

P.0. Box 3011

Napervitie, I1l1nois 60566-7011

Mr. Joseph Masin:

I an in recetpt of your Jity 19. 1994 letter in which you fnquired about the 6 NYCRR Part 360 (Part 360) pervitting issues relating to a potential biomass-to-ethanol conversion facility.

Based upon the Information you provided in your letter, the proposed conversion factijty would not be viewed as a recycing facility. but would be considered etther a refuse-derived fuel processing facility regulated under Subpart 360-3 or a nonspecific factitty regulated under Section 360. 1.9(b). A final determination as to the speciftc Part 360 regulatory requirements applicable to your conversion facility will depend upon the submission of additiona? information associated with both your conversion process and project. as well as the waste materlais proposed to be used in the process, $i . e .$. wood chips, other bionass wastes, and waste paper.

Should you desire to discuss your proposal further, please contact me at teTephone (518) 457.6603, or Mr. Jeffrey C. Schmitt, Bureau of Resource Recovery, at telephone (518) 457.7336 .

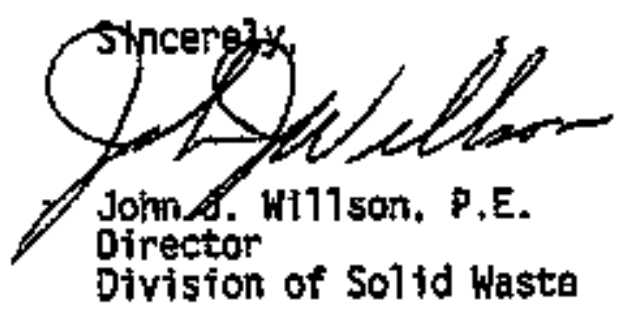


Jut-19-1994 09:02 FRW1 AFD - Maperulle E-3G TO TO

\section{ATTACHMENT 9-3}

July 19. 1994

John J. Whilson

Director, Division of Solid Waste

New York Department of Environmental Compliance

50 Walf Rogd

Albany, New York 12233-4010

\section{STATUS OF BIOMASS-TO-ETHANOL PADCESSING}

W* are interested in gettisg a determination of the NYDEC position an a process for converting blomass into ettand and other products. We are aware that the draft request for proposais to recyclo recovered paper for New York Cry (PIN 82794AD00069) specificatly identlfies an thancl facility as complyong technology, while exchuding direot buming techmologies. A reason for this may be that a biomass-ro ethanol process producas, es a main product, ethanot that can be used as a replacemant or supplement for gosoline. A liquid transportatlon fugl, Uke ethanol, is substantially olfferent from a solid fuel or power supply. Ethanol from a biomass, com, is alroady used in some gasolines sold in New York to help meet Clagn Air Act requirements and is supportive of the Renewable Oxygenate Standeris recently proposed by the EPA.

We ara involved with Stone \& Webster Engineering Corporation in the completion of a cost shating apreement with the Now Yark State Energy Research and Development Authority (Contract 3163ERTER-ER-94\} to Identify and evaluate sites in Now York Ciry and other Naw York State urban locaxions for a potential biortass-to-ethanol conversion facility. The proposed process could consume wood chips and other biomass wastes as weil as waste paper strearns. The quallit of the waste paper that could be used in the biomass-to-ethanol process tean be below that which is currently used for recvcling irto paper manufacture bacause iong fiber lengths need not be maintained. Commercialization of the process will develop benefieial uses for streams that would otherwise be consigned to landfils or decompose in fields. A byproduct of the blomass-to-ethanol process is composed of the unfermentable components in the feed. prinarily lionin. Afthough we expert that this byproduct stream will be used as a low-surfur solid fubl, Its weight will be less than half of the biornass feed stock rote.

Part of the NYSERDA study requires site-specific detemination of permitting and commenlty interest issues. A determination that your departinent wows a biomass-to-ethanol facility as compliant with the state's solid weste mandgement plan, is supportive of tatate recycling programs. is viewed more favorable than waste-to-energy processes, etc., would help resolve some of these issues. An expedient reply stating your view on biomass-to-othanol processing will allow the participants in this study to move a step further toward the determination of whether the construction of a commereial facility is economically attractive.

I would be pleased to give more detals on our process if that would help vour evaluation. I may be phoned at (70) 420-5416, or contacted via facsimile at (708) 420-5169. You atso may contact the Stone \& Webste" project manager, Henry Gestwirth, at (212) 290-6155.

Joseph Masin

ct: H. Gastwirth, SWEC

R. H. Walker 


\section{ATTACHMENT 9-4}

July 22,1954

Mr. Willian coider

Bureau Chiet

Bureau of Waste Reduction and Recycling

Department of Environmental Coneervation

50 Wolf Road

Albany, NY 12233-4015

Dear Mr. Cojder:

NYwix Information Resources Company is currently participating with Anoco Coxporation, Etone $E$ V Viabeter Engineering, ano Chempion International in a New York state gite evaluation atudy for poantble Amoco bionals-to-erhanol plant utjlizing biomars convereion technology. Thie projezt is being partially funded by grante from the New York seate Energy Reangrch and Developinent Author1ty and the Natientl Renewable Energy Labaratory.

MYNEX has two primary deliverablec under this project: i) A businese as aessment of ethanol as an slternative fuel in the Corporate vehicle fleet; 2) A buginesg asgegsment of the Amoco process a an alternative end-upe market for the recycling of a mixed paper wagte gtream.

As part of our research on the gecond i iene, we have besn conducting Interviewn with our recycling official contaste in New York City and in several upotate municipaileieo. Several of theae offick.e]s raised the following question as a

potential determining factor in their intereat in the Ancco proceari Does blomess convereicn technology qualify 89 recycling unfer the WYs DEC definitions Incorporated in the state's solid Veste Management plan?

Bome time ago, wh had a similar queption regarding tho dratug of compoeting under the state's Belid taste Management Plar. and we apprecintea your office's quiok reepones and interpretation that composing le mogt aseuredly considered recyciling unless the end-product is to be used as dally landfill cover. 
In order to make our current effort as complete and accurate ao poosible, could you please provide ue with anothar interpretation-this time regarding the DEC position on biomase converaion technology as a form of recyeling.

If you have any gueationa or need more information about the Amoco procege, pleane it we know.

gincerely,

Joth S. Baldguer

Director

Environmental Etalilis 


\section{ATTACHMENT 9-5}

SUMMAFY OF UCFARA WAETE GALULATONO

ANATtAI. 1993

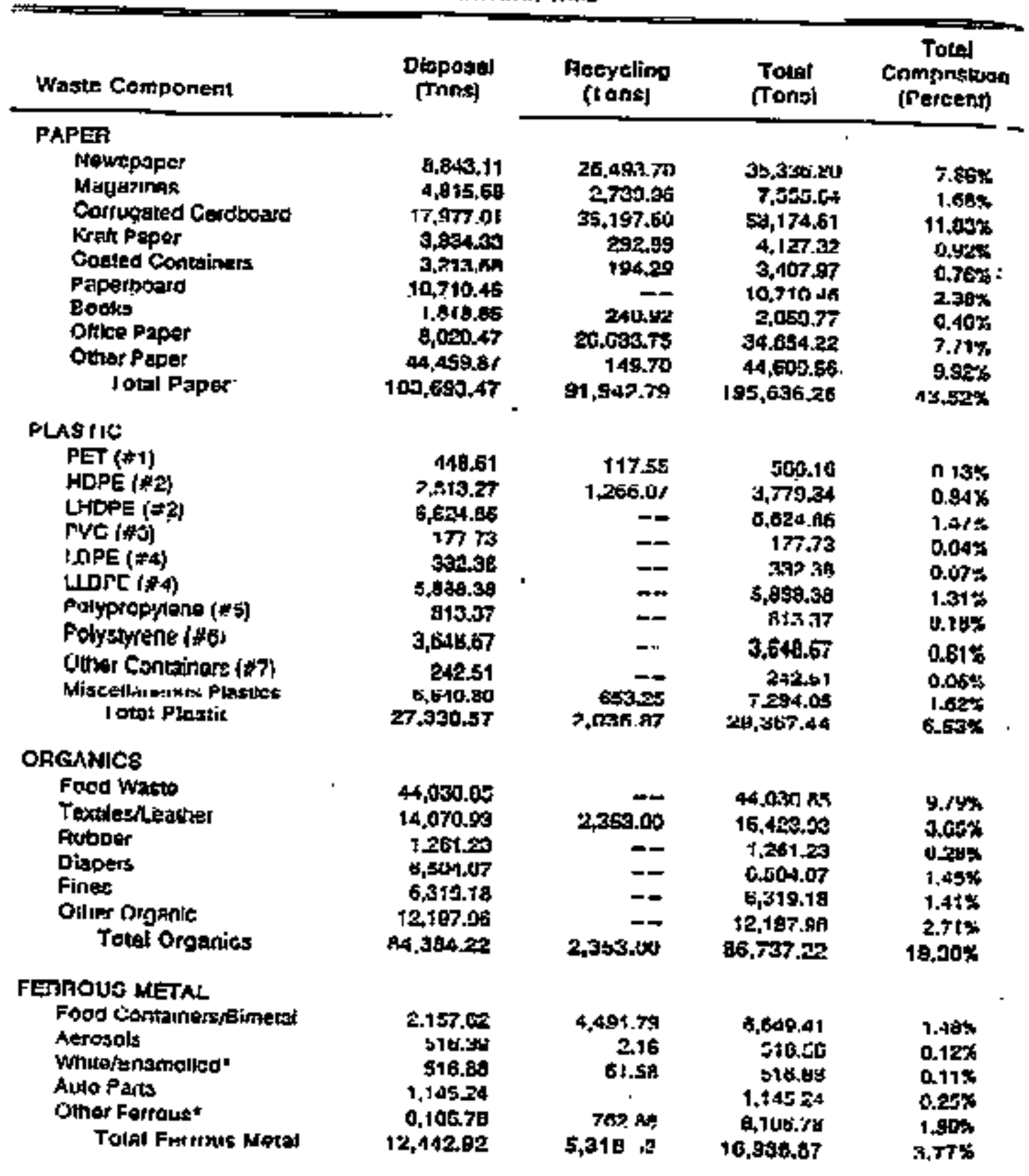




\section{ATTACIMENT 9-6}

\section{SUMMARY DF OCRRA WASTE CALCULATIONS}

ANNUAL T999

\begin{tabular}{|c|c|c|c|c|}
\hline Waste Component & $\begin{array}{l}\text { Disposal } \\
\text { (Tons) }\end{array}$ & $\begin{array}{l}\text { Pecycling } \\
\text { (Tons) }\end{array}$ & $\begin{array}{l}\text { Total } \\
\text { (Tons) }\end{array}$ & $\begin{array}{c}\text { Total } \\
\text { Composition } \\
\text { (Percent) }\end{array}$ \\
\hline \multicolumn{5}{|l|}{ NON-FERROUS METAL } \\
\hline Aluminum Foil & $\begin{array}{l}456.88 \\
764.19\end{array}$ & $\begin{array}{r}80.77 \\
--\end{array}$ & $\begin{array}{r}1,267.65 \\
763.19\end{array}$ & $\begin{array}{l}0.28 \% \\
0.17 \%\end{array}$ \\
\hline Other Aluminum & 398.14 & -- & 398.14 & $0.09 \%$ \\
\hline Other Non-Ferrous & 500.13 & . $\quad 3,142.75$ & 3.645 .88 & $0.81 \%$ \\
\hline Total Nor-Forrous Matad & $2,101.35$ & $3,973.52$ & 6.074 .86 & $t .35 \%$ \\
\hline \multicolumn{5}{|l|}{ GLASS - } \\
\hline Clear Contalners & $3,659.56$ & $3,5: 2.24$ & $7,171.80$ & $1.60 \%$ \\
\hline Green Containers & 281.25 & $779.06 \cdot$ & $1,060.91$ & $0.24 \%$ \\
\hline Brown Containers & 346.34 & 704.88 & $1,051.23$ & $0.23 \%$ \\
\hline Plate Glass & $464.41^{\circ}$ & -- & 464,41 & $0.10 \%$ \\
\hline Other Giass & 1.778 .93 & 9.426 .99 & $11,205.89$ & $2.49 \%$ \\
\hline . Total Glass & 6.530 .49 & $14,423.14$ & $20,953.63$ & $4.66 \%$ \\
\hline \multicolumn{5}{|l|}{ WOOD } \\
\hline Pałlets & $4,230.45$ & $4,033.96$ & $8,264.41$ & $1.84 \%$ \\
\hline Lumber & $5,920.07$ & -- & $\$ .920 .07$ & $1.32 \%$ \\
\hline Sturmps/Heavy Sections & 6.45 & -- & 6.45 & $0,00 \%$ \\
\hline Other Wood & $16,819.61$ & $2,453.45$ & $19,273.06$ & $4.29 \%$ \\
\hline Total Wood & 26.976 .57 & 6.487 .42 & 33.463 .99 & $7,44 \%$ \\
\hline \multicolumn{5}{|l|}{ INEAT } \\
\hline Asphatt & 21 ,ass.51 & -- & 21.835 .51 & $4.86 \%$ \\
\hline Concrete/Esuck/Rock & $4,578.31$ & -- & $4,578.31$ & $3.02 \%$ \\
\hline Dist & $2,506.90$ & 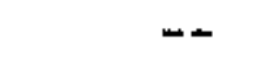 & $2,506.90$ & $0.55 \%$ \\
\hline $\begin{array}{l}\text { Other Inett } \\
\text { Total Inert }\end{array}$ & $\begin{array}{r}23.721 .39 \\
52.642 .09\end{array}$ & $0 .-\overline{0}$ & $\begin{array}{r}23,721.38 \\
52,642.09\end{array}$ & $\begin{array}{l}5.28 \% \\
11.71 \%\end{array}$ \\
\hline \multicolumn{5}{|l|}{ YARO WASTE } \\
\hline Leaves & $1,019.16$ & $1,207,09$ & $2,226.25$ & $0.50 \%$ \\
\hline Grass & 875.63 & $t, 207.09$ & $2,002.72$ & $0.46 \%$ \\
\hline Other Yard Waste & $1,658.99$ & 687.27 & $2,346.26$ & $0.52 \%$ \\
\hline Total Yaro Waste & $3,559.78$ & $3,101,45$ & $6,655,23$ & $1.48 \%$ \\
\hline \multicolumn{5}{|l|}{ HAZARDOUS } \\
\hline Load/Acid Banteries & 130.58 & $m$ & 130.58 & $0.09 \%$ \\
\hline Dry Cell Baneries & 120.61 & -- & $t 20.61$ & $0.03 \%$ \\
\hline Othet Hazardous & 810.10 & $\rightarrow$ & 810.10 & $0.18 \%$ \\
\hline Total Hazardous. & $1,061,29$ & 0.00 & $1,061.29$ & 0.246 \\
\hline TOTAL & $320,716.79$ & $\$ 29,636.59$ & $449.520 .8 \mathrm{~B}$ & $100.00 \%$ \\
\hline
\end{tabular}

- Pecycling tominges were nox roported for these components

- Porions of White Goods and OAher Fercous are segregated from the disposect waste stream by OCRRA personnel tor tocycling. 


\title{
ERIE COUNTY CRA
}

\author{
PLANNING AREA:
}

TOWNS:

\section{ALDEN}

CHEEKTOWAGA

CONCORD

HAMBUPG

NEWSTEAD

WALES

AUROPA

CLARENCE

EDEN

HOLLAND

N. COLINS

W. SENECA
BOSTON

COLDEN

ELMA

LANCASTEA

ORCHARO PAFK
BFANT'

COUNS

EVANS

MAPHบA

SARDINIA

VILLAGES:

AKAON

DEPEW

LANCASTEF

SPAINGVILE

\section{ALDEN}

E. AUROPA

N. COLNS
ANGGOLL

FARNHAM

OFCHARD PAFK
BLASDELI.

HAMBUAG

SLOAN

and

CITY OF LACKAWANNA 
ATTACHMENT 9-8

\begin{tabular}{|c|c|c|c|c|c|c|c|c|c|c|c|c|c|c|c|c|}
\hline & $\begin{array}{l}111 \\
x \\
0 \times 5\end{array}$ & navitar & $\begin{array}{c}\text { mingl } \\
\text { mate } \\
\text { prot }\end{array}$ & $\begin{array}{l}\text { aNtT } \\
\text { netr } \\
\text { 19ox }\end{array}$ & $\begin{array}{c}\text { LSW ask } \\
\text { iten }\end{array}$ & 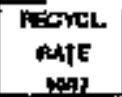 & पhएक & A & Fechle & ariा & | & Fquat. & $\begin{array}{l}C+\pi t \\
f \in 0 y\end{array}$ & now & Foctor. & : quy \\
\hline & & & & & Ob & & & & & & & & & & & \\
\hline 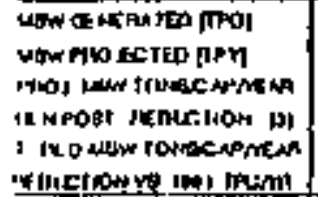 & & 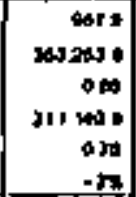 & & & 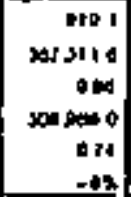 & & & 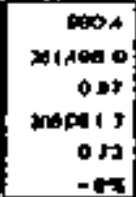 & & & 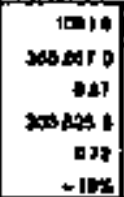 & & & 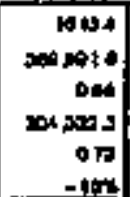 & & \\
\hline n & 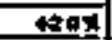 & inginis & 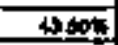 & 선하웅 & 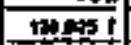 & 3수내: & 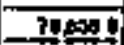 & Iritsis & ation & 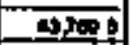 & inteont & ats & 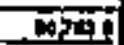 & vitelle & 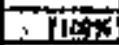 & $0+21$ \\
\hline 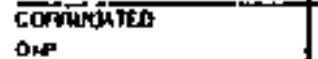 & nas & m, & 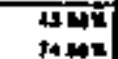 & 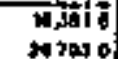 & 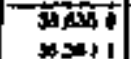 & 145x & $\begin{array}{l}201 \\
4 \geqslant 1\end{array}$ & 70, & $\begin{array}{l}\operatorname{nin} 1 \\
0,32 x\end{array}$ & mori is & $\operatorname{limi}_{x \rightarrow \infty}$ & $\begin{array}{l}\text { ifos } \\
\text { N194. }\end{array}$ & $\operatorname{tin} 5$ & 611 & 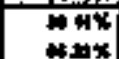 & 31001 \\
\hline 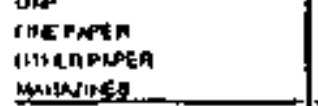 & $\begin{array}{l}110 \\
0 \times 4 \\
0 \times 4 \\
0.08\end{array}$ & 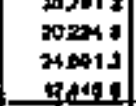 & 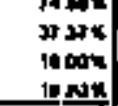 & 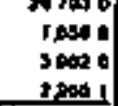 & 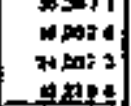 & 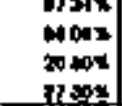 & 列, & 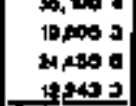 & 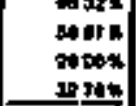 & 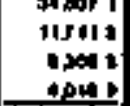 & 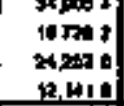 & 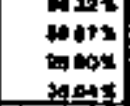 & 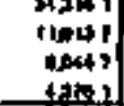 & 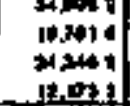 & 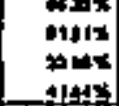 & 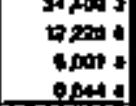 \\
\hline 要 & tos & Anest & 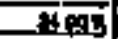 & $+1,09$ & 촐ㄹ & 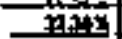 & INI4 & istes & $\overrightarrow{34}$ & 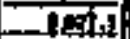 & 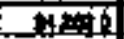 & 새마눈 & Itain & $A+1$ & rof & $21+1$ \\
\hline CUt & $35 \mathbf{x}$ & 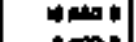 & $26 x$ & 10000 & w)ta 1 & Mrit & the of & torat & 0.0ㄴ? & onst. & thmi & ongst: & $\operatorname{Ton} 4$ & 10sid & $n+4 x$ & isend \\
\hline 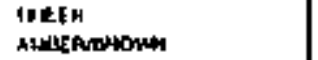 & 的的 & $\begin{array}{lll}-\infty & \infty \\
+\infty & 0\end{array}$ & 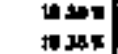 & 114 & 1900 & 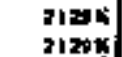 & $\lim _{40} 0$ & $\begin{array}{l}\cos 6 \\
\sin : 1\end{array}$ & 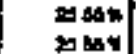 & 1,ion an & onas a & $\sin x$ & IpN & SATE O & 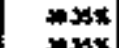 & 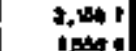 \\
\hline 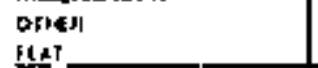 & $\begin{array}{ll}D \in x \\
\text { ose }\end{array}$ & (1) & 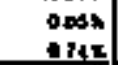 & $\begin{array}{r}\infty 2 \\
406\end{array}$ & $\begin{array}{l}\text { indit } \\
\text { ieniti }\end{array}$ & notis & and & apta: & $\begin{array}{l}10 x \\
100 x \\
1004\end{array}$ & not & 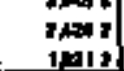 & 1aik & at & 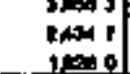 & 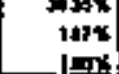 & 소 \\
\hline 슴 & $10 x$ & 98 & 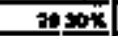 & 1 14as 1 & Anmen & Hats & tom & 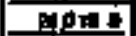 & Hets & t11004: & tist & sitex & s bat? & tint & 74 & 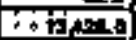 \\
\hline EAFOU CANTE & $30 \times$ & $0.25 \%$ & 5204 & $\operatorname{tin} 4$ & $\sin 6$ & $6+20$ & best & $\Leftrightarrow 4$ & 100 & 9277 & क्षापा & 19ets & 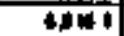 & $6+\infty 00$ & mosos & $7,0,4$ \\
\hline OHteAfrentoug & $43 x$ & 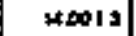 & $\omega m x$ & and ? & Agais al & nalu & $3 \rightarrow 02$ & n & $\infty \mathrm{H} *$ & | & 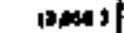 & ע & $A \Delta \Rightarrow 9$ & | & 54,4y & i 1 \\
\hline 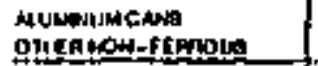 & $\begin{array}{l}0.8 \\
\text { Dax }\end{array}$ & $\begin{array}{l}1000 \text { ? } \\
+\operatorname{ses} 1 \\
\end{array}$ & $\begin{array}{l}n 00 x \\
170 x\end{array}$ & $\begin{array}{l}410 \\
0.0\end{array}$ & ins of & 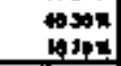 & 24 & ien of & 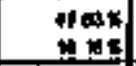 & wos & 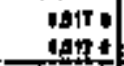 & 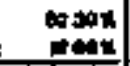 & 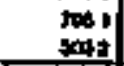 & Let 13 & 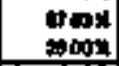 & A \\
\hline 을 & ton & 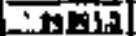 & $-11+5$ & 2101 & 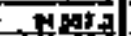 & tom & Bust & 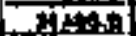 & stits & Aath & 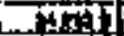 & $x^{2}+5$ & $\theta=1$ & Frida & 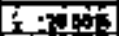 & 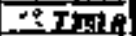 \\
\hline FXSTC COWTADEFA & 404 & $12, \pi 950$ & $\sin x$ & $2 \infty 11$ & Thtial & $\operatorname{tinx}$ & 3700 & thats & 2305 & 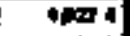 & is, wia & 345 & जiro & 17. & ins & $8 \operatorname{sen} 3$ \\
\hline ontenpula & $20 \times$ & $0.12 * 2$ & $2 \mathrm{mon}$ & 1ent? & $0 \times 006$ of & $420 \times$ & & 0.10 & $\cos 2$ & & | & 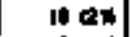 & 180 & $0,+\infty)$ & 45 & a] \\
\hline wan & 10: & 니닌소 & t $\cos$ & 은 & 30에 7 & $\tan$ & ind & $\mathrm{s} 000 \mathrm{t}$ & $\operatorname{son} 4$ & bus & 20 & then & 부료 & 30as] & IE & $\underline{z}$ \\
\hline 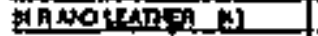 & 182 & tot? & 이난 & 5 & 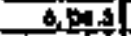 & $7 \sin$ & ats & ". & $\ln$ & stes: & risend & 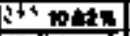 & ARpent? & 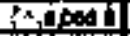 & Alysels & 부요 \\
\hline in & ton & tom & 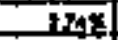 & 西1 & An & 49 & 望 & inges & Mis & atia & testint & T.4 & apt| & 3,0001 & 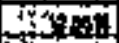 & ind \\
\hline 0 & 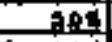 & $\therefore$ anda & $\therefore$ 1010 & 292 & tent & i.taly & nígle & $n$ & $\therefore$ anty & in stiti & ton & tints. & ent & AE영 & 4 & irit \\
\hline$t$ & ent & itsia & $\operatorname{lng}$ & indas & statal & ind & $\operatorname{lol} 1$ & 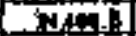 & in tines & thes & [ivand & Tatent & titan & INA & (n)tin: & Sidento \\
\hline 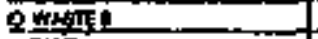 & 1004 & expint & 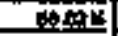 & कo, w1 & Apent & $\sin$ & $\Rightarrow 0$ & "sesta & Iftets & 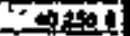 & 140 & 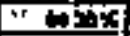 & istarlis & 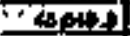 & TF" & $\therefore$-1101: \\
\hline TERE: & $\begin{array}{l}\text { DJu } \\
\text { 10x }\end{array}$ & $\begin{array}{ll}104000 \\
1600\end{array}$ & $\begin{array}{l}10015 \\
01005\end{array}$ & 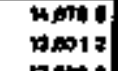 & 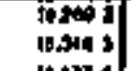 & $\begin{array}{l}\sin \theta \\
\sin x\end{array}$ & Tat? & $\begin{array}{ccc}0 & 0 \\
0 & 0\end{array}$ & $\begin{array}{l}0+\infty \\
40 \infty x\end{array}$ & 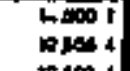 & 10, In sing & $\begin{array}{l}\sin 0 \\
\sin 1\end{array}$ & $\infty \%$ & क.m. & 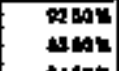 & 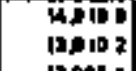 \\
\hline 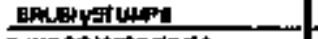 & 4 & 브믄 & 쑬 & 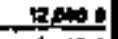 & 브란ㄷㄴ & $=$ & 里4 & 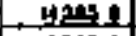 & - & & 4243 & ongex & & & lllaps & IPens \\
\hline 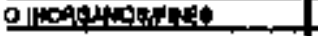 & 192 & t2id & intilt & $\div \sqrt{1.1}$ & 1all. & 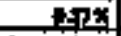 & -4 & -1204 & indetst & cis & Thats & of & & [a) & is 0 is & DEvint \\
\hline 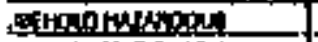 & 9.tㄴ. & tetenl & $\therefore$ I대느 & $2 \quad 21$ & $\therefore$ terit & $r$ otax & in & Stanin & $\because 3$ bat: & $\sin 112$ & 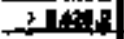 & S1 & a & Filax & 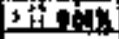 & mits \\
\hline 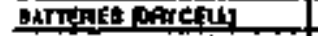 & $\cos x$ & Eact & 2서묘 & 31 & Fin & 푸료르. & \pm 1 & Prta & 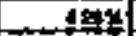 & 115 & _taj & is & & mat & $\tan x$ & \\
\hline coptris & I면? & 5.9114 & 문도 & int & 1 & Dis: & 14 & 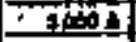 & id & $\sqrt{3}=\frac{164}{4}$ & is & 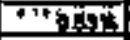 & 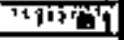 & 72 & DEtor & Biti2 \\
\hline 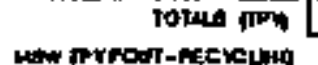 & $1000 \pi$ & | & a w w & 160704 & $\begin{array}{ll}2000000 \\
0\end{array}$ & $4 \mathbf{x}$ & Nipent & 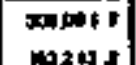 & $10 \mathrm{Nal}$ & tromol & $x+100$ & $\cos \pi$ & iondtet & | & mans & | \\
\hline \multirow{2}{*}{\multicolumn{2}{|c|}{ 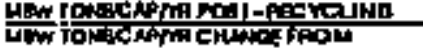 }} & एon & & & $\frac{14}{940}$ & & & $\frac{10}{01}$ & & & 19 & & & 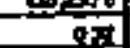 & & \\
\hline & \multicolumn{2}{|c|}{ 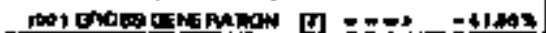 } & & & $\mathbf{n}$ & & & & & & & & & & & \\
\hline
\end{tabular}




\section{ATTACHMIENT 9.9}

Primary and Secondary Wasleshed Compostition Cafculated Irom 1997 Tonnages

\begin{tabular}{|c|c|c|c|c|}
\hline \multirow[b]{2}{*}{ 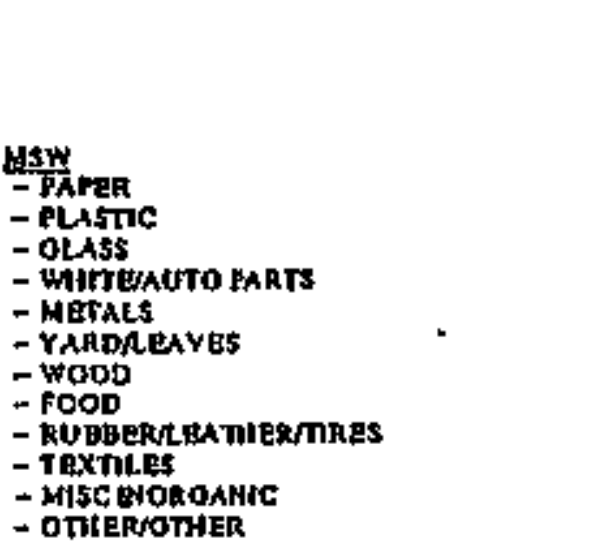 } & \multicolumn{2}{|c|}{ 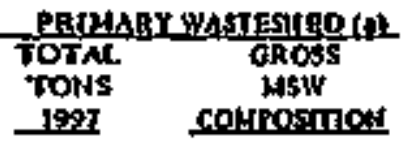 } & \multicolumn{2}{|c|}{ 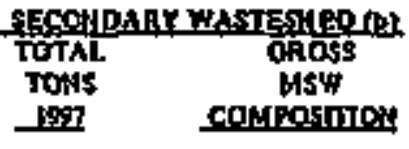 } \\
\hline & $\begin{array}{r}300,413 \\
55,179 \\
67,679 \\
4,112 \\
31,423 \\
95,311 \\
10,135 \\
62,796 \\
9,913 \\
19,441 \\
1,014 \\
24,145\end{array}$ & 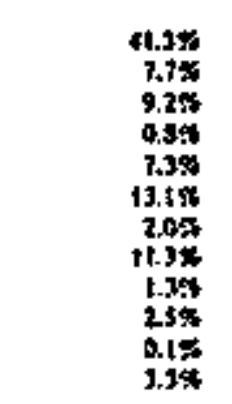 & $\begin{array}{r}212,969 \\
11,040 \\
11,437 \\
5,37 \\
31,796 \\
24,919 \\
17,595 \\
39,391 \\
6,197 \\
16,003 \\
9,170 \\
23,130\end{array}$ & 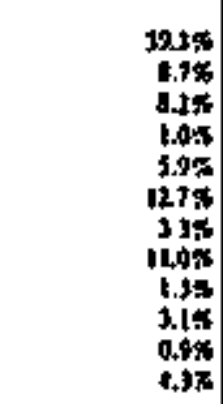 \\
\hline \multicolumn{5}{|c|}{ 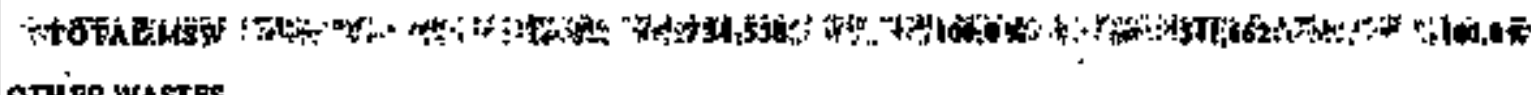 } \\
\hline 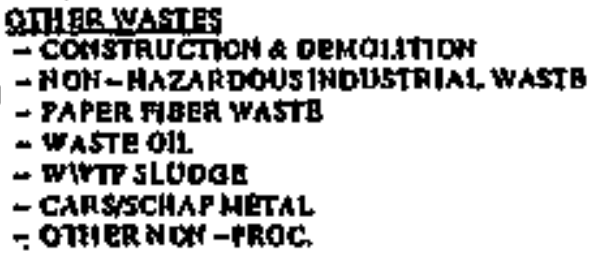 & $\begin{array}{r}135,519 \\
0 \\
127,414 \\
5,132 \\
04,193 \\
30,054 \\
40,431\end{array}$ & $\begin{array}{c}21.9 \% \\
0.05 \\
2.85 \\
124 \\
20.7 \% \\
2.05 \\
9.4 \%\end{array}$ & 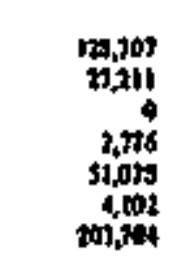 & $\begin{array}{r}30.48 \\
6.58 \\
0.05 \\
0.7 \% \\
1225 \\
1.048 \\
4.0 \%\end{array}$ \\
\hline \multicolumn{5}{|c|}{ 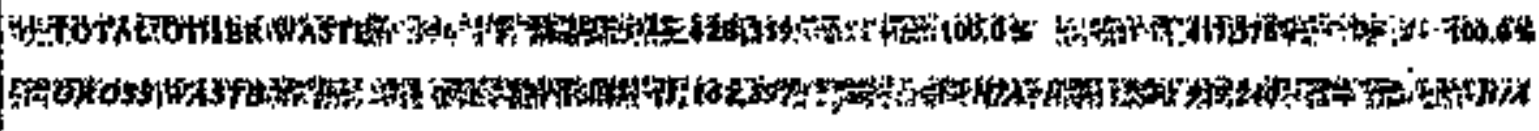 } \\
\hline \multicolumn{5}{|c|}{ 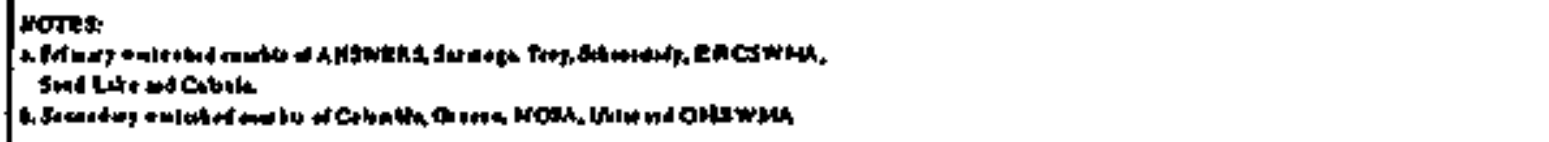 } \\
\hline
\end{tabular}




\section{ATTACHMENT 9-10}

PAPER GENERATION AND RECYCLING IN SCHENECTADY COUNTY

SCHENECTADY ESTIMATED GENERATION' 1993 ESTIMATED RECYCLING ${ }^{2}$

\section{COUNTY}

PAPER TOTAL.

\section{newspaper}

magazines

corrugaled

other paperboard

books

office paper

other paper
TPD TPY

$165 \quad 60,225$

$47 \quad 17,246$

$22 \quad 8,103$

$39 \quad 14,337$

$24 \quad 8,935$

$4 \quad 1,456$

$18 \quad 6,442$

$10 \quad 3,741$
TPY

8,369

5,260

161

1,305

1,643
19946 MONTH MRF SALES ${ }^{3}$

RATE

$13.90 \%$

$30.50 \%$

$1.99 \%$

$9.10 \%$

$0.00 \%$

$0.00 \%$

$25.50 \%$

$0.00 \%$
TPY

5.716

4,410

944

24

272

66
RATE

$9.49 \%$

$25.57 \%$

$0.00 \%$

$6.58 \%$

$0.00 \%$

$1.65 \%$

$4.22 \%$

$1.76 \%$

'Schenectady County Draft Integrated solid Waste Management Plan, Table 1.2.4-1, Willam F. Cosulich, June 1991. haulers.

¿Estimates reported by municipalities based on inforiation provided by private waste

'Bassd on receipts from sales of materlals. 
AECYCLING OF PAPER WASTES EY TYPE AND TOTAL In Lpsiata New York

\begin{tabular}{|c|c|c|c|c|c|c|c|c|c|c|}
\hline \multirow[b]{2}{*}{ MGEHGTKGTY } & \multirow[b]{2}{*}{ feEtion } & \multirow[b]{2}{*}{ Nans } & \multirow[b]{2}{*}{ Co:P } & \multicolumn{4}{|c|}{ WASTE INTONS PER YEAR } & \multirow[b]{2}{*}{ HKGRMDE } & \multirow[b]{2}{*}{ othtrin } & \multirow[b]{2}{*}{ TOTAL } \\
\hline & & & & MAs & $\begin{array}{l}\text { PHOAN } \\
\text { EOOQK }\end{array}$ & $\begin{array}{c}\text { MnKk } \\
\text { MadL }\end{array}$ & $\begin{array}{l}\text { PAPGA } \\
\text { BOARD }\end{array}$ & & & \\
\hline butchecto fin & 3 & \$500 & 5,457 & 0 & 0 & 0 & 0 & 2016 & $t, 64$ & 15,807 \\
\hline Drances. & 3 & 7,948 & ge & 0 & 13 & 0 & 0 & 0 & 3.6 & Q.400 \\
\hline Putien os & 3 & 2.7t1 & 329 & $2+B$ & 0 & 144 & 0 & $20 x$ & 0 & 3.500 \\
\hline Ponting Fo. & 3 & 9.9at2 & $5 \$ 1$ & $\mathbf{0}$ & 0 & 6 & 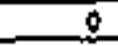 & 22 & 2018 & $134+3$ \\
\hline tosanca & 3 & 6016 & $e+\infty$ & 0 & 0 & 0 & 0 & 16 & 4 & 1,448 \\
\hline toptor Co. & 3 & $\$ 6,400$ & $3,0 \mathrm{st3}$ & 1,351 & 50 & 0 & 0 & 2.128 & $1 \% 1$ & 27,610 \\
\hline 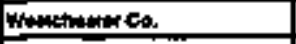 & 3 & 0 & 0 & 0 & 0 & 0 & 호 & 101 & 30,4 & $\leq .615$ \\
\hline 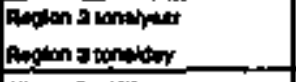 & & & & & & & & & & $\begin{array}{r}105,619 \\
209 \\
\end{array}$ \\
\hline y & 4 & 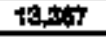 & 3.58 & 501 & 7 & 40 & $\overline{27}$ & 2,250 & E41 & 29,428 \\
\hline Cotribtates. & 4 & 1,129 & $3 x$ & a & $\mathbf{0}$ & 0 & 0 & 9 & 473 & 1.89 \\
\hline 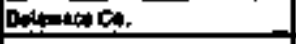 & 4 & BAd & 1,679 & 0 & $\mathbf{0}$ & 0 & $\underline{0}$ & 4,70 & 90, & 7,743 \\
\hline G:anco. & 4 & 906 & 48 & 0 & a & 0 & 0 & 32 & 0 & 1,308 \\
\hline 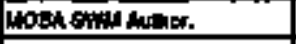 & 4 & 20.5 & 150 & $\mathbf{0}$ & 0 & 0 & $\underline{0}$ & D & 477 & $4,0.92$ \\
\hline Amminiter Oa & 4 & 1,316 & 413 & 3 & 0 & 0 & 0 & 8 & Gibs & 2340 \\
\hline fenmendifto & 4 & 5,734 & 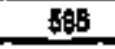 & 316 & 0 & 5tt. & 0 & 0 & 24 & 7,184 \\
\hline Ringlos a waster & & & & & & & & & & $\begin{array}{r}45,151 \\
124 \\
\end{array}$ \\
\hline coritomca & 5 & 41 & 1,024 & 84 & 0 & Q & 0 & 401 & 6 & 2,065 \\
\hline Con Co. & 5 & 153 & 354 & 24 & 0 & 0 & a & 15 & 0 & 545 \\
\hline Frandin Ginth & 6 & $\$ 6$ & $17 \%$ & 2⿺辶 & 0 & 0 & 0 & 30 & 34 & 65 \\
\hline Fulten fa & 6 & 670 & $28 \%$ & .0 & 6 & 0 & 0 & 0 & 22 & 1.464 \\
\hline 14:5140 & 5 & 20 & $\mathbf{8 0}$ & 0 & 0 & 0 & 0 & 9 & 0 & 464 \\
\hline OAl & 5 & 6.62 & 2504. & the.241 & 0 & a & 5,209 & 8 & 21 & 45,973 \\
\hline Whition & 5 & 1068 & 591 & 205 & 8 & 0 & 8 & 7 & b & 1.887 \\
\hline wulletefinga. & $\mathbf{5}$ & 564 & 89 & $\pi$ & 2 & 0 & 15 & 51 & 18 & 2562 \\
\hline 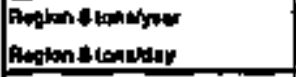 & & & & & & & & & & $\begin{array}{r}5544 \\
152 \\
\end{array}$ \\
\hline 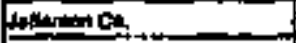 & 6 & 2558 & 1959 & 0 & 0 & 0 & 0 & D & t.384 & 191 \\
\hline 1. & E & 34 & 2065 & 0 & 0 & 0 & $\mathbf{0}$ & 29 & 9 & B83 \\
\hline 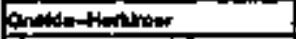 & 6 & 10,458 & $75 \times 9$ & $\operatorname{con}$ & 144 & $\theta$ & 365 & 448 & 313 & 19,925 \\
\hline 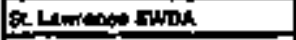 & 6 & $\boldsymbol{\sigma}$ & 9 & a & $\mathbf{0}$ & 0 & 0 & 0 & $9.7+8$ & 9,710 \\
\hline 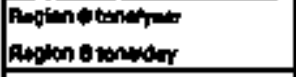 & & & & & & & & & & $\begin{array}{r}35,972 \\
93 \\
\end{array}$ \\
\hline Bromen Ca. & 7 & 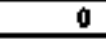 & 0 & o & 0 & 0 & 0 & 0 & 4 & 0 \\
\hline 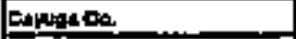 & 7 & 8 & 250 & 0 & $\mathbf{0}$ & 0 & 0 & $D$ & 0 & 3,557 \\
\hline cheningaten & 7 & esta & $1.10 t$ & 0 & 0 & 0 & 0 & 0 & 549 & 2,506 \\
\hline tordend Cor & 7 & 690 & 1200 & 0 & $\mathbf{0}$ & 0 & $\mathbf{0}$ & 0 & 196 & 2,236 \\
\hline 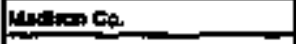 & 7 & 1,473 & 7351 & 0 & $\mathbf{0}$ & $\mathbf{D}$ & 0 & 0 & 1,065 & 4,649 \\
\hline 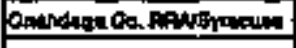 & 7 & 20,120 & 40,407 & 2,000 & 20 & sag & $\mathbf{0}$ & $20,0 \leqslant 5$ & int & g2,070 \\
\hline 型 & 7 & 3.54a & 2090 & $40 \mathrm{P}$ & 12 & 0 & 0 & 242 & 50 & 6342 \\
\hline Inocico. & 7 & 1,535 & 24 & 481 & $\mathbf{0}$ & 0 & 0 & $\mathbf{0}$ & 0 & 2000 \\
\hline fonglineos. & 7 & 1, & 1,550 & 0 & 0 & 0 & 0 & 124 & 1.867 & $5 \operatorname{ses}$ \\
\hline 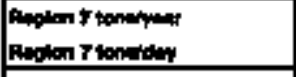 & & & & & & & & & & $\begin{array}{r}119,275 \\
307 \\
\end{array}$ \\
\hline Fingaca. & : & $3 \pm 27$ & 2,400 & 0 & 0 & $1,24 t$ & 60 & $\mathbf{3}$ & 0 & a,\$59 \\
\hline 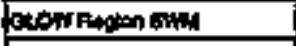 & 6 & 202 & 1,167 & $\mathbf{D}$ & $\mathbf{0}$ & 0 & 1 & 105 & 1 & $3 \leq 60$ \\
\hline 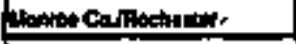 & $\mathbf{B}$ & 虾211 & 3,109 & 2,129 & 0 & $\mathbf{0}$ & 0 & 700 & $18 t 0$ & 425 \\
\hline Ont:loca. & 8 & 255 & 1389 & 0. & 9 & a & 0 & 0 & 304 & 4.35 \\
\hline 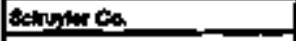 & $\theta$ & 72 & 129 & 0 & 0 & 0 & 0 & 4 & 5 & 205 \\
\hline 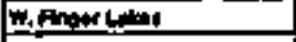 & $\theta$ & 3.675 & 1 료 & $\mathbf{0}$ & 0 & 260 & 0 & 40 & 5 & 5,247 \\
\hline Cantwin 4a. & E & $1+50$ & 1,572 & 0 & g & 0 & 0 & 15 & 4 & 2918 \\
\hline 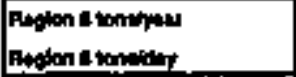 & & & & & & & & & & $\begin{array}{r}65.478 \\
182\end{array}$ \\
\hline 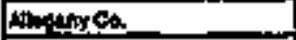 & $\mathbf{B}$ & 604 & 600 & 0 & $\theta$ & $\mathbf{0}$ & 0 & $\theta$ & 0 & 1,27 \\
\hline 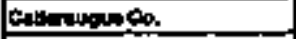 & $\underline{1}$ & 2.040 & 1.571 & 0 & 0 & 0 & 0 & 6 & 畕1 & 4,045 \\
\hline 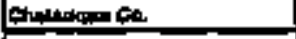 & 9 & 3,094 & $4,7 \%$ & $\mathbf{0}$ & 0 & 112 & 0 & 11 & 298 & 8.313 \\
\hline 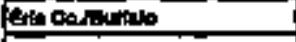 & 9 & $9 / 4 \pi$ & 1,765 & 30 & 0 & 0 & 2 & 153 & $195 \%$ & 31,00 \\
\hline Hances. & 9 & 4A00 & 7,245 & 0 & 0 & 0 & 0 & 0 & 0 & 1t.Eم \\
\hline 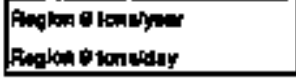 & & & & & & & & & & $\begin{array}{r}65,468 \\
155 \\
\end{array}$ \\
\hline
\end{tabular}

